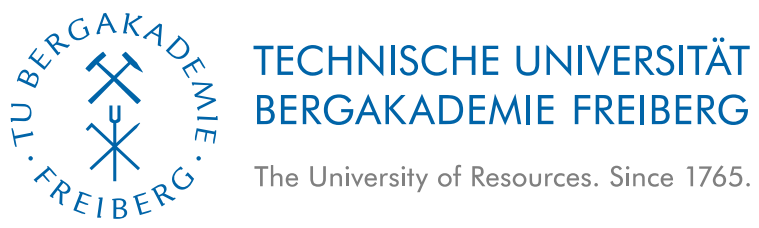

\title{
The influence of strong time-dependent oscillations on semilinear damped wave models
}

\author{
To the Faculty of Mathematics and Computer Science \\ of the Technische Universität Bergakademie Freiberg \\ is submitted this
}

\section{Thesis}

to attain the academic degree of

Doctor rerum naturalium

(Dr. rer. nat.)

submitted by M. Sc. Aslan, Halit Sevki

born on the December 1st, 1988 in Sanliurfa (Turkey)

Freiberg, June 15, 2020 



\section{Versicherung}

Hiermit versichere ich, dass ich die vorliegende Arbeit ohne unzulässige Hilfe Dritter und ohne Benutzung anderer als der angegebenen Hilfsmittel angefertigt habe; die aus fremden Quellen direkt oder indirekt übernommenen Gedanken sind als solche kenntlich gemacht.

Bei der Auswahl und Auswertung des Materials sowie bei der Herstellung des Manuskripts habe ich Unterstützungsleistungen von folgenden Personen erhalten:

$\diamond$ Prof. Dr. Michael Reissig

$\diamond$ Prof. Dr. Fumihiko Hirosawa

Weitere Personen waren an der Abfassung der vorliegenden Arbeit nicht beteiligt.

Die Hilfe eines Promotionsberaters habe ich nicht in Anspruch genommen. Weitere Personen haben von mir keine geldwerten Leistungen für Arbeiten erhalten, die nicht als solche kenntlich gemacht worden sind. Die Arbeit wurde bisher weder im Inland noch im Ausland in gleicher oder ähnlicher Form einer anderen Prüfungsbehörde vorgelegt.

15. Juni 2020

M. Sc. Halit Sevki Aslan 


\section{Declaration}

I hereby declare that I completed this work without any improper help from a third party and without using any aids other than those cited. All ideas derived directly or indirectly from other sources are identified as such.

In the selection and use of materials and in the writing of the manuscript I received support from the following persons:

$\diamond$ Prof. Dr. Michael Reissig

$\diamond$ Prof. Dr. Fumihiko Hirosawa

Persons other than those above did not contribute to the writing of this thesis.

I did not seek the help of a professional doctorate-consultant. Only those persons identified as having done so received any financial payment from me for any work done for me. This thesis has not previously been published in the same or a similar form in Germany or abroad. 


\section{Contents}

$\begin{array}{ll}\text { 1. Introduction } & 7\end{array}$

1.1. Motivation ........................... 7

1.1.1. Cauchy problems for wave models with constant coefficients . . . . . 8

1.1.2. Cauchy problems for wave models with time-dependent coefficients . . 9

1.2. Main goals, some results and plan of the thesis . . . . . . . . . . . . . 16

2. The influence of oscillations on linear damped wave equation with time-dependent $\begin{array}{ll}\text { coefficients } & 21\end{array}$

2.1. Introduction . . . . . . . . . . . . . . . . . . . . . 21

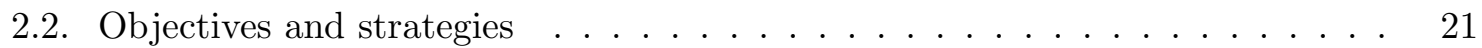

2.3. Assumptions . . . . . . . . . . . . . . . . . . . . . 22

2.3.1. Some examples . . . . . . . . . . . . . . . . 23

2.4. Representation of solutions . . . . . . . . . . . . . . 25

2.4.1. Considerations in the hyperbolic zone . . . . . . . . . . . 28

2.4.2. Considerations in the oscillation subzone . . . . . . . . . . . . 37

2.4.3. Considerations in the elliptic zone . . . . . . . . . . . . . 38

2.4.4. Considerations in the dissipative zone . . . . . . . . . . . . . 45

2.4.5. Considerations in the reduced zone . . . . . . . . . . . . . 50

2.5. Energy estimates of higher order . . . . . . . . . . . . . . . 51

2.5.1. A family of parameter-dependent linear Cauchy problems . . . . . . 51

2.5.2. Estimates for the multiplier $\hat{K}_{1} \ldots \ldots \ldots \ldots$. . . . . . . 59

2.5.3. Estimates for the multiplier $\hat{K}_{0} \ldots \ldots \ldots \ldots$. . . . . . . 64

2.5.4. Final estimates . . . . . . . . . . . . . . . . . 67

2.5.5. Matsumura-type estimates with additional regularity of the data . . . 68

2.5.6. Some examples . . . . . . . . . . . . . . . . 75

2.6. Construction of admissible oscillating functions . . . . . . . . . . . . 77

3. Global in time existence results for damped wave models with power nonlinearity $\mathbf{8 1}$

3.1. Introduction . . . . . . . . . . . . . . . . . 81

3.1.1. Some assumptions and tools . . . . . . . . . . . . . 81

3.2. Low regular data . . . . . . . . . . . . . . . . . . . . 87

3.2.1. Case of sub-exponential propagation speed . . . . . . . . . 87

3.2.2. Case of super-exponential propagation speed . . . . . . . . . . . 97

3.3. Data in energy space . . . . . . . . . . . . . . . . . . . 103

3.3.1. Case of sub-exponential propagation speed . . . . . . . . . . . 103

3.3.2. Case of super-exponential propagation speed . . . . . . . . . . . 120

3.4. High regular data . . . . . . . . . . . . . . . . . . . . . . . 131

3.4.1. Case of sub-exponential propagation speed . . . . . . . . . . . . 132

3.4.2. Case of super-exponential propagation speed . . . . . . . . . . . . 145

3.5. Large regular data . . . . . . . . . . . . . . . . . . . . . 153

3.5.1. Case of sub-exponential propagation speed . . . . . . . . . . . 153

3.5.2. Case of super-exponential propagation speed . . . . . . . . . . . 159

4. Global in time existence results for damped wave models with different power nonlinearities

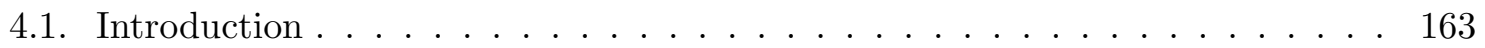

4.1.1. Philosophy of our approach . . . . . . . . . . . . 163 
4.2. Semilinear damped wave models of the derivative type power nonlinearity $\left|u_{t}\right|^{p} 164$

4.2.1. Case of sub-exponential propagation speed . . . . . . . . . . . 167

4.2.2. Case of super-exponential propagation speed . . . . . . . . . . . 180

4.3. Semilinear damped wave models of the generalized type power nonlinearity $\left.\left.|| D\right|^{a} u\right|^{p}$ and $a \in(0,1] \ldots \ldots \ldots \ldots \ldots \ldots \ldots$

4.3.1. Case of sub-exponential propagation speed in energy space . . . . . 187

4.3.2. Case of super-exponential propagation speed in energy space . . . . . 198

5. $L^{p}-L^{q}$ estimates for wave equations with strong time-dependent oscillations 207

5.1. Introduction . . . . . . . . . . . . . . . . . . . . . . . . 207

5.2. Representation of solutions . . . . . . . . . . . . . . . 208

5.2.1. Assumptions . . . . . . . . . . . . . . . . . . . . . 209

5.2.2. Considerations in the pseudo-differential zone . . . . . . . . . . . 210

5.2.3. Considerations in the oscillation subzone . . . . . . . . . . . . 211

5.2.4. Considerations in the hyperbolic zone . . . . . . . . . . . . 215

5.2.5. Some auxiliary estimates . . . . . . . . . . . . . . 217

5.3. $L^{p}-L^{q}$ estimates on the conjugate line . . . . . . . . . . . . 226

5.3.1. $L^{p}-L^{q}$ estimates for Fourier multipliers with amplitudes localized in

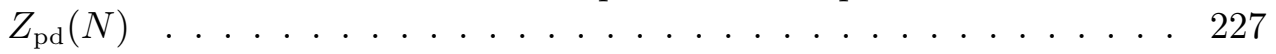

5.3.2. $L^{p}-L^{q}$ estimates for Fourier multipliers with amplitudes localized in

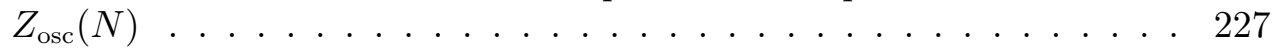

5.3.3. $L^{p}-L^{q}$ estimates for Fourier multipliers with amplitudes localized in

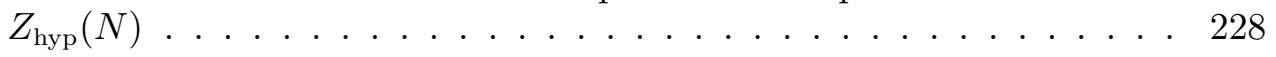

6. Further research topics 233

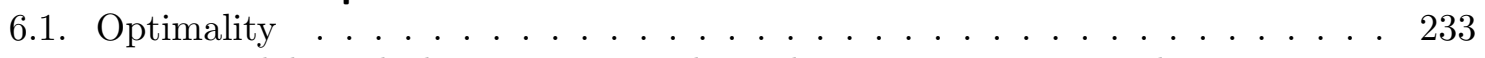

6.2. Wave models with decreasing time dependent propagation speed . . . . . . 233

6.3. Damped wave models with different oscillation functions . . . . . . . . . . . . 234

6.4. Weakly coupled systems of semilinear damped wave models . . . . . . . . . 234

A. Basic tools $\quad 235$

A.1. Basic inequalities . . . . . . . . . . . . . . . . . . . 235

A.2. The Peano-Baker formula . . . . . . . . . . . . . . 235

A.3. Faà di Bruno's formula . . . . . . . . . . . . . . . . . . . . . . . . . . 236

A.4. Some results from Interpolation Theory . . . . . . . . . . . . . . 237

A.5. Littman type lemma . . . . . . . . . . . . . . . . . . . . . 238

A.6. Hardy-Littlewood inequality . . . . . . . . . . . . . . . . . 238

A.7. New tools from Harmonic Analysis . . . . . . . . . . . . . . . . . 238

A.7.1. Bessel and Riesz potential spaces . . . . . . . . . . . . . . . . 239

A.7.2. Fractional Gagliardo-Nirenberg inequality . . . . . . . . . . . . . 239

A.7.3. Fractional Leibniz rule . . . . . . . . . . . . . . . . . . . 239

A.7.4. Fractional chain rule . . . . . . . . . . . . . . . . . . . 240

A.7.5. Fractional homogeneous Sobolev embeddings . . . . . . . . . . . . 240

A.7.6. Fractional powers . . . . . . . . . . . . . . . . . . . . 241

B. List of symbols and abbreviations 243

$\begin{array}{ll}\text { Bibliography } & 247\end{array}$ 


\section{Introduction}

\subsection{Motivation}

Nowadays, it has been widely investigated by many mathematicians the following Cauchy problem for semilinear wave models with time-dependent propagation speed and time-dependent damping term:

$$
\begin{cases}u_{t t}-a^{2}(t) \Delta u+b(t) u_{t}=f\left(u, u_{t}, \nabla_{x} u\right), & (t, x) \in[0, \infty) \times \mathbb{R}^{n} \\ u(0, x)=u_{0}(x), \quad u_{t}(0, x)=u_{1}(x), & x \in \mathbb{R}^{n}\end{cases}
$$

Many questions arise for qualitative properties of solutions to (1.1.1). Some of these questions we itemize below.

- Asymptotic behavior of the solutions.

We are interested in the long-time behavior of weak solutions to the Cauchy problem (1.1.1). In particular, under different assumptions the influence of the time-depending coefficients $a=a(t)$ and $b=b(t)$ in the speed of propagation and damping term, respectively, under reasonable regularity assumptions on the Cauchy data $\left(u_{0}, u_{1}\right)$ and the nonlinearity $f\left(u, u_{t}, \nabla_{x} u\right)$ we want to understand the long-time behavior (measured in terms of energies or certain norms) of solutions to (1.1.1).

- Decay properties.

The significant progress in the study of nonlinear hyperbolic differential equations (with or without damping term) is due to basic results on the decay in time of the solutions to the corresponding linear wave equations.

- Local (in time) or global (in time) well-posedness of the associated Cauchy problems. It is of interest to find suitable spaces for initial data to have local (in time) or even global (in time) existence, uniqueness and continuous dependence on initial data. The main arguments in the treatment are the contraction argument, together with some energy estimates for families of linear parameter-dependent Cauchy problems.

- Associated nonlinear Cauchy problems.

Here we are interested in models with nonlinear source term $f\left(u, u_{t}, \nabla_{x} u\right.$ ) (for instance the power source nonlinear term $f(u)=|u|^{p}$ with $p>1$ ). All the questions raised in the linear case may be extended to the nonlinear one. The main argument here is again the use of contraction principle.

- Global (in time) existence.

The nonlinear source term $f\left(u, u_{t}, \nabla_{x} u\right)$ may cause a blow-up behavior of suitable solutions. Thus, it seems to be reasonable to ask if under some assumptions the zero solution is a steady state solution. Then, there exist global (in time) small data Sobolev solutions.

- Blow-up and the control of life-span.

In the case of local (in time) existence results we may investigate whether the solution may blow-up in some finite time, as well as study the behavior of such life-span with respect to the parameters involved (for example, smallness of the initial data, regularity of the initial data). 
The items just listed show the essential importance of the estimates for the solutions to the Cauchy problem (1.1.1). There is an extensive literature about such results. We will sketch some of them as it is presented in [11] and state the progress that has taken place up to the present.

\subsubsection{Cauchy problems for wave models with constant coefficients}

Let us briefly recall some results about linear and semilinear Cauchy problems to wave models with and without classical damping term. In particular, we present some known results on energy estimates, $L^{p}-L^{q}$ decay estimates, global (in time) existence and blow-up results.

In order to study global (in time) existence of small data Sobolev solutions for semilinear equations, the key tool is estimates for solutions to the corresponding linear Cauchy problems. A systematic overview on global existence and uniqueness results can be found in books [11] and [31].

\section{Linear Cauchy problems for free wave equation}

We consider the following Cauchy problem for the homogeneous linear free wave equation:

$$
\begin{cases}u_{t t}-\Delta u=0, & (t, x) \in[0, \infty) \times \mathbb{R}^{n}, \\ u(0, x)=u_{0}(x), \quad u_{t}(0, x)=u_{1}(x), & x \in \mathbb{R}^{n} .\end{cases}
$$

The Cauchy problem (1.1.2) is $H^{s}$ well-posed, $s \in \mathbb{R}$, that is, for any $\left(u_{0}, u_{1}\right) \in H^{s} \times H^{s-1}$ there exists for all positive $T$ a uniquely determined energy solution $u \in \mathcal{C}\left([0, T], H^{s}\right) \cap$ $\mathcal{C}^{1}\left([0, T], H^{s-1}\right)$ which depends continuously on Cauchy data. by

If $u \in \mathcal{C}\left([0, T], H^{1}\right) \cap \mathcal{C}^{1}\left([0, T], L^{2}\right)$, then the total energy of the solution to (1.1.2) is defined

$$
\mathbb{E}_{W}(u)(t):=\frac{1}{2}\left(\left\|\nabla_{x} u(t, \cdot)\right\|_{L^{2}}^{2}+\left\|u_{t}(t, \cdot)\right\|_{L^{2}}^{2}\right)
$$

and it holds the conservation of the energy, that is, $\mathbb{E}_{W}(u)(t)=\mathbb{E}_{W}(u)(0)$ for all $t \geq 0$.

The study of $L^{p}-L^{q}$ decay estimates for solutions to (1.1.2) bases on classical papers $[29,41,42,45]$. Such decay estimates are called Strichartz type decay estimates for the energy $\mathbb{E}_{W}(u)(t)$ basing on the $L^{q}$ norm. In a precise formulation the estimate reads as follows:

$$
\left\|u_{t}(t, \cdot), \nabla_{x} u(t, \cdot)\right\|_{L^{q}} \leq C(1+t)^{-\frac{n-1}{2}\left(\frac{1}{p}-\frac{1}{q}\right)}\left(\left\|u_{0}\right\|_{W_{p}^{N_{p}+1}}+\left\|u_{1}\right\|_{W_{p}^{N_{p}}}\right)
$$

for $n \geq 2, p \in[1,2), \frac{1}{p}+\frac{1}{q}=1$ and $N_{p} \geq n\left(\frac{1}{p}-\frac{1}{q}\right)$. Here $W_{p}^{N_{p}}$ denotes the standard Sobolev space over $L^{p}$ with regularity $N_{p} \in \mathbb{N}$.

\section{Linear Cauchy problems for damped wave equation with constant coefficients}

Let us consider the following linear Cauchy problem for the classical damped wave equation:

$$
\begin{cases}u_{t t}-\Delta u+u_{t}=0, & (t, x) \in[0, \infty) \times \mathbb{R}^{n}, \\ u(0, x)=u_{0}(x), \quad u_{t}(0, x)=u_{1}(x), & x \in \mathbb{R}^{n} .\end{cases}
$$

In this case the solutions to (1.1.4) describe the wave propagation with friction, such as the telegraph equation and the heat conduction with finite propagation speed of perturbations.

The wave energy of Sobolev solutions to the Cauchy problem (1.1.4) is defined by

$$
\mathbb{E}_{W}(u)(t):=\frac{1}{2}\left(\left\|\nabla_{x} u(t, \cdot)\right\|_{L^{2}}^{2}+\left\|u_{t}(t, \cdot)\right\|_{L^{2}}^{2}\right) .
$$

Here $\mathbb{E}_{W}(u)(t)$ is monotonically decreasing. This fact follows from differentiation of the energy $\mathbb{E}_{W}(u)(t)$ with respect to $t$ and integration by parts. That is, it holds

$$
\frac{d}{d t} \mathbb{E}_{W}(u)(t)=\int_{\mathbb{R}^{n}}\left(u_{t} u_{t t}+\nabla u \cdot \nabla u_{t}\right) d x=\int_{\mathbb{R}^{n}}-u_{t}(t, x)^{2} d x \leq 0 .
$$


Thus, we can not expect energy conservation because of the damping term. This gives no information whether it tends to zero or remains positive for all times. However, it arises the question for the behavior of the energy for $t \rightarrow \infty$. Of special interest is the question whether the energy $\mathbb{E}_{W}(u)(t)$ tends to 0 for $t \rightarrow \infty$. Such a behavior is called decay.

Sharp decay estimates for solutions to the Cauchy problem (1.1.4) are proved in [23]. The author proved, that the solutions to the damped wave equation (1.1.4) satisfy the estimates

$$
\left\|D_{t}^{k} D_{x}^{\alpha} u(t, x)\right\|_{L^{2}} \leq C(1+t)^{-\frac{n}{2}\left(\frac{1}{m}-\frac{1}{2}\right)-\frac{|\alpha|}{2}-k}\left(\left\|u_{0}\right\|_{H^{k+|\alpha|}}+\left\|u_{1}\right\|_{H^{k+|\alpha|-1}}+\left\|\left(u_{0}, u_{1}\right)\right\|_{L^{m}}\right)
$$

for $t \geq 0$ and $m \in[1,2]$. Here the estimates are improved by assuming an additional $L^{m}$ regularity with $m \in[1,2]$.

There is a difference in the influence on the decay order between spatial and time derivatives like for estimates of solutions to the corresponding heat equation. We remark that the previous estimates coincide in the decay order with the corresponding estimates for the heat equation. The corresponding $L^{p}-L^{q}$ decay estimates to the Cauchy problem (1.1.4) are given as follows:

$$
\left\|u_{t}(t, \cdot), \nabla_{x} u(t, \cdot)\right\|_{L^{q}} \leq C(1+t)^{-\frac{n}{2}\left(\frac{1}{p}-\frac{1}{q}\right)-\frac{1}{2}}\left(\left\|u_{0}\right\|_{W_{p}^{N_{p}+1}}+\left\|u_{1}\right\|_{W_{p}^{N_{p}}}\right)
$$

for $n \geq 2, p \in[1,2), \frac{1}{p}+\frac{1}{q}=1$ and $N_{p} \geq n\left(\frac{1}{p}-\frac{1}{q}\right)$. That is, these decay rates correspond to estimates for the heat equation (see [30]). Thus, this parabolic structure can be expressed in terms of the so-called diffusion phenomenon and the asymptotic behavior of the solutions to the damped wave equation are related to corresponding solutions of the heat equation.

\section{Semilinear Cauchy problems for damped wave equation with constant coefficients}

Now we consider the following semilinear Cauchy problem for the classical damped wave equation:

$$
\begin{cases}u_{t t}-\Delta u+u_{t}=|u|^{p}, & (t, x) \in[0, \infty) \times \mathbb{R}^{n}, \\ u(0, x)=u_{0}(x), \quad u_{t}(0, x)=u_{1}(x), & x \in \mathbb{R}^{n} .\end{cases}
$$

There is a lot of effort from many authors devoted to the question of the global (in time) existence or the blow-up in a finite time of Sobolev solutions to the Cauchy problem (1.1.5). Small perturbations of the data in suitable Banach spaces preserve the property of the Cauchy problem to have globally (in time) Sobolev solutions. It turns out that there exists a critical exponent $p_{\text {crit }}$, a threshold between global and non-global (in time) existence of small data Sobolev solutions.

In the paper [44], by using the Matsumura estimates for solutions to the linear Cauchy problem (1.1.4), it is proved a global (in time) existence result for energy solutions to (1.1.5) assuming compactly supported data $\left(u_{0}, u_{1}\right) \in H^{1} \times L^{2}$ to be sufficiently small, $p>p_{\text {Fuj }}(n)=$ $1+\frac{2}{n}$ and $p \leq p_{\mathrm{GN}}(n)=\frac{n}{n-2}$ if $n \geq 3$. Moreover, the authors also proved a blow-up result for $1<p<p_{\text {Fuj }}(n)$, assuming the data satisfy some integral sign conditions.

Here $p_{\text {Fuj }}(n)$ denotes the so-called Fujita exponent, which is the the critical exponent of the semilinear heat equations. This indicates that the damping term drastically changes the asymptotic behavior of the solution to the wave equation. In other words, the solutions to (1.1.5) seem to behave more like solutions of the heat equation at large times.

Later on in [50], the author showed that the critical case $p=p_{\text {Fuj }}(n)$ belongs to the blow-up region applying the so-called test function method. This method bases on a contradiction argument and yields sharp results for models with a parabolic like decay for solutions. Moreover, in [21] the authors extended the global (in time) existence result to certain non-compactly supported initial data.

\subsubsection{Cauchy problems for wave models with time-dependent coefficients}

We consider now wave models with time-dependent coefficients. Results for such models may help getting a better understanding of classical results for constant coefficients models, as well, as giving hints to possible generalizations. 


\section{Linear Cauchy problems for wave equation with time-dependent speed of propagation}

Let us consider the following Cauchy problem for a wave equation with time-dependent propagation speed $a=a(t)$ :

$$
\begin{cases}u_{t t}-a^{2}(t) \Delta u=0, & (t, x) \in[0, \infty) \times \mathbb{R}^{n}, \\ u(0, x)=u_{0}(x), \quad u_{t}(0, x)=u_{1}(x), & x \in \mathbb{R}^{n} .\end{cases}
$$

Then, the total energy of the solution to (1.1.6) is defined by

$$
\mathbb{E}_{W, a}(u)(t):=\frac{1}{2}\left(a^{2}(t)\left\|\nabla_{x} u(t, \cdot)\right\|_{L^{2}}^{2}+\left\|u_{t}(t, \cdot)\right\|_{L^{2}}^{2}\right) .
$$

One can observe many different effects for the behavior of $\mathbb{E}_{W, a}(u)(t)$ as $t \rightarrow \infty$ according to properties of the speed of propagation $a=a(t)$.

If $0<a_{0} \leq a(t) \leq a_{1}<\infty$ for all $t \geq 0$, then the energy $\mathbb{E}_{W, a}(u)(t)$ is equivalent to

$$
\mathbb{E}_{W, 1}(u)(t)=\mathbb{E}_{W}(u)(t):=\frac{1}{2}\left(\left\|\nabla_{x} u(t, \cdot)\right\|_{L^{2}}^{2}+\left\|u_{t}(t, \cdot)\right\|_{L^{2}}^{2}\right) .
$$

Although $\mathbb{E}_{W}(u)(t)$ is a conserved quantity for the classical wave equation, oscillations of the time-dependent coefficient $a=a(t)$ may have a deteriorating influence on the energy behavior of solutions (see [7] and [35]). Namely, a time-dependent propagation speed can cause many difficulties. This is shown in [35] by means of the Cauchy problem

$$
\begin{cases}u_{t t}-(2+\sin t)^{2} \Delta u=0, & (t, x) \in[0, \infty) \times \mathbb{R}^{n}, \\ u(0, x)=u_{0}(x), \quad u_{t}(0, x)=u_{1}(x), & x \in \mathbb{R}^{n} .\end{cases}
$$

For an admissible solution $u$ of this Cauchy problem it follows immediately that

$$
\mathbb{E}_{W}(u)(t) \leq C \exp (c t) \mathbb{E}_{W}(u)(0)
$$

with constants $c, C>0$. The authors showed that this energy estimate cannot be substantially improved, even if $L^{p}-L^{q}$ estimates are considered. This means, that the oscillating coefficients have a deteriorating effect on energy estimates.

In [33], the authors examined this effect more closely for propagation speeds of the form

$$
a^{2}(t)=2+\sin \left((\log (t+30))^{\gamma}\right) .
$$

Then, for the given function $a=a(t)$ does not damage the energy estimate for a suitable $\gamma>0$. For this reason, it is necessary to control the oscillating behavior of the coefficients. Such a classification was proposed in [35] and can be used to control suitable energies. That is, if

$$
\left|a^{(k)}(t)\right| \leq C_{k}(1+t)^{-k}, \quad k=1,2,
$$

holds (due to the proposed classification, here only very slow oscillations are allowed), then the so-called generalized energy conservation law (GECL) holds (see [33]). This means, that there exist positive constants $C_{0}$ and $C_{1}$ such that the inequalities

$$
C_{1} \mathbb{E}_{W, a}(u)(0) \leq \mathbb{E}_{W, a}(u)(t) \leq C_{2} \mathbb{E}_{W, a}(u)(0)
$$

are valid for all $t \in(0, \infty)$, where the positive constants $C_{0}$ and $C_{1}$ are independent on the data. This (GECL) excludes decay and blow up behavior of $\mathbb{E}_{W, a}(u)(t)$ for $t \rightarrow \infty$.

One can allow faster oscillations of the coefficients if further structural properties of the coefficients are supposed. In the paper [16], even though oscillations are very fast, the author proved (GECL) to (1.1.6) provided that the following conditions to the coefficient $a=a(t)$ are satisfied:

$$
0<a_{1} \leq a(t) \leq a_{2},
$$




$$
\begin{gathered}
\left|a^{(k)}(t)\right| \leq C_{k}(1+t)^{-k \alpha}, \quad k=1,2, \cdots, M, \\
\int_{0}^{t}\left|a(\tau)-a_{\infty}\right| d \tau \leq C(1+t)^{\beta},
\end{gathered}
$$

for some real $a_{\infty}$ and $\beta \in(0,1)$ with $\alpha \geq \beta+\frac{1-\beta}{M}$. Here (1.1.9) is a so-called stabilization condition and by this condition one can get some benefit of higher order regularity of $a=a(t)$.

The situation becomes more complicated if one considers an unbounded propagation speed $a=a(t)$. If $a(t)>a_{0}>0$ is an increasing function satisfying a suitable control on the oscillations, the author proved in [1] the estimate

$$
\mathbb{E}_{W, a}(u)(t) \leq C_{1} a(t)\left(\mathbb{E}_{W, a}(u)(0)+\left\|u_{0}\right\|_{L^{2}}^{2}\right) .
$$

In the case of an increasing $a=a(t)$ in the derived energy estimate for $\mathbb{E}_{W, a}(u)(t)$ the $H^{1}$ norm of $u_{0}$ appears, not only the $L^{2}$ norm of its gradient as in the case of bounded $a=a(t)$.

It turns out that it is useful to write

$$
a(t)=\lambda(t) \omega(t)
$$

where

- $\lambda=\lambda(t)$ describes the increasing behavior (improving influence on estimates),

- $\omega=\omega(t)$ describes the oscillating behavior (deteriorating influence on estimates).

In series of papers $[34,35,37]$, the authors generalized the question for $L^{p}-L^{q}$ decay estimates to wave equations with $a(t)=\lambda(t) \omega(t)$. For a good survey see [32]. The authors proposed a classification based on the interplay between $\lambda(t)$ and $\omega(t)$ as follows.

Definition 1.1.1 (Speed of oscillations). We assume that there exists a real $\gamma \in[0,1]$ such that the following condition is satisfied:

$$
\left|\omega^{(k)}(t)\right| \leq C_{k}\left(\frac{\lambda(t)}{\Lambda(t)}(\log \Lambda(t))^{\gamma}\right)^{k}, \quad \text { for large } t, k \in \mathbb{N},
$$

where $\Lambda=\Lambda(t)$ is defined by $\Lambda(t):=\int_{0}^{t} \lambda(\tau) d \tau$. The parameter $\gamma$ controls the oscillations of $\omega$ as follows:

- if $\gamma=0$, then $\omega$ has very slow oscillations,

- if $0<\gamma<1$, then $\omega$ has slow oscillations,

- if $\gamma=1$, then $\omega$ has fast oscillations,

- and if the condition (1.1.10) is not satisfied for $\gamma=1$, then $\omega$ has very fast oscillations.

If the oscillations of $\omega$ are very slow, slow or fast by assuming some suitable assumptions on the smooth coefficient $\lambda=\lambda(t)$ one can expect $L^{p}-L^{q}$ decay estimates. If the oscillations are very fast, then one can not expect such decay estimates without assuming a suitable stabilization condition.

If (1.1.10) holds for $\gamma \in[0,1]$, then the $L^{p}-L^{q}$ decay estimate

$$
\left\|\left(u_{t}, \lambda(t) \nabla_{x} u\right)(t, \cdot)\right\|_{L^{q}} \leq C \sqrt{\lambda(t)}(\Lambda(t))^{\gamma_{0}-\frac{n-1}{2}\left(\frac{1}{p}-\frac{1}{q}\right)}\left(\left\|u_{0}\right\|_{W_{p}^{N_{p}+1}}+\left\|u_{1}\right\|_{W_{p}^{N_{p}}}\right)
$$

holds for the solution $u=u(t, x)$ to (1.1.6) with real value $\gamma_{0} \geq 0$, which depends on $\gamma$. Here $n \geq 2, p \in(1,2], \frac{1}{p}+\frac{1}{q}=1$ and $N_{p} \geq n\left(\frac{1}{p}-\frac{1}{q}\right)$. There is a connection between the oscillating behavior of $\omega$ and the loss of decay, that is, the size of $\gamma_{0}$ describes how the decay rate differs from the classical one stated in (1.1.2). 
In the paper [19], the authors studied the Cauchy problem (1.1.6) after introducing $a(t)=$ $\lambda(t) \omega(t)$ with a monotonously increasing shape function $\lambda=\lambda(t)$ and a (bounded) oscillating function $\omega=\omega(t)$. By using the $\mathcal{C}^{M}$ property of $\lambda=\lambda(t)$ and $\omega=\omega(t)$ and the idea of stabilization condition they proved the two sided estimate

$$
C_{0} \leq \frac{1}{\lambda(t)} \mathbb{E}_{\lambda}(u)(t) \leq C_{1},
$$

where the nonnegative constants $C_{0}$ and $C_{1}$ depend on the data and $\mathbb{E}_{\lambda}(u)(t)$ is defined as

$$
\mathbb{E}_{\lambda}(u)(t):=\frac{1}{2}\left(\lambda^{2}(t)\left\|\nabla_{x} u(t, \cdot)\right\|_{L^{2}}^{2}+\left\|u_{t}(t, \cdot)\right\|_{L^{2}}^{2}\right) .
$$

\section{Linear damped wave equations with time-dependent dissipation}

A further problem of interest is the Cauchy problem for the wave equation with timedependent dissipation

$$
\begin{cases}u_{t t}-\Delta u+b(t) u_{t}=0, & (t, x) \in[0, \infty) \times \mathbb{R}^{n}, \\ u(0, x)=u_{0}(x), \quad u_{t}(0, x)=u_{1}(x), & x \in \mathbb{R}^{n} .\end{cases}
$$

The term $b(t) u_{t}$ is called the damping term, which prevents the motion of the wave and reduces its energy, and the coefficient $b=b(t)$ represents the strength of the damping. The asymptotic behavior of solutions and their wave energy change according to the positive coefficient $b=b(t)$ in the damping term.

In the PhD thesis [46], the author proposed a classification of the time-dependent dissipation terms in the following way:

- scattering producing to the free wave equation,

- non-effective dissipation,

- effective dissipation,

- over-damping producing.

If the solution behaves asymptotically like that of the wave equation, that is, if the damping term in (1.1.12) has no essential influence on the behavior of the solution, then the solution scatters to that of the free wave equation when $t \rightarrow \infty$. This case is called scattering producing case. If the $L^{p}-L^{q}$ estimates of the solution to the Cauchy problem (1.1.12) are closely related to those of the solutions to the free wave equation, then the damping term is called non-effective. If the solution to the Cauchy problem (1.1.12) has the same decay behavior as the solution of the corresponding parabolic Cauchy problem

$$
\begin{cases}u_{t}=\frac{1}{b(t)} \Delta u, & (t, x) \in[0, \infty) \times \mathbb{R}^{n} \\ u(0, x)=u_{0}(x), & x \in \mathbb{R}^{n}\end{cases}
$$

that is, if the damping term has a stronger influence, then the damping term is called effective. Finally, if the energy of the solution has no any decay estimate, namely, if the damping term has too strong influence, then the damping term is called over-damping producing.

In general for the cases scattering or over-damping there is no energy decay. Particularly, the effectiveness to the model (1.1.12) is defined as follows.

Definition 1.1.2 (Effective dissipation). The damping term in (1.1.12) is called effective, if $b=b(t)$ satisfies the following assumptions:

(B1) $b(t)>0$ for any $t \geq 0$;

(B2) $\left|b^{(k)}(t)\right| \leq C_{k} b(t)\left(\frac{1}{1+t}\right)^{k}, k=1,2, \cdots, \ell$; 
(B3) $b(t)$ is monotonic;

(B4) $\frac{1}{b(t)} \notin L^{1}([0, \infty))$;

(B5) $\left|b^{\prime}(t)\right|=o\left(b^{2}(t)\right)$, that is, $t b(t) \rightarrow \infty$ as $t \rightarrow \infty$;

(B6) $\frac{1}{b(t)(1+t)^{2}} \in L^{1}([0, \infty))$.

In the case of effective dissipation, the $L^{p}-L^{q}$ decay estimates to the solution (1.1.12) and their derivatives were given in the following way in [46, 48]:

$$
\left\|D_{t}^{k} D_{x}^{\alpha} u(t, \cdot)\right\|_{L^{q}} \leq C \frac{1}{b^{k}(t)}\left(1+\int_{0}^{t} \frac{d \tau}{b(\tau)}\right)^{-\frac{n}{2}\left(\frac{1}{p}-\frac{1}{q}\right)-\frac{|\alpha|}{2}-k}\left(\left\|u_{0}\right\|_{W_{p}^{N_{p}+|\alpha|}}+\left\|u_{1}\right\|_{W_{p}^{N_{p}+|\alpha|-1}}\right),
$$

where $p \in[1,2], p q=p+q$ and the regularity $N_{p}>n\left(\frac{1}{p}-\frac{1}{q}\right)$. Note that this decay rate coincides with the corresponding estimate for the heat equation.

\section{Semilinear damped wave equation with time-dependent dissipation}

Next, we consider the following Cauchy problem for semilinear time-dependent damped wave equation:

$$
\begin{cases}u_{t t}-\Delta u+b(t) u_{t}=|u|^{p}, & (t, x) \in[0, \infty) \times \mathbb{R}^{n}, \\ u(0, x)=u_{0}(x), \quad u_{t}(0, x)=u_{1}(x), & x \in \mathbb{R}^{n} .\end{cases}
$$

Since the nonlinearity $|u|^{p}$ of (1.1.13) is a source term, in general the solution may blow up in finite time even if the initial data is sufficiently small.

In the paper [22], the authors proved that the critical exponent for solutions to the Cauchy problem (1.1.13) remains the same as for the Cauchy problem with $b(t)=1$ with special effective damping term $b(t) u_{t}=b_{0}(1+t)^{\beta} u_{t}$ for $-1<\beta<1$, provided that the small initial data belong to $H^{1} \times L^{2}$ with compact support. That is, the authors have obtained a blow-up result if $1<p \leq p_{\text {Fuj }}(n)$ and a global (in time) existence result if $p>p_{\text {Fuj }}(n)$. Later, a global (in time) existence result to (1.1.13) was extended in [10] to more general $b(t)$ satisfying a monotonicity condition and a polynomial-like behavior. Moreover, the authors relaxed the assumption of compactly supported with data from exponentially weighted energy spaces. The authors also dealt with initial data belonging to the class $\left(H^{1} \cap L^{1}\right) \times\left(L^{2} \cap L^{1}\right)$ when $n \leq 4$. In particular, the global (in time) existence holds for $p>p_{\mathrm{Fuj}}(n)$ and $p \leq p_{\mathrm{GN}}(n)=\frac{n}{n-2}$ with $n \geq 3$, if initial data are assumed to be small in exponentially weighted energy spaces. In the paper [8], the authors treated subcritical and critical case $1<p \leq p_{\text {Fuj }}(n)$. The authors proved that there is no global (in time) existence of small data solutions, under a suitable sign assumption.

\section{Linear damped wave equations with time-dependent speed of propagation and dissipation}

In the $\mathrm{PhD}$ thesis [1], the author studied the following linear Cauchy problem with timedependent propagation speed and dissipation (see also [2]):

$$
\begin{cases}u_{t t}-a^{2}(t) \Delta u+b(t) u_{t}=0, & (t, x) \in[0, \infty) \times \mathbb{R}^{n}, \\ u(0, x)=u_{0}(x), \quad u_{t}(0, x)=u_{1}(x), & x \in \mathbb{R}^{n} .\end{cases}
$$

Here $a=a(t)$ describes an increasing propagation speed of waves and $b(t) u_{t}$ describes a damping effect. More precisely, the author was interested in time-dependent coefficients $a=a(t)$ and $b=b(t)$ having very slow oscillations only.

The author proposed a classification of the damping term $b(t) u_{t}$ in terms of the increasing speed of propagation $a=a(t)$ as follows: 
- scattering producing to the wave equation,

- non-effective dissipation,

- effective dissipation,

- over-damping producing.

The increasing speed of propagation $a=a(t)$ has the following properties:

(A1) $a(t)>0$ and $a^{\prime}(t)>0$ for $t \in[0, \infty)$;

(A2) $a_{0} \frac{a(t)}{A(t)} \leq \frac{a^{\prime}(t)}{a(t)} \leq a_{1} \frac{a(t)}{A(t)}, a_{0}, a_{1}>0$;

(A3) $\left|a^{\prime \prime}(t)\right| \leq a_{2} a(t)\left(\frac{a(t)}{A(t)}\right)^{2}, a_{2}>0$.

Then, by studying the interaction between $a=a(t)$ and $b=b(t)$, the effective dissipation is defined as follows.

Definition 1.1.3 (Effective dissipation). The damping term $b(t) u_{t}$ in (1.1.14) is called effective, if the following assumptions are satisfied:

(B'1) $b(t)>0 \quad$ and $\quad b(t)=\mu(t) \frac{a(t)}{A(t)}$;

$$
\left|d_{t}^{k} \mu(t)\right| \leq C_{k} \mu(t)\left(\frac{a(t)}{A(t)}\right)^{k} \quad \text { for } k=1,2, \cdots, \ell
$$

(B'3) $\frac{\mu(t)}{A(t)}$ is monotonic and $\mu(t) \rightarrow \infty$ as $t \rightarrow \infty$;

(B'4) $\frac{a^{2}(t)}{b(t)}=\frac{a(t) A(t)}{\mu(t)} \notin L^{1}([0, \infty))$.

Thus, by the above effectiveness assumptions the following $L^{p}-L^{q}$ decay estimate holds to (1.1.14):

$$
\left\|\left(u_{t}, a(t) \nabla_{x} u\right)(t, \cdot)\right\|_{L^{q}} \leq C a(t)\left(1+\int_{0}^{t} \frac{a^{2}(\tau)}{b(\tau)} d \tau\right)^{-\frac{n}{2}\left(\frac{1}{p}-\frac{1}{q}\right)-\frac{1}{2}}\left(\left\|u_{0}\right\|_{W_{p}^{N_{p}}}+\left\|u_{1}\right\|_{W_{p}^{N_{p}-1}}\right),
$$

where $p \in[1,2], p q=p+q$ and the regularity $N_{p}>n\left(\frac{1}{p}-\frac{1}{q}\right)$.

This shows that the model (1.1.14), in the effective damping case is hinted to relations to parabolic models from the point of view of decay estimates for the long-time behavior of solutions and its energies.

\section{Semilinear damped wave equation with time-dependent speed of propagation and dissipation}

In the above cited $\mathrm{PhD}$ thesis the author studied also the following semilinear Cauchy problem for the damped wave equations with increasing time-dependent propagation speed and effective dissipation term (see also $[3,4]$ ):

$$
\begin{cases}u_{t t}-a^{2}(t) \Delta u+b(t) u_{t}=|u|^{p}, & (t, x) \in[0, \infty) \times \mathbb{R}^{n}, \\ u(0, x)=u_{0}(x), \quad u_{t}(0, x)=u_{1}(x), & x \in \mathbb{R}^{n} .\end{cases}
$$


In order to prove the global (in time) existence of small data Sobolev solutions to a given semilinear Cauchy problem, after using Duhamel's principle the estimates of solutions to the following family of parameter-dependent Cauchy problems are necessary to estimate:

$$
\begin{cases}u_{t t}-a^{2}(t) \Delta u+b(t) u_{t}=0, & (t, x) \in[s, \infty) \times \mathbb{R}^{n} \\ u(s, x)=0, \quad u_{t}(s, x)=g(s, x), & x \in \mathbb{R}^{n} .\end{cases}
$$

With the $\mathcal{C}^{2}$ property of the above given assumptions (B'1) to (B'4) and the additional assumption

$$
\frac{a^{2}(t)}{A^{2}(t) b(t)} \in L^{1}([0, \infty))
$$

the author derived the following estimates of solutions to the Cauchy problem (1.1.16):

$$
\begin{aligned}
\|u(t, \cdot)\|_{L^{2}} & \leq C \frac{1}{b(s)}\left(1+B_{a}(s, t)\right)^{-\frac{n}{2}\left(\frac{1}{m}-\frac{1}{2}\right)}\|g(s, \cdot)\|_{L^{m} \cap L^{2}}, \\
\left\|\nabla_{x} u(t, \cdot)\right\|_{L^{2}} & \leq C \frac{1}{b(s)}\left(1+B_{a}(s, t)\right)^{-\frac{n}{2}\left(\frac{1}{m}-\frac{1}{2}\right)-\frac{1}{2}}\|g(s, \cdot)\|_{L^{m} \cap L^{2}}, \\
\left\|u_{t}(t, \cdot)\right\|_{L^{2}} & \leq C \frac{a^{2}(t)}{b(s) b(t)}\left(1+B_{a}(s, t)\right)^{-\frac{n}{2}\left(\frac{1}{m}-\frac{1}{2}\right)-1}\|g(s, \cdot)\|_{L^{m} \cap L^{2}},
\end{aligned}
$$

where $m \in[1,2)$ and $B_{a}(s, t):=\int_{s}^{t} \frac{a^{2}(\tau)}{b(\tau)} d \tau$.

The author divided his considerations into two cases to derive results about global (in time) existence of small data Sobolev solutions to the Cauchy problem (1.1.15): the case of sub-exponential propagation speed and the case super-exponential propagation speed, which are defined by the aid of the following auxiliary functions:

$$
\nu(\delta, t):=\frac{a(t)}{A(t)} \frac{A(\delta t)}{a(\delta t)} \quad \text { and } \quad \nu(\delta):=\lim _{t \rightarrow \infty} \sup \nu(\delta, t), \quad \delta \in(0,1) .
$$

Then, the following classification is introduced:

- the case of sub-exponential propagation speed if $\nu(\delta) \lesssim 1$,

- the case of super-exponential propagation speed if $\nu(\delta)=\infty$.

By the additional assumption

(C1) $\mu^{\prime}(t) \leq \mu_{1} \mu(t) \frac{a(t)}{A(t)}, \quad \mu_{1} \in[0,2)$,

in the case of sub-exponential propagation speed, the result about global (in time) existence of small data Sobolev solutions is formulated by means of the following parameters:

$$
\begin{aligned}
& \bar{p}_{1}:=1+\left(1-\frac{2 a_{0}}{2+R}\right) \frac{2}{n} \\
& \bar{p}_{2}:=1+\left(1-\frac{\delta}{\nu(\delta)} \frac{2 a_{0}}{2+R}\right) \frac{2}{n}, \\
& \bar{p}_{3}:=\frac{1}{2}+\frac{1}{2\left(1-\frac{2+R}{2-\mu_{1}}+\frac{2+R}{2-\mu_{1}} \frac{\delta}{\nu(\delta)}\right)}+\left(\frac{1}{1-\frac{2+R}{2-\mu_{1}}+\frac{2+R}{2-\mu_{1}} \frac{\delta}{\nu(\delta)}}-\frac{\frac{2 a_{0}}{2+R} \frac{\delta}{\nu(\delta)}}{1-\frac{2+R}{2-\mu_{1}}+\frac{2+R}{2-\mu_{1}} \frac{\delta}{\nu(\delta)}}\right) \frac{2}{n} .
\end{aligned}
$$

Here the nonnegative constants $\mu_{1}$ is from condition (C1), $a_{0}$ is from condition (A2) and $R$ is from condition (B2) with $k=1$ and $\mu^{\prime}(t) / \mu(t) \geq-R a(t) / A(t)$.

In the case of sub-exponential propagation speed, under the following assumptions it has been proved the global (in time) existence of small data energy solutions belonging 
to $\mathcal{C}\left([0, \infty), H^{1}\right) \cap \mathcal{C}^{1}\left([0, \infty), L^{2}\right)$, where the initial data $\left(u_{0}, u_{1}\right)$ are assumed to belong to $\left(H^{1} \cap L^{1}\right) \times\left(L^{2} \cap L^{1}\right):$

$$
\frac{\delta}{\nu(\delta)}>\frac{(2+R) n}{8 a_{0}} \quad \text { and } \quad \begin{cases}p>\bar{p}, \quad p \geq 2 & \text { if } n=1,2 \\ 2 \leq p \leq 3=p_{\mathrm{GN}}(3) & \text { if } n=3 \\ p=2=p_{\mathrm{GN}}(4) & \text { if } n=4\end{cases}
$$

where $\bar{p}:=\max \left\{\bar{p}_{1} ; \bar{p}_{2} ; \bar{p}_{3}\right\}$ (see [3]).

On the other hand, in the case of super-exponential propagation speed, the following additional assumption was supposed to be satisfied:

(C2) The function $\theta(t):=\frac{A^{2}(t)}{\mu(t)}$ satisfies the following conditions:

- $\theta(t)$ is increasing,

- $\theta^{\prime}(t) \leq \alpha \theta(t) \frac{1}{\log A(t)} \frac{a(t)}{A(t)}, \quad \alpha>0$.

It has been proved the global (in time) existence of small data energy solutions belonging to $\mathcal{C}\left([0, \infty), H^{1}\right) \cap \mathcal{C}^{1}\left([0, \infty), L^{2}\right)$, where the initial data $\left(u_{0}, u_{1}\right)$ are assumed to belong to $\left(H^{1} \cap L^{1}\right) \times\left(L^{2} \cap L^{1}\right), \nu(\delta, t)=O(\log A(t))$ and

$$
\begin{cases}2 \leq p & \text { if } n=1,2 \\ 2 \leq p \leq 3=p_{\mathrm{GN}}(3) & \text { if } n=3 \\ p=2=p_{\mathrm{GN}}(4) & \text { if } n=4\end{cases}
$$

$($ see $[4])$.

\subsection{Main goals, some results and plan of the thesis}

In this thesis, we are interested in damped wave models with time-dependent propagation speed and time-dependent damping term both having a time-dependent oscillation term. More precisely, we are concerned with the Cauchy problem

$$
\begin{cases}u_{t t}-\lambda^{2}(t) \omega^{2}(t) \Delta u+\rho(t) \omega(t) u_{t}=0, & (t, x) \in[0, \infty) \times \mathbb{R}^{n}, \\ u(0, x)=u_{0}(x), \quad u_{t}(0, x)=u_{1}(x), & x \in \mathbb{R}^{n},\end{cases}
$$

and the corresponding semilinear Cauchy problem with some power source nonlinearities on the right-hand side, that is,

$$
\begin{cases}u_{t t}-\lambda^{2}(t) \omega^{2}(t) \Delta u+\rho(t) \omega(t) u_{t}=f(u), & (t, x) \in[0, \infty) \times \mathbb{R}^{n}, \\ u(0, x)=u_{0}(x), \quad u_{t}(0, x)=u_{1}(x), & x \in \mathbb{R}^{n} .\end{cases}
$$

To verify special properties of solutions ( $L^{p}-L^{q}$ estimates on the conjugate line) we restrict our considerations to the following Cauchy problem to the linear wave equation with time dependent speed of propagation:

$$
\begin{cases}u_{t t}-\lambda^{2}(t) \omega^{2}(t) \Delta u=0, & (t, x) \in[0, \infty) \times \mathbb{R}^{n}, \\ u(0, x)=u_{0}(x), \quad u_{t}(0, x)=u_{1}(x), & x \in \mathbb{R}^{n} .\end{cases}
$$

Here the time-dependent functions $\lambda=\lambda(t), \rho=\rho(t)$ and $\omega=\omega(t)$ are smooth and strictly positive functions. In particular, $\lambda$ is a monotonously increasing nontrivial shape function in the propagation speed, $\rho$ is a nontrivial shape function in the damping term and $\omega$ is a bounded oscillating function in both propagation speed and damping term. 
Throughout this thesis, we restrict ourselves to "effective-like" (due to the oscillating term $\omega=\omega(t)$ ) damping $\rho(t) \omega(t) u_{t}$ according to the classification in [2] and [48]. Here effective means that the solution to (1.2.1) behaves like that of a corresponding parabolic problem.

The main goal of the thesis is to understand the influence of stronger oscillations on Sobolev solutions to the linear models (1.2.1) and (1.2.3) and, consequently, to the semilinear model (1.2.2). Especially, due to the deteriorating influence of oscillations on solutions, a stabilization condition and higher order regularity of the time-dependent coefficients may compensate "bad behaviors" arising from oscillations. In this way, stabilization condition allows us to control a certain amount of very fast oscillations (see $[16,17,19]$ ).

From a mathematical point of view, it is an interesting problem to study how the oscillations in the propagation speed and the damping term affects the properties of the solutions with a stabilization condition. In other words, in this thesis, we investigate how solutions of the given linear and semilinear Cauchy problems differ from very fast oscillations with a stabilization condition to very slow oscillations.

In this thesis we will represent the coefficients $a=a(t)$ and $b=b(t)$ in (1.1.1) by the following products:

$$
a(t)=\lambda(t) \omega(t) \quad \text { and } \quad b(t)=\rho(t) \omega(t) .
$$

If we have in mind $\omega(t) \equiv 1$, then $a=a(t)$ and $b=b(t)$ have very slow oscillations. In this way, we will call the damping term with the oscillating function $\omega$ is an "effective-like" damping term.

\section{Some results of the thesis}

In this section, we collect the selected results described in this thesis.

In order to prove global (in time) existence of small data Sobolev solutions to the semilinear Cauchy problem (1.2.2), after using Duhamel's principle we need estimates of solutions to the following family of parameter-dependent Cauchy problems:

$$
\begin{cases}v_{t t}-\lambda^{2}(t) \omega^{2}(t) \Delta v+\rho(t) \omega(t) v_{t}=0, & (t, x) \in[s, \infty) \times \mathbb{R}^{n}, s \geq 0, \\ v(s, x)=0, \quad v_{t}(s, x)=g(s, x), & x \in \mathbb{R}^{n} .\end{cases}
$$

We derived in Section 2 the following estimates of solutions to the Cauchy problem (1.2.4) under the assumptions stated in Section 2.3:

$$
\begin{aligned}
&\|v(t, \cdot)\|_{\dot{H}^{\sigma}} \lesssim \frac{\Lambda(s)}{\lambda(s)}\left(1+B_{\lambda}(s, t)\right)^{-\frac{\sigma}{2}-\frac{n}{2}\left(\frac{1}{m}-\frac{1}{2}\right)}\|g(s, \cdot)\|_{L^{m} \cap H^{\max \{\sigma-1 ; 0\}}}, \\
&\left\|v_{t}(t, \cdot)\right\|_{\dot{H}^{\sigma}} \lesssim \frac{\Lambda(s)}{\lambda(s)} \max \left\{\frac{\lambda^{2}(t)}{\rho(t)}\left(1+B_{\lambda}(s, t)\right)^{-\frac{\sigma}{2}-\frac{n}{2}\left(\frac{1}{m}-\frac{1}{2}\right)-1} ;\right. \\
&\left.\lambda(t) F(\Lambda(t))\left(F^{2}(\Lambda(t))\right)^{-\frac{\sigma}{2}-\frac{n}{2}\left(\frac{1}{m}-\frac{1}{2}\right)-1}\right\}\|g(s, \cdot)\|_{L^{m} \cap H^{\sigma}},
\end{aligned}
$$

where $\sigma \in \mathbb{R}_{+}, m \in[1,2)$ and $B_{\lambda}(s, t):=\int_{0}^{t} \frac{\lambda^{2}(\tau)}{\rho(\tau)} d \tau$.

To derive these estimates we use primarily the WKB-analysis and the method of zones. In opposite to earlier literature, due to the influence arising from strong oscillations, we should modify the definition of the zones used in related models. Particularly, first, we shrink the hyperbolic zone by imposing an oscillation subzone. Next, in the same manner, we also need to get a smaller elliptic zone, however, this leads to enlarging the dissipative zone. Therefore, this brings competition between the estimates in $\left\|v_{t}(t, \cdot)\right\|_{\dot{H}^{\sigma}}$ deriving from the elliptic zone and the dissipative zone, respectively.

Main results for Sobolev solutions to the Cauchy problem (1.2.2) when $f(u)=|u|^{p}, p>1$, are derived in Section 3 together with higher order energy estimates of solutions and their partial derivatives. For the sake of simplicity, here we present our results for some typical examples in our approach, which divide into two cases: case of sub-exponential propagation speed and case of super-exponential propagation speed, respectively. We introduce only simplified results under the assumptions stated in Section 2.3 and Section 3.1.1 with the constructed admissible oscillation function in Section 2.6. 
- Case of sub-exponential propagation speed:

Let $\lambda(t)=(\alpha+1)(1+t)^{\alpha}, \alpha>0$. Then, we obtain

$$
\begin{aligned}
& \Lambda(t)=(1+t)^{\alpha+1} \quad \text { and } \Theta(t)=(1+t)^{\gamma+1}, \quad-1<\gamma<\alpha, \\
& \rho(t) \simeq(1+t)^{\beta-1}, \quad \alpha-\gamma<\beta<2 \alpha+2 \quad \text { and } \quad F(\Lambda(t)) \simeq(1+t)^{\alpha+2 \kappa-1}, \quad \frac{4-\beta}{4} \leq \kappa<1 .
\end{aligned}
$$

- Case of super-exponential propagation speed:

Let us choose $\lambda(t)=e^{t} e^{e^{t}}$. Then, we have

$$
\begin{aligned}
& \Lambda(t)=e^{e^{t}} \text { and } \Theta(t)=e^{r e^{t}}, \quad 0<r<1, \\
& \rho(t) \simeq e^{t} e^{q e^{t}}, \quad 1-r<q<2 \text { and } F(\Lambda(t)) \simeq e^{(1+2 \kappa) e^{t}}, \quad-\frac{q}{4} \leq \kappa<0 .
\end{aligned}
$$

We introduce the space

$$
\mathcal{D}_{m}^{\sigma}:=\left(H^{\sigma} \cap L^{m}\right) \times\left(H^{\max \{\sigma-1 ; 0\}} \cap L^{m}\right)
$$

with the norm

$$
\left\|\left(u_{0}, u_{1}\right)\right\|_{\mathcal{D}_{m}^{\sigma}}:=\left\|u_{0}\right\|_{H^{\sigma}}+\left\|u_{0}\right\|_{L^{m}}+\left\|u_{1}\right\|_{H^{\max \{\sigma-1 ; 0\}}}+\left\|u_{1}\right\|_{L^{m}},
$$

where $\sigma \in \mathbb{R}_{+}$and $m \in[1,2)$.

The space for initial data influences the choice of the space in which we look for solutions. The results for global (in time) existence of small data solutions to the Cauchy problem (1.2.2) are treated separately in the case of sub-exponential and the case super-exponential propagation speed, respectively.

- If the data has a low regularity

$$
\left(u_{0}, u_{1}\right) \in\left(H^{\sigma} \cap L^{m}\right) \times\left(L^{2} \cap L^{m}\right),
$$

where $\sigma \in(0,1)$ and $m \in[1,2)$, then we prove a global (in time) existence result of Sobolev solutions provided that the exponent $p$ belongs to some admissible interval and for the case of sub-exponential propagation speed also satisfies

$$
p>1+\left(\frac{\beta-\alpha+1}{\alpha+1}\right) \frac{2 m}{n} .
$$

Moreover, we have the following decay estimates in the case of sub-exponential propagation speed:

$$
\begin{aligned}
\|u(t, \cdot)\|_{L^{2}} & \lesssim(1+t)^{-(2 \alpha-\beta+2) \frac{n}{2}\left(\frac{1}{m}-\frac{1}{2}\right)}\left\|\left(u_{0}, u_{1}\right)\right\|_{\mathcal{D}_{m}^{\sigma}}, \\
\left\||D|^{\sigma} u(t, \cdot)\right\|_{L^{2}} & \lesssim(1+t)^{-(2 \alpha-\beta+2)\left(\frac{n}{2}\left(\frac{1}{m}-\frac{1}{2}\right)+\frac{\sigma}{2}\right)}\left\|\left(u_{0}, u_{1}\right)\right\|_{\mathcal{D}_{m}^{\sigma}} .
\end{aligned}
$$

In the case of super-exponential propagation speed we have the decay estimates

$$
\begin{aligned}
&\|u(t, \cdot)\|_{L^{2}} \lesssim e^{-(2-q) \frac{n}{2}\left(\frac{1}{m}-\frac{1}{2}\right) e^{t}}\left\|\left(u_{0}, u_{1}\right)\right\|_{\mathcal{D}_{m}^{\sigma}}, \\
&\left\||D|^{\sigma} u(t, \cdot)\right\|_{L^{2}} \lesssim e^{-(2-q)\left(\frac{n}{2}\left(\frac{1}{m}-\frac{1}{2}\right)+\frac{\sigma}{2}\right) e^{t}}\left\|\left(u_{0}, u_{1}\right)\right\|_{\mathcal{D}_{m}^{\sigma}} .
\end{aligned}
$$

Here the competition in the estimates of $\left\|u_{t}(t, \cdot)\right\|_{\dot{H}^{\sigma}}$ does not influence the admissible exponents $p$, since we do not have a classical energy solution.

- If the data are taken from energy space with additional regularity, that is,

$$
\left(u_{0}, u_{1}\right) \in\left(H^{1} \cap L^{m}\right) \times\left(L^{2} \cap L^{m}\right),
$$


then, the global (in time) existence of small data energy solutions can be proved for larger dimension $n$, where it is provided that the exponent $p$ belongs to some admissible interval and in the case of sub-exponential propagation speed the exponent $p$ also holds

$$
p>1+\left(\frac{\beta-\alpha+1}{\alpha+1}\right) \frac{2 m}{n} .
$$

Moreover, we have the estimates in the case of sub-exponential propagation speed

$$
\begin{aligned}
&\|u(t, \cdot)\|_{L^{2}} \lesssim(1+t)^{-(2 \alpha-\beta+2) \frac{n}{2}\left(\frac{1}{m}-\frac{1}{2}\right)}\left\|\left(u_{0}, u_{1}\right)\right\|_{\mathcal{D}_{m}^{1}}, \\
&\||D| u(t, \cdot)\|_{L^{2}} \lesssim(1+t)^{-(2 \alpha-\beta+2)\left(\frac{n}{2}\left(\frac{1}{m}-\frac{1}{2}\right)+\frac{1}{2}\right)}\left\|\left(u_{0}, u_{1}\right)\right\|_{\mathcal{D}_{m}^{1}}, \\
&\left\|u_{t}(t, \cdot)\right\|_{L^{2}} \lesssim \max \left\{(1+t)^{-(2 \alpha-\beta+2) \frac{n}{2}\left(\frac{1}{m}-\frac{1}{2}\right)-1} ;\right. \\
&\left.(1+t)^{-(2 \alpha+4 \kappa-2) \frac{n}{2}\left(\frac{1}{m}-\frac{1}{2}\right)-2 \kappa+1}\right\}\left\|\left(u_{0}, u_{1}\right)\right\|_{\mathcal{D}_{m}^{1}},
\end{aligned}
$$

and in the case of super-exponential propagation speed

$$
\begin{aligned}
\|u(t, \cdot)\|_{L^{2}} & \lesssim e^{-(2-q) \frac{n}{2}\left(\frac{1}{m}-\frac{1}{2}\right) e^{t}}\left\|\left(u_{0}, u_{1}\right)\right\|_{\mathcal{D}_{m}^{1}}, \\
\||D| u(t, \cdot)\|_{L^{2}} & \lesssim e^{-(2-q)\left(\frac{n}{2}\left(\frac{1}{m}-\frac{1}{2}\right)+\frac{1}{2}\right) e^{t}}\left\|\left(u_{0}, u_{1}\right)\right\|_{\mathcal{D}_{m}^{1}}, \\
\left\|u_{t}(t, \cdot)\right\|_{L^{2}} & \lesssim \max \left\{e^{t} e^{-(2-q) \frac{n}{2}\left(\frac{1}{m}-\frac{1}{2}\right) e^{t}} ; e^{t} e^{-2 \kappa e^{t}} e^{-(2+4 \kappa) \frac{n}{2}\left(\frac{1}{m}-\frac{1}{2}\right) e^{t}}\right\}\left\|\left(u_{0}, u_{1}\right)\right\|_{\mathcal{D}_{m}^{1}} .
\end{aligned}
$$

Here let us point out that it appears the competition between our estimates for time derivative of the solution $u$. If the first components are dominant, then we extend the results of [3] and [4] with $\omega \equiv 1$ by using additional regularity $L^{m}, m \in[1,2)$, for the data.

- Next, we treat the Cauchy problem (1.2.2) for

$$
\left(u_{0}, u_{1}\right) \in\left(H^{\sigma} \cap L^{m}\right) \times\left(H^{\sigma-1} \cap L^{m}\right),
$$

where $\sigma>1$. In addition to $p>1+\left(\frac{\beta-\alpha+1}{\alpha+1}\right) \frac{2 m}{n}$ and after using the fractional chain rule from Section A.7.4 in Appendix another condition comes into play which is $p>\lceil\sigma\rceil$ for any space dimension $n$. Moreover, we have the estimates in the case of sub-exponential propagation speed

$$
\begin{aligned}
&\|u(t, \cdot)\|_{L^{2}} \lesssim(1+t)^{-(2 \alpha-\beta+2) \frac{n}{2}\left(\frac{1}{m}-\frac{1}{2}\right)}\left\|\left(u_{0}, u_{1}\right)\right\|_{\mathcal{D}_{m}^{\sigma}} \\
&\||D| u(t, \cdot)\|_{L^{2}} \lesssim(1+t)^{-(2 \alpha-\beta+2)\left(\frac{n}{2}\left(\frac{1}{m}-\frac{1}{2}\right)+\frac{1}{2}\right)}\left\|\left(u_{0}, u_{1}\right)\right\|_{\mathcal{D}_{m}^{\sigma}}, \\
&\left\|u_{t}(t, \cdot)\right\|_{L^{2}} \lesssim \max \left\{(1+t)^{-(2 \alpha-\beta+2) \frac{n}{2}\left(\frac{1}{m}-\frac{1}{2}\right)-1} ;\right. \\
&\left.(1+t)^{-(2 \alpha+4 \kappa-2) \frac{n}{2}\left(\frac{1}{m}-\frac{1}{2}\right)-2 \kappa+1}\right\}\left\|\left(u_{0}, u_{1}\right)\right\|_{\mathcal{D}_{m}^{\sigma}}, \\
&\left\||D|^{\sigma-1} u_{t}(t, \cdot)\right\|_{L^{2}} \lesssim \max \left\{(1+t)^{-(2 \alpha-\beta+2)\left(\frac{n}{2}\left(\frac{1}{m}-\frac{1}{2}\right)+\frac{\sigma-1}{2}\right)-1} ;\right. \\
&\left.(1+t)^{-(2 \alpha+4 \kappa-2)\left(\frac{n}{2}\left(\frac{1}{m}-\frac{1}{2}\right)+\frac{\sigma-1}{2}\right)-2 \kappa+1}\right\}\left\|\left(u_{0}, u_{1}\right)\right\|_{\mathcal{D}_{m}^{\sigma}},
\end{aligned}
$$

and in the case of super-exponential propagation speed

$$
\begin{aligned}
&\|u(t, \cdot)\|_{L^{2}} \lesssim e^{-(2-q) \frac{n}{2}\left(\frac{1}{m}-\frac{1}{2}\right) e^{t}}\left\|\left(u_{0}, u_{1}\right)\right\|_{\mathcal{D}_{m}^{\sigma}}, \\
&\||D| u(t, \cdot)\|_{L^{2}} \lesssim e^{-(2-q)\left(\frac{n}{2}\left(\frac{1}{m}-\frac{1}{2}\right)+\frac{1}{2}\right) e^{t}}\left\|\left(u_{0}, u_{1}\right)\right\|_{\mathcal{D}_{m}^{\sigma}}, \\
&\left\|u_{t}(t, \cdot)\right\|_{L^{2}} \lesssim \max \left\{e^{t} e^{-(2-q) \frac{n}{2}\left(\frac{1}{m}-\frac{1}{2}\right) e^{t}} ; e^{t} e^{-2 \kappa e^{t}} e^{-(2+4 \kappa) \frac{n}{2}\left(\frac{1}{m}-\frac{1}{2}\right) e^{t}}\right\}\left\|\left(u_{0}, u_{1}\right)\right\|_{\mathcal{D}_{m}^{\sigma}}, \\
&\left\||D|^{\sigma-1} u_{t}(t, \cdot)\right\|_{L^{2}} \lesssim \max \left\{e^{t} e^{-(2-q)\left(\frac{n}{2}\left(\frac{1}{m}-\frac{1}{2}\right)+\frac{\sigma-1}{2}\right) e^{t}} ;\right. \\
&\left.e^{t} e^{-2 \kappa e^{t}} e^{-(2+4 \kappa)\left(\frac{n}{2}\left(\frac{1}{m}-\frac{1}{2}\right)+\frac{\sigma-1}{2}\right) e^{t}}\right\}\left\|\left(u_{0}, u_{1}\right)\right\|_{\mathcal{D}_{m}^{\sigma}} .
\end{aligned}
$$


- Finally, a particular case $\sigma>\frac{n}{2}+1$ is treated. Using fractional powers from Section A.7.6 in Appendix the condition $p>\lceil\sigma\rceil$ can be relaxed to $p>\sigma$.

\section{Plan of the thesis}

The plan of the thesis is organized as follows.

In Chapter 2, for the linear Cauchy problem $\left(L^{m} \cap L^{2}\right)-L^{2}, m \in[1,2)$, estimates are obtained for the solution and its time derivative. In order to prove these estimates, some known techniques are applied, such as, WKB-analysis, the method of zones. Besides, microenergies in different parts of the extended phase space, diagonalization procedure, symbol classes and their hierarchies play an important role. In this chapter, the approaches developed for the linear wave models with time-dependent coefficients with damping $[1,10,48,46]$ and without damping $[16,18,19,33]$ are applied.

In Chapter 3 several global (in time) existence results are proved in the case $f(u)=|u|^{p}$. These results are divided into two cases with respect to the behavior of the propagation speed: case of sub-exponential propagation speed and case of super-exponential propagation speed. Firstly, it is considered results for Sobolev solutions below the energy level. Then, results for energy solution and results for solutions with higher regularity than energy solutions are derived. In particular, for the results in spaces with higher regularity some tools from Harmonic Analysis are necessary to deal with the power nonlinearity in homogeneous Sobolev spaces of fractional order. Finally, results for large regular solutions are considered.

In Chapter 4 some global (in time) existence results are derived in the case $f(u)=\left|u_{t}\right|^{p}$ and $f(u)=\left.\left.|| D\right|^{a} u\right|^{p}$ with $a \in(0,1]$. If $f(u)=\left|u_{t}\right|^{p}$, we prove large regular Sobolev solutions to (1.2.2) in the cases of sub-exponential and super-exponential propagation speeds, respectively. These solutions are imbedded into $L^{\infty}$. So, we may use results on fractional powers. Moreover, if $f(u)=\left.\left.|| D\right|^{a} u\right|^{p}$ with $a \in(0,1]$, we obtain the global (in time) existence of small data energy solutions in the classical energy space in the cases of sub-exponential and super-exponential propagation speeds, respectively.

Chapter 5 is devoted to the Cauchy problem for the linear model in (1.2.3). We obtain $L^{p}-L^{q}$ estimates on the conjugate line for Sobolev solutions of the Cauchy problem (1.2.3) by following the approach in [33]. To get these $L^{p}-L^{q}$ estimates we apply the partial Fourier transformation and employ the stationary phase method to the corresponding Fourier multipliers. For these Fourier multipliers we divide our considerations into two steps: application of Hardy-Littlewood inequality and of a Littman type lemma.

Finally, we proposed in Chapter 6 some further research problems for wave and damped wave problems with time-dependent variables.

Concluding, in Appendix, we explain the notations used in this thesis, some useful lemmas and mainly known results concerning Fourier Analysis and Fractional Calculus. 


\section{The influence of oscillations on linear damped wave equation with time-dependent coefficients}

\subsection{Introduction}

In this chapter, we consider the asymptotic behavior of solutions to the following linear damped wave equation with time-dependent speed of propagation and time-dependent dissipation:

$$
\begin{cases}u_{t t}-\lambda^{2}(t) \omega^{2}(t) \Delta u+\rho(t) \omega(t) u_{t}=0, & (t, x) \in[0, \infty) \times \mathbb{R}^{n}, \\ u(0, x)=u_{0}(x), \quad u_{t}(0, x)=u_{1}(x), & x \in \mathbb{R}^{n},\end{cases}
$$

with suitable assumptions on the time-dependent coefficients $\lambda=\lambda(t), \rho=\rho(t)$ and $\omega=\omega(t)$. Here $\lambda=\lambda(t)$ and $\rho=\rho(t)$ are nontrivial shape functions and $\omega=\omega(t)$ is a smooth bounded oscillating function.

The main goal is to prove higher order energy estimates to solutions (2.1.1) taking account of the $\mathcal{C}^{M}$ properties of the coefficients together with a stabilization condition. Generally, without stabilization condition, for very fast oscillating coefficients one might expect to destroy the estimates, which are valid for very slow oscillating coefficients (see [7, 35]). Roughly speaking, it is expected to compensate "bad behavior" of oscillations by a stabilization condition.

This idea was at first developed in [16] to investigate the asymptotic behavior for the total energy of solutions of wave equations with time-dependent propagation speed. The stabilization condition is an essential condition to the coefficients for estimates in lower frequency part of the extended phase space to derive a benefit of the $\mathcal{C}^{M}$ property of the coefficients to energy estimates. That is, we can derive some improvements from the $\mathcal{C}^{M}$ properties of the coefficients by taking into account the stabilization condition.

\subsection{Objectives and strategies}

We are interested in understanding energy estimates of solutions $u=u(t, x)$ for the model (2.1.1) with oscillating coefficients. Let us explain our strategy.

- In the first step we apply the partial Fourier transformation and the "dissipative" transformation to reduce the partial differential equation to an ordinary differential equation for $v=v(t, \xi)$ parameterized by the frequency parameter $\xi$.

- We will divide the extended phase space into two regions by a monotonic separating curve, the hyperbolic region and the elliptic region.

- We will also divide both regions of the extended phase space into different zones. The stabilization condition allows us to use weaker assumptions on derivatives with respect to those from the paper [2] by shrinking the hyperbolic zone. Also by shrinking the elliptic zone we enlarge the dissipative zone.

- In different cases we propose a different WKB-analysis. The main tools to develop a suitable WKB-analysis are definition of zones, symbol classes, to carry out a diagonalization procedure, to estimate the fundamental solution and to glue the representations in different zones together. These steps provide estimates for the energies. 
- In the hyperbolic zone we apply the $\mathcal{C}^{M}$ theory and $M$ steps of the diagonalization procedure to cope with the stronger oscillations. So, the remainder becomes integrable. Then, we derive a suitable representation of the fundamental solution.

- In the oscillation subzone we relate our model problem with the model where $\omega(t) \equiv 1$ to get desired estimates by using the stabilization condition.

- In the elliptic zone, we will introduce an appropriate micro-energy to get a system of first order. After two steps of the diagonalization procedure the remainder matrix is integrable over this zone. We explain the matrix representation of the fundamental solution which entries can be estimated by deriving a refined estimate.

- In the dissipative zone introducing an appropriate micro-energy we get an integral representation by using the fundamental solution to a first order system.

- In the reduced zone, around the separating curve between the elliptic and the hyperbolic region the model has no special type. We define an appropriate micro-energy to get for it a system of first order. Then, we derive a representation of the fundamental solution.

- Finally, by using the gluing procedure in the cases where the separating curve is monotonously increasing or monotonously decreasing, we get the desired higher order energy estimates.

\subsection{Assumptions}

We assume the following conditions for $\lambda=\lambda(t)$ and $\omega=\omega(t)$ belonging to $\mathcal{C}^{M}([0, \infty))$ with $M \geq 2$ (here we follow some ideas of [19]):

(A1) $\lambda(t)>0$ and $\lambda^{\prime}(t)>0$ for all times $t>0$, and the derivatives of $\lambda$ satisfy the conditions

$$
\lambda_{0} \frac{\lambda(t)}{\Lambda(t)} \leq \frac{\lambda^{\prime}(t)}{\lambda(t)} \leq \lambda_{1} \frac{\lambda(t)}{\Lambda(t)}, \quad\left|d_{t}^{k} \lambda(t)\right| \leq \lambda_{k} \lambda(t)\left(\frac{\lambda(t)}{\Lambda(t)}\right)^{k}, \quad k=1,2, \cdots, M,
$$

where $\lambda_{0}$ and all $\lambda_{k}$ are positive constants and $\Lambda(t)=1+\int_{0}^{t} \lambda(\tau) d \tau$ is a primitive of $\lambda(t)$

(A2) $0<c_{0} \leq \omega(t) \leq c_{1}$ and the derivatives of $\omega$ satisfy the conditions

$$
\left|d_{t}^{k} \omega(t)\right| \leq \omega_{k} \Xi^{-k}(t), \quad k=1,2, \cdots, M,
$$

where all $\omega_{k}$ are positive constants and $\Xi=\Xi(t)$ is a positive, monotonous and continuous function satisfying the compatibility condition

$$
C_{1} \Theta(t) \leq \lambda(t) \Xi(t) \leq C_{2} \Lambda(t) .
$$

Here $\Theta=\Theta(t)$ is a strictly increasing continuous function with $\Theta(0)=1, \Theta(t)<\Lambda(t)$ for $t>0$ and $\Theta(t)=o(\Lambda(t))$;

(A3) $\omega=\omega(t)$ is $\lambda$-stabilizing towards 1 , that is,

$$
\int_{0}^{t} \lambda(\tau)|\omega(\tau)-1| d \tau \leq C_{3} \Theta(t)
$$

(A4) the following estimate holds:

$$
\int_{t}^{\infty} \lambda^{-M}(\tau) \Xi^{-M-1}(\tau) d \tau \leq C_{4} \Theta^{-M}(t) ;
$$


(A5) the function $F=F(\Lambda(t))$ is defined by

$$
\frac{1}{F(\Lambda(t))}=\int_{t}^{\infty} \lambda^{-1}(\tau) \Xi^{-2}(\tau) d \tau \quad \text { and } \quad F(\Lambda(t)) \rightarrow \infty \quad \text { as } \quad t \rightarrow \infty,
$$

where we assume that $\lambda^{-1}(t) \Xi^{-2}(t) \in L^{1}([0, \infty))$.

Cauchy problems with increasing speed of propagation have been considered in [36]. Following their approach condition (A1) is standard. Condition (A3) is a so-called stabilization condition, which allows us to control a certain amount of very fast oscillations. In particular, this stabilization condition describes an error made from the oscillating behavior of the coefficients.

Remark 2.3.1. If we consider very slow oscillations, that is, if we choose formally $\Theta(t) \equiv \Lambda(t)$ and $F(\Lambda(t)) \equiv \Lambda(t)$, then conditions (A2) to (A5) trivially hold and by these choices the stabilization condition disappears. Hence, the stabilization condition (A3) has a meaning only in the case $M \geq 2$.

We will restrict ourselves to "effective-like" damping case in the sense of [2] and [48]. Here effective means that the solution behaves like that of a corresponding parabolic equation. Now motivated by the considerations from [2], in order to study the interaction between the shape functions $\lambda=\lambda(t), \rho=\rho(t)$ and the oscillating function $\omega=\omega(t)$ we assume the following conditions:

(B1) $\rho(t)>0 \quad$ and $\quad \rho(t)=\mu(t) \frac{\lambda(t)}{\Lambda(t)}$;

(B2) $\left|d_{t}^{k} \mu(t)\right| \leq \mu_{k} \mu(t)\left(\frac{\lambda(t)}{\Lambda(t)}\right)^{k} \quad$ for $k=1,2, \cdots, M$, where all $\mu_{k}$ are positive constants;

(B3) $\frac{\mu(t)}{\Lambda(t)}$ is monotonic and $\mu(t) \rightarrow \infty$ for $t \rightarrow \infty$;

(B4) $\frac{\lambda^{2}(t)}{\rho(t)}=\frac{\lambda(t) \Lambda(t)}{\mu(t)} \notin L^{1}([0, \infty))$;

(B5) $\mu(t) \frac{\Theta(t)}{\Lambda(t)} \rightarrow \infty$ as $t \rightarrow \infty$, this implies that $\left|(\rho(t) \omega(t))^{\prime}\right|=o\left((\rho(t) \omega(t))^{2}\right)$ as $t \rightarrow \infty$;

(B6) $\int_{0}^{t} \frac{\lambda^{2}(\tau)}{\rho(\tau)} d \tau \leq C_{5} F^{2}(\Lambda(t))$, where $C_{5}$ is a positive constant.

The conditions (B3) and (B4) describe the effective damping case related to a given increasing propagation speed. In particular, (B4) excludes the over-damping case (see [2]). The condition (B5) allows us to control a certain amount of very fast oscillations in the damping term $\rho(t) \omega(t) u_{t}$.

Remark 2.3.2. If we consider very slow oscillations, then it holds $F(\Lambda(t)) \equiv \Lambda(t)$. Hence, the condition (B6) trivially holds, since we have

$$
\int_{0}^{t} \frac{\lambda^{2}(\tau)}{\rho(\tau)} d \tau=\int_{0}^{t} \frac{\lambda(\tau) \Lambda(\tau)}{\mu(\tau)} d \tau \leq \int_{0}^{t} \frac{\lambda(\tau) \Lambda(\tau)}{\mu_{0}} d \tau \lesssim \Lambda^{2}(t),
$$

where due to $\mu(t) \rightarrow \infty$ as $t \rightarrow \infty$, there exists a constant $\mu_{0}$ such that $\mu(t) \geq \mu_{0}$ for large t.

\subsubsection{Some examples}

Now let us discuss some typical examples related to suitable shape functions $\lambda=\lambda(t)$ and $\rho=\rho(t)$ in order to verify our conditions. 
Example 2.3.1 (Polynomial case). Let $\lambda(t)=(\alpha+1)(1+t)^{\alpha}$ with $\alpha>0$. Then, we get

$$
\Lambda(t)=(1+t)^{\alpha+1} \quad \text { and } \quad \rho(t)=(\alpha+1) \frac{\mu(t)}{1+t} .
$$

Now taking account of the stabilization condition (A3), we can choose

$$
\Theta(t)=(1+t)^{\gamma+1}, \quad-1<\gamma<\alpha .
$$

If we take $\mu(t)=(1+t)^{\beta+1}$ with $\alpha-\gamma-1<\beta<2 \alpha+1$, then we have

$$
\rho(t)=(\alpha+1)(1+t)^{\beta} .
$$

Moreover, from the condition (B5) we obtain

$$
\mu(t) \frac{\Theta(t)}{\Lambda(t)}=(1+t)^{\beta+\gamma-\alpha+1} .
$$

Hence, by these choices the conditions (A1) to (A3) and (B1) to (B5) are satisfied.

Example 2.3.2 (Exponential case). Let $\lambda(t)=e^{t}$. Then, we obtain

$$
\Lambda(t)=e^{t} \quad \text { and } \quad \rho(t)=\mu(t) .
$$

If we consider the stabilization condition (A3), we can take

$$
\Theta(t)=e^{r t}, \quad 0<r<1 .
$$

We choose $\rho(t)=\mu(t)=e^{q t}$ with $1-r<q<2$. Then, from the condition (B5) we have

$$
\mu(t) \frac{\Theta(t)}{\Lambda(t)}=e^{(q+r-1) t} .
$$

Hence, by these choices the conditions (A1) to (A3) and (B1) to (B5) are satisfied.

Example 2.3.3 (Super-exponential case). Let $\lambda(t)=e^{t} e^{e^{t}}$. Then, we get

$$
\Lambda(t)=e^{e^{t}} \quad \text { and } \quad \rho(t)=\mu(t) e^{t} .
$$

If we consider the stabilization condition (A3), we can choose

$$
\Theta(t)=e^{r e^{t}}, \quad 0<r<1 .
$$

Let us choose $\mu(t)=e^{q e^{t}}$ with $1-r<q<2$. Then we have

$$
\rho(t)=e^{q e^{t}} e^{t} .
$$

Moreover, from the condition (B5) we obtain

$$
\mu(t) \frac{\Theta(t)}{\Lambda(t)}=e^{(q+r-1) e^{t}} .
$$

Hence, by these choices the conditions (A1) to (A3) and (B1) to (B5) are satisfied.

Here we remark that the conditions (A4), (A5) and (B6) will be verify in the next section for these kind of examples. 


\subsection{Representation of solutions}

In this section we transform our Cauchy problem (2.1.1) with time-dependent propagation speed and dissipation to a Cauchy problem with time-dependent mass. We apply the partial Fourier transformation with respect to spatial variables to the Cauchy problem (2.1.1), we get that $\hat{u}=\hat{u}(t, \xi)=\mathcal{F}_{x \rightarrow \xi}(u(t, x))(t, \xi)$ solves

$$
\begin{cases}\hat{u}_{t t}+\lambda^{2}(t) \omega^{2}(t)|\xi|^{2} \hat{u}+\rho(t) \omega(t) \hat{u}_{t}=0, & (t, \xi) \in[0, \infty) \times \mathbb{R}^{n} \\ \hat{u}(0, \xi)=\hat{u}_{0}(\xi), \quad \hat{u}_{t}(0, \xi)=\hat{u}_{1}(\xi), & \xi \in \mathbb{R}^{n}\end{cases}
$$

Applying the transformation

$$
\hat{u}(t, \xi)=\exp \left(-\frac{1}{2} \int_{0}^{t} \rho(\tau) \omega(\tau) d \tau\right) v(t, \xi),
$$

transfers the Cauchy problem (2.4.1) into

$$
\begin{cases}v_{t t}+m(t, \xi) v=0, & (t, \xi) \in[0, \infty) \times \mathbb{R}^{n} \\ v(0, \xi)=v_{0}(\xi), \quad v_{t}(0, \xi)=v_{1}(\xi), & \xi \in \mathbb{R}^{n}\end{cases}
$$

where

$$
v_{0}(\xi)=\hat{u}_{0}(\xi) \text { and } v_{1}(\xi)=\frac{\rho(0) \omega(0)}{2} \hat{u}_{0}(\xi)+\hat{u}_{1}(\xi)
$$

and the coefficient $m=m(t, \xi)$ of the mass term which is defined by

$$
m(t, \xi):=\lambda^{2}(t) \omega^{2}(t)|\xi|^{2}-\frac{1}{4}(\rho(t) \omega(t))^{2}-\frac{1}{2}(\rho(t) \omega(t))^{\prime} .
$$

By condition (B1) we can rewrite the formula (2.4.3) as

$$
m(t, \xi)=\lambda^{2}(t) \omega^{2}(t)|\xi|^{2}-\frac{1}{4} \mu^{2}(t) \frac{\lambda^{2}(t) \omega^{2}(t)}{\Lambda^{2}(t)}-\frac{1}{2}\left(\mu(t) \frac{\lambda(t) \omega(t)}{\Lambda(t)}\right)^{\prime} .
$$

Due to conditions (B2), (B3) and (B5) we see that $(\rho(t) \omega(t))^{\prime}$ is a negligible term in (2.4.3), that is, it holds $\left|(\rho(t) \omega(t))^{\prime}\right|=o\left((\rho(t) \omega(t))^{2}\right)$ as $t \rightarrow \infty$. Indeed, we have

$$
\begin{aligned}
\frac{\left|(\rho(t) \omega(t))^{\prime}\right|}{(\rho(t) \omega(t))^{2}} & =\frac{\left|\left(\mu(t) \frac{\lambda(t)}{\Lambda(t)} \omega(t)\right)^{\prime}\right|}{\mu^{2}(t) \frac{\lambda^{2}(t)}{\Lambda^{2}(t)} \omega^{2}(t)} \leq \frac{\left|\mu^{\prime}(t)\right| \frac{\lambda(t)}{\Lambda(t)} \omega(t)+\lambda_{1} \mu(t) \frac{\lambda^{2}(t)}{\Lambda^{2}(t)} \omega(t)+\mu(t) \frac{\lambda(t)}{\Lambda(t)}\left|\omega^{\prime}(t)\right|}{\mu^{2}(t) \frac{\lambda^{2}(t)}{\Lambda^{2}(t)} \omega^{2}(t)} \\
& \lesssim \frac{1}{\mu(t)}+\frac{\Xi^{-1}(t)}{\mu(t) \frac{\lambda(t)}{\Lambda(t)}} \lesssim \frac{1}{\mu(t)}+\frac{\frac{\lambda(t)}{\Theta(t)}}{\mu(t) \frac{\lambda(t)}{\Lambda(t)}} \lesssim \frac{1}{\mu(t)}+\frac{1}{\mu(t) \frac{\Theta(t)}{\Lambda(t)}} \rightarrow 0 \quad \text { as } \quad t \rightarrow \infty .
\end{aligned}
$$

Motivated by the above considerations

$$
\lambda^{2}(t) \omega^{2}(t)|\xi|^{2}-\frac{1}{4} \rho^{2}(t) \omega^{2}(t)
$$

can be considered as the principal part of the coefficient $m=m(t, \xi)$ of the mass term $m(t, \xi) v$. Hence, we can introduce a separating curve as

$$
\Gamma:=\left\{(t, \xi) \in[0, \infty) \times \mathbb{R}^{n}:|\xi|=\frac{1}{2} \frac{\mu(t)}{\Lambda(t)}\right\}
$$

which divides the extended phase space into two regions, the hyperbolic region $\Pi_{\text {hyp }}$ and the elliptic region $\Pi_{\text {ell }}$, as follows:

$$
\begin{aligned}
\Pi_{\text {hyp }} & =\left\{(t, \xi) \in[0, \infty) \times \mathbb{R}^{n}:|\xi|>\frac{1}{2} \frac{\mu(t)}{\Lambda(t)}\right\}, \\
\Pi_{\text {ell }} & =\left\{(t, \xi) \in[0, \infty) \times \mathbb{R}^{n}:|\xi|<\frac{1}{2} \frac{\mu(t)}{\Lambda(t)}\right\} .
\end{aligned}
$$


Let us define the auxiliary weight function

$$
\langle\xi\rangle_{\lambda(t), \omega(t)}:=\sqrt{\left.\left|\lambda^{2}(t) \omega^{2}(t)\right| \xi\right|^{2}-\frac{\rho^{2}(t) \omega^{2}(t)}{4} \mid}=\sqrt{\left.\left|\lambda^{2}(t) \omega^{2}(t)\right| \xi\right|^{2}-\mu^{2}(t) \frac{\lambda^{2}(t) \omega^{2}(t)}{4 \Lambda^{2}(t)} \mid} .
$$

Proposition 2.4.1. It holds

$$
\partial_{t}\langle\xi\rangle_{\lambda(t), \omega(t)}= \pm \frac{\lambda(t) \omega(t)(\lambda(t) \omega(t))^{\prime}|\xi|^{2}-\left(\mu(t) \frac{\lambda(t) \omega(t)}{2 \Lambda(t)}\right)\left(\mu(t) \frac{\lambda(t) \omega(t)}{2 \Lambda(t)}\right)^{\prime}}{\langle\xi\rangle_{\lambda(t), \omega(t)}}
$$

and

$$
\partial_{|\xi|}\langle\xi\rangle_{\lambda(t), \omega(t)}= \pm \frac{\lambda^{2}(t) \omega^{2}(t)|\xi|}{\langle\xi\rangle_{\lambda(t), \omega(t)}}
$$

where the upper sign is taken in the hyperbolic region.

\section{Division of the extended phase space}

Now we will divide both regions of the extended phase space into some zones in order to organize the necessary steps of WKB-analysis. Here we follow some ideas of [2]. However, we have to restrict the considerations to a smaller hyperbolic zone and smaller elliptic zone in the extended phase space to cope with stronger oscillations in $\omega=\omega(t)$ in our approach.

The zones are defined as follows:

- hyperbolic zone:

$$
Z_{\text {hyp }}(N)=\left\{(t, \xi) \in[0, \infty) \times \mathbb{R}^{n}:\langle\xi\rangle_{\lambda(t), \omega(t)} \geq N \frac{\rho(t) \omega(t)}{2}\right\} \cap \Pi_{\text {hyp }},
$$

and $\Theta(t)|\xi| \geq N$;

- oscillation subzone:

$$
Z_{\text {osc }}(N, \varepsilon)=\left\{(t, \xi) \in[0, \infty) \times \mathbb{R}^{n}: \varepsilon \frac{\rho(t) \omega(t)}{2} \leq\langle\xi\rangle_{\lambda(t), \omega(t)} \leq N \frac{\rho(t) \omega(t)}{2}\right\} \cap \Pi_{\text {hyp }},
$$

$\Theta(t)|\xi| \leq N$ and $\Lambda(t)|\xi| \geq N$

- reduced zone:

$$
Z_{\mathrm{red}}(\varepsilon)=\left\{(t, \xi) \in[0, \infty) \times \mathbb{R}^{n}:\langle\xi\rangle_{\lambda(t), \omega(t)} \leq \varepsilon \frac{\rho(t) \omega(t)}{2}\right\}
$$

- elliptic zone:

$$
Z_{\mathrm{ell}}\left(d_{0}, \varepsilon\right)=\left\{(t, \xi) \in[0, \infty) \times \mathbb{R}^{n}:|\xi| \geq \frac{d_{0}}{F(\Lambda(t))}\right\} \cap\left\{\langle\xi\rangle_{\lambda(t), \omega(t)} \geq \varepsilon \frac{\rho(t) \omega(t)}{2}\right\} \cap \Pi_{\mathrm{ell}}
$$

- dissipative zone:

$$
Z_{\text {diss }}\left(d_{0}\right)=\left\{(t, \xi) \in[0, \infty) \times \mathbb{R}^{n}:|\xi| \leq \frac{d_{0}}{F(\Lambda(t))}\right\} \cap \Pi_{\text {ell }} .
$$

Here in general, $N$ is a large positive constant and $\varepsilon$ is a small positive constant. Both will be chosen later. Moreover, let us point out that to get a smaller hyperbolic zone we impose an oscillation subzone, however, shrinking the elliptic zone leads to enlarging the dissipative zone.

Let us introduce separating lines between these zones as follows: 
- by $t_{\text {diss }}=t_{\text {diss }}(|\xi|)$, we denote the separating line between the dissipative zone and the elliptic zone;

- by $t_{\text {ell }}=t_{\text {ell }}(|\xi|)$, we denote the separating line between the elliptic zone and the reduced zone;

- by $t_{\text {red }}=t_{\text {red }}(|\xi|)$, we denote the separating line between the reduced zone and oscillation subzone;

- by $t_{\mathrm{osc}}=t_{\mathrm{osc}}(|\xi|)$, we denote the separating line between the oscillation subzone and the hyperbolic zone.

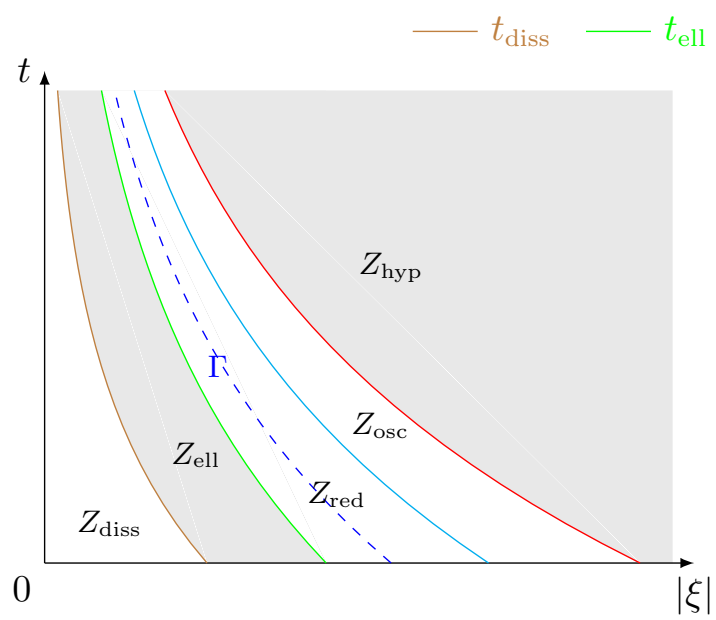

a. The case that $\mu(t) / \Lambda(t)$ is decreasing

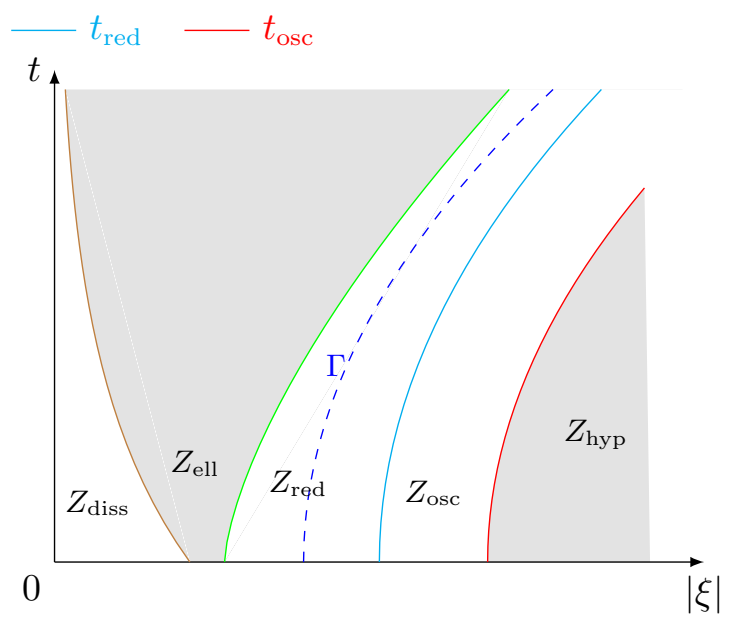

b. The case that $\mu(t) / \Lambda(t)$ is increasing

Fig. 2.1.: Division of extended phase space into zones

Let us introduce

$$
h_{1}(t, \xi)=\chi(|\xi| F(\Lambda(t))) \frac{\lambda(t)}{F(\Lambda(t))}+(1-\chi(|\xi| F(\Lambda(t)))) \lambda(t)|\xi|
$$

and

$$
h_{2}(t, \xi)=\chi\left(\frac{\langle\xi\rangle_{\lambda(t), \omega(t)}}{\varepsilon \frac{\rho(t) \omega(t)}{2}}\right) \varepsilon \frac{\rho(t) \omega(t)}{2}+\left(1-\chi\left(\frac{\langle\xi\rangle_{\lambda(t), \omega(t)}}{\varepsilon \frac{\rho(t) \omega(t)}{2}}\right)\right)\langle\xi\rangle_{\lambda(t), \omega(t)},
$$

where $\chi \in \mathcal{C}^{\infty}([0, \infty))$ such that $\chi(t)=1$ for $0 \leq t \leq \frac{1}{2}$ and $\chi(t)=0$ for $t \geq 1$.

Now let us define the micro-energy

$$
U(t, \xi):=\left(h_{1}(t, \xi) \hat{u}(t, \xi), D_{t} \hat{u}(t, \xi)\right)^{\mathrm{T}} .
$$

Then, we obtain from (2.4.1) the system of first order

$$
D_{t} U(t, \xi)=\underbrace{\left(\begin{array}{cc}
\frac{D_{t} h_{1}(t, \xi)}{h_{1}(t, \xi)} & h_{1}(t, \xi) \\
\frac{\lambda^{2}(t) \omega^{2}(t)|\xi|^{2}}{h_{1}(t, \xi)} & i \rho(t) \omega(t)
\end{array}\right)}_{A(t, \xi)} U(t, \xi)
$$

with the initial condition $U(0, \xi)=\left(h_{1}(0, \xi) \hat{u}(0, \xi), D_{t} \hat{u}(0, \xi)\right)^{\mathrm{T}}$.

On the other hand, we define the micro-energy

$$
V(t, \xi):=\left(h_{2}(t, \xi) v(t, \xi), D_{t} v(t, \xi)\right)^{\mathrm{T}} .
$$


Then, by (2.4.2) we obtain that $V=V(t, \xi)$ satisfies the following system of first order:

$$
D_{t} V(t, \xi)=\underbrace{\left(\begin{array}{cc}
\frac{D_{t} h_{2}(t, \xi)}{h_{2}(t, \xi)} & h_{2}(t, \xi) \\
\frac{m(t, \xi)}{h_{2}(t, \xi)} & 0
\end{array}\right)}_{A_{V}(t, \xi)} V(t, \xi)
$$

with the initial condition $V(0, \xi)=\left(h_{2}(0, \xi) v(0, \xi), D_{t} v(0, \xi)\right)^{\mathrm{T}}$.

Definition 2.4.1. For any $t \geq s \geq 0$, we denote by $E=E(t, s, \xi)$ and $E_{V}=E_{V}(t, s, \xi)$ the fundamental solutions of (2.4.6) and (2.4.7), respectively. That is, the matrix-valued functions which solve the Cauchy problems

$$
D_{t} E(t, s, \xi)=A(t, \xi) E(t, s, \xi), \quad E(s, s, \xi)=I
$$

and

$$
D_{t} E_{V}(t, s, \xi)=A_{V}(t, \xi) E_{V}(t, s, \xi), \quad E_{V}(s, s, \xi)=I,
$$

respectively.

Hence, it is easy to prove that we have $U(t, \xi)=E(t, 0, \xi)\left(h_{1}(0, \xi) \hat{u}(0, \xi), D_{t} \hat{u}(0, \xi)\right)^{\mathrm{T}}$ and $V(t, \xi)=E_{V}(t, 0, \xi)\left(h_{2}(0, \xi) v(0, \xi), D_{t} v(0, \xi)\right)^{\mathrm{T}}$. Moreover, for any $t_{2} \geq t_{1} \geq 0$ it holds $E\left(t, t_{1}, \xi\right)=E\left(t, t_{2}, \xi\right) E\left(t_{2}, t_{1}, \xi\right)$ and $E_{V}\left(t, t_{1}, \xi\right)=E_{V}\left(t, t_{2}, \xi\right) E_{V}\left(t_{2}, t_{1}, \xi\right)$.

Remark 2.4.1. By the previous considerations, after obtaining estimates for $E_{V}=E_{V}(t, s, \xi)$ it is sufficient to apply the backward transformation to the original Cauchy problem. That is, we transform back $E_{V}=E_{V}(t, s, \xi)$ to estimate the fundamental solution $E=E(t, s, \xi)$ which is related to a system of first order for the micro-energy $\left(\lambda(t)|\xi| \hat{u}, D_{t} \hat{u}\right)$, which gives the representation

$$
E(t, s, \xi)=T(t, \xi) E_{V}(t, s, \xi) T^{-1}(s, \xi),
$$

where the matrix $T(t, \xi)$ is defined in the following way:

$$
\left(\begin{array}{c}
\lambda(t)|\xi| \hat{u} \\
D_{t} \hat{u}
\end{array}\right)=\underbrace{\left(\begin{array}{cc}
\frac{\lambda(t)|\xi|}{\delta(t) h_{2}(t, \xi)} & 0 \\
i \frac{\rho(t) \omega(t)}{2 \delta(t) h_{2}(t, \xi)} & \frac{1}{\delta(t)}
\end{array}\right)}_{T(t, \xi)}\left(\begin{array}{c}
h_{2}(t, \xi) v \\
D_{t} v
\end{array}\right)
$$

with the inverse matrix

$$
T^{-1}(t, \xi)=\left(\begin{array}{cc}
\frac{\delta(t) h_{2}(t, \xi)}{\lambda(t)|\xi|} & 0 \\
-i \frac{\rho(t) \omega(t) \delta(t)}{2 \lambda(t)|\xi|} & \delta(t)
\end{array}\right),
$$

where the auxiliary function

$$
\delta(t):=\exp \left(\frac{1}{2} \int_{0}^{t} \rho(\tau) \omega(\tau) d \tau\right)
$$

is related to the damping term $\rho(t) \omega(t) \hat{u}_{t}$.

\subsubsection{Considerations in the hyperbolic zone}

In order to derive a representation of solutions in the hyperbolic zone we apply a diagonalization procedure to a first order system corresponding to the Cauchy problem (2.4.2). This procedure is well-known as WKB-analysis (WKB is an acronym of the physicists WentzelKramers-Brillouin).

Basically, we will consider a first order system with a coefficient matrix composed of a diagonal main part and remainder part. The goal of this diagonalization procedure is to keep 
the diagonal part in every step of the diagonalization. However, after every step we get a "better normwise estimate" for the remaining part in some scale of symbol classes.

An improvement of the diagonalization procedure was developed in [16]. The author performed more diagonalization steps in order to use structural properties of the coefficient matrices by assuming higher regularity of the entries of the matrix.

In the hyperbolic zone, we will apply $M$ steps of diagonalization procedure. After $M$ steps of diagonalization procedure, we can guarantee that the remainder part is uniformly integrable over the hyperbolic zone. Here we follow some ideas of $[16,18,19]$.

First of all let us introduce the following family of symbol classes in the hyperbolic zone.

Definition 2.4.2. A function $f=f(t, \xi)$ belongs to the hyperbolic symbol class $S_{N}^{\ell}\left\{m_{1}, m_{2}\right\}$ of limited smoothness if the estimates

$$
\left|D_{t}^{k} f(t, \xi)\right| \leq C_{k}\langle\xi\rangle_{\lambda(t), \omega(t)}^{m_{1}} \Xi(t)^{-m_{2}-k}
$$

are valid for all $(t, \xi) \in Z_{\text {hyp }}(N)$ and all $k=0,1, \cdots, \ell$ with $\ell \leq M$. Here $M$ is the order of the regularity of the time-dependent coefficients as well as the number of steps of the diagonalization procedure.

We note that in the hyperbolic zone $Z_{\text {hyp }}(N)$, the auxiliary symbol $\langle\xi\rangle_{\lambda(t), \omega(t)}$ can be estimated by

$$
\langle\xi\rangle_{\lambda(t), \omega(t)} \sim \lambda(t)|\xi|
$$

From the definition of the symbol classes we may conclude the following rules.

Proposition 2.4.2. The following statements are true:

1. $S_{N}^{\ell}\left\{m_{1}, m_{2}\right\}$ is a vector space for all nonnegative integers $\ell$;

2. $S_{N}^{\ell}\left\{m_{1}, m_{2}\right\} \cdot S_{N}^{\ell^{\prime}}\left\{m_{1}^{\prime}, m_{2}^{\prime}\right\} \hookrightarrow S_{N}^{\tilde{\ell}}\left\{m_{1}+m_{1}^{\prime}, m_{2}+m_{2}^{\prime}\right\}$ for all nonnegative integers $\ell$ and $\ell^{\prime}$ with $\tilde{\ell}=\min \left\{\ell, \ell^{\prime}\right\}$;

3. $D_{t}^{k} S_{N}^{\ell}\left\{m_{1}, m_{2}\right\} \hookrightarrow S_{N}^{\ell-k}\left\{m_{1}, m_{2}+k\right\}$ for all nonnegative integers $\ell$ with $k \leq \ell$;

4. $S_{N}^{0}\{-M, M+1\} \hookrightarrow L_{\xi}^{\infty} L_{t}^{1}\left(Z_{\text {hyp }}(N)\right)$ with $M$ from condition (A4).

Proof. We only verify the fourth property. Indeed, if $f=f(t, \xi) \in S_{N}^{0}\{-M, M+1\}$, then we have

$$
\begin{aligned}
\int_{t_{\mathrm{osc}}}^{\infty}|f(\tau, \xi)| d \tau & \lesssim \int_{t_{\mathrm{osc}}}^{\infty}\langle\xi\rangle_{\lambda(\tau), \omega(\tau)}^{-M} \Xi^{-M-1}(\tau) d \tau \lesssim \int_{t_{\mathrm{osc}}}^{\infty}|\xi|^{-M} \lambda^{-M}(\tau) \Xi^{-M-1}(\tau) d \tau \\
& \lesssim|\xi|^{-M} \Theta^{-M}\left(t_{\mathrm{osc}}\right) \leq \frac{1}{N^{M}}<\infty
\end{aligned}
$$

where we used (2.4.10), condition (A4) and the definition of the hyperbolic zone, respectively.

Proposition 2.4.3. Assume the conditions (A1), (A2) and (B1), (B2). Then, the following inequalities hold:

1. $\left|D_{t}^{k}\langle\xi\rangle_{\lambda(t), \omega(t)}\right| \lesssim\langle\xi\rangle_{\lambda(t), \omega(t)} \Xi^{-k}(t)$ for all $k=0,1, \cdots, \ell$ with $\ell \leq M$;

2. $\left|D_{t}^{k}(\rho(t) \omega(t))\right| \lesssim\langle\xi\rangle_{\lambda(t), \omega(t)} \Xi^{-k}(t)$ for all $k=0,1, \cdots, \ell$ with $\ell \leq M$.

In the hyperbolic zone we have $h_{2}(t, \xi)=\langle\xi\rangle_{\lambda(t), \omega(t)}$. We introduce the micro-energy $V=V(t, \xi)$ by

$$
V=\left(\langle\xi\rangle_{\lambda(t), \omega(t)} v, D_{t} v\right)^{\mathrm{T}}
$$

Then, it holds

$$
D_{t} V=\left(\begin{array}{cc}
0 & \langle\xi\rangle_{\lambda(t), \omega(t)} \\
\langle\xi\rangle_{\lambda(t), \omega(t)} & 0
\end{array}\right) V+\left(\begin{array}{cc}
\frac{D_{t}\langle\xi\rangle_{\lambda(t), \omega(t)}}{\langle\xi\rangle_{\lambda(t), \omega(t)}} & 0 \\
-\frac{(\rho(t) \omega(t))^{\prime}}{2\langle\xi\rangle_{\lambda(t), \omega(t)}} & 0
\end{array}\right) V .
$$


Let us carry out the first step of the diagonalization procedure. The eigenvalues of the first matrix are $\pm\langle\xi\rangle_{\lambda(t), \omega(t)}$. Thus, the matrix of eigenvectors $P$ and its inverse $P^{-1}$ are

$$
P=\left(\begin{array}{cc}
1 & -1 \\
1 & 1
\end{array}\right) \quad \text { and } \quad P^{-1}=\frac{1}{2}\left(\begin{array}{cc}
1 & 1 \\
-1 & 1
\end{array}\right)
$$

Defining $V^{(0)}:=P^{-1} V$, we get the transformed system

$$
D_{t} V^{(0)}=\left[\mathcal{D}_{0}(t, \xi)+\mathcal{R}_{0}(t, \xi)\right] V^{(0)},
$$

where

$$
\mathcal{D}_{0}(t, \xi)=\left(\begin{array}{cc}
\langle\xi\rangle_{\lambda(t), \omega(t)}+\frac{D_{t}\langle\xi\rangle_{\lambda(t), \omega(t)}}{2\langle\xi\rangle_{\lambda(t), \omega(t)}}-\frac{(\rho(t) \omega(t))^{\prime}}{4\langle\xi\rangle_{\lambda(t), \omega(t)}} & 0 \\
0 & -\langle\xi\rangle_{\lambda(t), \omega(t)}+\frac{D_{t}\langle\xi\rangle_{\lambda(t), \omega(t)}}{2\langle\xi\rangle_{\lambda(t), \omega(t)}}+\frac{(\rho(t) \omega(t))^{\prime}}{4\langle\xi\rangle_{\lambda(t), \omega(t)}}
\end{array}\right)
$$

and

$$
\mathcal{R}_{0}(t, \xi)=\left(\begin{array}{cc}
0 & -\frac{D_{t}\langle\xi\rangle_{\lambda(t), \omega(t)}}{2\langle\xi\rangle_{\lambda(t), \omega(t)}}+\frac{(\rho(t) \omega(t))^{\prime}}{4\langle\xi\rangle_{\lambda(t), \omega(t)}} \\
-\frac{D_{t}\langle\xi\rangle_{\lambda(t), \omega(t)}}{2\langle\xi\rangle_{\lambda(t), \omega(t)}}-\frac{(\rho(t) \omega(t))^{\prime}}{4\langle\xi\rangle_{\lambda(t), \omega(t)}} & 0
\end{array}\right) .
$$

Note that $\mathcal{R}_{0}(t, \xi) \in S_{N}^{M-1}\{0,1\}$. Now we want to carry out further steps of the diagonalization procedure. The goal is to transform the previous system such that the new matrix has diagonal structure and the new remainder belongs to a hyperbolic symbol class.

Lemma 2.4.4. There exists a zone constant $N>0$ such that for any $k=0,1, \cdots, M$ we can find matrices with the following properties:

- the matrices $N_{k}=N_{k}(t, \xi) \in S_{N}^{M-k}\{0,0\}$ are invertible and $N_{k}^{-1}=N_{k}^{-1}(t, \xi) \in$ $S_{N}^{M-k}\{0,0\}$

- the matrices $\mathcal{D}_{k}=\mathcal{D}_{k}(t, \xi) \in S_{N}^{M-k}\{1,0\}$ are diagonal and

$$
\begin{aligned}
& \mathcal{D}_{k}(t, \xi)=\operatorname{diag}\left(\tau_{k}^{+}(t, \xi), \tau_{k}^{-}(t, \xi)\right) \\
& \text { with }\left|\tau_{k}^{+}(t, \xi)-\tau_{k}^{-}(t, \xi)\right| \geq C_{k}\langle\xi\rangle_{\lambda(t), \omega(t)} ;
\end{aligned}
$$

- the matrices $\mathcal{R}_{k}=\mathcal{R}_{k}(t, \xi) \in S_{N}^{M-k}\{-k, k+1\}$ are antidiagonal;

all these matrices are defined in $Z_{\text {hyp }}(N)$ such that the operator identity

$$
\left(D_{t}-\mathcal{D}_{k}(t, \xi)-\mathcal{R}_{k}(t, \xi)\right) N_{k}(t, \xi)=N_{k}(t, \xi)\left(D_{t}-\mathcal{D}_{k+1}(t, \xi)-\mathcal{R}_{k+1}(t, \xi)\right)
$$

is valid.

Proof. The proof goes by direct construction. Let us denote the difference of the diagonal entries by

$$
\delta_{k}(t, \xi)=\tau_{k}^{+}(t, \xi)-\tau_{k}^{-}(t, \xi) .
$$

Assume that we have given a system by $D_{t} V^{(k)}=\left(\mathcal{D}_{k}(t, \xi)+\mathcal{R}_{k}(t, \xi)\right) V^{(k)}$ with

$$
\mathcal{D}_{k}(t, \xi)=\operatorname{diag}\left(\tau_{k}^{+}(t, \xi), \tau_{k}^{-}(t, \xi)\right) \in S_{N}^{M-k}\{1,0\}
$$

satisfying

$$
\left|\delta_{k}(t, \xi)\right|=\left|\tau_{k}^{+}(t, \xi)-\tau_{k}^{-}(t, \xi)\right| \geq C_{k}\langle\xi\rangle_{\lambda(t), \omega(t)}
$$

and an antidiagonal remainder $\mathcal{R}_{k}=\mathcal{R}_{k}(t, \xi) \in S_{N}^{M-k}\{-k, k+1\}$. Then, we can construct

$$
N^{(k)}(t, \xi)=\left(\begin{array}{cc}
0 & -\frac{\left(\mathcal{R}_{k}\right)_{12}}{\delta_{k}} \\
\frac{\left(\mathcal{R}_{k}\right)_{21}}{\delta_{k}} & 0
\end{array}\right) \in S_{N}^{M-k}\{-k-1, k+1\},
$$


such that $N_{k}(t, \xi)=I+N^{(k)}(t, \xi)$ is invertible for a sufficiently large zone constant $N$. Indeed,

$$
\begin{aligned}
\left|N^{(k)}(t, \xi)\right| & \lesssim\langle\xi\rangle_{\lambda(t), \omega(t)}^{-k-1} \Xi^{-k-1}(t) \lesssim \frac{1}{|\xi|^{k+1} \lambda^{k+1}(t) \Xi^{k+1}(t)} \\
& \lesssim \frac{1}{|\xi|^{k+1} \Theta^{k+1}(t)} \leq \frac{1}{N^{k+1}} \rightarrow 0 \text { as } N \rightarrow \infty .
\end{aligned}
$$

Furthermore, by construction

$$
\left[\mathcal{D}_{k}(t, \xi), N_{k}(t, \xi)\right]=-\mathcal{R}_{k}(t, \xi),
$$

such that

$$
\begin{aligned}
B^{(k+1)}(t, \xi) & =\left(D_{t}-\mathcal{D}_{k}(t, \xi)-\mathcal{R}_{k}(t, \xi)\right) N_{k}(t, \xi)-N_{k}(t, \xi)\left(D_{t}-\mathcal{D}_{k}(t, \xi)\right) \\
& =D_{t} N_{k}(t, \xi)-\left[\mathcal{D}_{k}(t, \xi), N_{k}(t, \xi)\right]-\mathcal{R}_{k}(t, \xi) N_{k}(t, \xi) \\
& =D_{t} N_{k}(t, \xi)-\mathcal{R}_{k}(t, \xi)\left(N_{k}(t, \xi)-I\right) \in S_{N}^{M-k-1}\{-k-1, k+2\} .
\end{aligned}
$$

Thus, by defining

$$
\mathcal{D}_{k+1}(t, \xi)=\mathcal{D}_{k}(t, \xi)-\operatorname{diag}\left(N_{k}^{-1}(t, \xi) B^{(k+1)}(t, \xi)\right)
$$

and

$$
\mathcal{R}_{k+1}(t, \xi)=\operatorname{diag}\left(N_{k}^{-1}(t, \xi) B^{(k+1)}(t, \xi)\right)-N_{k}^{-1}(t, \xi) B^{(k+1)}(t, \xi)
$$

we obtain the operator equation

$$
\left(D_{t}-\mathcal{D}_{k}(t, \xi)-\mathcal{R}_{k}(t, \xi)\right) N_{k}(t, \xi)=N_{k}(t, \xi)\left(D_{t}-\mathcal{D}_{k+1}(t, \xi)-\mathcal{R}_{k+1}(t, \xi)\right)
$$

with $\mathcal{D}_{k+1} \in S_{N}^{M-k-1}\{1,0\}$ and $\mathcal{R}_{k+1} \in S_{N}^{M-k-1}\{-k-1, k+2\}$. The estimate for $B^{(k+1)}(t, \xi)$ implies that

$$
\left|\tau_{k+1}^{+}(t, \xi)-\tau_{k+1}^{-}(t, \xi)\right| \geq\left|\tau_{k}^{+}(t, \xi)-\tau_{k}^{-}(t, \xi)\right|-\langle\xi\rangle_{\lambda(t), \omega(t)} \frac{C}{N}
$$

If we choose $N$ sufficiently large, then the statement is proved with $C_{k+1}:=C_{k}-\frac{C}{N}$.

Finally, we obtain for $k=M$ a remainder $\mathcal{R}_{M}=\mathcal{R}_{M}(t, \xi) \in S_{N}^{0}\{-M, M+1\}$, which is uniformly integrable over the hyperbolic zone.

To complete the derivation of our representation we need more information on the diagonal matrices $\mathcal{D}_{k}=\mathcal{D}_{k}(t, \xi)$.

Lemma 2.4.5. The difference of the diagonal entries of $\mathcal{D}_{k}=\mathcal{D}_{k}(t, \xi)$ is real for all $k=$ $0,1, \cdots, M-1$.

Proof. Let us prove this lemma by induction following the diagonalization scheme. We will show that the above statement and the following hypothesis

$\left(H_{k}\right) \mathcal{R}_{k}=\mathcal{R}_{k}(t, \xi)$ has the form $\mathcal{R}_{k}=i\left(\begin{array}{cc}0 & \overline{\beta_{k}} \\ \beta_{k} & 0\end{array}\right)$ with complex-valued $\beta_{k}(t, \xi)$ for all $k=0,1, \cdots, M-1$,

are both valid.

For $k=0$, by the definition of $\mathcal{D}_{0}$ and $\mathcal{R}_{0}$ we see that the assertion $\left(H_{0}\right)$ is satisfied with

$$
\beta_{0}=\beta_{0}(t, \xi)=\frac{\partial_{t}\langle\xi\rangle_{\lambda(t), \omega(t)}}{2\langle\xi\rangle_{\lambda(t), \omega(t)}}+i \frac{(\rho(t) \omega(t))^{\prime}}{4\langle\xi\rangle_{\lambda(t), \omega(t)}}
$$

and

$$
\tau_{0}^{ \pm}=\tau_{0}^{ \pm}(t, \xi)= \pm\langle\xi\rangle_{\lambda(t), \omega(t)}+\frac{1}{i} \frac{\partial_{t}\langle\xi\rangle_{\lambda(t), \omega(t)}}{2\langle\xi\rangle_{\lambda(t), \omega(t)}} \mp \frac{(\rho(t) \omega(t))^{\prime}}{4\langle\xi\rangle_{\lambda(t), \omega(t)}}
$$


Assume that $\left(H_{k}\right)$ is true and we will show that $\left(H_{k}\right)$ implies $\left(H_{k+1}\right)$. The construction implies

$$
N^{(k)}(t, \xi)=\frac{i}{\delta_{k}}\left(\begin{array}{cc}
0 & -\overline{\beta_{k}} \\
\beta_{k} & 0
\end{array}\right) \quad \text { with } \operatorname{det} N^{(k)}=\frac{\left|\beta_{k}\right|^{2}}{\delta_{k}^{2}} \leq c<1
$$

with a suitable choice of the zone constant $N$. Following the diagonalization scheme of $[16,18,19]$ and setting

$$
d_{k}=d_{k}(t, \xi):=\frac{\left|\beta_{k}(t, \xi)\right|^{2}}{\delta_{k}^{2}(t, \xi)}
$$

we obtain

$$
N_{k}^{-1}\left(\mathcal{D}_{k}+\mathcal{R}_{k}\right) N_{k}=\frac{1}{1-d_{k}}\left(\operatorname{diag}\left(\tau_{k}^{+}-d_{k} \tau_{k}^{+}-\delta_{k} d_{k}, \tau_{k}^{-}-d_{k} \tau_{k}^{-}+\delta_{k} d_{k}\right)+d_{k} \mathcal{R}_{k}\right)
$$

and

$$
N_{k}^{-1}\left(D_{t} N_{k}\right)=\frac{1}{1-d_{k}}\left[\left(\begin{array}{cc}
i \frac{\overline{\beta_{k}}}{\delta_{k}} \partial_{t} \frac{\beta_{k}}{\delta_{k}} & 0 \\
0 & i \frac{\beta_{k}}{\delta_{k}} \partial_{t} \overline{\frac{\beta_{k}}{\delta_{k}}}
\end{array}\right)+\left(\begin{array}{cc}
0 & -\partial_{t} \overline{\frac{\bar{\beta}_{k}}{\delta_{k}}} \\
\partial_{t} \frac{\beta_{k}}{\delta_{k}} & 0
\end{array}\right)\right]
$$

such that

$$
\operatorname{Re}\left(\frac{\beta_{k}}{\delta_{k}} \partial_{t} \frac{\overline{\beta_{k}}}{\delta_{k}}\right)=\frac{1}{2}\left(\frac{\beta_{k}}{\delta_{k}} \partial_{t} \frac{\overline{\beta_{k}}}{\delta_{k}}+\frac{\overline{\beta_{k}}}{\delta_{k}} \partial_{t} \frac{\beta_{k}}{\delta_{k}}\right)=\frac{1}{2} \partial_{t} d_{k}=\operatorname{Re}\left(\frac{\overline{\beta_{k}}}{\delta_{k}} \partial_{t} \frac{\beta_{k}}{\delta_{k}}\right)
$$

implies

$$
\tau_{k+1}^{ \pm}=\tau_{k}^{ \pm} \mp \frac{1}{1-d_{k}}\left(d_{k} \delta_{k}+\operatorname{Im}\left(\frac{\beta_{k}}{\delta_{k}} \partial_{t} \frac{\overline{\beta_{k}}}{\delta_{k}}\right)\right)-i \frac{\partial_{t} d_{k}}{2\left(d_{k}-1\right)} .
$$

Hence $\delta_{k+1}$ is real again and $\mathcal{R}_{k+1}$ satisfies $\left(H_{k+1}\right)$ and, therefore, both statements are true for all $k=0,1, \cdots, M-1$.

Now we want to construct the fundamental solution $E_{\text {hyp }}^{V}=E_{\text {hyp }}^{V}(t, s, \xi), 0 \leq s \leq t$, for the operator

$$
D_{t}-\mathcal{D}_{0}(t, \xi)-\mathcal{R}_{0}(t, \xi) .
$$

For this reason after $M$ steps of diagonalization it is sufficient to construct the fundamental solution satisfying the system

$$
D_{t} E_{M}(t, s, \xi)=\left(\mathcal{D}_{M}(t, \xi)+\mathcal{R}_{M}(t, \xi)\right) E_{M}(t, s, \xi), \quad E_{M}(s, s, \xi)=I .
$$

At first we solve the diagonal system

$$
D_{t} \mathcal{E}_{M}(t, s, \xi)=\mathcal{D}_{M}(t, s, \xi) \mathcal{E}_{M}(t, s, \xi), \quad \mathcal{E}_{M}(s, s, \xi)=I, \quad 0 \leq s \leq t .
$$

Its fundamental solution is given by

$$
\mathcal{E}_{M}(t, s, \xi)=\exp \left(i \int_{s}^{t} \mathcal{D}_{M}(\theta, \xi) d \theta\right)=\operatorname{diag}\left(e^{i \int_{s}^{t} \tau_{M}^{+}(\theta, \xi) d \theta}, e^{i \int_{s}^{t} \tau_{M}^{-}(\theta, \xi) d \theta}\right) .
$$

We make the ansatz $E_{M}(t, s, \xi)=\mathcal{E}_{M}(t, s, \xi) \mathcal{Q}_{M}(t, s, \xi)$ with a uniformly bounded and invertible matrix $\mathcal{Q}_{M}=\mathcal{Q}_{M}(t, s, \xi)$. It follows that the matrix $\mathcal{Q}_{M}=\mathcal{Q}_{M}(t, s, \xi)$ satisfies the system

$$
D_{t} \mathcal{Q}_{M}(t, s, \xi)=R_{M}(t, s, \xi) \mathcal{Q}_{M}(t, s, \xi), \quad \mathcal{Q}_{M}(s, s, \xi)=I
$$

with the coefficient matrix

$$
R_{M}(t, s, \xi)=\mathcal{E}_{M}(s, t, \xi) \mathcal{R}_{M}(t, \xi) \mathcal{E}_{M}(t, s, \xi) .
$$

Taking account of $\mathcal{R}_{M}(t, \xi) \in S_{N}^{0}\{-M, M+1\}$ we obtain

$$
\left|R_{M}(t, s, \xi)\right|=\left|\mathcal{R}_{M}(t, \xi)\right| \lesssim\langle\xi\rangle_{\lambda(t), \omega(t)}^{-M} \Xi^{-M-1}(t) \lesssim|\xi|^{-M} \lambda^{-M}(t) \Xi^{-M-1}(t) .
$$


The solution $\mathcal{Q}_{M}=\mathcal{Q}_{M}(t, s, \xi)$ can be represented as Peano-Baker series (see Appendix A.2.1)

$$
\mathcal{Q}_{M}(t, s, \xi)=I+\sum_{k=1}^{\infty} i^{k} \int_{s}^{t} R_{M}\left(t_{1}, s, \xi\right) \int_{s}^{t_{1}} R_{M}\left(t_{2}, s, \xi\right) \cdots \int_{s}^{t_{k-1}} R_{M}\left(t_{k}, s, \xi\right) d t_{k} \cdots d t_{1} .
$$

Then, we obtain the following statement.

Lemma 2.4.6. The fundamental solution $E_{h y p}^{V}=E_{h y p}^{V}(t, s, \xi)$ is representable in the following form:

$$
E_{\text {hyp }}^{V}(t, s, \xi)=P\left(\prod_{k=0}^{M-1} N_{k}(t, \xi)\right) \mathcal{E}_{M}(t, s, \xi) \mathcal{Q}_{M}(t, s, \xi)\left(\prod_{k=0}^{M-1} N_{k}^{-1}(s, \xi)\right) P^{-1}
$$

for all $(t, \xi),(s, \xi) \in Z_{\text {hyp }}(N)$, where

- the matrices $N_{k}=N_{k}(t, \xi)$ and $N_{k}^{-1}=N_{k}^{-1}(t, \xi)$ are uniformly bounded and invertible;

- the matrices $\mathcal{Q}_{M}=\mathcal{Q}_{M}(t, s, \xi)$ and $\mathcal{Q}_{M}^{-1}=\mathcal{Q}_{M}^{-1}(t, s, \xi)$ are uniformly bounded and invertible.

Proof. The standard construction of $\mathcal{Q}_{M}=\mathcal{Q}_{M}(t, s, \xi)$ in terms of a Peano-Baker series implies the uniform bounds for this matrix as follows:

$$
\begin{aligned}
\left|\mathcal{Q}_{M}(t, s, \xi)\right| & \leq \exp \left(\int_{s}^{t}\left|R_{M}(\theta, s, \xi)\right| d \theta\right) \leq \exp \left(\int_{t_{\mathrm{osc}}}^{\infty} C\langle\xi\rangle_{\lambda(\theta), \omega(\theta)}^{-M} \Xi^{-M-1}(\theta) d \theta\right) \\
& \leq \exp \left(\frac{C^{\prime}}{|\xi|^{M} \Theta^{M}\left(t_{\mathrm{osc}}\right)}\right) \leq \exp \left(\frac{C^{\prime}}{N^{M}}\right) \lesssim 1,
\end{aligned}
$$

where we used condition (A4) and the definition of $Z_{\text {hyp }}(N)$, respectively. Moreover, after applying Liouville theorem (cf. Theorem A.2.3) and the invariance of the trace under multiplication we get

$$
\operatorname{det} \mathcal{Q}_{M}(t, s, \xi)=\exp \left(i \int_{s}^{t} \operatorname{tr} R_{M}(\theta, s, \xi) d \theta\right)=\exp \left(i \int_{s}^{t} \operatorname{tr} \mathcal{R}_{M}(\theta, \xi) d \theta\right)=1
$$

and $\left|\mathcal{Q}_{M}^{-1}(t, s, \xi)\right| \lesssim 1$. The proof is complete.

The asymptotic behavior of the fundamental solution $E_{\text {hyp }}^{V}=E_{\text {hyp }}^{V}(t, s, \xi)$ is given by the following statement.

Lemma 2.4.7. Assume the conditions (A1) to (A4) and (B1) to (B3). Then, the fundamental solution $E_{\text {hyp }}^{V}=E_{\text {hyp }}^{V}(t, s, \xi)$, satisfies the estimate

$$
\left(\left|E_{h y p}^{V}(t, s, \xi)\right|\right) \lesssim \frac{\sqrt{\lambda(t)}}{\sqrt{\lambda(s)}}\left(\begin{array}{cc}
1 & 1 \\
1 & 1
\end{array}\right)
$$

uniformly for all $(s, \xi),(t, \xi) \in Z_{\text {hyp }}(N)$.

Proof. The statement of Lemma 2.4.6 implies that

$$
\left|E_{M}(t, s, \xi)\right| \lesssim\left|\mathcal{E}_{M}(t, s, \xi)\right|=\exp \left(-\int_{s}^{t} \operatorname{Im} \tau_{M}^{ \pm}(\theta, \xi) d \theta\right) \quad \text { for } t \rightarrow \infty
$$

uniformly for $(s, \xi),(t, \xi) \in Z_{\text {hyp }}(N)$. We can use our representation of $\tau_{M}^{ \pm}(t, \xi)$ to deduce

$$
\operatorname{Im} \tau_{M}^{+}(t, \xi)=\operatorname{Im} \tau_{M}^{-}(t, \xi)=-\frac{\partial_{t}\langle\xi\rangle_{\lambda(t), \omega(t)}}{2\langle\xi\rangle_{\lambda(t), \omega(t)}}-\sum_{j=1}^{M-1} \frac{\partial_{t} d_{j}(t, \xi)}{2\left(d_{j}(t, \xi)-1\right)},
$$


where $d_{j}=d_{j}(t, \xi)$ is defined in (2.4.12), such that

$$
\begin{aligned}
\exp \left(-\int_{s}^{t} \operatorname{Im} \tau_{M}^{ \pm}(\theta, \xi) d \theta\right) & =\exp \left(\int_{s}^{t} \frac{\partial_{\tau}\langle\xi\rangle_{\lambda(\tau), \omega(\tau)}}{2\langle\xi\rangle_{\lambda(\tau), \omega(\tau)}} d \tau\right) \prod_{j=1}^{M-1}\left(\frac{d_{j}(t, \xi)-1}{d_{j}(s, \xi)-1}\right)^{-\frac{1}{2}} \\
& \lesssim \frac{\sqrt{\langle\xi\rangle_{\lambda(t), \omega(t)}}}{\sqrt{\langle\xi\rangle_{\lambda(s), \omega(s)}}} \lesssim \frac{\sqrt{\lambda(t)|\xi|}}{\sqrt{\lambda(s)|\xi|}} \lesssim \frac{\sqrt{\lambda(t)}}{\sqrt{\lambda(s)}}
\end{aligned}
$$

This completes the proof.

After constructing the fundamental solution $E_{\text {hyp }}^{V}=E_{\text {hyp }}^{V}(t, s, \xi)$ we use the backward "dissipative" transformation to the Fourier transformed original Cauchy problem (2.4.1). Thus, we get the following representation of the fundamental solution $E_{\mathrm{hyp}}=E_{\mathrm{hyp}}(t, s, \xi)$ in the hyperbolic zone.

Corollary 2.4.8. Assume the conditions (A1) to (A4) and (B1) to (B3). Then, the fundamental solution $E_{\text {hyp }}=E_{\text {hyp }}(t, s, \xi)$ satisfies the estimate

$$
\left(\left|E_{\text {hyp }}(t, s, \xi)\right|\right) \lesssim \frac{\sqrt{\lambda(t)}}{\sqrt{\lambda(s)}} \exp \left(-\frac{1}{2} \int_{s}^{t} \rho(\theta) \omega(\theta) d \theta\right)\left(\begin{array}{cc}
1 & 1 \\
1 & 1
\end{array}\right)
$$

for all $(s, \xi),(t, \xi) \in Z_{\text {hyp }}(N)$.

\section{Some examples}

We complete this section with some examples for special coefficient functions in combination with the fourth property of Proposition 5.2.5.

Example 2.4.1 (Polynomial case). Let $\lambda(t)=(\alpha+1)(1+t)^{\alpha}, \alpha>0$. Then, we obtain

$$
\Lambda(t)=(1+t)^{\alpha+1} \text { and } \Theta(t)=(1+t)^{\gamma+1}, \quad-1<\gamma<\alpha .
$$

Moreover, we have

$$
\rho(t)=(\alpha+1)(1+t)^{\beta}, \quad \alpha-\gamma-1<\beta<2 \alpha+1 .
$$

Now from condition (A2) let us choose $\Xi(t)=(1+t)^{\kappa}$ with

$$
1>\kappa \geq \kappa_{M}=1-\alpha+\gamma+\frac{\alpha-\gamma}{M+1} .
$$

Let us suppose that the remainder $\mathcal{R}_{M}=\mathcal{R}_{M}(t, \xi)$ belongs to $S_{N}^{0}\{-M, M+1\}$ in $Z_{\text {hyp }}(N)$. Then, by using the definition of $Z_{\text {hyp }}(N)$, in particular, $(1+t)^{\gamma+1}|\xi| \geq N$, we get

$$
\begin{aligned}
\int_{t_{\mathrm{osc}}}^{\infty}\left|\mathcal{R}_{M}(\tau, \xi)\right| d \tau & \lesssim \int_{t_{\mathrm{osc}}}^{\infty}\langle\xi\rangle_{\lambda(\tau), \omega(\tau)}^{-M}(1+\tau)^{-\kappa(M+1)} d \tau \\
& \lesssim|\xi|^{-M} \int_{t_{\mathrm{osc}}}^{\infty}(1+\tau)^{-\alpha M-\kappa(M+1)} d \tau \lesssim|\xi|^{-M}\left(1+t_{\mathrm{osc}}\right)^{-\alpha M-\kappa(M+1)+1} \\
& \leq N^{-M}\left(1+t_{\mathrm{osc}}\right)^{M(\gamma+1)-\alpha M-\kappa(M+1)+1} \lesssim 1
\end{aligned}
$$

by the condition (2.4.16).

On the other hand, from condition (A5) we obtain

$$
F(\Lambda(t)) \simeq(1+t)^{\alpha+2 \kappa-1}, \quad \alpha+2 \kappa-1>0
$$

By (2.4.16) and (2.4.18) we have

$$
\frac{1-\alpha}{2}>1-\alpha+\gamma+\frac{\alpha-\gamma}{M+1}
$$


Finally, let us consider condition (B6). It holds that

$$
(1+t)^{2 \alpha-\beta+1} \lesssim(1+t)^{2 \alpha+4 \kappa-2}, \quad \kappa \geq \frac{3-\beta}{4} .
$$

We can see that from (2.4.18) and (2.4.19), since $\beta<2 \alpha+1$, it holds

$$
\frac{3-\beta}{4}>\frac{1-\alpha}{2} \text {. }
$$

Therefore, we obtain that the range for admissible $\kappa$ is given by

$$
\kappa \geq \frac{3-\beta}{4} \text {. }
$$

In the case $\alpha=\gamma$, that is, no stabilization condition holds, then (2.4.17) is satisfied with $M=1$, since in this case we have

$$
\Xi^{-1}(t)=\frac{\lambda(t)}{\Lambda(t)}=\frac{\alpha+1}{1+t}
$$

Therefore, the estimate (2.4.14) coincides with the estimates given in [2], in the case of very slow oscillations.

Example 2.4.2 (Exponential case). Let us choose $\lambda(t)=e^{t}$. Then, we have

$$
\Lambda(t)=e^{t} \quad \text { and } \quad \Theta(t)=e^{r t}, \quad 0<r<1 .
$$

Moreover, we have

$$
\rho(t)=e^{q t}, \quad 1-r<q<2 .
$$

Now from the assumption (A2) we choose $\Xi(t)=e^{\kappa t}$ with

$$
0>\kappa \geq \kappa_{M}=r-1+\frac{1-r}{M+1} .
$$

Let us suppose that the remainder $\mathcal{R}_{M}=\mathcal{R}_{M}(t, \xi)$ belongs to $S_{N}^{0}\{-M, M+1\}$ in $Z_{\text {hyp }}(N)$. By using the definition of $Z_{\text {hyp }}(N)$, in particular, $e^{r t}|\xi| \geq N$, we obtain

$$
\begin{aligned}
\int_{t_{\mathrm{osc}}}^{\infty}\left|\mathcal{R}_{M}(\tau, \xi)\right| d \tau & \lesssim \int_{t_{\mathrm{osc}}}^{\infty}\langle\xi\rangle_{\lambda(\tau), \omega(\tau)}^{-M} e^{-\kappa(M+1) \tau} d \tau \lesssim|\xi|^{-M} \int_{t_{\mathrm{osc}}}^{\infty} e^{-M \tau} e^{-\kappa(M+1) \tau} d \tau \\
& \lesssim|\xi|^{-M} e^{(-M-\kappa M-\kappa) t_{\mathrm{osc}}} \leq N^{-M} e^{r M t_{\mathrm{osc}}+(-M-\kappa M-\kappa) t_{\mathrm{osc}}} \lesssim 1
\end{aligned}
$$

by the condition (2.4.22).

On the other hand, from condition (A5) we obtain

$$
F(\Lambda(t)) \simeq e^{(1+2 \kappa) t}, \quad 1+2 \kappa>0 .
$$

By (2.4.22) and (2.4.24) we have

$$
-\frac{1}{2}>r-1+\frac{1-r}{M+1}
$$

Finally, let us consider condition (B6). It holds

$$
e^{(2-q) t} \lesssim e^{(2+4 \kappa) t}, \quad \kappa \geq-\frac{q}{4} .
$$

We can see that from (2.4.24) and (2.4.25), since $q<2$, it holds

$$
-\frac{q}{4}>-\frac{1}{2}
$$


For this reason, we obtain that the range for admissible $q$ is given by

$$
\kappa \geq-\frac{q}{4}
$$

We note that if $r=1$, that is, no stabilization condition holds, then (2.4.23) is satisfied with $M=1$, since in this case we have

$$
\Xi^{-1}(t)=\frac{\lambda(t)}{\Lambda(t)}=1
$$

Therefore, the estimate (2.4.14) coincides with the estimates given in [2], in the case of very slow oscillations.

Example 2.4.3 (Super-exponential case). We choose $\lambda(t)=e^{t} e^{e^{t}}$. Then, we have

$$
\Lambda(t)=e^{e^{t}} \text { and } \Theta(t)=e^{r e^{t}}, 0<r<1 .
$$

Moreover, we have

$$
\rho(t)=e^{t} e^{q e^{t}}, \quad 1-r<q<2 .
$$

Now from the assumption (A2) let us choose $\Xi(t)=e^{-t} e^{\kappa e^{t}}$ with

$$
0>\kappa \geq \kappa_{M}=r-1+\frac{1-r}{M+1} .
$$

If the remainder $\mathcal{R}_{M}$ belongs to $S_{N}^{0}\{-M, M+1\}$ in $Z_{\text {hyp }}(N)$, then by using the definition of $Z_{\text {hyp }}(N)$, in particular, $e^{r e^{t}}|\xi| \geq N$, we obtain

$$
\begin{aligned}
& \int_{t_{\mathrm{osc}}}^{\infty}\left|\mathcal{R}_{M}(\tau, \xi)\right| d \tau \lesssim \int_{t_{\mathrm{osc}}}^{\infty}\langle\xi\rangle_{\lambda(\tau), \omega(\tau)}^{-M} e^{(M+1) \tau} e^{-\kappa(M+1) e^{\tau}} d \tau \\
& \lesssim|\xi|^{-M} \int_{t_{\mathrm{osc}}}^{\infty} e^{-M \tau} e^{-M e^{\tau}} e^{(M+1) \tau} e^{-\kappa(M+1) e^{\tau}} d \tau=|\xi|^{-M} \int_{t_{\mathrm{osc}}}^{\infty} e^{\tau} e^{(-M-\kappa M-\kappa) e^{\tau}} d \tau \\
& \lesssim|\xi|^{-M} e^{(-M-\kappa M-\kappa) e^{t_{\mathrm{osc}}}} \leq N^{-M} e^{r M e^{t_{\mathrm{osc}}}} e^{(-M-\kappa M-\kappa) e^{t_{\mathrm{osc}}}} \lesssim 1,
\end{aligned}
$$

by the condition (2.4.28).

On the other hand, from condition (A5) we obtain

$$
F(\Lambda(t)) \simeq e^{(1+2 \kappa) e^{t}}, \quad 1+2 \kappa>0 .
$$

By (2.4.28) and (2.4.30) we have

$$
-\frac{1}{2}>r-1+\frac{1-r}{M+1} .
$$

Finally, let us consider the assumption (B6). It holds

$$
e^{(2-q) e^{t}} \lesssim e^{(2+4 \kappa) e^{t}}, \quad \kappa \geq-\frac{q}{4}
$$

We can see that from (2.4.30) and (2.4.31), since $q<2$, it holds

$$
-\frac{q}{4}>-\frac{1}{2} \text {. }
$$

For this reason, we obtain that the range for admissible $q$ is given by

$$
\kappa \geq-\frac{q}{4}
$$

We note that if $r=1$, that is, no stabilization condition holds, then, (2.4.29) is satisfied with $M=1$, since in this case we have

$$
\Xi^{-1}(t)=\frac{\lambda(t)}{\Lambda(t)}=e^{t}
$$

Therefore, the estimate (2.4.14) coincides with the estimates given in [2], in the case of very slow oscillations. 


\subsubsection{Considerations in the oscillation subzone}

Basically, what we have done in the hyperbolic zone was to cope with the stronger oscillating behavior of $\omega=\omega(t)$, we have applied more diagonalization steps and restricted our considerations to a smaller hyperbolic zone with respect to the one in the approach from [2]. In other words, we have already chosen the hyperbolic zone as large as possible. For this reason we have a zone between the reduced zone $Z_{\text {red }}(\varepsilon)$ and the hyperbolic zone $Z_{\text {hyp }}(N)$, the so-called oscillation subzone $Z_{\mathrm{osc}}(N, \varepsilon)$.

The following question arises:

How to estimate the fundamental solution $E_{\mathrm{osc}}=E_{\mathrm{osc}}(t, s, \xi)$ in the oscillation subzone $Z_{\text {osc }}(N, \varepsilon)$ ?

The basic ideas are taken from [18, 19]. Essentially, in $Z_{\mathrm{osc}}(N, \varepsilon)$ we relate the fundamental solution $E_{\mathrm{osc}}=E_{\mathrm{osc}}(t, s, \xi)$ to the fundamental solution $E_{\lambda}=E_{\lambda}(t, s, \xi)$ to the corresponding model with $\omega(t) \equiv 1$ and effective dissipation.

Note that $Z_{\text {osc }}(N, \varepsilon) \subset Z_{\text {hyp }}^{\lambda}(N)$ such that we can use the known estimates for $E_{\lambda}=$ $E_{\lambda}(t, s, \xi)$ from [2] (see Lemma 3.5 of [2]). This estimate reads as follows:

$$
\left(\left|E_{\lambda}(t, s, \xi)\right|\right) \lesssim \frac{\sqrt{\lambda(t)}}{\sqrt{\lambda(s)}} \exp \left(-\frac{1}{2} \int_{s}^{t} \rho(\theta) d \theta\right)\left(\begin{array}{cc}
1 & 1 \\
1 & 1
\end{array}\right) .
$$

Let us introduce the micro-energy $U=U(t, \xi)$ by

$$
U(t, \xi)=\left(\lambda(t)|\xi| \hat{u}, D_{t} \hat{u}\right)^{\mathrm{T}} .
$$

Then, we obtain from (2.4.1) the system of first order

$$
D_{t} U=\underbrace{\left(\begin{array}{cc}
\frac{D_{t} \lambda(t)}{\lambda(t)} & \lambda(t)|\xi| \\
\lambda(t) \omega^{2}(t)|\xi| & i \rho(t) \omega(t)
\end{array}\right)}_{A(t, \xi)} U .
$$

Our aim is to construct the corresponding fundamental solution, that is, the matrix-valued solution of the system

$$
D_{t} E_{\mathrm{osc}}(t, s, \xi)=A(t, \xi) E_{\mathrm{osc}}(t, s, \xi), \quad E_{\mathrm{osc}}(s, s, \xi)=I, \quad 0 \leq s \leq t .
$$

If we set formally $\omega(t) \equiv 1$ and define the micro-energy to the corresponding model by

$$
U_{\lambda}(t, \xi)=\left(\lambda(t)|\xi| \hat{u}, D_{t} \hat{u}\right)^{\mathrm{T}},
$$

then, it satisfies the system of first order

$$
D_{t} U_{\lambda}=\underbrace{\left(\begin{array}{cc}
\frac{D_{t} \lambda(t)}{\lambda(t)} & \lambda(t)|\xi| \\
\lambda(t)|\xi| & i \rho(t)
\end{array}\right)}_{A_{\lambda}(t, \xi)} U_{\lambda} .
$$

We denote the corresponding fundamental solution as $E_{\lambda}=E_{\lambda}(t, s, \xi)$, i.e., the solution to

$$
D_{t} E_{\lambda}(t, s, \xi)=A_{\lambda}(t, \xi) E_{\lambda}(t, s, \xi), \quad E_{\lambda}(s, s, \xi)=I, 0 \leq s \leq t .
$$

An estimate of the fundamental solution $E_{\lambda}=E_{\lambda}(t, s, \xi)$ is essentially given by the $\mathcal{C}^{2}$ theory. Hence, in $Z_{\mathrm{osc}}(N, \varepsilon)$ we relate $E_{\mathrm{osc}}(t, s, \xi)$ to $E_{\lambda}(t, s, \xi)$ and use the stabilization condition (A3).

Corollary 2.4.9. Assume conditions (A1) to (A3) and (B1). Then, the fundamental solution $E_{\text {osc }}=E_{\text {osc }}(t, s, \xi)$ satisfies the estimate

$$
\left(\left|E_{\text {osc }}(t, s, \xi)\right|\right) \lesssim \frac{\sqrt{\lambda(t)}}{\sqrt{\lambda(s)}} \exp \left(-\frac{1}{2} \int_{s}^{t} \rho(\theta) d \theta\right)\left(\begin{array}{ll}
1 & 1 \\
1 & 1
\end{array}\right)
$$

uniformly for $(s, \xi),(t, \xi) \in Z_{\text {osc }}(N, \varepsilon), 0 \leq s \leq t$. 
Proof. In order to relate $E_{\mathrm{osc}}=E_{\mathrm{osc}}(t, s, \xi)$ to $E_{\lambda}=E_{\lambda}(t, s, \xi)$ in $Z_{\mathrm{osc}}(N, \varepsilon)$ we make the ansatz

$$
E_{\mathrm{osc}}(t, s, \xi)=E_{\lambda}(t, s, \xi) \mathcal{Q}_{\mathrm{osc}}(t, s, \xi) .
$$

It follows that the matrix $\mathcal{Q}_{\mathrm{osc}}=\mathcal{Q}_{\mathrm{osc}}(t, s, \xi)$ satisfies

$$
D_{t} \mathcal{Q}_{\mathrm{osc}}(t, s, \xi)=E_{\lambda}(s, t, \xi)\left(A(t, \xi)-A_{\lambda}(t, \xi)\right) E_{\lambda}(t, s, \xi) \mathcal{Q}_{\mathrm{osc}}(t, s, \xi)
$$

with initial condition $\mathcal{Q}_{\text {osc }}=\mathcal{Q}_{\text {osc }}(s, s, \xi)=I$. Taking account of $E_{\lambda}^{-1}(t, s, \xi)=E_{\lambda}(s, t, \xi)$ we derive

$$
\begin{aligned}
\left|E_{\lambda}(s, t, \xi)\left(A(t, \xi)-A_{\lambda}(t, \xi)\right) E_{\lambda}(t, s, \xi)\right| & \lesssim\left|A(t, \xi)-A_{\lambda}(t, \xi)\right| \\
& \lesssim \lambda(t)|\xi|\left|\omega^{2}(t)-1\right|+\rho(t)|\omega(t)-1| \\
& \lesssim \lambda(t)|\xi||\omega(t)-1|+\mu(t) \frac{\lambda(t)}{\Lambda(t)}|\omega(t)-1| \\
& \lesssim \lambda(t)|\xi||\omega(t)-1|,
\end{aligned}
$$

where we used the definition of the hyperbolic region. Hence, applying the Peano-Baker formula and using the stabilization condition (A3), we get the uniform boundedness for the matrix $\mathcal{Q}_{\text {osc }}=\mathcal{Q}_{\text {osc }}(t, s, \xi)$ over $Z_{\text {osc }}(N, \varepsilon)$ as follows:

$$
\begin{aligned}
\left|\mathcal{Q}_{\mathrm{osc}}(t, s, \xi)\right| & \leq \exp \left(C|\xi| \int_{t_{\mathrm{red}}}^{t_{\mathrm{osc}}} \lambda(\tau)|\omega(\tau)-1| d \tau\right) \\
& \leq \exp \left(C^{\prime}|\xi| \Theta\left(t_{\mathrm{osc}}\right)\right) \lesssim 1
\end{aligned}
$$

Furthermore, we get $\left|\operatorname{det} \mathcal{Q}_{\text {osc }}(t, s, \xi)\right|=1$ from Liouville theorem and conclude that $\mathcal{Q}_{\text {osc }}=$ $\mathcal{Q}_{\text {osc }}(t, s, \xi)$ is uniformly invertible within $Z_{\text {osc }}(N, \varepsilon)$. Thus, we arrive at the desired estimate for the fundamental solution $E_{\mathrm{osc}}=E_{\mathrm{osc}}(t, s, \xi)$. This completes the proof.

\subsubsection{Considerations in the elliptic zone}

In a similar manner as in the hyperbolic zone, we will try to cope with the strong oscillating behavior of $\omega=\omega(t)$ by shrinking the elliptic zone. This leads to enlarging the dissipative zone in the extended phase space since we do not propose any oscillation subzone between these two zones.

In the elliptic zone we can follow the standard diagonalization procedure. However, contrary to the hyperbolic zone, we will not perform more diagonalization steps to obtain the fundamental solution. We follow some ideas of [2] and [48].

Now let us introduce the following family of symbol classes in the elliptic zone $Z_{\text {ell }}\left(d_{0}, \varepsilon\right)$. The following definition of symbol classes characterizes the necessary properties of the remainder.

Definition 2.4.3. A function $f=f(t, \xi)$ belongs to the elliptic symbol class $S^{\ell}\left\{m_{1}, m_{2}\right\}$ of limited smoothness if the derivatives of $f$ satisfy the estimates

$$
\left|D_{t}^{k} f(t, \xi)\right| \leq C_{k}\langle\xi\rangle_{\lambda(t), \omega(t)}^{m_{1}} \Xi(t)^{-m_{2}-k}
$$

for all $(t, \xi) \in Z_{\text {ell }}\left(d_{0}, \varepsilon\right)$ and all $k \leq \ell$ with $2 \leq \ell$.

Note that the auxiliary symbol $\langle\xi\rangle_{\lambda(t), \omega(t)}$ in $Z_{\text {ell }}\left(d_{0}, \varepsilon\right)$ can be estimated by

$$
\langle\xi\rangle_{\lambda(t), \omega(t)} \sim \frac{\rho(t)}{2} \sim \mu(t) \frac{\lambda(t)}{2 \Lambda(t)} .
$$

Some useful properties of the symbolic calculus are collected in the following proposition.

Proposition 2.4.10. The following statements are true: 
1. $S^{\ell}\left\{m_{1}, m_{2}\right\}$ is a vector space for all nonnegative integers $\ell$;

2. $S^{\ell}\left\{m_{1}, m_{2}\right\} \cdot S_{N}^{\ell^{\prime}}\left\{m_{1}^{\prime}, m_{2}^{\prime}\right\} \hookrightarrow S^{\tilde{\ell}}\left\{m_{1}+m_{1}^{\prime}, m_{2}+m_{2}^{\prime}\right\}$ for all nonnegative integers $\ell$ and $\ell^{\prime}$ with $\tilde{\ell}=\min \left\{\ell, \ell^{\prime}\right\}$;

3. $D_{t}^{k} S^{\ell}\left\{m_{1}, m_{2}\right\} \hookrightarrow S^{\ell-k}\left\{m_{1}, m_{2}+k\right\}$ for all nonnegative integers $\ell$ with $k \leq \ell$;

4. $S^{\ell-2}\{-1,2\} \hookrightarrow L_{\xi}^{\infty} L_{t}^{1}\left(Z_{\text {ell }}\left(d_{0}, \varepsilon\right)\right)$ with $\ell \geq 2$.

Proof. We only verify the integrability statement. Indeed, if $f=f(t, \xi) \in S^{\ell-2}\{-1,2\}$, then it holds

$$
\begin{aligned}
\int_{t_{\mathrm{diss}}}^{t_{\mathrm{ell}}}|f(\tau, \xi)| d \tau & \lesssim \int_{t_{\mathrm{diss}}}^{t_{\mathrm{ell}}} \frac{\Xi^{-2}(\tau)}{\langle\xi\rangle_{\lambda(\tau), \omega(\tau)}} d \tau \lesssim \int_{t_{\mathrm{diss}}}^{t_{\mathrm{ell}}} \frac{\Lambda(\tau)}{\mu(\tau) \lambda(\tau)} \frac{\lambda^{2}(\tau) F^{\prime}(\Lambda(\tau))}{F^{2}(\Lambda(\tau))} d \tau \\
& \lesssim \frac{1}{|\xi|} \int_{t_{\mathrm{diss}}}^{t_{\mathrm{ell}}} \frac{\lambda(\tau) F^{\prime}(\Lambda(\tau))}{F^{2}(\Lambda(\tau))} d \tau=-\left.\frac{1}{|\xi|} \frac{1}{F(\Lambda(\tau))}\right|_{t_{\mathrm{diss}}} ^{t_{\mathrm{ell}}} \lesssim \frac{1}{|\xi| F\left(\Lambda\left(t_{\mathrm{diss}}\right)\right)} \lesssim 1
\end{aligned}
$$

where we used (2.4.35), definition of the elliptic region, $\Xi(t)=\frac{F(\Lambda(t))}{\lambda(t) \sqrt{F^{\prime}(\Lambda(t))}}$ and $|\xi| F\left(\Lambda\left(t_{\text {diss }}\right)\right)=$ $d_{0}$, respectively.

In the elliptic zone we introduce the micro-energy $V=V(t, \xi)$ by

$$
V=\left(\langle\xi\rangle_{\lambda(t), \omega(t)} v, D_{t} v\right)^{\mathrm{T}}
$$

for all $t \geq s$ and $(t, \xi),(s, \xi) \in Z_{\text {ell }}\left(d_{0}, \varepsilon\right)$. Then, the corresponding first order system to the Cauchy problem (2.4.2), with respect to the micro-energy $V$, is stated as

$$
D_{t} V=\left(\begin{array}{cc}
0 & \langle\xi\rangle_{\lambda(t), \omega(t)} \\
-\langle\xi\rangle_{\lambda(t), \omega(t)} & 0
\end{array}\right) V+\left(\begin{array}{cc}
\frac{D_{t}\langle\xi\rangle_{\lambda(t), \omega(t)}}{\langle\xi\rangle_{\lambda(t), \omega(t)}} & 0 \\
-\frac{(\rho(t) \omega(t))^{\prime}}{2\langle\xi\rangle_{\lambda(t), \omega(t)}} & 0
\end{array}\right) V .
$$

Performing the diagonalization procedure we get after the second step of the diagonalization that the entries of the remainder matrix are uniformly integrable over the elliptic zone.

Step 1. We denote by $P$ the matrix

$$
P=\left(\begin{array}{cc}
i & -i \\
1 & 1
\end{array}\right)
$$

consisting of eigenvectors of the first matrix on the right-hand side with the inverse matrix

$$
P^{-1}=\frac{1}{2}\left(\begin{array}{cc}
-i & 1 \\
i & 1
\end{array}\right)
$$

Then, defining $V^{(0)}:=P^{-1} V$ we get the system

$$
D_{t} V^{(0)}=[\mathcal{D}(t, \xi)+\mathcal{R}(t, \xi)] V^{(0)},
$$

where

$$
\mathcal{D}(t, \xi)=\left(\begin{array}{cc}
-i\langle\xi\rangle_{\lambda(t), \omega(t)} & 0 \\
0 & i\langle\xi\rangle_{\lambda(t), \omega(t)}
\end{array}\right)
$$

and

$$
\mathcal{R}(t, \xi)=\frac{1}{2}\left(\begin{array}{cc}
\frac{D_{t}\langle\xi\rangle_{\lambda(t), \omega(t)}}{2\langle\xi\rangle_{\lambda(t), \omega(t)}}-i \frac{(\rho(t) \omega(t))^{\prime}}{4\langle\xi\rangle_{\lambda(t), \omega(t)}} & -\frac{D_{t}\langle\xi\rangle_{\lambda(t), \omega(t)}}{2\langle\xi\rangle_{\lambda(t), \omega(t)}}+i \frac{(\rho(t) \omega(t))^{\prime}}{4\langle\xi\rangle_{\lambda(t), \omega(t)}} \\
-\frac{D_{t}\langle\xi\rangle_{\lambda(t), \omega(t)}}{2\langle\xi\rangle_{\lambda(t), \omega(t)}}-i \frac{(\rho(t) \omega(t))^{\prime}}{4\langle\xi\rangle_{\lambda(t), \omega(t)}} & \frac{D_{t}\langle\xi\rangle_{\lambda(t), \omega(t)}}{2\langle\xi\rangle_{\lambda(t), \omega(t)}}+i \frac{(\rho(t) \omega(t))^{\prime}}{4\langle\xi\rangle_{\lambda(t), \omega(t)}}
\end{array}\right) .
$$

Then, we obtain

$$
\mathcal{D}(t, \xi) \in S^{M}\{1,0\}, \quad \mathcal{R}(t, \xi) \in S^{M-1}\{0,1\} .
$$


Step 2. Let us introduce $F_{0}(t, \xi)=\operatorname{diag} \mathcal{R}(t, \xi)$. Now we carry out the next step(s) of diagonalization. The difference of the diagonal entries of the matrix $\mathcal{D}(t, \xi)+F_{0}(t, \xi)$ is

$$
i \alpha(t, \xi)=2\langle\xi\rangle_{\lambda(t), \omega(t)}+\frac{(\rho(t) \omega(t))^{\prime}}{2\langle\xi\rangle_{\lambda(t), \omega(t)}} \sim 2\langle\xi\rangle_{\lambda(t), \omega(t)}+\frac{o\left(\rho^{2}(t) \omega^{2}(t)\right)}{2\langle\xi\rangle_{\lambda(t), \omega(t)}} \sim\langle\xi\rangle_{\lambda(t), \omega(t)}
$$

for $t \geq t_{0}$ with a sufficiently large $t_{0}=t_{0}(\varepsilon)$ by using $\left|(\rho(t) \omega(t))^{\prime}\right|=o\left(\rho^{2}(t) \omega^{2}(t)\right)$.

Now we can follow the usual diagonalization procedure. Therefore, we choose a matrix $N^{(1)}=N^{(1)}(t, \xi)$. The choice of this matrix was proposed in [49]. Let

$$
N^{(1)}(t, \xi)=\left(\begin{array}{cc}
0 & -\frac{\mathcal{R}_{12}}{\alpha(t, \xi)} \\
\frac{\mathcal{R}_{21}}{\alpha(t, \xi)} & 0
\end{array}\right) \sim\left(\begin{array}{cc}
0 & i \frac{D_{t}\langle\xi\rangle_{\lambda(t), \omega(t)}}{4\langle\xi\rangle_{\lambda(t), \omega(t)}^{2}}-\frac{(\rho(t) \omega(t))^{\prime}}{8\langle\xi\rangle_{\lambda(t), \omega(t)}^{2}} \\
i \frac{D_{t}\langle\xi\rangle_{\lambda(t), \omega(t)}}{4\langle\xi\rangle_{\lambda(t), \omega(t)}^{2}}+\frac{(\rho(t) \omega(t))^{\prime}}{8\langle\xi\rangle_{\lambda(t), \omega(t)}^{2}} & 0
\end{array}\right) .
$$

Taking into consideration the rules of the symbolic calculus we have

$$
N^{(1)}(t, \xi) \in S^{M-1}\{-1,1\} \quad \text { and } \quad N_{1}(t, \xi)=I+N^{(1)}(t, \xi) \in S^{M-1}\{0,0\} .
$$

For a sufficiently large time $t \geq t_{0}$ the matrix $N_{1}=N_{1}(t, \xi)$ is invertible with uniformly bounded inverse $N_{1}^{-1}=N_{1}^{-1}(t, \xi)$. Indeed, in the elliptic zone due to condition (B5), it holds

Let

$$
\left|N_{1}(t, \xi)-I\right| \leq \frac{\Xi^{-1}(t)}{\langle\xi\rangle_{\lambda(t), \omega(t)}} \lesssim \frac{\Lambda(t)}{\mu(t) \Theta(t)} \rightarrow 0 \quad \text { for } \quad t \rightarrow \infty
$$

$$
\begin{aligned}
B^{(1)}(t, \xi)= & D_{t} N^{(1)}(t, \xi)-\left(\mathcal{R}(t, \xi)-F_{0}(t, \xi)\right) N^{(1)}(t, \xi) \in S^{M-2}\{-1,2\} \\
& \mathcal{R}_{1}(t, \xi)=-N_{1}^{-1}(t, \xi) B^{(1)}(t, \xi) \in S^{M-2}\{-1,2\} .
\end{aligned}
$$

Then, we have the following operator identity:

$$
\left(D_{t}-\mathcal{D}(t, \xi)-\mathcal{R}(t, \xi)\right) N_{1}(t, \xi)=N_{1}(t, \xi)\left(D_{t}-\mathcal{D}(t, \xi)-F_{0}(t, \xi)-\mathcal{R}_{1}(t, \xi)\right) .
$$

Hence, the previous steps of the diagonalization give us the following lemma.

Lemma 2.4.11. Assume that $\lambda=\lambda(t), \omega=\omega(t)$ satisfy the conditions (A1), (A2) and (A5) and $\rho=\rho(t)$ and $\omega=\omega(t)$ satisfy the conditions (B1), (B2) and (B5). Then, there exists a sufficiently large $t_{0}$ such that in $Z_{\text {ell }}\left(d_{0}, \varepsilon\right)$ the following statements hold:

- $N_{1} \in S^{M-1}\{0,0\}$, invertible for $(t, \xi) \in Z_{\text {ell }}\left(d_{0}, \varepsilon\right)$ with $N_{1}^{-1} \in S^{M-1}\{0,0\}$;

- $F_{0}=\operatorname{diag}\left(\frac{D_{t}\langle\xi\rangle_{\lambda(t), \omega(t)}}{2\langle\xi\rangle_{\lambda(t), \omega(t)}}-i \frac{(\rho(t) \omega(t))^{\prime}}{4\langle\xi\rangle_{\lambda(t), \omega(t)}}, \frac{D_{t}\langle\xi\rangle_{\lambda(t), \omega(t)}}{2\langle\xi\rangle_{\lambda(t), \omega(t)}}+i \frac{(\rho(t) \omega(t))^{\prime}}{4\langle\xi\rangle_{\lambda(t), \omega(t)}}\right) \in S^{M-1}\{0,1\}$;

- $\mathcal{R}_{1} \in S^{M-2}\{-1,2\}$ with $M \geq 2$.

Moreover, the operator identity

$$
\left(D_{t}-\mathcal{D}(t, \xi)-\mathcal{R}(t, \xi)\right) N_{1}(t, \xi)=N_{1}(t, \xi)\left(D_{t}-\mathcal{D}(t, \xi)-F_{0}(t, \xi)-\mathcal{R}_{1}(t, \xi)\right)
$$

holds for all $(t, \xi) \in Z_{\text {ell }}\left(d_{0}, \varepsilon\right)$.

Step 3. Construction of the fundamental solution. In order to solve the transformed system and construct its fundamental solution we can not follow the considerations from the theory of the hyperbolic zone, since the main diagonal entries are purely imaginary.

Lemma 2.4.12. Assume the conditions (A1), (A2), (A5) and (B1), (B2), (B5). Then, the fundamental solution $E_{\text {ell }}^{V}=E_{\text {ell }}^{V}(t, s, \xi)$ to the transformed operator

$$
D_{t}-\mathcal{D}(t, \xi)-F_{0}(t, \xi)-\mathcal{R}_{1}(t, \xi)
$$

can be estimated by

$$
\left(\left|E_{e l l}^{V}(t, s, \xi)\right|\right) \lesssim \frac{\langle\xi\rangle_{\lambda(t), \omega(t)}}{\langle\xi\rangle_{\lambda(s), \omega(s)}} \exp \left(\int_{s}^{t}\langle\xi\rangle_{\lambda(\tau), \omega(\tau)} d \tau\right)\left(\begin{array}{ll}
1 & 1 \\
1 & 1
\end{array}\right),
$$

with $(t, \xi),(s, \xi) \in Z_{\text {ell }}\left(d_{0}, \varepsilon\right) \cap\left\{t \geq t_{0}(\varepsilon)\right\}, 0 \leq s \leq t$. 
Proof. We transform the system for $E_{\text {ell }}^{V}=E_{\text {ell }}^{V}(t, s, \xi)$ to an integral equation for a new matrix-valued function $\mathcal{Q}_{\text {ell }}=\mathcal{Q}_{\text {ell }}(t, s, \xi)$. If we differentiate the term

$$
\exp \left\{-i \int_{s}^{t}\left(\mathcal{D}(\tau, \xi)+F_{0}(\tau, \xi)\right) d \tau\right\} E_{\mathrm{ell}}^{V}(t, s, \xi),
$$

then we obtain

$$
\begin{aligned}
D_{t}\left(\exp \left\{-i \int_{s}^{t}\left(\mathcal{D}(\tau, \xi)+F_{0}(\tau, \xi)\right) d \tau\right\} E_{\mathrm{ell}}^{V}(t, s, \xi)\right) \\
=-\left(\mathcal{D}(t, \xi)+F_{0}(t, \xi)\right) \exp \left\{-i \int_{s}^{t}\left(\mathcal{D}(\tau, \xi)+F_{0}(\tau, \xi)\right) d \tau\right\} E_{\mathrm{ell}}^{V}(t, s, \xi) \\
\quad+\exp \left\{-i \int_{s}^{t}\left(\mathcal{D}(\tau, \xi)+F_{0}(\tau, \xi)\right) d \tau\right\}\left(\mathcal{D}(t, \xi)+F_{0}(t, \xi)+\mathcal{R}_{1}(t, \xi)\right) E_{\mathrm{ell}}^{V}(t, s, \xi) \\
=\exp \left\{-i \int_{s}^{t}\left(\mathcal{D}(\tau, \xi)+F_{0}(\tau, \xi)\right) d \tau\right\} \mathcal{R}_{1}(t, \xi) E_{\mathrm{ell}}^{V}(t, s, \xi) .
\end{aligned}
$$

Hence, by integration on the interval $[s, t]$, we obtain that $E_{\text {ell }}^{V}=E_{\text {ell }}^{V}(t, s, \xi)$ satisfies the following integral equation:

$$
\begin{aligned}
E_{\mathrm{ell}}^{V}(t, s, \xi)= & \exp \left\{i \int_{s}^{t}\left(\mathcal{D}(\tau, \xi)+F_{0}(\tau, \xi)\right) d \tau\right\} E_{\mathrm{ell}}^{V}(s, s, \xi) \\
& +i \int_{s}^{t} \exp \left\{i \int_{\theta}^{t}\left(\mathcal{D}(\tau, \xi)+F_{0}(\tau, \xi)\right) d \tau\right\} \mathcal{R}_{1}(\theta, \xi) E_{\mathrm{ell}}^{V}(\theta, s, \xi) d \theta
\end{aligned}
$$

In general, the exponential term is not bounded if $\rho=\rho(t)$ is increasing. In order to compensate this "bad behavior" we introduce a weight factor. Let us define

$$
\mathcal{Q}_{\mathrm{ell}}(t, s, \xi)=\exp \left\{-\int_{s}^{t} \beta(\tau, \xi) d \tau\right\} E_{\mathrm{ell}}^{V}(t, s, \xi),
$$

with a suitable $\beta=\beta(t, \xi)$. It satisfies the new integral equation

$$
\begin{aligned}
\mathcal{Q}_{\mathrm{ell}}(t, s, \xi)=\exp & \left\{\int_{s}^{t}\left(i \mathcal{D}(\tau, \xi)+i F_{0}(\tau, \xi)-\beta(\tau, \xi) I\right) d \tau\right\} \\
& +\int_{s}^{t} \exp \left\{\int_{\theta}^{t}\left(i \mathcal{D}(\tau, \xi)+i F_{0}(\tau, \xi)-\beta(\tau, \xi) I\right) d \tau\right\} \mathcal{R}_{1}(\theta, \xi) \mathcal{Q}_{\mathrm{ell}}(\theta, s, \xi) d \theta .
\end{aligned}
$$

The function $\mathcal{R}_{1}=\mathcal{R}_{1}(\theta, \xi) \in S^{M-2}\{-1,2\}$ is uniformly integrable for $M \geq 2$ over the elliptic zone by Proposition 2.4.10. Hence, if the exponential term is bounded, then the solution $\mathcal{Q}_{\text {ell }}=\mathcal{Q}_{\text {ell }}(t, s, \xi)$ of the integral equation is uniformly bounded over the elliptic zone for a suitable weight $\beta(t, \xi)$. It remains to see that the exponential term remains bounded and this is guaranteed by a sign condition on the exponent.

The main entries of the diagonal matrix $i \mathcal{D}(t, \xi)+i F_{0}(t, \xi)$ are given by

$$
\begin{aligned}
(I) & =\langle\xi\rangle_{\lambda(t), \omega(t)}+\frac{\partial_{t}\langle\xi\rangle_{\lambda(t), \omega(t)}}{2\langle\xi\rangle_{\lambda(t), \omega(t)}}+\frac{(\rho(t) \omega(t))^{\prime}}{4\langle\xi\rangle_{\lambda(t), \omega(t)}}, \\
(I I) & =-\langle\xi\rangle_{\lambda(t), \omega(t)}+\frac{\partial_{t}\langle\xi\rangle_{\lambda(t), \omega(t)}}{2\langle\xi\rangle_{\lambda(t), \omega(t)}}-\frac{(\rho(t) \omega(t))^{\prime}}{4\langle\xi\rangle_{\lambda(t), \omega(t)}} .
\end{aligned}
$$

For the difference $(I I)-(I)$ we get

$$
(I I)-(I)=-2\langle\xi\rangle_{\lambda(t), \omega(t)}-\frac{(\rho(t) \omega(t))^{\prime}}{2\langle\xi\rangle_{\lambda(t), \omega(t)}}=-\frac{\rho^{2}(t) \omega^{2}(t)+(\rho(t) \omega(t))^{\prime}-4 \lambda^{2}(t) \omega^{2}(t)|\xi|^{2}}{2\langle\xi\rangle_{\lambda(t), \omega(t)}} \leq 0
$$


in $Z_{\text {ell }}\left(d_{0}, \varepsilon\right)$ for $t \geq t_{0}$ by using $\left|(\rho(t) \omega(t))^{\prime}\right|=o\left(\rho^{2}(t) \omega^{2}(t)\right)$. It follows that the term $(I)$ is dominant. Therefore, we choose the weight $\beta(t, \xi)=(I)$. By this choice, we get

$$
i \mathcal{D}(\tau, \xi)+i F_{0}(\tau, \xi)-\beta(\tau, \xi) I=\left(\begin{array}{cc}
0 & 0 \\
0 & -2\langle\xi\rangle_{\lambda(\tau), \omega(\tau)}-\frac{(\rho(\tau) \omega(\tau))^{\prime}}{2\langle\xi\rangle_{\lambda(\tau), \omega(\tau)}}
\end{array}\right) .
$$

It follows

$$
\begin{aligned}
H(t, s, \xi) & =\exp \left\{\int_{s}^{t}\left(i \mathcal{D}(\tau, \xi)+i F_{0}(\tau, \xi)-\beta(\tau, \xi) I\right) d \tau\right\} \\
& =\operatorname{diag}\left(1, \exp \left\{\int_{s}^{t}\left(-2\langle\xi\rangle_{\lambda(\tau), \omega(\tau)}-\frac{(\rho(\tau) \omega(\tau))^{\prime}}{2\langle\xi\rangle_{\lambda(\tau), \omega(\tau)}}\right) d \tau\right\}\right) \rightarrow\left(\begin{array}{ll}
1 & 0 \\
0 & 0
\end{array}\right)
\end{aligned}
$$

as $t \rightarrow \infty$ for any fixed $s$. Hence, the matrix $H=H(t, s, \xi)$ is uniformly bounded for $(s, \xi),(t, \xi) \in Z_{\text {ell }}\left(d_{0}, \varepsilon\right)$. So, the representation of $\mathcal{Q}_{\text {ell }}=\mathcal{Q}_{\text {ell }}(t, s, \xi)$ by a Neumann series gives

$$
\begin{array}{r}
\mathcal{Q}_{\mathrm{ell}}(t, s, \xi)=H(t, s, \xi)+\sum_{k=1}^{\infty} i^{k} \int_{s}^{t} H\left(t, t_{1}, \xi\right) \mathcal{R}_{1}\left(t_{1}, \xi\right) \int_{s}^{t_{1}} H\left(t_{1}, t_{2}, \xi\right) \mathcal{R}_{1}\left(t_{2}, \xi\right) \\
\times \cdots \int_{s}^{t_{k-1}} H\left(t_{k-1}, t_{k}, \xi\right) \mathcal{R}_{1}\left(t_{k}, \xi\right) d t_{k} \cdots d t_{2} d t_{1}
\end{array}
$$

Then, convergence of this series is obtained from the symbol estimates, since $\left|\mathcal{R}_{1}(t, \xi)\right|$ is uniformly integrable over $Z_{\text {ell }}\left(d_{0}, \varepsilon\right)$. Hence, from the last considerations we may conclude

$$
\begin{aligned}
E_{\mathrm{ell}}^{V}(t, s, \xi) & =\exp \left\{\int_{s}^{t} \beta(\tau, \xi) d \tau\right\} \mathcal{Q}_{\mathrm{ell}}(t, s, \xi) \\
& =\exp \left\{\int_{s}^{t}\left(\langle\xi\rangle_{\lambda(\tau), \omega(\tau)}+\frac{\partial_{\tau}\langle\xi\rangle_{\lambda(\tau), \omega(\tau)}}{2\langle\xi\rangle_{\lambda(\tau), \omega(\tau)}}+\frac{(\rho(\tau) \omega(\tau))^{\prime}}{4\langle\xi\rangle_{\lambda(\tau), \omega(\tau)}}\right) d \tau\right\} \mathcal{Q}_{\mathrm{ell}}(t, s, \xi)
\end{aligned}
$$

where $\mathcal{Q}_{\text {ell }}=\mathcal{Q}_{\text {ell }}(t, s, \xi)$ is a uniformly bounded matrix. Then, it follows

$$
\begin{aligned}
\left(\left|E_{\mathrm{ell}}^{V}(t, s, \xi)\right|\right) & \lesssim \exp \left\{\int_{s}^{t}\left(\langle\xi\rangle_{\lambda(\tau), \omega(\tau)}+\frac{\partial_{\tau}\langle\xi\rangle_{\lambda(\tau), \omega(\tau)}}{2\langle\xi\rangle_{\lambda(\tau), \omega(\tau)}}+\frac{(\rho(\tau) \omega(\tau))^{\prime}}{2 \rho(\tau) \omega(\tau)}\right) d \tau\right\}\left(\begin{array}{cc}
1 & 1 \\
1 & 1
\end{array}\right) \\
& \lesssim \frac{\langle\xi\rangle_{\lambda(t), \omega(t)}}{\langle\xi\rangle_{\lambda(s), \omega(s)}} \exp \left\{\int_{s}^{t}\langle\xi\rangle_{\lambda(\tau), \omega(\tau)} d \tau\right\}\left(\begin{array}{cc}
1 & 1 \\
1 & 1
\end{array}\right) .
\end{aligned}
$$

This completes the proof.

Step 4. Transforming back to the original Cauchy problem. Now we want to obtain an estimate for the energy of the solution to our original Cauchy problem. For this reason we need to transform back to get an estimate of the fundamental solution $E_{\text {ell }}=$ $E_{\text {ell }}(t, s, \xi)$ which is related to a system of first order for the micro-energy $\left(\lambda(t)|\xi| \hat{u}, D_{t} \hat{u}\right)$.

Lemma 2.4.13. Under the conditions (B1) to (B3) the following holds:

1. in the elliptic zone it holds $\langle\xi\rangle_{\lambda(t), \omega(t)}-\frac{\rho(t) \omega(t)}{2} \leq-\frac{\lambda^{2}(t) \omega(t)|\xi|^{2}}{\rho(t)}$,

2. $\frac{\delta(s)}{\delta(t)} \exp \left(\int_{s}^{t}\langle\xi\rangle_{\lambda(\tau), \omega(\tau)} d \tau\right) \leq \exp \left(-|\xi|^{2} \int_{s}^{t} \frac{\lambda^{2}(\tau) \omega(\tau)}{\rho(\tau)} d \tau\right)$

where $\delta=\delta(t)=\exp \left(\frac{1}{2} \int_{0}^{t} \rho(\tau) \omega(\tau) d \tau\right)$. 
Proof. By using the property

$$
\sqrt{x+y} \leq \sqrt{x}+\frac{y}{2 \sqrt{x}}
$$

for any $x \geq 0$ and $y \geq-x$, the first statement is equivalent to the following inequality:

$$
\sqrt{\frac{\rho^{2}(t) \omega^{2}(t)}{4}-\lambda^{2}(t) \omega^{2}(t)|\xi|^{2}}-\frac{\rho(t) \omega(t)}{2} \leq-\frac{\lambda^{2}(t) \omega(t)|\xi|^{2}}{\rho(t)} .
$$

The second statement follows directly from the first one together with the definition of $\delta=$ $\delta(t)$.

From Lemma 2.4.12 we get inside the elliptic zone, that is, for $(t, \xi),(s, \xi) \in Z_{\text {ell }}\left(d_{0}, \varepsilon\right)$ the estimate

$$
\left(\left|E_{\mathrm{ell}}^{V}(t, s, \xi)\right|\right) \lesssim \frac{\rho(t) \omega(t)}{\rho(s) \omega(s)} \exp \left(\int_{s}^{t}\langle\xi\rangle_{\lambda(\tau), \omega(\tau)} d \tau\right)\left(\begin{array}{cc}
1 & 1 \\
1 & 1
\end{array}\right) .
$$

This yields in combination with (2.4.8) the estimate

$$
\begin{aligned}
& \left(\left|E_{\text {ell }}(t, s, \xi)\right|\right) \\
& \quad \lesssim\left(\begin{array}{cc}
\lambda(t)|\xi| & 0 \\
\rho(t) & \rho(t)
\end{array}\right) \exp \left(\int_{s}^{t}\left(\langle\xi\rangle_{\lambda(\tau), \omega(\tau)}-\frac{\rho(\tau) \omega(\tau)}{2}\right) d \tau\right)\left(\begin{array}{cc}
1 & 1 \\
1 & 1
\end{array}\right)\left(\begin{array}{cc}
\frac{1}{\lambda(s)|\xi|} & 0 \\
\frac{1}{\lambda(s)|\xi|} & \frac{1}{\rho(s)}
\end{array}\right) \\
& \quad \lesssim \exp \left(-|\xi|^{2} \int_{s}^{t} \frac{\lambda^{2}(\tau) \omega(\tau)}{\rho(\tau)} d \tau\right)\left(\begin{array}{cc}
\frac{\lambda(t)}{\lambda(s)} & \frac{\lambda(t)|\xi|}{\rho(s)} \\
\frac{\rho(t)}{\lambda(s)|\xi|} & \frac{\rho(t)}{\rho(s)}
\end{array}\right),
\end{aligned}
$$

where we used Lemma 2.4.13.

Remark 2.4.2. Taking into account the estimates (2.4.36), we see that the estimates for the first row are reasonable. However, the estimates for the second row seems to be not reasonable. Because, the estimate for $\left|E_{\text {ell }}^{(22)}\right|$ is only reasonable for decreasing coefficients $\rho(t)$ and the estimate for $\left|E_{\mathrm{ell}}^{(21)}\right|$ is not optimal since the upper bound for $\left|E_{\mathrm{ell}}^{(21)}\right|$ is not bounded in the elliptic zone. This "contradicts somehow" the damping effect in our model. For this reason we derive a refined estimate which we present in the next step.

\section{Step 5. A refined estimate for the fundamental solution in the elliptic zone}

Corollary 2.4.14. The fundamental solution $E_{\text {ell }}=E_{\text {ell }}(t, s, \xi)$ satisfies the following estimate:

$$
\left(\left|E_{\text {ell }}(t, s, \xi)\right|\right) \lesssim \exp \left(-|\xi|^{2} \int_{s}^{t} \frac{\lambda^{2}(\tau) \omega(\tau)}{\rho(\tau)} d \tau\right)\left(\begin{array}{cc}
\frac{\lambda(t)}{\lambda(s)} & \frac{\lambda(t)|\xi|}{\rho(s)} \\
\frac{\lambda^{2}(t)|\xi|}{\lambda(s) \rho(t)} & \frac{\lambda^{2}(t)|\xi|^{2}}{\rho(s) \rho(t)}
\end{array}\right)+\frac{\delta^{2}(s)}{\delta^{2}(t)}\left(\begin{array}{cc}
0 & 0 \\
0 & 1
\end{array}\right)
$$

for all $t \geq s$ and $(t, \xi),(s, \xi) \in Z_{\text {ell }}\left(d_{0}, \varepsilon\right)$.

Proof. Let us assume that $\Phi_{k}=\Phi_{k}(t, s, \xi), k=1,2$, are solutions to the equation

$$
\Phi_{t t}+\lambda^{2}(t) \omega^{2}(t)|\xi|^{2} \Phi+\rho(t) \omega(t) \Phi_{t}=0
$$

with initial values

$$
\Phi_{k}(s, s, \xi)=\delta_{1 k}, \quad \partial_{t} \Phi_{k}(s, s, \xi)=\delta_{2 k} .
$$

Then, we have

$$
\left(\begin{array}{c}
\lambda(t)|\xi| v(t, \xi) \\
D_{t} v(t, \xi)
\end{array}\right)=\left(\begin{array}{cc}
\frac{\lambda(t)}{\lambda(s)} \Phi_{1}(t, s, \xi) & i \lambda(t)|\xi| \Phi_{2}(t, s, \xi) \\
\frac{D_{t} \Phi_{1}(t, s, \xi)}{\lambda(s)|\xi|} & i D_{t} \Phi_{2}(t, s, \xi)
\end{array}\right)\left(\begin{array}{c}
\lambda(s)|\xi| v(s, \xi) \\
D_{t} v(s, \xi)
\end{array}\right) .
$$


Our basic idea is to relate the entries of the above given estimates to the multipliers $\Phi_{k}=$ $\Phi_{k}(t, s, \xi)$ and use Duhamel's formula to improve the estimates for the second row using estimates from the first one. Hence, if we compare with the estimates (2.4.36), then we obtain

$$
\begin{aligned}
\left|\Phi_{1}(t, s, \xi)\right| & \lesssim \exp \left(-|\xi|^{2} \int_{s}^{t} \frac{\lambda^{2}(\tau) \omega(\tau)}{\rho(\tau)} d \tau\right) \\
\left|\Phi_{2}(t, s, \xi)\right| & \lesssim \frac{1}{\rho(s)} \exp \left(-|\xi|^{2} \int_{s}^{t} \frac{\lambda^{2}(\tau) \omega(\tau)}{\rho(\tau)} d \tau\right) \\
\left|\partial_{t} \Phi_{1}(t, s, \xi)\right| & \lesssim \rho(t) \exp \left(-|\xi|^{2} \int_{s}^{t} \frac{\lambda^{2}(\tau) \omega(\tau)}{\rho(\tau)} d \tau\right) \\
\left|\partial_{t} \Phi_{2}(t, s, \xi)\right| & \lesssim \frac{\rho(t)}{\rho(s)} \exp \left(-|\xi|^{2} \int_{s}^{t} \frac{\lambda^{2}(\tau) \omega(\tau)}{\rho(\tau)} d \tau\right) .
\end{aligned}
$$

Let $\Psi_{k}=\Psi_{k}(t, s, \xi)=\partial_{t} \Phi_{k}(t, s, \xi), k=1,2$. Then, we obtain the equations of first order

$$
\partial_{t} \Psi_{k}+\rho(t) \omega(t) \Psi_{k}=-\lambda^{2}(t) \omega^{2}(t)|\xi|^{2} \Phi_{k}, \quad \Psi_{k}(s, s, \xi)=\delta_{2 k} .
$$

Standard calculations lead to

$$
\begin{aligned}
& \Psi_{1}(t, s, \xi)=-|\xi|^{2} \int_{s}^{t} \lambda^{2}(\tau) \omega^{2}(\tau) \frac{\delta^{2}(\tau)}{\delta^{2}(t)} \Phi_{1}(\tau, s, \xi) d \tau \\
& \Psi_{2}(t, s, \xi)=\frac{\delta^{2}(s)}{\delta^{2}(t)}-|\xi|^{2} \int_{s}^{t} \lambda^{2}(\tau) \omega^{2}(\tau) \frac{\delta^{2}(\tau)}{\delta^{2}(t)} \Phi_{2}(\tau, s, \xi) d \tau .
\end{aligned}
$$

If we are able to derive the desired estimate for $\left|\Psi_{1}(t, s, \xi)\right|$, then we conclude immediately the desired estimate for $\left|\Psi_{2}(t, s, \xi)\right|$. Using the estimates for $\left|\Phi_{1}(t, s, \xi)\right|$ and applying partial integration we get

$$
\begin{aligned}
\left|\Psi_{1}(t, s, \xi)\right| & \lesssim \frac{|\xi|^{2}}{\delta^{2}(t)} \int_{s}^{t} \lambda^{2}(\tau) \delta^{2}(\tau) \exp \left(-|\xi|^{2} \int_{s}^{\tau} \frac{\lambda^{2}(\theta) \omega(\theta)}{\rho(\theta)} d \theta\right) d \tau \\
& \lesssim \frac{\lambda^{2}(t)|\xi|^{2}}{\delta^{2}(t)} \int_{s}^{t} \delta^{2}(\tau) \exp \left(-|\xi|^{2} \int_{s}^{\tau} \frac{\lambda^{2}(\theta) \omega(\theta)}{\rho(\theta)} d \theta\right) d \tau \\
& \lesssim \frac{\lambda^{2}(t)|\xi|^{2}}{\delta^{2}(t)} \int_{s}^{t} \frac{1}{\rho(\tau) \omega(\tau)} \exp \left(-|\xi|^{2} \int_{s}^{\tau} \frac{\lambda^{2}(\theta) \omega(\theta)}{\rho(\theta)} d \theta\right) \partial_{\tau} \delta^{2}(\tau) d \tau \\
& \left.\lesssim \frac{\lambda^{2}(t)|\xi|^{2}}{\delta^{2}(t)}\left(\delta^{2}(\tau) \frac{1}{\rho(\tau) \omega(\tau)} \exp \left(-|\xi|^{2} \int_{s}^{\tau} \frac{\lambda^{2}(\theta) \omega(\theta)}{\rho(\theta)} d \theta\right)\right)\right|_{s} ^{t} \\
& +\frac{\lambda^{2}(t)|\xi|^{2}}{\delta^{2}(t)} \int_{s}^{t} \delta^{2}(\tau)(\underbrace{\left.\frac{(\rho(\tau) \omega(\tau))^{\prime}}{(\rho(\tau) \omega(\tau))^{2}}+\frac{\lambda^{2}(\tau)|\xi|^{2}}{\rho^{2}(\tau)}\right)}_{\lesssim C(\tau) \leq C_{0}<1} \exp \left(-|\xi|^{2} \int_{s}^{\tau} \frac{\lambda^{2}(\theta) \omega(\theta)}{\rho(\theta)} d \theta\right) d \tau \\
& \lesssim \frac{\lambda^{2}(t)|\xi|^{2}}{\rho(t)} \exp \left(-|\xi|^{2} \int_{s}^{\left.\int^{t} \frac{\lambda^{2}(\tau) \omega(\tau)}{\rho(\tau)} d \tau\right)-\frac{\lambda^{2}(t)|\xi|^{2}}{\rho(s)} \frac{\delta^{2}(s)}{\delta^{2}(t)}}\right.
\end{aligned}
$$

Here we have used $\lambda^{2}(t)|\xi|^{2} \leq \frac{\rho^{2}(t)}{4}\left(1-\varepsilon^{2}\right)$ from the definition of the elliptic zone and $\frac{(\rho(\tau) \omega(\tau))^{\prime}}{(\rho(\tau) \omega(\tau))^{2}}=o(1)$. Now we verify that

$$
\frac{\lambda^{2}(t)|\xi|^{2}}{\rho(s)} \frac{\delta^{2}(s)}{\delta^{2}(t)} \lesssim \frac{\lambda^{2}(t)|\xi|^{2}}{\rho(t)} \exp \left(-|\xi|^{2} \int_{s}^{t} \frac{\lambda^{2}(\tau) \omega(\tau)}{\rho(\tau)} d \tau\right)
$$


Then, it is sufficient to show that

$$
\begin{aligned}
C(t, s, \xi) & =\frac{\rho(s)}{\rho(t)} \exp \left(-|\xi|^{2} \int_{s}^{t} \frac{\lambda^{2}(\tau) \omega(\tau)}{\rho(\tau)} d \tau\right) \frac{\delta^{2}(t)}{\delta^{2}(s)} \\
& \sim \frac{\rho(s) \omega(s)}{\rho(t) \omega(t)} \exp \left(\int_{s}^{t}\left(\rho(\tau) \omega(\tau)-\frac{\lambda^{2}(\tau) \omega^{2}(\tau)|\xi|^{2}}{\rho(\tau) \omega(\tau)}\right) d \tau\right) \\
& =\exp \left(\int_{s}^{t}\left(\rho(\tau) \omega(\tau)-\frac{\lambda^{2}(\tau) \omega^{2}(\tau)|\xi|^{2}}{\rho(\tau) \omega(\tau)}-\frac{(\rho(\tau) \omega(\tau))^{\prime}}{\rho(\tau) \omega(\tau)}\right) d \tau\right) .
\end{aligned}
$$

By condition (B5), the argument of the exponential term in the previous statement is larger than 0 for $\tau>t_{0}$ sufficiently large. Hence, we have the desired estimate

$$
\left|\Psi_{1}(t, s, \xi)\right| \lesssim \frac{\lambda^{2}(t)|\xi|^{2}}{\rho(t)} \exp \left(-|\xi|^{2} \int_{s}^{t} \frac{\lambda^{2}(\tau) \omega(\tau)}{\rho(\tau)} d \tau\right)
$$

Similarly, we can estimate $\left|\Psi_{2}(t, s, \xi)\right|$ in the following way:

$$
\begin{aligned}
\left|\Psi_{2}(t, s, \xi)\right| & \lesssim \frac{\delta^{2}(s)}{\delta^{2}(t)}+\frac{|\xi|^{2}}{\delta^{2}(t)} \int_{s}^{t} \lambda^{2}(\tau) \delta^{2}(\tau) \frac{1}{\rho(s)} \exp \left(-|\xi|^{2} \int_{s}^{\tau} \frac{\lambda^{2}(\theta) \omega(\theta)}{\rho(\theta)} d \theta\right) d \tau \\
& \lesssim \frac{\delta^{2}(s)}{\delta^{2}(t)}+\frac{\lambda^{2}(t)|\xi|^{2}}{\rho(t) \rho(s)} \exp \left(-|\xi|^{2} \int_{s}^{t} \frac{\lambda^{2}(\tau) \omega(\tau)}{\rho(\tau)} d \tau\right)
\end{aligned}
$$

Summarizing all desired estimates are proved.

Remark 2.4.3. We are able to derive a refined estimate for the fundamental solution, because in the proof of Corollary 2.4.14 we use only estimates for $E_{\text {ell }}^{(11)}$ and $E_{\text {ell }}^{(12)}$ and both estimates seem to be optimal with our analytical tools.

Remark 2.4.4. If we choose a fixed $s$, then the first summand in (2.4.37) is dominated by the second one. Indeed, if we set $s=t_{\text {ell }}$, then by using $\lambda\left(t_{\text {ell }}\right)|\xi| \sim \rho\left(t_{\text {ell }}\right)$ we get the following estimate:

$$
\left(\left|E_{\mathrm{ell}}\left(t, t_{\mathrm{ell}}, \xi\right)\right|\right) \lesssim \exp \left(-|\xi|^{2} \int_{t_{\mathrm{ell}}}^{t} \frac{\lambda^{2}(\tau) \omega(\tau)}{\rho(\tau)} d \tau\right)\left(\begin{array}{cc}
\frac{\lambda(t)}{\lambda\left(t_{\mathrm{ell}}\right)} & \frac{\lambda(t)}{\lambda\left(t_{\mathrm{ell}}\right)} \\
\frac{\lambda^{2}(t)|\xi|}{\lambda\left(t_{\mathrm{ell}}\right) \rho(t)} & \frac{\lambda^{2}(t)|\xi|}{\lambda\left(t_{\mathrm{ell}}\right) \rho(t)}
\end{array}\right)
$$

\subsubsection{Considerations in the dissipative zone}

In the dissipative zone we define the micro-energy $U=U(t, \xi)$ by

$$
U=\left(\gamma(t) \hat{u}, D_{t} \hat{u}\right)^{\mathrm{T}}, \quad \gamma(t):=\frac{\lambda(t)}{F(\Lambda(t))},
$$

because we will later need to estimate $\lambda(t)|\xi| \hat{u}$ and it holds $\lambda(t)|\xi| \lesssim \frac{\lambda(t)}{F(\Lambda(t))}$ due to the definition of the dissipative zone. Then, the Fourier transformed Cauchy problem (2.4.1) leads to the system of first order

$$
D_{t} U=\underbrace{\left(\begin{array}{cc}
\frac{D_{t} \gamma(t)}{\gamma(t)} & \gamma(t) \\
\frac{\lambda^{2}(t) \omega^{2}(t)|\xi|^{2}}{\gamma(t)} & i \rho(t) \omega(t)
\end{array}\right)}_{A(t, \xi)} U
$$

We are interested in the fundamental solution

$$
E_{\text {diss }}=E_{\text {diss }}(t, s, \xi)=\left(\begin{array}{ll}
E_{\text {diss }}^{(11)} & E_{\text {diss }}^{(12)} \\
E_{\text {diss }}^{(21)} & E_{\text {diss }}^{(22)}
\end{array}\right)
$$


to the system (2.4.38), that is, the solution of

$$
D_{t} E_{\mathrm{diss}}(t, s, \xi)=A(t, \xi) E_{\mathrm{diss}}(t, s, \xi), \quad E_{\mathrm{diss}}(s, s, \xi)=I,
$$

for all $0 \leq s \leq t$ and $(t, \xi),(s, \xi) \in Z_{\text {diss }}\left(d_{0}\right)$. Thus, the solution $U=U(t, \xi)$ is represented as

$$
U(t, \xi)=E_{\text {diss }}(t, s, \xi) U(s, \xi) .
$$

We will use the auxiliary function

$$
\delta(t)=\exp \left(\frac{1}{2} \int_{0}^{t} \rho(\tau) \omega(\tau) d \tau\right)
$$

which is related to the entry $i \rho(t) \omega(t)$ of the coefficient matrix.

The entries $E_{\text {diss }}^{(k \ell)}(t, s, \xi), k, \ell=1,2$, of the fundamental solution $E_{\text {diss }}(t, s, \xi)$ satisfy the following system of Volterra integral equations for $k=1,2$ :

$$
\begin{aligned}
& D_{t} E_{\text {diss }}^{(1 \ell)}(t, s, \xi)=\frac{D_{t} \gamma(t)}{\gamma(t)} E_{\text {diss }}^{(1 \ell)}(t, s, \xi)+\gamma(t) E_{\text {diss }}^{(2 \ell)}(t, s, \xi), \\
& D_{t} E_{\text {diss }}^{(2 \ell)}(t, s, \xi)=\frac{\lambda^{2}(t) \omega^{2}(t)|\xi|^{2}}{\gamma(t)} E_{\text {diss }}^{(1 \ell)}(t, s, \xi)+i \rho(t) \omega(t) E_{\text {diss }}^{(2 \ell)}(t, s, \xi)
\end{aligned}
$$

together with their initial conditions

$$
\left(\begin{array}{ll}
E_{\text {diss }}^{(11)}(s, s, \xi) & E_{\text {diss }}^{(12)}(s, s, \xi) \\
E_{\text {diss }}^{(21)}(s, s, \xi) & E_{\text {diss }}^{(22)}(s, s, \xi)
\end{array}\right)=\left(\begin{array}{ll}
1 & 0 \\
0 & 1
\end{array}\right) .
$$

Then, by direct calculations we get

$$
\begin{aligned}
& E_{\text {diss }}^{(11)}(t, s, \xi)=\frac{\gamma(t)}{\gamma(s)}+i \gamma(t) \int_{s}^{t} E_{\text {diss }}^{(21)}(\tau, s, \xi) d \tau, \\
& E_{\text {diss }}^{(21)}(t, s, \xi)=\frac{i|\xi|^{2}}{\delta^{2}(t)} \int_{s}^{t} \frac{\lambda^{2}(\tau) \omega^{2}(\tau)}{\gamma(\tau)} \delta^{2}(\tau) E_{\text {diss }}^{(11)}(\tau, s, \xi) d \tau, \\
& E_{\text {diss }}^{(12)}(t, s, \xi)=i \gamma(t) \int_{s}^{t} E_{\text {diss }}^{(22)}(\tau, s, \xi) d \tau, \\
& E_{\text {diss }}^{(22)}(t, s, \xi)=\frac{\delta^{2}(s)}{\delta^{2}(t)}+\frac{i|\xi|^{2}}{\delta^{2}(t)} \int_{s}^{t} \frac{\lambda^{2}(\tau) \omega^{2}(\tau)}{\gamma(\tau)} \delta^{2}(\tau) E_{\text {diss }}^{(12)}(\tau, s, \xi) d \tau .
\end{aligned}
$$

The next lemma is important for deriving suitable estimates for the entries $E_{\text {diss }}^{(k \ell)}(t, s, \xi)$, $k, \ell=1,2$.

Lemma 2.4.15. The assumption (B3) implies $\frac{\lambda(t)}{\delta^{2}(t)} \in L^{1}\left(\mathbb{R}_{+}\right)$with

$$
\int_{t}^{\infty} \frac{\lambda(\tau)}{\delta^{2}(\tau)} d \tau \lesssim \frac{\Lambda(t)}{\delta^{2}(t)}
$$

Moreover, $\frac{\Lambda(t)}{\delta^{2}(t)}$ is monotonously decreasing for large $t$.

Proof. From $\mu(t) \rightarrow \infty$ as $t \rightarrow \infty$, it follows $\mu(t) \geq \frac{1+\varepsilon}{\omega(t)}$. Then, we may conclude that

$$
\delta^{2}(t)=\exp \left(\int_{0}^{t} \rho(\tau) \omega(\tau) d \tau\right) \geq \exp \left((1+\varepsilon) \int_{0}^{t} \frac{\lambda(\tau)}{\Lambda(\tau)} d \tau\right)=\Lambda^{1+\varepsilon}(t)
$$

which implies the integrability of $\frac{\lambda(t)}{\delta^{2}(t)}$. Furthermore, for large $t$ we have

$$
\begin{aligned}
\frac{1}{C_{\varepsilon}} \int_{t}^{\infty} \frac{\lambda(\tau)}{\delta^{2}(\tau)} d \tau & \leq \int_{t}^{\infty} \frac{\varepsilon}{\omega(\tau)} \frac{\lambda(\tau)}{\delta^{2}(\tau)} d \tau \leq \int_{t}^{\infty}\left(\mu(\tau)-\frac{1}{\omega(\tau)}\right) \frac{\lambda(\tau)}{\delta^{2}(\tau)} d \tau \\
& =\int_{t}^{\infty} \frac{\mu(\tau) \lambda(\tau) \omega(\tau)-\lambda(\tau)}{\omega(\tau) \delta^{2}(\tau)} d \tau \lesssim \int_{t}^{\infty} \frac{\mu(\tau) \lambda(\tau) \omega(\tau)-\lambda(\tau)}{\delta^{2}(\tau)} d \tau=\frac{\Lambda(t)}{\delta^{2}(t)}
\end{aligned}
$$


Moreover, we have

$$
\frac{d}{d t} \frac{\Lambda(t)}{\delta^{2}(t)}=\frac{\lambda(t)-\rho(t) \omega(t) \Lambda(t)}{\delta^{2}(t)}=\frac{\lambda(t)(1-\mu(t) \omega(t))}{\delta^{2}(t)}
$$

and $\mu(t) \geq \frac{1+\varepsilon}{\omega(t)}$ for large $t$, which implies that $\frac{\Lambda(t)}{\delta^{2}(t)}$ is decreasing for large $t$. This completes the proof.

In order to estimate the modulus $\left|E_{\text {diss }}^{(k \ell)}(t, s, \xi)\right|, k, \ell=1,2$, of the entries $E_{\text {diss }}^{(k \ell)}(t, s, \xi)$ we will use condition (B6).

Corollary 2.4.16. Assume conditions (A1) for $\lambda=\lambda(t)$, (A2) for $\omega=\omega(t)$, (B3) and (B6) for $\rho=\rho(t)$. Then, we have the following estimates in the dissipative zone:

$$
\left(\left|E_{\text {diss }}(t, s, \xi)\right|\right) \lesssim \frac{\lambda(t)}{F(\Lambda(t))}\left(\begin{array}{cc}
\frac{F(\Lambda(s))}{\lambda(s)} & \frac{\Lambda(s)}{\lambda(s)} \\
\frac{F(\Lambda(s))}{\lambda(s)} & \frac{\Lambda(s)}{\lambda(s)}
\end{array}\right)
$$

with $(s, \xi),(t, \xi) \in Z_{\text {diss }}\left(d_{0}\right)$ and $0 \leq s \leq t$.

Proof. First let us consider the first column, that is,

$$
\begin{aligned}
& E_{\text {diss }}^{(11)}(t, s, \xi)=\frac{\gamma(t)}{\gamma(s)}+i \gamma(t) \int_{s}^{t} E_{\text {diss }}^{(21)}(\tau, s, \xi) d \tau, \\
& E_{\text {diss }}^{(21)}(t, s, \xi)=\frac{i|\xi|^{2}}{\delta^{2}(t)} \int_{s}^{t} \frac{\lambda^{2}(\tau) \omega^{2}(\tau)}{\gamma(\tau)} \delta^{2}(\tau) E_{\text {diss }}^{(11)}(\tau, s, \xi) d \tau .
\end{aligned}
$$

Plugging the representation for $E_{\mathrm{diss}}^{(21)}(t, s, \xi)$ into the integral equation for $E_{\mathrm{diss}}^{(11)}(t, s, \xi)$ gives

$$
\begin{aligned}
E_{\mathrm{diss}}^{(11)}(t, s, \xi) & =\frac{\gamma(t)}{\gamma(s)}+i \gamma(t) \int_{s}^{t}\left(\frac{i|\xi|^{2}}{\delta^{2}(\tau)} \int_{s}^{\tau} \frac{\lambda^{2}(\theta) \omega^{2}(\theta)}{\gamma(\theta)} \delta^{2}(\theta) E_{\mathrm{diss}}^{(11)}(\theta, s, \xi) d \theta\right) d \tau \\
& =\frac{\gamma(t)}{\gamma(s)}-|\xi|^{2} \gamma(t) \int_{s}^{t} \int_{s}^{\tau} \lambda^{2}(\theta) \omega^{2}(\theta) \frac{\delta^{2}(\theta)}{\delta^{2}(\tau)} \frac{1}{\gamma(\theta)} E_{\mathrm{diss}}^{(11)}(\theta, s, \xi) d \theta d \tau
\end{aligned}
$$

It follows

$$
\frac{\gamma(s)}{\gamma(t)} E_{\mathrm{diss}}^{(11)}(t, s, \xi)=1-|\xi|^{2} \int_{s}^{t} \int_{s}^{\tau} \lambda^{2}(\theta) \omega^{2}(\theta) \frac{\delta^{2}(\theta)}{\delta^{2}(\tau)} \frac{\gamma(s)}{\gamma(\theta)} E_{\mathrm{diss}}^{(11)}(\theta, s, \xi) d \theta d \tau .
$$

By setting $y(t, s, \xi):=\frac{\gamma(s)}{\gamma(t)} E_{\text {diss }}^{(11)}(t, s, \xi)$ we obtain

$$
\begin{aligned}
y(t, s, \xi) & =1-|\xi|^{2} \int_{s}^{t} \int_{\theta}^{t} \lambda^{2}(\theta) \omega^{2}(\theta) \frac{\delta^{2}(\theta)}{\delta^{2}(\tau)} y(\theta, s, \xi) d \tau d \theta \\
& =1+|\xi|^{2} \int_{s}^{t} \lambda^{2}(\theta) \omega^{2}(\theta)\left[\int_{\theta}^{t} \frac{1}{\rho(\tau) \omega(\tau)} \partial_{\tau}\left(\frac{\delta^{2}(\theta)}{\delta^{2}(\tau)}\right) d \tau\right] y(\theta, s, \xi) d \theta \\
& =1+|\xi|^{2} \int_{s}^{t} \lambda^{2}(\theta) \omega^{2}(\theta)[\left.\frac{1}{\rho(\tau) \omega(\tau)} \frac{\delta^{2}(\theta)}{\delta^{2}(\tau)}\right|_{\theta} ^{t}+\int_{\theta}^{t} \underbrace{\frac{(\rho(\tau) \omega(\tau))^{\prime}}{(\rho(\tau) \omega(\tau))^{2}}}_{=o(1)} \frac{\delta^{2}(\theta)}{\delta^{2}(\tau)} d \tau] y(\theta, s, \xi) d \theta \\
& \approx 1+|\xi|^{2} \int_{s}^{t} \lambda^{2}(\theta) \omega^{2}(\theta)\left[\frac{1}{\rho(t) \omega(t)} \frac{\delta^{2}(\theta)}{\delta^{2}(t)}-\frac{1}{\rho(\theta) \omega(\theta)}\right] y(\theta, s, \xi) d \theta .
\end{aligned}
$$

Then, we have

$$
|y(t, s, \xi)| \lesssim 1+|\xi|^{2} \int_{s}^{t}\left(\frac{\lambda^{2}(\theta) \omega^{2}(\theta)}{\rho(t) \omega(t)} \frac{\delta^{2}(\theta)}{\delta^{2}(t)}+\frac{\lambda^{2}(\theta) \omega(\theta)}{\rho(\theta)}\right)|y(\theta, s, \xi)| d \theta .
$$


Applying Gronwall's inequality (cf. Lemma A.1.1) we get the estimate

$$
\begin{aligned}
|y(t, s, \xi)| & \lesssim \exp \left(|\xi|^{2} \int_{s}^{t} \frac{\lambda^{2}(\theta) \omega^{2}(\theta)}{\rho(t) \omega(t)} \frac{\delta^{2}(\theta)}{\delta^{2}(t)} d \theta+|\xi|^{2} \int_{s}^{t} \frac{\lambda^{2}(\theta) \omega(\theta)}{\rho(\theta)} d \theta\right) \\
& \lesssim \exp (\frac{\lambda^{2}(t)|\xi|^{2}}{\rho(t)} \frac{1}{\delta^{2}(t)} \int_{s}^{t} \frac{1}{\rho(\theta) \omega(\theta)} \underbrace{\rho(\theta) \omega(\theta) \delta^{2}(\theta)}_{\partial_{\theta} \delta^{2}(\theta)} d \theta+|\xi|^{2} \int_{s}^{t} \frac{\lambda^{2}(\theta)}{\rho(\theta)} d \theta) \\
& =\exp \left(\frac{\lambda^{2}(t)|\xi|^{2}}{\rho(t)} \frac{1}{\delta^{2}(t)}(\left.\frac{\delta^{2}(\theta)}{\rho(\theta) \omega(\theta)}\right|_{s} ^{t}+\int_{s}^{t} \underbrace{\frac{(\rho(\theta) \omega(\theta))^{\prime}}{(\rho(\theta) \omega(\theta))^{2}}}_{=o(1)} \delta^{2}(\theta) d \theta)+|\xi|^{2} \int_{s}^{t} \frac{\lambda^{2}(\theta)}{\rho(\theta)} d \theta\right) \\
& \lesssim \exp \left(\frac{\lambda^{2}(t)|\xi|^{2}}{\rho^{2}(t)}+|\xi|^{2} \int_{s}^{t} \frac{\lambda^{2}(\theta)}{\rho(\theta)} d \theta\right) \exp \left(\frac{\lambda^{2}(t)|\xi|^{2}}{\rho^{2}(t)}+\frac{d_{0}^{2}}{F^{2}(\Lambda(t))} \int_{s}^{t} \frac{\lambda^{2}(\theta)}{\rho(\theta)} d \theta\right) \lesssim 1 .
\end{aligned}
$$

Here we have used $\frac{(\rho(\theta) \omega(\theta))^{\prime}}{(\rho(\theta) \omega(\theta))^{2}}=o(1)$ from condition (B5), $\lambda(t)|\xi| \lesssim \rho(t)$ from the definition of the elliptic region, $|\xi| \leq \frac{d_{0}}{F(\Lambda(t))}$ from the definition of the dissipative zone and the condition (B6), respectively. Hence, we may conclude that

$$
\left|E_{\text {diss }}^{(11)}(t, s, \xi)\right| \lesssim \frac{\gamma(t)}{\gamma(s)}=\frac{\lambda(t)}{F(\Lambda(t))} \frac{F(\Lambda(s))}{\lambda(s)} .
$$

Now we consider $E_{\text {diss }}^{(21)}(t, s, \xi)$. By using the estimate for $\left|E_{\text {diss }}^{(11)}(t, s, \xi)\right|$ we obtain

$$
\begin{aligned}
\left|E_{\text {diss }}^{(21)}(t, s, \xi)\right| & \lesssim|\xi|^{2} \int_{s}^{t} \frac{\lambda^{2}(\tau)}{\gamma(\tau)} \frac{\delta^{2}(\tau)}{\delta^{2}(t)}\left|E_{\text {diss }}^{(11)}(\tau, s, \xi)\right| d \tau \\
& \lesssim \frac{1}{\gamma(s)}|\xi|^{2} \int_{s}^{t} \lambda^{2}(\tau) \frac{\delta^{2}(\tau)}{\delta^{2}(t)} d \tau \lesssim \frac{1}{\gamma(s)} \frac{\lambda^{2}(t)|\xi|^{2}}{\delta^{2}(t)} \int_{s}^{t} \delta^{2}(\tau) d \tau \\
& =\frac{1}{\gamma(s)} \frac{\lambda^{2}(t)|\xi|^{2}}{\delta^{2}(t)} \int_{s}^{t} \frac{1}{\rho(\tau) \omega(\tau)} \underbrace{\rho(\tau) \omega(\tau) \delta^{2}(\tau)}_{\partial_{\tau} \delta^{2}(\tau)} d \tau \\
& =\frac{1}{\gamma(s)} \frac{\lambda^{2}(t)|\xi|^{2}}{\delta^{2}(t)}(\left.\frac{1}{\rho(\tau) \omega(\tau)} \delta^{2}(\tau)\right|_{s} ^{t}+\int_{s}^{t} \underbrace{\frac{(\rho(\tau) \omega(\tau))^{\prime}}{(\rho(\tau) \omega(\tau))^{2}}}_{=o(1)} \delta^{2}(\tau) d \tau) \\
& \lesssim \frac{1}{\gamma(s)} \frac{\lambda^{2}(t)|\xi|^{2}}{\rho(t) \omega(t)} \lesssim \frac{1}{\gamma(s)} \lambda(t)|\xi| \lesssim \frac{F(\Lambda(s))}{\lambda(s)} \frac{\lambda(t)}{F(\Lambda(t))} .
\end{aligned}
$$

Here we used $\frac{(\rho(\theta) \omega(\theta))^{\prime}}{(\rho(\theta) \omega(\theta))^{2}}=o(1)$ from condition (B5), $\lambda(t)|\xi| \lesssim \rho(t)$ from the definition of the elliptic region and $|\xi| \leq \frac{d_{0}}{F(\Lambda(t))}$ from the definition of the dissipative zone, respectively.

Next we consider the entries of the second column. We have

$$
\begin{aligned}
E_{\text {diss }}^{(12)}(t, s, \xi) & =i \gamma(t) \int_{s}^{t} E_{\text {diss }}^{(22)}(\tau, s, \xi) d \tau \\
E_{\text {diss }}^{(22)}(t, s, \xi) & =\frac{\delta^{2}(s)}{\delta^{2}(t)}+\frac{i|\xi|^{2}}{\delta^{2}(t)} \int_{s}^{t} \frac{\lambda^{2}(\tau) \omega^{2}(\tau)}{\gamma(\tau)} \delta^{2}(\tau) E_{\text {diss }}^{(12)}(\tau, s, \xi) d \tau .
\end{aligned}
$$

Plugging the representation for $E_{\text {diss }}^{(22)}(t, s, \xi)$ into the integral equation for $E_{\text {diss }}^{(12)}(t, s, \xi)$ gives

$$
\begin{aligned}
E_{\mathrm{diss}}^{(12)}(t, s, \xi) & =i \gamma(t) \int_{s}^{t}\left(\frac{\delta^{2}(s)}{\delta^{2}(\tau)}+\frac{i|\xi|^{2}}{\delta^{2}(\tau)} \int_{s}^{\tau} \frac{\lambda^{2}(\theta) \omega^{2}(\theta)}{\gamma(\theta)} \delta^{2}(\theta) E_{\mathrm{diss}}^{(12)}(\theta, s, \xi) d \theta\right) d \tau \\
& =i \gamma(t) \delta^{2}(s) \int_{s}^{t} \frac{1}{\delta^{2}(\tau)} d \tau-|\xi|^{2} \gamma(t) \int_{s}^{t} \int_{s}^{\tau} \lambda^{2}(\theta) \omega^{2}(\theta) \frac{\delta^{2}(\theta)}{\delta^{2}(\tau)} \frac{1}{\gamma(\theta)} E_{\mathrm{diss}}^{(12)}(\theta, s, \xi) d \theta d \tau
\end{aligned}
$$


By setting $y(t, s, \xi):=\frac{1}{\gamma(t)} E_{\text {diss }}^{(12)}(t, s, \xi)$, it follows

$$
y(t, s, \xi)=i \delta^{2}(s) \int_{s}^{t} \frac{1}{\delta^{2}(\tau)} d \tau+|\xi|^{2} \int_{s}^{t} \lambda^{2}(\theta) \omega^{2}(\theta)\left[\int_{\theta}^{t} \frac{1}{\rho(\tau) \omega(\tau)} \partial_{\tau}\left(\frac{\delta^{2}(\theta)}{\delta^{2}(\tau)}\right) d \tau\right] y(\theta, s, \xi) d \theta
$$

In the same way as we did for $E_{\text {diss }}^{(11)}(t, s, \xi)$, after integration by parts we obtain

$$
|y(t, s, \xi)| \lesssim \delta^{2}(s) \int_{s}^{t} \frac{\lambda(\tau)}{\delta^{2}(\tau)} \frac{1}{\lambda(\tau)} d \tau+|\xi|^{2} \int_{s}^{t}\left(\frac{\lambda^{2}(\theta) \omega^{2}(\theta)}{\rho(t) \omega(t)} \frac{\delta^{2}(\theta)}{\delta^{2}(t)}+\frac{\lambda^{2}(\theta) \omega^{2}(\theta)}{\rho(\theta) \omega(\theta)}\right)|y(\theta, s, \xi)| d \theta .
$$

Now we can use again Gronwall's inequality, since the first integral can be estimated by $\frac{\Lambda(s)}{\delta^{2}(s)} \frac{1}{\lambda(s)}$ due to Lemma 2.4.15. Then, we get

$$
|y(t, s, \xi)| \lesssim \frac{\Lambda(s)}{\lambda(s)} \exp \left(|\xi|^{2} \int_{s}^{t} \frac{\lambda^{2}(\theta) \omega^{2}(\theta)}{\rho(t) \omega(t)} \frac{\delta^{2}(\theta)}{\delta^{2}(t)} d \theta+|\xi|^{2} \int_{s}^{t} \frac{\lambda^{2}(\theta) \omega(\theta)}{\rho(\theta)} d \theta\right) \lesssim \frac{\Lambda(s)}{\lambda(s)} .
$$

Thus, we obtain

$$
\left|E_{\text {diss }}^{(12)}(t, s, \xi)\right| \lesssim \gamma(t) \frac{\Lambda(s)}{\lambda(s)}=\frac{\lambda(t)}{F(\Lambda(t))} \frac{\Lambda(s)}{\lambda(s)} .
$$

Finally, let us consider $E_{\text {diss }}^{(22)}(t, s, \xi)$ by using the estimate for $\left|E_{\text {diss }}^{(12)}(t, s, \xi)\right|$. It holds

$$
\begin{aligned}
\left|E_{\text {diss }}^{(22)}(t, s, \xi)\right| & \lesssim \frac{\delta^{2}(s)}{\delta^{2}(t)}+|\xi|^{2} \int_{s}^{t} \lambda^{2}(\tau) \frac{\delta^{2}(\tau)}{\delta^{2}(t)} \frac{1}{\gamma(\tau)}\left|E_{\text {diss }}^{(12)}(\tau, s, \xi)\right| d \tau \\
& \lesssim \frac{\delta^{2}(s)}{\delta^{2}(t)}+\frac{\lambda^{2}(t)|\xi|^{2}}{\delta^{2}(t)} \frac{\Lambda(s)}{\lambda(s)} \int_{s}^{t} \delta^{2}(\tau) d \tau \\
& =\frac{\delta^{2}(s)}{\delta^{2}(t)}+\frac{\lambda^{2}(t)|\xi|^{2}}{\delta^{2}(t)} \frac{\Lambda(s)}{\lambda(s)} \int_{s}^{t} \frac{1}{\rho(\tau) \omega(\tau)} \underbrace{\rho(\tau) \omega(\tau) \delta^{2}(\tau)}_{\partial_{\tau} \delta^{2}(\tau)} d \tau \\
& =\frac{\delta^{2}(s)}{\delta^{2}(t)}+\frac{\lambda^{2}(t)|\xi|^{2}}{\delta^{2}(t)} \frac{\Lambda(s)}{\lambda(s)}(\left.\frac{1}{\rho(\tau) \omega(\tau)} \delta^{2}(\tau)\right|_{s} ^{t}+\int_{s}^{t} \underbrace{\frac{(\rho(\tau) \omega(\tau))^{\prime}}{(\rho(\tau) \omega(\tau))^{2}}}_{=o(1)} \delta^{2}(\tau) d \tau) \\
& \lesssim \frac{\delta^{2}(s)}{\delta^{2}(t)}+\frac{\lambda^{2}(t)|\xi|^{2}}{\rho(t) \omega(t)} \frac{\Lambda(s)}{\lambda(s)} \lesssim \frac{\delta^{2}(s)}{\delta^{2}(t)}+\frac{\Lambda(s)}{\lambda(s)} \lambda(t)|\xi| .
\end{aligned}
$$

In order to apply Lemma 2.4.15 we rewrite the last inequality as

$$
\begin{aligned}
\frac{\Lambda(t)}{\lambda(t)}\left|E_{\mathrm{diss}}^{(22)}(t, s, \xi)\right| & \lesssim \frac{\Lambda(t)}{\delta^{2}(t)} \frac{\delta^{2}(s)}{\lambda(t)}+\frac{\Lambda(s)}{\lambda(s)} \frac{\Lambda(t)}{\lambda(t)} \lambda(t)|\xi| \\
& \lesssim \frac{\Lambda(s)}{\lambda(s)}+\frac{\Lambda(s)}{\lambda(s)} \Lambda(t)|\xi| \lesssim \frac{\Lambda(s)}{\lambda(s)}+\frac{\Lambda(s)}{\lambda(s)} \frac{\Lambda(t)}{F(\Lambda(t))} .
\end{aligned}
$$

Here we used that $\frac{\Lambda(t)}{\delta^{2}(t)}$ is decreasing for large $t$ due to Lemma 2.4.15. Thus, we get

$$
\left|E_{\text {diss }}^{(22)}(t, s, \xi)\right| \lesssim \frac{\Lambda(s)}{\lambda(s)} \frac{\lambda(t)}{\Lambda(t)}+\frac{\Lambda(s)}{\lambda(s)} \frac{\Lambda(t)}{F(\Lambda(t))} \frac{\lambda(t)}{\Lambda(t)} \lesssim \frac{\lambda(t)}{F(\Lambda(t))} \frac{\Lambda(s)}{\lambda(s)},
$$

since $\Lambda(t) \geq F(\Lambda(t))$. This completes the proof. 


\subsubsection{Considerations in the reduced zone}

In the reduced zone we introduce the micro-energy $V=V(t, \xi)$ by

$$
V=\left(\varepsilon \frac{\rho(t) \omega(t)}{2} v, D_{t} v\right)^{\mathrm{T}} .
$$

Then, by (2.4.2) the function $V$ satisfies the following system:

$$
D_{t} V=\underbrace{\left(\begin{array}{cc}
\frac{D_{t}(\rho(t) \omega(t))}{\rho(t) \omega(t)} & \varepsilon \frac{\rho(t) \omega(t)}{2} \\
\frac{\lambda^{2}(t) \omega^{2}(t)|\xi|^{2}-\frac{1}{4}(\rho(t) \omega(t))^{2}-\frac{1}{2}(\rho(t) \omega(t))^{\prime}}{\varepsilon \frac{\rho(t) \omega(t)}{2}} & 0
\end{array}\right)}_{A_{V}(t, \xi)} V .
$$

We want to estimate the fundamental solution $E_{\mathrm{red}}^{V}=E_{\mathrm{red}}^{V}(t, s, \xi)$ to $(2.4 .39)$, that is, the solution to

$$
D_{t} E_{\text {red }}^{V}(t, s, \xi)=A_{V}(t, \xi) E_{\text {red }}^{V}(t, s, \xi), \quad E_{\text {red }}^{V}(s, s, \xi)=I .
$$

Due to $\left|(\rho(t) \omega(t))^{\prime}\right|=o\left((\rho(t) \omega(t))^{2}\right)$ for sufficiently large $t \geq t_{0}$, it holds

$$
\frac{\left|D_{t}(\rho(t) \omega(t))\right|}{\rho(t) \omega(t)} \lesssim \varepsilon \rho(t) \omega(t)
$$

Moreover, we have the estimate

$$
\langle\xi\rangle_{\lambda(t), \omega(t)} \lesssim \varepsilon \frac{\rho(t) \omega(t)}{2} .
$$

Hence, we obtain the following estimate:

$$
\frac{\left.\left|\lambda^{2}(t) \omega^{2}(t)\right| \xi\right|^{2}-\frac{1}{4}(\rho(t) \omega(t))^{2}-\frac{1}{2}(\rho(t) \omega(t))^{\prime} \mid}{\varepsilon \frac{\rho(t) \omega(t)}{2}}=\left|\frac{2\langle\xi\rangle_{\lambda(t) \omega(t)}^{2}}{\varepsilon \rho(t) \omega(t)}-\frac{(\rho(t) \omega(t))^{\prime}}{\varepsilon \rho(t) \omega(t)}\right| \lesssim \varepsilon \rho(t) \omega(t),
$$

where we used $\left|(\rho(t) \omega(t))^{\prime}\right|=o\left((\rho(t) \omega(t))^{2}\right)$.

Finally, the norm of the coefficient matrix of (2.4.39) can be estimated by $\varepsilon \rho(t) \omega(t)$ for sufficiently large $t$.

Summarizing, the following statement holds.

Lemma 2.4.17. Under the assumptions (B1), (B2) and (B5) the fundamental solution $E_{\text {red }}^{V}=E_{\text {red }}^{V}(t, s, \xi)$ to (2.4.39) satisfies the following estimate:

$$
\left(\left|E_{r e d}^{V}(t, s, \xi)\right|\right) \lesssim \exp \left(\varepsilon \int_{s}^{t} \rho(\tau) \omega(\tau) d \tau\right)\left(\begin{array}{ll}
1 & 1 \\
1 & 1
\end{array}\right)
$$

for all $t \geq s \geq t_{0}$ with sufficiently large $t_{0}=t_{0}(\varepsilon)$ and $(t, \xi),(s, \xi) \in Z_{\text {red }}(\varepsilon)$.

Remark 2.4.5. From the backward transformation we may conclude that the fundamental solution $E_{\text {red }}=E_{\text {red }}(t, s, \xi)$ can be estimated as follows:

$$
\left(\left|E_{\text {red }}(t, s, \xi)\right|\right) \lesssim \exp \left(-\frac{1}{2} \int_{s}^{t} \rho(\tau) \omega(\tau) d \tau\right)\left(\left|E_{\text {red }}^{V}(t, s, \xi)\right|\right) .
$$

Corollary 2.4.18. The fundamental solution $E_{\text {red }}=E_{\text {red }}(t, s, \xi)$ satisfies the following estimate in the reduced zone:

$$
\left(\left|E_{\text {red }}(t, s, \xi)\right|\right) \lesssim \exp \left(\left(\varepsilon-\frac{1}{2}\right) \int_{s}^{t} \rho(\tau) \omega(\tau) d \tau\right)\left(\begin{array}{cc}
1 & 1 \\
1 & 1
\end{array}\right)
$$

for $t \geq s \geq t_{0}$ with sufficiently large $t_{0}=t_{0}(\varepsilon)$ and $(t, \xi),(s, \xi) \in Z_{\text {red }}(\varepsilon)$. 


\subsection{Energy estimates of higher order}

The main goal of this section is to prove on the one hand higher order energy estimates to solutions of the following Cauchy problem:

$$
\begin{cases}u_{t t}-\lambda^{2}(t) \omega^{2}(t) \Delta u+\rho(t) \omega(t) u_{t}=0, & (t, x) \in[0, \infty) \times \mathbb{R}^{n}, \\ u(0, x)=u_{0}(x), \quad u_{t}(0, x)=u_{1}(x), & x \in \mathbb{R}^{n} .\end{cases}
$$

On the other hand, we want to derive higher order energy estimates for solutions to the following family of parameter-dependent Cauchy problems with suitable initial data $(0, g(s, x))$ :

$$
\begin{cases}v_{t t}-\lambda^{2}(t) \omega^{2}(t) \Delta v+\rho(t) \omega(t) v_{t}=0, & (t, x) \in[s, \infty) \times \mathbb{R}^{n}, \quad s \geq 0, \\ v(s, x)=0, \quad v_{t}(s, x)=g(s, x), & x \in \mathbb{R}^{n} .\end{cases}
$$

Here, in both cases we will assume some additional regularity of the data and our conditions (A1) to (A5) and (B1) to (B6) are satisfied which are given in Section 2.3.

The representation of the fundamental solutions obtained so far allows us to conclude estimates for the solution and their derivatives to the above Cauchy problems.

\subsubsection{A family of parameter-dependent linear Cauchy problems}

Let us consider the following family of parameter-dependent Cauchy problems:

$$
\begin{cases}u_{t t}-\lambda^{2}(t) \omega^{2}(t) \Delta u+\rho(t) \omega(t) u_{t}=0, & (t, x) \in[s, \infty) \times \mathbb{R}^{n}, s \geq 0, \\ u(s, x)=f(s, x), \quad u_{t}(s, x)=g(s, x), & x \in \mathbb{R}^{n} .\end{cases}
$$

We apply the partial Fourier transformation to (2.5.2) with respect to the spatial variables. Denoting by $\hat{u}=\hat{u}(t, \xi)$ the partial Fourier transformation $\mathcal{F}_{x \rightarrow \xi}(u)(t, \xi)$ we obtain

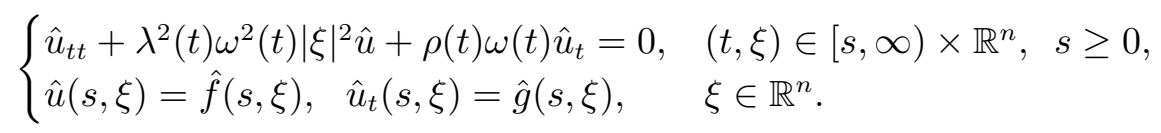

Now we make the change of variables

$$
\hat{y}(t, \xi)=\frac{\delta(t)}{\delta(s)} \hat{u}(t, \xi), \quad \delta(t):=\exp \left(\frac{1}{2} \int_{0}^{t} \rho(\tau) \omega(\tau) d \tau\right) .
$$

Then, we obtain the Cauchy problem

$$
\begin{cases}\hat{y}_{t t}+m(t, \xi) \hat{y}=0, & (t, \xi) \in[s, \infty) \times \mathbb{R}^{n}, s \geq 0, \\ \hat{y}(s, \xi)=\hat{f}(s, \xi), & \xi \in \mathbb{R}^{n}, \\ \hat{y}_{t}(s, \xi)=\frac{\rho(s) \omega(s)}{2} \hat{f}(s, \xi)+\hat{g}(s, \xi), & \xi \in \mathbb{R}^{n}\end{cases}
$$

where

$$
\begin{aligned}
m(t, \xi) & =\lambda^{2}(t) \omega^{2}(t)|\xi|^{2}-\frac{(\rho(t) \omega(t))^{2}}{4}-\frac{(\rho(t) \omega(t))^{\prime}}{2} \\
& =\lambda^{2}(t) \omega^{2}(t)|\xi|^{2}-\mu^{2}(t) \frac{\lambda^{2}(t) \omega^{2}(t)}{4 \Lambda^{2}(t)}-\left(\mu(t) \frac{\lambda(t) \omega(t)}{2 \Lambda(t)}\right)^{\prime} .
\end{aligned}
$$

Let us define the functions

$$
\eta(t):=\frac{\rho(t)}{2 \lambda(t)}=\frac{\mu(t)}{2 \Lambda(t)} \quad \text { and }\langle\xi\rangle_{\lambda(t), \omega(t)}:=\sqrt{\left.\left|\lambda^{2}(t) \omega^{2}(t)\right| \xi\right|^{2}-\rho^{2}(t) \omega^{2}(t) / 4 \mid} .
$$

In the same manner as in Section 2.4 we divide the extended phase space $[s, \infty) \times \mathbb{R}^{n}$ into the following zones: 
- hyperbolic zone:

$$
Z_{\text {hyp }}(N)=\left\{(t, \xi) \in[s, \infty) \times \mathbb{R}^{n}:\langle\xi\rangle_{\lambda(t), \omega(t)} \geq N \lambda(t) \omega(t) \eta(t)\right\} \cap \Pi_{\text {hyp }}
$$

and $\Theta(t)|\xi| \geq N$

- oscillation subzone:

$$
\begin{aligned}
& Z_{\mathrm{osc}}(N, \varepsilon)=\left\{(t, \xi) \in[s, \infty) \times \mathbb{R}^{n}: \varepsilon \lambda(t) \omega(t) \eta(t) \leq\langle\xi\rangle_{\lambda(t), \omega(t)} \leq N \lambda(t) \omega(t) \eta(t)\right\} \cap \Pi_{\mathrm{hyp}} \\
& \Theta(t)|\xi| \leq N \text { and } \Lambda(t)|\xi| \geq N
\end{aligned}
$$

- reduced zone:

$$
Z_{\mathrm{red}}(\varepsilon)=\left\{(t, \xi) \in[s, \infty) \times \mathbb{R}^{n}:\langle\xi\rangle_{\lambda(t), \omega(t)} \leq \varepsilon \lambda(t) \omega(t) \eta(t)\right\} ;
$$

- elliptic zone:

$$
Z_{\mathrm{ell}}\left(d_{0}, \varepsilon\right)=\left\{(t, \xi) \in[s, \infty) \times \mathbb{R}^{n}:|\xi| \geq \frac{d_{0}}{F(\Lambda(t))}\right\} \cap\left\{\langle\xi\rangle_{\lambda(t), \omega(t)} \geq \varepsilon \lambda(t) \omega(t) \eta(t)\right\} \cap \Pi_{\mathrm{ell}}
$$

- dissipative zone:

$$
Z_{\text {diss }}\left(d_{0}\right)=\left\{(t, \xi) \in[s, \infty) \times \mathbb{R}^{n}:|\xi| \leq \frac{d_{0}}{F(\Lambda(t))}\right\} \cap \Pi_{\text {ell }} .
$$

Definition 2.5.1. We denote by $B_{\lambda}=B_{\lambda}(s, t)$ the primitive of $\frac{\lambda^{2}(t)}{\rho(t)}$ which vanishes at $t=s$. So, it is defined by

$$
B_{\lambda}(s, t):=\int_{s}^{t} \frac{\lambda^{2}(\tau)}{\rho(\tau)} d \tau=B_{\lambda}(0, t)-B_{\lambda}(0, s) .
$$

We introduce the limit

$$
\eta_{\infty}:=\lim _{t \rightarrow \infty} \eta(t) \in[0, \infty] .
$$

This limit exists because of the monotonic behavior of $\eta=\eta(t)$.

Let us introduce the functions

$$
h_{1}=h_{1}(t, \xi)=\chi(|\xi| F(\Lambda(t))) \frac{\lambda(t)}{F(\Lambda(t))}+(1-\chi(|\xi| F(\Lambda(t)))) \lambda(t)|\xi|
$$

and

$$
h_{2}=h_{2}(t, \xi)=\chi\left(\frac{\langle\xi\rangle_{\lambda(t), \omega(t)}}{\varepsilon \frac{\rho(t) \omega(t)}{2}}\right) \varepsilon \frac{\rho(t) \omega(t)}{2}+\left(1-\chi\left(\frac{\langle\xi\rangle_{\lambda(t), \omega(t)}}{\varepsilon \frac{\rho(t) \omega(t)}{2}}\right)\right) \sqrt{|m(t, \xi)|},
$$

for our models (2.5.3) and (2.5.5), respectively. Here $\chi \in \mathcal{C}^{\infty}[0, \infty)$ is a localizing function with $\chi(\zeta)=1$ for $0 \leq \zeta \leq \frac{1}{2}$ and $\chi(\zeta)=0$ for $\zeta \geq 1$.

Remark 2.5.1. In $Z_{\text {diss }}\left(d_{0}\right)$ it is reasonable to choose $h_{1}(t, \xi)=\frac{\lambda(t)}{F(\Lambda(t))}$, since from the definition of $Z_{\text {diss }}\left(d_{0}\right)$ it holds $\lambda(t)|\xi| \lesssim \frac{\lambda(t)}{F(\Lambda(t))}$. Furthermore, from the definition of $Z_{\text {red }}(\varepsilon)$, for any $(t, \xi) \notin Z_{\text {red }}(\varepsilon)$, it holds $|m(t, \xi)| \geq C \varepsilon^{2} \frac{\rho^{2}(t) \omega^{2}(t)}{4}$. Therefore, $h_{2}(t, \xi) \geq C_{1} \varepsilon \frac{\rho(t) \omega(t)}{2}$. 
We define the energy

$$
U(t, \xi)=\left(h_{1}(t, \xi) \hat{u}(t, \xi), D_{t} \hat{u}(t, \xi)\right)^{\mathrm{T}} .
$$

Then, by using (2.5.3), $U=U(t, \xi)$ satisfies the system

$$
D_{t} U(t, \xi)=\underbrace{\left(\begin{array}{cc}
\frac{D_{t} h_{1}(t, \xi)}{h_{1}(t, \xi)} & h_{1}(t, \xi) \\
\frac{\lambda^{2}(t) \omega^{2}(t)|\xi|^{2}}{h_{1}(t, \xi)} & i \rho(t) \omega(t)
\end{array}\right)}_{A(t, \xi)} U(t, \xi) .
$$

For any $t \geq t_{1} \geq s$, we denote by $E=E\left(t, t_{1}, \xi\right)$ the fundamental solution to (2.5.8), that is, the matrix which solves

$$
D_{t} E\left(t, t_{1}, \xi\right)=A(t, \xi) E\left(t, t_{1}, \xi\right), \quad E\left(t_{1}, t_{1}, \xi\right)=I .
$$

For any $t \geq t_{2} \geq t_{1} \geq s$ we can write

$$
E\left(t, t_{1}, \xi\right)=E\left(t, t_{2}, \xi\right) E\left(t_{2}, t_{1}, \xi\right)
$$

On the other hand, we use the dissipative transformed Cauchy problem (2.5.5) and we define its energy by

$$
Y(t, \xi)=\left(h_{2}(t, \xi) \hat{y}(t, \xi), D_{t} \hat{y}(t, \xi)\right)^{\mathrm{T}} .
$$

Then, from (2.5.5) we have

$$
D_{t} Y(t, \xi)=\underbrace{\left(\begin{array}{cc}
\frac{D_{t} h_{2}(t, \xi)}{h_{2}(t, \xi)} & h_{2}(t, \xi) \\
\frac{m(t, \xi)}{h_{2}(t, \xi)} & 0
\end{array}\right)}_{A^{Y}(t, \xi)} Y(t, \xi) .
$$

We denote by $E^{Y}=E^{Y}\left(t, t_{1}, \xi\right)$ the fundamental solution to (2.5.9) for any $t \geq t_{1} \geq s$, i.e., the solution to

$$
D_{t} E^{Y}\left(t, t_{1}, \xi\right)=A^{Y}(t, \xi) E^{Y}\left(t, t_{1}, \xi\right), \quad E^{Y}\left(t_{1}, t_{1}, \xi\right)=I .
$$

For $t_{2} \geq t_{1}$ and $\left(t_{1}, \xi\right),\left(t_{2}, \xi\right) \in Z_{\text {hyp }}(N)$ we will introduce $E^{Y}\left(t_{2}, t_{1}, \xi\right)=E_{\text {hyp }}^{Y}\left(t_{2}, t_{1}, \xi\right)$ and we introduce corresponding notations in the other zones.

Remark 2.5.2. By proceeding like in the previous sections, we can obtain in each zone the same estimates for the fundamental solutions $E=E(t, s, \xi)$ and $E^{Y}=E^{Y}(t, s, \xi)$ to the models (2.5.3) and (2.5.5), respectively.

\section{Estimates in the zones}

In the oscillation subzone $Z_{\text {osc }}(N, \varepsilon)$, by the result from Corollary 2.4 .9 and for all $(s, \xi),(t, \xi) \in$ $Z_{\text {osc }}(N, \varepsilon)$ we have

$$
\left(\left|E_{\mathrm{osc}}(t, s, \xi)\right|\right) \lesssim \frac{\sqrt{\lambda(t)}}{\sqrt{\lambda(s)}} \exp \left(-\frac{1}{2} \int_{s}^{t} \rho(\tau) d \tau\right)\left(\begin{array}{cc}
1 & 1 \\
1 & 1
\end{array}\right)
$$

in the reduced zone $Z_{\text {red }}(\varepsilon)$, by the result from Corollary 2.4.18 and for all $(s, \xi),(t, \xi) \in$ $Z_{\text {red }}(\varepsilon)$ we have

$$
\left(\left|E_{\mathrm{red}}(t, s, \xi)\right|\right) \lesssim \exp \left(\left(C \varepsilon-\frac{1}{2}\right) \int_{s}^{t} \rho(\tau) \omega(\tau) d \tau\right)\left(\begin{array}{cc}
1 & 1 \\
1 & 1
\end{array}\right) ;
$$


in the dissipative zone $Z_{\text {diss }}\left(d_{0}\right)$, by the result from Corollary 2.4.16 and for all $(s, \xi),(t, \xi) \in$ $Z_{\text {diss }}\left(d_{0}\right)$ we have

$$
\left(\left|E_{\mathrm{diss}}(t, s, \xi)\right|\right) \lesssim \frac{\lambda(t)}{F(\Lambda(t))}\left(\begin{array}{cc}
\frac{F(\Lambda(s))}{\lambda(s)} & \frac{\Lambda(s)}{\lambda(s)} \\
\frac{F(\Lambda(s))}{\lambda(s)} & \frac{\Lambda(s)}{\lambda(s)}
\end{array}\right)
$$

in the hyperbolic zone $Z_{\text {hyp }}(N)$, by the result from Lemma 2.4.7 and for all $(s, \xi),(t, \xi) \in$ $Z_{\text {hyp }}(N)$ we have

$$
\left(\left|E_{\text {hyp }}^{Y}(t, s, \xi)\right|\right) \lesssim \frac{\sqrt{\lambda(t)}}{\sqrt{\lambda(s)}}\left(\begin{array}{cc}
1 & 1 \\
1 & 1
\end{array}\right)
$$

and finally, in the elliptic zone $Z_{\text {ell }}\left(d_{0}, \varepsilon\right)$, by the result from Lemma 2.4 .12 and for all $(s, \xi),(t, \xi) \in Z_{\text {ell }}\left(d_{0}, \varepsilon\right)$ we have

$$
\left(\left|E_{\mathrm{ell}}^{Y}(t, s, \xi)\right|\right) \lesssim \frac{\langle\xi\rangle_{\lambda(t), \omega(t)}}{\langle\xi\rangle_{\lambda(s), \omega(s)}} \exp \left(\int_{s}^{t}\langle\xi\rangle_{\lambda(\tau), \omega(\tau)} d \tau\right)\left(\begin{array}{cc}
1 & 1 \\
1 & 1
\end{array}\right)
$$

\section{Representation of the solutions}

Let us turn now to the Cauchy problem (2.5.2). We introduce $\hat{K}_{1}=\hat{K}_{1}(t, s, \xi)$ as the solution of the Cauchy problem (2.5.3) with initial conditions $\hat{u}(s, \xi)=0$ and $\hat{u}_{t}(s, \xi)=1$. Then, we have the following identity for $k=1,2$ :

$$
\begin{aligned}
\left(\begin{array}{cc}
\frac{\lambda(t)|\xi|}{h_{k}(t, \xi)} & 0 \\
0 & 1
\end{array}\right) \mathcal{E}^{k}(t, s, \xi)\left(\begin{array}{c}
0 \\
1
\end{array}\right) & =\left(\begin{array}{cc}
\frac{\lambda(t)|\xi|}{h_{k}(t, \xi)} & 0 \\
0 & 1
\end{array}\right)\left(\begin{array}{c}
h_{k}(t, \xi) \hat{K}_{1}(t, s, \xi) \\
D_{t} \hat{K}_{1}(t, s, \xi)
\end{array}\right) \\
& =\left(\begin{array}{c}
\lambda(t)|\xi| \hat{K}_{1}(t, s, \xi) \\
D_{t} \hat{K}_{1}(t, s, \xi)
\end{array}\right) .
\end{aligned}
$$

Moreover, it holds

$$
\begin{aligned}
\left(\begin{array}{cc}
\frac{\lambda(t)|\xi|}{h_{k}(t, \xi)} & 0 \\
0 & 1
\end{array}\right) \mathcal{E}^{k}(t, s, \xi)\left(\begin{array}{c}
0 \\
1
\end{array}\right) & =\left(\begin{array}{cc}
\frac{\lambda(t)|\xi|}{h_{k}(t, \xi)} & 0 \\
0 & 1
\end{array}\right)\left(\begin{array}{c}
\mathcal{E}_{12}^{k}(t, s, \xi) \\
\mathcal{E}_{22}^{k}(t, s, \xi)
\end{array}\right) \\
& =\left(\begin{array}{c}
\frac{\lambda(t)|\xi|}{h_{k}(t, \xi)} \mathcal{E}_{12}^{k}(t, s, \xi) \\
\mathcal{E}_{22}^{k}(t, s, \xi)
\end{array}\right)
\end{aligned}
$$

where $h_{1}=h_{1}(t, \xi)$ and $h_{2}=h_{2}(t, \xi)$ are defined in (2.5.6) and (2.5.7), respectively. Moreover, we define $\mathcal{E}^{1}(t, s, \xi):=E(t, s, \xi)$ for the system (2.5.8) and $\mathcal{E}^{2}(t, s, \xi):=E^{Y}(t, s, \xi)$ for the system $(2.5 .9)$.

The above relations allow us to transfer properties of $E=E(t, s, \xi)$ and $E^{Y}=E^{Y}(t, s, \xi)$ to $\hat{K}_{1}=\hat{K}_{1}(t, s, \xi)$ and vice versa. Thus, we obtain

$$
\begin{aligned}
\hat{K}_{1}(t, s, \xi) & =\frac{1}{h_{1}(t, \xi)} E_{12}(t, s, \xi), \\
D_{t} \hat{K}_{1}(t, s, \xi) & =E_{22}(t, s, \xi) .
\end{aligned}
$$

Moreover, we consider a representation of the solution $\hat{k}_{1}=\hat{k}_{1}(t, s, \xi)$ to $(2.5 .5)$ with the initial conditions $\hat{y}(s, \xi)=0$ and $\hat{y}_{t}(s, \xi)=1$. Then, we have

$$
\begin{aligned}
\hat{k}_{1}(t, s, \xi) & =\frac{1}{h_{2}(t, \xi)} E_{12}^{Y}(t, s, \xi), \\
D_{t} \hat{k}_{1}(t, s, \xi) & =E_{22}^{Y}(t, s, \xi) .
\end{aligned}
$$


Hence, by using (2.5.4) it holds

$$
\begin{aligned}
\hat{K}_{1}(t, s, \xi) & =\frac{\delta(s)}{\delta(t)} \hat{k}_{1}(t, s, \xi)=\frac{\delta(s)}{\delta(t)} \frac{E_{12}^{Y}(t, s, \xi)}{h_{2}(t, \xi)} \\
D_{t} \hat{K}_{1}(t, s, \xi) & =\frac{\delta(s)}{\delta(t)}\left(D_{t} \hat{k}_{1}(t, s, \xi)-\frac{\rho(t) \omega(t)}{2} \hat{k}_{1}(t, s, \xi)\right) \\
& =\frac{\delta(s)}{\delta(t)}\left(E_{22}^{Y}(t, s, \xi)-\frac{\rho(t) \omega(t)}{2 h_{2}(t, \xi)} E_{12}^{Y}(t, s, \xi)\right) .
\end{aligned}
$$

In the same way we consider $\hat{K}_{0}=\hat{K}_{0}(t, 0, \xi)$ as the solution of the Cauchy problem (2.5.3) with $s=0$ and initial conditions $\hat{u}(0, \xi)=1$ and $\hat{u}_{t}(0, \xi)=0$. Consequently, by the definition of the fundamental solution given in Definition 2.4.1 we have the following identity:

$$
\begin{aligned}
\left(\begin{array}{cc}
\frac{\lambda(t)|\xi|}{h_{k}(t, \xi)} & 0 \\
0 & 1
\end{array}\right) \mathcal{E}^{k}(t, 0, \xi)\left(\begin{array}{c}
h_{k}(0, \xi) \\
0
\end{array}\right) & =\left(\begin{array}{cc}
\frac{\lambda(t)|\xi|}{h_{k}(t, \xi)} & 0 \\
0 & 1
\end{array}\right)\left(\begin{array}{c}
h_{k}(t, \xi) \hat{K}_{0}(t, 0, \xi) \\
D_{t} \hat{K}_{0}(t, 0, \xi)
\end{array}\right) \\
& =\left(\begin{array}{c}
\lambda(t)|\xi| \hat{K}_{0}(t, 0, \xi) \\
D_{t} \hat{K}_{0}(t, 0, \xi)
\end{array}\right) .
\end{aligned}
$$

Moreover, it holds

$$
\begin{aligned}
\left(\begin{array}{cc}
\frac{\lambda(t)|\xi|}{h_{k}(t, \xi)} & 0 \\
0 & 1
\end{array}\right) \mathcal{E}^{k}(t, 0, \xi)\left(\begin{array}{c}
h_{k}(0, \xi) \\
0
\end{array}\right) & =\left(\begin{array}{cc}
\frac{\lambda(t)|\xi|}{h_{k}(t, \xi)} & 0 \\
0 & 1
\end{array}\right)\left(\begin{array}{c}
h_{k}(0, \xi) \mathcal{E}_{11}^{k}(t, 0, \xi) \\
h_{k}(0, \xi) \mathcal{E}_{21}^{k}(t, 0, \xi)
\end{array}\right) \\
& =\left(\begin{array}{c}
\frac{h_{k}(0, \xi)}{h_{k}(t, \xi)} \lambda(t)|\xi| \mathcal{E}_{11}^{k}(t, 0, \xi) \\
h_{k}(0, \xi) \mathcal{E}_{21}^{k}(t, 0, \xi)
\end{array}\right)
\end{aligned}
$$

where $h_{1}=h_{1}(t, \xi)$ and $h_{2}=h_{2}(t, \xi)$ are defined in (2.5.6) and (2.5.7), respectively. Moreover, we define $\mathcal{E}^{1}=\mathcal{E}^{1}(t, 0, \xi):=E(t, 0, \xi)$ for the system $(2.5 .8)$ and $\mathcal{E}^{2}=\mathcal{E}^{2}(t, 0, \xi):=E^{Y}(t, 0, \xi)$ for the system (2.5.9).

The above relations allow us to transfer properties of $E=E(t, 0, \xi)$ and $E^{Y}=E^{Y}(t, 0, \xi)$ to $\hat{K}_{0}=\hat{K}_{0}(t, 0, \xi)$. Then, we get

$$
\begin{aligned}
\hat{K}_{0}(t, 0, \xi) & =\frac{h_{1}(0, \xi)}{h_{1}(t, \xi)} E_{11}(t, 0, \xi), \\
D_{t} \hat{K}_{0}(t, 0, \xi) & =h_{1}(0, \xi) E_{21}(t, 0, \xi) .
\end{aligned}
$$

Moreover, we consider a representation of the solution $\hat{k}_{0}=\hat{k}_{0}(t, 0, \xi)$ to $(2.5 .5)$ with $s=0$ and the initial conditions $\hat{y}(0, \xi)=1$ and $\hat{y}_{t}(0, \xi)=0$. Then, we have

$$
\begin{aligned}
\hat{k}_{0}(t, 0, \xi) & =\frac{h_{2}(0, \xi)}{h_{2}(t, \xi)} E_{11}^{Y}(t, 0, \xi), \\
D_{t} \hat{k}_{0}(t, 0, \xi) & =h_{2}(0, \xi) E_{21}^{Y}(t, 0, \xi) .
\end{aligned}
$$

By using (2.5.4), it holds

$$
\begin{aligned}
\hat{K}_{0}(t, 0, \xi) & =\frac{1}{\delta(t)} \hat{k}_{0}(t, 0, \xi)=\frac{1}{\delta(t)} \frac{h_{2}(0, \xi)}{h_{2}(t, \xi)} E_{11}^{Y}(t, 0, \xi), \\
D_{t} \hat{K}_{0}(t, 0, \xi) & =\frac{1}{\delta(t)}\left(D_{t} \hat{k}_{0}(t, 0, \xi)-\frac{\rho(t) \omega(t)}{2} \hat{k}_{0}(t, 0, \xi)\right) \\
& =\frac{h_{2}(0, \xi)}{\delta(t)}\left(E_{21}^{Y}(t, 0, \xi)-\frac{\rho(t) \omega(t)}{2 h_{2}(t, \xi)} E_{11}^{Y}(t, 0, \xi)\right) .
\end{aligned}
$$

In our further approach we will distinguish between four cases:

$$
\eta(t) \searrow 0, \quad \eta(t) \searrow \eta_{\infty}>0, \quad \eta(t) \nearrow \eta_{\infty}>0 \quad \text { and } \quad \eta(t) \nearrow \infty .
$$


The division of the extended phase space is given in the next figures in all these four cases.

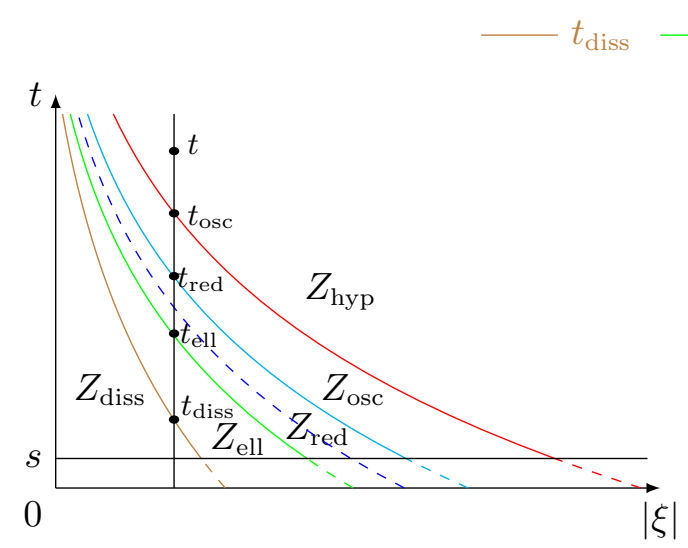

Fig. 2.2.: The case $\eta(t) \searrow 0$

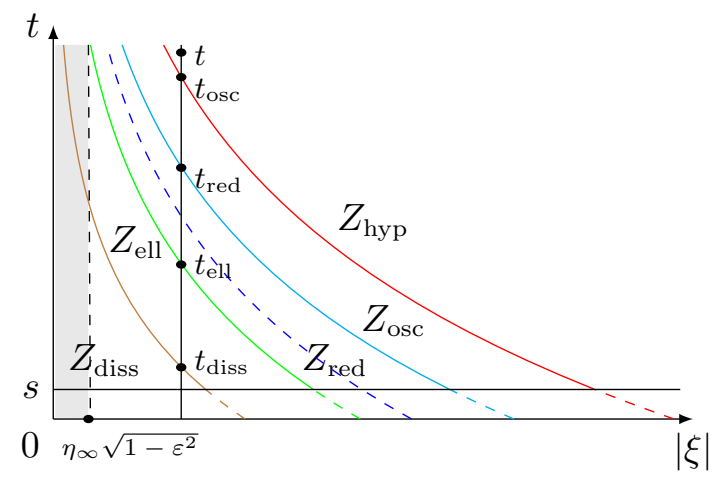

Fig. 2.3.: The case $\eta(t) \searrow \eta_{\infty}>0$

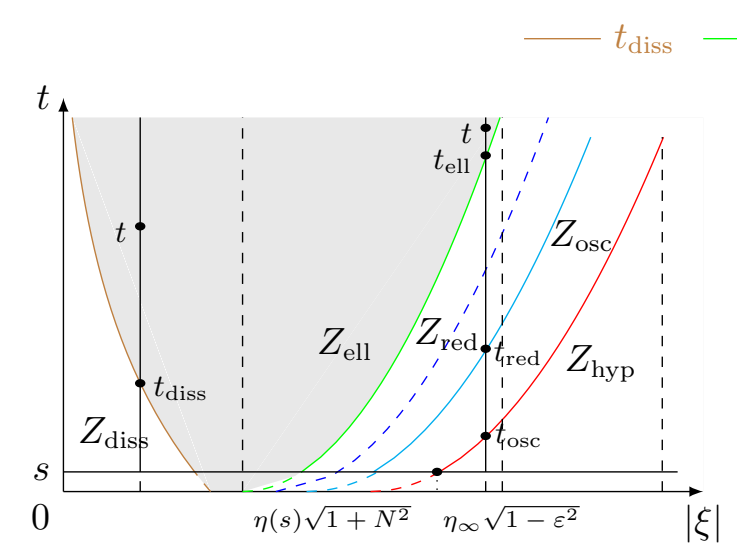

Fig. 2.4.: The case $\eta(t) \nearrow \eta_{\infty}>0$

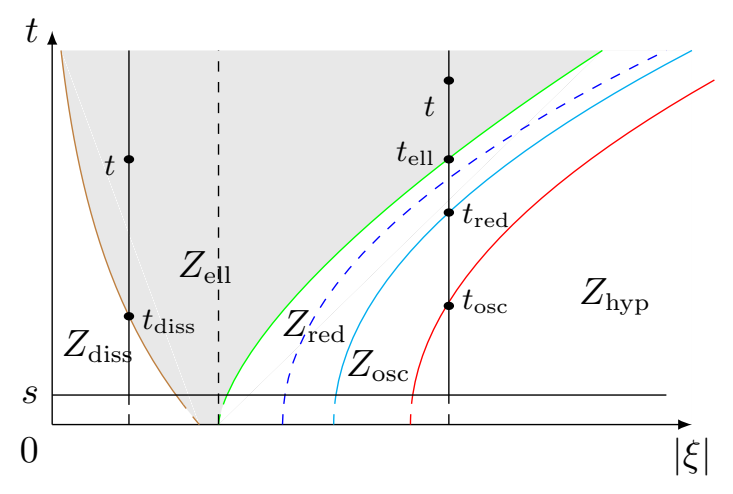

Fig. 2.5.: The case $\eta(t) \nearrow \infty$

We first consider the case that $\eta=\eta(t)$ is decreasing, that is, $\eta(t) \searrow \eta_{\infty}$ with $\eta_{\infty} \in[0, \infty)$ and $(s, \xi) \in Z_{\text {diss }}\left(d_{0}\right)$, i.e., $|\xi| \leq \frac{d_{0}}{F(\Lambda(s))}$. Then, we have the following representations:

- If $|\xi|>\eta_{\infty} \sqrt{1+N^{2}}$, then there exist parameters $t_{\mathrm{osc}}>t_{\mathrm{red}}>t_{\mathrm{ell}}>t_{\mathrm{diss}} \geq s$, such that for any $t \geq t_{\text {osc }}$ it holds

$$
\begin{aligned}
E(t, s, \xi)=E_{\mathrm{hyp}}\left(t, t_{\mathrm{osc}}, \xi\right) E_{\mathrm{osc}}\left(t_{\mathrm{osc}},\right. & \left.t_{\mathrm{red}}, \xi\right) E_{\mathrm{red}}\left(t_{\mathrm{red}}, t_{\mathrm{ell}}, \xi\right) \\
& \times E_{\mathrm{ell}}\left(t_{\mathrm{ell}}, t_{\mathrm{diss}}, \xi\right) E_{\mathrm{diss}}\left(t_{\mathrm{diss}}, s, \xi\right) .
\end{aligned}
$$

- If $\eta_{\infty} \sqrt{1+\varepsilon^{2}}<|\xi| \leq \eta_{\infty} \sqrt{1+N^{2}}$, then there exist parameters $t_{\text {red }}>t_{\text {ell }}>t_{\text {diss }} \geq s$, such that for any $t \geq t_{\text {red }}$ it holds

$$
E(t, s, \xi)=E_{\mathrm{osc}}\left(t, t_{\mathrm{red}}, \xi\right) E_{\mathrm{red}}\left(t_{\mathrm{red}}, t_{\mathrm{ell}}, \xi\right) E_{\mathrm{ell}}\left(t_{\mathrm{ell}}, t_{\mathrm{diss}}, \xi\right) E_{\mathrm{diss}}\left(t_{\mathrm{diss}}, s, \xi\right)
$$

- If $\eta_{\infty} \sqrt{1-\varepsilon^{2}}<|\xi| \leq \eta_{\infty} \sqrt{1+\varepsilon^{2}}$, then there exist parameters $t_{\text {ell }}>t_{\text {diss }} \geq s$, such that for any $t \geq t_{\text {ell }}$ it holds

$$
E(t, s, \xi)=E_{\mathrm{red}}\left(t, t_{\mathrm{ell}}, \xi\right) E_{\mathrm{ell}}\left(t_{\mathrm{ell}}, t_{\mathrm{diss}}, \xi\right) E_{\mathrm{diss}}\left(t_{\mathrm{diss}}, s, \xi\right) .
$$


- If $\frac{d_{0}}{F(\Lambda(s))}<|\xi| \leq \eta_{\infty} \sqrt{1-\varepsilon^{2}}$, then there exists parameters $t_{\text {diss }} \geq s$, such that for any $t \geq t_{\text {diss }}$ it holds

$$
E(t, s, \xi)=E_{\text {ell }}\left(t, t_{\text {diss }}, \xi\right) E_{\text {diss }}\left(t_{\text {diss }}, s, \xi\right) .
$$

- If $|\xi| \leq \frac{d_{0}}{F(\Lambda(s))}$, then $E(t, s, \xi)=E_{\text {diss }}(t, s, \xi)$.

If we consider $|\xi| \geq \eta(s) \sqrt{1+N^{2}}$, then we get $E(t, s, \xi)=E_{\text {hyp }}(t, s, \xi)$ for any $t \in[s, \infty)$. In the same way, if we take $(s, \xi)$ in another zone, we can obtain $E=E(t, s, \xi)$ as a glue of the fundamental solutions in each zone.

Now we consider the case that $\eta=\eta(t)$ is increasing, that is, $\eta(t) \nearrow \eta_{\infty}$ with $\eta_{\infty} \in(0,+\infty]$. However, there is big difference in the localization of these zones (see Figures 2.4 and 2.5). On the one hand we glue the estimates $Z_{\text {ell }}\left(d_{0}, \varepsilon\right)$ with $Z_{\text {diss }}\left(d_{0}\right)$ for $t \geq t_{\text {diss }} \geq s$. Therefore, we have the following relation:

$$
E(t, s, \xi)=E_{\text {ell }}\left(t, t_{\text {diss }}, \xi\right) E_{\text {diss }}\left(t_{\text {diss }}, s, \xi\right) .
$$

On the other hand, if $(s, \xi) \in Z_{\text {hyp }}(N)$, then the situation is reversed according to the decreasing case. Hence, we have the following representations for the fundamental solution $E=E(t, s, \xi):$

- If $|\xi| \leq \eta_{\infty} \sqrt{1-\varepsilon^{2}}$, then there exist parameters $t_{\mathrm{red}}>t_{\mathrm{osc}}>t_{\mathrm{hyp}} \geq s$, such that for any $t \geq t_{\text {red }}$ it holds

$$
E(t, s, \xi)=E_{\text {ell }}\left(t, t_{\text {red }}, \xi\right) E_{\text {red }}\left(t_{\text {red }}, t_{\text {osc }}, \xi\right) E_{\text {osc }}\left(t_{\text {osc }}, t_{\text {hyp }}, \xi\right) E_{\text {hyp }}\left(t_{\text {hyp }}, s, \xi\right) .
$$

- If $\eta_{\infty} \sqrt{1-\varepsilon^{2}}<|\xi| \leq \eta_{\infty} \sqrt{1+\varepsilon^{2}}$, then there exist parameters $t_{\mathrm{osc}}>t_{\mathrm{hyp}} \geq s$, such that for any $t \geq t_{\text {osc }}$ it holds

$$
E(t, s, \xi)=E_{\mathrm{red}}\left(t, t_{\mathrm{osc}}, \xi\right) E_{\mathrm{osc}}\left(t_{\mathrm{osc}}, t_{\mathrm{hyp}}, \xi\right) E_{\mathrm{hyp}}\left(t_{\mathrm{hyp}}, s, \xi\right) .
$$

- If $\eta_{\infty} \sqrt{1+\varepsilon^{2}}<|\xi| \leq \eta_{\infty} \sqrt{1+N^{2}}$, then there exist parameters $t_{\mathrm{hyp}} \geq s$, such that for any $t \geq t_{\text {hyp }}$ it holds

$$
E(t, s, \xi)=E_{\mathrm{osc}}\left(t, t_{\mathrm{hyp}}, \xi\right) E_{\mathrm{hyp}}\left(t_{\mathrm{hyp}}, s, \xi\right) .
$$

- If $|\xi|>\eta_{\infty} \sqrt{1+N^{2}}$, then $E(t, s, \xi)=E_{\text {hyp }}(t, s, \xi)$,

for the case $\eta(s) \sqrt{1+N^{2}} \leq|\xi| \leq \eta_{\infty} \sqrt{1-\varepsilon^{2}}$ (if this set is not empty).

In the same way, if we take $(s, \xi)$ in another zone, we can obtain $E=E(t, s, \xi)$ as a glue of the fundamental solutions in each zone.

\section{Estimates for the multipliers and time derivative of the multipliers}

In order to estimate the norm of the solution of our original Cauchy problem we need to estimate our multipliers $\left|\hat{K}_{1}(t, s, \xi)\right|$ and $\left|\hat{K}_{0}(t, 0, \xi)\right|$ in each zone of the extended phase space. Applying a more refined approach in the elliptic zone $Z_{\text {ell }}\left(d_{0}, \varepsilon\right)$, we can derive estimates for $\left|\partial_{t} \hat{K}_{1}(t, s, \xi)\right|$ and $\left|\partial_{t} \hat{K}_{0}(t, 0, \xi)\right|$ as well.

Introducing

$$
\sigma(t):=\exp \left(\frac{1}{2} \int_{0}^{t} \rho(\tau) d \tau\right)
$$

we consider the following estimates of $\left|\hat{K}_{1}(t, s, \xi)\right|$ in $Z_{\text {osc }}(N, \varepsilon), Z_{\text {red }}(\varepsilon)$ and $Z_{\text {hyp }}(N)$ as $K_{1}^{\text {osc }}(t, s, \xi), K_{1}^{\text {red }}(t, s, \xi)$ and $K_{1}^{\text {hyp }}(t, s, \xi)$, respectively:

In $Z_{\text {osc }}(N, \varepsilon)$ we have $h_{1}(t, \xi)=\lambda(t)|\xi|$. Then, it holds

$$
\left|K_{1}^{\mathrm{osc}}(t, s, \xi)\right| \leq \frac{1}{\lambda(t)|\xi|} \frac{\sqrt{\lambda(t)}}{\sqrt{\lambda(s)}} \frac{\sigma(s)}{\sigma(t)}=\frac{1}{|\xi|} \frac{1}{\sqrt{\lambda(t)} \sqrt{\lambda(s)}} \frac{\sigma(s)}{\sigma(t)} .
$$


In $Z_{\text {red }}(\varepsilon)$ we have $h_{2}(t, \xi) \sim \varepsilon \frac{\rho(t) \omega(t)}{2} \sim \lambda(t)|\xi|$. Then, it holds

$$
\left|K_{1}^{\mathrm{red}}(t, s, \xi)\right| \lesssim \frac{1}{\lambda(t)|\xi|}\left(\frac{\delta(s)}{\delta(t)}\right)^{1-2 \beta}
$$

where we choose $\varepsilon>0$ such that $\beta:=C \varepsilon<\frac{1}{2}$.

In $Z_{\text {hyp }}(N)$ we have $h_{2}(t, \xi)=\langle\xi\rangle_{\lambda(t), \omega(t)} \sim \lambda(t)|\xi|$. Then, it holds

$$
\left|K_{1}^{\mathrm{hyp}}(t, s, \xi)\right| \lesssim \frac{1}{\lambda(t)|\xi|} \frac{\sqrt{\lambda(t)}}{\sqrt{\lambda(s)}} \frac{\delta(s)}{\delta(t)}=\frac{1}{|\xi|} \frac{1}{\sqrt{\lambda(t)} \sqrt{\lambda(s)}} \frac{\delta(s)}{\delta(t)} .
$$

It is clear that we can uniformly estimate $\left|K_{1}^{\text {osc }}(t, s, \xi)\right|$ and $\left|K_{1}^{\text {hyp }}(t, s, \xi)\right|$ by the upper bound from the estimate of $\left|K_{1}^{\text {red }}(t, s, \xi)\right|$ in (2.5.27), which is the worst among (2.5.26) to (2.5.28). Indeed, by (2.5.26) and (2.5.27) we obtain the following estimate:

$$
\frac{\sqrt{\lambda(t)}}{\sigma(t)^{C}} \lesssim \frac{\sqrt{\lambda(s)}}{\sigma(s)^{C}}
$$

where $C:=1-c_{0}(1-2 \beta)$ and $c_{0}$ is a sufficiently small positive constant from condition (A2). In order to prove this estimate we will show that the function

$$
h(t)=\frac{\lambda(t)}{\sigma(t)^{2 C}}
$$

is decreasing for large $t$. Indeed, we have

$$
h^{\prime}(t)=\frac{\lambda^{\prime}(t)-C \rho(t) \lambda(t)}{\sigma(t)^{2 C}} \leq \frac{\lambda_{1} \frac{\lambda^{2}(t)}{\Lambda(t)}-C \mu(t) \frac{\lambda^{2}(t)}{\Lambda(t)}}{\sigma(t)^{2 C}} .
$$

Due to the condition (B3), we have $\mu(t) \rightarrow \infty$ as $t \rightarrow \infty$. Therefore, we obtain $h^{\prime}(t) \leq 0$ for large $t$. In the same way, from (2.5.27) and (2.5.28) we get

$$
\frac{\sqrt{\lambda(t)}}{\delta(t)^{2 \beta}} \lesssim \frac{\sqrt{\lambda(s)}}{\delta(s)^{2 \beta}}
$$

Thus, we can glue $Z_{\text {red }}(\varepsilon)$ to the hyperbolic region and we define new regions by

$$
\begin{aligned}
\Pi_{\text {hyp }}(N, \varepsilon) & =Z_{\text {red }}(\varepsilon) \cup Z_{\text {osc }}(N, \varepsilon) \cup Z_{\text {hyp }}(N), \\
\Pi_{\text {ell }}\left(d_{0}, \varepsilon\right) & =Z_{\text {ell }}\left(d_{0}, \varepsilon\right) \cup Z_{\text {diss }}\left(d_{0}\right),
\end{aligned}
$$

respectively.

We denote by $t_{\text {diss }}=t_{\text {diss }}(|\xi|)$ the separating line between $Z_{\text {ell }}\left(d_{0}, \varepsilon\right)$ and $Z_{\text {diss }}\left(d_{0}\right)$ and by $t_{|\xi|}=t(|\xi|)$ the separating curve between $\Pi_{\text {ell }}\left(d_{0}, \varepsilon\right)$ and $\Pi_{\text {hyp }}(N, \varepsilon)$. This curve is given by

$$
\eta^{2}\left(t_{|\xi|}\right)-|\xi|^{2}=\varepsilon^{2} \eta^{2}\left(t_{|\xi|}\right), \quad \text { i.e., } \quad t_{|\xi|}=\eta^{-1}\left(\frac{|\xi|}{\sqrt{1-\varepsilon^{2}}}\right) .
$$

In order to obtain energy estimates, first we establish some auxiliary estimates.

Lemma 2.5.1. Under the conditions (A1), (B1), (B2) and (B6) the following estimates hold:

1. Supposing $|\xi| F\left(\Lambda\left(t_{\text {diss }}\right)\right) \sim 1$ it holds

$$
\exp \left(-C|\xi|^{2} \int_{0}^{t_{d i s s}} \frac{\lambda^{2}(\tau)}{\rho(\tau)} d \tau\right) \sim 1 .
$$


2. Supposing $|\xi|=\eta\left(t_{|\xi|}\right) \sqrt{1-\varepsilon^{2}}$ it holds

$$
\left|d_{|\xi|} t_{|\xi|}\right| \gtrsim \frac{\mu\left(t_{|\xi|}\right)}{|\xi| \rho\left(t_{|\xi|}\right)}
$$

Proof. 1. We have the following estimate from the definition of $Z_{\text {diss }}\left(d_{0}\right)$ and condition (B6):

$$
|\xi|^{2} \int_{0}^{t_{\mathrm{diss}}} \frac{\lambda^{2}(\tau)}{\rho(\tau)} d \tau \lesssim \frac{1}{F^{2}\left(\Lambda\left(t_{\mathrm{diss}}\right)\right)} \int_{0}^{t_{\mathrm{diss}}} \frac{\lambda^{2}(\tau)}{\rho(\tau)} d \tau \lesssim 1
$$

2. We get the following estimate by using the definition of the separating curve $t_{|\xi|}$ :

$$
|\xi|=\sqrt{1-\varepsilon^{2}} \frac{\rho\left(t_{|\xi|}\right)}{\lambda\left(t_{|\xi|}\right)}=\sqrt{1-\varepsilon^{2}} \frac{\mu\left(t_{|\xi|}\right)}{\Lambda\left(t_{|\xi|}\right)} .
$$

Then, we get

$$
\frac{d|\xi|}{d t_{|\xi|}}=\sqrt{1-\varepsilon^{2}} \frac{d}{d t_{|\xi|}}\left(\frac{\mu\left(t_{|\xi|}\right)}{\Lambda\left(t_{|\xi|}\right)}\right)
$$

Hence, it holds

$$
\begin{aligned}
\left|d_{|\xi|} t_{|\xi|}\right|^{-1} & \lesssim\left|\frac{\mu^{\prime}\left(t_{|\xi|}\right) \Lambda\left(t_{|\xi|}\right)-\mu\left(t_{|\xi|}\right) \lambda\left(t_{|\xi|}\right)}{\Lambda^{2}\left(t_{|\xi|}\right)}\right| \lesssim \frac{\mu\left(t_{|\xi|}\right) \frac{\lambda\left(t_{|\xi|}\right)}{\Lambda\left(t_{|\xi|}\right)} \Lambda\left(t_{|\xi|}\right)+\mu\left(t_{|\xi|}\right) \lambda\left(t_{|\xi|}\right)}{\Lambda^{2}\left(t_{|\xi|}\right)} \\
& \lesssim \frac{\mu\left(t_{|\xi|}\right)}{\Lambda\left(t_{|\xi|}\right)} \frac{\lambda\left(t_{|\xi|}\right)}{\Lambda\left(t_{|\xi|}\right)} \sim|\xi| \frac{\lambda\left(t_{|\xi|}\right)}{\Lambda\left(t_{|\xi|}\right)}=|\xi| \frac{\rho\left(t_{|\xi|}\right)}{\mu\left(t_{|\xi|}\right)}
\end{aligned}
$$

This completes the proof.

\subsubsection{Estimates for the multiplier $\hat{K}_{1}$}

Now we distinguish between two cases related to the setting of the zones in the extended phase space for a general $s \geq 0$.

\section{Small frequencies}

In this case if $\eta=\eta(t)$ is decreasing, in general all zones appear (see Figures 2.2 and 2.3). Otherwise, if $\eta=\eta(t)$ is increasing we have two different parts of the phase space (see Figures 2.4 and 2.5). Then, we have the following three cases:

Case 1: $0 \leq s \leq t \leq t_{\text {diss }}$

In this case $(t, \xi)$ and $(s, \xi)$ belong to $Z_{\text {diss }}\left(d_{0}\right)$. It holds $h_{1}(t, \xi)=\frac{\lambda(t)}{F(\Lambda(t))}$. Then, we have the following estimates for all $t \in\left[s, t_{\text {diss }}\right]$ :

$$
\begin{aligned}
\left|\hat{K}_{1}(t, s, \xi)\right| & \lesssim \frac{\Lambda(s)}{\lambda(s)} \\
\left|\partial_{t} \hat{K}_{1}(t, s, \xi)\right| & \lesssim \frac{\lambda(t)}{F(\Lambda(t))} \frac{\Lambda(s)}{\lambda(s)} .
\end{aligned}
$$

Case 2: $0 \leq s \leq t_{\text {diss }} \leq t \leq t_{|\xi|}$

First let us consider the case $(s, \xi),(t, \xi) \in Z_{\text {ell }}\left(d_{0}, \varepsilon\right)$, where it holds $h_{2}(t, \xi) \sim \rho(t)$. Then, from Corollary 2.4.14 we have the following estimates:

$$
\begin{aligned}
\left|\hat{K}_{1}(t, s, \xi)\right| & \lesssim \frac{1}{\rho(s)} \exp \left(-C|\xi|^{2} B_{\lambda}(s, t)\right), \\
\left|\partial_{t} \hat{K}_{1}(t, s, \xi)\right| & \lesssim \frac{\lambda^{2}(t)|\xi|^{2}}{\rho(s) \rho(t)} \exp \left(-C|\xi|^{2} B_{\lambda}(s, t)\right) .
\end{aligned}
$$

Now we will glue the estimates in $Z_{\text {ell }}\left(d_{0}, \varepsilon\right)$ from Corollary 2.4.14 and in $Z_{\text {diss }}\left(d_{0}\right)$ from Corollary 2.4.16. That is, we use the representations (2.5.21) and (2.5.22). Hence, we arrive at the following statement. 
Lemma 2.5.2. The following estimates hold for all $(s, \xi) \in Z_{\text {diss }}\left(d_{0}\right)$ and $t \in\left[t_{\text {diss }}, t_{|\xi|}\right]$ :

$$
\begin{aligned}
\left|\hat{K}_{1}(t, s, \xi)\right| & \lesssim \frac{\Lambda(s)}{\lambda(s)} \exp \left(-C|\xi|^{2} B_{\lambda}(s, t)\right), \\
\left|\partial_{t} \hat{K}_{1}(t, s, \xi)\right| & \lesssim \frac{\lambda^{2}(t)|\xi|^{2}}{\rho(t)} \frac{\Lambda(s)}{\lambda(s)} \exp \left(-C|\xi|^{2} B_{\lambda}(s, t)\right) .
\end{aligned}
$$

Proof. The fundamental solution $E=E(t, s, \xi)$ can be represented as

$$
E(t, s, \xi)=E_{\text {ell }}\left(t, t_{\text {diss }}, \xi\right) E_{\text {diss }}\left(t_{\text {diss }}, s, \xi\right)
$$

for all $(s, \xi) \in Z_{\mathrm{diss}}\left(d_{0}\right)$ and $t \in\left[t_{\mathrm{diss}}, t_{|\xi|}\right]$. Then, we have

$$
\begin{aligned}
& (|E(t, s, \xi)|) \\
& \quad \lesssim\left(\left|E_{\text {ell }}\left(t, t_{\text {diss }}, \xi\right)\right|\right)\left(\left|E_{\text {diss }}\left(t_{\text {diss }}, s, \xi\right)\right|\right) \\
& \quad \lesssim \exp \left(-C|\xi|^{2} B_{\lambda}\left(t_{\text {diss }}, t\right)\right)\left(\begin{array}{cc}
\frac{\lambda(t)}{\lambda\left(t_{\text {diss }}\right)} & \frac{\lambda(t)|\xi|}{\rho\left(t_{\text {dis }}\right.} \\
\frac{\lambda^{2}(t)|\xi|}{\lambda\left(t_{\text {diss }}\right) \rho(t)} & \frac{\lambda^{2}(t)|\xi|^{2}}{\rho\left(t_{\text {diss }}\right) \rho(t)}
\end{array}\right) \frac{\lambda\left(t_{\text {diss }}\right)}{F\left(\Lambda\left(t_{\text {diss }}\right)\right)}\left(\begin{array}{cc}
\frac{F(\Lambda(s))}{\lambda(s)} & \frac{\Lambda(s)}{\lambda(s)} \\
\frac{F(\Lambda(s))}{\lambda(s)} & \frac{\Lambda(s)}{\lambda(s)}
\end{array}\right) \\
& \quad \lesssim \exp \left(-C|\xi|^{2} B_{\lambda}(s, t)\right)\left(\begin{array}{cc}
\lambda(t)|\xi| \frac{F(\Lambda(s))}{\lambda(s)} & \lambda(t)|\xi| \frac{\Lambda(s)}{\lambda(s)} \\
\frac{\lambda^{2}(t)|\xi|^{2}}{\rho(t)} \frac{F(\Lambda(s))}{\lambda(s)} & \frac{\lambda^{2}(t)|\xi|^{2}}{\rho(t)} \frac{\Lambda(s)}{\lambda(s)}
\end{array}\right)
\end{aligned}
$$

where we used $\lambda\left(t_{\text {diss }}\right)|\xi| \lesssim \rho\left(t_{\text {diss }}\right)$ and $|\xi| F\left(\Lambda\left(t_{\text {diss }}\right)\right) \sim 1$. Moreover, due to the first statement of Lemma 2.5.1 we can extend $B_{\lambda}\left(t_{\text {diss }}, t\right)$ to $B_{\lambda}(s, t)$. Hence, from the estimate (2.5.29) we may conclude

$$
\begin{aligned}
\left|\hat{K}_{1}(t, s, \xi)\right| & \lesssim \frac{1}{\rho(t)}\left|E_{12}(t, s, \xi)\right| \lesssim \frac{\Lambda(s)}{\lambda(s)} \exp \left(-C|\xi|^{2} B_{\lambda}(s, t)\right), \\
\left|\partial_{t} \hat{K}_{1}(t, s, \xi)\right| & \lesssim\left|E_{22}(t, s, \xi)\right| \lesssim \frac{\lambda^{2}(t)|\xi|^{2}}{\rho(t)} \frac{\Lambda(s)}{\lambda(s)} \exp \left(-C|\xi|^{2} B_{\lambda}(s, t)\right),
\end{aligned}
$$

respectively. Here for the estimate $\left|\hat{K}_{1}(t, s, \xi)\right|$ we have used $\lambda(t)|\xi| \lesssim \rho(t)$ from the definition of $\Pi_{\text {ell }}\left(d_{0}, \varepsilon\right)$. This completes the proof.

Case 3: $t \geq t_{|\xi|}$

Firstly, let us consider $(s, \xi),(t, \xi) \in \Pi_{\text {hyp }}(N, \varepsilon)$. Then, from the estimate in $Z_{\text {red }}(\varepsilon)$ after taking account of $h_{2}(t, \xi) \sim \lambda(t)|\xi|$ we get the following estimates:

$$
\begin{aligned}
\left|\hat{K}_{1}(t, s, \xi)\right| & \lesssim \frac{1}{\lambda(t)|\xi|}\left(\frac{\delta(s)}{\delta(t)}\right)^{1-2 \beta} \\
\left|\partial_{t} \hat{K}_{1}(t, s, \xi)\right| & \lesssim\left(\frac{\delta(s)}{\delta(t)}\right)^{1-2 \beta} .
\end{aligned}
$$

To derive the corresponding estimates for $t \in\left[t_{|\xi|}, \infty\right)$ we shall estimate the term

$$
S(t,|\xi|):=\exp \left(-C|\xi|^{2} \int_{s}^{t_{|\xi|}} \frac{\lambda^{2}(\tau) \omega(\tau)}{\rho(\tau)} d \tau\right) \exp \left(-\frac{1}{2} \int_{t_{|\xi|}}^{t} \rho(\tau) \omega(\tau) d \tau\right) .
$$

This term explains the competition of influences from different zones.

Lemma 2.5.3. For any $t \geq t_{|\xi|}$ and for a sufficiently small positive constant $C$ the function $S=S(t,|\xi|)$ satisfies the following estimate:

$$
S(t,|\xi|) \leq \max _{\xi \in \mathbb{R}^{n}}\left\{\exp \left(-C|\xi|^{2} \int_{s}^{t} \frac{\lambda^{2}(\tau) \omega(\tau)}{\rho(\tau)} d \tau\right)\right\}
$$


Proof. First we show that the first partial derivative $\partial_{|\xi|} S(t,|\xi|)$ is negative for $|\xi|$ small. For this reason we calculate

$$
\begin{aligned}
\partial_{|\xi|} & S(t,|\xi|) \\
& =S(t,|\xi|)\left(-2 C|\xi| \int_{s}^{t_{|\xi|}} \frac{\lambda^{2}(\tau) \omega(\tau)}{\rho(\tau)} d \tau-C \frac{\lambda^{2}\left(t_{|\xi|}\right) \omega\left(t_{|\xi|}\right)|\xi|^{2}}{\rho\left(t_{|\xi|}\right)} d_{|\xi|} t_{|\xi|}+\frac{\rho\left(t_{|\xi|}\right) \omega\left(t_{|\xi|}\right)}{2} d_{|\xi|} t_{|\xi|}\right) \\
& \leq S(t,|\xi|)\left(-C \frac{\lambda^{2}\left(t_{|\xi|}\right) \omega^{2}\left(t_{|\xi|}\right)|\xi|^{2}}{\rho\left(t_{|\xi|}\right) \omega\left(t_{|\xi|}\right)} d_{|\xi|} t_{|\xi|}+\frac{\rho\left(t_{|\xi|}\right) \omega\left(t_{|\xi|}\right)}{2} d_{|\xi|} t_{|\xi|}\right) \\
& =S(t,|\xi|)\left(\left(2-C\left(1-\varepsilon^{2}\right)\right) \frac{\rho\left(t_{|\xi|}\right) \omega\left(t_{|\xi|}\right)}{4} d_{|\xi|} t_{|\xi|}\right)
\end{aligned}
$$

where we used

$$
\frac{\lambda^{2}\left(t_{|\xi|}\right) \omega^{2}\left(t_{|\xi|}\right)|\xi|^{2}}{\rho\left(t_{|\xi|}\right) \omega\left(t_{|\xi|}\right)}=\frac{\rho\left(t_{|\xi|}\right) \omega\left(t_{|\xi|}\right)}{4}\left(1-\varepsilon^{2}\right)
$$

Hence, sufficiently small positive constants $\varepsilon$ and $C$ guarantee that $\left(2-C\left(1-\varepsilon^{2}\right)\right) \frac{\rho\left(t_{|\xi|}\right) \omega\left(t_{|\xi|}\right)}{4}>$ 0. On the other hand, by using the second statement of Lemma 2.5.1 we have

$$
d_{|\xi|} t_{|\xi|}<0, \quad\left|d_{|\xi|} t_{|\xi|}\right| \geq \frac{\mu\left(t_{|\xi|}\right)}{|\xi| \rho\left(t_{|\xi|}\right)} \text { and } \mu\left(t_{|\xi|}\right) \rightarrow \infty \text { for }|\xi| \rightarrow 0 .
$$

This shows the decreasing behavior of the function $S=S(t,|\xi|)$ in $|\xi|$. Now let us fix $t>0$. Then, the function $S=S(t,|\xi|)$ takes its maximum for $|\tilde{\xi}|$ satisfying $t=t_{\mid \tilde{\xi}}$, that is, the second integral vanishes in $S(t,|\xi|)$. This completes the proof.

Now we consider the case $(s, \xi) \in Z_{\text {ell }}\left(d_{0}, \varepsilon\right)$ and $(t, \xi) \in \Pi_{\text {hyp }}(N, \varepsilon)$. Then, we will glue the estimates from $Z_{\text {red }}(\varepsilon)$ and $Z_{\text {ell }}\left(d_{0}, \varepsilon\right)$. Hence, we obtain the following statement.

Lemma 2.5.4. The following estimates hold for all $(s, \xi) \in Z_{\text {ell }}\left(d_{0}, \varepsilon\right)$ and $t \in\left[t_{|\xi|}, \infty\right)$ :

$$
\begin{aligned}
\left|\hat{K}_{1}(t, s, \xi)\right| & \lesssim \frac{1}{\rho(s)} \exp \left(-C^{\prime}|\xi|^{2} B_{\lambda}(s, t)\right), \\
\left|\partial_{t} \hat{K}_{1}(t, s, \xi)\right| & \lesssim \frac{\lambda^{2}(t)|\xi|^{2}}{\rho(s) \rho(t)} \exp \left(-C^{\prime}|\xi|^{2} B_{\lambda}(s, t)\right) .
\end{aligned}
$$

Proof. We have the following estimates for all $(s, \xi) \in Z_{\text {ell }}\left(d_{0}, \varepsilon\right)$ and $t \in\left[t_{|\xi|}, \infty\right)$ :

$$
\begin{aligned}
(|E(t, s, \xi)|) & \lesssim\left(\left|E_{\text {red }}\left(t, t_{|\xi|}, \xi\right)\right|\right)\left(\left|E_{\text {ell }}\left(t_{|\xi|}, s, \xi\right)\right|\right) \\
& \lesssim\left(\frac{\delta\left(t_{|\xi|}\right)}{\delta(t)}\right)^{C_{2}}\left(\begin{array}{cc}
1 & 1 \\
1 & 1
\end{array}\right) \exp \left(-C_{1}|\xi|^{2} B_{\lambda}\left(s, t_{|\xi|}\right)\right)\left(\begin{array}{cc}
\frac{\lambda\left(t_{|\xi|}\right)}{\lambda(s)} & \frac{\lambda\left(t_{|\xi|}\right)|\xi|}{\rho(s)} \\
\frac{\lambda^{2}\left(t_{|\xi|}\right)|\xi|}{\lambda(s) \rho\left(t_{|\xi|}\right)} & \frac{\lambda^{2}\left(t_{|\xi|}|\xi|^{2}\right.}{\rho(s) \rho\left(t_{|\xi|}\right)}
\end{array}\right) \\
& \lesssim \exp \left(-C^{\prime}|\xi|^{2} B_{\lambda}(s, t)\right)\left(\begin{array}{cc}
\frac{\lambda\left(t_{|\xi|}\right)}{\lambda(s)} & \frac{\lambda\left(t_{|\xi|} \mid\right)|\xi|}{\rho(s)} \\
\frac{\lambda\left(t_{|\xi|}\right)}{\lambda(s)} & \frac{\lambda\left(t_{|\xi|}\right)|\xi|}{\rho(s)}
\end{array}\right)
\end{aligned}
$$

where we used $\rho\left(t_{|\xi|}\right) \sim \lambda\left(t_{|\xi|}\right)|\xi|$ and Lemma 2.5.3 with

$$
\exp \left(-C_{1}|\xi|^{2} B_{\lambda}\left(s, t_{|\xi|}\right)\right)\left(\frac{\delta\left(t_{|\xi|}\right)}{\delta(t)}\right)^{C_{2}} \leq \exp \left(-C^{\prime}|\xi|^{2} B_{\lambda}(s, t)\right),
$$

here $C^{\prime}:=\min \left\{C_{1}, C_{2}\right\}$. Hence, from the estimate (2.5.30) we get

$$
\begin{aligned}
\left|\hat{K}_{1}(t, s, \xi)\right| & \lesssim \frac{1}{\lambda(t)|\xi|} \frac{\lambda\left(t_{|\xi|}\right)|\xi|}{\rho(s)} \exp \left(-C^{\prime}|\xi|^{2} B_{\lambda}(s, t)\right) \\
& \lesssim \frac{1}{\rho(s)} \exp \left(-C^{\prime}|\xi|^{2} B_{\lambda}(s, t)\right)
\end{aligned}
$$


for all $t \geq t_{|\xi|}$. Similarly, for the estimate $\left|\partial_{t} \hat{K}_{1}(t, s, \xi)\right|$ we have

$$
\begin{aligned}
\left|\partial_{t} \hat{K}_{1}(t, s, \xi)\right| & \lesssim \frac{\lambda\left(t_{|\xi|}\right)|\xi|}{\rho(s)} \exp \left(-C^{\prime}|\xi|^{2} B_{\lambda}(s, t)\right) \\
& \lesssim \frac{\lambda^{2}(t)|\xi|^{2}}{\rho(s) \rho(t)} \exp \left(-C^{\prime}|\xi|^{2} B_{\lambda}(s, t)\right)
\end{aligned}
$$

for all $t \geq t_{|\xi|}$. This completes the proof.

Finally, for small frequencies it remains to glue the estimates in $\Pi_{\text {hyp }}(N, \varepsilon), Z_{\text {ell }}\left(d_{0}, \varepsilon\right)$ and $Z_{\text {diss }}\left(d_{0}\right)$. In this case we use the representations $(2.5 .18),(2.5 .19)$ and (2.5.20). We remark that this case comes into play only if $\eta=\eta(t)$ is decreasing.

We have already obtained in (2.5.29) the estimate after gluing of the estimates in $Z_{\mathrm{ell}}\left(d_{0}, \varepsilon\right)$ and $Z_{\text {diss }}\left(d_{0}\right)$. Denoting the glued propagator by $(|\mathcal{E}(t, s, \xi)|)$ we will only glue the estimate in $Z_{\text {red }}(\varepsilon)$ with the estimate (2.5.29). Hence, we have the following statement.

Lemma 2.5.5. The following estimates hold for all $t \in\left[t_{|\xi|}, \infty\right)$ :

$$
\begin{aligned}
\left|\hat{K}_{1}(t, s, \xi)\right| & \lesssim \frac{\Lambda(s)}{\lambda(s)} \exp \left(-C^{\prime}|\xi|^{2} B_{\lambda}(s, t)\right), \\
\left|\partial_{t} \hat{K}_{1}(t, s, \xi)\right| & \lesssim \frac{\lambda^{2}(t)|\xi|^{2}}{\rho(t)} \frac{\Lambda(s)}{\lambda(s)} \exp \left(-C^{\prime}|\xi|^{2} B_{\lambda}(s, t)\right) .
\end{aligned}
$$

Proof. We have the following estimate in $Z_{\text {red }}(\varepsilon)$ :

$$
\left(\left|E_{\mathrm{red}}(t, s, \xi)\right|\right) \lesssim\left(\frac{\delta(s)}{\delta(t)}\right)^{1-2 \beta}\left(\begin{array}{cc}
1 & 1 \\
1 & 1
\end{array}\right)
$$

Then, by using the estimate (2.5.29) we obtain

$$
\begin{aligned}
(\mid E & (t, s, \xi) \mid) \\
& \lesssim\left(\left|E_{\mathrm{red}}\left(t, t_{|\xi|}, \xi\right)\right|\right)\left(\left|\mathcal{E}\left(t_{|\xi|}, s, \xi\right)\right|\right) \\
& \lesssim\left(\frac{\delta\left(t_{|\xi|}\right)}{\delta(t)}\right)^{C_{2}}\left(\begin{array}{cc}
1 & 1 \\
1 & 1
\end{array}\right) \exp \left(-C_{1}|\xi|^{2} B_{\lambda}\left(s, t_{|\xi|}\right)\right)\left(\begin{array}{cc}
\lambda\left(t_{|\xi|}\right)|\xi| \frac{F(\Lambda(s))}{\lambda(s)} & \lambda\left(t_{|\xi|}\right)|\xi| \frac{\Lambda(s)}{\lambda(s)} \\
\frac{\lambda^{2}\left(t_{|\xi|}\right)|\xi|^{2}}{\rho\left(t_{|\xi|}\right)} \frac{F(\Lambda(s))}{\lambda(s)} & \frac{\lambda^{2}\left(t_{|\xi|}\right)|\xi|^{2}}{\rho\left(t_{|\xi|}\right)} \frac{\Lambda(s)}{\lambda(s)}
\end{array}\right) \\
& \lesssim \exp \left(-C^{\prime}|\xi|^{2} B_{\lambda}(s, t)\right)\left(\begin{array}{ll}
\lambda\left(t_{|\xi|}\right)|\xi| \frac{F(\Lambda(s))}{\lambda(s)} & \lambda\left(t_{|\xi|}\right)|\xi| \frac{\Lambda(s)}{\lambda(s)} \\
\lambda\left(t_{|\xi|}\right)|\xi| \frac{F(\Lambda(s))}{\lambda(s)} & \lambda\left(t_{|\xi|}\right)|\xi| \frac{\Lambda(s)}{\lambda(s)}
\end{array}\right)
\end{aligned}
$$

for all $t \geq t_{|\xi|}$. Here we have used $\lambda\left(t_{|\xi|}\right)|\xi| \sim \rho\left(t_{|\xi|}\right)$ and by Lemma 2.5.3

$$
\exp \left(-C_{1}|\xi|^{2} B_{\lambda}\left(s, t_{|\xi|}\right)\right)\left(\frac{\delta\left(t_{|\xi|}\right)}{\delta(t)}\right)^{C_{2}} \leq \exp \left(-C^{\prime}|\xi|^{2} B_{\lambda}(s, t)\right),
$$

where $C^{\prime}=\min \left\{C_{1}, C_{2}\right\}$. Hence, from the estimate (2.5.31) we obtain

$$
\begin{aligned}
\left|\hat{K}_{1}(t, s, \xi)\right| & \lesssim \frac{1}{\lambda(t)|\xi|} \lambda\left(t_{|\xi|}\right)|\xi| \frac{\Lambda(s)}{\lambda(s)} \exp \left(-C^{\prime}|\xi|^{2} B_{\lambda}(s, t)\right) \\
& \lesssim \frac{\Lambda(s)}{\lambda(s)} \exp \left(-C^{\prime}|\xi|^{2} B_{\lambda}(s, t)\right)
\end{aligned}
$$

for all $t \geq t_{|\xi|}$. In a similar way, for estimating $\left|\partial_{t} \hat{K}_{1}(t, s, \xi)\right|$ we get

$$
\begin{aligned}
\left|\partial_{t} \hat{K}_{1}(t, s, \xi)\right| & \lesssim \lambda\left(t_{|\xi|}\right)|\xi| \frac{\Lambda(s)}{\lambda(s)} \exp \left(-C^{\prime}|\xi|^{2} B_{\lambda}(s, t)\right) \\
& \lesssim \lambda(t)|\xi| \frac{\Lambda(s)}{\lambda(s)} \exp \left(-C^{\prime}|\xi|^{2} B_{\lambda}(s, t)\right) \\
& \lesssim \frac{\lambda^{2}(t)|\xi|^{2}}{\rho(t)} \frac{\Lambda(s)}{\lambda(s)} \exp \left(-C^{\prime}|\xi|^{2} B_{\lambda}(s, t)\right)
\end{aligned}
$$

for all $t \geq t_{|\xi|}$. This completes the proof. 


\section{Large frequencies}

Case $4: 0 \leq s \leq t \leq t_{|\xi|}$

If $\eta=\eta(t)$ is increasing, then $(t, \xi)$ and $(s, \xi)$ belong to $\Pi_{\text {hyp }}(N, \varepsilon)$. Taking $h_{2}(t, \xi) \sim \lambda(t)|\xi|$ we have the following estimates:

$$
\begin{aligned}
\left|\hat{K}_{1}(t, s, \xi)\right| & \lesssim \frac{1}{\lambda(t)|\xi|}\left(\frac{\delta(s)}{\delta(t)}\right)^{1-2 \beta} \\
\left|\partial_{t} \hat{K}_{1}(t, s, \xi)\right| & \lesssim\left(\frac{\delta(s)}{\delta(t)}\right)^{1-2 \beta} .
\end{aligned}
$$

We remark that the estimates (2.5.32) and (2.5.33) remain true for large frequencies in the case that $\eta=\eta(t)$ is decreasing. If $\eta=\eta(t)$ is decreasing, then we have only $Z_{\text {hyp }}(N)$ for large frequencies.

Case 5: $0 \leq s \leq t_{|\xi|} \leq t$

In this case we use the representation (2.5.23). We remark that this case comes into play only if $\eta=\eta(t)$ is increasing and there is no separating line if $|\xi| \geq \eta_{\infty} \sqrt{1-\varepsilon^{2}}$. Then, we have the following statement.

Lemma 2.5.6. The following estimates hold for all $t \in\left[t_{|\xi|}, \infty\right)$ :

$$
\begin{aligned}
\left|\hat{K}_{1}(t, s, \xi)\right| & \lesssim \frac{1}{\rho(s)} \exp \left(-C^{\prime}|\xi|^{2} B_{\lambda}(s, t)\right), \\
\left|\partial_{t} \hat{K}_{1}(t, s, \xi)\right| & \lesssim \frac{\lambda(t)|\xi|}{\rho(s)} \exp \left(-C^{\prime}|\xi|^{2} B_{\lambda}(s, t)\right) .
\end{aligned}
$$

Proof. We know that the estimate from $\Pi_{\text {hyp }}(N)$ comes from $Z_{\text {red }}(\varepsilon)$. Therefore, taking into consideration the representation

$$
E(t, s, \xi)=E_{\text {ell }}\left(t, t_{\mathrm{red}}, \xi\right) E_{\mathrm{red}}\left(t_{\mathrm{red}}, t_{\mathrm{osc}}, \xi\right) E_{\mathrm{osc}}\left(t_{\mathrm{osc}}, t_{\mathrm{hyp}}, \xi\right) E_{\mathrm{hyp}}\left(t_{\mathrm{hyp}}, s, \xi\right)
$$

leads to the estimate

$$
\begin{aligned}
(|E(t, s, \xi)|) & \lesssim\left(\left|E_{\text {ell }}\left(t, t_{|\xi|}, \xi\right)\right|\right)\left(\left|E_{\text {red }}\left(t_{|\xi|}, s, \xi\right)\right|\right) \\
& \lesssim \exp \left(-C_{1}|\xi|^{2} B_{\lambda}\left(t_{|\xi|}, t\right)\right)\left(\begin{array}{cc}
\frac{\lambda(t)}{\lambda\left(t_{|\xi|}\right)} & \frac{\lambda(t)|\xi|}{\rho\left(t_{|\xi|}\right.} \\
\frac{\lambda^{2}(t)|\xi|}{\lambda\left(t_{|\xi|}\right) \rho(t)} & \frac{\lambda^{2}(t)|\xi|^{2}}{\rho\left(t_{|\xi|}\right) \rho(t)}
\end{array}\right)\left(\frac{\delta(s)}{\delta\left(t_{|\xi|}\right)}\right)^{C_{2}}\left(\begin{array}{cc}
1 & 1 \\
1 & 1
\end{array}\right) \\
& \lesssim \exp \left(-C^{\prime}|\xi|^{2} B_{\lambda}(s, t)\right)\left(\begin{array}{cc}
\frac{\lambda(t)}{\lambda\left(t_{|\xi|}\right)} & \frac{\lambda(t)}{\lambda(t|\xi|)} \\
\frac{\lambda^{2}(t)|\xi|}{\lambda\left(t_{|\xi|}\right) \rho(t)} & \frac{\lambda^{2}(t)|\xi|}{\lambda\left(t_{|\xi|}\right) \rho(t)}
\end{array}\right)
\end{aligned}
$$

where we used $\rho\left(t_{|\xi|}\right) \sim \lambda\left(t_{|\xi|}\right)|\xi|$ and by Lemma 2.5.3 we may conclude

$$
\exp \left(-C_{1}|\xi|^{2} B_{\lambda}\left(t_{|\xi|}, t\right)\right)\left(\frac{\delta(s)}{\delta\left(t_{|\xi|}\right)}\right)^{C_{2}} \leq \exp \left(-C^{\prime}|\xi|^{2} B_{\lambda}(s, t)\right),
$$

where $C^{\prime}=\min \left\{C_{1}, C_{2}\right\}$. Hence, from the estimate (2.5.34) we obtain the following estimate for $\left|\hat{K}_{1}(t, s, \xi)\right|$ :

$$
\begin{aligned}
\left|\hat{K}_{1}(t, s, \xi)\right| & \lesssim \frac{1}{\rho(t)} \frac{\lambda(t)}{\lambda\left(t_{|\xi|}\right)}\left(-C^{\prime}|\xi|^{2} B_{\lambda}(s, t)\right) \lesssim \frac{1}{\lambda\left(t_{|\xi|}\right)|\xi|} \exp \left(-C^{\prime}|\xi|^{2} B_{\lambda}(s, t)\right) \\
& \lesssim \frac{1}{\lambda(s) \eta(s)} \exp \left(-C^{\prime}|\xi|^{2} B_{\lambda}(s, t)\right) \lesssim \frac{1}{\rho(s)} \exp \left(-C^{\prime}|\xi|^{2} B_{\lambda}(s, t)\right) .
\end{aligned}
$$


Similarly, for estimating $\left|\partial_{t} \hat{K}_{1}(t, s, \xi)\right|$ we have

$$
\begin{aligned}
\left|\partial_{t} \hat{K}_{1}(t, s, \xi)\right| & \lesssim \frac{\lambda^{2}(t)|\xi|}{\lambda\left(t_{|\xi|}\right) \rho(t)} \exp \left(-C^{\prime}|\xi|^{2} B_{\lambda}(s, t)\right) \\
& \lesssim \frac{\lambda^{2}(t)|\xi|^{2}}{\lambda(s)|\xi| \rho(t)} \exp \left(-C^{\prime}|\xi|^{2} B_{\lambda}(s, t)\right) \\
& \lesssim \frac{\lambda(t)^{2}|\xi|^{2}}{\rho(s) \rho(t)} \exp \left(-C^{\prime}|\xi|^{2} B_{\lambda}(s, t)\right) .
\end{aligned}
$$

Here for both estimates we used $\eta(s) \sqrt{1-\varepsilon^{2}} \leq|\xi| \leq \eta(t) \sqrt{1-\varepsilon^{2}}$, since $\eta=\eta(t)$ is increasing. This completes the proof.

\subsubsection{Estimates for the multiplier $\hat{K}_{0}$}

In the same way as we derived estimates for the multiplier $\hat{K}_{1}$ and its partial time derivative $\partial_{t} \hat{K}_{1}$ we will derive estimates for $\hat{K}_{0}=\hat{K}_{0}(t, 0, \xi)$ and $\partial_{t} \hat{K}_{0}=\partial_{t} \hat{K}_{0}(t, 0, \xi)$ with $s=0$.

\section{Small frequencies}

Case 1: $t \leq t_{\text {diss }}$

In this case $(t, \xi)$ belongs to $Z_{\text {diss }}\left(d_{0}\right)$. It holds $h_{1}(t, \xi)=\frac{\lambda(t)}{F(\Lambda(t))}$. Then, we have the following estimates for all $t \in\left[0, t_{\text {diss }}\right]$ :

Case 2: $t_{\text {diss }} \leq t \leq t_{|\xi|}$

$$
\begin{aligned}
\left|\hat{K}_{0}(t, 0, \xi)\right| & \leq \frac{h_{1}(0, \xi)}{h_{1}(t, \xi)}\left|E_{11}(t, 0, \xi)\right| \lesssim 1, \\
\left|\partial_{t} \hat{K}_{0}(t, 0, \xi)\right| & \leq h_{1}(0, \xi)\left|E_{21}(t, 0, \xi)\right| \lesssim \frac{\lambda(t)}{F(\Lambda(t))} .
\end{aligned}
$$

In this case we need to glue the estimates in $Z_{\text {ell }}\left(d_{0}, \varepsilon\right)$ from Corollary 2.4 .14 and in $Z_{\text {diss }}\left(d_{0}\right)$ from Corollary 2.4.16 with $s=0$. Then, by using the representations (2.5.21) and (2.5.22) for $s=0$ we get the following statement.

Lemma 2.5.7. The following estimates hold for all $t \in\left[t_{d i s s}, t_{|\xi|}\right]$ :

$$
\begin{aligned}
\left|\hat{K}_{0}(t, 0, \xi)\right| & \lesssim \exp \left(-C|\xi|^{2} B_{\lambda}(0, t)\right), \\
\left|\partial_{t} \hat{K}_{0}(t, 0, \xi)\right| & \lesssim \frac{\lambda^{2}(t)|\xi|^{2}}{\rho(t)} \exp \left(-C|\xi|^{2} B_{\lambda}(0, t)\right) .
\end{aligned}
$$

Proof. We can proceed in a similar way as in the proof of Lemma 2.5.2. Then, from the estimate (2.5.29) with $s=0$ and taking $h_{2}(t, \xi) \sim \rho(t)$ we immediately get the following estimates:

$$
\begin{aligned}
\left|\hat{K}_{0}(t, 0, \xi)\right| & \leq \frac{h_{2}(0, \xi)}{h_{2}(t, \xi)}\left|E_{11}(t, 0, \xi)\right| \lesssim \exp \left(-C|\xi|^{2} B_{\lambda}(0, t)\right), \\
\left|\partial_{t} \hat{K}_{0}(t, 0, \xi)\right| \leq h_{2}(0, \xi)\left|E_{21}(t, 0, \xi)\right| & \lesssim \frac{\lambda^{2}(t)|\xi|^{2}}{\rho(t)} \exp \left(-C|\xi|^{2} B_{\lambda}(0, t)\right),
\end{aligned}
$$

respectively. This completes the proof.

\section{Case 3: $t \geq t_{|\xi|}$}

Firstly let us consider $(t, \xi) \in \Pi_{\text {hyp }}(N, \varepsilon)$. Then, from the estimates in $Z_{\text {hyp }}(N)$ and after taking account of $h_{2}(t, \xi) \sim \lambda(t)|\xi|$ we get

$$
\begin{aligned}
\left|\hat{K}_{0}(t, 0, \xi)\right| & \leq \frac{h_{2}(0, \xi)}{h_{2}(t, \xi)}\left|E_{11}(t, 0, \xi)\right| \lesssim \frac{1}{\lambda(t)}\left(\frac{1}{\delta(t)}\right)^{1-2 \beta}, \\
\left|\partial_{t} \hat{K}_{0}(t, 0, \xi)\right| & \leq h_{2}(0, \xi)\left|E_{21}(t, 0, \xi)\right| \lesssim|\xi|\left(\frac{1}{\delta(t)}\right)^{1-2 \beta} .
\end{aligned}
$$


Now we glue the estimates from $\Pi_{\text {hyp }}(N, \varepsilon)$ and $Z_{\text {ell }}\left(d_{0}, \varepsilon\right)$. Hence, we obtain the following statement for $\frac{d_{0}}{F(\Lambda(t))} \leq|\xi|$ and small frequencies.

Lemma 2.5.8. The following estimates hold for all $t \in\left[t_{|\xi|}, \infty\right)$ and $\frac{d_{0}}{F(\Lambda(t))} \leq|\xi|$ :

$$
\begin{aligned}
\left|\hat{K}_{0}(t, 0, \xi)\right| & \lesssim \exp \left(-C^{\prime}|\xi|^{2} B_{\lambda}(0, t)\right) \\
\left|\partial_{t} \hat{K}_{0}(t, 0, \xi)\right| & \lesssim \frac{\lambda^{2}(t)|\xi|^{2}}{\rho(t)} \exp \left(-C^{\prime}|\xi|^{2} B_{\lambda}(0, t)\right) .
\end{aligned}
$$

Proof. We apply a similar argument to the proof of Lemma 2.5.4. Then, from the estimate (2.5.30) with $s=0$ and taking into consideration $h_{2}(t, \xi) \sim \lambda(t)|\xi|$ we have

$$
\begin{aligned}
\left|\hat{K}_{0}(t, 0, \xi)\right| & \lesssim \frac{1}{\lambda(t)} \lambda\left(t_{|\xi|}\right)\left(-C^{\prime}|\xi|^{2} B_{\lambda}(0, t)\right) \\
& \lesssim \exp \left(-C^{\prime}|\xi|^{2} B_{\lambda}(0, t)\right)
\end{aligned}
$$

and

$$
\begin{aligned}
\left|\partial_{t} \hat{K}_{0}(t, 0, \xi)\right| & \lesssim|\xi| \lambda\left(t_{|\xi|}\right) \exp \left(-C^{\prime}|\xi|^{2} B_{\lambda}(0, t)\right) \\
& \lesssim \frac{\lambda^{2}(t)|\xi|^{2}}{\rho(t)} \exp \left(-C^{\prime}|\xi|^{2} B_{\lambda}(0, t)\right)
\end{aligned}
$$

for all $t \geq t_{|\xi|}$. This completes the proof.

Finally, it remains to glue the estimates in $\Pi_{\text {hyp }}(N, \varepsilon), Z_{\text {ell }}\left(d_{0}, \varepsilon\right)$ and $Z_{\text {diss }}\left(d_{0}\right)$ for small frequencies. In this case we use the representations (2.5.18), (2.5.19) and (2.5.20) for $s=0$. We remark that this case comes into play only if $\eta=\eta(t)$ is decreasing. Hence, we have the following statement.

Lemma 2.5.9. The following estimates hold for all $t \in\left[t_{|\xi|}, \infty\right)$ :

$$
\begin{aligned}
\left|\hat{K}_{0}(t, 0, \xi)\right| & \lesssim \exp \left(-C^{\prime}|\xi|^{2} B_{\lambda}(0, t)\right) \\
\left|\partial_{t} \hat{K}_{0}(t, 0, \xi)\right| & \lesssim \frac{\lambda^{2}(t)|\xi|^{2}}{\rho(t)} \exp \left(-C^{\prime}|\xi|^{2} B_{\lambda}(0, t)\right) .
\end{aligned}
$$

Proof. We can derive estimates for $\left|\hat{K}_{0}(t, 0, \xi)\right|$ and $\left|\partial_{t} \hat{K}_{0}(t, 0, \xi)\right|$ in a similar way as we did in the proof to Lemma 2.5.5. Hence, from the estimate (2.5.31) with $s=0$ and taking account of $h_{2}(t, \xi) \sim \lambda(t)|\xi|$ we obtain

$$
\begin{aligned}
\left|\hat{K}_{0}(t, 0, \xi)\right| & \lesssim \frac{\lambda(0)}{\lambda(t)} \lambda\left(t_{|\xi|}\right)|\xi|\left(-C^{\prime}|\xi|^{2} B_{\lambda}(0, t)\right) \\
& \lesssim \exp \left(-C^{\prime}|\xi|^{2} B_{\lambda}(0, t)\right)
\end{aligned}
$$

and

$$
\begin{aligned}
\left|\partial_{t} \hat{K}_{0}(t, 0, \xi)\right| & \lesssim \lambda(0)|\xi| \lambda\left(t_{|\xi|}\right)|\xi| \exp \left(-C^{\prime}|\xi|^{2} B_{\lambda}(0, t)\right) \\
& \lesssim \lambda(t)|\xi| \exp \left(-C^{\prime}|\xi|^{2} B_{\lambda}(0, t)\right) \\
& \lesssim \frac{\lambda^{2}(t)|\xi|^{2}}{\rho(t)} \exp \left(-C^{\prime}|\xi|^{2} B_{\lambda}(0, t)\right)
\end{aligned}
$$

This completes the proof. 


\section{Large frequencies}

Case 4: $t \leq t_{|\xi|}$

In this case if $\eta=\eta(t)$ is increasing, then $(t, \xi)$ belongs to $\Pi_{\text {hyp }}(N, \varepsilon)$. Here it holds $h_{2}(t, \xi) \sim \lambda(t)|\xi|$. Hence, we have the following estimates:

$$
\begin{gathered}
\left|\hat{K}_{0}(t, 0, \xi)\right| \leq \frac{h_{2}(0, \xi)}{h_{2}(t, \xi)}\left|E_{11}(t, 0, \xi)\right| \lesssim \frac{1}{\lambda(t)}\left(\frac{1}{\delta(t)}\right)^{1-2 \beta}, \\
\left|\partial_{t} \hat{K}_{0}(t, 0, \xi)\right| \leq h_{1}(0, \xi)\left|E_{21}(t, 0, \xi)\right| \lesssim|\xi|\left(\frac{1}{\delta(t)}\right)^{1-2 \beta} .
\end{gathered}
$$

We note that these estimates hold for large frequencies if $\eta=\eta(t)$ is decreasing, too. If $\eta=\eta(t)$ is decreasing, then we have only $Z_{\text {hyp }}(N)$ for large frequencies.

Case 5: $t \geq t_{|\xi|}$

Firstly let us consider the case $(t, \xi) \in Z_{\text {ell }}\left(d_{0}, \varepsilon\right)$. Here it holds $h_{2}(t, \xi) \sim \rho(t)$. This case appears in the case that $\eta=\eta(t)$ is increasing (see Figures 2.4 and 2.5). Then, from Corollary 2.4.14 we have the following estimates with $s=0$ :

$$
\begin{aligned}
\left|\hat{K}_{0}(t, 0, \xi)\right| & \leq \frac{h_{2}(0, \xi)}{h_{2}(t, \xi)}\left|E_{11}(t, 0, \xi)\right| \lesssim \exp \left(-C|\xi|^{2} B_{\lambda}(0, t)\right), \\
\left|\partial_{t} \hat{K}_{0}(t, 0, \xi)\right| & \leq h_{2}(0, \xi)\left|E_{21}(t, 0, \xi)\right| \lesssim \frac{\lambda^{2}(t)|\xi|^{2}}{\rho(t)} \exp \left(-C|\xi|^{2} B_{\lambda}(0, t)\right),
\end{aligned}
$$

where we used $\eta(0) \sqrt{1-\varepsilon^{2}} \leq|\xi| \leq \eta(t) \sqrt{1-\varepsilon^{2}}$. We note that in this case if $\eta=\eta(t)$ is decreasing, then the above estimates remain valid, since the set of admissible parameters forms in this case a compact set in the extended phase space.

Now taking into consideration (2.5.23) for $s=0$ we have

$$
E(t, 0, \xi)=E_{\text {ell }}\left(t, t_{\mathrm{red}}, \xi\right) E_{\mathrm{red}}\left(t_{\mathrm{red}}, t_{\mathrm{osc}}, \xi\right) E_{\mathrm{osc}}\left(t_{\mathrm{osc}}, t_{\mathrm{hyp}}, \xi\right) E_{\mathrm{hyp}}\left(t_{\mathrm{hyp}}, 0, \xi\right) \text {. }
$$

We remark that this case comes into play only if $\eta=\eta(t)$ is increasing. Then, we have the following statement.

Lemma 2.5.10. The following estimates hold for all $t \in\left[t_{|\xi|}, \infty\right)$ :

$$
\begin{aligned}
\left|\hat{K}_{0}(t, 0, \xi)\right| & \lesssim \exp \left(-C^{\prime}|\xi|^{2} B_{\lambda}(0, t)\right), \\
\left|\partial_{t} \hat{K}_{0}(t, 0, \xi)\right| & \lesssim \frac{\lambda^{2}(t)|\xi|^{2}}{\rho(t)} \exp \left(-C^{\prime}|\xi|^{2} B_{\lambda}(0, t)\right) .
\end{aligned}
$$

Proof. In order to derive the estimates for $\left|\hat{K}_{0}(t, 0, \xi)\right|$ and $\left|\partial_{t} \hat{K}_{0}(t, 0, \xi)\right|$ we can follow the proof to Lemma 2.5.6. Then, from the estimate (2.5.34) with $s=0$ and taking $h_{2}(t, \xi) \sim \rho(t)$ we have

$$
\begin{aligned}
\left|\hat{K}_{0}(t, 0, \xi)\right| & \lesssim \frac{1}{\rho(t)} \frac{\lambda(t)}{\lambda\left(t_{|\xi|}\right)} \exp \left(-C|\xi|^{2} B_{\lambda}(0, t)\right) \\
& \lesssim \frac{1}{\lambda\left(t_{|\xi|}\right)|\xi|} \exp \left(-C^{\prime}|\xi|^{2} B_{\lambda}(0, t)\right) \\
& \lesssim \exp \left(-C^{\prime}|\xi|^{2} B_{\lambda}(0, t)\right) .
\end{aligned}
$$

To estimate $\left|\partial_{t} \hat{K}_{0}(t, 0, \xi)\right|$ we proceed as follows:

$$
\begin{aligned}
\left|\partial_{t} \hat{K}_{0}(t, 0, \xi)\right| & \lesssim \frac{\lambda^{2}(t)|\xi|}{\lambda\left(t_{|\xi|}\right) \rho(t)} \exp \left(-C|\xi|^{2} B_{\lambda}(0, t)\right) \\
& \lesssim \frac{\lambda^{2}(t)|\xi|^{2}}{\lambda\left(t_{|\xi|}\right)|\xi| \rho(t)} \exp \left(-C|\xi|^{2} B_{\lambda}(0, t)\right) \\
& \lesssim \frac{\lambda^{2}(t)|\xi|^{2}}{\rho(t)} \exp \left(-C|\xi|^{2} B_{\lambda}(0, t)\right) .
\end{aligned}
$$

Here in both estimates we have used the monotonicity of $\lambda=\lambda(t)$ and since $\eta=\eta(t)$ is increasing $\eta(0) \sqrt{1-\varepsilon^{2}} \leq|\xi| \leq \eta(t) \sqrt{1-\varepsilon^{2}}$. This completes the proof. 


\subsubsection{Final estimates}

Let us define

$$
\Omega(s, t):=\max \{\eta(s), \eta(t)\} \sqrt{1-\varepsilon^{2}}
$$

for any $t \geq s$ and $s \in[0, \infty)$.

Remark 2.5.3. We distinguish between small and large frequencies. Small frequencies satisfy the condition $|\xi| \leq \Omega(s, t)$, while, large frequencies satisfy the condition $|\xi| \geq \Omega(s, t)$.

Summarizing we arrived at the following statements for the estimates of $\left|\hat{K}_{1}(t, s, \xi)\right|$ and $\left|\partial_{t} \hat{K}_{1}(t, s, \xi)\right|$ with $t \geq s \geq 0$.

Corollary 2.5.11. If $|\xi| \geq \Omega(s, t)$, then we have the following estimates:

$$
\begin{aligned}
\left|\hat{K}_{1}(t, s, \xi)\right| & \lesssim \frac{1}{\lambda(t)|\xi|}\left(\frac{\delta(s)}{\delta(t)}\right)^{1-2 \beta} \\
\left|\partial_{t} \hat{K}_{1}(t, s, \xi)\right| & \lesssim\left(\frac{\delta(s)}{\delta(t)}\right)^{1-2 \beta} .
\end{aligned}
$$

If $\frac{d_{0}}{F(\Lambda(t))} \leq|\xi| \leq \Omega(s, t)$, then we have the following estimates:

$$
\begin{aligned}
\left|\hat{K}_{1}(t, s, \xi)\right| & \lesssim \frac{\Lambda(s)}{\lambda(s)} \exp \left(-C^{\prime}|\xi|^{2} B_{\lambda}(s, t)\right) \\
\left|\partial_{t} \hat{K}_{1}(t, s, \xi)\right| & \lesssim \frac{\lambda^{2}(t)|\xi|^{2}}{\rho(t)} \frac{\Lambda(s)}{\lambda(s)} \exp \left(-C^{\prime}|\xi|^{2} B_{\lambda}(s, t)\right)
\end{aligned}
$$

If $|\xi| \leq \frac{d_{0}}{F(\Lambda(t))}$, then we have the following estimates:

$$
\begin{aligned}
\left|\hat{K}_{1}(t, s, \xi)\right| & \lesssim \frac{\Lambda(s)}{\lambda(s)}, \\
\left|\partial_{t} \hat{K}_{1}(t, s, \xi)\right| & \lesssim \frac{\Lambda(s)}{\lambda(s)} \frac{\lambda(t)}{F(\Lambda(t))} .
\end{aligned}
$$

We have similar results for the estimates of $\left|\hat{K}_{0}(t, 0, \xi)\right|$ and $\left|\partial_{t} \hat{K}_{0}(t, 0, \xi)\right|$.

Corollary 2.5.12. If $|\xi| \geq \Omega(0, t)$, then we have the following estimates:

$$
\begin{aligned}
\left|\hat{K}_{0}(t, 0, \xi)\right| & \lesssim \frac{1}{\lambda(t)}\left(\frac{1}{\delta(t)}\right)^{1-2 \beta}, \\
\left|\partial_{t} \hat{K}_{0}(t, 0, \xi)\right| & \lesssim|\xi|\left(\frac{1}{\delta(t)}\right)^{1-2 \beta} .
\end{aligned}
$$

If $\frac{d_{0}}{F(\Lambda(t))} \leq|\xi| \leq \Omega(0, t)$, then we have the following estimates:

$$
\begin{aligned}
\left|\hat{K}_{0}(t, 0, \xi)\right| & \lesssim \exp \left(-C^{\prime}|\xi|^{2} B_{\lambda}(0, t)\right) \\
\left|\partial_{t} \hat{K}_{0}(t, 0, \xi)\right| & \lesssim \frac{\lambda^{2}(t)|\xi|^{2}}{\rho(t)} \exp \left(-C^{\prime}|\xi|^{2} B_{\lambda}(0, t)\right)
\end{aligned}
$$

If $|\xi| \leq \frac{d_{0}}{F(\Lambda(t))}$, then we have the following estimates:

$$
\begin{aligned}
\left|\hat{K}_{0}(t, 0, \xi)\right| & \lesssim 1 \\
\left|\partial_{t} \hat{K}_{0}(t, 0, \xi)\right| & \lesssim \frac{\lambda(t)}{F(\Lambda(t))}
\end{aligned}
$$




\subsubsection{Matsumura-type estimates with additional regularity of the data}

In this section let us consider the following two Cauchy problems:

$$
\begin{cases}v_{t t}-\lambda^{2}(t) \omega^{2}(t) \Delta v+\rho(t) \omega(t) v_{t}=0, & (t, x) \in[s, \infty) \times \mathbb{R}^{n}, s \geq 0 \\ v(s, x)=0, \quad v_{t}(s, x)=g(s, x), & x \in \mathbb{R}^{n}\end{cases}
$$

and

$$
\begin{cases}w_{t t}-\lambda^{2}(t) \omega^{2}(t) \Delta w+\rho(t) \omega(t) w_{t}=0, & (t, x) \in[0, \infty) \times \mathbb{R}^{n}, \\ w(0, x)=f(x), \quad w_{t}(0, x)=0, & x \in \mathbb{R}^{n} .\end{cases}
$$

We have denoted by $K_{1}=K_{1}(t, s, x)$ and $K_{0}=K_{0}(t, 0, x)$ are the solutions to the corresponding linear Cauchy problems (2.5.47) and (2.5.48) with initial data $g=\delta_{0}$ and $f=\delta_{0}$, respectively, where $\delta_{0}$ is the Dirac distribution with respect to spatial variables in $x=0$.

Using these notations for the solution $u=u(t, x)$ to the Cauchy problem

$$
\begin{cases}u_{t t}-\lambda^{2}(t) \omega^{2}(t) \Delta u+\rho(t) \omega(t) u_{t}=0, & (t, x) \in[0, \infty) \times \mathbb{R}^{n}, \\ u(0, x)=f(x), \quad u_{t}(0, x)=g(x), & x \in \mathbb{R}^{n}\end{cases}
$$

we have

$$
u(t, x)=K_{0}(t, 0, x) *_{(x)} f(x)+K_{1}(t, 0, x) *{ }_{(x)} g(x) .
$$

Thus, we may conclude the following estimates for the solutions of $v=v(t, x)$ and $w=w(t, x)$ :

$$
\|v(t, \cdot)\|_{L^{2}}=\|\hat{v}(t, \cdot)\|_{L^{2}} \leq\left\|\hat{K}_{1}(t, s, \xi) \hat{g}(s, \xi)\right\|_{L^{2}}
$$

and

$$
\|w(t, \cdot)\|_{L^{2}}=\|\hat{w}(t, \cdot)\|_{L^{2}} \leq\left\|\hat{K}_{0}(t, 0, \xi) \hat{f}(\xi)\right\|_{L^{2}}
$$

In order to estimate the $L^{2}$ norm of $\partial_{t}^{\ell} \partial_{x}^{\sigma} K_{1}(t, s, x) *_{(x)} g(s, x)$ and $\partial_{t}^{\ell} \partial_{x}^{\sigma} K_{0}(t, 0, x) *(x) f(x)$ for $\ell=0,1$ and for any $\sigma \geq 0$, we can follow the techniques used in [4], [10] and [23].

\section{Matsumura-type estimates with $s \geq 0$}

In this case we assume additional $L^{m}$ regularity for the data, with $m \in[1,2)$ in order to prove estimates of solutions and their first partial derivatives to the Cauchy problem (2.5.47).

We have the following statements for large and small frequencies.

Lemma 2.5.13. We have the following cases for large frequencies $|\xi| \geq \Omega(s, t)$ :

1. If $\eta=\eta(t)$ is increasing the following estimates hold for $\sigma+\ell \geq 1, \ell=0,1$ and $t \geq s \geq 0$ :

$$
\left\||\xi|^{\sigma} \partial_{t}^{\ell} \hat{K}_{1}(t, s, \cdot) \hat{g}(s, \cdot)\right\|_{L^{2}(|\xi| \geq \Omega(s, t))} \lesssim \frac{\lambda^{\ell}(t)}{\lambda(s)}\left(\frac{\delta(s)}{\delta(t)}\right)^{1-2 \beta}\|g(s, \cdot)\|_{H^{\sigma+\ell-1}} .
$$

Moreover, if $\sigma=\ell=0$ we have the estimate

$$
\left\|\hat{K}_{1}(t, s, \cdot) \hat{g}(s, \cdot)\right\|_{L^{2}(|\xi| \geq \Omega(s, t))} \lesssim \frac{1}{\eta(t) \lambda(s)}\left(\frac{\delta(s)}{\delta(t)}\right)^{1-2 \beta}\|g(s, \cdot)\|_{L^{2}} .
$$

2. If $\eta=\eta(t)$ is decreasing the following estimates hold for $\sigma+\ell \geq 1, \ell=0,1$ and $t \geq s \geq 0$

$$
\left\||\xi|^{\sigma} \partial_{t}^{\ell} \hat{K}_{1}(t, s, \cdot) \hat{g}(s, \cdot)\right\|_{L^{2}(|\xi| \geq \Omega(s, t))} \lesssim \frac{\lambda^{\ell}(t)}{\lambda(s)}\left(\frac{\delta(s)}{\delta(t)}\right)^{1-2 \beta}\|g(s, \cdot)\|_{H^{\sigma+\ell-1}} .
$$

Moreover, if $\sigma=\ell=0$ we have the estimate

$$
\left\|\hat{K}_{1}(t, s, \cdot) \hat{g}(s, \cdot)\right\|_{L^{2}(|\xi| \geq \Omega(s, t))} \lesssim \frac{1}{\eta(s) \lambda(s)}\left(\frac{\delta(s)}{\delta(t)}\right)^{1-2 \beta}\|g(s, \cdot)\|_{L^{2}} .
$$


Proof. We have the following estimate for $\sigma+\ell \geq 1$ :

$$
\begin{aligned}
& \left\||\xi|^{\sigma} \partial_{t}^{\ell} \hat{K}_{1}(t, s, \cdot) \hat{g}(s, \cdot)\right\|_{L^{2}(|\xi| \geq \Omega(s, t))} \\
& \quad \leq\left\||\xi|^{1-\ell} \partial_{t}^{\ell} \hat{K}_{1}(t, s, \xi)\right\|_{L^{\infty}(|\xi| \geq \Omega(s, t))}\left\||\xi|^{\sigma+\ell-1} \hat{g}(s, \cdot)\right\|_{L^{2}(|\xi| \geq \Omega(s, t))}
\end{aligned}
$$

The second term on the right-hand side can be estimated by $\|g(s, \cdot)\|_{H^{\sigma+\ell-1}}$, since $\sigma+\ell \geq 1$. Now let us consider the $L^{\infty}$ norm of $K_{1}(t, s, \xi)$ and its derivatives with respect to $t$. Indeed, for $\ell=0$ by using the estimate (2.5.35) we get

$$
|\xi|\left|\hat{K}_{1}(t, s, \xi)\right| \lesssim \frac{1}{\lambda(t)}\left(\frac{\delta(s)}{\delta(t)}\right)^{1-2 \beta} \lesssim \frac{1}{\lambda(s)}\left(\frac{\delta(s)}{\delta(t)}\right)^{1-2 \beta}
$$

and for $\ell=1$ by using the estimate (2.5.36) we get

$$
\left|\partial_{t} \hat{K}_{1}(t, s, \xi)\right| \lesssim\left(\frac{\delta(s)}{\delta(t)}\right)^{1-2 \beta}=\frac{\lambda(t)}{\lambda(t)}\left(\frac{\delta(s)}{\delta(t)}\right)^{1-2 \beta} \lesssim \frac{\lambda(t)}{\lambda(s)}\left(\frac{\delta(s)}{\delta(t)}\right)^{1-2 \beta}
$$

Let $\sigma=\ell=0$. Then, we use

$$
\frac{1}{|\xi|} \leq \frac{1}{\Omega(s, t)}
$$

For this reason we have the estimate

$$
\left|\hat{K}_{1}(t, s, \xi)\right| \lesssim \frac{1}{|\xi|} \frac{1}{\lambda(t)}\left(\frac{\delta(s)}{\delta(t)}\right)^{1-2 \beta} \lesssim \frac{1}{\Omega(s, t)} \frac{1}{\lambda(s)}\left(\frac{\delta(s)}{\delta(t)}\right)^{1-2 \beta}
$$

Finally, we arrive at

$$
\left\|\hat{K}_{1}(t, s, \cdot) \hat{g}(s, \cdot)\right\|_{\left(L^{2}|\xi| \geq \Omega(s, t)\right)} \lesssim \frac{1}{\Omega(s, t)} \frac{1}{\lambda(s)}\left(\frac{\delta(s)}{\delta(t)}\right)^{1-2 \beta}\|g(s, \cdot)\|_{L^{2}} .
$$

This completes the proof.

Lemma 2.5.14. The following estimates hold for small frequencies $\frac{d_{0}}{F(\Lambda(t))} \leq|\xi| \leq \Omega(s, t)$ :

$$
\begin{aligned}
\left\||\xi|^{\sigma} \partial_{t}^{\ell} \hat{K}_{1}(t, s, \cdot) \hat{g}(s, \cdot)\right\|_{L^{2}\left(\frac{d_{0}}{F(\Lambda(t))} \leq|\xi| \leq \Omega(s, t)\right.} & \\
& \lesssim \frac{\lambda^{2 \ell}(t)}{\rho^{\ell}(t)} \frac{\Lambda(s)}{\lambda(s)}\left(B_{\lambda}(s, t)\right)^{-l}\left(B_{\lambda}(s, t)\right)^{-\frac{\sigma}{2}-\frac{n}{2}\left(\frac{1}{m}-\frac{1}{2}\right)}\|g(s, \cdot)\|_{L^{m}}
\end{aligned}
$$

for any $\sigma \geq 0, t \geq s \geq 0$ and $\ell=0,1$, where $m \in[1,2)$.

Proof. Let $1 \leq m<2$ and we choose $m^{\prime}$ and $p$ such that $\frac{1}{m}+\frac{1}{m^{\prime}}=1$ and $\frac{1}{p}+\frac{1}{m^{\prime}}=\frac{1}{2}$. Therefore, it holds $\frac{1}{p}=\frac{1}{m}-\frac{1}{2}$. Then, applying Hölder's inequality with $\frac{2}{p}+\frac{2}{m^{\prime}}=1$ we obtain the following estimate:

$$
\begin{aligned}
& \left\||\xi|^{\sigma} \partial_{t}^{\ell} \hat{K}_{1}(t, s, \cdot) \hat{g}(s, \cdot)\right\|_{L^{2}\left(\frac{d_{0}}{F(\Lambda(t))} \leq|\xi| \leq \Omega(s, t)\right.} \\
& \quad \leq\left\||\xi|^{\sigma} \partial_{t}^{\ell} \hat{K}_{1}(t, s, \cdot)\right\|_{L^{p}\left(\frac{d_{0}}{F(\Lambda(t))} \leq|\xi| \leq \Omega(s, t)\right.}\|\hat{g}(s, \cdot)\|_{L^{m^{\prime}}\left(\frac{d_{0}}{F(\Lambda(t))} \leq|\xi| \leq \Omega(s, t)\right)} .
\end{aligned}
$$

We can estimate $\|\hat{g}(s, \cdot)\|_{L^{m^{\prime}}}$ by $\|g(s, \cdot)\|_{L^{m}}$ due to the Hausdorff-Young inequality. Therefore, we have only to control the $L^{p}$ norm of the multiplier. Thanks to (2.5.37) and (2.5.38) we have the following estimate:

$$
\begin{aligned}
& \left\||\xi|^{\sigma} \partial_{t}^{\ell} \hat{K}_{1}(t, s, \cdot)\right\|_{L^{p}\left(\frac{d_{0}}{F(\Lambda(t))} \leq|\xi| \leq \Omega(s, t)\right)} \\
& \quad \lesssim \frac{\lambda^{2 \ell}(t)}{\rho^{\ell}(t)} \frac{\Lambda(s)}{\lambda(s)}\left(\int_{\left\{\frac{d_{0}}{F(\Lambda(t))} \leq|\xi| \leq \Omega(s, t)\right\}}|\xi|^{p(\sigma+\ell)} \exp \left(-C p|\xi|^{2} B_{\lambda}(s, t)\right) d \xi\right)^{\frac{1}{p}} .
\end{aligned}
$$


Let us apply the change of variables

$$
r=C p|\xi|^{2} B_{\lambda}(s, t), \quad d r=2 C p|\xi| d|\xi| B_{\lambda}(s, t) .
$$

Then, we have

$$
\begin{aligned}
& \int_{\left\{\frac{d_{0}}{F(\Lambda(t))} \leq|\xi| \leq \Omega(s, t)\right\}}|\xi|^{p(\sigma+2 \ell)} \exp \left(-C p|\xi|^{2} B_{\lambda}(s, t)\right) d \xi \\
& \lesssim\left(B_{\lambda}(s, t)\right)^{-\frac{p(\sigma+2 \ell)+n}{2}} \int_{0}^{\infty} r^{\frac{p(\sigma+2 \ell)+n}{2}-1} e^{-r} d r .
\end{aligned}
$$

The integral on the right-hand side is bounded and we get the function

$$
\frac{\lambda^{2 \ell}(t)}{\rho^{\ell}(t)} \frac{\Lambda(s)}{\lambda(s)}\left(B_{\lambda}(s, t)\right)^{-\frac{\sigma}{2}-\ell-\frac{n}{2 p}}=\frac{\lambda^{2 \ell}(t)}{\rho^{\ell}(t)} \frac{\Lambda(s)}{\lambda(s)}\left(B_{\lambda}(s, t)\right)^{-\ell}\left(B_{\lambda}(s, t)\right)^{-\frac{\sigma}{2}-\frac{n}{2}\left(\frac{1}{m}-\frac{1}{2}\right)} .
$$

This completes the proof.

Lemma 2.5.15. The following estimates hold for small frequencies $|\xi| \leq \frac{d_{0}}{F(\Lambda(t))}$ :

$$
\begin{aligned}
& \left\||\xi|^{\sigma} \partial_{t}^{\ell} \hat{K}_{1}(t, s, \cdot) \hat{g}(s, \cdot)\right\|_{L^{2}\left(|\xi| \leq \frac{d_{0}}{F(\Lambda(t))}\right)} \\
& \quad \lesssim \lambda^{\ell}(t) F^{\ell}(\Lambda(t)) \frac{\Lambda(s)}{\lambda(s)}\left(F^{2}(\Lambda(t))\right)^{-\ell}\left(F^{2}(\Lambda(t))\right)^{-\frac{\sigma}{2}-\frac{n}{2}\left(\frac{1}{m}-\frac{1}{2}\right)}\|g(s, \cdot)\|_{L^{m}}
\end{aligned}
$$

for any $\sigma \geq 0, t \geq s \geq 0$ and $\ell=0,1$, where $m \in[1,2)$.

Proof. In the same way as in the proof of the the previous lemma we have the following estimate:

$$
\left\||\xi|^{\sigma} \partial_{t}^{\ell} \hat{K}_{1}(t, s, \cdot) \hat{g}(s, \cdot)\right\|_{L^{2}\left(|\xi| \leq \frac{d_{0}}{F(\Lambda(t))}\right)} \leq\left\||\xi|^{\sigma} \partial_{t}^{\ell} \hat{K}_{1}(t, s, \cdot)\right\|_{L^{p}\left(|\xi| \leq \frac{d_{0}}{F(\Lambda(t))}\right)}\|\hat{g}(s, \cdot)\|_{L^{m^{\prime}}\left(|\xi| \leq \frac{d_{0}}{F(\Lambda(t))}\right)} .
$$

Thanks to the estimates (2.5.39) and (2.5.40) we obtain

$$
\begin{aligned}
& \left\||\xi|^{\sigma} \partial_{t}^{\ell} \hat{K}_{1}(t, s, \cdot)\right\|_{L^{p}\left(|\xi| \leq \frac{d_{0}}{F(\Lambda(t))}\right)} \\
& \quad \lesssim \frac{\Lambda(s)}{\lambda(s)} \frac{\lambda^{\ell}(t)}{F^{\ell}(\Lambda(t))}\left(\int_{\left\{|\xi| \leq \frac{d_{0}}{F(\Lambda(t))}\right.}|\xi|^{\sigma p} d \xi\right)^{\frac{1}{p}} \lesssim \frac{\Lambda(s)}{\lambda(s)} \frac{\lambda^{\ell}(t)}{F^{\ell}(\Lambda(t))}\left(\int_{0}^{\frac{d_{0}}{F(\Lambda(t))}}|\xi|^{\sigma p+n-1} d|\xi|\right)^{\frac{1}{p}} \\
& \quad \lesssim \frac{\Lambda(s)}{\lambda(s)} \frac{\lambda^{\ell}(t)}{F^{\ell}(\Lambda(t))}\left(\frac{d_{0}}{F(\Lambda(t))}\right)^{\sigma \frac{n}{p}} \lesssim \frac{\Lambda(s)}{\lambda(s)} \frac{\lambda^{\ell}(t)}{F^{\ell}(\Lambda(t))}\left(F^{2}(\Lambda(t))\right)^{-\frac{\sigma}{2}-\frac{n}{2}\left(\frac{1}{m}-\frac{1}{2}\right)} .
\end{aligned}
$$

This completes the proof.

The main result for the family of one-parameter dependent Cauchy problems (2.5.47) follows from the statements of the Lemmas 2.5.13, 2.5.14 and 2.5.15.

Theorem 2.5.16 (Main theorem with $s \geq 0$ ). Let us assume that $\lambda=\lambda(t), \rho=\rho(t)$ and $\omega=\omega(t)$ satisfy the conditions $(\boldsymbol{A} 1)$ to $(\boldsymbol{A} 5)$ and $(\boldsymbol{B 1})$ to $(\boldsymbol{B} 6)$. Then, the solution $v=v(t, x)$ to the Cauchy problem (2.5.47) satisfies the following Matsumura-type estimates for $t \geq s, m \in[1,2)$ and $\sigma \geq 0$ :

$$
\begin{aligned}
&\|v(t, \cdot)\|_{\dot{H}^{\sigma}} \lesssim \frac{\Lambda(s)}{\lambda(s)}\left(1+B_{\lambda}(s, t)\right)^{-\frac{\sigma}{2}-\frac{n}{2}\left(\frac{1}{m}-\frac{1}{2}\right)}\|g(s, \cdot)\|_{\left.L^{m} \cap H^{[\sigma-1}\right]_{+}}, \\
&\left\|v_{t}(t, \cdot)\right\|_{\dot{H}^{\sigma}} \lesssim \frac{\Lambda(s)}{\lambda(s)} \max \left\{\frac{\lambda^{2}(t)}{\rho(t)}\left(1+B_{\lambda}(s, t)\right)^{-\frac{\sigma}{2}-\frac{n}{2}\left(\frac{1}{m}-\frac{1}{2}\right)-1} ;\right. \\
&\left.\lambda(t) F(\Lambda(t))\left(F^{2}(\Lambda(t))\right)^{-\frac{\sigma}{2}-\frac{n}{2}\left(\frac{1}{m}-\frac{1}{2}\right)-1}\right\}\|g(s, \cdot)\|_{L^{m} \cap H^{\sigma}} .
\end{aligned}
$$


Proof. Combining and comparing all the estimates coming from three different parts of the extended phase space we arrive at the higher order energy estimates of solutions to the Cauchy problem (2.5.47). We have a "potential type estimate" because the estimates for small frequencies ("potential type estimate") are faster than the estimates for large frequencies ("exponential type estimate"). That is, here we take into consideration that the estimates in (2.5.53) and (2.5.54) are worse than the estimates from (2.5.49) to (2.5.52), respectively. On the other hand, due to condition (B6) we have the estimate

$$
B_{\lambda}(s, t) \leq B_{\lambda}(0, t) \lesssim F^{2}(\Lambda(t)) .
$$

Hence, from (2.5.53) and (2.5.54) for $\ell=0$ we obtain

$$
\left(F^{2}(\Lambda(t))\right)^{-\frac{\sigma}{2}-\frac{n}{2}\left(\frac{1}{m}-\frac{1}{2}\right)} \lesssim\left(B_{\lambda}(s, t)\right)^{-\frac{\sigma}{2}-\frac{n}{2}\left(\frac{1}{m}-\frac{1}{2}\right)} .
$$

Hence, we may conclude that the relevant behavior for the estimate of $v=v(t, x)$ is coming from the elliptic zone and the estimate of $v_{t}=v_{t}(t, x)$ is coming from the elliptic zone and dissipative zone. Moreover, the regularity of the data is coming from the influence of the large frequencies from (2.5.49) to (2.5.52).

Let us introduce $C_{\sigma}:=\frac{\sigma}{2}+\frac{n}{2}\left(\frac{1}{m}-\frac{1}{2}\right)$. We distinguish between two cases as follows:

Case 1: $\eta=\eta(t)$ is increasing

In this case we shall compare the estimates (2.5.49) and (2.5.50) with (2.5.53). Moreover, we will compare the estimates (2.5.49) and (2.5.50) with (2.5.54). Then, we have the following cases:

Case 1.1. Comparison of the estimates (2.5.49) and (2.5.53)

In this case we compare the term

$$
\frac{\lambda^{\ell}(t)}{\lambda(s)}\left(\frac{\delta(s)}{\delta(t)}\right)^{1-2 \beta}=\frac{\lambda^{\ell}(t)}{\lambda(s)}\left(\frac{\delta(s)}{\delta(t)}\right)^{2 C_{1}}=\frac{\lambda^{\ell}(t)}{\lambda(s)} \exp \left(-C_{1} \int_{s}^{t} \rho(\tau) \omega(\tau) d \tau\right)
$$

from (2.5.49) with the term

$$
\frac{\lambda^{2 \ell}(t)}{\rho^{\ell}(t)} \frac{\Lambda(s)}{\lambda(s)}\left(B_{\lambda}(s, t)\right)^{-\ell}\left(B_{\lambda}(s, t)\right)^{-\frac{\sigma}{2}-\frac{n}{2}\left(\frac{1}{m}-\frac{1}{2}\right)}=\frac{\lambda^{2 \ell}(t)}{\rho^{\ell}(t)} \frac{\Lambda(s)}{\lambda(s)}\left(B_{\lambda}(s, t)\right)^{-C_{\sigma}-\ell}
$$

from (2.5.53). We will show that

$$
\frac{\lambda^{\ell}(t)}{\lambda(s)}\left(\frac{\delta(s)}{\delta(t)}\right)^{2 C_{1}} \lesssim \frac{\lambda^{2 \ell}(t)}{\rho^{\ell}(t)} \frac{\Lambda(s)}{\lambda(s)}\left(B_{\lambda}(s, t)\right)^{-C_{\sigma}-\ell} .
$$

It follows

$$
\left(\frac{\delta(s)}{\delta(t)}\right)^{2 C_{1}} \lesssim \frac{1}{\eta^{\ell}(t)} \Lambda(s)\left(B_{\lambda}(s, t)\right)^{-C_{\sigma}-\ell}
$$

Due to $\eta(t)$ is increasing, then there exists a constant $t_{0}$ such that $C=\eta\left(t_{0}\right) \leq \eta(t)$ uniformly for all $t \geq t_{0}$. Thus, we have

$$
\rho^{2}(t)=\lambda^{2}(t) \eta^{2}(t) \geq C^{2} \lambda^{2}(t), \quad \text { which implies } \quad \rho(t) \geq C^{2} \frac{\lambda^{2}(t)}{\rho(t)}
$$

for all $t \geq t_{0}$. This brings the desired dominance.

Case 1.2. Comparison of the estimates (2.5.50) and (2.5.53)

In this case we have $\sigma=\ell=0$. Then, we will show that

$$
\frac{1}{\eta(t) \lambda(s)}\left(\frac{\delta(s)}{\delta(t)}\right)^{2 C_{1}} \lesssim \frac{\Lambda(s)}{\lambda(s)}\left(B_{\lambda}(s, t)\right)^{-C_{\sigma}}
$$


Due to the considerations in the Case 1.1 and since $\eta(t)$ is increasing, this estimate holds for all $t \geq s$.

Case 1.3. Comparison of the estimates (2.5.49) and (2.5.54)

In this case we compare the term

$$
\frac{\lambda^{\ell}(t)}{\lambda(s)}\left(\frac{\delta(s)}{\delta(t)}\right)^{2 C_{1}}=\frac{\lambda^{\ell}(t)}{\lambda(s)} \exp \left(-C_{1} \int_{s}^{t} \rho(\tau) \omega(\tau) d \tau\right)
$$

from (2.5.49) with the term

$$
\lambda^{\ell}(t) F^{\ell}(\Lambda(t)) \frac{\Lambda(s)}{\lambda(s)}\left(F^{2}(\Lambda(t))\right)^{-\ell}\left(F^{2}(\Lambda(t))\right)^{-\frac{\sigma}{2}-\frac{n}{2}\left(\frac{1}{m}-\frac{1}{2}\right)}=\lambda^{\ell}(t) \frac{\Lambda(s)}{\lambda(s)} F(\Lambda(t))^{-2 C_{\sigma}-\frac{\ell}{2}}
$$

from (2.5.54). We want to show that

$$
\frac{\lambda^{\ell}(t)}{\lambda(s)}\left(\frac{\delta(s)}{\delta(t)}\right)^{2 C_{1}} \lesssim \lambda^{\ell}(t) \frac{\Lambda(s)}{\lambda(s)} F(\Lambda(t))^{-2 C_{\sigma}-\frac{\ell}{2}} .
$$

So, it suffices to consider the monotonicity of the function

$$
F(\Lambda(t))^{-2 C_{\sigma}-\frac{\ell}{2}}\left(\frac{\delta(t)}{\delta(s)}\right)^{2 C_{1}}
$$

We form the derivative of this function

$$
\begin{aligned}
& \partial_{t}\left[F(\Lambda(t))^{-2 C_{\sigma}-\frac{\ell}{2}}\left(\frac{\delta(t)}{\delta(s)}\right)^{2 C_{1}}\right] \\
& =\left(-2 C_{\sigma}-\frac{\ell}{2}\right) F(\Lambda(t))^{-2 C_{\sigma}-\frac{\ell}{2}-1} \lambda(t) F^{\prime}(\Lambda(t))\left(\frac{\delta(t)}{\delta(s)}\right)^{2 C_{1}}+C_{1} \rho(t) \omega(t)\left(\frac{\delta(t)}{\delta(s)}\right)^{2 C_{1}} F(\Lambda(t))^{-2 C_{\sigma}-\frac{\ell}{2}} \\
& =F(\Lambda(t))^{-2 C_{\sigma}-\frac{\ell}{2}-1} \lambda(t) F^{\prime}(\Lambda(t))\left(\frac{\delta(t)}{\delta(s)}\right)^{2 C_{1}}\left(-2 C_{\sigma}-\frac{\ell}{2}+C_{1} \omega(t) \frac{\mu(t)}{\Lambda(t)} \frac{F(\Lambda(t))}{F^{\prime}(\Lambda(t))}\right) \\
& =F(\Lambda(t))^{-2 C_{\sigma}-\frac{\ell}{2}-1} \lambda(t) F^{\prime}(\Lambda(t))\left(\frac{\delta(t)}{\delta(s)}\right)^{2 C_{1}}\left(-2 C_{\sigma}-\frac{\ell}{2}+C_{1} \omega(t) \frac{\mu(t)}{\Lambda(t)} \frac{\lambda(t) \Xi(t)}{\sqrt{F^{\prime}(\Lambda(t))}}\right),
\end{aligned}
$$

where we used condition (A5), that is, $\Xi(t)=\frac{F(\Lambda(t))}{\lambda(t) \sqrt{F^{\prime}(\Lambda(t))}}$. Here we can see that the last term in the last bracket tends to $\infty$ as $t$ tends to $\infty$. Because, from condition (A2) we have $0<c_{0} \leq \omega(t) \leq c_{1}$ and $\Theta(t) \lesssim \lambda(t) \Xi(t) \lesssim \Lambda(t)$. For this reason we obtain

$$
\omega(t) \frac{\mu(t)}{\Lambda(t)} \frac{\lambda(t) \Xi(t)}{\sqrt{F^{\prime}(\Lambda(t))}} \gtrsim \mu(t) \frac{\Theta(t)}{\Lambda(t)} \frac{1}{\sqrt{F^{\prime}(\Lambda(t))}},
$$

where by the condition (B5) we have $\mu(t) \frac{\Theta(t)}{\Lambda(t)} \rightarrow \infty$ as $t \rightarrow \infty$. Moreover, from condition (A5) we have

$$
\frac{1}{\sqrt{F^{\prime}(\Lambda(t))}}=\frac{\lambda(t) \Xi(t)}{F(\Lambda(t))} \gtrsim \frac{\Theta(t)}{F(\Lambda(t))}
$$

Thus, we arrive at the expected comparison.

Case 1.4. Comparison of the estimates (2.5.50) and (2.5.54)

In this case we have $\sigma=\ell=0$. We want to show the estimate

$$
\frac{1}{\eta(t) \lambda(s)}\left(\frac{\delta(s)}{\delta(t)}\right)^{2 C_{1}} \lesssim \frac{\Lambda(s)}{\lambda(s)} F(\Lambda(t))^{-2 C_{\sigma}} .
$$

Hence, due to the Case 1.3 and since $\eta(t)$ is increasing, this estimate holds.

Case 2: $\eta=\eta(t)$ is decreasing 
Now we need to compare (2.5.51) and (2.5.52) with (2.5.53). Moreover, we will compare the estimates (2.5.51) and (2.5.52) with (2.5.54).

Case 2.1. Comparison of the estimates (2.5.51) and (2.5.53)

In this case let us compare the term

$$
\frac{\lambda^{\ell}(t)}{\lambda(s)}\left(\frac{\delta(s)}{\delta(t)}\right)^{1-2 \beta}=\frac{\lambda^{\ell}(t)}{\lambda(s)}\left(\frac{\delta(s)}{\delta(t)}\right)^{2 C_{1}}=\frac{\lambda^{\ell}(t)}{\lambda(s)} \exp \left(-C_{1} \int_{s}^{t} \rho(\tau) \omega(\tau) d \tau\right)
$$

from (2.5.51) with the term

$$
\frac{\lambda^{2 \ell}(t)}{\rho^{\ell}(t)} \frac{\Lambda(s)}{\lambda(s)}\left(B_{\lambda}(s, t)\right)^{-\ell}\left(B_{\lambda}(s, t)\right)^{-\frac{\sigma}{2}-\frac{n}{2}\left(\frac{1}{m}-\frac{1}{2}\right)}=\frac{\lambda^{2 \ell}(t)}{\rho^{\ell}(t)} \frac{\Lambda(s)}{\lambda(s)}\left(B_{\lambda}(s, t)\right)^{-C_{\sigma}-\ell}
$$

from (2.5.53). We will verify that the following estimate holds:

$$
\frac{\lambda^{\ell}(t)}{\lambda(s)}\left(\frac{\delta(s)}{\delta(t)}\right)^{2 C_{1}} \lesssim \frac{\lambda^{2 \ell}(t)}{\rho^{\ell}(t)} \frac{\Lambda(s)}{\lambda(s)}\left(B_{\lambda}(s, t)\right)^{-C_{\sigma}-\ell} .
$$

It follows

$$
1 \lesssim \frac{1}{\eta^{\ell}(t)} \Lambda(s)\left(\frac{\delta(t)}{\delta(s)}\right)^{2 C_{1}}\left(B_{\lambda}(s, t)\right)^{-C_{\sigma}-\ell}
$$

Then, we consider the monotonicity of the function

$$
\left(\frac{\delta(t)}{\delta(s)}\right)^{2 C_{1}}\left(B_{\lambda}(s, t)\right)^{-C_{\sigma}-\ell}
$$

We calculate the derivative of this function as follows:

$$
\begin{aligned}
\partial_{t}[ & \left.\left(\frac{\delta(t)}{\delta(s)}\right)^{2 C_{1}}\left(B_{\lambda}(s, t)\right)^{-C_{\sigma}-\ell}\right] \\
& =C_{1} \rho(t) \omega(t)\left(\frac{\delta(t)}{\delta(s)}\right)^{2 C_{1}}\left(B_{\lambda}(s, t)\right)^{-C_{\sigma}-\ell}\left(-C_{\sigma}-\ell\right)\left(\frac{\delta(t)}{\delta(s)}\right)^{2 C_{1}}\left(B_{\lambda}(s, t)\right)^{-C_{\sigma}-\ell-1} \frac{\lambda^{2}(t)}{\rho(t)} \\
& =\left(\frac{\delta(t)}{\delta(s)}\right)^{2 C_{1}}\left(B_{\lambda}(s, t)\right)^{-C_{\sigma}-\ell-1} \frac{\lambda^{2}(t)}{\rho(t)}\left(\frac{C_{1} \rho^{2}(t) \omega(t)}{\lambda^{2}(t)} B_{\lambda}(s, t)-C_{\sigma}-\ell\right) .
\end{aligned}
$$

Now we can see that the first term in the last bracket tends to $\infty$ as $t$ tends to $\infty$. Indeed, we have

$$
\begin{aligned}
\frac{\rho^{2}(t) \omega(t)}{\lambda^{2}(t)} B_{\lambda}(s, t) & =\frac{\mu^{2}(t) \omega(t)}{\Lambda^{2}(t)} \int_{s}^{t} \frac{\lambda(\tau) \Lambda(\tau)}{\mu(\tau)} d \tau \\
& =\frac{\mu^{2}(t) \omega(t)}{\Lambda^{2}(t)}\left(\frac{\Lambda^{2}(t)}{2 \mu(t)}-\frac{\Lambda^{2}(s)}{2 \mu(s)}+\int_{s}^{t} \frac{\mu^{\prime}(\tau) \Lambda^{2}(\tau)}{2 \mu^{2}(\tau)} d \tau\right) \\
& \geq \frac{\mu(\tau) \omega(\tau)}{2}-\frac{\mu^{2}(t) \omega(t)}{2 \Lambda^{2}(t)} \frac{\Lambda^{2}(s)}{\mu(s)}-\frac{\mu_{1} \mu^{2}(t) \omega(t)}{2 \Lambda^{2}(t)} \int_{s}^{t} \frac{\lambda(\tau) \Lambda(\tau)}{\mu(\tau)} d \tau,
\end{aligned}
$$

where we used condition (B2), that is, $\left|\mu^{\prime}(t)\right| \leq \mu_{1} \mu(t) \lambda(t) / \Lambda(t)$. From the last estimate we get

$$
\begin{aligned}
\frac{\mu^{2}(t)}{\Lambda^{2}(t)} \int_{s}^{t} \frac{\lambda(\tau) \Lambda(\tau)}{\mu(\tau)} d \tau & \geq\left(\frac{1}{2+\mu_{1}}\right)\left(\mu(t)-\frac{\mu^{2}(t)}{\Lambda^{2}(t)} \frac{\Lambda^{2}(s)}{\mu(s)}\right) \\
& \geq\left(\frac{1}{2+\mu_{1}}\right)\left(\mu(t)-\frac{\mu(t)}{\Lambda(t)} \Lambda(s)\right) \gtrsim \mu(t) .
\end{aligned}
$$

Here we have used the function $\eta(t)=\frac{\mu(t)}{2 \Lambda(t)}$ is decreasing and the function $\Lambda(t)$ is increasing. Thus, for a sufficiently large time $t_{0}$ the expression (2.5.55) is positive for all $t \geq t_{0}$. This yields our expected comparison. 
Case 2.2. Comparison of the estimates (2.5.52) and (2.5.53)

In this case we have $\sigma=\ell=0$. Then, we will show that

$$
\frac{1}{\lambda(s) \eta(s)}\left(\frac{\delta(s)}{\delta(t)}\right)^{2 C_{1}} \lesssim \frac{\Lambda(s)}{\lambda(s)}\left(B_{\lambda}(s, t)\right)^{-C_{\sigma}}
$$

It follows

$$
1 \lesssim \mu(s)\left(\frac{\delta(t)}{\delta(s)}\right)^{2 C_{1}}\left(B_{\lambda}(s, t)\right)^{-C_{\sigma}} .
$$

Therefore, this estimate is satisfied due to the considerations in the Case 2.1.

Case 2.3. Comparison of the estimates (2.5.51) and (2.5.54)

This case can be shown in the same way as in the Case 1.3.

Case 2.4. Comparison of the estimates (2.5.52) and (2.5.54)

In this case we have $\sigma=\ell=0$. Then, we will show that

$$
\frac{1}{\lambda(s) \eta(s)}\left(\frac{\delta(s)}{\delta(t)}\right)^{2 C_{1}} \lesssim \frac{\Lambda(s)}{\lambda(s)} F(\Lambda(t))^{-2 C_{\sigma}}
$$

Hence, from the considerations in the Case 1.3 this estimate holds for all $t \geq s$.

In this way we have proved the desired statements. This completes the proof.

Remark 2.5.4. We note that in our estimates we replace $B_{\lambda}(0, t)$ by $1+B_{\lambda}(0, t)$. This can be done modulo a compact set in the extended phase space. Such a compact set will never influence the desired estimates.

\section{Matsumura-type estimates with $s=0$}

We use the same approach to estimate $\hat{K}_{0}(t, 0, \xi)$ and $\partial_{t} \hat{K}_{0}(t, 0, \xi)$. Again we assume additional $L^{m}$ regularity for the data, with $m \in[1,2)$ in order to prove energy estimates for the solutions and their first partial derivative in time to the Cauchy problem (2.5.48). We have the following statements.

Lemma 2.5.17. The following estimates hold for large frequencies $|\xi| \geq \Omega(0, t)$ :

$$
\left\||\xi|^{\sigma} \partial_{t}^{\ell} \hat{K}_{0}(t, 0, \cdot) \hat{f}\right\|_{L^{2}(|\xi| \geq \Omega(0, t))} \lesssim \lambda^{\ell}(t)\left(\frac{1}{\delta(t)}\right)^{1-2 \beta}\|f\|_{H^{\sigma+\ell}}
$$

for any $\sigma \geq 0$ and $\ell=0,1$.

Lemma 2.5.18. The following estimates hold for the small frequencies $\frac{d_{0}}{F(\Lambda(t))} \leq|\xi| \leq \Omega(0, t)$ :

$$
\begin{aligned}
&\left\||\xi|^{\sigma} \partial_{t}^{\ell} \hat{K}_{0}(t, 0, \cdot) \hat{f}\right\|_{L^{2}\left(\frac{d_{0}}{F(\Lambda(t))} \leq|\xi| \leq \Omega(0, t)\right.} \\
& \lesssim \frac{\lambda^{2 \ell}(t)}{\rho^{\ell}(t)}\left(B_{\lambda}(0, t)\right)^{-\ell}\left(B_{\lambda}(0, t)\right)^{-\frac{\sigma}{2}-\frac{n}{2}\left(\frac{1}{m}-\frac{1}{2}\right)}\|f\|_{L^{m}}
\end{aligned}
$$

for any $\sigma \geq 0$ and $\ell=0,1$, where $m \in[1,2)$.

Lemma 2.5.19. The following estimates hold for small frequencies $|\xi| \leq \frac{d_{0}}{F(\Lambda(t))}$ :

$$
\begin{aligned}
\left\||\xi|^{\sigma} \partial_{t}^{\ell} \hat{K}_{0}(t, 0, \cdot) \hat{f}\right\|_{L^{2}\left(|\xi| \leq \frac{d_{0}}{F(\Lambda(t))}\right)} & \\
& \lesssim \lambda^{\ell}(t) F^{\ell}(\Lambda(t))\left(F^{2}(\Lambda(t))\right)^{-\ell}\left(F^{2}(\Lambda(t))\right)^{-\frac{\sigma}{2}-\frac{n}{2}\left(\frac{1}{m}-\frac{1}{2}\right)}\|f\|_{L^{m}}
\end{aligned}
$$

for any $\sigma \geq 0$ and $\ell=0,1$, where $m \in[1,2)$. 
The main result for the Cauchy problem (2.5.48) follows from Lemmas 2.5.17, 2.5.18 and 2.5.19. The estimate that we obtained in (2.5.57) and (2.5.58) are worse than the estimate that we have for large frequencies in (2.5.56). Moreover, due to condition (B6) from (2.5.57) and (2.5.58) for $\ell=0$ we have the estimate

$$
\left(F^{2}(\Lambda(t))\right)^{-\frac{\sigma}{2}-\frac{n}{2}\left(\frac{1}{m}-\frac{1}{2}\right)} \lesssim\left(B_{\lambda}(0, t)\right)^{-\frac{\sigma}{2}-\frac{n}{2}\left(\frac{1}{m}-\frac{1}{2}\right)} .
$$

The comparison arguments for small and large frequencies can be verified in a similar way as in the proof of Theorem 2.5.16 with $s=0$. Thus, we get the following result.

Theorem 2.5.20. Let us assume that $\lambda=\lambda(t), \rho=\rho(t)$ and $\omega=\omega(t)$ satisfy the conditions (A1) to (A5) and (B1) to (B6). Then, the solution to the Cauchy problem (2.5.48) satisfies the following estimates for $\sigma \geq 0$ and $m \in[1,2)$ :

$$
\begin{aligned}
&\|u(t, \cdot)\|_{\dot{H}^{\sigma}} \lesssim\left(1+B_{\lambda}(0, t)\right)^{-\frac{\sigma}{2}-\frac{n}{2}\left(\frac{1}{m}-\frac{1}{2}\right)}\|f\|_{L^{m} \cap H^{\sigma}}, \\
&\left\|u_{t}(t, \cdot)\right\|_{\dot{H}^{\sigma}} \lesssim \max \left\{\frac{\lambda^{2}(t)}{\rho(t)}\left(1+B_{\lambda}(0, t)\right)^{-\frac{\sigma}{2}-\frac{n}{2}\left(\frac{1}{m}-\frac{1}{2}\right)-1} ;\right. \\
&\left.\lambda(t) F(\Lambda(t))\left(F^{2}(\Lambda(t))\right)^{-\frac{\sigma}{2}-\frac{n}{2}\left(\frac{1}{m}-\frac{1}{2}\right)-1}\right\}\|f\|_{L^{m} \cap H^{\sigma+1}} .
\end{aligned}
$$

We know that the solution $u=u(t, x)$ to the Cauchy problem

$$
\begin{cases}u_{t t}-\lambda^{2}(t) \omega^{2}(t) \Delta u+\rho(t) \omega(t) u_{t}=0, & (t, x) \in[0, \infty) \times \mathbb{R}^{n}, \\ u(0, x)=u_{0}(x), \quad u_{t}(0, x)=u_{1}(x), & x \in \mathbb{R}^{n} .\end{cases}
$$

can be represented as

$$
u(t, x)=K_{0}(t, 0, x) *_{(x)} u_{0}(x)+K_{1}(t, 0, x) *_{(x)} u_{1}(x) .
$$

Summarizing, we obtain the following statement.

Theorem 2.5.21 (Main theorem with $s=0$ ). We assume that $\lambda=\lambda(t), \rho=\rho(t)$ and $\omega=\omega(t)$ satisfy the conditions $(\boldsymbol{A} \mathbf{1})$ to $(\boldsymbol{A} 5)$ and $(\boldsymbol{B 1})$ to $(\boldsymbol{B} 6)$. Then, the solution to the Cauchy problem (2.5.59) satisfies the following estimates with $m \in[1,2)$ and $\sigma \geq 0$ :

$$
\begin{aligned}
\|u(t, \cdot)\|_{\dot{H}^{\sigma}} \lesssim(1+ & \left.B_{\lambda}(0, t)\right)^{-\frac{\sigma}{2}-\frac{n}{2}\left(\frac{1}{m}-\frac{1}{2}\right)}\left(\left\|u_{0}\right\|_{L^{m} \cap H^{\sigma}}+\left\|u_{1}\right\|_{L^{m} \cap H^{[\sigma-1]_{+}}}\right), \\
\left\|u_{t}(t, \cdot)\right\|_{\dot{H}^{\sigma}} \lesssim \max \{ & \frac{\lambda^{2}(t)}{\rho(t)}\left(1+B_{\lambda}(0, t)\right)^{-\frac{\sigma}{2}-\frac{n}{2}\left(\frac{1}{m}-\frac{1}{2}\right)-1} ; \\
& \left.\lambda(t) F(\Lambda(t))\left(F^{2}(\Lambda(t))\right)^{-\frac{\sigma}{2}-\frac{n}{2}\left(\frac{1}{m}-\frac{1}{2}\right)-1}\right\}\left(\left\|u_{0}\right\|_{L^{m} \cap H^{\sigma+1}}+\left\|u_{1}\right\|_{L^{m} \cap H^{\sigma}}\right) .
\end{aligned}
$$

\subsubsection{Some examples}

We will conclude this section with some examples for special coefficients.

Example 2.5.1 (Polynomial case). Let us choose $\lambda(t)=(\alpha+1)(1+t)^{\alpha}, \alpha>0$. So, we have

$$
\Lambda(t)=(1+t)^{\alpha+1} \text { and } \Theta(t)=(1+t)^{\gamma+1}, \quad-1<\gamma<\alpha .
$$

Moreover, we have

$$
\rho(t)=\frac{(\alpha+1)^{2}}{2 \alpha-\beta+1}(1+t)^{\beta}, \quad \alpha-\gamma-1<\beta<2 \alpha+1 .
$$

Now we choose $\Xi(t)=(1+t)^{\kappa}$ with $1>\kappa \geq \frac{3-\beta}{4}$. Then, we obtain

$$
F(\Lambda(t)) \simeq(1+t)^{\alpha+2 \kappa-1} .
$$


Hence, the hypotheses of Theorem 2.5.21 are satisfied.

Then, all the above choices give us the following estimates:

$$
\|u(t, \cdot)\|_{\dot{H}^{\sigma}} \lesssim(1+t)^{-(2 \alpha-\beta+1)\left(\frac{n}{2}\left(\frac{1}{m}-\frac{1}{2}\right)+\frac{\sigma}{2}\right)}\left(\left\|u_{0}\right\|_{L^{m} \cap H^{\sigma}}+\left\|u_{1}\right\|_{L^{m} \cap H^{[\sigma-1]_{+}}}\right)
$$

and

$$
\begin{aligned}
\left\|u_{t}(t, \cdot)\right\|_{\dot{H}^{\sigma}} \lesssim & \max \left\{(1+t)^{-(2 \alpha-\beta+1)\left(\frac{n}{2}\left(\frac{1}{m}-\frac{1}{2}\right)+\frac{\sigma}{2}\right)-1} ;(1+t)^{-(2 \alpha+4 \kappa-2)\left(\frac{n}{2}\left(\frac{1}{m}-\frac{1}{2}\right)+\frac{\sigma}{2}\right)-2 \kappa+1}\right\} \\
& \times\left(\left\|u_{0}\right\|_{L^{m} \cap H^{\sigma+1}}+\left\|u_{1}\right\|_{L^{m} \cap H^{\sigma}}\right) .
\end{aligned}
$$

Now let us consider the competition between the estimates in (2.5.60). We introduce $C_{\sigma, n, m}:=$ $\frac{n}{2}\left(\frac{1}{m}-\frac{1}{2}\right)+\frac{\sigma}{2}$. If

$$
-(2 \alpha-\beta+1) C_{\sigma, n, m}-1<-(2 \alpha+4 \kappa-2) C_{\sigma, n, m}-2 \kappa+1
$$

holds, then we have

$$
\left\|u_{t}(t, \cdot)\right\|_{\dot{H}^{\sigma}} \lesssim(1+t)^{-(2 \alpha+4 \kappa-2)\left(\frac{n}{2}\left(\frac{1}{m}-\frac{1}{2}\right)+\frac{\sigma}{2}\right)-2 \kappa+1}\left(\left\|u_{0}\right\|_{L^{m} \cap H^{\sigma+1}}+\left\|u_{1}\right\|_{L^{m} \cap H^{\sigma}}\right) .
$$

On the other hand, if

$$
-(2 \alpha-\beta+1) C_{\sigma, n, m}-1>-(2 \alpha+4 \kappa-2) C_{\sigma, n, m}-2 \kappa+1
$$

holds, then we get

$$
\left\|u_{t}(t, \cdot)\right\|_{\dot{H}^{\sigma}} \lesssim(1+t)^{-(2 \alpha-\beta+1)\left(\frac{n}{2}\left(\frac{1}{m}-\frac{1}{2}\right)+\frac{\sigma}{2}\right)-1}\left(\left\|u_{0}\right\|_{L^{m} \cap H^{\sigma+1}}+\left\|u_{1}\right\|_{L^{m} \cap H^{\sigma}}\right) .
$$

We note that if we set formally $\kappa=1$, then we have $F(\Lambda(t)) \equiv \Lambda(t)$ and $\Theta(t) \equiv \Lambda(t)$. Hence, the last estimate is satisfied with $-1<\beta$ and and we say that this estimate coincides with the estimate in the case of very slow oscillations.

Example 2.5.2 (Exponential case). Let us choose $\lambda(t)=e^{t}$. So, we have

$$
\Lambda(t)=e^{t} \text { and } \Theta(t)=e^{r t}, \quad 0<r<1 .
$$

Moreover, we have

$$
\rho(t)=\frac{1}{2-q} e^{q t}, \quad 1-r<q<2 .
$$

Now we choose $\Xi(t)=e^{\kappa t}$ with $0>\kappa \geq-\frac{q}{4}$. Then, we obtain

$$
F(\Lambda(t)) \simeq e^{(1+2 \kappa) t} .
$$

Hence, the hypotheses of Theorem 2.5.21 are satisfied.

Therefore, all the above choices give us the following estimates:

$$
\|u(t, \cdot)\|_{\dot{H}^{\sigma}} \lesssim e^{-(2-q)\left(\frac{n}{2}\left(\frac{1}{m}-\frac{1}{2}\right)+\frac{\sigma}{2}\right) t}\left(\left\|u_{0}\right\|_{L^{m} \cap H^{\sigma}}+\left\|u_{1}\right\|_{L^{m} \cap H^{[\sigma-1]_{+}}}\right)
$$

and

$$
\begin{aligned}
\left\|u_{t}(t, \cdot)\right\|_{\dot{H}^{\sigma}} \lesssim \max & \left\{e^{-(2-q)\left(\frac{n}{2}\left(\frac{1}{m}-\frac{1}{2}\right)+\frac{\sigma}{2}\right) t} ; e^{-2 \kappa t} e^{-(2+4 \kappa)\left(\frac{n}{2}\left(\frac{1}{m}-\frac{1}{2}\right)+\frac{\sigma}{2}\right) t}\right\} \\
\times & \left(\left\|u_{0}\right\|_{L^{m} \cap H^{\sigma+1}}+\left\|u_{1}\right\|_{L^{m} \cap H^{\sigma}}\right) .
\end{aligned}
$$

Now let us consider the competition between the estimates in (2.5.61) introducing $C_{\sigma, n, m}=$ $\frac{\sigma}{2}+\frac{n}{2}\left(\frac{1}{m}-\frac{1}{2}\right)$. If

$$
-(2-q) C_{\sigma, n, m}<-(2+4 \kappa) C_{\sigma, n, m}-2 \kappa
$$

holds, then we have the estimate

$$
\left\|u_{t}(t, \cdot)\right\|_{\dot{H}^{\sigma}} \lesssim e^{-2 \kappa t} e^{-(2+4 \kappa)\left(\frac{n}{2}\left(\frac{1}{m}-\frac{1}{2}\right)+\frac{\sigma}{2}\right) t}\left(\left\|u_{0}\right\|_{L^{m} \cap H^{\sigma+1}}+\left\|u_{1}\right\|_{L^{m} \cap H^{\sigma}}\right) .
$$


Whereas, if

$$
-(2-q) C_{\sigma, n, m}>-(2+4 \kappa) C_{\sigma, n, m}-2 \kappa
$$

holds, then we have the estimate

$$
\left\|u_{t}(t, \cdot)\right\|_{\dot{H}^{\sigma}} \lesssim e^{-(2-q)\left(\frac{n}{2}\left(\frac{1}{m}-\frac{1}{2}\right)+\frac{\sigma}{2}\right) t}\left(\left\|u_{0}\right\|_{L^{m} \cap H^{\sigma+1}}+\left\|u_{1}\right\|_{L^{m} \cap H^{\sigma}}\right) .
$$

We note that if we set formally $\kappa=0$, then we have $F(\Lambda(t)) \equiv \Lambda(t)$ and $\Theta(t) \equiv \Lambda(t)$. Hence, the last estimate is satisfied with $0<q$ and and we say that this estimate coincides with the estimate in the case of very slow oscillations.

Example 2.5.3 (Super-exponential case). Let us choose $\lambda(t)=e^{t} e^{e^{t}}$. So, we have

$$
\Lambda(t)=e^{e^{t}} \quad \text { and } \quad \Theta(t)=e^{r e^{t}}, \quad 0<r<1 .
$$

Moreover, we have

$$
\rho(t)=\frac{1}{2-q} e^{t} e^{q e^{t}}, \quad 1-r<q<2 .
$$

Now we choose $\Xi(t)=e^{-t} e^{\kappa e^{t}}$ with $0>\kappa \geq-\frac{q}{4}$. Then, we get

$$
F(\Lambda(t)) \simeq e^{(1+2 \kappa) e^{t}}
$$

Hence, the hypotheses of Theorem 2.5.21 are satisfied.

By the above choices we get the following estimates:

$$
\|u(t, \cdot)\|_{\dot{H}^{\sigma}} \lesssim e^{-(2-q)\left(\frac{n}{2}\left(\frac{1}{m}-\frac{1}{2}\right)+\frac{\sigma}{2}\right) e^{t}}\left(\left\|u_{0}\right\|_{L^{m} \cap H^{\sigma}}+\left\|u_{1}\right\|_{L^{m} \cap H^{[\sigma-1]_{+}}}\right),
$$

and

$$
\begin{aligned}
\left\|u_{t}(t, \cdot)\right\|_{\dot{H}^{\sigma}} \lesssim \max & \left\{e^{t} e^{-(2-q)\left(\frac{n}{2}\left(\frac{1}{m}-\frac{1}{2}\right)+\frac{\sigma}{2}\right) e^{t}} ; e^{-2 \kappa e^{t}} e^{-(2+4 \kappa)\left(\frac{n}{2}\left(\frac{1}{m}-\frac{1}{2}\right)+\frac{\sigma}{2}\right) e^{t}}\right\} \\
& \times\left(\left\|u_{0}\right\|_{L^{m} \cap H^{\sigma+1}}+\left\|u_{1}\right\|_{L^{m} \cap H^{\sigma}}\right) .
\end{aligned}
$$

Now let us consider the competition between the estimates in (2.5.62). If

$$
-(2-q) C_{\sigma, n, m}<-(2+4 \kappa) C_{\sigma, n, m}-2 \kappa
$$

holds, then we have the estimate

$$
\left\|u_{t}(t, \cdot)\right\|_{\dot{H}^{\sigma}} \lesssim e^{-2 \kappa e^{t}} e^{-(2+4 \kappa)\left(\frac{n}{2}\left(\frac{1}{m}-\frac{1}{2}\right)+\frac{\sigma}{2}\right) e^{t}}\left(\left\|u_{0}\right\|_{L^{m} \cap H^{\sigma+1}}+\left\|u_{1}\right\|_{L^{m} \cap H^{\sigma}}\right) .
$$

While, if

$$
-(2-q) C_{\sigma, n, m}>-(2+4 \kappa) C_{\sigma, n, m}-2 \kappa
$$

holds, then we have the estimate

$$
\left\|u_{t}(t, \cdot)\right\|_{\dot{H}^{\sigma}} \lesssim e^{t} e^{-(2-q)\left(\frac{n}{2}\left(\frac{1}{m}-\frac{1}{2}\right)+\frac{\sigma}{2}\right) e^{t}}\left(\left\|u_{0}\right\|_{L^{m} \cap H^{\sigma+1}}+\left\|u_{1}\right\|_{L^{m} \cap H^{\sigma}}\right) .
$$

We note that if we set formally $\kappa=0$, then we have $F(\Lambda(t)) \equiv \Lambda(t)$ and $\Theta(t) \equiv \Lambda(t)$. Hence, the last estimate is satisfied with $0<q$ and and we say that this estimate coincides with the estimate in the case of very slow oscillations.

\subsection{Construction of admissible oscillating functions}

Now we present an admissible nontrivial oscillating function $\omega=\omega(t)$ in the coefficients of our model (2.1.1) satisfying the hypotheses of Theorems 2.5.16, 2.5.20 and 2.5.21. The construction of the function $\omega=\omega(t)$ was done in [19]. 
In order to construct a nontrivial function $\omega=\omega(t)$ satisfying conditions (A2), (A3) and (B5) let us choose the positive sequences $\left\{t_{j}\right\}_{j},\left\{\delta_{j}\right\}_{j}$ and $\left\{\eta_{j}\right\}_{j}$ in the following way:

$$
t_{j} \rightarrow \infty, \quad \delta_{j} \leq \Delta t_{j}:=t_{j+1}-t_{j} \quad \text { and } \quad \eta_{j} \leq 1
$$

and a function $\psi \in \mathcal{C}_{0}^{M}(\mathbb{R})$ with

$$
\operatorname{supp} \psi \subseteq[0,1], \quad-1<\psi(t)<1 \quad \text { and } \quad \int_{0}^{1}|\psi(t)| d t=\frac{1}{2} .
$$

Then, we define

$$
\omega(t)=1+\sum_{j=1}^{\infty} \eta_{j} \psi\left(\frac{t-t_{j}}{\delta_{j}}\right) .
$$

The last sum is convergent, because by (2.6.1) for each $t$ at most one term is present. Furthermore, if $c_{0}=\min _{[0,1]} \psi(t)$ and $c_{1}=\max _{[0,1]} \psi(t)$, then we get the bounds

$$
0<1+c_{0} \leq \omega(t) \leq 1+c_{1} .
$$

For (A2) we can take

$$
C_{1}^{-1} \Xi\left(t_{j}\right) \leq \delta_{j} \leq C_{1} \Xi\left(t_{j}\right)
$$

and the sequence $\left\{t_{j}\right\}_{j}$ satisfying

$$
C_{2}^{-1} \lambda\left(t_{k+1}\right) \leq \lambda\left(t_{k}\right) \leq C_{2} \lambda\left(t_{k+1}\right) \quad \text { and } \quad C_{3}^{-1} \Lambda\left(t_{k+1}\right) \leq \Lambda\left(t_{k}\right) \leq C_{3} \Lambda\left(t_{k+1}\right)
$$

with positive constants $C_{j}, j=1,2,3$, which are independent of $k$. Indeed, by (2.6.1) and the definition of $\omega=\omega(t)$ we have

$$
\omega(t)=1+\eta_{k} \psi\left(\frac{t-t_{k}}{\delta_{k}}\right) \quad \text { for all } \quad t \in\left[t_{k}, t_{k+1}\right] .
$$

Taking account of (2.6.2) and (2.6.3) it follows

$$
\left|d_{t}^{i} \omega(t)\right| \leq C_{i} \frac{\eta_{k}}{\delta_{k}^{i}} \leq \tilde{C}_{i} \Xi^{-i}(t) \quad \text { for all } t \in\left[t_{k}, t_{k+1}\right], \quad i=1, \cdots, M .
$$

Using again the definition of $\omega(t)$ we may conclude for all $t \in\left[t_{k}, t_{k+1}\right]$

$$
\int_{0}^{t} \lambda(s)|\omega(s)-1| d s=\sum_{j=1}^{k} \eta_{j} \int_{t_{j}}^{t_{j+1}} \lambda(s)\left|\psi\left(\frac{s-t_{j}}{\delta_{j}}\right)\right| d s \leq \frac{1}{2} \sum_{j=1}^{k} \eta_{j} \delta_{j} \lambda\left(t_{j+1}\right) .
$$

This implies that the stabilization condition (A3) is ensured if we assume $\eta_{j} \delta_{j}$ are small enough. Indeed,

$$
\Theta\left(t_{k+1}\right) \approx \sum_{j=1}^{k} \eta_{j} \delta_{j} \lambda\left(t_{j+1}\right)=o\left(\sum_{j=1}^{k} \lambda\left(t_{j}\right) \Delta\left(t_{j}\right)\right) .
$$

Example 2.6.1 (Polynomial case). We consider $\lambda(t)=(\alpha+1)(1+t)^{\alpha}, \alpha>0$. In order to define one admissible function $\omega=\omega(t)$ let us choose the parameters $\alpha, \gamma$ and $\kappa$ from Example 2.4.1 and positive sequences $\left\{t_{j}\right\}_{j},\left\{\delta_{j}\right\}_{j}$ and $\left\{\eta_{j}\right\}_{j}$ in the following way:

$$
t_{j}=2^{j}, \quad \delta_{j}=2^{\kappa j} \leq \Delta t_{j}=t_{j+1}-t_{j}=2^{j} \quad \text { and } \quad \eta_{j}=2^{j(\gamma-\alpha-\kappa)} .
$$

From (2.4.16) and (2.4.20) we have

$$
1>\kappa \geq \frac{3-\beta}{4}>\kappa_{M}=1-\alpha+\gamma+\frac{\alpha-\gamma}{M+1} .
$$

Therefore, from the last relations we get $\gamma-\alpha-\kappa<0$ and this implies that we have $0<\eta_{j} \leq 1$. Moreover, the stabilization condition (A3) is satisfied, since

$$
\eta_{j} \delta_{j}=2^{j(\gamma-\alpha)}<1,
$$

where $\gamma-\alpha<0$ from (2.4.15). 
Example 2.6.2 (Exponential case). We consider $\lambda(t)=e^{t}$. Let us choose the parameters $r$ and $\kappa$ from Example 2.4.2 and positive sequences $\left\{t_{j}\right\}_{j},\left\{\delta_{j}\right\}_{j}$ and $\left\{\eta_{j}\right\}_{j}$ as follows:

$$
t_{j}=j, \quad \delta_{j}=e^{\kappa j} \leq \Delta t_{j}=t_{j+1}-t_{j} \quad \text { and } \quad \eta_{j}=e^{j(r-\kappa-1)} .
$$

From (2.4.22) and (2.4.26) we have

$$
0>\kappa \geq-\frac{q}{4}>\kappa_{M}=r-1+\frac{1-r}{M+1} .
$$

Therefore, from the last relations we get $\kappa<0$ and $r-\kappa-1<0$. These imply that we have $\delta_{j}<1$ and $0<\eta_{j} \leq 1$, respectively. Moreover, the stabilization condition (A3) is satisfied, because

$$
\eta_{j} \delta_{j}=e^{j(r-1)}<1,
$$

where $r-1<0$ from $(2.4 .21)$.

Example 2.6.3 (Super-exponential case). We consider $\lambda(t)=e^{t} e^{e^{t}}$. Let us choose the parameters $r$ and $\kappa$ from Example 2.4.3 and positive sequences $\left\{t_{j}\right\}_{j},\left\{\delta_{j}\right\}_{j}$ and $\left\{\eta_{j}\right\}_{j}$ as follows:

$$
t_{j}=e^{j}, \quad \delta_{j}=e^{-j} e^{\kappa e^{j}} \leq \Delta t_{j}=t_{j+1}-t_{j} \quad \text { and } \quad \eta_{j}=e^{(r-\kappa-1) e^{j}} .
$$

From (2.4.28) and (2.4.32) we have

$$
0>\kappa \geq-\frac{q}{4}>\kappa_{M}=r-1+\frac{1-r}{M+1} .
$$

Therefore, from the last relations we get $\kappa<0$ and $r-\kappa-1<0$. These imply that we have $\delta_{j}<1$ and $0<\eta_{j} \leq 1$, respectively. Moreover, the stabilization condition (A3) is satisfied, since

$$
\eta_{j} \delta_{j}=e^{-j} e^{(r-1) e^{j}}<1,
$$

where $r-1<0$ from $(2.4 .27)$. 



\section{Global in time existence results for damped wave models with power nonlinearity}

\subsection{Introduction}

In this chapter we will consider the following semilinear Cauchy problem with time-dependent speed of propagation and "effective-like" time-dependent dissipation together with an oscillating term:

$$
\begin{cases}u_{t t}-\lambda^{2}(t) \omega^{2}(t) \Delta u+\rho(t) \omega(t) u_{t}=|u|^{p}, & (t, x) \in[0, \infty) \times \mathbb{R}^{n} \\ u(0, x)=u_{0}(x), \quad u_{t}(0, x)=u_{1}(x), & x \in \mathbb{R}^{n}\end{cases}
$$

Here we assume that the coefficients $\lambda=\lambda(t), \rho=\rho(t)$ and $\omega=\omega(t)$ satisfy the assumptions (A1) to (A5) and (B1) to (B6) which are given in Section 2.3 and $p>1$.

In the present chapter our aim is to prove some global (in time) existence results of small data solutions to the Cauchy problem (3.1.1) with additional regularity of the data. In order to prove these results our essential tools will be Banach's fixed point argument and energy estimates for the solutions to the linear Cauchy problem with vanishing right-hand side. Moreover, the space for initial data influences the choice of the space in which we look for solutions. The considerations are divided into two cases depending on the behavior of the propagation speed: the case of sub-exponential propagation speed and the case of superexponential propagation speed, which are proposed in [3] and [4], respectively.

In Theorems 3.2.1 and 3.2.2 we assume low regularity of the data with additional $L^{m}$ regularity, $m \in[1,2)$, for the case of sub-exponential and the case of super-exponential propagation speed, respectively. So, we prove existence results for Sobolev solutions without having a classical energy, that is, we do not have in general the total gradient of a solution in $L^{2}$.

In Theorems 3.3.1 and 3.3.2 we suppose data in the energy space $H^{1} \times L^{2}$ with additional $L^{m}$ regularity, $m \in[1,2)$, to the case of sub-exponential and the case of super-exponential propagation speed, respectively.

Next, in Theorems 3.4.1 and 3.4.2 we consider suitable higher regularity for the data with additional $L^{m}$ regularity, $m \in[1,2)$, for the case of sub-exponential and the case of superexponential propagation speed, respectively. In order to deal with the power nonlinearity in fractional Sobolev spaces, we employ in Section 3.4 some fractional inequalities, as the fractional chain rule and the fractional Leibniz rule, that will be explained in Appendix A.7.

Finally, in Theorems 3.5.1 and 3.5.2 we obtain large regular solutions to (3.1.1) for the case of sub-exponential and the case of super-exponential propagation speed, respectively. These solutions are imbedded into $L^{\infty}$. So, we may use results on fractional powers from Section A.7.6 in the Appendix.

\subsubsection{Some assumptions and tools}

Let us define for any $\sigma \geq 0$ and $m \in[1,2)$ the space of data

$$
\mathcal{D}_{m}^{\sigma}:=\left(L^{m} \cap H^{\sigma}\right) \times\left(L^{m} \cap H^{\max \{\sigma-1 ; 0\}}\right)
$$

with the norm

$$
\left\|\left(u_{0}, u_{1}\right)\right\|_{\mathcal{D}_{m}^{\sigma}}:=\left\|u_{0}\right\|_{L^{m}}+\left\|u_{0}\right\|_{H^{\sigma}}+\left\|u_{1}\right\|_{L^{m}}+\left\|u_{1}\right\|_{H^{\max \{\sigma-1 ; 0\}}} .
$$


Let us introduce the function $B_{\lambda}=B_{\lambda}(s, t)$ for $0 \leq s \leq t$ by

$$
B_{\lambda}(s, t):=\int_{s}^{t} \frac{\lambda^{2}(\tau)}{\rho(\tau)} d \tau=B_{\lambda}(0, t)-B_{\lambda}(0, s) .
$$

We have the following classification for the behavior of the propagation speed as in [3] and [4]: case of sub-exponential propagation speed and case of super-exponential propagation speed. We define for $\delta \in(0,1)$ and for large $t$ the auxiliary functions

$$
\nu(\delta, t):=\frac{\lambda(t)}{\Lambda(t)} \frac{\Lambda(\delta t)}{\lambda(\delta t)} \quad \text { and } \quad \nu(\delta):=\lim _{t \rightarrow \infty} \sup \nu(\delta, t)
$$

In this case we define

- the case of sub-exponential propagation speed if $\nu(\delta) \lesssim 1$,

- the case of super-exponential propagation if $\nu(\delta)=\infty$.

Moreover, due to the strong oscillating behavior of the time-dependent coefficients and a competition between several later derived estimates, now we will also introduce the case of oscillating sub-exponential propagation speed and the case of oscillating super-exponential propagation speed by using the following auxiliary functions for $\delta \in(0,1)$ and for large $t$ :

$$
\vartheta(\delta, t):=\frac{F(\Lambda(t))}{\lambda(t) \Xi^{2}(t)} \frac{\Lambda(\delta t)}{\lambda(\delta t)} \text { and } \vartheta(\delta):=\lim _{t \rightarrow \infty} \sup \vartheta(\delta, t) .
$$

We define

- the case of oscillating sub-exponential propagation speed if $\vartheta(\delta) \lesssim 1$,

- the case of oscillating super-exponential propagation speed if $\vartheta(\delta)=\infty$.

Remark 3.1.1. We see that assuming a suitable control of the oscillations, that is, in particular, if we choose formally $\omega(t) \equiv 1$, then we get $F(\Lambda(t))=\Lambda(t)$ and $\Xi(t)=\frac{\Lambda(t)}{\lambda(t)}$. Therefore, we immediately conclude that the case of oscillating sub-exponential and the case of oscillating super-exponential propagation speed coincide with the case of sub-exponential and the case of super-exponential propagation speed, respectively. Namely, in this case we formally have $\vartheta(\delta, t)=\nu(\delta, t)$.

\section{A family of parameter-dependent linear Cauchy problems}

In order to prove the global (in time) existence of small data Sobolev solutions to given semilinear Cauchy problems after using Duhamel's principle we need estimates of Sobolev solutions to the following family of parameter-dependent Cauchy problems:

$$
\begin{cases}v_{t t}-\lambda^{2}(t) \omega^{2}(t) \Delta v+\rho(t) \omega(t) v_{t}=0, & (t, x) \in[s, \infty) \times \mathbb{R}^{n} \\ v(s, x)=0, \quad v_{t}(s, x)=g(s, x), & x \in \mathbb{R}^{n}\end{cases}
$$

Moreover, the corresponding linear model to (3.1.1) with vanishing right-hand side is

$$
\begin{cases}u_{t t}-\lambda^{2}(t) \omega^{2}(t) \Delta u+\rho(t) \omega(t) u_{t}=0, & (t, x) \in[0, \infty) \times \mathbb{R}^{n} \\ u(0, x)=u_{0}(x), \quad u_{t}(0, x)=u_{1}(x), & x \in \mathbb{R}^{n} .\end{cases}
$$

We remark that the linear Cauchy problems (3.1.4) and (3.1.5) have been extensively discussed in the previous chapter providing a detailed analysis of the time asymptotic behavior. The latter, in turn, provides the expectations on the semilinear Cauchy problems studied here. 
Theorem 3.1.1. Assume that $\lambda, \rho$ and $\omega$ satisfy the conditions $(\boldsymbol{A} 1)$ to $(\boldsymbol{A} 5)$ and $(\boldsymbol{B} 1)$ to (B6). Then, the Sobolev solutions to the Cauchy problem (3.1.4) satisfy the following estimates for $t \geq s$ and data from the energy space $(\sigma=1)$ with additional $L^{m}$ regularity, $m \in[1,2)$ :

$$
\begin{aligned}
&\|v(t, \cdot)\|_{L^{2}} \lesssim \frac{\Lambda(s)}{\lambda(s)}\left(1+B_{\lambda}(s, t)\right)^{-\frac{n}{2}\left(\frac{1}{m}-\frac{1}{2}\right)}\|g(s, \cdot)\|_{L^{m} \cap L^{2}}, \\
&\||D| v(t, \cdot)\|_{L^{2}} \lesssim \frac{\Lambda(s)}{\lambda(s)}\left(1+B_{\lambda}(s, t)\right)^{-\frac{n}{2}\left(\frac{1}{m}-\frac{1}{2}\right)-\frac{1}{2}}\|g(s, \cdot)\|_{L^{m} \cap L^{2}}, \\
&\left\|v_{t}(t, \cdot)\right\|_{L^{2}} \lesssim \frac{\Lambda(s)}{\lambda(s)} \max \left\{\frac{\lambda^{2}(t)}{\rho(t)}\left(1+B_{\lambda}(s, t)\right)^{-\frac{n}{2}\left(\frac{1}{m}-\frac{1}{2}\right)-1} ;\right. \\
&\left.\lambda(t) F(\Lambda(t))\left(F^{2}(\Lambda(t))\right)^{-\frac{n}{2}\left(\frac{1}{m}-\frac{1}{2}\right)-1}\right\}\|g(s, \cdot)\|_{L^{m} \cap L^{2}},
\end{aligned}
$$

for higher regular data $(\sigma>1)$ with additional $L^{m}$ regularity, $m \in[1,2)$ :

$$
\begin{aligned}
&\|v(t, \cdot)\|_{L^{2}} \lesssim \frac{\Lambda(s)}{\lambda(s)}\left(1+B_{\lambda}(s, t)\right)^{-\frac{n}{2}\left(\frac{1}{m}-\frac{1}{2}\right)}\|g(s, \cdot)\|_{L^{m} \cap H^{\sigma-1}}, \\
&\left\||D|^{\sigma} v(t, \cdot)\right\|_{L^{2}} \lesssim \frac{\Lambda(s)}{\lambda(s)}\left(1+B_{\lambda}(s, t)\right)^{-\frac{n}{2}\left(\frac{1}{m}-\frac{1}{2}\right)-\frac{\sigma}{2}}\|g(s, \cdot)\|_{L^{m} \cap H^{\sigma-1}} \\
&\left\|v_{t}(t, \cdot)\right\|_{L^{2}} \lesssim \frac{\Lambda(s)}{\lambda(s)} \max \left\{\frac{\lambda^{2}(t)}{\rho(t)}\left(1+B_{\lambda}(s, t)\right)^{-\frac{n}{2}\left(\frac{1}{m}-\frac{1}{2}\right)-1} ;\right. \\
&\left.\lambda(t) F(\Lambda(t))\left(F^{2}(\Lambda(t))\right)^{-\frac{n}{2}\left(\frac{1}{m}-\frac{1}{2}\right)-1}\right\}\|g(s, \cdot)\|_{L^{m} \cap H^{\sigma-1}}, \\
&\left\||D|^{\sigma-1} v_{t}(t, \cdot)\right\|_{L^{2}} \lesssim \frac{\Lambda(s)}{\lambda(s)} \max \left\{\frac{\lambda^{2}(t)}{\rho(t)}\left(1+B_{\lambda}(s, t)\right)^{-\frac{n}{2}\left(\frac{1}{m}-\frac{1}{2}\right)-\frac{\sigma-1}{2}-1} ;\right. \\
&\left.\lambda(t) F(\Lambda(t))\left(F^{2}(\Lambda(t))\right)^{-\frac{n}{2}\left(\frac{1}{m}-\frac{1}{2}\right)-\frac{\sigma-1}{2}-1}\right\}\|g(s, \cdot)\|_{L^{m} \cap H^{\sigma-1}} .
\end{aligned}
$$

Theorem 3.1.2. We assume that $\lambda, \rho$ and $\omega$ satisfy the conditions $(\boldsymbol{A} 1)$ to $(\boldsymbol{A} 5)$ and $(\boldsymbol{B} 1)$ to (B6). Then, the Sobolev solutions to the Cauchy problem (3.1.5) satisfy the following estimates with $m \in[1,2)$ and data from the energy space $(\sigma=1)$ :

$$
\begin{aligned}
&\|u(t, \cdot)\|_{L^{2}} \lesssim\left(1+B_{\lambda}(0, t)\right)^{-\frac{n}{2}\left(\frac{1}{m}-\frac{1}{2}\right)}\left\|\left(u_{0}, u_{1}\right)\right\|_{\mathcal{D}_{m}^{1}}, \\
&\||D| u(t, \cdot)\|_{L^{2}} \lesssim\left(1+B_{\lambda}(0, t)\right)^{-\frac{n}{2}\left(\frac{1}{m}-\frac{1}{2}\right)-\frac{1}{2}}\left\|\left(u_{0}, u_{1}\right)\right\|_{\mathcal{D}_{m}^{1}}, \\
&\left\|u_{t}(t, \cdot)\right\|_{L^{2}} \lesssim \max \left\{\frac{\lambda^{2}(t)}{\rho(t)}\left(1+B_{\lambda}(0, t)\right)^{-\frac{n}{2}\left(\frac{1}{m}-\frac{1}{2}\right)-1} ;\right. \\
&\left.\lambda(t) F(\Lambda(t))\left(F^{2}(\Lambda(t))\right)^{-\frac{n}{2}\left(\frac{1}{m}-\frac{1}{2}\right)-1}\right\}\left\|\left(u_{0}, u_{1}\right)\right\|_{\mathcal{D}_{m}^{1}},
\end{aligned}
$$

for higher regular data $(\sigma>1)$ :

$$
\begin{aligned}
\|u(t, \cdot)\|_{L^{2}} \lesssim\left(1+B_{\lambda}(0, t)\right)^{-\frac{n}{2}\left(\frac{1}{m}-\frac{1}{2}\right)}\left\|\left(u_{0}, u_{1}\right)\right\|_{\mathcal{D}_{m}^{\sigma}}, \\
\left\||D|^{\sigma} u(t, \cdot)\right\|_{L^{2}} \lesssim\left(1+B_{\lambda}(0, t)\right)^{-\frac{n}{2}\left(\frac{1}{m}-\frac{1}{2}\right)-\frac{\sigma}{2}}\left\|\left(u_{0}, u_{1}\right)\right\|_{\mathcal{D}_{m}^{\sigma}}, \\
\left\|u_{t}(t, \cdot)\right\|_{L^{2}} \lesssim \max \left\{\frac{\lambda^{2}(t)}{\rho(t)}\left(1+B_{\lambda}(0, t)\right)^{-\frac{n}{2}\left(\frac{1}{m}-\frac{1}{2}\right)-1} ;\right. \\
\left.\lambda(t) F(\Lambda(t))\left(F^{2}(\Lambda(t))\right)^{-\frac{n}{2}\left(\frac{1}{m}-\frac{1}{2}\right)-1}\right\}\left\|\left(u_{0}, u_{1}\right)\right\|_{\mathcal{D}_{m}^{\sigma}}, \\
\left\||D|^{\sigma-1} u_{t}(t, \cdot)\right\|_{L^{2}} \lesssim \max \left\{\frac{\lambda^{2}(t)}{\rho(t)}\left(1+B_{\lambda}(0, t)\right)^{-\frac{n}{2}\left(\frac{1}{m}-\frac{1}{2}\right)-\frac{\sigma-1}{2}-1} ;\right. \\
\left.\lambda(t) F(\Lambda(t))\left(F^{2}(\Lambda(t))\right)^{-\frac{n}{2}\left(\frac{1}{m}-\frac{1}{2}\right)-\frac{\sigma-1}{2}-1}\right\}\left\|\left(u_{0}, u_{1}\right)\right\|_{\mathcal{D}_{m}^{\sigma}} .
\end{aligned}
$$


In Theorem 3.1.1 the estimates for $\left\|v_{t}(t, \cdot)\right\|_{L^{2}}$ and $\left\||D|^{\sigma-1} v_{t}(t, \cdot)\right\|_{L^{2}}$ are in competition to some other estimates. Therefore, in order to avoid some difficulties in the treatment for the semilinear Cauchy problem (3.1.1) we suppose that the following condition holds:

(C1) We assume that either the first component or the second component in the estimates for $\left\|v_{t}(t, \cdot)\right\|_{L^{2}}$ and $\left\||D|^{\sigma-1} v_{t}(t, \cdot)\right\|_{L^{2}}$ is dominant for all $t \geq s \geq 0$ and $\sigma \geq 1$.

We define the following functions:

$$
\begin{aligned}
& \Phi_{1, m}(s, t)=\max \left\{\frac{\lambda^{2}(t)}{\rho(t)}\left(1+B_{\lambda}(s, t)\right)^{-\frac{n}{2}\left(\frac{1}{m}-\frac{1}{2}\right)-1} ;\right. \\
&\left.\lambda(t) F(\Lambda(t))\left(F^{2}(\Lambda(t))\right)^{-\frac{n}{2}\left(\frac{1}{m}-\frac{1}{2}\right)-1}\right\}, \\
& \Phi_{1}(s, t)=\max \left\{\frac{\lambda^{2}(t)}{\rho(t)}\left(1+B_{\lambda}(s, t)\right)^{-1} ; \lambda(t) F(\Lambda(t))\left(F^{2}(\Lambda(t))\right)^{-1}\right\}, \\
& \Phi_{2, m}(s, t)=\max \left\{\frac{\lambda^{2}(t)}{\rho(t)}\left(1+B_{\lambda}(s, t)\right)^{-\frac{n}{2}\left(\frac{1}{m}-\frac{1}{2}\right)-\frac{\sigma-1}{2}-1} ;\right. \\
&\left.\lambda(t) F(\Lambda(t))\left(F^{2}(\Lambda(t))\right)^{-\frac{n}{2}\left(\frac{1}{m}-\frac{1}{2}\right)-\frac{\sigma-1}{2}-1}\right\}, \\
& \Phi_{2}(s, t)=\max \left\{\frac{\lambda^{2}(t)}{\rho(t)}\left(1+B_{\lambda}(s, t)\right)^{-\frac{\sigma-1}{2}-1} ; \lambda(t) F(\Lambda(t))\left(F^{2}(\Lambda(t))\right)^{-\frac{\sigma-1}{2}-1}\right\} .
\end{aligned}
$$

We will show in our approach that condition (C1) is really satisfied in both cases, the case of oscillating sub-exponential and the case of oscillating super-exponential propagation speed by some typical examples.

\section{Case of sub-exponential propagation speed}

We assume the following additional condition in order to get some useful estimates for $B_{\lambda}(s, t)$, which will come into play in the treatment of the semilinear Cauchy problem (3.1.1):

(C2) There exists a constant $\mu_{1} \in[0,2)$ such that

$$
\mu^{\prime}(t) \leq \mu_{1} \mu(t) \frac{\lambda(t)}{\Lambda(t)} \text { for } t \geq 0
$$

Then, we have the following statement.

Lemma 3.1.3. We assume that the conditions (B1) to (B4) and (C2) are satisfied. Then, for a fixed $\delta$ with $\delta \in(0,1)$ the following inequalities hold:

$$
\begin{gathered}
\frac{1}{2+R}\left(\frac{\Lambda^{2}(t)}{\mu(t)}-\frac{\Lambda^{2}(s)}{\mu(s)}\right) \leq B_{\lambda}(s, t) \leq \frac{1}{2-\mu_{1}}\left(\frac{\Lambda^{2}(t)}{\mu(t)}-\frac{\Lambda^{2}(s)}{\mu(s)}\right) \text { for } s \in[0, t], \\
B_{\lambda}(s, t) \approx B_{\lambda}(0, t) \text { for } s \in[0, \delta t] \\
\left(\frac{\Lambda(\delta t)}{\Lambda(t)}\right)^{2+R} B_{\lambda}(0, t) \leq B_{\lambda}(0, s) \leq B_{\lambda}(0, t) \text { for } s \in[\delta t, t] .
\end{gathered}
$$

Here we used condition (B2) for $k=1$ such that there exist constants $R \geq 0$ and $\tilde{R} \geq 0$ satisfying

$$
-R \mu(t) \frac{\lambda(t)}{\Lambda(t)} \leq \mu^{\prime}(t) \leq \tilde{R} \mu(t) \frac{\lambda(t)}{\Lambda(t)} .
$$

We note that if $\mu=\mu(t)$ is an increasing function, then we can choose $R=0$.

For the proof see [3]. 


\section{Case of super-exponential propagation speed}

Similarly to what we have done in the previous case, now we use the following condition in order to get some useful estimates for $B_{\lambda}(s, t)$ :

(C3) The function $\phi(t)=\frac{\Lambda^{2}(t)}{\mu(t)}$ satisfies the following conditions:

- $\phi(t)$ is increasing,

- $\phi^{\prime}(t) \leq \alpha \phi(t) \frac{\lambda(t)}{\Lambda(t)} \frac{1}{\log \Lambda(t)}, \alpha>0$.

Under these conditions we have the following statement.

Lemma 3.1.4. Let us assume that the conditions (B1) to (B4) and (C3) are satisfied. Then, there exists a fixed $\delta$ with $\delta \in(0,1)$ such that the following inequalities of $B_{\lambda}(s, t)$ hold:

$$
\begin{gathered}
\frac{1}{\alpha+2}(\phi(t) \log \Lambda(t)-\phi(s) \log \Lambda(s)) \\
\leq B_{\lambda}(s, t) \leq \phi(t) \log \Lambda(t)-\phi(s) \log \Lambda(s) \text { for } s \in[0, t], \\
B_{\lambda}(s, t) \approx B_{\lambda}(0, t) \text { for } s \in[0, \delta t] .
\end{gathered}
$$

For the proof see [4].

Besides conditions (C2) and (C3), now we assume that the following condition is satisfied which will be used later in both cases:

(C4) The function $\frac{\mu(t)}{\lambda^{2}(t)}$ satisfies the following conditions:

- $\frac{\mu(t)}{\lambda^{2}(t)}$ is decreasing,

- there exist positive constants $\beta_{0}$ and $\beta_{1}$ such that the following inequalities hold:

$$
-\beta_{0} \frac{\mu(t)}{\lambda(t) \Lambda(t)} \leq\left(\frac{\mu(t)}{\lambda^{2}(t)}\right)^{\prime} \leq-\beta_{1} \frac{\mu(t)}{\lambda(t) \Lambda(t)} .
$$

These estimates, together with the estimates of Lemma 3.1.3 and Lemma 3.1.4, will play an important role in the proof of global (in time) existence theorems for the case of subexponential and the case of super-exponential propagation speed, respectively.

Remark 3.1.2. By using conditions (B2) and (C2) we obtain the following two estimates in order to specify the positive constants $\beta_{0}$ and $\beta_{1}$ :

$$
\begin{aligned}
\left(\frac{\mu(t)}{\lambda^{2}(t)}\right)^{\prime} & =\frac{\mu^{\prime}(t) \lambda^{2}(t)-2 \mu(t) \lambda(t) \lambda^{\prime}(t)}{\lambda^{4}(t)} \\
& \leq \frac{\mu_{1} \mu(t) \frac{\lambda(t)}{\Lambda(t)} \lambda^{2}(t)-2 \lambda_{0} \mu(t) \lambda(t) \frac{\lambda^{2}(t)}{\Lambda(t)}}{\lambda^{4}(t)}=\left(\mu_{1}-2 \lambda_{0}\right) \frac{\mu(t)}{\lambda(t) \Lambda(t)} .
\end{aligned}
$$

On the other hand, we have

$$
\begin{aligned}
\left(\frac{\mu(t)}{\lambda^{2}(t)}\right)^{\prime} & =\frac{\mu^{\prime}(t) \lambda^{2}(t)-2 \mu(t) \lambda(t) \lambda^{\prime}(t)}{\lambda^{4}(t)} \\
& \geq \frac{-R \mu(t) \frac{\lambda(t)}{\Lambda(t)} \lambda^{2}(t)-2 \lambda_{1} \mu(t) \lambda(t) \frac{\lambda^{2}(t)}{\Lambda(t)}}{\lambda^{4}(t)}=\left(-R-2 \lambda_{1}\right) \frac{\mu(t)}{\lambda(t) \Lambda(t)} .
\end{aligned}
$$

Therefore, we can introduce $\beta_{0}$ and $\beta_{1}$ such that

$$
\beta_{0} \geq R+2 \lambda_{1} \text { and } \beta_{1} \leq 2 \lambda_{0}-\mu_{1} .
$$


Finally, we have the following auxiliary estimates, which will be used later in both the case of sub-exponential and the case of super-exponential propagation speed.

Lemma 3.1.5. We assume that condition $(\boldsymbol{C} 4)$ holds. Then, from Lemma 3.1 .3 and Lemma 3.1.4, respectively, we have the following estimates for all $s \in[0, t]$ :

$$
\begin{aligned}
& \frac{d\left(\frac{\mu(s)}{\lambda^{2}(s)}\left(1+B_{\lambda}(0, s)\right)^{-\alpha_{2}(p)}\right)}{d s} \lesssim \frac{\mu(s)}{\lambda^{2}(s)}\left(1+B_{\lambda}(0, s)\right)^{-\alpha_{2}(p)-1} \frac{d\left(1+B_{\lambda}(0, s)\right)}{d s} \\
& \frac{d\left(\frac{\mu(s)}{\lambda^{2}(s)}\left(1+B_{\lambda}(0, s)\right)^{-\alpha_{2}(p)}\right)}{d s} \gtrsim-\frac{\mu(s)}{\lambda^{2}(s)}\left(1+B_{\lambda}(0, s)\right)^{-\alpha_{2}(p)-1} \frac{d\left(1+B_{\lambda}(0, s)\right)}{d s}
\end{aligned}
$$

where $\alpha_{2}(p)=\frac{n}{2 m} p-\frac{n}{4}>0$.

Proof. First, let us prove this lemma for the case of sub-exponential propagation speed by using the estimates (3.1.17) from Lemma 3.1.3. So, we obtain

$$
\begin{aligned}
& \frac{d\left(\frac{\mu(s)}{\lambda^{2}(s)}\left(1+B_{\lambda}(0, s)\right)^{-\alpha_{2}(p)}\right)}{d s} \\
& \leq \frac{\mu(s)}{\lambda^{2}(s)}\left(1+B_{\lambda}(0, s)\right)^{-\alpha_{2}(p)}\left(\beta_{1} \frac{\lambda(s) \Lambda(s)}{\mu(s)} \frac{\mu(s)}{\Lambda^{2}(s)}+\alpha_{2}(p) \frac{d\left(1+B_{\lambda}(0, s)\right)}{d s} \frac{1}{1+B_{\lambda}(0, s)}\right) \\
& \lesssim \frac{\mu(s)}{\lambda^{2}(s)}\left(1+B_{\lambda}(0, s)\right)^{-\alpha_{2}(p)} \frac{d\left(1+B_{\lambda}(0, s)\right)}{d s} \frac{1}{1+B_{\lambda}(0, s)} .
\end{aligned}
$$

Moreover, we have

$$
\begin{aligned}
& \frac{d\left(\frac{\mu(s)}{\lambda^{2}(s)}\left(1+B_{\lambda}(0, s)\right)^{-\alpha_{2}(p)}\right)}{d s} \\
& \geq \frac{\mu(s)}{\lambda^{2}(s)}\left(1+B_{\lambda}(0, s)\right)^{-\alpha_{2}(p)}\left(-\beta_{0} \frac{\lambda(s) \Lambda(s)}{\mu(s)} \frac{\mu(s)}{\Lambda^{2}(s)}-\alpha_{2}(p) \frac{d\left(1+B_{\lambda}(0, s)\right)}{d s} \frac{1}{1+B_{\lambda}(0, s)}\right) \\
& \gtrsim-\frac{\mu(s)}{\lambda^{2}(s)}\left(1+B_{\lambda}(0, s)\right)^{-\alpha_{2}(p)} \frac{d\left(1+B_{\lambda}(0, s)\right)}{d s} \frac{1}{1+B_{\lambda}(0, s)} .
\end{aligned}
$$

On the other hand, for the case of super-exponential propagation speed we get the following estimates using the estimates (3.1.20) from Lemma 3.1.4:

$$
\begin{aligned}
& \frac{d\left(\frac{\mu(s)}{\lambda^{2}(s)}\left(1+B_{\lambda}(0, s)\right)^{-\alpha_{2}(p)}\right)}{d s} \\
& \leq \frac{\mu(s)}{\lambda^{2}(s)}\left(1+B_{\lambda}(0, s)\right)^{-\alpha_{2}(p)}\left(\beta_{1} \frac{\lambda(s) \Lambda(s)}{\mu(s)} \frac{\mu(s)}{\Lambda^{2}(s)} \frac{1}{\log \Lambda(s)}+\alpha_{2}(p) \frac{d\left(1+B_{\lambda}(0, s)\right)}{d s} \frac{1}{1+B_{\lambda}(0, s)}\right) \\
& \lesssim \frac{\mu(s)}{\lambda^{2}(s)}\left(1+B_{\lambda}(0, s)\right)^{-\alpha_{2}(p)} \frac{d\left(1+B_{\lambda}(0, s)\right)}{d s} \frac{1}{1+B_{\lambda}(0, s)} .
\end{aligned}
$$

Moreover, we find

$$
\begin{aligned}
& \frac{d\left(\frac{\mu(s)}{\lambda^{2}(s)}\left(1+B_{\lambda}(0, s)\right)^{-\alpha_{2}(p)}\right)}{d s} \\
& \geq \frac{\mu(s)}{\lambda^{2}(s)}\left(1+B_{\lambda}(0, s)\right)^{-\alpha_{2}(p)}\left(-\beta_{0} \frac{\lambda(s) \Lambda(s)}{\mu(s)} \frac{\mu(s)}{\Lambda^{2}(s)}-\alpha_{2}(p) \frac{d\left(1+B_{\lambda}(0, s)\right)}{d s} \frac{1}{1+B_{\lambda}(0, s)}\right) \\
& \gtrsim-\frac{\mu(s)}{\lambda^{2}(s)}\left(1+B_{\lambda}(0, s)\right)^{-\alpha_{2}(p)} \frac{d\left(1+B_{\lambda}(0, s)\right)}{d s} \frac{1}{1+B_{\lambda}(0, s)},
\end{aligned}
$$

where we used

$$
\frac{1}{1+B_{\lambda}(0, s)} \leq(\alpha+2) \frac{\mu(s)}{\Lambda^{2}(s)} \frac{1}{\log \Lambda(s)} \leq(\alpha+2) \frac{\mu(s)}{\Lambda^{2}(s)} .
$$

The proof is completed. 


\subsection{Low regular data}

In this section we are interested in the case of low regularity for the data, that is, $L^{2}$ regularity under the energy threshold with additional $L^{m}$ regularity, $m \in[1,2)$. More precisely, we will assume $\left(u_{0}, u_{1}\right) \in \mathcal{D}_{m}^{\sigma}$ with $\sigma \in(0,1)$. Therefore, we will consider the solution as a Sobolev solution in $\mathcal{C}\left([0, \infty), H^{\sigma}\right)$ only.

\subsubsection{Case of sub-exponential propagation speed}

We define the following parameters:

$$
\begin{aligned}
p_{\mathrm{Fuj}, m}(n) & :=1+\frac{2 m}{n} \quad \text { for } n \geq 1, \\
p_{1, m}(n) & :=1+\left(1-\frac{\beta_{1}}{2+R}\right) \frac{2 m}{n} \quad \text { for } n \geq 1, \\
p_{2, m}(n) & :=\frac{m}{2}+\frac{1-\frac{m}{2}}{1-\frac{2+R}{2-\mu_{1}}+\frac{2+R}{2-\mu_{1}} \frac{\delta}{\nu(\delta)}}+\left(\frac{1-\frac{\beta_{1}}{2+R} \frac{\delta}{\nu(\delta)}}{1-\frac{2+R}{2-\mu_{1}}+\frac{2+R}{2-\mu_{1}} \frac{\delta}{\nu(\delta)}}\right) \frac{2 m}{n} \quad \text { for } n \geq 1, \\
p_{\mathrm{GN}, \sigma}(n) & :=\frac{n}{n-2 \sigma} \quad \text { for } n>2 \sigma .
\end{aligned}
$$

We have the following result for the case of sub-exponential propagation speed.

Theorem 3.2.1. Let us assume that the Hypotheses (A1) to (A5), (B1) to (B6), (C2) and $\left(C_{4}\right)$, and $\nu(\delta) \lesssim 1$ are satisfied. Let $\left(u_{0}, u_{1}\right) \in \mathcal{D}_{m}^{\sigma}$ with $\sigma \in(0,1), m \in[1,2)$ and $n \leq \frac{4 \sigma}{2-m}$. Moreover, we suppose that the exponent $p$ satisfies

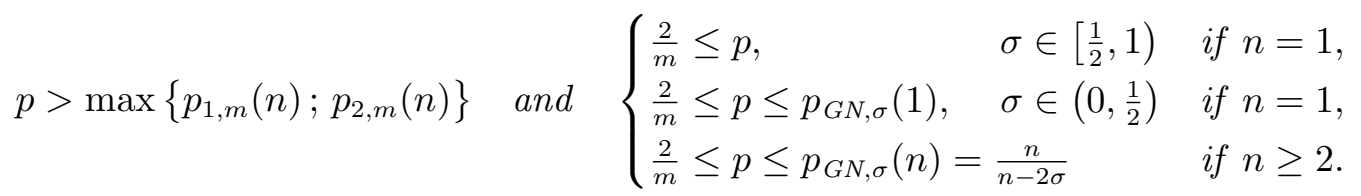

Under these assumptions there exists a constant $\varepsilon_{0}>0$ such that, for all $\left(u_{0}, u_{1}\right) \in \mathcal{D}_{m}^{\sigma}$ with $\left\|\left(u_{0}, u_{1}\right)\right\|_{\mathcal{D}_{m}^{\sigma}} \leq \varepsilon_{0}$, there exists a uniquely determined globally (in time) Sobolev solution

$$
u \in \mathcal{C}\left([0, \infty), H^{\sigma}\right)
$$

to the Cauchy problem (3.1.1).

Furthermore, there exists a constant $C>0$ such that the solution satisfies the following decay estimates:

$$
\begin{aligned}
\|u(t, \cdot)\|_{L^{2}} & \leq C\left(1+B_{\lambda}(0, t)\right)^{-\frac{n}{2}\left(\frac{1}{m}-\frac{1}{2}\right)}\left\|\left(u_{0}, u_{1}\right)\right\|_{\mathcal{D}_{m}^{\sigma}}, \\
\left\||D|^{\sigma} u(t, \cdot)\right\|_{L^{2}} & \leq C\left(1+B_{\lambda}(0, t)\right)^{-\frac{n}{2}\left(\frac{1}{m}-\frac{1}{2}\right)-\frac{\sigma}{2}}\left\|\left(u_{0}, u_{1}\right)\right\|_{\mathcal{D}_{m}^{\sigma}} .
\end{aligned}
$$

Proof. We define the space of solutions $X(t)$ by

$$
X(t)=\mathcal{C}\left([0, t], H^{\sigma}\right)
$$

with the norm

$$
\|u\|_{X(t)}=\sup _{0 \leq \tau \leq t}\left[\left(1+B_{\lambda}(0, \tau)\right)^{\frac{n}{2}\left(\frac{1}{m}-\frac{1}{2}\right)}\|u(\tau, \cdot)\|_{L^{2}}+\left(1+B_{\lambda}(0, \tau)\right)^{\frac{\sigma}{2}+\frac{n}{2}\left(\frac{1}{m}-\frac{1}{2}\right)}\left\||D|^{\sigma} u(\tau, \cdot)\right\|_{L^{2}}\right] .
$$

We remark that if $u \in X(t)$, then $\|u\|_{X(\tau)} \leq\|u\|_{X(t)}$ for any $0 \leq \tau \leq t$. Let us underline here that the norm of the solution space $X(t)$ is defined according to the estimates for solutions of the corresponding linear Cauchy problem with vanishing right-hand side in Theorem 3.1.2. 
Let us introduce the operator $N$ for any $u \in X(t)$ by

$$
N: u \in X(t) \rightarrow N u=N u(t, x):=u^{\mathrm{lin}}(t, x)+u^{\mathrm{nl}}(t, x) .
$$

We denote by $K_{0}(t, 0, x)$ and $K_{1}(t, 0, x)$ the fundamental solutions to the linear equation, that is,

$$
u^{\operatorname{lin}}(t, x):=K_{0}(t, 0, x) *(x) u_{0}(x)+K_{1}(t, 0, x) *_{(x)} u_{1}(x)
$$

is the solution to the linear Cauchy problem

$$
\begin{cases}u_{t t}-\lambda^{2}(t) \omega^{2}(t) \Delta u+\rho(t) \omega(t) u_{t}=0, & (t, x) \in[0, \infty) \times \mathbb{R}^{n}, \\ u(0, x)=u_{0}(x), \quad u_{t}(0, x)=u_{1}(x), & x \in \mathbb{R}^{n} .\end{cases}
$$

On the other hand, by Duhamel's principle

$$
u^{\mathrm{nl}}(t, x):=\int_{0}^{t} K_{1}(t, s, x) *{ }_{(x)} f(s, x) d s
$$

is the solution to the non-homogeneous Cauchy problem

$$
\begin{cases}u_{t t}-\lambda^{2}(t) \omega^{2}(t) \Delta u+\rho(t) \omega(t) u_{t}=f(t, x), & (t, x) \in[0, \infty) \times \mathbb{R}^{n}, \\ u(0, x)=0, \quad u_{t}(0, x)=0, & x \in \mathbb{R}^{n}\end{cases}
$$

Therefore, we formally set

$$
N u(t, x)=K_{0}(t, 0, x) *{ }_{(x)} u_{0}(x)+K_{1}(t, 0, x) *_{(x)} u_{1}(x)+\int_{0}^{t} K_{1}(t, s, x) *_{(x)} f(s, x) d s
$$

and try to find fixed point $u=N u$ of the mapping $N: X(t) \rightarrow X(t)$. We show that the mapping $N$ satisfies the following two estimates:

$$
\begin{aligned}
\|N u\|_{X(t)} & \leq C_{0}\left\|\left(u_{0}, u_{1}\right)\right\|_{\mathcal{D}_{m}^{\sigma}}+C_{1}(t)\|u\|_{X(t)}^{p}, \\
\|N u-N v\|_{X(t)} & \leq C_{2}(t)\|u-v\|_{X(t)}\left(\|u\|_{X(t)}^{p-1}+\|v\|_{X(t)}^{p-1}\right),
\end{aligned}
$$

where $C_{1}(t), C_{2}(t) \rightarrow 0$ for $t \rightarrow 0$ and $C_{1}(t), C_{2}(t) \leq C$ for all $t \in[0, \infty)$. These estimates indicate the existence of a unique solution of $u=N u$. We can use the idea of proving Banach's fixed point theorem. Therefore, to complete the proof it remains only to establish (3.2.2) and (3.2.3). In this way we get the global (in time) existence of small data solutions and local (in time) existence of large data solutions.

Firstly, let us begin to prove the estimate (3.2.2). From the estimates of Theorem 3.1.2 and the definition of the norm of the solution space $X(t)$ we have

$$
\begin{aligned}
&\left\|u^{\operatorname{lin}}\right\|_{X(t)}=\sup _{0 \leq \tau \leq t}[\left(1+B_{\lambda}(0, \tau)\right)^{\frac{n}{2}\left(\frac{1}{m}-\frac{1}{2}\right)}\left\|u^{\operatorname{lin}}(\tau, \cdot)\right\|_{L^{2}} \\
&\left.+\left(1+B_{\lambda}(0, \tau)\right)^{\frac{\sigma}{2}+\frac{n}{2}\left(\frac{1}{m}-\frac{1}{2}\right)}\left\||D|^{\sigma} u^{\operatorname{lin}}(\tau, \cdot)\right\|_{L^{2}}\right] \\
& \lesssim \sup _{0 \leq \tau \leq t}\left[\left(1+B_{\lambda}(0, \tau)\right)^{\frac{n}{2}\left(\frac{1}{m}-\frac{1}{2}\right)}\left(1+B_{\lambda}(0, \tau)\right)^{-\frac{n}{2}\left(\frac{1}{m}-\frac{1}{2}\right)}\left\|\left(u_{0}, u_{1}\right)\right\|_{\mathcal{D}_{m}^{\sigma}}\right. \\
&\left.\quad+\left(1+B_{\lambda}(0, \tau)\right)^{\frac{\sigma}{2}+\frac{n}{2}\left(\frac{1}{m}-\frac{1}{2}\right)}\left(1+B_{\lambda}(0, \tau)\right)^{-\frac{\sigma}{2}-\frac{n}{2}\left(\frac{1}{m}-\frac{1}{2}\right)}\left\|\left(u_{0}, u_{1}\right)\right\|_{\mathcal{D}_{m}^{\sigma}}\right] \\
& \lesssim\left\|\left(u_{0}, u_{1}\right)\right\|_{\mathcal{D}_{m}^{\sigma}} .
\end{aligned}
$$

Consequently, we get

$$
\left\|u^{\operatorname{lin}}\right\|_{X(t)} \lesssim\left\|\left(u_{0}, u_{1}\right)\right\|_{\mathcal{D}_{m}^{\sigma}} .
$$

Then, in order to conclude the proof of (3.2.2) it remains to prove the following inequality:

$$
\left\|u^{\mathrm{nl}}\right\|_{X(t)} \lesssim\|u\|_{X(t)}^{p} .
$$


First we have that

$$
\left\||D|^{\sigma} u^{\mathrm{nl}}(t, \cdot)\right\|_{L^{2}} \leq C \int_{0}^{t}\left\||D|^{\sigma}\left(K_{1}(t, s, x) *_{(x)}|u(s, x)|^{p}\right)\right\|_{L^{2}} d s .
$$

Now, we can use two different strategies to estimate the integral term in $[0, t]$ by splitting this interval into $[0, \delta t]$ and $[\delta t, t]$, where $\delta$ is fixed and $\delta \in(0,1)$. In particular, if $s \in[0, \delta t]$, we use $\left(L^{m} \cap L^{2}\right)-L^{2}$ estimates with $m \in[1,2)$ and if $s \in[\delta t, t]$ we use $L^{2}-L^{2}$ estimates from Theorem 2.5.16. So, we have the estimate

$$
\begin{aligned}
\left\||D|^{\sigma} u^{\mathrm{nl}}(t, \cdot)\right\|_{L^{2}} \leq & C \int_{0}^{\delta t} \frac{\Lambda(s)}{\lambda(s)}\left(1+B_{\lambda}(s, t)\right)^{-\frac{\sigma}{2}-\frac{n}{2}\left(\frac{1}{m}-\frac{1}{2}\right)}\left\||u(s, \cdot)|^{p}\right\|_{L^{m} \cap L^{2}} d s \\
& +C \int_{\delta t}^{t} \frac{\Lambda(s)}{\lambda(s)}\left(1+B_{\lambda}(s, t)\right)^{-\frac{\sigma}{2}}\left\||u(s, \cdot)|^{p}\right\|_{L^{2}} d s .
\end{aligned}
$$

Using

$$
\left\||u(s, \cdot)|^{p}\right\|_{L^{m} \cap L^{2}} \lesssim\left\||u(s, \cdot)|^{p}\right\|_{L^{m}}+\left\||u(s, \cdot)|^{p}\right\|_{L^{2}}=\|u(s, \cdot)\|_{L^{m p}}^{p}+\|u(s, \cdot)\|_{L^{2 p}}^{p},
$$

Gagliardo-Nirenberg inequality comes into play. We can estimate

$$
\begin{aligned}
\|u(s, \cdot)\|_{L^{m p}}^{p} & \lesssim\left\||D|^{\sigma} u(s, \cdot)\right\|_{L^{2}}^{p \theta_{\sigma}(m p)}\|u(s, \cdot)\|_{L^{2}}^{p\left(1-\theta_{\sigma}(m p)\right)}, \\
\|u(s, \cdot)\|_{L^{2 p}}^{p} & \lesssim\left\||D|^{\sigma} u(s, \cdot)\right\|_{L^{2}}^{p \theta_{\sigma}(2 p)}\|u(s, \cdot)\|_{L^{2}}^{p\left(1-\theta_{\sigma}(2 p)\right)},
\end{aligned}
$$

where

$$
\theta_{\sigma}(m p)=\frac{n}{\sigma}\left(\frac{1}{2}-\frac{1}{m p}\right) \in[0,1], \quad \theta_{\sigma}(2 p)=\frac{n}{\sigma}\left(\frac{1}{2}-\frac{1}{2 p}\right) \in[0,1] .
$$

Therefore, the requisite $\theta_{\sigma}(m p) \geq 0$ implies that $p \geq \frac{2}{m}$ and the requisite $\theta_{\sigma}(2 p) \leq 1$ implies that $p \leq p_{\mathrm{GN}, \sigma}(n)=\frac{n}{n-2 \sigma}$ for $n>2 \sigma$. Then, by using (3.2.6), (3.2.7) and the norm of the solution space $X(t)$ we obtain the following estimates:

$$
\begin{aligned}
\|u(s, \cdot)\|_{L^{m p}}^{p} & \lesssim\left(1+B_{\lambda}(0, s)\right)^{-\frac{n}{2}\left(\frac{1}{m}-\frac{1}{2}\right) p-\frac{\sigma}{2} p \theta_{\sigma}(m p)}\|u\|_{X(s)}^{p} \\
& =\left(1+B_{\lambda}(0, s)\right)^{-\frac{n}{2 m} p+\frac{n}{2 m}}\|u\|_{X(s)}^{p}, \\
\|u(s, \cdot)\|_{L^{2 p}}^{p} & \lesssim\left(1+B_{\lambda}(0, s)\right)^{-\frac{n}{2}\left(\frac{1}{m}-\frac{1}{2}\right) p-\frac{\sigma}{2} p \theta_{\sigma}(2 p)}\|u\|_{X(s)}^{p} \\
& =\left(1+B_{\lambda}(0, s)\right)^{-\frac{n}{2 m} p+\frac{n}{4}}\|u\|_{X(s)}^{p} .
\end{aligned}
$$

Hence, we can estimate $\left\||u(s, \cdot)|^{p}\right\|_{L^{m} \cap L^{2}}$ as follows:

$$
\left\||u(s, \cdot)|^{p}\right\|_{L^{m} \cap L^{2}} \lesssim\left(1+B_{\lambda}(0, s)\right)^{-\frac{n}{2 m} p+\frac{n}{2 m}}\|u\|_{X(s)}^{p},
$$

since it holds $\theta_{\sigma}(m p)<\theta_{\sigma}(2 p)$. Plugging (3.2.9) and (3.2.10) into (3.2.5) and using $\|u\|_{X(s)} \leq$ $\|u\|_{X(t)}$ for any $0 \leq s \leq t$, we get

$$
\begin{aligned}
\left\||D|^{\sigma} u^{\mathrm{nl}}(t, \cdot)\right\|_{L^{2}} \leq & C\|u\|_{X(t)}^{p} \underbrace{\int_{0}^{\delta t} \frac{\Lambda(s)}{\lambda(s)}\left(1+B_{\lambda}(s, t)\right)^{-\frac{\sigma}{2}-\frac{n}{2}\left(\frac{1}{m}-\frac{1}{2}\right)}\left(1+B_{\lambda}(0, s)\right)^{-\frac{n}{2 m} p+\frac{n}{2 m}} d s}_{\mathbf{A}} \\
& +C\|u\|_{X(t)}^{p} \underbrace{\int_{\delta t}^{t} \frac{\Lambda(s)}{\lambda(s)}\left(1+B_{\lambda}(s, t)\right)^{-\frac{\sigma}{2}}\left(1+B_{\lambda}(0, s)\right)^{-\frac{n}{2 m} p+\frac{n}{4}} d s}_{\mathbf{B}} .
\end{aligned}
$$

Now we want to estimate the integral terms $\mathbf{A}$ and $\mathbf{B}$ in order to get the desired estimates. Let us consider

$$
\mathbf{A} \lesssim\left(1+B_{\lambda}(0, t)\right)^{-\frac{\sigma}{2}-\frac{n}{2}\left(\frac{1}{m}-\frac{1}{2}\right)} \underbrace{\int_{0}^{\delta t} \frac{\Lambda(s)}{\lambda(s)}\left(1+B_{\lambda}(0, s)\right)^{-\frac{n}{2 m} p+\frac{n}{2 m}} d s}_{A_{1}(t)}
$$


where we used (3.1.18). Denoting $\alpha_{1}(p):=\frac{n}{2 m} p-\frac{n}{2 m}$ we get for $\alpha_{1}(p) \neq 1$ the relation

$$
\begin{aligned}
A_{1}(t)= & \int_{0}^{\delta t} \frac{\mu(s)}{\lambda^{2}(s)}\left(1+B_{\lambda}(0, s)\right)^{-\alpha_{1}(p)} d\left(1+B_{\lambda}(0, s)\right) \\
= & \left.\frac{1}{1-\alpha_{1}(p)} \frac{\mu(s)}{\lambda^{2}(s)}\left(1+B_{\lambda}(0, s)\right)^{-\alpha_{1}(p)+1}\right|_{0} ^{\delta t} \\
& -\frac{1}{1-\alpha_{1}(p)} \int_{0}^{\delta t}\left(1+B_{\lambda}(0, s)\right)^{-\alpha_{1}(p)+1} d\left(\frac{\mu(s)}{\lambda^{2}(s)}\right) .
\end{aligned}
$$

Now we shall distinguish between three cases.

Case 1: $\alpha_{1}(p)>1$, i.e., $p>1+\frac{2 m}{n}$. In this case it holds

$$
\begin{aligned}
A_{1}(t) \leq & \left.\frac{1}{1-\alpha_{1}(p)} \frac{\mu(s)}{\lambda^{2}(s)}\left(1+B_{\lambda}(0, s)\right)^{-\alpha_{1}(p)+1}\right|_{0} ^{\delta t} \\
& -\frac{\beta_{1}}{\alpha_{1}(p)-1} \int_{0}^{\delta t}\left(1+B_{\lambda}(0, s)\right)^{-\alpha_{1}(p)}\left(1+B_{\lambda}(0, s)\right) \frac{\mu(s)}{\lambda(s) \Lambda(s)} d s \\
\leq & \left.\frac{1}{1-\alpha_{1}(p)} \frac{\mu(s)}{\lambda^{2}(s)}\left(1+B_{\lambda}(0, s)\right)^{-\alpha_{1}(p)+1}\right|_{0} ^{\delta t} \\
& -\frac{\beta_{1}}{\left(\alpha_{1}(p)-1\right)(2+R)} \int_{0}^{\delta t}\left(1+B_{\lambda}(0, s)\right)^{-\alpha_{1}(p)} \frac{\Lambda^{2}(s)}{\mu(s)} \frac{\mu(s)}{\lambda(s) \Lambda(s)} d s \\
\leq & \left.\frac{1}{1-\alpha_{1}(p)} \frac{\mu(s)}{\lambda^{2}(s)}\left(1+B_{\lambda}(0, s)\right)^{-\alpha_{1}(p)+1}\right|_{0} ^{\delta t} \\
& -\frac{\beta_{1}}{\left(\alpha_{1}(p)-1\right)(2+R)} \underbrace{\int_{0}^{\delta t} \frac{\Lambda(s)}{\lambda(s)}\left(1+B_{\lambda}(0, s)\right)^{-\alpha_{1}(p)} d s}_{A_{1}(t)},
\end{aligned}
$$

where we used condition (C4) and (3.1.17), respectively. Thus, from the last estimate of $A_{1}=A_{1}(t)$ we obtain

$$
\left(1+\frac{\beta_{1}}{\left(\alpha_{1}(p)-1\right)(2+R)}\right) A_{1}(t) \lesssim \frac{\mu(0)}{\lambda^{2}(0)}-\frac{\mu(\delta t)}{\lambda^{2}(\delta t)}\left(1+B_{\lambda}(0, \delta t)\right)^{-\alpha_{1}(p)+1} .
$$

It implies that

$$
A_{1}(t) \lesssim \frac{\mu(0)}{\lambda^{2}(0)}-\frac{\mu(\delta t)}{\lambda^{2}(\delta t)}\left(1+B_{\lambda}(0, \delta t)\right)^{-\alpha_{1}(p)+1} .
$$

Case 2: $\alpha_{1}(p)<1$. In this case we have the estimate

$$
\begin{aligned}
& A_{1}(t) \geq\left.\frac{1}{1-\alpha_{1}(p)} \frac{\mu(s)}{\lambda^{2}(s)}\left(1+B_{\lambda}(0, s)\right)^{-\alpha_{1}(p)+1}\right|_{0} ^{\delta t} \\
& +\frac{\beta_{1}}{1-\alpha_{1}(p)} \int_{0}^{\delta t}\left(1+B_{\lambda}(0, s)\right)^{-\alpha_{1}(p)}\left(1+B_{\lambda}(0, s)\right) \frac{\mu(s)}{\lambda(s) \Lambda(s)} d s \\
& \geq\left.\frac{1}{1-\alpha_{1}(p)} \frac{\mu(s)}{\lambda^{2}(s)}\left(1+B_{\lambda}(0, s)\right)^{-\alpha_{1}(p)+1}\right|_{0} ^{\delta t} \\
& +\frac{\beta_{1}}{\left(1-\alpha_{1}(p)\right)(2+R)} \int_{0}^{\delta t}\left(1+B_{\lambda}(0, s)\right)^{-\alpha_{1}(p)} \frac{\Lambda^{2}(s)}{\mu(s)} \frac{\mu(s)}{\lambda(s) \Lambda(s)} d s \\
& =\left.\frac{1}{1-\alpha_{1}(p)} \frac{\mu(s)}{\lambda^{2}(s)}\left(1+B_{\lambda}(0, s)\right)^{-\alpha_{1}(p)+1}\right|_{0} ^{\delta t} \\
& +\frac{\beta_{1}}{\left(1-\alpha_{1}(p)\right)(2+R)} \underbrace{\int_{0}^{\delta t} \frac{\Lambda(s)}{\lambda(s)}\left(1+B_{\lambda}(0, s)\right)^{-\alpha_{1}(p)} d s}_{A_{1}(t)} .
\end{aligned}
$$


Then, it follows

$$
\left(1-\frac{\beta_{1}}{\left(1-\alpha_{1}(p)\right)(2+R)}\right) A_{1}(t) \gtrsim \frac{\mu(\delta t)}{\lambda^{2}(\delta t)}\left(1+B_{\lambda}(0, \delta t)\right)^{-\alpha_{1}(p)+1}-\frac{\mu(0)}{\lambda^{2}(0)} .
$$

It implies that

$$
\left(\frac{\beta_{1}}{\left(1-\alpha_{1}(p)\right)(2+R)}-1\right) A_{1} \lesssim \frac{\mu(0)}{\lambda^{2}(0)}-\frac{\mu(\delta t)}{\lambda^{2}(\delta t)}\left(1+B_{\lambda}(0, \delta t)\right)^{-\alpha_{1}(p)+1} .
$$

We can guarantee that $A_{1}=A_{1}(t)$ is bounded by the condition $p>p_{1, m}(n)$. Because, if

$$
\frac{\beta_{1}}{\left(1-\alpha_{1}(p)\right)(2+R)}-1>0, \quad \text { then } \quad p>1+\left(1-\frac{\beta_{1}}{2+R}\right) \frac{2 m}{n},
$$

which we have assumed in the statement of the theorem. Otherwise, we need to show that

$$
\mathcal{A}_{1}(\delta t):=\frac{\mu(\delta t)}{\lambda^{2}(\delta t)}\left(1+B_{\lambda}(0, \delta t)\right)^{-\alpha_{1}(p)+1}
$$

is strictly decreasing in $t$. Indeed, taking the derivative of function $\mathcal{A}_{1}=\mathcal{A}_{1}(t)$ we get

$$
\begin{aligned}
\mathcal{A}_{1}^{\prime}(t) & =\left(\frac{\mu(t)}{\lambda^{2}(t)}\right)^{\prime}\left(1+B_{\lambda}(0, t)\right)^{-\alpha_{1}(p)+1}+\left(-\alpha_{1}(p)+1\right) \frac{\mu(t)}{\lambda^{2}(t)} \frac{\lambda^{2}(t)}{\rho(t)}\left(1+B_{\lambda}(0, t)\right)^{-\alpha_{1}(p)} \\
& \leq\left[-\beta_{1} \frac{\mu(t)}{\lambda(t) \Lambda(t)}\left(1+B_{\lambda}(0, t)\right)+\left(-\alpha_{1}(p)+1\right) \frac{\Lambda(t)}{\lambda(t)}\right]\left(1+B_{\lambda}(0, t)\right)^{-\alpha_{1}(p)} \\
& \leq\left[-\frac{\beta_{1}}{2+R} \frac{\mu(t)}{\lambda(t) \Lambda(t)} \frac{\Lambda^{2}(t)}{\mu(t)}+\left(-\alpha_{1}(p)+1\right) \frac{\Lambda(t)}{\lambda(t)}\right]\left(1+B_{\lambda}(0, t)\right)^{-\alpha_{1}(p)} \\
& \leq\left[-\frac{\beta_{1}}{2+R}-\alpha_{1}(p)+1\right] \frac{\Lambda(t)}{\lambda(t)}\left(1+B_{\lambda}(0, t)\right)^{-\alpha_{1}(p)} .
\end{aligned}
$$

The condition $p>p_{1, m}(n)$ implies that $\mathcal{A}_{1}^{\prime}(t)<0$. This shows us that the right-hand side of (3.2.12) is bounded.

Case 3: $\alpha_{1}(p)=1$. In this final case we have

$$
\begin{aligned}
A_{1}(t) & =\int_{0}^{\delta t} \frac{\Lambda(s)}{\lambda(s)}\left(1+B_{\lambda}(0, s)\right)^{-1} d s \lesssim \int_{0}^{\delta t} \frac{\Lambda(s)}{\lambda(s)} \frac{\mu(s)}{\Lambda^{2}(s)} d s=\int_{0}^{\delta t} \frac{\mu(s)}{\lambda^{2}(s)} \frac{\lambda(s)}{\Lambda(s)} d s \\
& =\left.\frac{\mu(s)}{\lambda^{2}(s)} \log \Lambda(s)\right|_{0} ^{\delta t}-\int_{0}^{\delta t} \log \Lambda(s) d\left(\frac{\mu(s)}{\lambda^{2}(s)}\right) \\
& \left.\lesssim \frac{\mu(s)}{\lambda^{2}(s)} \log \Lambda(s)\right|_{0} ^{\delta t}+\int_{0}^{\delta t} \frac{\mu(s)}{\lambda^{2}(s)} \log \Lambda(s) \frac{\lambda(s)}{\Lambda(s)} d s,
\end{aligned}
$$

where we have used (3.1.17) and condition (C4), respectively. Now we will show that for a sufficiently small positive constant $\varepsilon$ we have the estimate

$$
\frac{\mu(t)}{\lambda^{2}(t)} \log \Lambda(t) \lesssim \Lambda^{-\varepsilon}(t)
$$

Therefore, we form the derivative of the function

$$
\mathcal{A}_{2}(t):=\frac{\mu(t)}{\lambda^{2}(t)} \Lambda^{\varepsilon}(t) \log \Lambda(t)
$$

Using condition (C4) it holds

$$
\begin{aligned}
\mathcal{A}_{2}^{\prime}(t) & =\left(\frac{\mu(t)}{\lambda^{2}(t)}\right)^{\prime} \Lambda^{\varepsilon}(t) \log \Lambda(t)+\varepsilon \frac{\mu(t)}{\lambda^{2}(t)} \lambda(t) \Lambda^{\varepsilon-1}(t) \log \Lambda(t)+\frac{\mu(t)}{\lambda^{2}(t)} \frac{\lambda(t)}{\Lambda(t)} \Lambda^{\varepsilon}(t) \\
& \leq\left[-\beta_{1} \frac{\mu(t)}{\lambda(t) \Lambda(t)} \log \Lambda(t)+\varepsilon \frac{\mu(t)}{\lambda(t) \Lambda(t)} \log \Lambda(t)+\frac{\mu(t)}{\lambda(t) \Lambda(t)}\right] \Lambda^{\varepsilon}(t) \\
& \leq\left[-\beta_{1}+\varepsilon+\frac{1}{\log \Lambda(t)}\right] \frac{\mu(t)}{\lambda(t) \Lambda(t)} \log \Lambda(t) \Lambda^{\varepsilon}(t) .
\end{aligned}
$$


Hence, for a sufficiently large time $t$ and sufficiently small constant $\varepsilon>0$ we get $\mathcal{A}_{2}^{\prime}(t)<0$. This shows the decreasing behavior of $\mathcal{A}_{2}=\mathcal{A}_{2}(t)$. Hence, we obtain that $A_{1}=A_{1}(t)$ is uniformly bounded.

Summarizing, from the above three cases we get our desired estimate

$$
\mathbf{A} \lesssim\left(1+B_{\lambda}(0, t)\right)^{-\frac{\sigma}{2}-\frac{n}{2}\left(\frac{1}{m}-\frac{1}{2}\right)} .
$$

Now let us consider the case $s \in[\delta t, t]$. Denoting $\alpha_{2}(p):=\frac{n}{2 m} p-\frac{n}{4}$ it holds

$$
\begin{aligned}
\mathbf{B}= & \int_{\delta t}^{t} \frac{\Lambda(s)}{\lambda(s)}\left(1+B_{\lambda}(s, t)\right)^{-\frac{\sigma}{2}}\left(1+B_{\lambda}(0, s)\right)^{-\alpha_{2}(p)} d s \\
= & -\int_{\delta t}^{t} \frac{\mu(s)}{\lambda^{2}(s)}\left(1+B_{\lambda}(0, s)\right)^{-\alpha_{2}(p)}\left(1+B_{\lambda}(s, t)\right)^{-\frac{\sigma}{2}} d\left(1+B_{\lambda}(s, t)\right) \\
= & \left.\frac{-1}{1-\frac{\sigma}{2}} \frac{\mu(s)}{\lambda^{2}(s)}\left(1+B_{\lambda}(0, s)\right)^{-\alpha_{2}(p)}\left(1+B_{\lambda}(s, t)\right)^{-\frac{\sigma}{2}+1}\right|_{\delta t} ^{t} \\
& +\frac{1}{1-\frac{\sigma}{2}} \int_{\delta t}^{t}\left(1+B_{\lambda}(s, t)\right)^{-\frac{\sigma}{2}+1} d\left(\frac{\mu(s)}{\lambda^{2}(s)}\left(1+B_{\lambda}(0, s)\right)^{-\alpha_{2}(p)}\right) \\
\lesssim & \left.\frac{-1}{1-\frac{\sigma}{2}} \frac{\mu(s)}{\lambda^{2}(s)}\left(1+B_{\lambda}(0, s)\right)^{-\alpha_{2}(p)}\left(1+B_{\lambda}(s, t)\right)^{-\frac{\sigma}{2}+1}\right|_{\delta t} ^{t} \\
& +\frac{1}{1-\frac{\sigma}{2}} \int_{\delta t}^{t} \frac{\mu(s)}{\lambda^{2}(s)}\left(1+B_{\lambda}(0, s)\right)^{-\alpha_{2}(p)}\left(1+B_{\lambda}(s, t)\right)^{-\frac{\sigma}{2}+1} \frac{d\left(1+B_{\lambda}(0, s)\right)}{1+B_{\lambda}(0, s)}
\end{aligned}
$$

where we used Lemma 3.1.5. Then, we obtain

$$
\begin{aligned}
\mathbf{B} \lesssim & \frac{\mu(\delta t)}{\lambda^{2}(\delta t)}\left(1+B_{\lambda}(0, \delta t)\right)^{-\alpha_{2}(p)}\left(1+B_{\lambda}(\delta t, t)\right)^{-\frac{\sigma}{2}+1} \\
& +\int_{\delta t}^{t} \frac{\mu(s)}{\lambda^{2}(s)}\left(1+B_{\lambda}(0, s)\right)^{-\alpha_{2}(p)}\left(1+B_{\lambda}(s, t)\right)^{-\frac{\sigma}{2}+1} \frac{d\left(1+B_{\lambda}(0, s)\right)}{1+B_{\lambda}(0, s)}
\end{aligned}
$$

Hence, due to $1-\frac{\sigma}{2}>0$ and multiplying both sides of the previous inequality by the function $\left(1+B_{\lambda}(0, t)\right)^{\frac{\sigma}{2}+\frac{n}{2}\left(\frac{1}{m}-\frac{1}{2}\right)}$, we find

$$
\begin{aligned}
\left(1+B_{\lambda}(0, t)\right)^{\frac{\sigma}{2}+\frac{n}{2}\left(\frac{1}{m}-\frac{1}{2}\right)} \mathbf{B} & \lesssim \underbrace{\frac{\mu(\delta t)}{\lambda^{2}(\delta t)}\left(1+B_{\lambda}(0, \delta t)\right)^{-\alpha_{2}(p)}\left(1+B_{\lambda}(0, t)\right)^{\frac{n}{2}\left(\frac{1}{m}-\frac{1}{2}\right)+1}}_{B_{1}(t)} \\
& +\underbrace{\int_{\delta t}^{t} \frac{\mu(s)}{\lambda^{2}(s)}\left(1+B_{\lambda}(0, s)\right)^{-\alpha_{2}(p)}\left(1+B_{\lambda}(0, t)\right)^{\frac{n}{2}\left(\frac{1}{m}-\frac{1}{2}\right)+1} \frac{d\left(1+B_{\lambda}(0, s)\right)}{1+B_{\lambda}(0, s)}}_{B_{2}(t)} .
\end{aligned}
$$

In order to prove that $B_{1}=B_{1}(t)$ and $B_{2}=B_{2}(t)$ are uniformly bounded it is sufficient to show for a small positive constant $\epsilon$ we have the estimate

$$
\frac{\mu(\delta t)}{\lambda^{2}(\delta t)}\left(1+B_{\lambda}(0, \delta t)\right)^{-\alpha_{2}(p)}\left(1+B_{\lambda}(0, t)\right)^{\frac{n}{2}\left(\frac{1}{m}-\frac{1}{2}\right)+1} \lesssim\left(1+B_{\lambda}(0, \delta t)\right)^{-\epsilon} .
$$

Hence, we will show that the following function is bounded:

$$
\frac{\mu(\delta t)}{\lambda^{2}(\delta t)}\left(1+B_{\lambda}(0, \delta t)\right)^{-\alpha_{2}(p)+\epsilon}\left(1+B_{\lambda}(0, t)\right)^{\frac{n}{2}\left(\frac{1}{m}-\frac{1}{2}\right)+1} .
$$


Taking account of the estimates of $B_{\lambda}(s, t)$ in (3.1.19) we obtain

$$
\begin{aligned}
\frac{\mu(\delta t)}{\lambda^{2}(\delta t)} & \left(1+B_{\lambda}(0, \delta t)\right)^{-\alpha_{2}(p)+\epsilon}\left(1+B_{\lambda}(0, t)\right)^{\frac{n}{2}\left(\frac{1}{m}-\frac{1}{2}\right)+1} \\
& \leq \frac{\mu(\delta t)}{\lambda^{2}(\delta t)}\left(1+B_{\lambda}(0, t)\right)^{\frac{n}{2}\left(\frac{1}{m}-\frac{1}{2}\right)+1-\alpha_{2}(p)+\epsilon}\left(\frac{\Lambda(\delta t)}{\Lambda(t)}\right)^{(2+R)\left(-\alpha_{2}(p)+\epsilon\right)}:=\mathcal{B}_{1}(t) .
\end{aligned}
$$

In order to show the monotonicity of this function we form the derivative of $\mathcal{B}_{1}=\mathcal{B}_{1}(t)$. It holds

$$
\begin{aligned}
\mathcal{B}_{1}^{\prime}(t)= & \underbrace{\left(\frac{\mu(\delta t)}{\lambda^{2}(\delta t)}\right)^{\prime}\left(1+B_{\lambda}(0, t)\right)^{\frac{n}{2}\left(\frac{1}{m}-\frac{1}{2}\right)+1-\alpha_{2}(p)+\epsilon}\left(\frac{\Lambda(\delta t)}{\Lambda(t)}\right)^{(2+R)\left(-\alpha_{2}(p)+\epsilon\right)}}_{I_{1}(t)} \\
& +\underbrace{\left(\left(1+B_{\lambda}(0, t)\right)^{\frac{n}{2}\left(\frac{1}{m}-\frac{1}{2}\right)+1-\alpha_{2}(p)+\epsilon}\left(\frac{\Lambda(\delta t)}{\Lambda(t)}\right)^{(2+R)\left(-\alpha_{2}(p)+\epsilon\right)}\right)^{\prime} \frac{\mu(\delta t)}{\lambda^{2}(\delta t)}}_{I_{2}(t)} .
\end{aligned}
$$

Then, $I_{1}=I_{1}(t)$ satisfies the following estimates:

$$
\begin{aligned}
I_{1}(t) & \leq-\delta \beta_{1} \frac{\mu(\delta t)}{\lambda^{2}(\delta t)} \frac{\lambda(\delta t)}{\Lambda(\delta t)}\left(1+B_{\lambda}(0, t)\right)\left(1+B_{\lambda}(0, t)\right)^{\frac{n}{2}\left(\frac{1}{m}-\frac{1}{2}\right)-\alpha_{2}(p)+\epsilon}\left(\frac{\Lambda(\delta t)}{\Lambda(t)}\right)^{(2+R)\left(-\alpha_{2}(p)+\epsilon\right)} \\
& \leq-\frac{\delta \beta_{1}}{2+R} \frac{\lambda(\delta t)}{\Lambda(\delta t)} \frac{\Lambda^{2}(t)}{\mu(t)}\left(1+B_{\lambda}(0, t)\right)^{\frac{n}{2}\left(\frac{1}{m}-\frac{1}{2}\right)-\alpha_{2}(p)+\epsilon}\left(\frac{\Lambda(\delta t)}{\Lambda(t)}\right)^{(2+R)\left(-\alpha_{2}(p)+\epsilon\right)} \frac{\mu(\delta t)}{\lambda^{2}(\delta t)} .
\end{aligned}
$$

On the other hand, $I_{2}=I_{2}(t)$ satisfies the following estimates:

$$
\begin{aligned}
I_{2}(t)=( & \left.\frac{n}{2}\left(\frac{1}{m}-\frac{1}{2}\right)+1-\alpha_{2}(p)+\epsilon\right) \frac{\lambda^{2}(t)}{\rho(t)}\left(1+B_{\lambda}(0, t)\right)^{\frac{n}{2}\left(\frac{1}{m}-\frac{1}{2}\right)-\alpha_{2}(p)+\epsilon} \\
& \times\left(\frac{\Lambda(\delta t)}{\Lambda(t)}\right)^{(2+R)\left(-\alpha_{2}(p)+\epsilon\right)} \frac{\mu(\delta t)}{\lambda^{2}(\delta t)}+(2+R)\left(-\alpha_{2}(p)+\epsilon\right)\left(\frac{\delta \lambda(\delta t) \Lambda(t)-\lambda(t) \Lambda(\delta t)}{\Lambda^{2}(t)}\right) \\
& \times\left(\frac{\Lambda(\delta t)}{\Lambda(t)}\right)^{(2+R)\left(-\alpha_{2}(p)+\epsilon\right)-1}\left(1+B_{\lambda}(0, t)\right)^{\frac{n}{2}\left(\frac{1}{m}-\frac{1}{2}\right)+1-\alpha_{2}(p)+\epsilon} \frac{\mu(\delta t)}{\lambda^{2}(\delta t)} \\
\leq & \left(\frac{n}{2}\left(\frac{1}{m}-\frac{1}{2}\right)+1-\alpha_{2}(p)+\epsilon\right) \frac{\Lambda^{2}(t)}{\mu(t)} \frac{\lambda(\delta t)}{\Lambda(\delta t)} \frac{\lambda(t)}{\Lambda(t)} \frac{\Lambda(\delta t)}{\lambda(\delta t)} \\
& \left.+(2+R)\left(\alpha_{2}(p)-\epsilon\right) \frac{1}{2-\mu_{1}} \frac{\Lambda^{2}(t)}{\mu(t)} \frac{\Lambda(t)}{\Lambda(\delta t)} \frac{\lambda(\delta t)}{\Lambda(t)}\left(\frac{\lambda(t)}{\Lambda(t)} \frac{\Lambda(\delta t)}{\lambda(\delta t)}-\delta\right)\right] \\
& \times\left(\frac{\Lambda(\delta t)}{\Lambda(t)}\right)(2+R)\left(-\alpha_{2}(p)+\epsilon\right) \\
\leq[ & \left(\frac{n}{2}\left(\frac{1}{m}-\frac{1}{2}\right)+1-B_{\lambda}(0, t)\right)^{\frac{n}{2}\left(\frac{1}{m}-\frac{1}{2}\right)-\alpha_{2}(p)+\epsilon \frac{\mu(\delta t)}{\lambda^{2}(\delta t)}} \\
& \times \frac{\Lambda^{2}(t)}{\mu(t)} \frac{\lambda(\delta t)}{\Lambda(\delta t)}\left(\frac{\Lambda(\delta t)}{\Lambda(t)}\right)^{(2+R)\left(-\alpha_{2}(p)+\epsilon\right)}\left(1+B_{\lambda}(0, t)\right)^{\frac{n}{2}\left(\frac{1}{m}-\frac{1}{2}\right)-\alpha_{2}(p)+\epsilon} \frac{\mu(\delta t)}{\lambda^{2}(\delta t)} .
\end{aligned}
$$

Hence, we get

$$
\begin{aligned}
\mathcal{B}_{1}^{\prime}(t)= & I_{1}(t)+I_{2}(t) \\
\leq & {\left[-\frac{\delta \beta_{1}}{2+R}+\left(\frac{n}{2}\left(\frac{1}{m}-\frac{1}{2}\right)+1-\alpha_{2}(p)+\epsilon\right) \nu(\delta, t)+\left(\alpha_{2}(p)-\epsilon\right) \frac{2+R}{2-\mu_{1}}(\nu(\delta, t)-\delta)\right] } \\
& \quad \times \frac{\Lambda^{2}(t)}{\mu(t)} \frac{\lambda(\delta t)}{\Lambda(\delta t)}\left(\frac{\Lambda(\delta t)}{\Lambda(t)}\right)^{(2+R)\left(-\alpha_{2}(p)+\epsilon\right)}\left(1+B_{\lambda}(0, t)\right)^{\frac{n}{2}\left(\frac{1}{m}-\frac{1}{2}\right)-\alpha_{2}(p)+\epsilon} \frac{\mu(\delta t)}{\lambda^{2}(\delta t)} .
\end{aligned}
$$


Since $p>p_{2, m}(n)$, for large time $t$ and a small positive constant $\epsilon$ we may conclude that

$$
-\delta \frac{\beta_{1}}{2+R}+\left(\frac{n}{2}\left(\frac{1}{m}-\frac{1}{2}\right)+1-\alpha_{2}(p)\right) \nu(\delta)+\alpha_{2}(p) \frac{2+R}{2-\mu_{1}}(\nu(\delta)-\delta)<0 .
$$

This implies that $\mathcal{B}_{1}^{\prime}(t)<0$. Therefore, for all $p>p_{2, m}(n)$ we have

$$
\mathbf{B} \lesssim\left(1+B_{\lambda}(0, t)\right)^{-\frac{\sigma}{2}-\frac{n}{2}\left(\frac{1}{m}-\frac{1}{2}\right)} .
$$

Summarizing, from (3.2.14) and (3.2.16) we arrive at the estimate

$$
\left\||D|^{\sigma} u^{\mathrm{nl}}(t, \cdot)\right\|_{L^{2}} \lesssim\left(1+B_{\lambda}(0, t)\right)^{-\frac{\sigma}{2}-\frac{n}{2}\left(\frac{1}{m}-\frac{1}{2}\right)}\|u\|_{X(t)}^{p} .
$$

In the same way one can derive

$$
\left\|u^{\mathrm{nl}}(t, \cdot)\right\|_{L^{2}} \lesssim\left(1+B_{\lambda}(0, t)\right)^{-\frac{n}{2}\left(\frac{1}{m}-\frac{1}{2}\right)}\|u\|_{X(t)}^{p} .
$$

Using the norm of the solution space $X(t)$ and (3.2.17) and (3.2.18) we get

$$
\begin{aligned}
&\left\|u^{\mathrm{nl}}\right\|_{X(t)}= \sup _{0 \leq \tau \leq t}\left[\left(1+B_{\lambda}(0, \tau)\right)^{\frac{n}{2}\left(\frac{1}{m}-\frac{1}{2}\right)}\left\|u^{\mathrm{nl}}(\tau, \cdot)\right\|_{L^{2}}\right. \\
&\left.\quad+\left(1+B_{\lambda}(0, \tau)\right)^{\frac{\sigma}{2}+\frac{n}{2}\left(\frac{1}{m}-\frac{1}{2}\right)}\left\||D|^{\sigma} u^{\mathrm{nl}}(\tau, \cdot)\right\|_{L^{2}}\right] \\
& \lesssim \sup _{0 \leq \tau \leq t}\left[\left(1+B_{\lambda}(0, \tau)\right)^{\frac{n}{2}\left(\frac{1}{m}-\frac{1}{2}\right)}\left(1+B_{\lambda}(0, \tau)\right)^{-\frac{n}{2}\left(\frac{1}{m}-\frac{1}{2}\right)}\|u\|_{X(t)}^{p}\right. \\
&\left.\quad+\left(1+B_{\lambda}(0, \tau)\right)^{\frac{\sigma}{2}+\frac{n}{2}\left(\frac{1}{m}-\frac{1}{2}\right)}\left(1+B_{\lambda}(0, \tau)\right)^{-\frac{\sigma}{2}-\frac{n}{2}\left(\frac{1}{m}-\frac{1}{2}\right)}\|u\|_{X(t)}^{p}\right] \\
& \lesssim\|u\|_{X(t)}^{p} .
\end{aligned}
$$

So, this gives the desired estimate and together with (3.2.4) the estimate (3.2.2) is proved.

Now let us prove (3.2.3). We have that

$$
\|N u-N v\|_{X(t)}=\left\|\int_{0}^{t} K_{1}(t, s, x) *_{(x)}\left(|u(s, x)|^{p}-|v(s, x)|^{p}\right) d s\right\|_{X(t)} .
$$

Thanks to the estimates for the solutions to the family of parameter dependent Cauchy problem we can estimate

$$
\begin{aligned}
& \left\||D|^{\sigma} K_{1}(t, s, x) *_{(x)}\left(|u(s, x)|^{p}-|v(s, x)|^{p}\right)\right\|_{L^{2}} \\
& \quad \lesssim \begin{cases}\frac{\Lambda(s)}{\lambda(s)}\left(1+B_{\lambda}(s, t)\right)^{-\frac{\sigma}{2}-\frac{n}{2}\left(\frac{1}{m}-\frac{1}{2}\right)}\left\||u(s, x)|^{p}-|v(s, x)|^{p}\right\|_{L^{m} \cap L^{2}}, & s \in[0, \delta t], \\
\frac{\Lambda(s)}{\lambda(s)}\left(1+B_{\lambda}(s, t)\right)^{-\frac{\sigma}{2}}\left\||u(s, x)|^{p}-|v(s, x)|^{p}\right\|_{L^{2}}, & s \in[\delta t, t] .\end{cases}
\end{aligned}
$$

So, we use the fact that

$$
\left.|| u(s, x)\right|^{p}-|v(s, x)|^{p}|\lesssim| u(s, x)-v(s, x) \mid\left(|u(s, x)|^{p-1}+|v(s, x)|^{p-1}\right) .
$$

By Hölder's inequality we obtain

$$
\begin{aligned}
\left\||u(s, \cdot)|^{p}-|v(s, \cdot)|^{p}\right\|_{L^{m}} & \lesssim\|u(s, \cdot)-v(s, \cdot)\|_{L^{m p}}\left(\|u(s, \cdot)\|_{L^{m p}}^{p-1}+\|v(s, \cdot)\|_{L^{m p}}^{p-1}\right), \\
\left\||u(s, \cdot)|^{p}-|v(s, \cdot)|^{p}\right\|_{L^{2}} & \lesssim\|u(s, \cdot)-v(s, \cdot)\|_{L^{2 p}}\left(\|u(s, \cdot)\|_{L^{2 p}}^{p-1}+\|v(s, \cdot)\|_{L^{2 p}}^{p-1}\right) .
\end{aligned}
$$

In a similar way to the proof of (3.2.2) we use again Gagliardo-Nirenberg inequality to the following terms:

$$
\|u(s, \cdot)-v(s, \cdot)\|_{L^{q}}, \quad\|u(s, \cdot)\|_{L^{q}} \quad \text { and } \quad\|v(s, \cdot)\|_{L^{q}}
$$


with $q=m p$ and $q=2 p$. Then, we obtain the following estimates as we did for (3.2.8) and $(3.2 .9)$ :

$$
\begin{aligned}
\|u(s, \cdot)-v(s, \cdot)\|_{L^{m p}} & \lesssim\left(1+B_{\lambda}(0, s)\right)^{-\frac{n}{2 m}+\frac{n}{2 m p}}\|u-v\|_{X(s)}, \\
\|u(s, \cdot)-v(s, \cdot)\|_{L^{2 p}} & \lesssim\left(1+B_{\lambda}(0, s)\right)^{-\frac{n}{2 m}+\frac{n}{4 p}}\|u-v\|_{X(s)}, \\
\|u(s, \cdot)\|_{L^{m p}}^{p-1} & \lesssim\left(1+B_{\lambda}(0, s)\right)^{\left(-\frac{n}{2 m}+\frac{n}{2 m p}\right)(p-1)}\|u\|_{X(s)}^{p-1}, \\
\|u(s, \cdot)\|_{L^{2 p}}^{p-1} & \lesssim\left(1+B_{\lambda}(0, s)\right)^{\left(-\frac{n}{2 m}+\frac{n}{4 p}\right)(p-1)}\|u\|_{X(s)}^{p-1}, \\
\|v(s, \cdot)\|_{L^{m p}}^{p-1} & \lesssim\left(1+B_{\lambda}(0, s)\right)^{\left(-\frac{n}{2 m}+\frac{n}{2 m p}\right)(p-1)}\|v\|_{X(s)}^{p-1}, \\
\|v(s, \cdot)\|_{L^{2 p}}^{p-1} & \lesssim\left(1+B_{\lambda}(0, s)\right)^{\left(-\frac{n}{2 m}+\frac{n}{4 p}\right)(p-1)}\|v\|_{X(s)}^{p-1} .
\end{aligned}
$$

Then, we get

$$
\begin{aligned}
\left\||u(s, \cdot)|^{p}-|v(s, \cdot)|^{p}\right\|_{L^{m}} & \lesssim\left(1+B_{\lambda}(0, s)\right)^{-\frac{n}{2 m} p+\frac{n}{2 m}}\|u-v\|_{X(s)}\left(\|u\|_{X(s)}^{p-1}+\|v\|_{X(s)}^{p-1}\right), \\
\left\||u(s, \cdot)|^{p}-|v(s, \cdot)|^{p}\right\|_{L^{2}} & \lesssim\left(1+B_{\lambda}(0, s)\right)^{-\frac{n}{2 m} p+\frac{n}{4}}\|u-v\|_{X(s)}\left(\|u\|_{X(s)}^{p-1}+\|v\|_{X(s)}^{p-1}\right) .
\end{aligned}
$$

After plugging the previous two estimates into (3.2.19) we arrive at the following inequality:

$$
\begin{aligned}
\||D|^{\sigma}(N u & -N v)(t, \cdot)\left\|_{L^{2}} \lesssim\right\| u-v \|_{X(s)}\left(\|u\|_{X(s)}^{p-1}+\|v\|_{X(s)}^{p-1}\right) \\
& \times\left(\int_{0}^{\delta t} \frac{\Lambda(s)}{\lambda(s)}\left(1+B_{\lambda}(s, t)\right)^{-\frac{\sigma}{2}-\frac{n}{2}\left(\frac{1}{m}-\frac{1}{2}\right)}\left(1+B_{\lambda}(0, s)\right)^{-\frac{n}{2 m} p+\frac{n}{2 m}} d s\right. \\
& \left.\quad+\int_{\delta t}^{t} \frac{\Lambda(s)}{\lambda(s)}\left(1+B_{\lambda}(s, t)\right)^{-\frac{\sigma}{2}}\left(1+B_{\lambda}(0, s)\right)^{-\frac{n}{2 m} p+\frac{n}{4}} d s\right) .
\end{aligned}
$$

Applying the same ideas as we did to estimate $\left\||D|^{\sigma} u^{\mathrm{nl}}(t, \cdot)\right\|_{L^{2}}$ one can get the following estimates for $p>p_{1, m}(n)$ and $p>p_{2, m}(n)$ :

$$
\begin{aligned}
\left\||D|^{\sigma}(N u-N v)(t, \cdot)\right\|_{L^{2}} & \lesssim\left(1+B_{\lambda}(0, t)\right)^{-\frac{\sigma}{2}-\frac{n}{2}\left(\frac{1}{m}-\frac{1}{2}\right)}\|u-v\|_{X(t)}\left(\|u\|_{X(t)}^{p-1}+\|v\|_{X(t)}^{p-1}\right) \\
\|(N u-N v)(t, \cdot)\|_{L^{2}} & \lesssim\left(1+B_{\lambda}(0, t)\right)^{-\frac{n}{2}\left(\frac{1}{m}-\frac{1}{2}\right)}\|u-v\|_{X(t)}\left(\|u\|_{X(t)}^{p-1}+\|v\|_{X(t)}^{p-1}\right) .
\end{aligned}
$$

Hence, from the definition of $X(t)$, we can conclude the proof of the statement (3.2.3). In this way the proof of the theorem is completed.

Remark 3.2.1. In the last theorem we can see from the definitions of $p_{1, m}(n)$ and $p_{2, m}(n)$ if

$$
\lim _{\delta \rightarrow 1} \nu(\delta)=1
$$

then we have

$$
p_{1, m}(n)=p_{2, m}(n)=1+\left(1-\frac{\beta_{1}}{2+R}\right) \frac{2 m}{n} .
$$

Example 3.2.1 (Polynomial case). Let $\lambda(t)=(\alpha+1)(1+t)^{\alpha}, \alpha>0$. Then, we get

$$
\Lambda(t)=(1+t)^{\alpha+1} \text { and } \Theta(t)=(1+t)^{\gamma+1},-1<\gamma<\alpha .
$$

Moreover, choosing

$$
\mu(t)=(1+t)^{\beta}, \quad \alpha-\gamma<\beta<2 \alpha+2,
$$

we have

$$
\rho(t)=(\alpha+1)(1+t)^{\beta-1},
$$


where by condition (C2) we can define $\beta:=\mu_{1}(\alpha+1)$. Due to condition (C4), the parameter $\beta_{1}$ satisfies

$$
0<\beta_{1} \leq \frac{2 \alpha-\beta}{\alpha+1}, \quad \beta<2 \alpha
$$

Now applying Theorem 3.2.1 we also get $\nu(\delta)=\delta$ and $R=0$ (we can choose $R=0$, since in this case $\mu=\mu(t)$ is increasing). Therefore, taking account of the parameters $p_{1, m}(n)$ and $p_{2, m}(n)$ the "critical exponent" is

$$
p_{1, m}(n)=p_{2, m}(n)=1+\left(\frac{\beta-\alpha+1}{\alpha+1}\right) \frac{2 m}{n} .
$$

Consequently, we have the global (in time) existence of small data solutions for

$$
p>1+\left(\frac{\beta-\alpha+1}{\alpha+1}\right) \frac{2 m}{n}:=p_{m}(n) \text { and } \begin{cases}\frac{2}{m} \leq p, & \sigma \in\left[\frac{1}{2}, 1\right) \\ \frac{2}{m} \leq p \leq p_{\mathrm{GN}, \sigma}(1)=\frac{1}{1-2 \sigma}, & \text { if } n=1, \\ \frac{2}{m} \leq p \leq p_{\mathrm{GN}, \sigma}(n)=\frac{n}{n-2 \sigma}, & \text { if } n=1,\end{cases}
$$

The restriction for $n$ depends heavily on the choice of the parameters $m, \alpha, \beta$ and $\sigma$. Let us denote $r:=\frac{\beta-\alpha+1}{\alpha+1}$ and we remark that $r<1$, since $\beta<2 \alpha$. Then, we have the following statements for $n \geq 1$ :

1. If $m \in\left[1, \frac{-n+\sqrt{n^{2}+16 r n}}{4 r}\right)$, then $\max \left\{\frac{2}{m} ; p_{m}(n)\right\}=\frac{2}{m}$ and the restriction of the dimension $n$ will be $n \leq \frac{4 \sigma}{2-m}$.

2. If $m \in\left[\frac{-n+\sqrt{n^{2}+16 r n}}{4 r}, 2\right)$, then $\max \left\{\frac{2}{m} ; p_{m}(n)\right\}=p_{m}(n)$ and the restriction of the dimension $n$ will be $n<\frac{2 r m \sigma}{r m-\sigma}$.

Let us discuss in the following table the conditions for $p$ in some special cases which depend on the parameters $n, m, \sigma$ and $r$ to get the admissible range for $p$. We are interested in the case $n \leq 5$ only.

\begin{tabular}{|c|c|c|c|c|}
\hline$n$ & $m$ & $\sigma$ & $r$ & Admissible range for $p$ \\
\hline \multirow{2}{*}{$n=1$} & $m \in\left[\frac{-1+\sqrt{1+16 r}}{4 r}, 2\right)$ & $\sigma \in\left(\frac{r m}{1+2 r m}, 1\right)$ & $r \in(0,1)$ & $1+2 m r<p<\infty$ \\
\cline { 2 - 5 } & $m \in\left[\frac{-1+\sqrt{1+16 r}}{4 r}, 2\right)$ & $\sigma \in\left(\frac{r m}{1+2 r m}, \frac{1}{2}\right)$ & $r \in(0,1)$ & $1+2 m r<p \leq \frac{1}{1-2 \sigma}$ \\
\hline \multirow{2}{*}{$n=1$} & $m \in\left[1, \frac{-1+\sqrt{1+16 r}}{4 r}\right)$ & $\sigma \in\left[\frac{2-m}{4}, 1\right)$ & $r \in\left(0, \frac{1}{2}\right)$ & $\frac{2}{m} \leq p<\infty$ \\
\cline { 2 - 5 } & $m \in\left[1, \frac{-1+\sqrt{1+16 r}}{4 r}\right)$ & $\sigma \in\left[\frac{2-m}{4}, \frac{1}{2}\right)$ & $r \in\left(0, \frac{1}{2}\right)$ & $\frac{2}{m} \leq p \leq \frac{1}{1-2 \sigma}$ \\
\hline \multirow{2}{*}{$n=2$} & $m \in\left[\frac{-1+\sqrt{1+8 r}}{2 r}, 2\right)$ & $\sigma \in\left[\frac{r m}{1+r m}, 1\right)$ & $r \in(0,1)$ & $1+r m<p \leq \frac{1}{1-\sigma}$ \\
\cline { 2 - 5 } & $m \in\left[1, \frac{-1+\sqrt{1+8 r}}{2 r}\right)$ & $\sigma \in\left[\frac{2-m}{2}, 1\right)$ & $r \in(0,1)$ & $\frac{2}{m} \leq p \leq \frac{1}{1-\sigma}$ \\
\hline \multirow{2}{*}{$n=3$} & $m \in\left[\frac{-3+\sqrt{9+48 r}}{4 r}, 2\right)$ & $\sigma \in\left(\frac{3 r m}{2 r m+3}, 1\right)$ & $r \in\left(0, \frac{3}{m}\right)$ & $1+\frac{2 r m}{3}<p \leq \frac{3}{3-2 \sigma}$ \\
\cline { 2 - 5 } & $m \in\left[1, \frac{-3+\sqrt{9+48 r}}{4 r}\right)$ & $\sigma \in\left[\frac{6-3 m}{4}, 1\right)$ & $r \in\left(0, \frac{3}{2}\right)$ & $\frac{2}{m} \leq p \leq \frac{3}{3-2 \sigma}$ \\
\hline \multirow{2}{*}{$n=4$} & $m \in\left[1, \frac{-1+\sqrt{1+4 r}}{r}\right)$ & $\sigma \in\left(\frac{2 r m}{r m+2}, 1\right)$ & $r \in\left(0, \frac{2}{m}\right)$ & $1+\frac{r m}{2}<p \leq \frac{2}{2-\sigma}$ \\
\cline { 2 - 5 } & $m \in\left[1, \frac{-1+\sqrt{1+4 r}}{r}\right)$ & $\sigma \in[2-m, 1)$ & $r \in(0,1)$ & $\frac{2}{m} \leq p \leq \frac{2}{2-\sigma}$ \\
\hline \multirow{2}{*}{$n=5$} & $m \in\left[\frac{-5+\sqrt{25+80 r}}{4 r}, 2\right)$ & $\sigma \in\left[\frac{5 m r}{2 r m+5}, 1\right)$ & $r \in\left(0, \frac{5}{3 m}\right)$ & $1+\frac{2 r m}{5}<p \leq \frac{5}{5-2 \sigma}$ \\
\cline { 2 - 5 } & $m \in\left[\frac{6}{5}, \frac{-5+\sqrt{25+80 r}}{4 r}\right)$ & $\sigma \in\left[\frac{10-5 m}{4}, 1\right)$ & $r \in\left(0, \frac{53}{72}\right)$ & $\frac{2}{m} \leq p \leq \frac{5}{5-2 \sigma}$ \\
\hline
\end{tabular}

Tab. 3.1.: Admissible range for the parameters 
Remark 3.2.2. We can see that we have a benefit of the additional $L^{m}$ regularity of the data. It allows to have results for higher dimensions $n$. We note that the previous case with additional $L^{1}$ regularity can be proved for $n \leq 4$ only.

Example 3.2.2 (Exponential case). Let us choose $\lambda(t)=e^{t}$. Then, we obtain

$$
\Lambda(t)=e^{t} \quad \text { and } \quad \Theta(t)=e^{r t}, \quad 0<r<1 .
$$

Moreover, we choose

$$
\mu(t)=e^{\mu_{1} t}, \quad 1-r<\mu_{1}<2 .
$$

By condition (C4), the parameter $\beta_{1}$ satisfies

$$
0<\beta_{1} \leq 2-\mu_{1}, \quad \mu_{1}<2 .
$$

Applying Theorem 3.2.1 we get $\nu(\delta)=1$ and $R=0$ (we can choose $R=0$, since in this case $\mu=\mu(t)$ is increasing). Therefore, taking account of $p_{1, m}(n)$ and $p_{2, m}(n)$ we obtain

$$
\begin{aligned}
& p_{1, m}(n)=1+\left(1-\frac{\beta_{1}}{2}\right) \frac{2 m}{n}, \\
& p_{2, m}(n)=\frac{m}{2}+\frac{1-\frac{m}{2}}{1-\frac{2}{2-\mu_{1}}(1-\delta)}+\left(\frac{1-\frac{\beta_{1}}{2}}{1-\frac{2}{2-\mu_{1}}(1-\delta)}\right) \frac{2 m}{n} .
\end{aligned}
$$

Choosing $\delta$ close to 1 gives

$$
p_{1, m}(n)=p_{2, m}(n)=1+\left(1-\frac{\beta_{1}}{2}\right) \frac{2 m}{n} .
$$

So, we have the global (in time) existence of small data solutions for

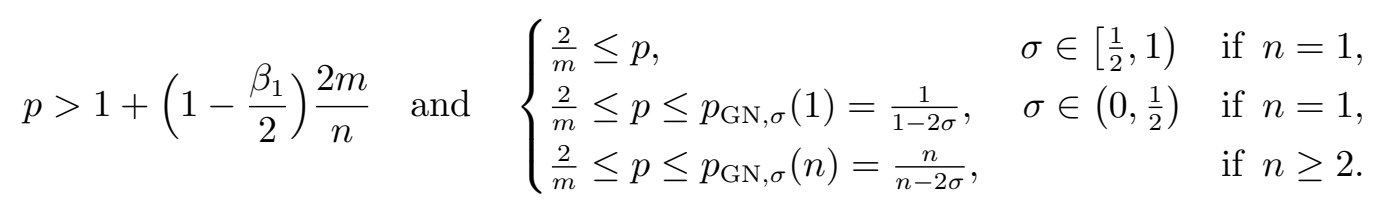

\subsubsection{Case of super-exponential propagation speed}

In a similar way as in the previous section, now we will discuss low regularity for the data in the case of super-exponential propagation speed. We have the following statement.

Theorem 3.2.2. Assume that the Hypotheses (A1) to (A5), (B1) to (B6), (C3) and $(\boldsymbol{C} 4)$, and $\nu(\delta)=\infty$ are satisfied. Let us choose the data $\left(u_{0}, u_{1}\right) \in \mathcal{D}_{m}^{\sigma}$ with $\sigma \in(0,1)$ and $m \in[1,2)$. Moreover, we suppose that the following condition for the function $\nu=\nu(\delta, t)$ holds:

$$
\nu(\delta, t)=o(\log \Lambda(t))
$$

Let

$$
n \leq \frac{4 \sigma}{2-m}
$$

and that the exponent $p$ satisfies

$$
\left\{\begin{array}{lll}
\frac{2}{m} \leq p, & \sigma \in\left[\frac{1}{2}, 1\right) & \text { if } n=1, \\
\frac{2}{m} \leq p \leq p_{G N, \sigma}(1)=\frac{1}{1-2 \sigma}, & \sigma \in\left(0, \frac{1}{2}\right) & \text { if } n=1, \\
\frac{2}{m} \leq p \leq p_{G N, \sigma}(n)=\frac{n}{n-2 \sigma} & & \text { if } n \geq 2 .
\end{array}\right.
$$

Then, there exists a constant $\varepsilon_{0}>0$ such that, for all $\left(u_{0}, u_{1}\right) \in \mathcal{D}_{m}^{\sigma}$ with $\left\|\left(u_{0}, u_{1}\right)\right\|_{\mathcal{D}_{m}^{\sigma}} \leq \varepsilon_{0}$, there exists a uniquely determined globally (in time) Sobolev solution

$$
u \in \mathcal{C}\left([0, \infty), H^{\sigma}\right)
$$


to the Cauchy problem (3.1.1).

Moreover, there exists a constant $C>0$ such that the solution satisfies the following decay estimates:

$$
\begin{aligned}
\|u(t, \cdot)\|_{L^{2}} & \leq C\left(1+B_{\lambda}(0, t)\right)^{-\frac{n}{2}\left(\frac{1}{m}-\frac{1}{2}\right)}\left\|\left(u_{0}, u_{1}\right)\right\|_{\mathcal{D}_{m}^{\sigma}}, \\
\left\||D|^{\sigma} u(t, \cdot)\right\|_{L^{2}} & \leq C\left(1+B_{\lambda}(0, t)\right)^{-\frac{\sigma}{2}-\frac{n}{2}\left(\frac{1}{m}-\frac{1}{2}\right)}\left\|\left(u_{0}, u_{1}\right)\right\|_{\mathcal{D}_{m}^{\sigma}} .
\end{aligned}
$$

Proof. The proof of this theorem is similar to the proof of Theorem 3.2.1. The goal is to show that the integrals $\mathbf{A}$ and $\mathbf{B}$ in the estimate

$$
\begin{aligned}
\left\||D|^{\sigma} u^{\mathrm{nl}}(t, \cdot)\right\|_{L^{2}} \leq & C\|u\|_{X(t)}^{p} \underbrace{\int_{0}^{\delta t} \frac{\Lambda(s)}{\lambda(s)}\left(1+B_{\lambda}(s, t)\right)^{-\frac{\sigma}{2}-\frac{n}{2}\left(\frac{1}{m}-\frac{1}{2}\right)}\left(1+B_{\lambda}(0, s)\right)^{-\frac{n}{2 m} p+\frac{n}{2 m}} d s}_{\mathbf{A}} \\
& +C\|u\|_{X(t)}^{p} \underbrace{\int_{\delta t}^{t} \frac{\Lambda(s)}{\lambda(s)}\left(1+B_{\lambda}(s, t)\right)^{-\frac{\sigma}{2}}\left(1+B_{\lambda}(0, s)\right)^{-\frac{n}{2 m} p+\frac{n}{4}} d s}_{\mathbf{B}}
\end{aligned}
$$

satisfy the desired decay estimates with the condition (C3). Hence, we will only establish the estimates

$$
\begin{aligned}
\|N u\|_{X(t)} & \lesssim\left\|\left(u_{0}, u_{1}\right)\right\|_{\mathcal{D}_{m}^{\sigma}}+\|u\|_{X(t)}^{p}, \\
\|N u-N v\|_{X(t)} & \lesssim\|u-v\|_{X(t)}\left(\|u\|_{X(t)}^{p-1}+\|v\|_{X(t)}^{p-1}\right) .
\end{aligned}
$$

From the definition of the norm of the solution space $X(t)$ and the estimates of Theorem 3.1.2 one can immediately conclude

$$
\left\|u^{\operatorname{lin}}\right\|_{X(t)} \lesssim\left\|\left(u_{0}, u_{1}\right)\right\|_{\mathcal{D}_{m}^{\sigma}} .
$$

To prove the first inequality it remains to estimate $\left\|u^{\mathrm{n} l}\right\|_{X(t)}$. Firstly, we have

$$
\mathbf{A} \lesssim\left(1+B_{\lambda}(0, t)\right)^{-\frac{\sigma}{2}-\frac{n}{2}\left(\frac{1}{m}-\frac{1}{2}\right)} \underbrace{\int_{0}^{\delta t} \frac{\Lambda(s)}{\lambda(s)}\left(1+B_{\lambda}(0, s)\right)^{-\frac{n}{2 m} p+\frac{n}{2 m}} d s}_{\bar{A}_{1}(t)}
$$

where we used (3.1.21). Let us define $\alpha_{1}(p):=\frac{n}{2 m} p-\frac{n}{2 m}$ and discuss the following cases:

Case 1: $\alpha_{1}(p) \neq 1$. In this case, it holds

$$
\begin{aligned}
\bar{A}_{1}(t)= & \int_{0}^{\delta t} \frac{\mu(s)}{\lambda^{2}(s)}\left(1+B_{\lambda}(0, s)\right)^{-\alpha_{1}(p)} d\left(1+B_{\lambda}(0, s)\right) \\
= & \left.\frac{1}{1-\alpha_{1}(p)} \frac{\mu(s)}{\lambda^{2}(s)}\left(1+B_{\lambda}(0, s)\right)^{-\alpha_{1}(p)+1}\right|_{0} ^{\delta t} \\
& -\frac{1}{1-\alpha_{1}(p)} \int_{0}^{\delta t}\left(1+B_{\lambda}(0, s)\right)^{-\alpha_{1}(p)+1} d\left(\frac{\mu(s)}{\lambda^{2}(s)}\right) \\
\lesssim & \frac{1}{1-\alpha_{1}(p)}\left(\frac{\mu(\delta t)}{\lambda^{2}(\delta t)}\left(1+B_{\lambda}(0, \delta t)\right)^{-\alpha_{1}(p)+1}-\frac{\mu(0)}{\lambda^{2}(0)}\right) \\
& +\frac{1}{1-\alpha_{1}(p)} \int_{0}^{\delta t} \frac{\mu(s)}{\lambda^{2}(s)}\left(1+B_{\lambda}(0, s)\right)^{-\alpha_{1}(p)+1} \frac{\lambda(s)}{\Lambda(s)} d s,
\end{aligned}
$$

where we used condition (C4). Now we shall show the integral $\bar{A}_{1}=\bar{A}_{1}(t)$ is bounded. For this reason, by choosing an arbitrary small positive constant $\varepsilon$ we want to verify the inequality

$$
\frac{\mu(t)}{\lambda^{2}(t)}\left(1+B_{\lambda}(0, t)\right)^{-\alpha_{1}(p)+1} \lesssim \Lambda(t)^{-\varepsilon} .
$$


Then, we need to show that the following function is bounded:

$$
\overline{\mathcal{A}}_{1}(t):=\frac{\mu(t)}{\lambda^{2}(t)} \Lambda(t)^{\varepsilon}\left(1+B_{\lambda}(0, t)\right)^{-\alpha_{1}(p)+1} .
$$

Let us consider the monotonicity of $\overline{\mathcal{A}}_{1}=\overline{\mathcal{A}}_{1}(t)$ by taking its derivative. Indeed, by using condition (C4) and (3.1.20) we have

$$
\begin{aligned}
\overline{\mathcal{A}}_{1}^{\prime}(t)= & \left(\frac{\mu(t)}{\lambda^{2}(t)}\right)^{\prime} \Lambda(t)^{\varepsilon}\left(1+B_{\lambda}(0, t)\right)^{-\alpha_{1}(p)+1}+\varepsilon \frac{\mu(t)}{\lambda^{2}(t)} \lambda(t) \Lambda(t)^{\varepsilon-1}\left(1+B_{\lambda}(0, t)\right)^{-\alpha_{1}(p)+1} \\
& +\left(1-\alpha_{1}(p)\right) \frac{\mu(t)}{\lambda^{2}(t)} \Lambda(t)^{\varepsilon} \frac{\lambda(t) \Lambda(t)}{\mu(t)}\left(1+B_{\lambda}(0, t)\right)^{-\alpha_{1}(p)} \\
\leq & {\left[\left(-\beta_{1}+\varepsilon\right) \frac{\mu(t)}{\lambda(t) \Lambda(t)}\left(1+B_{\lambda}(0, t)\right)+\left(1-\alpha_{1}(p)\right) \frac{\Lambda(t)}{\lambda(t)}\right]\left(1+B_{\lambda}(0, t)\right)^{-\alpha_{1}(p)} \Lambda(t)^{\varepsilon} } \\
\leq & {\left[\left(\frac{-\beta_{1}+\varepsilon}{\alpha+2}\right) \frac{\mu(t)}{\lambda(t) \Lambda(t)} \frac{\Lambda^{2}(t)}{\mu(t)} \log \Lambda(t)+\left(1-\alpha_{1}(p)\right) \frac{\Lambda(t)}{\lambda(t)}\right]\left(1+B_{\lambda}(0, t)\right)^{-\alpha_{1}(p)} \Lambda(t)^{\varepsilon} } \\
\leq & {\left[\left(\frac{-\beta_{1}+\varepsilon}{\alpha+2}\right) \log \Lambda(t)+\left(1-\alpha_{1}(p)\right)\right]\left(1+B_{\lambda}(0, t)\right)^{-\alpha_{1}(p)} \frac{\Lambda(t)}{\lambda(t)} \Lambda(t)^{\varepsilon} . }
\end{aligned}
$$

This inequality implies $\overline{\mathcal{A}}_{1}^{\prime}(t)<0$ for a small positive constant $\varepsilon$ and for large $t$. Hence, we get

$$
\int_{0}^{\delta t} \frac{\mu(s)}{\lambda^{2}(s)}\left(1+B_{\lambda}(0, s)\right)^{-\alpha_{1}(p)+1} \frac{\lambda(s)}{\Lambda(s)} d s \lesssim \int_{0}^{\delta t} \Lambda(s)^{-\varepsilon-1} \lambda(s) d s \lesssim 1 .
$$

Therefore, we may conclude that $\bar{A}_{1}=\bar{A}_{1}(t)$ is uniformly bounded.

Case 2: $\alpha_{1}(p)=1$. In this case we have

$$
\begin{aligned}
\bar{A}_{1}(t) & =\int_{0}^{\delta t} \frac{\Lambda(s)}{\lambda(s)}\left(1+B_{\lambda}(0, s)\right)^{-1} d s \lesssim \int_{0}^{\delta t} \frac{\Lambda(s)}{\lambda(s)} \frac{\mu(s)}{\Lambda^{2}(s)} \frac{1}{\log \Lambda(s)} d s \\
& \leq \int_{0}^{\delta t} \frac{\mu(s)}{\lambda^{2}(s)} \frac{\lambda(s)}{\Lambda(s)} d s=\left.\frac{\mu(s)}{\lambda^{2}(s)} \log \Lambda(s)\right|_{0} ^{\delta t}-\int_{0}^{\delta t} \log \Lambda(s) d\left(\frac{\mu(s)}{\lambda^{2}(s)}\right) \\
& \leq\left.\frac{\mu(s)}{\lambda^{2}(s)} \log \Lambda(s)\right|_{0} ^{\delta t}+\beta_{0} \int_{0}^{\delta t} \frac{\mu(s)}{\lambda^{2}(s)} \log \Lambda(s) \frac{\lambda(s)}{\Lambda(s)} d s,
\end{aligned}
$$

where we used (3.1.20) and condition (C4), respectively. Then, we show that for a sufficiently small constant $\varepsilon>0$ we have the estimate

$$
\frac{\mu(t)}{\lambda^{2}(t)} \log \Lambda(t) \lesssim \Lambda^{-\varepsilon}(t)
$$

Therefore, let us compute the derivative of the function

$$
\overline{\mathcal{A}}_{2}(t):=\frac{\mu(t)}{\lambda^{2}(t)} \Lambda^{\varepsilon}(t) \log \Lambda(t)
$$

It follows

$$
\begin{aligned}
\overline{\mathcal{A}}_{2}^{\prime}(t) & =\left(\frac{\mu(t)}{\lambda^{2}(t)}\right)^{\prime} \Lambda^{\varepsilon}(t) \log \Lambda(t)+\varepsilon \frac{\mu(t)}{\lambda^{2}(t)} \lambda(t) \Lambda^{\varepsilon-1}(t) \log \Lambda(t)+\frac{\mu(t)}{\lambda^{2}(t)} \Lambda^{\varepsilon}(t) \frac{\lambda(t)}{\Lambda(t)} \\
& \leq\left[-\beta_{1} \frac{\mu(t)}{\lambda(t) \Lambda(t)} \log \Lambda(t)+\varepsilon \frac{\mu(t)}{\lambda(t) \Lambda(t)} \log \Lambda(t)+\frac{\mu(t)}{\lambda(t) \Lambda(t)}\right] \Lambda^{\varepsilon}(t) \\
& \leq\left[\left(-\beta_{1}+\varepsilon\right) \log \Lambda(t)+1\right] \frac{\mu(t)}{\lambda(t) \Lambda(t)} \Lambda^{\varepsilon}(t) .
\end{aligned}
$$


Then, for sufficiently large time $t$ and sufficiently small $\varepsilon>0$ we get $\overline{\mathcal{A}}_{2}^{\prime}(t)<0$. This shows the decreasing behavior of $\overline{\mathcal{A}}_{2}=\overline{\mathcal{A}}_{2}(t)$. Hence, we obtain that $\bar{A}_{1}=\bar{A}_{1}(t)$ is uniformly bounded.

Consequently, we arrive at the following desired estimate in both cases:

$$
\mathbf{A} \lesssim\left(1+B_{\lambda}(0, t)\right)^{-\frac{\sigma}{2}-\frac{n}{2}\left(\frac{1}{m}-\frac{1}{2}\right)} .
$$

Now let us consider the case $s \in[\delta t, t]$. Defining $\alpha_{2}(p):=\frac{n}{2 m} p-\frac{n}{4}$ it holds

$$
\begin{aligned}
\mathbf{B}= & \int_{\delta t}^{t} \frac{\Lambda(s)}{\lambda(s)}\left(1+B_{\lambda}(s, t)\right)^{-\frac{\sigma}{2}}\left(1+B_{\lambda}(0, s)\right)^{-\alpha_{2}(p)} d s \\
= & -\int_{\delta t}^{t} \frac{\mu(s)}{\lambda^{2}(s)}\left(1+B_{\lambda}(0, s)\right)^{-\alpha_{2}(p)}\left(1+B_{\lambda}(s, t)\right)^{-\frac{\sigma}{2}} d\left(1+B_{\lambda}(s, t)\right) \\
= & -\left.\frac{1}{1-\frac{\sigma}{2}} \frac{\mu(s)}{\lambda^{2}(s)}\left(1+B_{\lambda}(0, s)\right)^{-\alpha_{2}(p)}\left(1+B_{\lambda}(s, t)\right)^{-\frac{\sigma}{2}+1}\right|_{\delta t} ^{t} \\
& +\frac{1}{1-\frac{\sigma}{2}} \int_{\delta t}^{t}\left(1+B_{\lambda}(s, t)\right)^{-\frac{\sigma}{2}+1} d\left(\frac{\mu(s)}{\lambda^{2}(s)}\left(1+B_{\lambda}(0, s)\right)^{-\alpha_{2}(p)}\right) .
\end{aligned}
$$

Using Lemma 3.1.5, we obtain

$$
\begin{aligned}
\mathbf{B} \lesssim & \frac{\mu(\delta t)}{\lambda^{2}(\delta t)}\left(1+B_{\lambda}(0, \delta t)\right)^{-\alpha_{2}(p)}\left(1+B_{\lambda}(0, t)\right)^{-\frac{\sigma}{2}+1} \\
& +\int_{\delta t}^{t} \frac{\mu(s)}{\lambda^{2}(s)}\left(1+B_{\lambda}(0, s)\right)^{-\alpha_{2}(p)}\left(1+B_{\lambda}(0, t)\right)^{-\frac{\sigma}{2}+1} \frac{d\left(1+B_{\lambda}(0, s)\right)}{1+B_{\lambda}(0, s)}
\end{aligned}
$$

since $1-\frac{\sigma}{2}>0$. Multiplying both sides of the last estimate by $\left(1+B_{\lambda}(0, t)\right)^{\frac{\sigma}{2}+\frac{n}{2}\left(\frac{1}{m}-\frac{1}{2}\right)}$, we find

$$
\begin{aligned}
\left(1+B_{\lambda}(0, t)\right)^{\frac{\sigma}{2}+\frac{n}{2}\left(\frac{1}{m}-\frac{1}{2}\right)} \mathbf{B} & \lesssim \underbrace{\frac{\mu(\delta t)}{\lambda^{2}(\delta t)}\left(1+B_{\lambda}(0, \delta t)\right)^{-\alpha_{2}(p)}\left(1+B_{\lambda}(0, t)\right)^{\frac{n}{2}\left(\frac{1}{m}-\frac{1}{2}\right)+1}}_{\bar{B}_{1}(t)} \\
& +\underbrace{\int_{\delta t}^{t} \frac{\mu(s)}{\lambda^{2}(s)}\left(1+B_{\lambda}(0, s)\right)^{-\alpha_{2}(p)}\left(1+B_{\lambda}(0, t)\right)^{\frac{n}{2}\left(\frac{1}{m}-\frac{1}{2}\right)+1} \frac{d\left(1+B_{\lambda}(0, s)\right)}{1+B_{\lambda}(0, s)}}_{\bar{B}_{2}(t)} .
\end{aligned}
$$

We will prove that on the right-hand side of the previous inequality $\bar{B}_{1}=\bar{B}_{1}(t)$ and $\bar{B}_{2}=$ $\bar{B}_{2}(t)$ are uniformly bounded. Therefore, we will show that for a constant $\gamma>0$ we have

$$
\frac{\mu(\delta t)}{\lambda^{2}(\delta t)}\left(1+B_{\lambda}(0, \delta t)\right)^{-\alpha_{2}(p)}\left(1+B_{\lambda}(0, t)\right)^{\frac{n}{2}\left(\frac{1}{m}-\frac{1}{2}\right)+1} \lesssim\left(1+B_{\lambda}(0, \delta t)\right)^{-\gamma} .
$$

For this reason we will check the monotonicity of the following function:

$$
\overline{\mathcal{B}}_{1}(t):=\frac{\mu(\delta t)}{\lambda^{2}(\delta t)}\left(1+B_{\lambda}(0, \delta t)\right)^{-\alpha_{2}(p)+\gamma}\left(1+B_{\lambda}(0, t)\right)^{\frac{n}{2}\left(\frac{1}{m}-\frac{1}{2}\right)+1} .
$$

Indeed, by taking the derivative of $\overline{\mathcal{B}}_{1}=\overline{\mathcal{B}}_{1}(t)$ we get

$$
\begin{aligned}
\overline{\mathcal{B}}_{1}^{\prime}(t)= & \underbrace{\left(\frac{\mu(\delta t)}{\lambda^{2}(\delta t)}\right)^{\prime}\left(1+B_{\lambda}(0, \delta t)\right)^{-\alpha_{2}(p)+\gamma}\left(1+B_{\lambda}(0, t)\right)^{\frac{n}{2}\left(\frac{1}{m}-\frac{1}{2}\right)+1}}_{\bar{I}_{1}(t)} \\
& +\underbrace{\left(\left(1+B_{\lambda}(0, \delta t)\right)^{-\alpha_{2}(p)+\gamma}\left(1+B_{\lambda}(0, t)\right)^{\frac{n}{2}\left(\frac{1}{m}-\frac{1}{2}\right)+1}\right)^{\prime} \frac{\mu(\delta t)}{\lambda^{2}(\delta t)}}_{\bar{I}_{2}(t)} .
\end{aligned}
$$


$\bar{I}_{1}=\bar{I}_{1}(t)$ satisfies the following estimate:

$$
\begin{aligned}
\bar{I}_{1}(t) \leq & -\delta \beta_{1} \frac{\mu(\delta t)}{\lambda(\delta t) \Lambda(\delta t)}\left(1+B_{\lambda}(0, \delta t)\right)^{-\alpha_{2}(p)+\gamma}\left(1+B_{\lambda}(0, t)\right)^{\frac{n}{2}\left(\frac{1}{m}-\frac{1}{2}\right)+1} \\
\leq & -\delta \beta_{1} \frac{\mu(\delta t)}{\lambda(\delta t) \Lambda(\delta t)}\left(1+B_{\lambda}(0, \delta t)\right)\left(1+B_{\lambda}(0, t)\right) \\
& \times\left(1+B_{\lambda}(0, \delta t)\right)^{-\alpha_{2}(p)+\gamma-1}\left(1+B_{\lambda}(0, t)\right)^{\frac{n}{2}\left(\frac{1}{m}-\frac{1}{2}\right)} \\
\leq & -\delta \beta_{1} \frac{\mu(\delta t)}{\lambda^{2}(\delta t)} \frac{\lambda(\delta t)}{\Lambda(\delta t)} \phi(\delta t) \log \Lambda(\delta t) \phi(t) \log \Lambda(t) \\
& \times\left(1+B_{\lambda}(0, \delta t)\right)^{-\alpha_{2}(p)+\gamma-1}\left(1+B_{\lambda}(0, t)\right)^{\frac{n}{2}\left(\frac{1}{m}-\frac{1}{2}\right)}
\end{aligned}
$$

where we used (3.1.20). On the other hand, for $\bar{I}_{2}=\bar{I}_{2}(t)$ we get

$$
\begin{aligned}
\bar{I}_{2}(t)= & {\left[\left(-\alpha_{2}(p)+\gamma\right) \frac{\lambda(\delta t) \Lambda(\delta t)}{\mu(\delta t)}\left(1+B_{\lambda}(0, t)\right)+\left(\frac{n}{2}\left(\frac{1}{m}-\frac{1}{2}\right)+1\right) \frac{\lambda(t) \Lambda(t)}{\mu(t)}\left(1+B_{\lambda}(0, \delta t)\right)\right] } \\
& \times\left(1+B_{\lambda}(0, \delta t)\right)^{-\alpha_{2}(p)+\gamma-1}\left(1+B_{\lambda}(0, t)\right)^{\frac{n}{2}\left(\frac{1}{m}-\frac{1}{2}\right)} \frac{\mu(\delta t)}{\lambda^{2}(\delta t)} \\
\leq & {\left[\frac{-\alpha_{2}(p)+\gamma}{\alpha+2} \frac{\lambda(\delta t) \Lambda(\delta t)}{\mu(\delta t)} \phi(t) \log \Lambda(t)+\left(\frac{n}{2}\left(\frac{1}{m}-\frac{1}{2}\right)+1\right) \frac{\lambda(t) \Lambda(t)}{\mu(t)} \phi(\delta t) \log \Lambda(\delta t)\right] } \\
& \times\left(1+B_{\lambda}(0, \delta t)\right)^{-\alpha_{2}(p)+\gamma-1}\left(1+B_{\lambda}(0, t)\right)^{\frac{n}{2}\left(\frac{1}{m}-\frac{1}{2}\right)} \frac{\mu(\delta t)}{\lambda^{2}(\delta t)} \\
\leq & {\left[\frac{-\alpha_{2}(p)+\gamma}{\alpha+2} \frac{\lambda(\delta t)}{\Lambda(\delta t)} \phi(t) \phi(\delta t) \log \Lambda(t)+\left(\frac{n}{2}\left(\frac{1}{m}-\frac{1}{2}\right)+1\right) \frac{\lambda(t)}{\Lambda(t)} \phi(t) \phi(\delta t) \log \Lambda(\delta t)\right] } \\
& \times\left(1+B_{\lambda}(0, \delta t)\right)^{-\alpha_{2}(p)+\gamma-1}\left(1+B_{\lambda}(0, t)\right)^{\frac{n}{2}\left(\frac{1}{m}-\frac{1}{2}\right)} \frac{\mu(\delta t)}{\lambda^{2}(\delta t)} \\
\leq & {\left[\frac{-\alpha_{2}(p)+\gamma}{\alpha+2} \frac{1}{\log \Lambda(\delta t)}+\left(\frac{n}{2}\left(\frac{1}{m}-\frac{1}{2}\right)+1\right) \frac{\lambda(t)}{\Lambda(t)} \frac{\Lambda(\delta t)}{\lambda(\delta t)} \frac{1}{\log \Lambda(t)}\right] \frac{\lambda(\delta t)}{\Lambda(\delta t)} } \\
& \times \phi(t) \phi(\delta t) \log \Lambda(t) \log \Lambda(\delta t)\left(1+B_{\lambda}(0, \delta t)\right)^{-\alpha_{2}(p)+\gamma-1}\left(1+B_{\lambda}(0, t)\right)^{\frac{n}{2}\left(\frac{1}{m}-\frac{1}{2}\right)} \frac{\mu(\delta t)}{\lambda^{2}(\delta t)} .
\end{aligned}
$$

Here we have used condition (C4) and (3.1.20). Hence, we have

$$
\begin{aligned}
\overline{\mathcal{B}}_{1}^{\prime}(t)= & \bar{I}_{1}(t)+\bar{I}_{2}(t) \\
\leq & {\left[-\delta \beta_{1}+\frac{-\alpha_{2}(p)+\gamma}{\alpha+2} \frac{1}{\log \Lambda(\delta t)}+\left(\frac{n}{2}\left(\frac{1}{m}-\frac{1}{2}\right)+1\right) \frac{\lambda(t)}{\Lambda(t)} \frac{\Lambda(\delta t)}{\lambda(\delta t)} \frac{1}{\log \Lambda(t)}\right] \frac{\lambda(\delta t)}{\Lambda(\delta t)} } \\
& \times \phi(t) \phi(\delta t) \log \Lambda(t) \log \Lambda(\delta t)\left(1+B_{\lambda}(0, \delta t)\right)^{-\alpha_{2}(p)+\gamma-1}\left(1+B_{\lambda}(0, t)\right)^{\frac{n}{2}\left(\frac{1}{m}-\frac{1}{2}\right)} \frac{\mu(\delta t)}{\lambda^{2}(\delta t)} .
\end{aligned}
$$

Now we can use the assumption (3.2.20), which implies $\overline{\mathcal{B}}_{1}^{\prime}(t)=\bar{I}_{1}(t)+\bar{I}_{2}(t)<0$ for large $t$. This shows that $\overline{\mathcal{B}}_{1}=\overline{\mathcal{B}}_{1}(t)$ is bounded for large $t$. Moreover, for $\bar{B}_{2}=\bar{B}_{2}(t)$ by using the estimate (3.2.29) we immediately get

$$
\bar{B}_{2}(t) \lesssim \int_{\delta t}^{t}\left(1+B_{\lambda}(0, s)\right)^{-\gamma-1} d\left(1+B_{\lambda}(0, s)\right)=\frac{1}{\gamma\left(1+B_{\lambda}(0, \delta t)\right)^{\gamma}}-\frac{1}{\gamma\left(1+B_{\lambda}(0, t)\right)^{\gamma}} \leq C .
$$

Consequently, we obtain the desired estimate

$$
\mathbf{B} \lesssim\left(1+B_{\lambda}(0, t)\right)^{-\frac{\sigma}{2}-\frac{n}{2}\left(\frac{1}{m}-\frac{1}{2}\right)} .
$$

From (3.2.28) and (3.2.31) we may conclude

$$
\left\||D|^{\sigma} u^{\mathrm{nl}}(t, \cdot)\right\|_{L^{2}} \lesssim\left(1+B_{\lambda}(0, t)\right)^{-\frac{\sigma}{2}-\frac{n}{2}\left(\frac{1}{m}-\frac{1}{2}\right)}\|u\|_{X(t)}^{p} .
$$


Similarly, we can get

$$
\left\|u^{\mathrm{nl}}(t, \cdot)\right\|_{L^{2}} \lesssim\left(1+B_{\lambda}(0, t)\right)^{-\frac{n}{2}\left(\frac{1}{m}-\frac{1}{2}\right)}\|u\|_{X(t)}^{p} .
$$

Replacing the estimates (3.2.32) and (3.2.33) in the norm of the solution space $X(t)$ we obtain

$$
\left\|u^{\mathrm{nl}}\right\|_{X(t)} \lesssim\|u\|_{X(t)}^{p} .
$$

Hence, by (3.2.25) and (3.2.34) the proof of (3.2.23) is completed.

Now let us prove (3.2.24). Following the same steps of the proof of Theorem 3.2.1 we get

$$
\begin{aligned}
\||D|^{\sigma}(N u & -N v)(t, \cdot)\left\|_{L^{2}} \lesssim\right\| u-v \|_{X(t)}\left(\|u\|_{X(t)}^{p-1}+\|v\|_{X(t)}^{p-1}\right) \\
& \times\left(\int_{0}^{\delta t} \frac{\Lambda(s)}{\lambda(s)}\left(1+B_{\lambda}(s, t)\right)^{-\frac{\sigma}{2}-\frac{n}{2}\left(\frac{1}{m}-\frac{1}{2}\right)}\left(1+B_{\lambda}(0, s)\right)^{-\frac{n}{2 m} p+\frac{n}{2 m}} d s\right. \\
& \left.\quad+\int_{\delta t}^{t} \frac{\Lambda(s)}{\lambda(s)}\left(1+B_{\lambda}(s, t)\right)^{-\frac{\sigma}{2}}\left(1+B_{\lambda}(0, s)\right)^{-\frac{n}{2 m} p+\frac{n}{4}} d s\right) .
\end{aligned}
$$

Proceeding as for proving $\left\||D|^{\sigma} u^{\mathrm{nl}}(t, \cdot)\right\|_{L^{2}}$, we have

$$
\begin{aligned}
\left\||D|^{\sigma}(N u-N v)(t, \cdot)\right\|_{L^{2}} & \lesssim\left(1+B_{\lambda}(0, t)\right)^{-\frac{\sigma}{2}-\frac{n}{2}\left(\frac{1}{m}-\frac{1}{2}\right)}\|u-v\|_{X(t)}\left(\|u\|_{X(t)}^{p-1}+\|v\|_{X(t)}^{p-1}\right), \\
\|(N u-N v)(t, \cdot)\|_{L^{2}} & \lesssim\left(1+B_{\lambda}(0, t)\right)^{-\frac{n}{2}\left(\frac{1}{m}-\frac{1}{2}\right)}\|u-v\|_{X(t)}\left(\|u\|_{X(t)}^{p-1}+\|v\|_{X(t)}^{p-1}\right) .
\end{aligned}
$$

Using these estimates in the norm of $X(t)$ we obtain (3.2.24). This concludes the proof.

Remark 3.2.3. Now let us discuss in the following table the conditions (3.2.21) and (3.2.22) of the last theorem in some special cases which depend on the parameters $n, m$ and $\sigma$ to get the admissible range for $p$. We are interested in the case $n \leq 6$ only.

\begin{tabular}{|c|c|c|c|}
\hline$n$ & $m$ & Regularity $\sigma$ & Admissible range for $p$ \\
\hline \multirow{2}{*}{$n=1$} & $m \in[1,2)$ & $\sigma \in\left[\frac{1}{2}, 1\right)$ & $\frac{2}{m} \leq p<\infty$ \\
\cline { 2 - 4 } & $m \in[1,2)$ & $\sigma \in\left[\frac{2-m}{4}, \frac{1}{2}\right)$ & $\frac{2}{m} \leq p \leq \frac{1}{1-2 \sigma}$ \\
\hline$n=2$ & $m \in[1,2)$ & $\sigma \in\left[\frac{2-m}{2}, 1\right)$ & $\frac{2}{m} \leq p \leq \frac{1}{1-\sigma}$ \\
\hline$n=3$ & $m \in[1,2)$ & $\sigma \in\left[\frac{6-3 m}{4}, 1\right)$ & $\frac{2}{m} \leq p \leq \frac{3}{3-2 \sigma}$ \\
\hline$n=4$ & $m \in(1,2)$ & $\sigma \in[2-m, 1)$ & $\frac{2}{m} \leq p \leq \frac{2}{2-\sigma}$ \\
\hline$n=5$ & $m \in\left(\frac{6}{5}, 2\right)$ & $\sigma \in\left[\frac{10-5 m}{4}, 1\right)$ & $\frac{2}{m} \leq p \leq \frac{5}{5-2 \sigma}$ \\
\hline$n=6$ & $m \in\left(\frac{4}{3}, 2\right)$ & $\sigma \in\left[\frac{6-3 m}{2}, 1\right)$ & $\frac{2}{m} \leq p \leq \frac{3}{3-\sigma}$ \\
\hline
\end{tabular}

Tab. 3.2.: Admissible range for the parameters

Remark 3.2.4. We can see that we have a benefit of the additional $L^{m}$ regularity of the data. It allows to have results for higher dimensions $n$. We note that the previous case with additional $L^{1}$ regularity can be proved for $n \leq 4$ only.

Example 3.2.3. We choose $\lambda(t)=e^{t} e^{e^{t}}$. Then, we have

$$
\Lambda(t)=e^{e^{t}}, \quad \Theta(t)=e^{r e^{t}}, \quad 0<r<1 .
$$

Moreover, since $\phi(t)=\frac{\Lambda^{2}(t)}{\mu(t)}$ is an increasing function from condition (C3) choosing

$$
\mu(t)=\frac{e^{2 e^{t}}}{e^{\alpha t}}, \quad \alpha>0
$$


we get $\rho(t)=\mu(t) e^{t}=e^{(1-\alpha) t} e^{2 e^{t}}$.

Applying Theorem 3.2.2 we obtain $\nu(\delta)=\infty$, namely, we are in the case of superexponential propagation speed. Then, using the condition (3.2.20) we obtain

$$
\nu(\delta, t) \frac{1}{\log \Lambda(t)}=\frac{\lambda(t)}{\Lambda(t)} \frac{\Lambda(\delta t)}{\lambda(\delta t)} \frac{1}{\log \Lambda(t)}=\frac{e^{t} e^{e^{t}}}{e^{e^{t}}} \frac{e^{e^{\delta t}}}{e^{\delta t} e^{e^{\delta t}}} \frac{1}{e^{t}}=\frac{1}{e^{\delta t}} .
$$

So, we have global (in time) existence of small data solutions for

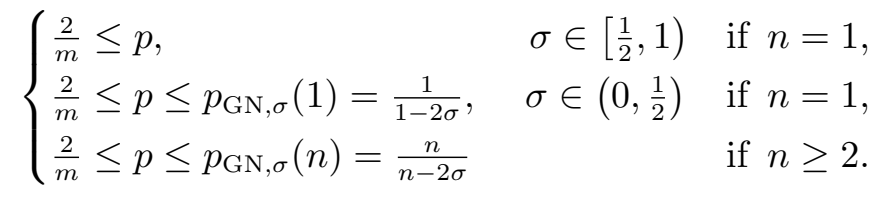

Example 3.2.4. Let us choose $\lambda(t)=e^{t} e^{e^{t}} e^{e^{e^{t}}}$. Then, we have

$$
\Lambda(t)=e^{e^{e^{t}}}, \quad \Theta(t)=e^{r e^{e^{t}}}, \quad 0<r<1 .
$$

Moreover, we choose the function $\mu=\mu(t)$ satisfying condition (C3) in the following way:

$$
\mu(t)=\frac{e^{2 e^{e^{t}}}}{e^{\alpha e^{t}}}, \quad \alpha>0
$$

we get

$$
\rho(t)=\mu(t) e^{t} e^{e^{t}}=e^{t} e^{(1-\alpha) e^{t}} e^{2 e^{e^{t}}} .
$$

Applying Theorem 3.2.2 we obtain $\nu(\delta)=\infty$, namely, we are in the case of superexponential propagation speed. Hence, by using the condition (3.2.20) we find

$$
\nu(\delta, t) \frac{1}{\log \Lambda(t)}=\frac{\lambda(t)}{\Lambda(t)} \frac{\Lambda(\delta t)}{\lambda(\delta t)} \frac{1}{\log \Lambda(t)}=\frac{e^{t} e^{e^{t}} e^{e^{e^{t}}}}{e^{e^{e^{t}}}} \frac{e^{e^{e^{\delta t}}}}{e^{\delta t} e^{e^{\delta t}} e^{e^{e^{\delta t}}}} \frac{1}{e^{e^{t}}}=\frac{e^{t}}{e^{\delta t} e^{e^{\delta t}}} .
$$

So, we have the global (in time) existence of small data solutions for

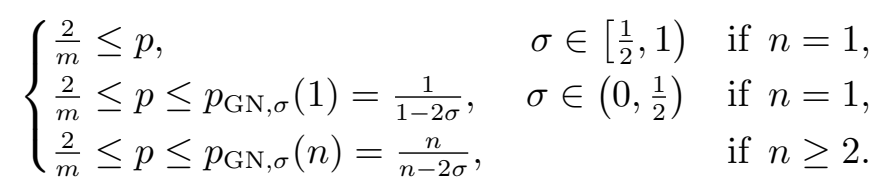

\subsection{Data in energy space}

In this section we are interested in the case $\sigma=1$ which corresponds to the classical energy space $H^{1} \times L^{2}$ with additional $L^{m}$ regularity, i.e., the data are assumed in $\mathcal{D}_{m}^{1}$ with $m \in[1,2)$.

\subsubsection{Case of sub-exponential propagation speed}

We define the following parameters:

$$
\begin{aligned}
& p_{\mathrm{Fuj}, m}(n):=1+\frac{2 m}{n} \quad \text { for } n \geq 1, \\
& \tilde{p}_{1, m}(n):=1+\left(1-\frac{\beta_{1}}{2+R}\right) \frac{2 m}{n} \quad \text { for } n \geq 1, \\
& \tilde{p}_{2, m}(n):=1+\left(1-\frac{\delta}{\nu(\delta)} \frac{\beta_{1}}{2+R}\right) \frac{2 m}{n} \quad \text { for } n \geq 1, \\
& \tilde{p}_{3, m}(n) \\
& :=\max \left\{\frac{m}{2}+\frac{1-\frac{m}{2}}{1-\frac{2+R}{2-\mu_{1}}+\frac{2+R}{2-\mu_{1}} \frac{\delta}{\nu(\delta)}}+\left(\frac{1-\frac{\beta_{1}}{2+R} \frac{\delta}{\nu(\delta)}}{1-\frac{2+R}{2-\mu_{1}}+\frac{2+R}{2-\mu_{1}} \frac{\delta}{\nu(\delta)}}\right) \frac{2 m}{n} ; 1+\left(1-\frac{\delta}{\vartheta(\delta)} \frac{\beta_{1}}{2}\right) \frac{2 m}{n}\right\} \\
& p_{\mathrm{GN}}(n):=\frac{n}{n-2} \text { for } n \geq 3,
\end{aligned}
$$


where we set

$$
\tilde{p}_{m}(n):=\max \left\{\tilde{p}_{1, m}(n) ; \tilde{p}_{2, m}(n) ; \tilde{p}_{3, m}(n)\right\} .
$$

Because of the data belongs to $\mathcal{D}_{m}^{1}$, we apply the classical Gagliardo-Nirenberg inequality in order to estimate the nonlinear term in (3.1.1) for the $L^{2}$ norm and the $L^{m}$ norm, $m \in[1,2)$. Therefore, from the application of the classical Gagliardo-Nirenberg inequality the restrictions for the exponent $p$ of the nonlinear term implies the conditions $p \geq \frac{2}{m}$ and $p \leq \frac{n}{n-2}$ if $n \geq 3$. Moreover, we require that the admissible range for the exponent $p$ satisfies the condition $p>\tilde{p}_{m}(n)$ in order to guarantee the integrability and boundedness of some terms in the energy estimates.

Theorem 3.3.1. Assume that the Hypotheses (A1) to (A5), (B1) to (B6), (C1), (C2) and $(\boldsymbol{C} 4)$ are satisfied. Let $\nu(\delta) \lesssim 1$ and $\vartheta(\delta) \lesssim 1$, where $\nu=\nu(\delta)$ and $\vartheta=\vartheta(\delta)$ are defined in (3.1.2) and (3.1.3), respectively. We suppose that $\left(u_{0}, u_{1}\right) \in \mathcal{D}_{m}^{1}$ with $m \in[1,2), n \leq \frac{4}{2-m}$ and that the exponent $p$ satisfies

$$
p>\tilde{p}_{m}(n) \quad \text { and } \quad \begin{cases}\frac{2}{m} \leq p<\infty & \text { if } n=1,2, \\ \frac{2}{m} \leq p \leq p_{G N}(n)=\frac{n}{n-2} & \text { if } n \geq 3,\end{cases}
$$

where $\tilde{p}_{m}(n)$ is defined in (3.3.1). Finally, we consider the following condition:

$$
\frac{\beta_{1}}{\frac{n}{2}\left(\frac{1}{m}-\frac{1}{2}\right)}>\max \left\{(2+R) \frac{\nu(\delta)}{\delta} ; 2 \frac{\vartheta(\delta)}{\delta}\right\},
$$

where the parameters $\beta_{1}$ and $R$ are from condition ( $\left.\boldsymbol{C} 4\right)$ and (3.1.17), respectively. Under these assumptions there exists a constant $\varepsilon_{0}>0$ such that, if $\left\|\left(u_{0}, u_{1}\right)\right\|_{\mathcal{D}_{m}^{1}} \leq \varepsilon_{0}$, then there exists a uniquely determined globally (in time) energy solution to the Cauchy problem (3.1.1) in

$$
\mathcal{C}\left([0, \infty), H^{1}\right) \cap \mathcal{C}^{1}\left([0, \infty), L^{2}\right) .
$$

Furthermore, there exists a constant $C>0$ such that the solution satisfies the estimates

$$
\begin{aligned}
&\|u(t, \cdot)\|_{L^{2}} \leq C\left(1+B_{\lambda}(0, t)\right)^{-\frac{n}{2}\left(\frac{1}{m}-\frac{1}{2}\right)}\left\|\left(u_{0}, u_{1}\right)\right\|_{\mathcal{D}_{m}^{1}}, \\
&\||D| u(t, \cdot)\|_{L^{2}} \leq C\left(1+B_{\lambda}(0, t)\right)^{-\frac{n}{2}\left(\frac{1}{m}-\frac{1}{2}\right)-\frac{1}{2}}\left\|\left(u_{0}, u_{1}\right)\right\|_{\mathcal{D}_{m}^{1}}, \\
&\left\|u_{t}(t, \cdot)\right\|_{L^{2}} \leq C \max \left\{\frac{\lambda^{2}(t)}{\rho(t)}\left(1+B_{\lambda}(0, t)\right)^{-\frac{n}{2}\left(\frac{1}{m}-\frac{1}{2}\right)-1} ;\right. \\
&\left.\lambda(t) F(\Lambda(t))\left(F^{2}(\Lambda(t))\right)^{-\frac{n}{2}\left(\frac{1}{m}-\frac{1}{2}\right)-1}\right\}\left\|\left(u_{0}, u_{1}\right)\right\|_{\mathcal{D}_{m}^{1}} .
\end{aligned}
$$

Remark 3.3.1. Let us compare the parameters $p_{1, m}(n)$ and $p_{2, m}(n)$ from Theorem 3.2.1, where $\sigma \in(0,1)$ and parameters $\tilde{p}_{1, m}(n), \tilde{p}_{2, m}(n)$ and $\tilde{p}_{3, m}(n)$ from Theorem 3.3.1, where $\sigma=1$.

- For the parameters $p_{1, m}(n)$ and $\tilde{p}_{1, m}(n)$, we have $p_{1, m}(n)=\tilde{p}_{1, m}(n)$.

- The parameter $\tilde{p}_{2, m}(n)$ appears only in Theorem 3.3.1. Because, in this theorem we have $\left\|u^{\mathrm{nl}}(t, \cdot)\right\|_{L^{2}}$, however in Theorem 3.2.1 we have $\left\||D|^{\sigma} u^{\mathrm{nl}}(t, \cdot)\right\|_{L^{2}}$ with $\sigma \in(0,1)$. Namely, in the case low regular data we do not have the total gradient of the solution in $L^{2}$.

- The first component of the parameter $\tilde{p}_{3, m}(n)$ coincide with the parameter $p_{2, m}(n)$. However, when we deal with the estimates of the norm $\left\|u_{t}^{\mathrm{nl}}(t, \cdot)\right\|_{L^{2}}$, due to the competition between several estimates, which appears only in the energy solution, we find also the second component of the parameter $\tilde{p}_{3, m}(n)$ having the competition between the first one.

- Finally, we have also a difference between Theorems 3.2.1 and 3.3.1 with the condition (3.3.2). This condition comes into play in the treatment of $\left\|u^{\mathrm{nl}}(t, \cdot)\right\|$ and $\left\|u_{t}^{\mathrm{nl}}(t, \cdot)\right\|$, which do not appear in Theorem 3.2.1. 
Proof of Theorem 3.3.1. Let us introduce the space of solutions $X(t)$ by

$$
X(t)=\mathcal{C}\left([0, t], H^{1}\right) \cap \mathcal{C}^{1}\left([0, t], L^{2}\right)
$$

with the norm

$$
\begin{aligned}
\|u\|_{X(t)}=\sup _{0 \leq \tau \leq t}[ & \left(1+B_{\lambda}(0, \tau)\right)^{\frac{n}{2}\left(\frac{1}{m}-\frac{1}{2}\right)}\|u(\tau, \cdot)\|_{L^{2}} \\
& \left.+\left(1+B_{\lambda}(0, \tau)\right)^{\frac{n}{2}\left(\frac{1}{m}-\frac{1}{2}\right)+\frac{1}{2}}\||D| u(\tau, \cdot)\|_{L^{2}}+\Phi_{1, m}^{-1}(0, \tau)\left\|u_{t}(\tau, \cdot)\right\|_{L^{2}}\right],
\end{aligned}
$$

where $\Phi_{1, m}=\Phi_{1, m}(0, \tau)$ is defined in (3.1.13) with $s=0$ and $t=\tau$.

Let $N$ be the operator which is defined by (3.2.1). Therefore, for any $u \in X(t)$ we have

$$
N u(t, x)=K_{0}(t, 0, x) *_{(x)} u_{0}(x)+K_{1}(t, 0, x) *_{(x)} u_{1}(x)+\int_{0}^{t} K_{1}(t, s, x) *_{(x)}|u(s, x)|^{p} d s .
$$

Our goal is to prove the existence of a fixed point for the operator $N$. We know that $\left(X(t),\|\cdot\|_{X(t)}\right)$ is a Banach space. Then, in order to use Banach's fixed point theorem we shall prove for $u, v \in X(t)$ and for any $\left(u_{0}, u_{1}\right) \in \mathcal{D}_{m}^{1}$ the following two estimates uniformly with respect to $t \in[0, \infty)$ :

$$
\begin{aligned}
\|N u\|_{X(t)} & \lesssim\left\|\left(u_{0}, u_{1}\right)\right\|_{\mathcal{D}_{m}^{1}}+\|u\|_{X(t)}^{p}, \\
\|N u-N v\|_{X(t)} & \lesssim\|u-v\|_{X(t)}\left(\|u\|_{X(t)}^{p-1}+\|v\|_{X(t)}^{p-1}\right) .
\end{aligned}
$$

After these considerations we know that to show the global (in time) existence for small data solutions is equivalent to show the inequalities (3.3.3) and (3.3.4).

Let us begin with inequality (3.3.3). Basically, using the estimates for solutions to the linear Cauchy problem and taking account of the norm of the solution space $X(t)$ we obtain immediately

$$
\left\|u^{\operatorname{lin}}\right\|_{X(t)} \lesssim\left\|\left(u_{0}, u_{1}\right)\right\|_{\mathcal{D}_{m}^{1}} .
$$

To complete the proof of (3.3.3) we have to estimate $\left\|u^{\mathrm{nl}}\right\|_{X(t)}$. Then, we use

$$
\left\||D|^{j} \partial_{t}^{\ell} u^{\mathrm{nl}}(t, \cdot)\right\|_{L^{2}} \leq C \int_{0}^{t}\left\||D|^{j} \partial_{t}^{\ell}\left(K_{1}(t, s, x) *_{(x)}|u(s, x)|^{p}\right)\right\|_{L^{2}} d s
$$

for $j+\ell=0,1$. Hence, we can estimate the integral term over $[0, t]$ by splitting this interval into $[0, \delta t]$ and $[\delta t, t]$, where $\delta$ is fixed and $\delta \in(0,1)$. In particular, if $s \in[0, \delta t]$ we use Theorem 3.1.1 with $m \in[1,2)$ and if $s \in[\delta t, t]$ we use it for $m=2$ only. So, it follows

$$
\begin{aligned}
\left\||D|^{j} \partial_{t}^{\ell} u^{\mathrm{nl}}(t, \cdot)\right\|_{L^{2}} \leq & C \int_{0}^{\delta t} \Phi_{1, m}^{\ell}(s, t) \frac{\Lambda(s)}{\lambda(s)}\left(1+B_{\lambda}(s, t)\right)^{(\ell-1) \frac{n}{2}\left(\frac{1}{m}-\frac{1}{2}\right)-\frac{j}{2}}\left\||u(s, \cdot)|^{p}\right\|_{L^{m} \cap L^{2}} d s \\
& +C \int_{\delta t}^{t} \Phi_{1}^{\ell}(s, t) \frac{\Lambda(s)}{\lambda(s)}\left(1+B_{\lambda}(s, t)\right)^{-\frac{j}{2}}\left\||u(s, \cdot)|^{p}\right\|_{L^{2}} d s
\end{aligned}
$$

for $j+\ell=0,1$. Here in the case $\ell=0$ we define $\Phi_{1, m}^{0}(s, t) \equiv 1$ and $\Phi_{1}^{0}(s, t) \equiv 1$ and, in the case $\ell=1$ the functions $\Phi_{1, m}^{1}(s, t)=\Phi_{1, m}(s, t)$ and $\Phi_{1}^{1}(s, t)=\Phi_{1}(s, t)$ are defined in (3.1.13) and (3.1.14), respectively.

Using

$$
\left\||u(s, \cdot)|^{p}\right\|_{L^{m} \cap L^{2}} \lesssim\left\||u(s, \cdot)|^{p}\right\|_{L^{m}}+\left\||u(s, \cdot)|^{p}\right\|_{L^{2}}=\|u(s, \cdot)\|_{L^{m p}}^{p}+\|u(s, \cdot)\|_{L^{2 p}}^{p}
$$

and applying the Gagliardo-Nirenberg inequality we get

$$
\begin{aligned}
\|u(s, \cdot)\|_{L^{m p}}^{p} & \lesssim\||D| u(s, \cdot)\|_{L^{2}}^{p \theta(m p)}\|u(s, \cdot)\|_{L^{2}}^{p(1-\theta(m p))}, \\
\|u(s, \cdot)\|_{L^{2 p}}^{p} & \lesssim\|D \mid u(s, \cdot)\|_{L^{2}}^{p \theta(2 p)}\|u(s, \cdot)\|_{L^{2}}^{p(1-\theta(2 p))},
\end{aligned}
$$


where $\theta(m p)=n\left(\frac{1}{2}-\frac{1}{m p}\right) \in[0,1]$ and $\theta(2 p)=n\left(\frac{1}{2}-\frac{1}{2 p}\right)$. Therefore, $\theta(m p) \geq 0$ implies $p \geq \frac{2}{m}$ and $\theta(2 p) \leq 1$ implies $p \leq p_{\mathrm{GN}}(n)=\frac{n}{n-2}$ for $n \geq 3$. Now we are able to estimate $\left\||u(s, \cdot)|^{p}\right\|_{L^{m} \cap L^{2}}$ by using (3.3.7), (3.3.8) and the definition of the norm of the solution space $X(t)$. We have the estimates

$$
\begin{aligned}
\left\||u(s, \cdot)|^{p}\right\|_{L^{2}} & \lesssim\left(1+B_{\lambda}(0, s)\right)^{-\frac{n}{2}\left(\frac{1}{m}-\frac{1}{2}\right) p-\frac{1}{2} p \theta(2 p)}\|u\|_{X(s)}^{p} \\
& =\left(1+B_{\lambda}(0, s)\right)^{-\frac{n}{2 m} p+\frac{n}{4}}\|u\|_{X(s)}^{p}, \\
\left\||u(s, \cdot)|^{p}\right\|_{L^{m} \cap L^{2}} & \lesssim\left(1+B_{\lambda}(0, s)\right)^{-\frac{n}{2}\left(\frac{1}{m}-\frac{1}{2}\right) p-\frac{1}{2} p \theta(m p)}\|u\|_{X(s)}^{p} \\
& =\left(1+B_{\lambda}(0, s)\right)^{-\frac{n}{2 m} p+\frac{n}{2 m}}\|u\|_{X(s)}^{p},
\end{aligned}
$$

since it holds $\theta(m p)<\theta(2 p)$. Therefore, plugging (3.3.9) and (3.3.10) into (3.3.6) and using $\|u\|_{X(s)} \leq\|u\|_{X(t)}$ for $0 \leq s \leq t$ we find

$$
\begin{aligned}
\left\||D|^{j} \partial_{t}^{\ell} u^{\mathrm{nl}}(t, \cdot)\right\|_{L^{2}} & \\
\leq & C\|u\|_{X(t)}^{p} \underbrace{\int_{0}^{\delta t} \Phi_{1, m}^{\ell}(s, t) \frac{\Lambda(s)}{\lambda(s)}\left(1+B_{\lambda}(s, t)\right)^{(\ell-1) \frac{n}{2}\left(\frac{1}{m}-\frac{1}{2}\right)-\frac{j}{2}}\left(1+B_{\lambda}(0, s)\right)^{-\frac{n}{2 m} p+\frac{n}{2 m}} d s}_{\mathbf{D}} \\
& +C\|u\|_{X(t)}^{p} \underbrace{\int_{\delta t}^{t} \Phi_{1}^{\ell}(s, t) \frac{\Lambda(s)}{\lambda(s)}\left(1+B_{\lambda}(s, t)\right)^{-\frac{j}{2}}\left(1+B_{\lambda}(0, s)\right)^{-\frac{n}{2 m} p+\frac{n}{4}} d s}_{\mathbf{E}} .
\end{aligned}
$$

We begin to estimate the integral $\mathbf{D}$. In this case, introducing $\alpha_{1}(p):=\frac{n}{2 m} p-\frac{n}{2 m}$ and using (3.1.18), we have

$$
\mathbf{D} \lesssim \Phi_{1, m}^{\ell}(0, t)\left(1+B_{\lambda}(0, t)\right)^{(\ell-1) \frac{n}{2}\left(\frac{1}{m}-\frac{1}{2}\right)-\frac{j}{2}} \underbrace{\int_{0}^{\delta t} \frac{\Lambda(s)}{\lambda(s)}\left(1+B_{\lambda}(0, s)\right)^{-\alpha_{1}(p)} d s}_{D_{1}(t)}
$$

for $j+\ell=0,1$. We have already shown in the proof of Theorem 3.2.1 the condition $p>\tilde{p}_{1, m}(n)$ guarantees that the integral term $A_{1}=A_{1}(t)$ in (3.2.11), which coincides with $D_{1}=D_{1}(t)$, remains bounded. Thus, for all $p>\tilde{p}_{1, m}(n)$ and for $j+\ell=0,1$ we get the estimate

$$
\mathbf{D} \lesssim \Phi_{1, m}^{\ell}(0, t)\left(1+B_{\lambda}(0, t)\right)^{(\ell-1) \frac{n}{2}\left(\frac{1}{m}-\frac{1}{2}\right)-\frac{j}{2}} .
$$

Now let us consider the integral E. We have

$$
\begin{aligned}
\mathbf{E} & =\int_{\delta t}^{t} \Phi_{1}^{\ell}(s, t) \frac{\Lambda(s)}{\lambda(s)}\left(1+B_{\lambda}(s, t)\right)^{-\frac{j}{2}}\left(1+B_{\lambda}(0, s)\right)^{-\alpha_{2}(p)} d s \\
& =-\int_{\delta t}^{t} \Phi_{1}^{\ell}(s, t) \frac{\mu(s)}{\lambda^{2}(s)}\left(1+B_{\lambda}(0, s)\right)^{-\alpha_{2}(p)}\left(1+B_{\lambda}(s, t)\right)^{-\frac{j}{2}} d\left(1+B_{\lambda}(s, t)\right),
\end{aligned}
$$

where $\alpha_{2}(p):=\frac{n}{2 m} p-\frac{n}{4}$. To obtain the desired estimates we consider separately the cases $j+\ell=0,1$.

For $j=\ell=0$ and $\alpha_{2}(p) \neq 1$ we get

$$
\begin{aligned}
\mathbf{E} & =\int_{\delta t}^{t} \frac{\mu(s)}{\lambda^{2}(s)}\left(1+B_{\lambda}(0, s)\right)^{-\alpha_{2}(p)} d\left(1+B_{\lambda}(0, s)\right) \\
& =\left.\frac{1}{1-\alpha_{2}(p)} \frac{\mu(s)}{\lambda^{2}(s)}\left(1+B_{\lambda}(0, s)\right)^{-\alpha_{2}(p)+1}\right|_{\delta t} ^{t}-\frac{1}{1-\alpha_{2}(p)} \int_{\delta t}^{t}\left(1+B_{\lambda}(0, s)\right)^{-\alpha_{2}(p)+1} d\left(\frac{\mu(s)}{\lambda^{2}(s)}\right) .
\end{aligned}
$$


Case 1: $\alpha_{2}(p)>1$. In this case it holds

$$
\begin{aligned}
\mathbf{E} \leq & \left.\frac{1}{1-\alpha_{2}(p)} \frac{\mu(s)}{\lambda^{2}(s)}\left(1+B_{\lambda}(0, s)\right)^{-\alpha_{2}(p)+1}\right|_{\delta t} ^{t} \\
& +\frac{\beta_{1}}{1-\alpha_{2}(p)} \int_{\delta t}^{t}\left(1+B_{\lambda}(0, s)\right)^{-\alpha_{2}(p)}\left(1+B_{\lambda}(0, s)\right) \frac{\mu(s)}{\lambda(s) \Lambda(s)} d s \\
\leq & \left.\frac{1}{1-\alpha_{2}(p)} \frac{\mu(s)}{\lambda^{2}(s)}\left(1+B_{\lambda}(0, s)\right)^{-\alpha_{2}(p)+1}\right|_{\delta t} ^{t} \\
& +\frac{\beta_{1}}{\left(1-\alpha_{2}(p)\right)(2+R)} \int_{\delta t}^{t}\left(1+B_{\lambda}(0, s)\right)^{-\alpha_{2}(p)} \frac{\Lambda^{2}(s)}{\mu(s)} \frac{\mu(s)}{\lambda(s) \Lambda(s)} d s \\
\leq & \left.\frac{1}{1-\alpha_{2}(p)} \frac{\mu(s)}{\lambda^{2}(s)}\left(1+B_{\lambda}(0, s)\right)^{-\alpha_{2}(p)+1}\right|_{\delta t} ^{t} \\
& +\frac{\beta_{1}}{\left(1-\alpha_{2}(p)\right)(2+R)} \underbrace{\int_{\delta t}^{t} \frac{\Lambda(s)}{\lambda(s)}\left(1+B_{\lambda}(0, s)\right)^{-\alpha_{2}(p)} d s}_{\mathbf{E}},
\end{aligned}
$$

where we used condition (C4) and (3.1.17), respectively. Then, we get

$$
\left(1+\frac{\beta_{1}}{\left(\alpha_{2}(p)-1\right)(2+R)}\right) \mathbf{E} \lesssim \frac{\mu(\delta t)}{\lambda^{2}(\delta t)}\left(1+B_{\lambda}(0, \delta t)\right)^{-\alpha_{2}(p)+1}-\frac{\mu(t)}{\lambda^{2}(t)}\left(1+B_{\lambda}(0, t)\right)^{-\alpha_{2}(p)+1} .
$$

It follows

$$
\mathbf{E} \lesssim \frac{\mu(\delta t)}{\lambda^{2}(\delta t)}\left(1+B_{\lambda}(0, \delta t)\right)^{-\alpha_{2}(p)+1}-\frac{\mu(t)}{\lambda^{2}(t)}\left(1+B_{\lambda}(0, t)\right)^{-\alpha_{2}(p)+1} .
$$

Case 2: $\alpha_{2}(p)<1$. In this case we have the estimates

$$
\begin{aligned}
\mathbf{E} \geq & \left.\frac{1}{1-\alpha_{2}(p)} \frac{\mu(s)}{\lambda^{2}(s)}\left(1+B_{\lambda}(0, s)\right)^{-\alpha_{2}(p)+1}\right|_{\delta t} ^{t} \\
& +\frac{\beta_{1}}{1-\alpha_{2}(p)} \int_{\delta t}^{t}\left(1+B_{\lambda}(0, s)\right)^{-\alpha_{2}(p)}\left(1+B_{\lambda}(0, s)\right) \frac{\mu(s)}{\lambda(s) \Lambda(s)} d s \\
\geq & \left.\frac{1}{1-\alpha_{2}(p)} \frac{\mu(s)}{\lambda^{2}(s)}\left(1+B_{\lambda}(0, s)\right)^{-\alpha_{2}(p)+1}\right|_{\delta t} ^{t} \\
& +\frac{\beta_{1}}{\left(1-\alpha_{2}(p)\right)(2+R)} \int_{\delta t}^{t}\left(1+B_{\lambda}(0, s)\right)^{-\alpha_{2}(p)} \frac{\Lambda^{2}(s)}{\mu(s)} \frac{\mu(s)}{\lambda(s) \Lambda(s)} d s \\
\geq & \left.\frac{1}{1-\alpha_{2}(p)} \frac{\mu(s)}{\lambda^{2}(s)}\left(1+B_{\lambda}(0, s)\right)^{-\alpha_{2}(p)+1}\right|_{\delta t} ^{t} \\
& +\frac{\beta_{1}}{\left(1-\alpha_{2}(p)\right)(2+R)} \underbrace{\int_{\delta t}^{t} \frac{\Lambda(s)}{\lambda(s)}\left(1+B_{\lambda}(0, s)\right)^{-\alpha_{2}(p)} d s}_{\mathbf{E}} .
\end{aligned}
$$

This implies

$$
\left(\frac{\beta_{1}}{\left(1-\alpha_{2}(p)\right)(2+R)}-1\right) \mathbf{E} \lesssim \frac{\mu(\delta t)}{\lambda^{2}(\delta t)}\left(1+B_{\lambda}(0, \delta t)\right)^{-\alpha_{2}(p)+1}-\frac{\mu(t)}{\lambda^{2}(t)}\left(1+B_{\lambda}(0, t)\right)^{-\alpha_{2}(p)+1} .
$$

Here we can guarantee that

$$
\frac{\beta_{1}}{\left(1-\alpha_{2}(p)\right)(2+R)}-1>0, \quad \text { if } \quad p>\frac{m}{2}+\left(1-\frac{\beta_{1}}{2+R}\right) \frac{2 m}{n} .
$$

It is clear that the above condition for $p$ is satisfied by the condition $p>\tilde{p}_{1, m}(n)$. Then, for all $p>\tilde{p}_{1, m}(n)$ it holds

$$
\mathbf{E} \lesssim \frac{\mu(\delta t)}{\lambda^{2}(\delta t)}\left(1+B_{\lambda}(0, \delta t)\right)^{-\alpha_{2}(p)+1}-\frac{\mu(t)}{\lambda^{2}(t)}\left(1+B_{\lambda}(0, t)\right)^{-\alpha_{2}(p)+1} .
$$


Now in order to get our desired estimate for $\mathbf{E}$ in the case $j=\ell=0$ we will show that the following estimate holds:

$$
\begin{aligned}
(1 & \left.+B_{\lambda}(0, t)\right)^{\frac{n}{2}\left(\frac{1}{m}-\frac{1}{2}\right)} \mathbf{E} \\
& \lesssim \frac{\mu(\delta t)}{\lambda^{2}(\delta t)}\left(1+B_{\lambda}(0, t)\right)^{\frac{n}{2}\left(\frac{1}{m}-\frac{1}{2}\right)}\left(1+B_{\lambda}(0, \delta t)\right)^{-\alpha_{2}(p)+1}-\frac{\mu(t)}{\lambda^{2}(t)}\left(1+B_{\lambda}(0, t)\right)^{-\alpha_{1}(p)+1} \lesssim 1,
\end{aligned}
$$

where we used the fact that $\alpha_{2}(p)=\alpha_{1}(p)+\frac{n}{2}\left(\frac{1}{m}-\frac{1}{2}\right)$. The second summand on the right-hand side of the previous estimate, that is,

$$
\mathcal{E}_{1}(t):=\frac{\mu(t)}{\lambda^{2}(t)}\left(1+B_{\lambda}(0, t)\right)^{-\alpha_{1}(p)+1}
$$

is bounded due to the condition $p>\tilde{p}_{1, m}(n)$. Indeed, we have

$$
\begin{aligned}
\mathcal{E}_{1}^{\prime}(t) & =\left(\frac{\mu(t)}{\lambda^{2}(t)}\right)^{\prime}\left(1+B_{\lambda}(0, t)\right)^{-\alpha_{1}(p)+1}+\left(-\alpha_{1}(p)+1\right) \frac{\mu(t)}{\lambda^{2}(t)} \frac{\lambda^{2}(t)}{\rho(t)}\left(1+B_{\lambda}(0, t)\right)^{-\alpha_{1}(p)} \\
& \leq\left[-\beta_{1} \frac{\mu(t)}{\lambda(t) \Lambda(t)}\left(1+B_{\lambda}(0, t)\right)+\left(-\alpha_{1}(p)+1\right) \frac{\Lambda(t)}{\lambda(t)}\right]\left(1+B_{\lambda}(0, t)\right)^{-\alpha_{1}(p)} \\
& \leq\left[-\frac{\beta_{1}}{2+R}-\alpha_{1}(p)+1\right] \frac{\Lambda(t)}{\lambda(t)}\left(1+B_{\lambda}(0, t)\right)^{-\alpha_{1}(p)} .
\end{aligned}
$$

Then, we can get

$$
\frac{-\beta_{1}}{2+R}-\alpha_{1}(p)+1<0, \quad \text { if } \quad p>1+\left(1-\frac{\beta_{1}}{2+R}\right) \frac{2 m}{n} .
$$

Hence, we have $\mathcal{E}_{1}^{\prime}(t)<0$ by $p>\tilde{p}_{1, m}(n)$, namely, $\mathcal{E}_{1}=\mathcal{E}_{1}(t)$ is decreasing. Therefore, it remains to ensure the boundedness of the term

$$
\frac{\mu(\delta t)}{\lambda^{2}(\delta t)}\left(1+B_{\lambda}(0, t)\right)^{\frac{n}{2}\left(\frac{1}{m}-\frac{1}{2}\right)}\left(1+B_{\lambda}(0, \delta t)\right)^{-\alpha_{2}(p)+1}
$$

in both cases $1-\alpha_{2}(p)>0$ and $1-\alpha_{2}(p)<0$. In the case $1-\alpha_{2}(p)>0$ we have

$$
\begin{aligned}
& \frac{\mu(\delta t)}{\lambda^{2}(\delta t)}\left(1+B_{\lambda}(0, t)\right)^{\frac{n}{2}\left(\frac{1}{m}-\frac{1}{2}\right)}\left(1+B_{\lambda}(0, \delta t)\right)^{-\alpha_{2}(p)+1} \\
& \quad \lesssim \frac{\mu(\delta t)}{\lambda^{2}(\delta t)}\left(1+B_{\lambda}(0, t)\right)^{\frac{n}{2}\left(\frac{1}{m}-\frac{1}{2}\right)-\alpha_{2}(p)+1}=\frac{\mu(\delta t)}{\lambda^{2}(\delta t)}\left(1+B_{\lambda}(0, t)\right)^{-\alpha_{1}(p)+1}:=\mathcal{E}_{2}(t) .
\end{aligned}
$$

Now in order to guarantee that the function $\mathcal{E}_{2}=\mathcal{E}_{2}(t)$ is decreasing we compute its derivative as follows:

$$
\begin{aligned}
\mathcal{E}_{2}^{\prime}(t) & =\left(\frac{\mu(\delta t)}{\lambda^{2}(\delta t)}\right)^{\prime}\left(1+B_{\lambda}(0, t)\right)^{-\alpha_{1}(p)+1}+\left(-\alpha_{1}(p)+1\right) \frac{\mu(\delta t)}{\lambda^{2}(\delta t)} \frac{\lambda^{2}(t)}{\rho(t)}\left(1+B_{\lambda}(0, t)\right)^{-\alpha_{1}(p)} \\
& \leq\left[-\delta \beta_{1} \frac{\mu(\delta t)}{\lambda^{2}(\delta t)} \frac{\lambda(\delta t)}{\Lambda(\delta t)}\left(1+B_{\lambda}(0, t)+\left(-\alpha_{1}(p)+1\right) \frac{\mu(\delta t)}{\lambda^{2}(\delta t)} \frac{\lambda^{2}(t)}{\rho(t)}\right]\left(1+B_{\lambda}(0, t)\right)^{-\alpha_{1}(p)}\right. \\
& \leq\left[-\frac{\delta \beta_{1}}{2+R} \frac{\mu(\delta t)}{\lambda^{2}(\delta t)} \frac{\lambda(\delta t)}{\Lambda(\delta t)} \frac{\Lambda^{2}(t)}{\mu(t)}+\left(-\alpha_{1}(p)+1\right) \frac{\mu(\delta t)}{\lambda^{2}(\delta t)} \frac{\lambda(t)}{\Lambda(t)} \frac{\Lambda^{2}(t)}{\mu(t)}\right]\left(1+B_{\lambda}(0, t)\right)^{-\alpha_{1}(p)} \\
& \leq\left[-\frac{\delta \beta_{1}}{2+R}+\left(-\alpha_{1}(p)+1\right) \nu(\delta, t)\right] \frac{\mu(\delta t)}{\lambda^{2}(\delta t)} \frac{\lambda(\delta t)}{\Lambda(\delta t)} \frac{\Lambda^{2}(t)}{\mu(t)}\left(1+B_{\lambda}(0, t)\right)^{-\alpha_{1}(p)} .
\end{aligned}
$$

Then, for a large time $t$ we can guarantee that $\mathcal{E}_{2}^{\prime}(t)<0$ by

$$
\frac{-\delta \beta_{1}}{2+R}+\left(-\alpha_{1}(p)+1\right) \nu(\delta)<0
$$


and this can be concluded from $p>\tilde{p}_{2, m}(n)$. In the other case $1-\alpha_{2}(p)<0$, our desired estimate can be obtained directly from the case $1-\alpha_{2}(p)>0$. Therefore, for all $p>\tilde{p}_{1, m}(n)$ and $p>\tilde{p}_{2, m}(n)$ we arrive at the expected estimate

$$
\mathbf{E} \lesssim\left(1+B_{\lambda}(0, t)\right)^{-\frac{n}{2}\left(\frac{1}{m}-\frac{1}{2}\right)} .
$$

Case 3: $\alpha_{2}(p)=1$. In this case it holds

$$
\begin{aligned}
\mathbf{E} & =\int_{\delta t}^{t} \frac{\Lambda(s)}{\lambda(s)}\left(1+B_{\lambda}(0, s)\right)^{-1} d s \lesssim \int_{\delta t}^{t} \frac{\Lambda(s)}{\lambda(s)} \frac{\mu(s)}{\Lambda^{2}(s)} d s \\
& =\int_{\delta t}^{t} \frac{\mu(s)}{\lambda^{2}(s)} \frac{\lambda(s)}{\Lambda(s)} d s=\left.\frac{\mu(s)}{\lambda^{2}(s)} \log \Lambda(s)\right|_{\delta t} ^{t}-\int_{\delta t}^{t} \log \Lambda(s) d\left(\frac{\mu(s)}{\lambda^{2}(s)}\right) \\
& \lesssim \frac{\mu(t)}{\lambda^{2}(t)} \log \Lambda(t)-\frac{\mu(\delta t)}{\lambda^{2}(\delta t)} \log \Lambda(\delta t)+\int_{\delta t}^{t} \frac{\mu(s)}{\lambda^{2}(s)} \log \Lambda(s) \frac{\lambda(s)}{\Lambda(s)} d s
\end{aligned}
$$

where we have used (3.1.17) and condition (C4), respectively. Then, it follows

$$
\begin{aligned}
\left(1+B_{\lambda}(0, t)\right)^{\frac{n}{2}\left(\frac{1}{m}-\frac{1}{2}\right)} \mathbf{E} \lesssim & \frac{\mu(t)}{\lambda^{2}(t)}\left(1+B_{\lambda}(0, t)\right)^{\frac{n}{2}\left(\frac{1}{m}-\frac{1}{2}\right)} \log \Lambda(t) \\
& +\int_{\delta t}^{t} \frac{\mu(s)}{\lambda^{2}(s)}\left(1+B_{\lambda}(0, t)\right)^{\frac{n}{2}\left(\frac{1}{m}-\frac{1}{2}\right)} \log \Lambda(s) \frac{\lambda(s)}{\Lambda(s)} d s .
\end{aligned}
$$

In order to prove that $\left(1+B_{\lambda}(0, t)\right)^{\frac{n}{2}\left(\frac{1}{m}-\frac{1}{2}\right)} \mathbf{E}$ is bounded, we will show that for a sufficiently small positive constant $\varepsilon$ we have the estimate

$$
\frac{\mu(\delta t)}{\lambda^{2}(\delta t)}\left(1+B_{\lambda}(0, t)\right)^{\frac{n}{2}\left(\frac{1}{m}-\frac{1}{2}\right)} \log \Lambda(\delta t) \lesssim \Lambda^{-\varepsilon}(\delta t) .
$$

So, it suffices to form the derivative of the function

$$
\mathcal{E}_{3}(t):=\frac{\mu(\delta t)}{\lambda^{2}(\delta t)} \Lambda^{\varepsilon}(\delta t)\left(1+B_{\lambda}(0, t)\right)^{\frac{n}{2}\left(\frac{1}{m}-\frac{1}{2}\right)} \log \Lambda(\delta t) .
$$

Indeed, it holds

$$
\begin{aligned}
\mathcal{E}_{3}^{\prime}(t)= & \left(\frac{\mu(\delta t)}{\lambda^{2}(\delta t)}\right)^{\prime} \Lambda^{\varepsilon}(\delta t)\left(1+B_{\lambda}(0, t)\right)^{\frac{n}{2}\left(\frac{1}{m}-\frac{1}{2}\right)} \log \Lambda(\delta t) \\
& +\varepsilon \delta \frac{\mu(\delta t)}{\lambda^{2}(\delta t)} \lambda(\delta t) \Lambda^{\varepsilon-1}(\delta t)\left(1+B_{\lambda}(0, t)\right)^{\frac{n}{2}\left(\frac{1}{m}-\frac{1}{2}\right)} \log \Lambda(\delta t) \\
& +\frac{n}{2}\left(\frac{1}{m}-\frac{1}{2}\right) \frac{\mu(\delta t)}{\lambda^{2}(\delta t)} \Lambda^{\varepsilon}(\delta t) \frac{\lambda^{2}(t)}{\rho(t)}\left(1+B_{\lambda}(0, t)\right)^{\frac{n}{2}\left(\frac{1}{m}-\frac{1}{2}\right)-1} \log \Lambda(\delta t) \\
& +\delta \frac{\mu(\delta t)}{\lambda^{2}(\delta t)} \Lambda^{\varepsilon}(\delta t)\left(1+B_{\lambda}(0, t)\right)^{\frac{n}{2}\left(\frac{1}{m}-\frac{1}{2}\right)} \frac{\lambda(\delta t)}{\Lambda(\delta t)} \\
\leq & {\left[-\delta \beta_{1} \frac{\lambda(\delta t)}{\Lambda(\delta t)}+\varepsilon \delta \frac{\lambda(\delta t)}{\Lambda(\delta t)}+\frac{n}{2}\left(\frac{1}{m}-\frac{1}{2}\right) \frac{\lambda^{2}(t)}{\rho(t)} \frac{1}{1+B_{\lambda}(0, t)}+\delta \frac{\lambda(\delta t)}{\Lambda(\delta t)} \frac{1}{\log \Lambda(\delta t)}\right] } \\
& \times \frac{\mu(\delta t)}{\lambda^{2}(\delta t)} \Lambda^{\varepsilon}(\delta t)\left(1+B_{\lambda}(0, t)\right)^{\frac{n}{2}\left(\frac{1}{m}-\frac{1}{2}\right)} \log \Lambda(\delta t) \\
\leq & {\left[-\delta \beta_{1} \frac{\lambda(\delta t)}{\Lambda(\delta t)}+\varepsilon \delta \frac{\lambda(\delta t)}{\Lambda(\delta t)}+(2+R) \frac{n}{2}\left(\frac{1}{m}-\frac{1}{2}\right) \frac{\lambda(t)}{\Lambda(t)}+\delta \frac{\lambda(\delta t)}{\Lambda(\delta t)} \frac{1}{\log \Lambda(\delta t)}\right] } \\
& \times \frac{\mu(\delta t)}{\lambda^{2}(\delta t)} \Lambda^{\varepsilon}(\delta t)\left(1+B_{\lambda}(0, t)\right)^{\frac{n}{2}\left(\frac{1}{m}-\frac{1}{2}\right)} \log \Lambda(\delta t) \\
\leq & {\left[-\beta_{1}+\varepsilon+(2+R) \frac{n}{2}\left(\frac{1}{m}-\frac{1}{2}\right) \frac{\nu(\delta, t)}{\delta}+\frac{1}{\log \Lambda(\delta t)}\right] } \\
& \times \delta \frac{\mu(\delta t)}{\lambda^{2}(\delta t)} \frac{\lambda(\delta t)}{\Lambda(\delta t)} \Lambda^{\varepsilon}(\delta t)\left(1+B_{\lambda}(0, t)\right)^{\frac{n}{2}\left(\frac{1}{m}-\frac{1}{2}\right)} \log \Lambda(\delta t) .
\end{aligned}
$$


Then, for sufficiently large time $t$ and sufficiently small constant $\varepsilon>0$, if

$$
(2+R) \frac{n}{2}\left(\frac{1}{m}-\frac{1}{2}\right) \frac{\nu(\delta)}{\delta}-\beta_{1}<0,
$$

which is related to condition (3.3.2), we get $\mathcal{E}_{3}^{\prime}(t)<0$. This shows the decreasing behavior of $\mathcal{E}_{3}=\mathcal{E}_{3}(t)$. Hence, we obtain

$$
\int_{\delta t}^{t} \frac{\mu(s)}{\lambda^{2}(s)}\left(1+B_{\lambda}(0, t)\right)^{\frac{n}{2}\left(\frac{1}{m}-\frac{1}{2}\right)} \log \Lambda(s) \frac{\lambda(s)}{\Lambda(s)} d s \lesssim \int_{\delta t}^{t} \Lambda^{-\varepsilon-1}(s) \lambda(s) d s \lesssim 1 .
$$

In the same way, by condition (3.3.2) we can also derive

$$
\frac{\mu(t)}{\lambda^{2}(t)}\left(1+B_{\lambda}(0, t)\right)^{\frac{n}{2}\left(\frac{1}{m}-\frac{1}{2}\right)} \log \Lambda(t) \lesssim \frac{\mu(\delta t)}{\lambda^{2}(\delta t)}\left(1+B_{\lambda}(0, t)\right)^{\frac{n}{2}\left(\frac{1}{m}-\frac{1}{2}\right)} \log \Lambda(t) \lesssim \Lambda^{-\varepsilon}(\delta t) .
$$

Consequently, in the case $j=\ell=0$ we obtain the desired estimate

$$
\mathbf{E} \lesssim\left(1+B_{\lambda}(0, t)\right)^{-\frac{n}{2}\left(\frac{1}{m}-\frac{1}{2}\right)} .
$$

Now let us consider the case $j=1$ and $\ell=0$ for the integral $\mathbf{E}$. In this case we have

$$
\begin{aligned}
\mathbf{E}= & -\int_{\delta t}^{t} \frac{\mu(s)}{\lambda^{2}(s)}\left(1+B_{\lambda}(0, s)\right)^{-\alpha_{2}(p)}\left(1+B_{\lambda}(s, t)\right)^{-\frac{1}{2}} d\left(1+B_{\lambda}(s, t)\right) \\
= & -\left.2 \frac{\mu(s)}{\lambda^{2}(s)}\left(1+B_{\lambda}(0, s)\right)^{-\alpha_{2}(p)}\left(1+B_{\lambda}(s, t)\right)^{\frac{1}{2}}\right|_{\delta t} ^{t} \\
& +2 \int_{\delta t}^{t}\left(1+B_{\lambda}(s, t)\right)^{\frac{1}{2}} d\left(\frac{\mu(s)}{\lambda^{2}(s)}\left(1+B_{\lambda}(0, s)\right)^{-\alpha_{2}(p)}\right) \\
\lesssim & -\left.\frac{\mu(s)}{\lambda^{2}(s)}\left(1+B_{\lambda}(0, s)\right)^{-\alpha_{2}(p)}\left(1+B_{\lambda}(s, t)\right)^{\frac{1}{2}}\right|_{\delta t} ^{t} \\
& +\int_{\delta t}^{t} \frac{\mu(s)}{\lambda^{2}(s)}\left(1+B_{\lambda}(0, s)\right)^{-\alpha_{2}(p)}\left(1+B_{\lambda}(s, t)\right)^{\frac{1}{2}} \frac{d\left(1+B_{\lambda}(0, s)\right)}{1+B_{\lambda}(0, s)},
\end{aligned}
$$

where we used Lemma 3.1.5. Then, we get

$$
\begin{aligned}
(1+ & \left.B_{\lambda}(0, t)\right)^{\frac{n}{2}\left(\frac{1}{m}-\frac{1}{2}\right)+\frac{1}{2}} \mathbf{E} \\
\lesssim & \frac{\mu(\delta t)}{\lambda^{2}(\delta t)}\left(1+B_{\lambda}(0, \delta t)\right)^{-\alpha_{2}(p)}\left(1+B_{\lambda}(\delta t, t)\right)^{\frac{1}{2}}\left(1+B_{\lambda}(0, t)\right)^{\frac{n}{2}\left(\frac{1}{m}-\frac{1}{2}\right)+\frac{1}{2}} \\
& +\left(1+B_{\lambda}(0, t)\right)^{\frac{n}{2}\left(\frac{1}{m}-\frac{1}{2}\right)+\frac{1}{2}} \int_{\delta t}^{t} \frac{\mu(s)}{\lambda^{2}(s)}\left(1+B_{\lambda}(0, s)\right)^{-\alpha_{2}(p)}\left(1+B_{\lambda}(s, t)\right)^{\frac{1}{2}} \frac{d\left(1+B_{\lambda}(0, s)\right)}{1+B_{\lambda}(0, s)} .
\end{aligned}
$$

Hence, it follows

$$
\begin{aligned}
\left(1+B_{\lambda}(0, t)\right)^{\frac{n}{2}\left(\frac{1}{m}-\frac{1}{2}\right)+\frac{1}{2}} \mathbf{E} & \lesssim \underbrace{\frac{\mu(\delta t)}{\lambda^{2}(\delta t)}\left(1+B_{\lambda}(0, \delta t)\right)^{-\alpha_{2}(p)}\left(1+B_{\lambda}(0, t)\right)^{\frac{n}{2}\left(\frac{1}{m}-\frac{1}{2}\right)+1}}_{E_{1}(t)} \\
& +\underbrace{\int_{\delta t}^{t} \frac{\mu(s)}{\lambda^{2}(s)}\left(1+B_{\lambda}(0, s)\right)^{-\alpha_{2}(p)}\left(1+B_{\lambda}(0, t)\right)^{\frac{n}{2}\left(\frac{1}{m}-\frac{1}{2}\right)+1} \frac{d\left(1+B_{\lambda}(0, s)\right)}{1+B_{\lambda}(0, s)}}_{E_{2}(t)}
\end{aligned}
$$

In order to prove that $E_{1}=E_{1}(t)$ and $E_{2}=E_{2}(t)$ are uniformly bounded it is sufficient to show that for small positive constant $\varepsilon$ we have

$$
\frac{\mu(\delta t)}{\lambda^{2}(\delta t)}\left(1+B_{\lambda}(0, \delta t)\right)^{-\alpha_{2}(p)}\left(1+B_{\lambda}(0, t)\right)^{\frac{n}{2}\left(\frac{1}{m}-\frac{1}{2}\right)+1} \lesssim\left(1+B_{\lambda}(0, \delta t)\right)^{-\varepsilon} .
$$


Then, employing (3.1.19) we get

$$
\begin{aligned}
& \frac{\mu(\delta t)}{\lambda^{2}(\delta t)}\left(1+B_{\lambda}(0, \delta t)\right)^{-\alpha_{2}(p)+\varepsilon}\left(1+B_{\lambda}(0, t)\right)^{\frac{n}{2}\left(\frac{1}{m}-\frac{1}{2}\right)+1} \\
& \quad \leq \frac{\mu(\delta t)}{\lambda^{2}(\delta t)}\left(1+B_{\lambda}(0, t)\right)^{\frac{n}{2}\left(\frac{1}{m}-\frac{1}{2}\right)+1-\alpha_{2}(p)+\varepsilon}\left(\frac{\Lambda(\delta t)}{\Lambda(t)}\right)^{(2+R)\left(-\alpha_{2}(p)+\varepsilon\right)}:=\mathcal{E}_{4}(t) .
\end{aligned}
$$

We may conclude immediately that the function $\mathcal{E}_{4}=\mathcal{E}_{4}(t)$ is bounded by the aid of the condition $p>\tilde{p}_{3, m}(n)$, because this function coincides with the function $\mathcal{B}_{1}=\mathcal{B}_{1}(t)$ in (3.2.15). Consequently, for the case $j=1$ and $\ell=0$ we obtain the expected estimate

$$
\mathbf{E} \lesssim\left(1+B_{\lambda}(0, t)\right)^{-\frac{n}{2}\left(\frac{1}{m}-\frac{1}{2}\right)-\frac{1}{2}} .
$$
have

Finally, it remains to consider the case $j=0$ and $\ell=1$ for the integral $\mathbf{E}$. In this case we

$$
\mathbf{E}=\int_{\delta t}^{t} \Phi_{1}(s, t) \frac{\Lambda(s)}{\lambda(s)}\left(1+B_{\lambda}(0, s)\right)^{-\frac{n}{2 m} p+\frac{n}{4}} d s,
$$

where $\Phi_{1}=\Phi_{1}(s, t)$ is defined in (3.1.14). Due to the competition between the estimates in $\Phi_{1}=\Phi_{1}(s, t)$, from condition (C1) we are interested in the following two cases only for all $s \in[\delta t, t]:$

Case $a: \Phi_{1}(s, t)=\frac{\lambda^{2}(t)}{\rho(t)}\left(1+B_{\lambda}(s, t)\right)^{-1}$. Then, it follows

$$
\begin{aligned}
\mathbf{E}= & \frac{\lambda^{2}(t)}{\rho(t)} \int_{\delta t}^{t} \frac{\Lambda(s)}{\lambda(s)}\left(1+B_{\lambda}(s, t)\right)^{-1}\left(1+B_{\lambda}(0, s)\right)^{-\alpha_{2}(p)} d s \\
=- & \frac{\lambda^{2}(t)}{\rho(t)} \int_{\delta t}^{t} \frac{\mu(s)}{\lambda^{2}(s)}\left(1+B_{\lambda}(0, s)\right)^{-\alpha_{2}(p)} \frac{d\left(1+B_{\lambda}(s, t)\right)}{1+B_{\lambda}(s, t)} \\
=- & \left.\frac{\lambda^{2}(t)}{\rho(t)} \frac{\mu(s)}{\lambda^{2}(s)}\left(1+B_{\lambda}(0, s)\right)^{-\alpha_{2}(p)} \log \left(1+B_{\lambda}(s, t)\right)\right|_{\delta t} ^{t} \\
& +\frac{\lambda^{2}(t)}{\rho(t)} \int_{\delta t}^{t} \log \left(1+B_{\lambda}(s, t)\right) d\left(\frac{\mu(s)}{\lambda^{2}(s)}\left(1+B_{\lambda}(0, s)\right)^{-\alpha_{2}(p)}\right) \\
\lesssim- & \left.\frac{\lambda^{2}(t)}{\rho(t)} \frac{\mu(s)}{\lambda^{2}(s)}\left(1+B_{\lambda}(0, s)\right)^{-\alpha_{2}(p)} \log \left(1+B_{\lambda}(s, t)\right)\right|_{\delta t} ^{t} \\
& +\frac{\lambda^{2}(t)}{\rho(t)} \int_{\delta t}^{t} \frac{\mu(s)}{\lambda^{2}(s)}\left(1+B_{\lambda}(0, s)\right)^{-\alpha_{2}(p)} \log \left(1+B_{\lambda}(s, t)\right) \frac{d\left(1+B_{\lambda}(0, s)\right)}{1+B_{\lambda}(0, s)} .
\end{aligned}
$$

Thus, we have

$$
\begin{aligned}
& \left(1+B_{\lambda}(0, t)\right)^{\frac{n}{2}\left(\frac{1}{m}-\frac{1}{2}\right)+1} \frac{\rho(t)}{\lambda^{2}(t)} \mathbf{E} \\
& \lesssim \underbrace{\frac{\mu(\delta t)}{\lambda^{2}(\delta t)}\left(1+B_{\lambda}(0, \delta t)\right)^{-\alpha_{2}(p)}\left(1+B_{\lambda}(0, t)\right)^{\frac{n}{2}\left(\frac{1}{m}-\frac{1}{2}\right)+1} \log \left(1+B_{\lambda}(0, t)\right)}_{E_{3}(t)} \\
& +\underbrace{\int_{\delta t}^{t} \frac{\mu(s)}{\lambda^{2}(s)}\left(1+B_{\lambda}(0, s)\right)^{-\alpha_{2}(p)}\left(1+B_{\lambda}(0, t)\right)^{\frac{n}{2}\left(\frac{1}{m}-\frac{1}{2}\right)+1} \log \left(1+B_{\lambda}(0, t)\right) \frac{d\left(1+B_{\lambda}(0, s)\right)}{1+B_{\lambda}(0, s)}}_{E_{4}(t)} .
\end{aligned}
$$

In order to prove that $E_{3}=E_{3}(t)$ and $E_{4}=E_{4}(t)$ are bounded we will show that for a small positive constant $\varepsilon$ we have

$$
\frac{\mu(\delta t)}{\lambda^{2}(\delta t)}\left(1+B_{\lambda}(0, \delta t)\right)^{-\alpha_{2}(p)}\left(1+B_{\lambda}(0, t)\right)^{\frac{n}{2}\left(\frac{1}{m}-\frac{1}{2}\right)+1} \log \left(1+B_{\lambda}(0, t)\right) \lesssim\left(1+B_{\lambda}(0, \delta t)\right)^{-\varepsilon} .
$$


This implies to deal with

$$
\frac{\mu(\delta t)}{\lambda^{2}(\delta t)}\left(1+B_{\lambda}(0, \delta t)\right)^{-\alpha_{2}(p)+\varepsilon}\left(1+B_{\lambda}(0, t)\right)^{\frac{n}{2}\left(\frac{1}{m}-\frac{1}{2}\right)+1} \log \left(1+B_{\lambda}(0, t)\right) .
$$

Hence, for the boundedness of $E_{3}=E_{3}(t)$ we have

$$
E_{3}(t) \lesssim \frac{\log \left(1+B_{\lambda}(0, t)\right)}{\left(1+B_{\lambda}(0, \delta t)\right)^{\varepsilon}} \mathcal{E}_{4}(t) \lesssim \frac{\log \left(1+B_{\lambda}(0, t)\right)}{\left(1+B_{\lambda}(0, \delta t)\right)^{\varepsilon}} \lesssim 1
$$

for large $t$. Here we used the boundedness of $\mathcal{E}_{4}=\mathcal{E}_{4}(t)$ which is given in (3.3.16) and l'Hospital's rule (cf. condition (B4)) for

$$
\lim _{t \rightarrow \infty} \sup \frac{\log \left(1+B_{\lambda}(0, t)\right)}{\left(1+B_{\lambda}(0, \delta t)\right)^{\varepsilon}} \lesssim \lim _{t \rightarrow \infty} \sup \frac{\nu(\delta, t)}{\left(1+B_{\lambda}(0, \delta t)\right)^{\varepsilon}} \lesssim 1,
$$

respectively. On the other hand, for $E_{4}=E_{4}(t)$ we have

$$
\begin{aligned}
E_{4} & \lesssim \log \left(1+B_{\lambda}(0, t)\right) \int_{\delta t}^{t}\left(1+B_{\lambda}(0, s)\right)^{-\varepsilon-1} d\left(1+B_{\lambda}(0, s)\right) \\
& =\frac{\log \left(1+B_{\lambda}(0, t)\right)}{\varepsilon\left(1+B_{\lambda}(0, \delta t)\right)^{\varepsilon}}-\frac{\log \left(1+B_{\lambda}(0, t)\right)}{\varepsilon\left(1+B_{\lambda}(0, t)\right)^{\varepsilon}} \lesssim 1
\end{aligned}
$$

where we used (3.3.18). Therefore, in the Case a we obtain the desired estimate

$$
\mathbf{E} \lesssim \frac{\lambda^{2}(t)}{\rho(t)}\left(1+B_{\lambda}(0, t)\right)^{-\frac{n}{2}\left(\frac{1}{m}-\frac{1}{2}\right)-1} .
$$

Summarizing, in the Case a from (3.3.14), (3.3.15), (3.3.17) and (3.3.19) for $j+\ell=0,1$ we arrive at

$$
\mathbf{E} \lesssim \frac{\lambda^{2 \ell}(t)}{\rho^{\ell}(t)}\left(1+B_{\lambda}(0, t)\right)^{-\frac{n}{2}\left(\frac{1}{m}-\frac{1}{2}\right)-\frac{j}{2}-\ell} .
$$

Case $b: \Phi_{1}(s, t)=\frac{\lambda(t)}{F(\Lambda(t))}$. In this case we have

$$
\mathbf{E}=\frac{\lambda(t)}{F(\Lambda(t))} \int_{\delta t}^{t} \frac{\Lambda(s)}{\lambda(s)}\left(1+B_{\lambda}(0, s)\right)^{-\alpha_{2}(p)} d s .
$$

Case b.1: $\alpha_{2}(p)>1$. In a similar way as we did in the Case 1 we have

$$
\frac{F(\Lambda(t))}{\lambda(t)} \mathbf{E} \lesssim \frac{\mu(\delta t)}{\lambda^{2}(\delta t)}\left(1+B_{\lambda}(0, \delta t)\right)^{-\alpha_{2}(p)+1}-\frac{\mu(t)}{\lambda^{2}(t)}\left(1+B_{\lambda}(0, t)\right)^{-\alpha_{2}(p)+1} .
$$

Case b.2: $\alpha_{2}(p)<1$. Similarly as in the Case 2 for $p>\tilde{p}_{1, m}(n)$ we conclude

$$
\frac{F(\Lambda(t))}{\lambda(t)} \mathbf{E} \lesssim \frac{\mu(\delta t)}{\lambda^{2}(\delta t)}\left(1+B_{\lambda}(0, \delta t)\right)^{-\alpha_{2}(p)+1}-\frac{\mu(t)}{\lambda^{2}(t)}\left(1+B_{\lambda}(0, t)\right)^{-\alpha_{2}(p)+1} .
$$

Then, it follows

$$
\begin{aligned}
& \frac{F(\Lambda(t))}{\lambda(t)}\left(F^{2}(\Lambda(t))\right)^{\frac{n}{2}\left(\frac{1}{m}-\frac{1}{2}\right)} \mathbf{E} \\
& \quad \lesssim \frac{\mu(\delta t)}{\lambda^{2}(\delta t)}\left(F^{2}(\Lambda(t))\right)^{\frac{n}{2}\left(\frac{1}{m}-\frac{1}{2}\right)}\left(1+B_{\lambda}(0, \delta t)\right)^{-\alpha_{2}(p)+1}-\frac{\mu(t)}{\lambda^{2}(t)}\left(1+B_{\lambda}(0, t)\right)^{\frac{n}{2}\left(\frac{1}{m}-\frac{1}{2}\right)-\alpha_{2}(p)+1}
\end{aligned}
$$


where we used condition (B6), that is, $B_{\lambda}(0, t) \lesssim F^{2}(\Lambda(t))$. We have shown in (3.3.13) by using the condition $p>\tilde{p}_{1, m}(n)$ that the second summand on the right-hand side of the previous inequality, that is,

$$
\frac{\mu(t)}{\lambda^{2}(t)}\left(1+B_{\lambda}(0, t)\right)^{\frac{n}{2}\left(\frac{1}{m}-\frac{1}{2}\right)-\alpha_{2}(p)+1}
$$

is bounded. For this reason let us consider the first term only, that is,

$$
\frac{\mu(\delta t)}{\lambda^{2}(\delta t)}\left(F^{2}(\Lambda(t))\right)^{\frac{n}{2}\left(\frac{1}{m}-\frac{1}{2}\right)}\left(1+B_{\lambda}(0, \delta t)\right)^{-\alpha_{2}(p)+1}
$$

in both cases $\alpha_{2}(p)<1$ and $\alpha_{2}(p)>1$. For the case $\alpha_{2}(p)<1$ we have

$$
\begin{aligned}
& \frac{\mu(\delta t)}{\lambda^{2}(\delta t)}\left(F^{2}(\Lambda(t))\right)^{\frac{n}{2}\left(\frac{1}{m}-\frac{1}{2}\right)}\left(1+B_{\lambda}(0, \delta t)\right)^{-\alpha_{2}(p)+1} \\
& \quad \lesssim \frac{\mu(\delta t)}{\lambda^{2}(\delta t)}\left(F^{2}(\Lambda(t))\right)^{\frac{n}{2}\left(\frac{1}{m}-\frac{1}{2}\right)-\alpha_{2}(p)+1}=\frac{\mu(\delta t)}{\lambda^{2}(\delta t)}\left(F^{2}(\Lambda(t))\right)^{-\alpha_{1}(p)+1}:=\mathcal{E}_{5}(t)
\end{aligned}
$$

where again we used condition (B6) and $\alpha_{2}(p)=\alpha_{1}(p)+\frac{n}{2}\left(\frac{1}{m}-\frac{1}{2}\right)$. Now if we form the derivative of the function $\mathcal{E}_{5}=\mathcal{E}_{5}(t)$ we get

$$
\begin{aligned}
\mathcal{E}_{5}^{\prime}(t) & =\left(\frac{\mu(\delta t)}{\lambda^{2}(\delta t)}\right)^{\prime} F(\Lambda(t))^{-2 \alpha_{1}(p)+2}+2\left(-\alpha_{1}(p)+1\right) \frac{\mu(\delta t)}{\lambda^{2}(\delta t)} \lambda(t) F^{\prime}(\Lambda(t)) F(\Lambda(t))^{-2 \alpha_{1}(p)+1} \\
& \leq\left[-\delta \beta_{1} \frac{\mu(\delta t)}{\lambda^{2}(\delta t)} \frac{\lambda(\delta t)}{\Lambda(\delta t)}+2\left(-\alpha_{1}(p)+1\right) \frac{\mu(\delta t)}{\lambda^{2}(\delta t)} \frac{\lambda(t)}{F(\Lambda(t))} F^{\prime}(\Lambda(t))\right] F(\Lambda(t))^{-2 \alpha_{1}(p)+2} \\
& \leq\left[-\delta \beta_{1}+2\left(-\alpha_{1}(p)+1\right) \frac{\lambda(t)}{F(\Lambda(t))} \frac{F^{2}(\Lambda(t))}{\lambda^{2}(t) \Xi^{2}(t)} \frac{\Lambda(\delta t)}{\lambda(\delta t)} \frac{\mu(\delta t)}{\lambda^{2}(\delta t)} \frac{\lambda(\delta t)}{\Lambda(\delta t)} F(\Lambda(t))^{-2 \alpha_{1}(p)+2}\right. \\
& \leq\left[-\delta \beta_{1}+2\left(-\alpha_{1}(p)+1\right) \frac{F(\Lambda(t))}{\lambda(t) \Xi^{2}(t)} \frac{\Lambda(\delta t)}{\lambda(\delta t)}\right] \frac{\mu(\delta t)}{\lambda^{2}(\delta t)} \frac{\lambda(\delta t)}{\Lambda(\delta t)} F(\Lambda(t))^{-2 \alpha_{1}(p)+2} \\
& \leq\left[-\delta \beta_{1}+2\left(-\alpha_{1}(p)+1\right) \vartheta(\delta, t)\right] \frac{\mu(\delta t)}{\lambda^{2}(\delta t)} \frac{\lambda(\delta t)}{\Lambda(\delta t)} F(\Lambda(t))^{-2 \alpha_{1}(p)+2},
\end{aligned}
$$

where from condition (A5) we used $F^{\prime}(\Lambda(t))=\frac{F^{2}(\Lambda(t))}{\lambda^{2}(t) \Xi^{2}(t)}$. Then, for a large time $t$ by using $p>\tilde{p}_{3, m}(n)$ we obtain

$$
-\delta \beta_{1}+2\left(-\alpha_{1}(p)+1\right) \vartheta(\delta)<0,
$$

which implies $\mathcal{E}_{5}^{\prime}(t)<0$. Similarly, in the case $\alpha_{2}(p)>1$ the desired estimate can be derived as in the case $\alpha_{2}(p)<1$. For this reason, we obtain the expected estimate

$$
\mathbf{E} \lesssim \lambda(t) F(\Lambda(t))\left(F^{2}(\Lambda(t))\right)^{-\frac{n}{2}\left(\frac{1}{m}-\frac{1}{2}\right)-1} .
$$

Case b.3: $\alpha_{2}(p)=1$. In the same way as in the Case 3 we obtain

$$
\frac{F(\Lambda(t))}{\lambda(t)} \mathbf{E} \lesssim \frac{\mu(t)}{\lambda^{2}(t)} \log \Lambda(t)-\frac{\mu(\delta t)}{\lambda^{2}(\delta t)} \log \Lambda(\delta t)+\int_{\delta t}^{t} \frac{\mu(s)}{\lambda^{2}(s)} \log \Lambda(s) \frac{\lambda(s)}{\Lambda(s)} d s .
$$

Then, we get

$$
\begin{aligned}
\frac{F(\Lambda(t))}{\lambda(t)}\left(F^{2}(\Lambda(t))\right)^{\frac{n}{2}\left(\frac{1}{m}-\frac{1}{2}\right)} \mathbf{E} \lesssim & \frac{\mu(t)}{\lambda^{2}(t)}\left(F^{2}(\Lambda(t))\right)^{\frac{n}{2}\left(\frac{1}{m}-\frac{1}{2}\right)} \log \Lambda(t) \\
& +\int_{\delta t}^{t} \frac{\mu(s)}{\lambda^{2}(s)}\left(F^{2}(\Lambda(t))\right)^{\frac{n}{2}\left(\frac{1}{m}-\frac{1}{2}\right)} \log \Lambda(s) \frac{\lambda(s)}{\Lambda(s)} d s .
\end{aligned}
$$


In order to show the desired estimate we assume that there exists a sufficiently small positive constant $\varepsilon$ such that we have the estimate

$$
\frac{\mu(\delta t)}{\lambda^{2}(\delta t)}\left(F^{2}(\Lambda(t))\right)^{\frac{n}{2}\left(\frac{1}{m}-\frac{1}{2}\right)} \log \Lambda(\delta t) \lesssim \Lambda^{-\varepsilon}(\delta t)
$$

Thus, it is sufficient to consider the derivative of the function

$$
\mathcal{E}_{6}(t):=\frac{\mu(\delta t)}{\lambda^{2}(\delta t)} \Lambda^{\varepsilon}(\delta t)\left(F^{2}(\Lambda(t))\right)^{\frac{n}{2}\left(\frac{1}{m}-\frac{1}{2}\right)} \log \Lambda(\delta t) .
$$

It follows

$$
\begin{aligned}
\mathcal{E}_{6}^{\prime}(t)= & \left(\frac{\mu(\delta t)}{\lambda^{2}(\delta t)}\right)^{\prime} \Lambda^{\varepsilon}(\delta t) F(\Lambda(t))^{n\left(\frac{1}{m}-\frac{1}{2}\right)} \log \Lambda(\delta t) \\
& +\delta \varepsilon \frac{\mu(\delta t)}{\lambda^{2}(\delta t)} \lambda(\delta t) \Lambda^{\varepsilon-1}(\delta t) F(\Lambda(t))^{n\left(\frac{1}{m}-\frac{1}{2}\right)} \log \Lambda(\delta t) \\
& +n\left(\frac{1}{m}-\frac{1}{2}\right) \frac{\mu(\delta t)}{\lambda^{2}(\delta t)} \Lambda^{\varepsilon}(\delta t) \lambda(t) F^{\prime}(\Lambda(t)) F(\Lambda(t))^{n\left(\frac{1}{m}-\frac{1}{2}\right)-1} \log \Lambda(\delta t) \\
& +\delta \frac{\mu(\delta t)}{\lambda^{2}(\delta t)} \Lambda^{\varepsilon}(\delta t) F(\Lambda(t))^{n\left(\frac{1}{m}-\frac{1}{2}\right)} \frac{\lambda(\delta t)}{\Lambda(\delta t)} \\
\leq & {\left[-\beta_{1} \frac{\lambda(\delta t)}{\Lambda(\delta t)}+\varepsilon \frac{\lambda(\delta t)}{\Lambda(\delta t)}+n\left(\frac{1}{m}-\frac{1}{2}\right) \frac{1}{\delta} \frac{\lambda(t)}{F(\Lambda(t))} F^{\prime}(\Lambda(t))+\frac{\lambda(\delta t)}{\Lambda(\delta t)} \frac{1}{\log \Lambda(\delta t)}\right] } \\
& \times \delta \frac{\mu(\delta t)}{\lambda^{2}(\delta t)} \Lambda^{\varepsilon}(\delta t)(F(\Lambda(t)))^{n\left(\frac{1}{m}-\frac{1}{2}\right)} \log \Lambda(\delta t) \\
\leq & \left.-\beta_{1}+\varepsilon+n\left(\frac{1}{m}-\frac{1}{2}\right) \frac{1}{\delta} \frac{\lambda(t)}{F(\Lambda(t))} \frac{F^{2}(\Lambda(t))}{\lambda^{2}(t) \Xi^{2}(t)} \frac{\Lambda(\delta t)}{\lambda(\delta t)}+\frac{1}{\log \Lambda(\delta t)}\right] \\
& \times \frac{\mu(\delta t)}{\lambda^{2}(\delta t)} \frac{\lambda(\delta t)}{\Lambda(\delta t)} \Lambda^{\varepsilon}(\delta t) F(\Lambda(t))^{n\left(\frac{1}{m}-\frac{1}{2}\right)} \log \Lambda(\delta t) \\
\leq & {\left[-\beta_{1}+\varepsilon+n\left(\frac{1}{m}-\frac{1}{2}\right) \frac{\vartheta(\delta, t)}{\delta}+\frac{1}{\log \Lambda(\delta t)}\right] } \\
& \times \delta \frac{\mu(\delta t)}{\lambda^{2}(\delta t)} \frac{\lambda(\delta t)}{\Lambda(\delta t)} \Lambda^{\varepsilon}(\delta t) F(\Lambda(t))^{n\left(\frac{1}{m}-\frac{1}{2}\right)} \log \Lambda(\delta t) .
\end{aligned}
$$

Here from condition (A5) we used $F^{\prime}(\Lambda(t))=\frac{F^{2}(\Lambda(t))}{\lambda^{2}(t) \Xi^{2}(t)}$. Hence, for a small constant $\varepsilon>0$ and for large time $t$ after taking account of

$$
-\beta_{1}+\varepsilon+n\left(\frac{1}{m}-\frac{1}{2}\right) \frac{\vartheta(\delta)}{\delta}<0
$$

which is related to condition (3.3.2), we have $\mathcal{E}_{6}^{\prime}(t)<0$. Analogously, using the decreasing behavior of the function $\frac{\mu(t)}{\lambda^{2}(t)}$ one can also prove by (3.3.2) the estimate

$$
\frac{\mu(t)}{\lambda^{2}(t)}\left(F^{2}(\Lambda(t))\right)^{\frac{n}{2}\left(\frac{1}{m}-\frac{1}{2}\right)} \log \Lambda(t) \lesssim \frac{\mu(\delta t)}{\lambda^{2}(\delta t)}\left(F^{2}(\Lambda(t))\right)^{\frac{n}{2}\left(\frac{1}{m}-\frac{1}{2}\right)} \log \Lambda(t) \lesssim \Lambda^{-\varepsilon}(\delta t) .
$$

Thus, we get

$$
\mathbf{E} \lesssim \lambda(t) F(\Lambda(t))\left(F^{2}(\Lambda(t))\right)^{-\frac{n}{2}\left(\frac{1}{m}-\frac{1}{2}\right)-1} .
$$

Summarizing, from (3.3.20), (3.3.22) and (3.3.24) for $j=0$ and $\ell=1$ we get

$$
\mathbf{E} \lesssim \max \left\{\frac{\lambda^{2}(t)}{\rho(t)}\left(1+B_{\lambda}(0, t)\right)^{-\frac{n}{2}\left(\frac{1}{m}-\frac{1}{2}\right)-1} ; \lambda(t) F(\Lambda(t))\left(F^{2}(\Lambda(t))\right)^{-\frac{n}{2}\left(\frac{1}{m}-\frac{1}{2}\right)-1}\right\} .
$$


Consequently, from (3.3.12), (3.3.20) and (3.3.25) we arrive at the statement

$$
\left\||D|^{j} \partial_{t}^{\ell} u^{\mathrm{nl}}(t, \cdot)\right\|_{L^{2}} \lesssim\|u\|_{X(t)}^{p} \Phi_{1, m}^{\ell}(0, t)\left(1+B_{\lambda}(0, t)\right)^{(\ell-1) \frac{n}{2}\left(\frac{1}{m}-\frac{1}{2}\right)-\frac{j}{2}},
$$

where $\Phi_{1, m}^{1}(0, t)=\Phi_{1, m}(0, t)$ is defined in (3.1.13) for $s=0$ and $\Phi_{1, m}^{0}(0, t)=1$. Replacing the estimates for $j+\ell=0,1$ in the norm of the solution space $X(t)$ we obtain

$$
\left\|u^{\mathrm{nl}}\right\|_{X(t)} \lesssim\|u\|_{X(t)}^{p}
$$

Then, after the estimates (3.3.5) and (3.3.26) imply (3.3.3).

Now let us prove (3.3.4). We have

$$
\|N u-N v\|_{X(t)}=\left\|\int_{0}^{t} K_{1}(t, s, x) *_{(x)}\left(|u(s, x)|^{p}-|v(s, x)|^{p}\right) d s\right\|_{X(t)} .
$$

Thanks to the estimates for the solutions to the family of linear parameter dependent Cauchy problems with vanishing right-hand side we can estimate

$$
\begin{aligned}
& \left\||D|^{j} \partial_{t}^{\ell} K_{1}(t, s, x) *_{(x)}\left(|u(s, x)|^{p}-|v(s, x)|^{p}\right)\right\|_{L^{2}} \\
& \quad \lesssim \begin{cases}\Phi_{1, m}^{\ell}(s, t) \frac{\Lambda(s)}{\lambda(s)}\left(1+B_{\lambda}(s, t)\right)^{(\ell-1) \frac{n}{2}\left(\frac{1}{m}-\frac{1}{2}\right)-\frac{j}{2}}\left\||u(s, x)|^{p}-|v(s, x)|^{p}\right\|_{L^{m} \cap L^{2}}, & s \in[0, \delta t], \\
\Phi_{1}^{\ell}(s, t) \frac{\Lambda(s)}{\lambda(s)}\left(1+B_{\lambda}(s, t)\right)^{-\frac{j}{2}}\left\||u(s, x)|^{p}-|v(s, x)|^{p}\right\|_{L^{2}}, & s \in[\delta t, t] .\end{cases}
\end{aligned}
$$

We use the fact that

$$
\left.|| u(s, x)\right|^{p}-|v(s, x)|^{p}|\lesssim| u(s, x)-v(s, x) \mid\left(|u(s, x)|^{p-1}+|v(s, x)|^{p-1}\right) .
$$

By Hölder's inequality we obtain

$$
\begin{aligned}
\left\||u(s, \cdot)|^{p}-|v(s, \cdot)|^{p}\right\|_{L^{m}} & \lesssim\|u(s, \cdot)-v(s, \cdot)\|_{L^{m p}}\left(\|u(s, \cdot)\|_{L^{m p}}^{p-1}+\|v(s, \cdot)\|_{L^{m p}}^{p-1}\right), \\
\left\||u(s, \cdot)|^{p}-|v(s, \cdot)|^{p}\right\|_{L^{2}} & \lesssim\|u(s, \cdot)-v(s, \cdot)\|_{L^{2 p}}\left(\|u(s, \cdot)\|_{L^{2 p}}^{p-1}+\|v(s, \cdot)\|_{L^{2 p}}^{p-1}\right) .
\end{aligned}
$$

In a similar way to the proof of (3.3.3) we use again Gagliardo-Nirenberg inequality to the following terms:

$$
\|u(s, \cdot)-v(s, \cdot)\|_{L^{q}}, \quad\|u(s, \cdot)\|_{L^{q}}, \quad\|v(s, \cdot)\|_{L^{q}}
$$

with $q=m p$ and $q=2 p$. Summarizing, we arrive at the following inequality:

$$
\begin{aligned}
\left\||D|^{j} \partial_{t}^{\ell}(N u-N v)\right\|_{L^{2}} \lesssim\|u-v\|_{X(t)}\left(\|u\|_{X(t)}^{p-1}+\|v\|_{X(t)}^{p-1}\right) \\
\quad \times\left(\int_{0}^{\delta t} \Phi_{1, m}^{\ell}(s, t) \frac{\Lambda(s)}{\lambda(s)}\left(1+B_{\lambda}(s, t)\right)^{(\ell-1) \frac{n}{2}\left(\frac{1}{m}-\frac{1}{2}\right)-\frac{j}{2}}\left(1+B_{\lambda}(0, s)\right)^{-\frac{n}{2 m} p+\frac{n}{2 m}} d s\right. \\
\left.\quad+\int_{\delta t}^{t} \Phi_{1}^{\ell}(s, t) \frac{\Lambda(s)}{\lambda(s)}\left(1+B_{\lambda}(s, t)\right)^{-\frac{j}{2}}\left(1+B_{\lambda}(0, s)\right)^{-\frac{n}{2 m} p+\frac{n}{4}} d s\right) .
\end{aligned}
$$

Thus, we can repeat the same arguments to the proof of (3.3.3) in order to estimate these two integrals employing the conditions $p>\tilde{p}_{m}(n)$ and (3.3.2). Finally, we may conclude the proof of the statement (3.3.4) from the definition of the norm of $X(t)$. In this way the proof of the theorem is completed.

Remark 3.3.2. Let us choose formally $\omega(t) \equiv 1$. Then, we have $\Lambda(t)=\Theta(t)=F(\Lambda(t))$ and $\nu(\delta)=\vartheta(\delta)$. Let $\mu=\mu(t)$ be an arbitrary function satisfying the assumptions (B1) to (B6), (C2) and (C4). Hence, choosing $\beta_{1}=2 \lambda_{0}$ and applying Theorem 3.3.1 with $m=1$ we can see that the definition of parameters $\tilde{p}_{1, m}(n)$ to $\tilde{p}_{3, m}(n)$ are related to the results of the paper [3]. 
Remark 3.3.3. From the parameters $\tilde{p}_{1, m}(n), \tilde{p}_{2, m}(n)$ and $\tilde{p}_{3, m}(n)$ of the last theorem we can see that if the condition

$$
\lim _{\delta \rightarrow 1} \nu(\delta)=1
$$

holds, then we have

$$
\tilde{p}_{1, m}(n)=\tilde{p}_{2, m}(n)=1+\left(1-\frac{\beta_{1}}{2+R}\right) \frac{2 m}{n}
$$

and

$$
\tilde{p}_{3, m}(n)=\max \left\{1+\left(1-\frac{\beta_{1}}{2+R}\right) \frac{2 m}{n} ; 1+\left(1-\frac{1}{\vartheta(\delta)}\right) \frac{2 m}{n}\right\} .
$$

Moreover, the condition (3.3.2) holds

$$
\frac{\beta_{1}}{\frac{n}{2}\left(\frac{1}{m}-\frac{1}{2}\right)}>\max \{2+R ; 2 \vartheta(\delta)\}
$$

On the other hand, if

$$
\lim _{\delta \rightarrow 1} \nu(\delta)=\lim _{\delta \rightarrow 1} \vartheta(\delta)=1
$$

then we get

$$
\tilde{p}_{1, m}(n)=\tilde{p}_{2, m}(n)=\tilde{p}_{3, m}(n)=1+\left(1-\frac{\beta_{1}}{2+R}\right) \frac{2 m}{n},
$$

where the condition (3.3.2) yields

$$
\beta_{1}>(2+R) \frac{n}{2}\left(\frac{1}{m}-\frac{1}{2}\right) .
$$

Remark 3.3.4. Let us point out that the phenomenon of loss of decay appears in order to discuss some typical examples in the case of sub-exponential propagation speed. We will propose some decay rates for solutions or some of their derivatives to the semilinear models which are worse than those given for the solutions to the corresponding linear models with vanishing right-hand side to treat the semilinear model (3.1.1), that is, we allow a loss of decay. This strategy comes into play to get some advantages when dealing with the competition between the estimates in (3.1.13) and (3.1.14). Here let us remark that if we allow a loss of decay in Theorem 3.3.1, this idea does not influence the conditions of this theorem.

Example 3.3.1 (Polynomial case). Let $\lambda(t)=(\alpha+1)(1+t)^{\alpha}, \alpha>0$. Then, we obtain

$$
\Lambda(t)=(1+t)^{\alpha+1} \text { and } \Theta(t)=(1+t)^{\gamma+1}, \quad-1<\gamma<\alpha .
$$

If we choose

$$
\mu(t)=(1+t)^{\beta}, \quad \alpha-\gamma<\beta<2 \alpha+2,
$$

then, we get

$$
\rho(t)=(\alpha+1)(1+t)^{\beta-1},
$$

where due to condition (C2) we define $\beta:=\mu_{1}(\alpha+1)$ with $\mu_{1} \in[0,2)$. Moreover, taking

$$
\Xi(t)=(1+t)^{\kappa}, \quad 1>\kappa \geq \frac{4-\beta}{4}
$$

we find

$$
F(\Lambda(t)) \simeq(1+t)^{\alpha+2 \kappa-1} .
$$

Finally, due to condition (C4), $\beta_{1}$ satisfies

$$
0<\beta_{1} \leq \frac{2 \alpha-\beta}{\alpha+1}, \quad \beta<2 \alpha .
$$


Taking account of the construction of the oscillating functions as in Section 2.6, the function $\omega=\omega(t)$ is defined in the following way:

$$
\omega(t)=1+\varphi_{k} \psi\left(\frac{t-t_{k}}{v_{k}}\right) \text { for all } t \in\left[t_{k}, t_{k+1}\right]
$$

where

$$
t_{k}:=2^{k}, \quad v_{k}:=2^{\kappa k} \leq \Delta t_{k}:=t_{k+1}-t_{k}=2^{k}, \quad \varphi_{k}:=2^{k(\gamma-\alpha-\kappa)} \leq 1 .
$$

Moreover, $\psi \in \mathcal{C}_{0}^{M}(\mathbb{R})$ and it holds

$$
\operatorname{supp} \psi \subseteq[0,1], \quad-1<\psi(t)<1 \quad \text { and } \quad \int_{0}^{1}|\psi(t)| d t=\frac{1}{2} .
$$

Then, the oscillating function $\omega=\omega(t)$ satisfies the related estimates in condition (A2). Moreover, the stabilization condition (A3) is satisfied, since $v_{k} \varphi_{k}$ is small enough. Indeed, we have $v_{k} \varphi_{k}=2^{k(\gamma-\alpha)}<1$.

Now let us consider

$$
\tilde{\Phi}_{1}(s, t)=\max \left\{\frac{\lambda^{2}(t)}{\rho(t)}\left(1+B_{\lambda}(s, t)\right)^{-1+\gamma_{1}} ; \lambda(t) F(\Lambda(t))\left(F^{2}(\Lambda(t))\right)^{-1+\gamma_{2}}\right\},
$$

which appears in the proof of Theorem 3.3.1 for $j=0$ and $\ell=1$. Here $\gamma_{1}$ and $\gamma_{2}$ are positive constants and these constants stand for the loss of decay in comparison with the corresponding decay estimates for the time derivative of the solution $u$ to the linear Cauchy problem with vanishing right-hand side. In this case, we get a benefit of allowing a loss of decay.

As we did in the proof, from condition (C1) we will only consider the following two cases of the function $\tilde{\Phi}_{1}=\tilde{\Phi}_{1}(s, t)$ for $s \in[\delta t, t]$ :

Case a: $\tilde{\Phi}_{1}(s, t)=\frac{\lambda^{2}(t)}{\rho(t)}\left(1+B_{\lambda}(s, t)\right)^{-1+\gamma_{1}} \quad$ for $\quad s \in[\delta t, t]$

This case implies that we have

$$
\frac{\lambda(t)}{\rho(t)}\left(1+B_{\lambda}(s, t)\right)^{-1+\gamma_{1}} \geq F(\Lambda(t))\left(F^{2}(\Lambda(t))\right)^{-1+\gamma_{2}} .
$$

Then, it follows

$$
\left(1+(1+t)^{2 \alpha-\beta+2}-(1+s)^{2 \alpha-\beta+2}\right)^{-1+\gamma_{1}} \geq(1+t)^{\beta-2 \kappa-2 \alpha+2 \gamma_{2}(\alpha+2 \kappa-1)} .
$$

Therefore, if we assume $\beta-2 \kappa-2 \alpha+2 \gamma_{2}(\alpha+2 \kappa-1) \leq 0$ and $\gamma_{1} \geq 1$, then for $s \in[\delta t, t]$ the first component in (3.3.28) becomes dominant.

Here let us remark that if we do not allow a loss of decay, namely, if we choose $\gamma_{1}=\gamma_{2}=0$, then we have

$$
(1+s)^{2 \alpha-\beta+2} \geq 1+(1+t)^{2 \alpha-\beta+2}-(1+t)^{2 \alpha+2 \kappa-\beta} .
$$

In order to show that we have one case which implies that the first component is dominant in (3.3.28), we have to assume $2 \alpha+2 \kappa-\beta \geq 2 \alpha-\beta+2$ in the the last inequality, namely, $\kappa \geq 1$ which contradicts the choices of $\kappa$. For this reason, we assume a loss of decay in $L^{2}-L^{2}$ estimates for the time derivative of the solution $u$ to the linear Cauchy problem with vanishing right-hand side to get the desired estimates.

Case b: $\tilde{\Phi}_{1}(s, t)=\lambda(t) F(\Lambda(t))\left(F^{2}(\Lambda(t))\right)^{-1+\gamma_{2}} \quad$ for $s \in[\delta t, t]$

Then, we have

$$
\frac{\lambda(t)}{\rho(t)}\left(1+B_{\lambda}(s, t)\right)^{-1+\gamma_{1}} \leq F(\Lambda(t))\left(F^{2}(\Lambda(t))\right)^{-1+\gamma_{2}} .
$$


Assuming $\gamma_{2} \geq \gamma_{1}$ for $s \in[\delta t, t]$ it follows

$$
\left(1+(1+t)^{2 \alpha-\beta+2}-(1+s)^{2 \alpha-\beta+2}\right)^{-1+\gamma_{1}} \leq(1+t)^{\beta-2 \kappa-2 \alpha+2 \gamma_{2}(\alpha+2 \kappa-1)} .
$$

Then, this shows that the second component is dominant in (3.3.28).

Applying Theorem 3.3.1 we also get $\nu(\delta)=\vartheta(\delta)=\delta$ and $R=0$ (we can choose $R=0$, since $\mu(t)$ is increasing). Moreover, taking into consideration the condition (3.3.2) for $\alpha_{2}(p)=$ $\frac{n}{2 m} p-\frac{n}{4}=1$ we have

$$
\beta_{1}>n\left(\frac{1}{m}-\frac{1}{2}\right) .
$$

This implies that we have

$$
n\left(\frac{1}{m}-\frac{1}{2}\right)<\beta_{1} \leq \frac{2 \alpha-\beta}{\alpha+1}, \quad \beta<2 \alpha .
$$

Therefore, the "critical" exponent is

$$
\tilde{p}_{1, m}(n)=\tilde{p}_{2, m}(n)=\tilde{p}_{3, m}(n)=1+\left(\frac{\beta-\alpha+1}{\alpha+1}\right) \frac{2 m}{n}=\tilde{p}_{m}(n) .
$$

Consequently, we have the global (in time) existence of small data solutions for

$$
p>1+\left(\frac{\beta-\alpha+1}{\alpha+1}\right) \frac{2 m}{n} \quad \text { and } \quad \begin{cases}\frac{2}{m} \leq p & \text { if } \quad n=1,2 \\ \frac{2}{m} \leq p \leq p_{\mathrm{GN}}(n)=\frac{n}{n-2} & \text { if } \quad n \geq 3\end{cases}
$$

We remark that by (3.3.27) we can see that we have $\frac{\beta-\alpha+1}{\alpha+1}<1$. For this reason, we have $\tilde{p}_{m}(n)<p_{\text {Fuj }, m}(n)$.

Example 3.3.2 (Exponential case). Let us choose $\lambda(t)=e^{t}$. Then, we obtain

$$
\Lambda(t)=e^{t} \quad \text { and } \quad \Theta(t)=e^{r t}, \quad 0<r<1 .
$$

Moreover, we take

$$
\mu(t)=\rho(t)=e^{\mu_{1} t}, \quad 1-r<\mu_{1}<2 .
$$

Now taking

$$
\Xi(t)=e^{\kappa t}, \quad 0>\kappa \geq-\frac{\mu_{1}}{4}
$$

we get

$$
F(\Lambda(t)) \simeq e^{(1+2 \kappa) t}
$$

The condition (C4) implies that

$$
0<\beta_{1} \leq 2-\mu_{1} .
$$

Now let us define the oscillating function, as we explained in Section, 2.6 as follows:

$$
\omega(t)=1+\varphi_{k} \psi\left(\frac{t-t_{k}}{v_{k}}\right) \text { for all } t \in\left[t_{k}, t_{k+1}\right],
$$

where

$$
t_{k}:=k, \quad v_{k}:=e^{\kappa k} \leq \Delta t_{k}:=t_{k+1}-t_{k}, \quad \varphi_{k}:=e^{k(r-\kappa-1)} \leq 1 .
$$

Moreover, $\psi \in \mathcal{C}_{0}^{M}(\mathbb{R})$ satisfies

$$
\operatorname{supp} \psi \subseteq[0,1], \quad-1<\psi(t)<1 \quad \text { and } \quad \int_{0}^{1}|\psi(t)| d t=\frac{1}{2} .
$$


Then, the oscillating function $\omega=\omega(t)$ fulfills the related estimates in condition (A2). Moreover, the stabilization condition (A3) is satisfied, since $v_{k} \varphi_{k}$ is small enough. Indeed, we have $v_{k} \varphi_{k}=e^{k(r-1)}<1$.

Now let us consider the case

$$
\tilde{\Phi}_{1}(s, t)=\max \left\{\frac{\lambda^{2}(t)}{\rho(t)}\left(1+B_{\lambda}(s, t)\right)^{-1+\gamma_{1}} ; \lambda(t) F(\Lambda(t))\left(F^{2}(\Lambda(t))\right)^{-1+\gamma_{2}}\right\} .
$$

Here $\gamma_{1}$ and $\gamma_{2}$ are positive constants which describe the loss of decay in comparison with the corresponding decay estimates for the time derivative of the solution $u$ to the linear Cauchy problem with vanishing right-hand side.

As we did in the proof of Theorem 3.3.1 from condition (C1) we will distinguish between two cases only.

Case a: $\tilde{\Phi}_{1}(s, t)=\frac{\lambda^{2}(t)}{\rho(t)}\left(1+B_{\lambda}(s, t)\right)^{-1+\gamma_{1}} \quad$ for $\quad s \in[\delta t, t]$

This case implies that we have

$$
\frac{\lambda(t)}{\rho(t)}\left(1+B_{\lambda}(s, t)\right)^{-1+\gamma_{1}} \geq F(\Lambda(t))\left(F^{2}(\Lambda(t))\right)^{-1+\gamma_{2}} .
$$

If we assume $\mu_{1}-2 \kappa-2+2 \gamma_{2}(1+2 \kappa) \leq 0$ and $\gamma_{1} \geq 1$, then for $s \in[\delta t, t]$ it holds

$$
\left(1+e^{\left(2-\mu_{1}\right) t}-e^{\left(2-\mu_{1}\right) s}\right)^{-1+\gamma_{1}} \geq e^{\left(\mu_{1}-2 \kappa-2+2 \gamma_{2}(1+2 \kappa)\right) t} .
$$

Therefore, this shows that the first component is always dominant in (3.3.31), because we have a benefit of allowing a loss of decay.

Case b: $\tilde{\Phi}_{1}(s, t)=\lambda(t) F(\Lambda(t))\left(F^{2}(\Lambda(t))\right)^{-1+\gamma_{2}} \quad$ for $\quad s \in[\delta t, t]$

This case implies

$$
\frac{\lambda(t)}{\rho(t)}\left(1+B_{\lambda}(s, t)\right)^{-1+\gamma_{1}} \leq F(\Lambda(t))\left(F^{2}(\Lambda(t))\right)^{-1+\gamma_{2}} .
$$

Assuming $\gamma_{2} \geq \gamma_{1}$ for $s \in[\delta t, t]$ it holds

$$
\left(1+e^{\left(2-\mu_{1}\right) t}-e^{\left(2-\mu_{1}\right) s}\right)^{-1+\gamma_{1}} \leq e^{\left(\mu_{1}-2 \kappa-2+2 \gamma_{2}(1+2 \kappa)\right) t} .
$$

Then, this shows that the second component is dominant in (3.3.31).

Now applying Theorem 3.3.1 we also find $\nu(\delta)=\vartheta(\delta)=1$ and $R=0$ (here we can choose $R=0$, since $\mu(t)$ is increasing). Moreover, the condition (3.3.2) for $\alpha_{2}(p)=\frac{n}{2 m} p-\frac{n}{4}=1$ is satisfied with

$$
\frac{\beta_{1}}{n\left(\frac{1}{m}-\frac{1}{2}\right)}>\frac{1}{\delta}
$$

So, we have

$$
\frac{n}{\delta}\left(\frac{1}{m}-\frac{1}{2}\right)<\beta_{1} \leq 2-\mu_{1}
$$

Therefore, we get

$$
\begin{aligned}
& \tilde{p}_{1, m}(n)=1+\left(1-\frac{\beta_{1}}{2}\right) \frac{2 m}{n}, \\
& \tilde{p}_{2, m}(n)=1+\left(1-\delta \frac{\beta_{1}}{2}\right) \frac{2 m}{n}, \\
& \bar{p}_{3, m}(n)=\frac{m}{2}+\frac{1-\frac{m}{2}}{1-\frac{2}{2-\mu_{1}}(1-\delta)}+\left(\frac{1-\delta \frac{\beta_{1}}{2}}{1-\frac{2}{2-\mu_{1}}(1-\delta)}\right) \frac{2 m}{n} .
\end{aligned}
$$


Choosing $\delta$ close to 1 gives

$$
\tilde{p}_{m}(n)=1+\left(1-\frac{\beta_{1}}{2}\right) \frac{2 m}{n}+\varepsilon
$$

with a positive arbitrary small $\varepsilon$. So, we have the global (in time) existence of small data solutions for

$$
p>1+\left(1-\frac{\beta_{1}}{2}\right) \frac{2 m}{n}+\varepsilon \quad \text { and } \quad \begin{cases}\frac{2}{m} \leq p & \text { if } n=1,2, \\ \frac{2}{m} \leq p \leq p_{\mathrm{GN}}(n)=\frac{n}{n-2} & \text { if } n \geq 3 .\end{cases}
$$

\subsubsection{Case of super-exponential propagation speed}

Now we will deal with the case of super-exponential propagation speed where the data belong to energy space $H^{1} \times L^{2}$ with additional $L^{m}$ regularity, $m \in[1,2)$.

Theorem 3.3.2. We assume that the Hypotheses (A1) to (A5), (B1) to (B6), (C1), (C3) and (C4) hold. Let $\nu(\delta)=\infty$ and $\vartheta(\delta)=\infty$, and we suppose that the following condition for the functions $\nu=\nu(\delta, t)$ and $\vartheta=\vartheta(\delta, t)$ (see (3.1.2) and (3.1.3)) holds:

$$
\max \{\nu(\delta, t) ; \vartheta(\delta, t)\}=o(\log \Lambda(t)) .
$$

We consider $\left(u_{0}, u_{1}\right) \in \mathcal{D}_{m}^{1}$ with $m \in[1,2), n \leq \frac{4}{2-m}$ and that the exponent $p$ satisfies

$$
\begin{cases}\frac{2}{m} \leq p<\infty & \text { if } n=1,2, \\ \frac{2}{m} \leq p \leq p_{G N}(n)=\frac{n}{n-2} & \text { if } n \geq 3 .\end{cases}
$$

Then, there exists a constant $\varepsilon_{0}>0$ such that for all $\left(u_{0}, u_{1}\right) \in \mathcal{D}_{m}^{1}$ with $\left\|\left(u_{0}, u_{1}\right)\right\|_{\mathcal{D}_{m}^{1}} \leq \varepsilon_{0}$, there is a uniquely determined globally (in time) energy solution to the Cauchy problem (3.1.1) in

$$
\mathcal{C}\left([0, \infty), H^{1}\right) \cap \mathcal{C}^{1}\left([0, \infty), L^{2}\right) .
$$

Furthermore, there exists a constant $C>0$ such that the solution satisfies the following estimates:

$$
\begin{aligned}
&\|u(t, \cdot)\|_{L^{2}} \leq C\left(1+B_{\lambda}(0, t)\right)^{-\frac{n}{2}\left(\frac{1}{m}-\frac{1}{2}\right)}\left\|\left(u_{0}, u_{1}\right)\right\|_{\mathcal{D}_{m}^{1}}, \\
&\||D| u(t, \cdot)\|_{L^{2}} \leq C\left(1+B_{\lambda}(0, t)\right)^{-\frac{n}{2}\left(\frac{1}{m}-\frac{1}{2}\right)-\frac{1}{2}}\left\|\left(u_{0}, u_{1}\right)\right\|_{\mathcal{D}_{m}^{1}}, \\
&\left\|u_{t}(t, \cdot)\right\|_{L^{2}} \leq C \max \left\{\frac{\lambda^{2}(t)}{\rho(t)}\left(1+B_{\lambda}(0, t)\right)^{-\frac{n}{2}\left(\frac{1}{m}-\frac{1}{2}\right)-1} ;\right. \\
&\left.\lambda(t) F(\Lambda(t))\left(F^{2}(\Lambda(t))\right)^{-\frac{n}{2}\left(\frac{1}{m}-\frac{1}{2}\right)-1}\right\}\left\|\left(u_{0}, u_{1}\right)\right\|_{\mathcal{D}_{m}^{1}} .
\end{aligned}
$$

Proof. We proceed in the same way as we did in the proof of Theorem 3.3.1 to verify that the integrals $\mathbf{D}$ and $\mathbf{E}$, which are given in (3.3.11), fulfill the desired estimates. For this reason our goal is only to prove the following inequalities:

$$
\begin{aligned}
\|N u\|_{X(t)} & \lesssim\left\|\left(u_{0}, u_{1}\right)\right\|_{\mathcal{D}_{m}^{1}}+\|u\|_{X(t)}^{p}, \\
\|N u-N v\|_{X(t)} & \lesssim\|u-v\|_{X(t)}\left(\|u\|_{X(t)}^{p-1}+\|v\|_{X(t)}^{p-1}\right) .
\end{aligned}
$$

Let us start with inequality (3.3.34). To complete the proof of (3.3.34) we have to estimate $\left\|u^{\mathrm{nl}}(t, \cdot)\right\|_{X(t)}$. For this reason, we begin with the integral $\mathbf{D}$ for $j+\ell=0,1$, that is,

$$
\mathbf{D} \lesssim \Phi_{1, m}^{\ell}(0, t)\left(1+B_{\lambda}(0, t)\right)^{(\ell-1) \frac{n}{2}\left(\frac{1}{m}-\frac{1}{2}\right)-\frac{j}{2}} \underbrace{\int_{0}^{\delta t} \frac{\Lambda(s)}{\lambda(s)}\left(1+B_{\lambda}(0, s)\right)^{-\frac{n}{2 m} p+\frac{n}{2 m}} d s}_{\bar{D}_{1}(t)},
$$


where we used (3.1.21). Here $\Phi_{1, m}^{0}(0, t) \equiv 1$ and $\Phi_{1, m}^{1}(0, t)=\Phi_{1, m}(0, t)$ is defined in (3.1.13) for $s=0$. Here the integral $\bar{D}_{1}=\bar{D}_{1}(t)$ coincides with the integral $\bar{A}_{1}=\bar{A}_{1}(t)$ in (3.2.26), which has been already shown that it is uniformly bounded in the proof of Theorem 3.2.2. So, $\bar{D}_{1}=\bar{D}_{1}(t)$ is uniformly bounded, too. Therefore, we may conclude the following desired estimate for $j+\ell=0,1$ :

$$
\mathbf{D} \lesssim \Phi_{1, m}^{\ell}(0, t)\left(1+B_{\lambda}(0, t)\right)^{(\ell-1) \frac{n}{2}\left(\frac{1}{m}-\frac{1}{2}\right)-\frac{j}{2}} .
$$

Now let us consider the integral $\mathbf{E}$, that is,

$$
\begin{aligned}
\mathbf{E} & =\int_{\delta t}^{t} \Phi_{1}^{\ell}(s, t) \frac{\Lambda(s)}{\lambda(s)}\left(1+B_{\lambda}(s, t)\right)^{-\frac{j}{2}}\left(1+B_{\lambda}(0, s)\right)^{-\frac{n}{2 m} p+\frac{n}{4}} d s \\
& =-\int_{\delta t}^{t} \Phi_{1}^{\ell}(s, t) \frac{\mu(s)}{\lambda^{2}(s)}\left(1+B_{\lambda}(0, s)\right)^{-\alpha_{2}(p)}\left(1+B_{\lambda}(s, t)\right)^{-\frac{j}{2}} d\left(1+B_{\lambda}(s, t)\right),
\end{aligned}
$$

where $\alpha_{2}(p):=\frac{n}{2 m} p-\frac{n}{4}$. We define for $\ell=0$ the function $\Phi_{1}^{0}(s, t) \equiv 1$ and for $\ell=1$ the function $\Phi_{1}^{1}(s, t) \stackrel{2 m}{=} \Phi_{1}(s, t)$ is defined in (3.1.14).

For $j=\ell=0$ we will discuss the cases $\alpha_{2}(p) \neq 1$ and $\alpha_{2}(p)=1$, respectively.

Case 1: $\alpha_{2}(p) \neq 1$. In this case, by using condition $(\mathbf{C} 4)$ we have

$$
\begin{aligned}
\mathbf{E} & =\int_{\delta t}^{t} \frac{\Lambda(s)}{\lambda(s)}\left(1+B_{\lambda}(0, s)\right)^{-\alpha_{2}(p)} d s=\int_{\delta t}^{t} \frac{\mu(s)}{\lambda^{2}(s)}\left(1+B_{\lambda}(0, s)\right)^{-\alpha_{2}(p)} d\left(1+B_{\lambda}(0, s)\right) \\
& =\frac{1}{1-\alpha_{2}(p)}\left[\left.\frac{\mu(s)}{\lambda^{2}(s)}\left(1+B_{\lambda}(0, s)\right)^{-\alpha_{2}(p)+1}\right|_{\delta t} ^{t}-\int_{\delta t}^{t}\left(1+B_{\lambda}(0, s)\right)^{-\alpha_{2}(p)+1} d\left(\frac{\mu(s)}{\lambda^{2}(s)}\right)\right] \\
& \lesssim \frac{1}{1-\alpha_{2}(p)}\left[\left.\frac{\mu(s)}{\lambda^{2}(s)}\left(1+B_{\lambda}(0, s)\right)^{-\alpha_{2}(p)+1}\right|_{\delta t} ^{t}+\int_{\delta t}^{t} \frac{\mu(s)}{\lambda^{2}(s)}\left(1+B_{\lambda}(0, s)\right)^{-\alpha_{2}(p)+1} \frac{\lambda(s)}{\Lambda(s)} d s\right] .
\end{aligned}
$$

Then, it follows

$$
\begin{aligned}
\left(1+B_{\lambda}(0, t)\right)^{\frac{n}{2}\left(\frac{1}{m}-\frac{1}{2}\right)} \mathbf{E} \lesssim & \underbrace{\frac{\mu(t)}{\lambda^{2}(t)}\left(1+B_{\lambda}(0, t)\right)^{-\alpha_{2}(p)+1}\left(1+B_{\lambda}(0, t)\right)^{\frac{n}{2}\left(\frac{1}{m}-\frac{1}{2}\right)}}_{\bar{E}_{1}(t)} \\
& +\underbrace{\frac{\mu(\delta t)}{\lambda^{2}(\delta t)}\left(1+B_{\lambda}(0, \delta t)\right)^{-\alpha_{2}(p)+1}\left(1+B_{\lambda}(0, t)\right)^{\frac{n}{2}\left(\frac{1}{m}-\frac{1}{2}\right)}}_{\bar{E}_{2}(t)} \\
& +\underbrace{\int_{\delta t}^{t} \frac{\mu(s)}{\lambda^{2}(s)}\left(1+B_{\lambda}(0, s)\right)^{-\alpha_{2}(p)+1}\left(1+B_{\lambda}(0, t)\right)^{\frac{n}{2}\left(\frac{1}{m}-\frac{1}{2}\right)} \frac{\lambda(s)}{\Lambda(s)} d s}_{\bar{E}_{3}(t)} .
\end{aligned}
$$

Since $\alpha_{2}(p)=\alpha_{1}(p)+\frac{n}{2}\left(\frac{1}{m}-\frac{1}{2}\right)$, we may conclude for large $t$ the relation

$$
\bar{E}_{1}(t)=\frac{\mu(t)}{\lambda^{2}(t)}\left(1+B_{\lambda}(0, t)\right)^{-\alpha_{1}(p)+1}=\overline{\mathcal{E}}_{1}(t) \Lambda(t)^{-\varepsilon} \lesssim \Lambda(t)^{-\varepsilon} \lesssim 1,
$$

where $\overline{\mathcal{E}}_{1}=\overline{\mathcal{E}}_{1}(t)$ is defined by

$$
\overline{\mathcal{E}}_{1}(t):=\frac{\mu(t)}{\lambda^{2}(t)} \Lambda^{\varepsilon}(t)\left(1+B_{\lambda}(0, t)\right)^{-\alpha_{1}(p)+1} .
$$

It is shown in (3.2.27) that $\overline{\mathcal{E}}_{1}=\overline{\mathcal{E}}_{1}(t)$ is decreasing. So, we find that $\bar{E}_{1}=\bar{E}_{1}(t)$ is bounded.

Now in order to prove that $\bar{E}_{2}=\bar{E}_{2}(t)$ and $\bar{E}_{3}=\bar{E}_{3}(t)$ are both uniformly bounded it is sufficient to show that for a small positive constant $\epsilon$ we have

$$
\frac{\mu(\delta t)}{\lambda^{2}(\delta t)}\left(1+B_{\lambda}(0, \delta t)\right)^{-\alpha_{2}(p)+1}\left(1+B_{\lambda}(0, t)\right)^{\frac{n}{2}\left(\frac{1}{m}-\frac{1}{2}\right)} \lesssim \Lambda(\delta t)^{-\epsilon} .
$$


Therefore, we will consider the monotonicity of the function

$$
\overline{\mathcal{E}}_{2}(t):=\frac{\mu(\delta t)}{\lambda^{2}(\delta t)} \Lambda(\delta t)^{\epsilon}\left(1+B_{\lambda}(0, \delta t)\right)^{-\alpha_{2}(p)+1}\left(1+B_{\lambda}(0, t)\right)^{\frac{n}{2}\left(\frac{1}{m}-\frac{1}{2}\right)} .
$$

Performing the derivative of the function $\overline{\mathcal{E}}_{2}=\overline{\mathcal{E}}_{2}(t)$ we obtain

$$
\begin{aligned}
\overline{\mathcal{E}}_{2}^{\prime}(t)= & \left(\frac{\mu(\delta t)}{\lambda^{2}(\delta t)}\right)^{\prime} \Lambda(\delta t)^{\epsilon}\left(1+B_{\lambda}(0, \delta t)\right)^{-\alpha_{2}(p)+1}\left(1+B_{\lambda}(0, t)\right)^{\frac{n}{2}\left(\frac{1}{m}-\frac{1}{2}\right)} \\
& +\epsilon \delta \frac{\mu(\delta t)}{\lambda^{2}(\delta t)} \lambda(\delta t) \Lambda(\delta t)^{\epsilon-1}\left(1+B_{\lambda}(0, \delta t)\right)^{-\alpha_{2}(p)+1}\left(1+B_{\lambda}(0, t)\right)^{\frac{n}{2}\left(\frac{1}{m}-\frac{1}{2}\right)} \\
& +\left(-\alpha_{2}(p)+1\right) \frac{\mu(\delta t)}{\lambda^{2}(\delta t)} \Lambda(\delta t)^{\epsilon} \frac{\lambda(\delta t) \Lambda(\delta t)}{\mu(\delta t)}\left(1+B_{\lambda}(0, \delta t)\right)^{-\alpha_{2}(p)}\left(1+B_{\lambda}(0, t)\right)^{\frac{n}{2}\left(\frac{1}{m}-\frac{1}{2}\right)} \\
& +\frac{n}{2}\left(\frac{1}{m}-\frac{1}{2}\right) \frac{\mu(\delta t)}{\lambda^{2}(\delta t)} \Lambda(\delta t)^{\epsilon}\left(1+B_{\lambda}(0, \delta t)\right)^{-\alpha_{2}(p)+1} \frac{\lambda(t) \Lambda(t)}{\mu(t)}\left(1+B_{\lambda}(0, t)\right)^{\frac{n}{2}\left(\frac{1}{m}-\frac{1}{2}\right)-1} \\
\leq & {\left[-\delta \beta_{1} \frac{\lambda(\delta t)}{\Lambda(\delta t)}+\epsilon \delta \frac{\lambda(\delta t)}{\Lambda(\delta t)}+\left(-\alpha_{2}(p)+1\right) \frac{\lambda(\delta t)}{\Lambda(\delta t)} \frac{1}{\log \Lambda(\delta t)}+\frac{n}{2}\left(\frac{1}{m}-\frac{1}{2}\right) \frac{\lambda(t)}{\Lambda(t)} \frac{1}{\log \Lambda(t)}\right] } \\
& \times \phi(t) \log \Lambda(t) \phi(\delta t) \log \Lambda(\delta t) \frac{\mu(\delta t)}{\lambda^{2}(\delta t)} \Lambda(\delta t)^{\epsilon}\left(1+B_{\lambda}(0, \delta t)\right)^{-\alpha_{2}(p)}\left(1+B_{\lambda}(0, t)\right)^{\frac{n}{2}\left(\frac{1}{m}-\frac{1}{2}\right)-1} \\
\leq & \left.-\delta \beta_{1}+\epsilon \delta+\left(-\alpha_{2}(p)+1\right) \frac{1}{\log \Lambda(\delta t)}+\frac{n}{2}\left(\frac{1}{m}-\frac{1}{2}\right) \frac{\lambda(t)}{\Lambda(t)} \frac{\Lambda(\delta t)}{\lambda(\delta t)} \frac{1}{\log \Lambda(t)}\right] \frac{\lambda(\delta t)}{\Lambda(\delta t)} \\
& \times \phi(t) \log \Lambda(t) \phi(\delta t) \log \Lambda(\delta t) \frac{\mu(\delta t)}{\lambda^{2}(\delta t)} \Lambda(\delta t)^{\epsilon}\left(1+B_{\lambda}(0, \delta t)\right)^{-\alpha_{2}(p)}\left(1+B_{\lambda}(0, t)\right)^{\frac{n}{2}\left(\frac{1}{m}-\frac{1}{2}\right)-1} .
\end{aligned}
$$

Here we used the condition (C4) and (3.1.20) with condition (C3), respectively. Now employing the condition (3.3.32), we immediately get $\overline{\mathcal{E}}_{2}^{\prime}(t)<0$ for large $t$. This implies that $\bar{E}_{2}=\bar{E}_{2}(t)$ is bounded and using (3.3.38) we find

$$
\bar{E}_{3}(t) \lesssim \int_{\delta t}^{t} \Lambda(s)^{-\epsilon-1} \lambda(s) d s \lesssim 1
$$

Hence, we may conclude in the case $j=\ell=0$ the estimate

$$
\mathbf{E} \lesssim\left(1+B_{\lambda}(0, t)\right)^{-\frac{n}{2}\left(\frac{1}{m}-\frac{1}{2}\right)} .
$$

Case 2: $\alpha_{2}(p)=1$. In this case it follows

$$
\begin{aligned}
\mathbf{E} & =\int_{\delta t}^{t} \frac{\Lambda(s)}{\lambda(s)}\left(1+B_{\lambda}(0, s)\right)^{-1} d s=\int_{\delta t}^{t} \frac{\mu(s)}{\lambda^{2}(s)} d\left(\log \left(1+B_{\lambda}(0, s)\right)\right) \\
& =\left.\frac{\mu(s)}{\lambda^{2}(s)} \log \left(1+B_{\lambda}(0, s)\right)\right|_{\delta t} ^{t}-\int_{\delta t}^{t} \log \left(1+B_{\lambda}(0, s)\right) d\left(\frac{\mu(s)}{\lambda^{2}(s)}\right) \\
& \leq \frac{\mu(t)}{\lambda^{2}(t)} \log \left(1+B_{\lambda}(0, t)\right)-\frac{\mu(\delta t)}{\lambda^{2}(\delta t)} \log \left(1+B_{\lambda}(0, \delta t)\right)+\beta_{0} \int_{\delta t}^{t} \frac{\mu(s)}{\lambda^{2}(s)} \log \left(1+B_{\lambda}(0, s)\right) \frac{\lambda(s)}{\Lambda(s)} d s,
\end{aligned}
$$

where we used condition (C4). Then, it holds

$$
\begin{aligned}
\left(1+B_{\lambda}(0, t)\right)^{\frac{n}{2}\left(\frac{1}{m}-\frac{1}{2}\right)} \mathbf{E} \leq & \frac{\mu(t)}{\lambda^{2}(t)} \log \left(1+B_{\lambda}(0, t)\right)\left(1+B_{\lambda}(0, t)\right)^{\frac{n}{2}\left(\frac{1}{m}-\frac{1}{2}\right)} \\
& +\beta_{0} \int_{\delta t}^{t} \frac{\mu(s)}{\lambda^{2}(s)} \log \left(1+B_{\lambda}(0, s)\right)\left(1+B_{\lambda}(0, t)\right)^{\frac{n}{2}\left(\frac{1}{m}-\frac{1}{2}\right)} \frac{\lambda(s)}{\Lambda(s)} d s .
\end{aligned}
$$

Similarly, in order to prove $\left(1+B_{\lambda}(0, t)\right)^{\frac{n}{2}\left(\frac{1}{m}-\frac{1}{2}\right)} \mathbf{E}$ is uniformly bounded we will show that for a sufficiently small positive constant $\epsilon$ we have the estimate

$$
\frac{\mu(\delta t)}{\lambda^{2}(\delta t)} \log \left(1+B_{\lambda}(0, \delta t)\right)\left(1+B_{\lambda}(0, t)\right)^{\frac{n}{2}\left(\frac{1}{m}-\frac{1}{2}\right)} \lesssim \Lambda(\delta t)^{-\epsilon} .
$$


Then, for an arbitrary positive constant $r$ we have

$$
\begin{aligned}
\frac{\mu(\delta t)}{\lambda^{2}(\delta t)} & \Lambda(\delta t)^{\epsilon} \log \left(1+B_{\lambda}(0, \delta t)\right)\left(1+B_{\lambda}(0, t)\right)^{\frac{n}{2}\left(\frac{1}{m}-\frac{1}{2}\right)} \\
& =\frac{\mu(\delta t)}{\lambda^{2}(\delta t)} \Lambda(\delta t)^{\epsilon} \frac{\log \left(1+B_{\lambda}(0, \delta t)\right)}{\left(1+B_{\lambda}(0, \delta t)\right)^{r}}\left(1+B_{\lambda}(0, \delta t)\right)^{r}\left(1+B_{\lambda}(0, t)\right)^{\frac{n}{2}\left(\frac{1}{m}-\frac{1}{2}\right)} \\
& \lesssim \frac{\mu(\delta t)}{\lambda^{2}(\delta t)} \Lambda(\delta t)^{\epsilon}\left(1+B_{\lambda}(0, \delta t)\right)^{r}\left(1+B_{\lambda}(0, t)\right)^{\frac{n}{2}\left(\frac{1}{m}-\frac{1}{2}\right)},
\end{aligned}
$$

where we have employed l'Hospital rule (cf. condition (B4)) as follows:

$$
\begin{aligned}
\lim _{t \rightarrow \infty} \sup \frac{\log \left(1+B_{\lambda}(0, \delta t)\right)}{\left(1+B_{\lambda}(0, \delta t)\right)^{r}} & =\lim _{t \rightarrow \infty} \sup \frac{\frac{\lambda(\delta t) \Lambda(\delta t)}{\mu(\delta t)}}{r \delta\left(1+B_{\lambda}(0, \delta t)\right) \frac{\lambda(\delta t) \Lambda(\delta t)}{\mu(\delta t)}\left(1+B_{\lambda}(0, \delta t)\right)^{r-1}} \\
& =\lim _{t \rightarrow \infty} \sup \frac{1}{r \delta\left(1+B_{\lambda}(0, \delta t)\right)^{r}} \lesssim 1 .
\end{aligned}
$$

Therefore, taking account of the monotonic behavior of $\overline{\mathcal{E}}_{2}=\overline{\mathcal{E}}_{2}(t)$ we find the desired estimate

$$
\mathbf{E} \lesssim\left(1+B_{\lambda}(0, t)\right)^{-\frac{n}{2}\left(\frac{1}{m}-\frac{1}{2}\right)} .
$$

Now let us consider (3.3.37) in the case $j=1$ and $\ell=0$. Then, we get

$$
\begin{aligned}
\mathbf{E}= & \int_{\delta t}^{t} \frac{\Lambda(s)}{\lambda(s)}\left(1+B_{\lambda}(s, t)\right)^{-\frac{1}{2}}\left(1+B_{\lambda}(0, s)\right)^{-\alpha_{2}(p)} d s \\
= & -\int_{\delta t}^{t} \frac{\mu(s)}{\lambda^{2}(s)}\left(1+B_{\lambda}(0, s)\right)^{-\alpha_{2}(p)}\left(1+B_{\lambda}(s, t)\right)^{-\frac{1}{2}} d\left(1+B_{\lambda}(s, t)\right) \\
= & -\left.2 \frac{\mu(s)}{\lambda^{2}(s)}\left(1+B_{\lambda}(0, s)\right)^{-\alpha_{2}(p)}\left(1+B_{\lambda}(s, t)\right)^{\frac{1}{2}}\right|_{\delta t} ^{t} \\
& +2 \int_{\delta t}^{t}\left(1+B_{\lambda}(s, t)\right)^{\frac{1}{2}} d\left(\frac{\mu(s)}{\lambda^{2}(s)}\left(1+B_{\lambda}(0, s)\right)^{-\alpha_{2}(p)}\right) \\
\lesssim & -\left.\frac{\mu(s)}{\lambda^{2}(s)}\left(1+B_{\lambda}(0, s)\right)^{-\alpha_{2}(p)}\left(1+B_{\lambda}(s, t)\right)^{\frac{1}{2}}\right|_{\delta t} ^{t} \\
& +\int_{\delta t}^{t} \frac{\mu(s)}{\lambda^{2}(s)}\left(1+B_{\lambda}(0, s)\right)^{-\alpha_{2}(p)}\left(1+B_{\lambda}(s, t)\right)^{\frac{1}{2}} \frac{d\left(1+B_{\lambda}(0, s)\right)}{1+B_{\lambda}(0, s)},
\end{aligned}
$$

where we have used Lemma 3.1.5. After, multiplying both sides of the last estimate by $\left(1+B_{\lambda}(0, t)\right)^{\frac{n}{2}\left(\frac{1}{m}-\frac{1}{2}\right)+\frac{1}{2}}$, we find

$$
\begin{aligned}
\left(1+B_{\lambda}(0, t)\right)^{\frac{n}{2}\left(\frac{1}{m}-\frac{1}{2}\right)+\frac{1}{2}} \mathbf{E} & \\
\lesssim & \frac{\mu(\delta t)}{\lambda^{2}(\delta t)}\left(1+B_{\lambda}(0, \delta t)\right)^{-\alpha_{2}(p)}\left(1+B_{\lambda}(0, t)\right)^{\frac{n}{2}\left(\frac{1}{m}-\frac{1}{2}\right)+1} \\
& \quad+\int_{\delta t}^{t} \frac{\mu(s)}{\lambda^{2}(s)}\left(1+B_{\lambda}(0, s)\right)^{-\alpha_{2}(p)}\left(1+B_{\lambda}(0, t)\right)^{\frac{n}{2}\left(\frac{1}{m}-\frac{1}{2}\right)+1} \frac{d\left(1+B_{\lambda}(0, s)\right)}{1+B_{\lambda}(0, s)} .
\end{aligned}
$$

To derive the desired estimates for the two summands on the right-hand side of the previous inequality, employing (3.3.32), we proceed in the same way as we did in the proof of Theorem 3.2.2 to estimate the terms $\bar{B}_{1}=\bar{B}_{1}(t)$ and $\bar{B}_{2}=\bar{B}_{2}(t)$. Summarizing, we have the estimate

$$
\mathbf{E} \lesssim\left(1+B_{\lambda}(0, t)\right)^{-\frac{n}{2}\left(\frac{1}{m}-\frac{1}{2}\right)-\frac{1}{2}} .
$$


Finally, we will consider (3.3.37) in the case $j=0$ and $\ell=1$. So, we have

$$
\mathbf{E}=\int_{\delta t}^{t} \Phi_{1}(s, t) \frac{\Lambda(s)}{\lambda(s)}\left(1+B_{\lambda}(0, s)\right)^{-\alpha_{2}(p)} d s
$$

where $\Phi_{1}=\Phi_{1}(s, t)$ is defined in (3.1.14). Employing condition (C1) we are only interested in the following two cases, which explains that we have either the first component or the second component is dominant in $\Phi_{1}=\Phi_{1}(s, t)$ for all $s \in[\delta t, t]$.

Case $a: \Phi_{1}(s, t)=\frac{\lambda^{2}(t)}{\rho(t)}\left(1+B_{\lambda}(s, t)\right)^{-1}$. In this case it holds

$$
\begin{aligned}
\mathbf{E}= & \frac{\lambda^{2}(t)}{\rho(t)} \int_{\delta t}^{t} \frac{\Lambda(s)}{\lambda(s)}\left(1+B_{\lambda}(s, t)\right)^{-1}\left(1+B_{\lambda}(0, s)\right)^{-\alpha_{2}(p)} d s \\
= & -\frac{\lambda^{2}(t)}{\rho(t)} \int_{\delta t}^{t} \frac{\mu(s)}{\lambda^{2}(s)}\left(1+B_{\lambda}(0, s)\right)^{-\alpha_{2}(p)} \frac{d\left(1+B_{\lambda}(s, t)\right)}{1+B_{\lambda}(s, t)} \\
= & -\left.\frac{\lambda^{2}(t)}{\rho(t)} \frac{\mu(s)}{\lambda^{2}(s)}\left(1+B_{\lambda}(0, s)\right)^{-\alpha_{2}(p)} \log \left(1+B_{\lambda}(s, t)\right)\right|_{\delta t} ^{t} \\
& +\frac{\lambda^{2}(t)}{\rho(t)} \int_{\delta t}^{t} \log \left(1+B_{\lambda}(s, t)\right) d\left(\frac{\mu(s)}{\lambda^{2}(s)}\left(1+B_{\lambda}(0, s)\right)^{-\alpha_{2}(p)}\right) \\
\lesssim & -\left.\frac{\lambda^{2}(t)}{\rho(t)} \frac{\mu(s)}{\lambda^{2}(s)}\left(1+B_{\lambda}(0, s)\right)^{-\alpha_{2}(p)} \log \left(1+B_{\lambda}(s, t)\right)\right|_{\delta t} ^{t} \\
& +\frac{\lambda^{2}(t)}{\rho(t)} \int_{\delta t}^{t} \frac{\mu(s)}{\lambda^{2}(s)}\left(1+B_{\lambda}(0, s)\right)^{-\alpha_{2}(p)} \log \left(1+B_{\lambda}(s, t)\right) \frac{d\left(1+B_{\lambda}(0, s)\right)}{1+B_{\lambda}(0, s)} .
\end{aligned}
$$

Then, we have

$$
\begin{aligned}
\frac{\rho(t)}{\lambda^{2}(t)}(1+ & \left.B_{\lambda}(0, t)\right)^{\frac{n}{2}\left(\frac{1}{m}-\frac{1}{2}\right)+1} \mathbf{E} \\
\lesssim & \underbrace{\frac{\mu(\delta t)}{\lambda^{2}(\delta t)}\left(1+B_{\lambda}(0, \delta t)\right)^{-\alpha_{2}(p)} \log \left(1+B_{\lambda}(0, t)\right)\left(1+B_{\lambda}(0, t)\right)^{\frac{n}{2}\left(\frac{1}{m}-\frac{1}{2}\right)+1}}_{\bar{E}_{4}(t)} \\
& +\underbrace{\int_{\delta t}^{t} \frac{\mu(s)}{\lambda^{2}(s)} \frac{\log \left(1+B_{\lambda}(0, t)\right)\left(1+B_{\lambda}(0, t)\right)^{\frac{n}{2}\left(\frac{1}{m}-\frac{1}{2}\right)+1}}{\left(1+B_{\lambda}(0, s)\right)^{\alpha_{2}(p)}} \frac{d\left(1+B_{\lambda}(0, s)\right)}{1+B_{\lambda}(0, s)}}_{\bar{E}_{5}(t)} .
\end{aligned}
$$

After applying the l'Hospital rule for $\gamma>1$ (cf. condition (B4)) we find

$$
\begin{aligned}
\lim _{t \rightarrow \infty} \sup \frac{\log \left(1+B_{\lambda}(0, t)\right)}{\left(1+B_{\lambda}(0, \delta t)\right)^{\gamma}} & =\lim _{t \rightarrow \infty} \sup \frac{\frac{\lambda(t) \Lambda(t)}{\mu(t)}}{\gamma \delta\left(1+B_{\lambda}(0, t)\right) \frac{\lambda(\delta t) \Lambda(\delta t)}{\mu(\delta t)}\left(1+B_{\lambda}(0, \delta t)\right)^{\gamma-1}} \\
& \lesssim \lim _{t \rightarrow \infty} \sup \frac{\frac{\lambda(t)}{\Lambda(t)} \phi(t)}{\phi(t) \log \Lambda(t) \frac{\lambda(\delta t)}{\Lambda(\delta t)} \phi(\delta t)\left(1+B_{\lambda}(0, \delta t)\right)^{\gamma-1}} \\
& \lesssim \lim _{t \rightarrow \infty} \sup \frac{\lambda(t)}{\Lambda(t)} \frac{\Lambda(\delta t)}{\lambda(\delta t)} \frac{1}{\phi(\delta t) \log \Lambda(t)} \frac{1}{\left(1+B_{\lambda}(0, \delta t)\right)^{\gamma-1}} \lesssim 1,
\end{aligned}
$$

where we used (3.1.20) with condition (C3). Therefore, to prove $\bar{E}_{4}=\bar{E}_{4}(t)$ and $\bar{E}_{5}=\bar{E}_{5}(t)$ are bounded we use the estimate

$$
\frac{\mu(\delta t)}{\lambda^{2}(\delta t)}\left(1+B_{\lambda}(0, \delta t)\right)^{-\alpha_{2}(p)}\left(1+B_{\lambda}(0, t)\right)^{\frac{n}{2}\left(\frac{1}{m}-\frac{1}{2}\right)+1} \lesssim\left(1+B_{\lambda}(0, \delta t)\right)^{-\gamma} .
$$


Then, we have

$$
\overline{\mathcal{E}}_{4}(t):=\frac{\mu(\delta t)}{\lambda^{2}(\delta t)}\left(1+B_{\lambda}(0, \delta t)\right)^{-\alpha_{2}(p)+\gamma}\left(1+B_{\lambda}(0, t)\right)^{\frac{n}{2}\left(\frac{1}{m}-\frac{1}{2}\right)+1} \lesssim 1 .
$$

To prove the boundedness of the function $\overline{\mathcal{E}}_{4}=\overline{\mathcal{E}}_{4}(t)$ we follow the proof of $\overline{\mathcal{B}}_{1}=\overline{\mathcal{B}}_{1}(t)$ which is defined in (3.2.30). Hence, by using (3.3.42) we get

$$
\begin{aligned}
\bar{E}_{4}(t) & =\frac{\log \left(1+B_{\lambda}(0, t)\right)}{\left(1+B_{\lambda}(0, \delta t)\right)^{\gamma}} \frac{\mu(\delta t)}{\lambda^{2}(\delta t)}\left(1+B_{\lambda}(0, \delta t)\right)^{-\alpha_{2}(p)+\gamma}\left(1+B_{\lambda}(0, t)\right)^{\frac{n}{2}\left(\frac{1}{m}-\frac{1}{2}\right)+1} \\
& =\frac{\log \left(1+B_{\lambda}(0, t)\right)}{\left(1+B_{\lambda}(0, \delta t)\right)^{\gamma}} \overline{\mathcal{E}}_{4}(t) \lesssim \frac{\log \left(1+B_{\lambda}(0, t)\right)}{\left(1+B_{\lambda}(0, \delta t)\right)^{\gamma}} \lesssim 1
\end{aligned}
$$

for large $t$. Moreover, for $\bar{E}_{5}=\bar{E}_{5}(t)$ using (3.3.43) and (3.3.44), respectively, we obtain

$$
\begin{aligned}
\bar{E}_{5}(t) & \lesssim \log \left(1+B_{\lambda}(0, t)\right) \int_{\delta t}^{t}\left(1+B_{\lambda}(0, s)\right)^{-\gamma-1} d\left(1+B_{\lambda}(0, s)\right) \\
& =\frac{\log \left(1+B_{\lambda}(0, t)\right)}{\gamma\left(1+B_{\lambda}(0, \delta t)\right)^{\gamma}}-\frac{\log \left(1+B_{\lambda}(0, t)\right)}{\gamma\left(1+B_{\lambda}(0, t)\right)^{\gamma}} \lesssim 1
\end{aligned}
$$

where we used again the l'Hospital rule from (3.3.42). Consequently, we obtain that $\bar{E}_{4}=$ $\bar{E}_{4}(t)$ and $\bar{E}_{5}=\bar{E}_{5}(t)$ are uniformly bounded. This implies

$$
\mathbf{E} \lesssim \frac{\lambda^{2}(t)}{\rho(t)}\left(1+B_{\lambda}(0, t)\right)^{-\frac{n}{2}\left(\frac{1}{m}-\frac{1}{2}\right)-1}
$$

Taking account of (3.3.39), (3.3.40), (3.3.41) and (3.3.45), in the Case $a$ and $j+\ell=0,1$ we arrive at the estimate

$$
\mathbf{E} \lesssim \frac{\lambda^{2 \ell}(t)}{\rho^{\ell}(t)}\left(1+B_{\lambda}(0, t)\right)^{-\frac{n}{2}\left(\frac{1}{m}-\frac{1}{2}\right)-\frac{j}{2}-\ell} .
$$

Case b: $\Phi_{1}(s, t)=\frac{\lambda(t)}{F(\Lambda(t))}$. In this case we have

$$
\begin{aligned}
\mathbf{E} & =\frac{\lambda(t)}{F(\Lambda(t))} \int_{\delta t}^{t} \frac{\Lambda(s)}{\lambda(s)}\left(1+B_{\lambda}(0, s)\right)^{-\alpha_{2}(p)} d s \\
& =\frac{\lambda(t)}{F(\Lambda(t))} \int_{\delta t}^{t} \frac{\mu(s)}{\lambda^{2}(s)}\left(1+B_{\lambda}(0, s)\right)^{-\alpha_{2}(p)} d\left(1+B_{\lambda}(0, s)\right) .
\end{aligned}
$$

Now we distinguish between two cases.

Case b.1: $\alpha_{2}(p) \neq 1$. It holds

$$
\begin{aligned}
\frac{F(\Lambda(t))}{\lambda(t)}\left(F^{2}(\Lambda(t))\right)^{\frac{n}{2}\left(\frac{1}{m}-\frac{1}{2}\right)} \mathbf{E} \lesssim & \underbrace{\frac{\mu(t)}{\lambda^{2}(t)}\left(1+B_{\lambda}(0, t)\right)^{-\alpha_{2}(p)+1}\left(F^{2}(\Lambda(t))\right)^{\frac{n}{2}\left(\frac{1}{m}-\frac{1}{2}\right)}}_{\bar{F}_{1}(t)} \\
& +\underbrace{\frac{\mu(\delta t)}{\lambda^{2}(\delta t)}\left(1+B_{\lambda}(0, \delta t)\right)^{-\alpha_{2}(p)+1}\left(F^{2}(\Lambda(t))\right)^{\frac{n}{2}\left(\frac{1}{m}-\frac{1}{2}\right)}}_{\bar{F}_{2}(t)} \\
& +\underbrace{\int_{\delta t}^{t} \frac{\mu(s)}{\lambda^{2}(s)}\left(1+B_{\lambda}(0, s)\right)^{-\alpha_{2}(p)+1}\left(F^{2}(\Lambda(t))\right)^{\frac{n}{2}\left(\frac{1}{m}-\frac{1}{2}\right)}}_{\bar{F}_{3}(t)} \frac{\lambda(s)}{\Lambda(s)} d s .
\end{aligned}
$$


Let us begin to estimate $\bar{F}_{1}=\bar{F}_{1}(t)$ for both cases $\alpha_{2}(p)>1$ and $\alpha_{2}(p)<1$. If $\alpha_{2}(p)>1$, then we have

$$
\bar{F}_{1}(t) \leq \frac{\mu(t)}{\lambda^{2}(t)}\left(F^{2}(\Lambda(t))\right)^{\frac{n}{2}\left(\frac{1}{m}-\frac{1}{2}\right)} \leq \frac{\mu(\delta t)}{\lambda^{2}(\delta t)}\left(F^{2}(\Lambda(t))\right)^{\frac{n}{2}\left(\frac{1}{m}-\frac{1}{2}\right)}:=\overline{\mathcal{F}}_{1}(t),
$$

where we used the decreasing behavior of the function $\frac{\mu(t)}{\lambda^{2}(t)}$ from condition (C4). Now we form the derivative of the function $\overline{\mathcal{F}}_{1}=\overline{\mathcal{F}}_{1}(t)$. It follows

$$
\begin{aligned}
\overline{\mathcal{F}}_{1}^{\prime}(t) & =\left(\frac{\mu(\delta t)}{\lambda^{2}(\delta t)}\right)^{\prime} F(\Lambda(t))^{n\left(\frac{1}{m}-\frac{1}{2}\right)}+n\left(\frac{1}{m}-\frac{1}{2}\right) \frac{\mu(\delta t)}{\lambda^{2}(\delta t)} \lambda(t) F^{\prime}(\Lambda(t)) F(\Lambda(t))^{n\left(\frac{1}{m}-\frac{1}{2}\right)-1} \\
& \leq\left[-\delta \beta_{1} \frac{\lambda(\delta t)}{\Lambda(\delta t)}+n\left(\frac{1}{m}-\frac{1}{2}\right) \frac{\lambda(t)}{F(\Lambda(t))} F^{\prime}(\Lambda(t))\right] \frac{\mu(\delta t)}{\lambda^{2}(\delta t)} F(\Lambda(t))^{n\left(\frac{1}{m}-\frac{1}{2}\right)} \\
& \leq\left[-\delta \beta_{1}+n\left(\frac{1}{m}-\frac{1}{2}\right) \frac{\lambda(t)}{F(\Lambda(t))} \frac{F^{2}(\Lambda(t))}{\lambda^{2}(t) \Xi^{2}(t)} \frac{\Lambda(\delta t)}{\lambda(\delta t)}\right] \frac{\mu(\delta t)}{\lambda^{2}(\delta t)} \frac{\lambda(\delta t)}{\Lambda(\delta t)} F(\Lambda(t))^{n\left(\frac{1}{m}-\frac{1}{2}\right)} \\
& \leq\left[-\delta \beta_{1}+n\left(\frac{1}{m}-\frac{1}{2}\right) \frac{F(\Lambda(t))}{\lambda(t) \Xi^{2}(t)} \frac{\Lambda(\delta t)}{\lambda(\delta t)}\right] \frac{\mu(\delta t)}{\lambda^{2}(\delta t)} \frac{\lambda(\delta t)}{\Lambda(\delta t)} F(\Lambda(t))^{n\left(\frac{1}{m}-\frac{1}{2}\right)} \\
& \leq\left[-\delta \beta_{1} \frac{1}{\log \Lambda(t)}+n\left(\frac{1}{m}-\frac{1}{2}\right) \vartheta(\delta, t) \frac{1}{\log \Lambda(t)}\right] \frac{\mu(\delta t)}{\lambda^{2}(\delta t)} \frac{\lambda(\delta t)}{\Lambda(\delta t)} \log \Lambda(t) F(\Lambda(t))^{n\left(\frac{1}{m}-\frac{1}{2}\right)},
\end{aligned}
$$

where from condition (A5) we used $F^{\prime}(\Lambda(t))=\frac{F^{2}(\Lambda(t))}{\lambda^{2}(t) \Xi^{2}(t)}$. Therefore, by using (3.3.32) we find $\overline{\mathcal{F}}_{1}^{\prime}(t)<0$ for large $t$. Analogously, if $\alpha_{2}(p)<1$, by using condition (B6) we get

$$
\begin{aligned}
\bar{F}_{1}(t) & \leq \frac{\mu(t)}{\lambda^{2}(t)}\left(F^{2}(\Lambda(t))\right)^{-\alpha_{2}(p)+1}\left(F^{2}(\Lambda(t))\right)^{\frac{n}{2}\left(\frac{1}{m}-\frac{1}{2}\right)} \\
& \leq \frac{\mu(t)}{\lambda^{2}(t)}\left(F^{2}(\Lambda(t))\right)^{-\alpha_{1}(p)+1} \leq \frac{\mu(\delta t)}{\lambda^{2}(\delta t)}\left(F^{2}(\Lambda(t))\right)^{-\alpha_{1}(p)+1}:=\overline{\mathcal{F}}_{2}(t) .
\end{aligned}
$$

Hence, in the same way we obtain

$$
\overline{\mathcal{F}}_{2}^{\prime}(t) \leq\left[-\frac{\delta \beta_{1}}{\log \Lambda(t)}+2\left(-\alpha_{1}(p)+1\right) \frac{\vartheta(\delta, t)}{\log \Lambda(t)}\right] \frac{\mu(\delta t)}{\lambda^{2}(\delta t)} \frac{\lambda(\delta t)}{\Lambda(\delta t)} \log \Lambda(t)(F(\Lambda(t)))^{-2 \alpha_{1}(p)+2} .
$$

Thus, if $\alpha_{2}(p)<1$, employing the condition (3.3.32) we may conclude that $\bar{F}_{1}=\bar{F}_{1}(t)$ is uniformly bounded for large $t$.

Now, in order to show that $\bar{F}_{2}=\bar{F}_{2}(t)$ and $\bar{F}_{3}=\bar{F}_{3}(t)$ are uniformly bounded we verify

$$
\frac{\mu(\delta t)}{\lambda^{2}(\delta t)}\left(1+B_{\lambda}(0, \delta t)\right)^{-\alpha_{2}(p)+1}\left(F^{2}(\Lambda(t))\right)^{\frac{n}{2}\left(\frac{1}{m}-\frac{1}{2}\right)} \lesssim \Lambda(\delta t)^{-\epsilon},
$$

with an arbitrary small positive constant $\epsilon$. Then, we may conclude

$$
\frac{\mu(\delta t)}{\lambda^{2}(\delta t)} \Lambda(\delta t)^{\epsilon}\left(1+B_{\lambda}(0, \delta t)\right)^{-\alpha_{2}(p)+1}\left(F^{2}(\Lambda(t))\right)^{\frac{n}{2}\left(\frac{1}{m}-\frac{1}{2}\right)} \lesssim 1,
$$

in both cases $\alpha_{2}(p)<1$ and $\alpha_{2}(p)>1$. In the case $\alpha_{2}(p)<1$ we have

$$
\begin{aligned}
& \frac{\mu(\delta t)}{\lambda^{2}(\delta t)} \Lambda(\delta t)^{\epsilon}\left(1+B_{\lambda}(0, \delta t)\right)^{-\alpha_{2}(p)+1}\left(F^{2}(\Lambda(t))\right)^{\frac{n}{2}\left(\frac{1}{m}-\frac{1}{2}\right)} \\
& \quad \lesssim \frac{\mu(\delta t)}{\lambda^{2}(\delta t)} \Lambda(\delta t)^{\epsilon}\left(F^{2}(\Lambda(t))\right)^{\frac{n}{2}\left(\frac{1}{m}-\frac{1}{2}\right)-\alpha_{2}(p)+1}=\frac{\mu(\delta t)}{\lambda^{2}(\delta t)} \Lambda(\delta t)^{\epsilon}\left(F^{2}(\Lambda(t))\right)^{-\alpha_{1}(p)+1}:=\overline{\mathcal{F}}_{3}(t),
\end{aligned}
$$

where we used condition (B6). We can estimate the derivative of the function $\overline{\mathcal{F}}_{3}=\overline{\mathcal{F}}_{3}(t)$ as follows:

$$
\overline{\mathcal{F}}_{3}^{\prime}(t) \leq\left[-\frac{\delta\left(\beta_{1}-\epsilon\right)}{\log \Lambda(t)}+2\left(-\alpha_{1}(p)+1\right) \frac{\vartheta(\delta, t)}{\log \Lambda(t)}\right] \frac{\mu(\delta t)}{\lambda^{2}(\delta t)} \frac{\lambda(\delta t)}{\Lambda(\delta t)} \log \Lambda(t) \Lambda(\delta t)^{\epsilon}(F(\Lambda(t)))^{-2 \alpha_{1}(p)+2} .
$$


Therefore, employing (3.3.32) we obtain that $\overline{\mathcal{F}}_{3}^{\prime}(t)<0$ for large $t$. On the other hand, if $\alpha_{2}(p)>1$, then one can derive for large $t$ the estimate

$$
\frac{\mu(\delta t)}{\lambda^{2}(\delta t)} \Lambda(\delta t)^{\epsilon}\left(1+B_{\lambda}(0, \delta t)\right)^{-\alpha_{2}(p)+1}\left(F^{2}(\Lambda(t))\right)^{\frac{n}{2}\left(\frac{1}{m}-\frac{1}{2}\right)} \lesssim \frac{\mu(\delta t)}{\lambda^{2}(\delta t)} \Lambda(\delta t)^{\epsilon}\left(F^{2}(\Lambda(t))\right)^{\frac{n}{2}\left(\frac{1}{m}-\frac{1}{2}\right)} \lesssim 1 .
$$

Summarizing, in the case $\alpha_{2}(p) \neq 1$ we obtain the desired estimate

$$
\mathbf{E} \lesssim \lambda(t) F(\Lambda(t))\left(F^{2}(\Lambda(t))\right)^{-\frac{n}{2}\left(\frac{1}{m}-\frac{1}{2}\right)-1} .
$$

Case b.2: $\alpha_{2}(p)=1$. In this case it holds

$$
\begin{aligned}
\frac{F(\Lambda(t))}{\lambda(t)}\left(F^{2}(\Lambda(t))\right)^{\frac{n}{2}\left(\frac{1}{m}-\frac{1}{2}\right)} \mathbf{E} \lesssim & \underbrace{\frac{\mu(t)}{\lambda^{2}(t)} \log \left(1+B_{\lambda}(0, t)\right)\left(F^{2}(\Lambda(t))\right)^{\frac{n}{2}\left(\frac{1}{m}-\frac{1}{2}\right)}}_{\bar{F}_{4}(t)} \\
& +\underbrace{\frac{\mu(\delta t)}{\lambda^{2}(\delta t)} \log \left(1+B_{\lambda}(0, \delta t)\right)\left(F^{2}(\Lambda(t))\right)^{\frac{n}{2}\left(\frac{1}{m}-\frac{1}{2}\right)}}_{\bar{F}_{5}(t)} \\
& +\underbrace{\int_{\delta t}^{t} \frac{\mu(s)}{\lambda^{2}(s)} \log \left(1+B_{\lambda}(0, s)\right)\left(F^{2}(\Lambda(t))\right)^{\frac{n}{2}\left(\frac{1}{m}-\frac{1}{2}\right)} \frac{\lambda(s)}{\Lambda(s)} d s}_{\bar{F}_{6}(t)} .
\end{aligned}
$$

Applying the l'Hospital rule for $r>1$ (cf. condition (B4)) we find

$$
\begin{aligned}
\lim _{t \rightarrow \infty} \sup \frac{\log \left(1+B_{\lambda}(0, t)\right)}{\left(1+B_{\lambda}(0, \delta t)\right)^{r}} & =\lim _{t \rightarrow \infty} \sup \frac{\frac{\lambda(t) \Lambda(t)}{\mu(t)}}{r \delta\left(1+B_{\lambda}(0, t)\right) \frac{\lambda(\delta t) \Lambda(\delta t)}{\mu(\delta t)}\left(1+B_{\lambda}(0, \delta t)\right)^{r-1}} \\
& \lesssim \lim _{t \rightarrow \infty} \sup \frac{\frac{\lambda(t)}{\Lambda(t)} \phi(t)}{\phi(t) \log \Lambda(t) \frac{\lambda(\delta t)}{\Lambda(\delta t)} \phi(\delta t)\left(1+B_{\lambda}(0, \delta t)\right)^{r-1}} \\
& \lesssim \lim _{t \rightarrow \infty} \sup \frac{\lambda(t)}{\Lambda(t)} \frac{\Lambda(\delta t)}{\lambda(\delta t)} \frac{1}{\phi(\delta t) \log \Lambda(t)} \frac{1}{\left(1+B_{\lambda}(0, \delta t)\right)^{r-1}} \lesssim 1
\end{aligned}
$$

where we used (3.1.20) with condition (C3). Therefore, in order to estimate $\bar{F}_{4}=\bar{F}_{4}(t)$, by using the estimates of (3.3.48) we find

$$
\begin{aligned}
\bar{F}_{4}(t) & \lesssim \frac{\mu(\delta t)}{\lambda^{2}(\delta t)} \frac{\log \left(1+B_{\lambda}(0, t)\right)}{\left(1+B_{\lambda}(0, \delta t)\right)^{r}}\left(1+B_{\lambda}(0, \delta t)\right)^{r}\left(F^{2}(\Lambda(t))\right)^{\frac{n}{2}\left(\frac{1}{m}-\frac{1}{2}\right)} \\
& \lesssim \frac{\mu(\delta t)}{\lambda^{2}(\delta t)}\left(1+B_{\lambda}(0, \delta t)\right)^{r}\left(F^{2}(\Lambda(t))\right)^{\frac{n}{2}\left(\frac{1}{m}-\frac{1}{2}\right)} .
\end{aligned}
$$

Now, using condition (B6) we continue in the same way to $\overline{\mathcal{F}}_{2}=\overline{\mathcal{F}}_{2}(t)$ and we may conclude

$$
\bar{F}_{4}(t) \lesssim \frac{\mu(\delta t)}{\lambda^{2}(\delta t)}\left(1+B_{\lambda}(0, \delta t)\right)^{r}\left(F^{2}(\Lambda(t))\right)^{\frac{n}{2}\left(\frac{1}{m}-\frac{1}{2}\right)} \lesssim \frac{\mu(\delta t)}{\lambda^{2}(\delta t)}\left(F^{2}(\Lambda(t))\right)^{\frac{n}{2}\left(\frac{1}{m}-\frac{1}{2}\right)+r} \lesssim 1 .
$$

Now in order to estimate $\bar{F}_{5}=\bar{F}_{5}(t)$ and $\bar{F}_{6}=\bar{F}_{6}(t)$ we verify the estimate

$$
\frac{\mu(\delta t)}{\lambda^{2}(\delta t)} \log \left(1+B_{\lambda}(0, \delta t)\right)\left(F^{2}(\Lambda(t))\right)^{\frac{n}{2}\left(\frac{1}{m}-\frac{1}{2}\right)} \lesssim \Lambda(\delta t)^{-\epsilon},
$$

with an arbitrary small positive constant $\epsilon$. Then, using again (3.3.48) and proceeding in the 
same way to estimate $\overline{\mathcal{F}}_{3}=\overline{\mathcal{F}}_{3}(t)$ for large $t$, we have

$$
\begin{aligned}
\frac{\mu(\delta t)}{\lambda^{2}(\delta t)} & \Lambda(\delta t)^{\epsilon} \log \left(1+B_{\lambda}(0, \delta t)\right)\left(F^{2}(\Lambda(t))\right)^{\frac{n}{2}\left(\frac{1}{m}-\frac{1}{2}\right)} \\
& =\frac{\mu(\delta t)}{\lambda^{2}(\delta t)} \Lambda(\delta t)^{\epsilon} \frac{\log \left(1+B_{\lambda}(0, t)\right)}{\left(1+B_{\lambda}(0, \delta t)\right)^{r}}\left(1+B_{\lambda}(0, \delta t)\right)^{r}\left(F^{2}(\Lambda(t))\right)^{\frac{n}{2}\left(\frac{1}{m}-\frac{1}{2}\right)} \\
& \leq \frac{\mu(\delta t)}{\lambda^{2}(\delta t)} \Lambda(\delta t)^{\epsilon}\left(F^{2}(\Lambda(t))\right)^{\frac{n}{2}\left(\frac{1}{m}-\frac{1}{2}\right)+r} \lesssim 1
\end{aligned}
$$

Consequently, in the case $\alpha_{2}(p)=1$ we obtain the desired estimate

$$
\mathbf{E} \lesssim \lambda(t) F(\Lambda(t))\left(F^{2}(\Lambda(t))\right)^{-\frac{n}{2}\left(\frac{1}{m}-\frac{1}{2}\right)-1} .
$$

Now taking account of (3.3.39), (3.3.40), (3.3.41), (3.3.47) and (3.3.49) in the Case $b$ and $j+\ell=0,1$ we obtain the estimate

$$
\mathbf{E} \lesssim \lambda^{\ell}(t) F^{\ell}(\Lambda(t))\left(1+B_{\lambda}(0, t)\right)^{-\frac{n}{2}\left(\frac{1}{m}-\frac{1}{2}\right)-\frac{j}{2}-\ell} .
$$

Combining (3.3.36) and (3.3.50) we may conclude

$$
\left\||D|^{j} \partial_{t}^{\ell} u^{\mathrm{nl}}(t, \cdot)\right\|_{L^{2}} \lesssim\|u\|_{X(t)}^{p} \Phi_{1, m}^{\ell}(0, t)\left(1+B_{\lambda}(0, t)\right)^{(\ell-1) \frac{n}{2}\left(\frac{1}{m}-\frac{1}{2}\right)-\frac{j}{2}},
$$

where $\Phi_{1, m}^{1}(0, t)=\Phi_{1, m}(0, t)$ is defined in (3.1.13) for $s=0$. Replacing the estimates for $j+\ell=0,1$ in the norm of the solution space $X(t)$ we obtain

$$
\left\|u^{\mathrm{n} 1}\right\|_{X(t)} \lesssim\|u\|_{X(t)}^{p} .
$$

In order to prove (3.3.35) proceeding in the same way as in the proof of Theorem 3.3.1, we get the following estimate:

$$
\begin{aligned}
\left\||D|^{j} \partial_{t}^{\ell}(N u-N v)\right\|_{L^{2}} \lesssim\|u-v\|_{X(t)}\left(\|u\|_{X(t)}^{p-1}+\|v\|_{X(t)}^{p-1}\right) \\
\quad \times\left(\int_{0}^{\delta t} \Phi_{1, m}^{\ell}(s, t) \frac{\Lambda(s)}{\lambda(s)}\left(1+B_{\lambda}(s, t)\right)^{(\ell-1) \frac{n}{2}\left(\frac{1}{m}-\frac{1}{2}\right)-\frac{j}{2}}\left(1+B_{\lambda}(0, s)\right)^{-\frac{n}{2 m} p+\frac{n}{2 m}} d s\right. \\
\left.\quad+\int_{\delta t}^{t} \Phi_{1}^{\ell}(s, t) \frac{\Lambda(s)}{\lambda(s)}\left(1+B_{\lambda}(s, t)\right)^{-\frac{j}{2}}\left(1+B_{\lambda}(0, s)\right)^{-\frac{n}{2 m} p+\frac{n}{4}} d s\right) .
\end{aligned}
$$

Thus, we can repeat the same arguments as in the proof of (3.3.34) to estimate these two integrals. Finally, we can conclude the proof of the statement (3.3.35) from the definition of the norm of $X(t)$. This completes the proof.

Remark 3.3.5. Let us choose formally $\omega(t) \equiv 1$. Then, we have $\Lambda(t)=\Theta(t)=F(\Lambda(t))$ and $\nu(\delta)=\vartheta(\delta)$. Let $\mu=\mu(t)$ be an arbitrary function satisfying the conditions (B1) to (B6), (C3) and (C4). Hence, choosing $\beta_{1}=2 \lambda_{0}$ and applying Theorem 3.3.2 with $m=1$ we can see that our results are related to the results of the paper [4].

Remark 3.3.6. We will discuss some examples in the case of super-exponential propagation speed. Proceeding in the same way as we did in the examples to the case of sub-exponential propagation speed, we take the derived energy estimates for the solutions to the corresponding linear Cauchy problem (3.1.1) with vanishing right-hand side and allow in the solution spaces some parameters describing a loss of decay. Therefore, we will get some flexibility when we deal with the competition between the estimates in (3.1.13) and (3.1.14) with the following examples. We remark that if we allow a loss of decay in Theorem 3.3.2, this idea does not influence the conditions of this theorem. 
Example 3.3.3. Let us choose $\lambda(t)=e^{t} e^{e^{t}}$. Then, we have

$$
\Lambda(t)=e^{e^{t}}, \quad \Theta(t)=e^{r e^{t}}, \quad 0<r<1 .
$$

Moreover, choosing

$$
\mu(t)=e^{q e^{t}}, \quad 1-r<q<2
$$

we get

$$
\rho(t)=e^{t} \mu(t)=e^{t} e^{q e^{t}} .
$$

Now we take $\Xi(t)=e^{-t} e^{\kappa e^{t}}$ with $0>\kappa \geq-\frac{q}{4}$. Then, we get

$$
F(\Lambda(t)) \simeq e^{(1+2 \kappa) e^{t}} .
$$

Now let us define the oscillating function as we constructed in Section 2.6 as follows:

$$
\omega(t)=1+\varphi_{k} \psi\left(\frac{t-t_{k}}{v_{k}}\right) \text { for all } t \in\left[t_{k}, t_{k+1}\right],
$$

where

$$
t_{k}:=e^{k}, \quad v_{k}:=e^{-k} e^{\kappa e^{k}} \leq \Delta t_{k}:=t_{k+1}-t_{k}, \quad \varphi_{k}:=e^{(r-\kappa-1) e^{k}} \leq 1 .
$$

Here $\psi \in \mathcal{C}_{0}^{M}(\mathbb{R})$ satisfies

$$
\operatorname{supp} \psi \subseteq[0,1], \quad-1<\psi(t)<1 \quad \text { and } \quad \int_{0}^{1}|\psi(t)| d t=\frac{1}{2} .
$$

Then, the oscillating function $\omega=\omega(t)$ satisfies the related estimates in condition (A2). Moreover, the stabilization condition (A3) is satisfied, because $v_{k} \varphi_{k}$ is small enough. Indeed, we have $v_{k} \varphi_{k}=e^{-k} e^{(r-1) e^{k}}<1$.

Now let us consider the case

$$
\tilde{\Phi}_{1}(s, t)=\max \left\{\frac{\lambda^{2}(t)}{\rho(t)}\left(1+B_{\lambda}(s, t)\right)^{-1+\gamma_{1}} ; \lambda(t) F(\Lambda(t))\left(F^{2}(\Lambda(t))\right)^{-1+\gamma_{2}}\right\}, s \in[\delta t, t],
$$

which appears in the proof of the last theorem in the case $j=0$ and $\ell=1$ (with $\gamma_{1}=\gamma_{2}=0$ ). Here $\gamma_{1}$ and $\gamma_{2}$ are positive constants and these constants stand for the loss of decay in comparison with the corresponding decay estimates for the time derivative of the solution $u$ to the linear Cauchy problem with vanishing right-hand side. In this case, we get a benefit of allowing a loss of decay (cf. Remark 3.3.6).

As we mentioned in the proof of the last theorem, from condition (C1) we will only consider the following two cases from the definition of the function $\Phi_{1}=\Phi_{1}(s, t)$ in (3.1.14):

Case a: $\tilde{\Phi}_{1}(s, t)=\frac{\lambda^{2}(t)}{\rho(t)}\left(1+B_{\lambda}(s, t)\right)^{-1+\gamma_{1}}$. This case implies that we have

$$
\frac{\lambda(t)}{\rho(t)}\left(1+B_{\lambda}(s, t)\right)^{-1+\gamma_{1}} \geq F(\Lambda(t))\left(F^{2}(\Lambda(t))\right)^{-1+\gamma_{2}} .
$$

If we assume $q-2 \kappa-2+2 \gamma_{2}(1+2 \kappa) \leq 0$ and $\gamma_{1} \geq 1$, then for $s \in[\delta t, t]$ it holds

$$
\left(1+e^{(2-q) e^{t}}-e^{(2-q) e^{s}}\right)^{-1+\gamma_{1}} \geq e^{\left(q-2 \kappa-2+2 \gamma_{2}(1+2 \kappa)\right) e^{t}},
$$

which shows that the first component is dominant in (3.3.51).

We remark that if we do not allow the loss of decay, namely, if we choose $\gamma_{1}=\gamma_{2}=0$, then we have

$$
e^{(2-q) e^{s}} \geq 1+e^{(2-q) e^{t}}-e^{(2 \kappa-q+2) e^{t}} .
$$


Therefore, in order to show that we have one case which implies that the first component is dominant in (3.3.51), we have to assume $2 \kappa-q+2 \geq 2-q$ from the last inequality, that is, $\kappa \geq 0$, which contradicts the choices of $\kappa$. For this reason, we allow a loss of decay in the $L^{2}-L^{2}$ estimates for the time derivative of the solution $u$ to the linear Cauchy problem with vanishing right-hand side to get the desired estimates.

Case b: $\tilde{\Phi}_{1}(s, t)=\lambda(t) F(\Lambda(t))\left(F^{2}(\Lambda(t))\right)^{-1+\gamma_{2}}$. This case implies

$$
\frac{\lambda(t)}{\rho(t)}\left(1+B_{\lambda}(s, t)\right)^{-1+\gamma_{1}} \leq F(\Lambda(t))\left(F^{2}(\Lambda(t))\right)^{-1+\gamma_{2}} .
$$

Assuming $\gamma_{2} \geq \gamma_{1}$ for the case $s \in[\delta t, t]$ it holds

$$
\left(1+e^{(2-q) e^{t}}-e^{(2-q) e^{s}}\right)^{-1+\gamma_{1}} \leq e^{\left(q-2 \kappa-2+2 \gamma_{2}(1+2 \kappa)\right) e^{t}} .
$$

Then, this shows that the second component is dominant in (3.3.51).

Hence, applying Theorem 3.3.2 we obtain $\nu(\delta)=\infty$ and

$$
\nu(\delta, t) \frac{1}{\log \Lambda(t)}=\frac{\lambda(t)}{\Lambda(t)} \frac{\Lambda(\delta t)}{\lambda(\delta t)} \frac{1}{\log \Lambda(t)}=\frac{e^{t} e^{e^{t}}}{e^{e^{t}}} \frac{e^{e^{\delta t}}}{e^{\delta t} e^{e^{\delta t}}} \frac{1}{e^{t}}=\frac{1}{e^{\delta t}} .
$$

Moreover, we have $\vartheta(\delta)=\infty$ and

$$
\vartheta(\delta, t) \frac{1}{\log \Lambda(t)}=\frac{F(\Lambda(t))}{\lambda(t) \Xi^{2}(t)} \frac{\Lambda(\delta t)}{\lambda(\delta t)} \frac{1}{\log \Lambda(t)}=\frac{e^{(1+2 \kappa) e^{t}}}{e^{t} e^{e^{t}} e^{-2 t} e^{2 \kappa e^{t}}} \frac{e^{e^{\delta t}}}{e^{\delta t} e^{e^{\delta t}}} \frac{1}{e^{t}}=\frac{1}{e^{\delta t}} .
$$

So, we have the global (in time) existence of small data solutions for

$$
\begin{cases}\frac{2}{m} \leq p & \text { if } n=1,2, \\ \frac{2}{m} \leq p \leq p_{\mathrm{GN}}(n)=\frac{n}{n-2} & \text { if } n \geq 3 .\end{cases}
$$

Example 3.3.4. We choose $\lambda(t)=e^{t} e^{e^{t}} e^{e^{e^{t}}}$. Then, we have

$$
\Lambda(t)=e^{e^{e^{t}}}, \quad \Theta(t)=e^{r e^{e^{t}}}, \quad 0<r<1 .
$$

Moreover, choosing

$$
\mu(t)=e^{q e^{e^{t}}}, \quad 1-r<q<2
$$

we get

$$
\rho(t)=e^{t} e^{e^{t}} \mu(t)=e^{t} e^{e^{t}} e^{q e^{e^{t}}} .
$$

Now we take $\Xi(t)=e^{-t} e^{-e^{t}} e^{\kappa e^{e^{t}}}$ with $0>\kappa \geq-\frac{q}{4}$. Then, we get

$$
F(\Lambda(t)) \simeq e^{(1+2 \kappa) e^{e^{t}}}
$$

Now let us define the oscillating function as we constructed in Section 2.6 as follows:

$$
\omega(t)=1+\varphi_{k} \psi\left(\frac{t-t_{k}}{v_{k}}\right) \text { for all } t \in\left[t_{k}, t_{k+1}\right],
$$

where

$$
t_{k}:=e^{e^{k}}, \quad v_{k}:=e^{-k} e^{-e^{k}} e^{\kappa e^{e^{k}}} \leq \Delta t_{k}:=t_{k+1}-t_{k}, \quad \varphi_{k}:=e^{(r-\kappa-1) e^{e^{k}}} \leq 1 .
$$

Here $\psi \in \mathcal{C}_{0}^{M}(\mathbb{R})$ satisfies

$$
\operatorname{supp} \psi \subseteq[0,1], \quad-1<\psi(t)<1 \quad \text { and } \quad \int_{0}^{1}|\psi(t)| d t=\frac{1}{2} .
$$


Then, the oscillating function $\omega=\omega(t)$ satisfies the related estimates in condition (A2). Moreover, the stabilization condition (A3) is satisfied, since $v_{k} \varphi_{k}$ is small enough. Indeed, we have $v_{k} \varphi_{k}=e^{-k} e^{-e^{k}} e^{(r-1) e^{e^{k}}}<1$.

Now let us consider the case

$$
\tilde{\Phi}_{1}(s, t)=\max \left\{\frac{\lambda^{2}(t)}{\rho(t)}\left(1+B_{\lambda}(s, t)\right)^{-1+\gamma_{1}} ; \lambda(t) F(\Lambda(t))\left(F^{2}(\Lambda(t))\right)^{-1+\gamma_{2}}\right\}, s \in[\delta t, t] .
$$

As we did in the proof of Theorem 3.3.2 we will distinguish between two cases only.

Case a: $\tilde{\Phi}_{1}(s, t)=\frac{\lambda^{2}(t)}{\rho(t)}\left(1+B_{\lambda}(s, t)\right)^{-1+\gamma_{1}}$. This case implies that we have

$$
\frac{\lambda(t)}{\rho(t)}\left(1+B_{\lambda}(s, t)\right)^{-1+\gamma_{1}} \geq F(\Lambda(t))\left(F^{2}(\Lambda(t))\right)^{-1+\gamma_{2}} .
$$

If we assume $q-2 \kappa-2+2 \gamma_{2}(1+2 \kappa) \leq 0$ and $\gamma_{1} \geq 1$, then for $s \in[\delta t, t]$ it holds

$$
\left(1+e^{(2-q) e^{e^{t}}}-e^{(2-q) e^{e^{s}}}\right)^{-1+\gamma_{1}} \geq e^{\left(q-2 \kappa-2+2 \gamma_{2}(1+2 \kappa)\right) e^{e^{t}}} .
$$

Therefore, this shows that the first component is dominant in (3.3.52).

Case b: $\tilde{\Phi}_{1}(s, t)=\lambda(t) F(\Lambda(t))\left(F^{2}(\Lambda(t))\right)^{-1+\gamma_{2}}$. This case implies

$$
\frac{\lambda(t)}{\rho(t)}\left(1+B_{\lambda}(s, t)\right)^{-1+\gamma_{1}} \leq F(\Lambda(t))\left(F^{2}(\Lambda(t))\right)^{-1+\gamma_{2}} .
$$

Assuming $\gamma_{2} \geq \gamma_{1}$ in the case $s \in[\delta t, t]$ it holds

$$
\left(1+e^{(2-q) e^{e^{t}}}-e^{(2-q) e^{e^{s}}}\right)^{-1+\gamma_{1}} \leq e^{\left(q-2 \kappa-2+2 \gamma_{2}(1+2 \kappa)\right) e^{e^{t}}} .
$$

Then, this shows that the second component is dominant in (3.3.52).

Applying Theorem 3.3.2 we obtain $\nu(\delta)=\infty$ and

$$
\nu(\delta, t) \frac{1}{\log \Lambda(t)}=\frac{e^{t} e^{e^{t}} e^{e^{e^{t}}}}{e^{e^{e^{t}}}} \frac{e^{e^{e^{\delta t}}}}{e^{\delta t} e^{e^{\delta t}} e^{e^{e^{\delta t}}}} \frac{1}{e^{e^{t}}}=\frac{e^{t}}{e^{\delta t} e^{e^{\delta t}}} .
$$

Moreover, we have $\vartheta(\delta)=\infty$ and

$$
\vartheta(\delta, t) \frac{1}{\log \Lambda(t)}=\frac{e^{(1+2 \kappa) e^{e^{t}}}}{e^{t} e^{e^{t}} e^{e^{e^{t}}} e^{-2 t} e^{-2 e^{t}} e^{2 \kappa e^{e^{t}}}} \frac{e^{e^{e^{\delta t}}}}{e^{\delta t} e^{e^{\delta t}} e^{e^{\delta t}}} \frac{1}{e^{e^{t}}}=\frac{e^{t}}{e^{\delta t} e^{e^{\delta t}}} .
$$

So, we have the global (in time) existence of small data solutions for

$$
\begin{cases}\frac{2}{m} \leq p & \text { if } n=1,2 \\ \frac{2}{m} \leq p \leq p_{\mathrm{GN}}(n)=\frac{n}{n-2} & \text { if } n \geq 3\end{cases}
$$

\subsection{High regular data}

In Sections 3.3.1 and 3.3.2 we discussed the Cauchy problem (3.1.1), that is,

$$
\begin{cases}u_{t t}-\lambda^{2}(t) \omega^{2}(t) \Delta u+\rho(t) \omega(t) u_{t}=|u|^{p}, & (t, x) \in[0, \infty) \times \mathbb{R}^{n} \\ u(0, x)=u_{0}(x), \quad u_{t}(0, x)=u_{1}(x), & x \in \mathbb{R}^{n},\end{cases}
$$


taking the data from the energy space $H^{1} \times L^{2}$ with additional $L^{m}$ regularity, $m \in[1,2)$, in the case of sub-exponential and case of super-exponential propagation speed, respectively. Therefore, in order to estimate the nonlinear term in (3.3.6) we derived for the exponent $p$ the conditions $p \geq \frac{2}{m}$ and $p \leq \frac{n}{n-2}$ if $n \geq 3$ from the applications of the classical GagliardoNirenberg inequality with the norms $L^{m}$ and $L^{2}$, respectively.

The goal of this section is to study again the Cauchy problem (3.1.1), but now with suitable higher regularity for the data

$$
\left(u_{0}, u_{1}\right) \in H^{\sigma} \times H^{\sigma-1}, \quad \sigma \in\left(1,1+\frac{n}{2}\right]
$$

with an additional regularity $L^{m}, m \in[1,2)$. In particular, by assuming higher regularity for the data we want to weaken the condition on $p$ from above, that is, the data will be considered in $H^{\sigma} \times H^{\sigma-1}$, with $\sigma>1$ and additional $L^{m}$ regularity.

In the present section we shall use fractional tools from Harmonic Analysis such as fractional Gagliargo-Nirenberg inequality, the fractional chain rule and the fractional Leibniz rule, which are explained in Appendix A.7. These tools play an important role to prove our global (in time) existence results (more details can be found in [28]).

\subsubsection{Case of sub-exponential propagation speed}

We define the following parameters:

$$
\begin{aligned}
& \hat{p}_{1, m}(n):=1+\left(\frac{\sigma+1}{2}-\frac{\beta_{1}}{2+R}\right) \frac{2 m}{n} \quad \text { for } n \geq 1, \\
& \hat{p}_{2, m}(n):=1+\left(1-\frac{\delta}{\nu(\delta)} \frac{\beta_{1}}{2+R}\right) \frac{2 m}{n} \quad \text { for } n \geq 1, \\
& \hat{p}_{3, m}(n) \\
& \quad:=\max \left\{\frac{m}{2}+\frac{1-\frac{m}{2}}{1-\frac{2+R}{2-\mu_{1}}+\frac{2+R}{2-\mu_{1}} \frac{\delta}{\nu(\delta)}}+\left(\frac{\frac{\sigma+1}{2}-\frac{\beta_{1}}{2+R} \frac{\delta}{\nu(\delta)}}{1-\frac{2+R}{2-\mu_{1}}+\frac{2+R}{2-\mu_{1}} \frac{\delta}{\nu(\delta)}}\right) \frac{2 m}{n} ; 1+\left(1-\frac{\delta}{\vartheta(\delta)} \frac{\beta_{1}}{2}\right) \frac{2 m}{n}\right\} \\
& \quad \text { for } \quad n \geq 1,
\end{aligned}
$$

where we set

$$
\hat{p}_{m}(n):=\max \left\{\hat{p}_{1, m}(n) ; \hat{p}_{2, m}(n) ; \hat{p}_{3, m}(n)\right\} .
$$

We state the main result for the case of sub-exponential propagation speed with suitable higher regularity of the data in the following way.

Theorem 3.4.1. Let us assume that the Hypotheses (A1) to (A5), (B1) to (B6), (C1), (C2) and (C4) are satisfied. Let $\nu(\delta) \lesssim 1$ and $\vartheta(\delta) \lesssim 1$, where $\nu=\nu(\delta)$ and $\vartheta=\vartheta(\delta)$ are defined in (3.1.2) and (3.1.3), respectively. We suppose that the data $\left(u_{0}, u_{1}\right) \in \mathcal{D}_{m}^{\sigma}$ with $\sigma \in\left(1,1+\frac{n}{2}\right]$ and $m \in[1,2)$, and that the exponent $p$ satisfies

$$
p>\max \left\{\lceil\sigma\rceil ; \frac{2}{m}\right\} \quad \text { and } \quad \hat{p}_{m}(n)<p \leq 1+\frac{2}{n-2 \sigma} \quad \text { if } \quad n>2 \sigma .
$$

Moreover, we assume that the following condition holds:

$$
\frac{\beta_{1}}{\frac{n}{2}\left(\frac{1}{m}-\frac{1}{2}\right)}>\max \left\{(2+R) \frac{\nu(\delta)}{\delta} ; 2 \frac{\vartheta(\delta)}{\delta}\right\}
$$

where the parameter $\beta_{1}$ is from condition $(\boldsymbol{C} 4)$ and the parameter $R$ is from (3.1.17).

Then, there exists a constant $\varepsilon_{0}>0$ such that for all $\left(u_{0}, u_{1}\right) \in \mathcal{D}_{m}^{\sigma}$ with $\left\|\left(u_{0}, u_{1}\right)\right\|_{\mathcal{D}_{m}^{\sigma}} \leq \varepsilon_{0}$, there is a uniquely determined energy solution

$$
u \in \mathcal{C}\left([0, \infty), H^{\sigma}\right) \cap \mathcal{C}^{1}\left([0, \infty), H^{\sigma-1}\right)
$$


to the Cauchy problem (3.1.1).

Furthermore, there exists a constant $C>0$ such that the solution satisfies the estimates

$$
\begin{aligned}
&\|u(t, \cdot)\|_{L^{2}} \leq C\left(1+B_{\lambda}(0, t)\right)^{-\frac{n}{2}\left(\frac{1}{m}-\frac{1}{2}\right)}\left\|\left(u_{0}, u_{1}\right)\right\|_{\mathcal{D}_{m}^{\sigma}}, \\
&\left\||D|^{\sigma} u(t, \cdot)\right\|_{L^{2}} \leq C\left(1+B_{\lambda}(0, t)\right)^{-\frac{n}{2}\left(\frac{1}{m}-\frac{1}{2}\right)-\frac{\sigma}{2}}\left\|\left(u_{0}, u_{1}\right)\right\|_{\mathcal{D}_{m}^{\sigma}}, \\
&\left\|u_{t}(t, \cdot)\right\|_{L^{2}} \leq C \max \left\{\frac{\lambda^{2}(t)}{\rho(t)}\left(1+B_{\lambda}(0, t)\right)^{-\frac{n}{2}\left(\frac{1}{m}-\frac{1}{2}\right)-1} ;\right. \\
&\left.\lambda(t) F(\Lambda(t))\left(F^{2}(\Lambda(t))\right)^{-\frac{n}{2}\left(\frac{1}{m}-\frac{1}{2}\right)-1}\right\}\left\|\left(u_{0}, u_{1}\right)\right\|_{\mathcal{D}_{m}^{\sigma}}, \\
&\left\||D|^{\sigma-1} u_{t}(t, \cdot)\right\|_{L^{2}} \leq C \max \left\{\frac{\lambda^{2}(t)}{\rho(t)}\left(1+B_{\lambda}(0, t)\right)^{-\frac{n}{2}\left(\frac{1}{m}-\frac{1}{2}\right)-\frac{\sigma-1}{2}-1} ;\right. \\
&\left.\lambda(t) F(\Lambda(t))\left(F^{2}(\Lambda(t))\right)^{-\frac{n}{2}\left(\frac{1}{m}-\frac{1}{2}\right)-\frac{\sigma-1}{2}-1}\right\}\left\|\left(u_{0}, u_{1}\right)\right\|_{\mathcal{D}_{m}^{\sigma}} .
\end{aligned}
$$

Proof. Let us proceed analogously to the proof of Theorem 3.3.1. We introduce the family of spaces

$$
X(t)=\mathcal{C}\left([0, t], H^{\sigma}\right) \cap \mathcal{C}^{1}\left([0, t], H^{\sigma-1}\right)
$$

with the norm

$$
\begin{aligned}
\|u(\tau, \cdot)\|_{X(t)}=\sup _{0 \leq \tau \leq t}[ & \left(1+B_{\lambda}(0, \tau)\right)^{\frac{n}{2}\left(\frac{1}{m}-\frac{1}{2}\right)}\|u(\tau, \cdot)\|_{L^{2}} \\
& +\left(1+B_{\lambda}(0, \tau)\right)^{\frac{n}{2}\left(\frac{1}{m}-\frac{1}{2}\right)+\frac{\sigma}{2}}\left\||D|^{\sigma} u(\tau, \cdot)\right\|_{L^{2}} \\
& \left.+\Phi_{1, m}^{-1}(0, \tau)\left\|u_{t}(\tau, \cdot)\right\|_{L^{2}}+\Phi_{2, m}^{-1}(0, \tau)\left\||D|^{\sigma-1} u_{t}(\tau, \cdot)\right\|_{L^{2}}\right] .
\end{aligned}
$$

Here $\Phi_{1, m}=\Phi_{1, m}(0, \tau)$ and $\Phi_{2, m}=\Phi_{2, m}(0, \tau)$ are defined in (3.1.13) and (3.1.15), respectively for $s=0$ and $t=\tau$.

We consider once again the integral operator $N$ which is defined in (3.2.1). We know that the space $X(t)$ is complete. For this reason, by standard arguments the uniqueness, local and global (in time) existence can be concluded from the following two inequalities:

$$
\begin{aligned}
\|N u\|_{X(t)} & \lesssim C_{0}\left\|\left(u_{0}, u_{1}\right)\right\|_{\mathcal{D}_{m}^{\sigma}}+C_{1}(t)\|u\|_{X(t)}^{p}, \\
\|N u-N v\|_{X(t)} & \lesssim C_{2}(t)\|u-v\|_{X(t)}\left(\|u\|_{X(t)}^{p-1}+\|v\|_{X(t)}^{p-1}\right),
\end{aligned}
$$

where $C_{1}(t), C_{2}(t) \rightarrow 0$ when $t \rightarrow 0$ and $C_{1}(t), C_{2}(t) \leq C$ for all $t \in[0, \infty)$.

Let us start with the proof of inequality (3.4.4). We see that, if we apply Theorem 3.1.2 for the solution

$$
u^{\operatorname{lin}}(t, x)=K_{0}(t, 0, x) *_{(x)} u_{0}(x)+K_{1}(t, 0, x) *_{(x)} u_{1}(x),
$$

of the linear Cauchy problem with vanishing right-hand side, we immediately obtain

$$
\left\|u^{\text {lin }}\right\|_{X(t)} \lesssim\left\|\left(u_{0}, u_{1}\right)\right\|_{D_{m}^{\sigma}} .
$$

In order to complete the proof of (3.4.4) let us continue with the "nonlinear part" of the solution $u$, namely

$$
u^{\mathrm{nl}}(t, x)=\int_{0}^{t} K_{1}(t, s, x) *(x)|u(s, x)|^{p} d s
$$

as in the proof of Theorem 3.3.1. For this reason we have to control the norms

$$
\left\|u^{\mathrm{nl}}(t, \cdot)\right\|_{L^{2}}, \quad\left\||D|^{\sigma} u^{\mathrm{nl}}(t, \cdot)\right\|_{L^{2}}, \quad\left\|u_{t}^{\mathrm{nl}}(t, \cdot)\right\|_{L^{2}} \quad \text { and } \quad\left\||D|^{\sigma-1} u_{t}^{\mathrm{nl}}(t, \cdot)\right\|_{L^{2}} \cdot
$$

Let us begin to estimate the norm $\left\||D|^{\sigma} u^{\mathrm{nl}}(t, \cdot)\right\|_{L^{2}}$. We have

$$
\left\||D|^{\sigma} u^{\mathrm{nl}}(t, \cdot)\right\|_{L^{2}} \lesssim \int_{0}^{t}\left\||D|^{\sigma}\left(K_{1}(t, s, x) *_{(x)}|u(s, x)|^{p}\right)\right\|_{L^{m} \cap L^{2}} d s .
$$


In order to control the norm $\left\||D|^{\sigma} u^{\mathrm{nl}}(t, \cdot)\right\|_{L^{2}}$ we use the $\left(L^{m} \cap L^{2}\right)-L^{2}$ estimates if $s \in[0, \delta t]$ and $L^{2}-L^{2}$ estimates if $s \in[\delta t, t]$ from Theorem 3.1.1. Therefore, we have the following estimate:

$$
\begin{aligned}
\left\||D|^{\sigma} u^{\mathrm{nl}}(t, \cdot)\right\|_{L^{2}} \lesssim & \int_{0}^{\delta t} \frac{\Lambda(s)}{\lambda(s)}\left(1+B_{\lambda}(s, t)\right)^{-\frac{n}{2}\left(\frac{1}{m}-\frac{1}{2}\right)-\frac{\sigma}{2}}\left\||u(s, \cdot)|^{p}\right\|_{L^{m} \cap L^{2} \cap \dot{H}^{\sigma-1}} d s \\
& +\int_{\delta t}^{t} \frac{\Lambda(s)}{\lambda(s)}\left(1+B_{\lambda}(s, t)\right)^{-\frac{\sigma}{2}}\left\||u(s, \cdot)|^{p}\right\|_{L^{2} \cap \dot{H}^{\sigma-1}} d s .
\end{aligned}
$$

Now we have to estimate the following norms:

$$
\left\||u(s, \cdot)|^{p}\right\|_{L^{m}}, \quad\left\||u(s, \cdot)|^{p}\right\|_{L^{2}} \text { and } \quad\left\||u(s, \cdot)|^{p}\right\|_{\dot{H}^{\sigma-1}} .
$$

For the first two norms, by using the classical Gagliardo-Nirenberg inequality we obtain

$$
\begin{aligned}
\left\||u(s, \cdot)|^{p}\right\|_{L^{m}} & =\|u(s, \cdot)\|_{L^{m p}}^{p} \lesssim\left\||D|^{\sigma} u(s, \cdot)\right\|_{L^{2}}^{p \theta_{\sigma}(m p)}\|u(s, \cdot)\|_{L^{2}}^{p\left(1-\theta_{\sigma}(m p)\right)}, \\
\left\||u(s, \cdot)|^{p}\right\|_{L^{2}} & =\|u(s, \cdot)\|_{L^{2 p}}^{p} \lesssim\left\||D|^{\sigma} u(s, \cdot)\right\|_{L^{2}}^{p \theta_{\sigma}(2 p)}\|u(s, \cdot)\|_{L^{2}}^{p\left(1-\theta_{\sigma}(2 p)\right)},
\end{aligned}
$$

where $\theta_{\sigma}(m p)=\frac{n}{\sigma}\left(\frac{1}{2}-\frac{1}{m p}\right) \in[0,1]$ and $\theta_{\sigma}(2 p)=\frac{n}{\sigma}\left(\frac{1}{2}-\frac{1}{2 p}\right) \in[0,1]$. Thus, we get the following conditions for the exponent $p$ :

$$
\begin{cases}\frac{2}{m} \leq p & \text { if } n \leq 2 \sigma, \\ \frac{2}{m} \leq p \leq \frac{n}{n-2 \sigma} & \text { if } n>2 \sigma .\end{cases}
$$

By using the norm of the solution space $X(t)$ we get

$$
\begin{aligned}
\|u(s, \cdot)\|_{L^{m p}}^{p} & \lesssim\left(1+B_{\lambda}(0, s)\right)^{-\frac{n}{2 m} p+\frac{n}{2 m}}\|u\|_{X(s)}^{p}, \\
\|u(s, \cdot)\|_{L^{2 p}}^{p} & \lesssim\left(1+B_{\lambda}(0, s)\right)^{-\frac{n}{2 m} p+\frac{n}{4}}\|u\|_{X(s)}^{p} .
\end{aligned}
$$

Now we want to estimate the norm $\left\||u(s, \cdot)|^{p}\right\|_{\dot{H}^{\sigma-1}}$. Using the fractional chain rule from Proposition A.7.7, we get

$$
\left\||D|^{\sigma-1}|u(s, \cdot)|^{p}\right\|_{L^{2}} \lesssim\|u(s, \cdot)\|_{L^{q_{1}}}^{p-1}\left\||D|^{\sigma-1} u(s, \cdot)\right\|_{L^{q_{2}}} \quad \text { for } \quad p>\lceil\sigma-1\rceil,
$$

with

$$
\frac{p-1}{q_{1}}+\frac{1}{q_{2}}=\frac{1}{2}
$$

We can estimate the two norms that appear on the right-hand side of the previous inequality by using the classical (cf. Corollary A.1.3) and fractional Gagliardo-Nirenberg inequality (cf. Corollary A.7.3), respectively. Therefore, we obtain

$$
\begin{aligned}
\||u(s, \cdot)|\|_{L^{q_{1}}} & \lesssim\left\||D|^{\sigma} u(s, \cdot)\right\|_{L^{2}}^{\theta_{\sigma}\left(q_{1}\right)}\|u(s, \cdot)\|_{L^{2}}^{1-\theta_{\sigma}\left(q_{1}\right)} \\
& \lesssim\left(1+B_{\lambda}(0, s)\right)^{-\frac{n}{2 m}+\frac{n}{2 q_{1}}}\|u\|_{X(s)}, \\
\left\||D|^{\sigma-1} u(s, \cdot)\right\|_{L^{q_{2}}} & \lesssim\left\||D|^{\sigma} u(s, \cdot)\right\|_{L^{2}-\theta^{2}\left(q_{2}\right)}^{\theta_{\sigma-1}}\|u(s, \cdot)\|_{L^{2}}^{1-\theta_{\sigma-1, \sigma}\left(q_{2}\right)} \\
& \lesssim\left(1+B_{\lambda}(0, s)\right)^{-\frac{n}{2 m}+\frac{n}{2 q_{2}}-\frac{\sigma-1}{2}}\|u\|_{X(s)},
\end{aligned}
$$

where $\theta_{\sigma}\left(q_{1}\right)=\frac{n}{\sigma}\left(\frac{1}{2}-\frac{1}{q_{1}}\right) \in[0,1]$ and $\theta_{\sigma-1, \sigma}\left(q_{2}\right)=\frac{n}{\sigma}\left(\frac{1}{2}-\frac{1}{q_{2}}+\frac{\sigma-1}{n}\right) \in\left[\frac{\sigma-1}{\sigma}, 1\right]$. Consequently, by using (3.4.9) we get

$$
\begin{aligned}
\left\||u(s, \cdot)|^{p}\right\|_{\dot{H}^{\sigma-1}} & \lesssim\left(1+B_{\lambda}(0, s)\right)^{\left(-\frac{n}{2 m}+\frac{n}{2 q_{1}}\right)(p-1)}\left(1+B_{\lambda}(0, s)\right)^{-\frac{n}{2 m}+\frac{n}{2 q_{2}}-\frac{\sigma-1}{2}}\|u\|_{X(s)}^{p} \\
& =\left(1+B_{\lambda}(0, s)\right)^{-\frac{n}{2 m} p+\frac{n}{4}-\frac{\sigma-1}{2}}\|u\|_{X(s)}^{p} .
\end{aligned}
$$


The requirements on $\theta_{\sigma}\left(q_{1}\right)$ and $\theta_{\sigma-1, \sigma}\left(q_{2}\right)$ imply the following conditions for $q_{1}$ and $q_{2}$ :

$$
\left\{\begin{array} { l l } 
{ 2 \leq q _ { 1 } } & { \text { if } n \leq 2 \sigma , } \\
{ 2 \leq q _ { 1 } \leq \frac { 2 n } { n - 2 \sigma } } & { \text { if } n > 2 \sigma , }
\end{array} \quad \left\{\begin{array}{ll}
2 \leq q_{2} & \text { if } n \leq 2, \\
2 \leq q_{2} \leq \frac{2 n}{n-2} & \text { if } n>2 .
\end{array}\right.\right.
$$

Then, we obtain for the sum $\frac{p-1}{q_{1}}+\frac{1}{q_{2}}$ the following restrictions:

$$
\begin{cases}\frac{p-1}{q_{1}}+\frac{1}{q_{2}} \in\left[0, \frac{p}{2}\right] & \text { if } n \leq 2, \\ \frac{p-1}{q_{1}}+\frac{1}{q_{2}} \in\left[\frac{n-2}{2 n}, \frac{p}{2}\right] & \text { if } 2<n \leq 2 \sigma, \\ \frac{p-1}{q_{1}}+\frac{1}{q_{2}} \in\left[\frac{(n-2 \sigma)(p-1)}{2 n}+\frac{n-2}{2 n}, \frac{p}{2}\right] & \text { if } n>2 \sigma .\end{cases}
$$

The conditions on $q_{1}$ and $q_{2}$ can be written in the following way:

$$
\left\{\begin{array} { l l } 
{ \frac { 1 } { q _ { 1 } } \in ( 0 , \frac { 1 } { 2 } ] } & { \text { if } n \leq 2 \sigma , } \\
{ \frac { 1 } { q _ { 1 } } \in [ \frac { 1 } { 2 } - \frac { \sigma } { n } , \frac { 1 } { 2 } ] } & { \text { if } n > 2 \sigma , }
\end{array} \quad \left\{\begin{array}{ll}
\frac{1}{q_{2}} \in\left(0, \frac{1}{2}\right] & \text { if } n \leq 2, \\
\frac{1}{q_{2}} \in\left[\frac{1}{2}-\frac{1}{n}, \frac{1}{2}\right] & \text { if } n>2 .
\end{array}\right.\right.
$$

Since the relation $\frac{1}{q_{2}}=\frac{1}{2}-\frac{p-1}{q_{1}}$, we can write the condition on $q_{1}$ equivalently as a condition on $q_{2}$, namely,

$$
\begin{cases}\frac{1}{q_{2}} \in\left[1-\frac{p}{2}, \frac{1}{2}\right) & \text { if } n \leq 2 \sigma, \\ \frac{1}{q_{2}} \in\left[1-\frac{p}{2}, 1-\frac{\sigma}{n}+\left(\frac{\sigma}{n}-\frac{1}{2}\right) p\right] & \text { if } n>2 \sigma .\end{cases}
$$

We should point out that the interval for $\frac{1}{q_{2}}$ is not empty, since $p>1$. Hence, in order to guarantee the existence of $q_{1}$ and $q_{2}$ it is sufficient to intersect the two intervals for $q_{2}$ to become non-empty.

Firstly, let us consider the case $n>2 \sigma$. In this case, taking account of the intervals of $q_{2}$, we should verify that

$$
\left[\frac{1}{2}-\frac{1}{n}, \frac{1}{2}\right] \cap\left[1-\frac{p}{2}, 1-\frac{\sigma}{n}+\left(\frac{\sigma}{n}-\frac{1}{2}\right) p\right] \neq \emptyset
$$

Therefore, we have

$$
\frac{1}{2}-\frac{1}{n} \in\left[1-\frac{p}{2}, 1-\frac{\sigma}{n}+\left(\frac{\sigma}{n}-\frac{1}{2}\right) p\right], \text { that is, } 1+\frac{2}{n} \leq p \leq 1+\frac{2}{n-2 \sigma},
$$

or

$$
1-\frac{p}{2} \in\left[\frac{1}{2}-\frac{1}{n}, \frac{1}{2}\right], \text { that is, } 1<p \leq 1+\frac{2}{n} .
$$

These two conditions for $p$ imply

$$
1<p \leq 1+\frac{2}{n-2 \sigma}
$$

which is one of the assumptions of the theorem.

Now, we consider the cases $2<n \leq 2 \sigma$ and $n \leq 2$. In these cases we should verify that

$$
\left[1-\frac{p}{2}, \frac{1}{2}\right) \cap\left[\frac{1}{2}-\frac{1}{n}, \frac{1}{2}\right] \neq \emptyset \quad \text { and }\left[1-\frac{p}{2}, \frac{1}{2}\right) \cap\left(0, \frac{1}{2}\right] \neq \emptyset
$$

respectively. Therefore, these two conditions are satisfied if and only if $p>1$.

Consequently, there exist suitable parameters $q_{1}$ and $q_{2}$.

Summarizing, from (3.4.7), (3.4.8) and (3.4.10), and $\|u\|_{X(s)} \leq\|u\|_{X(t)}$ for $0 \leq s \leq t$ we arrive at the estimate

$$
\begin{aligned}
\left\||D|^{\sigma} u^{\mathrm{nl}}(t, \cdot)\right\|_{L^{2}} \lesssim & \|u\|_{X(t)}^{p} \underbrace{\int_{0}^{\delta t} \frac{\Lambda(s)}{\lambda(s)}\left(1+B_{\lambda}(s, t)\right)^{-\frac{n}{2}\left(\frac{1}{m}-\frac{1}{2}\right)-\frac{\sigma}{2}}\left(1+B_{\lambda}(0, s)\right)^{-\frac{n}{2 m} p+\frac{n}{2 m}} d s}_{\mathbf{G}} \\
& +\|u\|_{X(t)}^{p} \underbrace{\int_{\delta t}^{t} \frac{\Lambda(s)}{\lambda(s)}\left(1+B_{\lambda}(s, t)\right)^{-\frac{\sigma}{2}}\left(1+B_{\lambda}(0, s)\right)^{-\frac{n}{2 m} p+\frac{n}{4}} d s}_{\mathbf{H}} .
\end{aligned}
$$


Let us consider the integral $\mathbf{G}$. In this case we have

$$
\mathbf{G} \lesssim\left(1+B_{\lambda}(0, t)\right)^{-\frac{n}{2}\left(\frac{1}{m}-\frac{1}{2}\right)-\frac{\sigma}{2}} \underbrace{\int_{0}^{\delta t} \frac{\Lambda(s)}{\lambda(s)}\left(1+B_{\lambda}(0, s)\right)^{-\frac{n}{2 m} p+\frac{n}{2 m}} d s}_{G_{1}(t)},
$$

where we used (3.1.18). We have already shown in the proof of Theorem 3.2.1 that the condition $p>1+\left(1-\frac{\beta_{1}}{2+R}\right) \frac{2 m}{n}$ guarantees that the integral term $A_{1}=A_{1}(t)$, which is given in (3.2.11) and coincides with $G_{1}=G_{1}(t)$, remains bounded. For this reason, since we have

$$
p>1+\left(\frac{\sigma+1}{2}-\frac{\beta_{1}}{2+R}\right) \frac{2 m}{n}>1+\left(1-\frac{\beta_{1}}{2+R}\right) \frac{2 m}{n},
$$

by the condition $p>\hat{p}_{1, m}(n)$ we may conclude the estimate

$$
\mathbf{G} \lesssim\left(1+B_{\lambda}(0, t)\right)^{-\frac{n}{2}\left(\frac{1}{m}-\frac{1}{2}\right)-\frac{\sigma}{2}} .
$$

Now we consider the integral $\mathbf{H}$. Introducing $\alpha_{2}(p):=\frac{n}{2 m} p-\frac{n}{4}$ we have

$$
\begin{aligned}
\mathbf{H}= & -\int_{\delta t}^{t} \frac{\mu(s)}{\lambda^{2}(s)}\left(1+B_{\lambda}(0, s)\right)^{-\alpha_{2}(p)}\left(1+B_{\lambda}(s, t)\right)^{-\frac{\sigma}{2}} d\left(1+B_{\lambda}(s, t)\right) \\
= & \left.\frac{-1}{1-\frac{\sigma}{2}} \frac{\mu(s)}{\lambda^{2}(s)}\left(1+B_{\lambda}(0, s)\right)^{-\alpha_{2}(p)}\left(1+B_{\lambda}(s, t)\right)^{-\frac{\sigma}{2}+1}\right|_{\delta t} ^{t} \\
& +\frac{1}{1-\frac{\sigma}{2}} \int_{\delta t}^{t}\left(1+B_{\lambda}(s, t)\right)^{-\frac{\sigma}{2}+1} d\left(\frac{\mu(s)}{\lambda^{2}(s)}\left(1+B_{\lambda}(0, s)\right)^{-\alpha_{2}(p)}\right) .
\end{aligned}
$$

We shall distinguish between the following cases:

Case 1: $-\frac{\sigma}{2}+1>0$. It follows

$$
\begin{aligned}
\mathbf{H} \lesssim & -\frac{\mu(t)}{\lambda^{2}(t)}\left(1+B_{\lambda}(0, t)\right)^{-\alpha_{2}(p)}+\frac{\mu(\delta t)}{\lambda^{2}(\delta t)}\left(1+B_{\lambda}(0, \delta t)\right)^{-\alpha_{2}(p)}\left(1+B_{\lambda}(\delta t, t)\right)^{-\frac{\sigma}{2}+1} \\
& +\int_{\delta t}^{t} \frac{\mu(s)}{\lambda^{2}(s)}\left(1+B_{\lambda}(0, s)\right)^{-\alpha_{2}(p)}\left(1+B_{\lambda}(s, t)\right)^{-\frac{\sigma}{2}+1} \frac{d\left(1+B_{\lambda}(0, s)\right)}{1+B_{\lambda}(0, s)}
\end{aligned}
$$

where we used Lemma 3.1.5. So, we have

$$
\begin{aligned}
\left(1+B_{\lambda}(0, t)\right)^{\frac{n}{2}\left(\frac{1}{m}-\frac{1}{2}\right)+\frac{\sigma}{2}} \mathbf{H} & \\
\lesssim & \underbrace{\frac{\mu(\delta t)}{\lambda^{2}(\delta t)}\left(1+B_{\lambda}(0, \delta t)\right)^{-\alpha_{2}(p)}\left(1+B_{\lambda}(0, t)\right)^{\frac{n}{2}\left(\frac{1}{m}-\frac{1}{2}\right)+1}}_{H_{1}(t)} \\
& +\underbrace{\int_{\delta t}^{t} \frac{\mu(s)}{\lambda^{2}(s)}\left(1+B_{\lambda}(0, s)\right)^{-\alpha_{2}(p)}\left(1+B_{\lambda}(0, t)\right)^{\frac{n}{2}\left(\frac{1}{m}-\frac{1}{2}\right)+1} \frac{d\left(1+B_{\lambda}(0, s)\right)}{1+B_{\lambda}(0, s)}}_{H_{2}(t)} .
\end{aligned}
$$

In order to show that $H_{1}=H_{1}(t)$ and $H_{2}=H_{2}(t)$ are uniformly bounded we suppose that there exists a positive constant $\varepsilon$ such that we have

$$
\frac{\mu(\delta t)}{\lambda^{2}(\delta t)}\left(1+B_{\lambda}(0, \delta t)\right)^{-\alpha_{2}(p)}\left(1+B_{\lambda}(0, t)\right)^{\frac{n}{2}\left(\frac{1}{m}-\frac{1}{2}\right)+1} \lesssim\left(1+B_{\lambda}(0, \delta t)\right)^{-\varepsilon} .
$$

Employing (3.1.19), from the previous estimate we have

$$
\begin{aligned}
& \frac{\mu(\delta t)}{\lambda^{2}(\delta t)}\left(1+B_{\lambda}(0, \delta t)\right)^{-\alpha_{2}(p)+\varepsilon}\left(1+B_{\lambda}(0, t)\right)^{\frac{n}{2}\left(\frac{1}{m}-\frac{1}{2}\right)+1} \\
& \quad \leq \frac{\mu(\delta t)}{\lambda^{2}(\delta t)}\left(1+B_{\lambda}(0, t)\right)^{\frac{n}{2}\left(\frac{1}{m}-\frac{1}{2}\right)+1-\alpha_{2}(p)+\varepsilon}\left(\frac{\Lambda(\delta t)}{\Lambda(t)}\right)^{(2+R)\left(-\alpha_{2}(p)+\varepsilon\right)}:=\mathcal{H}_{1}(t) .
\end{aligned}
$$


Analogously as we did in the proof of Theorem 3.2.1 for deriving the decreasing behavior of the function $\mathcal{B}_{1}=\mathcal{B}_{1}(t)$, which is given in (3.2.15), we may conclude that $\mathcal{H}_{1}^{\prime}(t)<0$ by $p>\hat{p}_{3, m}(n)$. This gives the expected estimate for $H_{1}=H_{1}(t)$. Moreover, using (3.4.16) we obtain the desired estimate for $H_{2}=H_{2}(t)$, as well.

Case 2: $-\frac{\sigma}{2}+1<0$. In this case it holds

$$
\begin{aligned}
\mathbf{H} \lesssim & \frac{\mu(t)}{\lambda^{2}(t)}\left(1+B_{\lambda}(0, t)\right)^{-\alpha_{2}(p)}-\frac{\mu(\delta t)}{\lambda^{2}(\delta t)}\left(1+B_{\lambda}(0, \delta t)\right)^{-\alpha_{2}(p)}\left(1+B_{\lambda}(\delta t, t)\right)^{-\frac{\sigma}{2}+1} \\
& +\int_{\delta t}^{t} \frac{\mu(s)}{\lambda^{2}(s)}\left(1+B_{\lambda}(0, s)\right)^{-\alpha_{2}(p)}\left(1+B_{\lambda}(s, t)\right)^{-\frac{\sigma}{2}+1} \frac{d\left(1+B_{\lambda}(0, s)\right)}{1+B_{\lambda}(0, s)}
\end{aligned}
$$

where we used again Lemma 3.1.5. Then, we have

$$
\begin{aligned}
\left(1+B_{\lambda}(0, t)\right)^{\frac{n}{2}\left(\frac{1}{m}-\frac{1}{2}\right)+\frac{\sigma}{2}} \mathbf{H} & \\
\lesssim & \underbrace{\frac{\mu(t)}{\lambda^{2}(t)}\left(1+B_{\lambda}(0, t)\right)^{-\alpha_{2}(p)+\frac{n}{2}\left(\frac{1}{m}-\frac{1}{2}\right)+\frac{\sigma}{2}}}_{H_{3}(t)} \\
& +\underbrace{\int_{\delta t}^{t} \frac{\mu(s)}{\lambda^{2}(s)}\left(1+B_{\lambda}(0, s)\right)^{-\alpha_{2}(p)}\left(1+B_{\lambda}(0, t)\right)^{\frac{n}{2}\left(\frac{1}{m}-\frac{1}{2}\right)+\frac{\sigma}{2}} \frac{d\left(1+B_{\lambda}(0, s)\right)}{1+B_{\lambda}(0, s)}}_{H_{4}(t)} .
\end{aligned}
$$

Using $\alpha_{2}(p)=\alpha_{1}(p)+\frac{n}{2}\left(\frac{1}{m}-\frac{1}{2}\right)$ we find

$$
H_{3}(t)=\frac{\mu(t)}{\lambda^{2}(t)}\left(1+B_{\lambda}(0, t)\right)^{-\alpha_{1}(p)+\frac{\sigma}{2}}
$$

Then, we consider the monotonicity of this function as follows:

$$
\begin{aligned}
H_{3}^{\prime}(t) & =\left(\frac{\mu(t)}{\lambda^{2}(t)}\right)^{\prime}\left(1+B_{\lambda}(0, t)\right)^{-\alpha_{1}(p)+\frac{\sigma}{2}}+\left(-\alpha_{1}(p)+\frac{\sigma}{2}\right) \frac{\mu(t)}{\lambda^{2}(t)} \frac{\lambda^{2}(t)}{\rho(t)}\left(1+B_{\lambda}(0, t)\right)^{-\alpha_{1}(p)+\frac{\sigma}{2}-1} \\
& \leq\left[-\beta_{1} \frac{\mu(t)}{\lambda(t) \Lambda(t)}\left(1+B_{\lambda}(0, t)\right)+\left(-\alpha_{1}(p)+\frac{\sigma}{2}\right) \frac{\Lambda(t)}{\lambda(t)}\right]\left(1+B_{\lambda}(0, t)\right)^{-\alpha_{1}(p)+\frac{\sigma}{2}-1} \\
& \leq\left[-\frac{\beta_{1}}{2+R}-\alpha_{1}(p)+\frac{\sigma}{2}\right] \frac{\Lambda(t)}{\lambda(t)}\left(1+B_{\lambda}(0, t)\right)^{-\alpha_{1}(p)+\frac{\sigma}{2}-1},
\end{aligned}
$$

where we used condition (C4) and (3.1.17), respectively. Then, we get $H_{3}^{\prime}(t)<0$ if

$$
\frac{-\beta_{1}}{2+R}-\alpha_{1}(p)+\frac{\sigma}{2}<0, \quad \text { that is, } \quad p>1+\left(\frac{\sigma}{2}-\frac{\beta_{1}}{2+R}\right) \frac{2 m}{n} .
$$

This condition holds by $p>\hat{p}_{1, m}(n)$ and we find that $H_{3}=H_{3}(t)$ is strictly decreasing. Similarly to $H_{1}=H_{1}(t)$ and $H_{2}=H_{2}(t)$, we can show that $H_{4}=H_{4}(t)$ is uniformly bounded.

Case 3: $-\frac{\sigma}{2}+1=0$. In this case we have

$$
\begin{aligned}
\mathbf{H}= & -\int_{\delta t}^{t} \frac{\mu(s)}{\lambda^{2}(s)}\left(1+B_{\lambda}(0, s)\right)^{-\alpha_{2}(p)} \frac{d\left(1+B_{\lambda}(s, t)\right)}{1+B_{\lambda}(s, t)} \\
= & -\left.\frac{\mu(s)}{\lambda^{2}(s)}\left(1+B_{\lambda}(0, s)\right)^{-\alpha_{2}(p)} \log \left(1+B_{\lambda}(s, t)\right)\right|_{\delta t} ^{t} \\
& +\int_{\delta t}^{t} \log \left(1+B_{\lambda}(s, t)\right) d\left(\frac{\mu(s)}{\lambda^{2}(s)}\left(1+B_{\lambda}(0, s)\right)^{-\alpha_{2}(p)}\right) \\
\lesssim & -\left.\frac{\mu(s)}{\lambda^{2}(s)}\left(1+B_{\lambda}(0, s)\right)^{-\alpha_{2}(p)} \log \left(1+B_{\lambda}(s, t)\right)\right|_{\delta t} ^{t} \\
& +\int_{\delta t}^{t} \frac{\mu(s)}{\lambda^{2}(s)}\left(1+B_{\lambda}(0, s)\right)^{-\alpha_{2}(p)} \log \left(1+B_{\lambda}(s, t)\right) \frac{d\left(1+B_{\lambda}(0, s)\right)}{1+B_{\lambda}(0, s)} .
\end{aligned}
$$


Thus, with $\frac{\sigma}{2}=1$ we have

$$
\begin{aligned}
& \left(1+B_{\lambda}(0, t)\right)^{\frac{n}{2}\left(\frac{1}{m}-\frac{1}{2}\right)+\frac{\sigma}{2}} \mathbf{H} \\
& \lesssim \underbrace{\frac{\mu(\delta t)}{\lambda^{2}(\delta t)}\left(1+B_{\lambda}(0, \delta t)\right)^{-\alpha_{2}(p)}\left(1+B_{\lambda}(0, t)\right)^{\frac{n}{2}\left(\frac{1}{m}-\frac{1}{2}\right)+1} \log \left(1+B_{\lambda}(0, t)\right)}_{H_{5}(t)} \\
& +\underbrace{\int_{\delta t}^{t} \frac{\mu(s)}{\lambda^{2}(s)}\left(1+B_{\lambda}(0, s)\right)^{-\alpha_{2}(p)}\left(1+B_{\lambda}(0, t)\right)^{\frac{n}{2}\left(\frac{1}{m}-\frac{1}{2}\right)+1} \log \left(1+B_{\lambda}(0, t)\right) \frac{d\left(1+B_{\lambda}(0, s)\right)}{1+B_{\lambda}(0, s)}}_{H_{6}(t)} .
\end{aligned}
$$

We want to verify that $H_{5}=H_{5}(t)$ and $H_{6}=H_{6}(t)$ are uniformly bounded as we did for $H_{1}=H_{1}(t)$ and $H_{2}=H_{2}(t)$. For $H_{5}=H_{5}(t)$ we find

$$
H_{5}(t) \lesssim \frac{\log \left(1+B_{\lambda}(0, t)\right)}{\left(1+B_{\lambda}(0, \delta t)\right)^{\varepsilon}} \mathcal{H}_{1}(t) \lesssim \frac{\log \left(1+B_{\lambda}(0, t)\right)}{\left(1+B_{\lambda}(0, \delta t)\right)^{\varepsilon}} \lesssim 1
$$

with an arbitrarily chosen small positive constant $\varepsilon$ and for large $t$. Here we have used the boundedness of $\mathcal{H}_{1}=\mathcal{H}_{1}(t)$ which is given in (3.4.17) and l'Hospital's rule (cf. with condition (B4)) as follows:

$$
\lim _{t \rightarrow \infty} \sup \frac{\log \left(1+B_{\lambda}(0, t)\right)}{\left(1+B_{\lambda}(0, \delta t)\right)^{\varepsilon}} \lesssim \lim _{t \rightarrow \infty} \sup \frac{\nu(\delta, t)}{\left(1+B_{\lambda}(0, \delta t)\right)^{\epsilon}} \lesssim 1,
$$

respectively. On the other hand, for $H_{6}=H_{6}(t)$ using (3.4.16) and the previous estimate, we find

$$
\begin{aligned}
H_{6}(t) & \lesssim \log \left(1+B_{\lambda}(0, t)\right) \int_{\delta t}^{t}\left(1+B_{\lambda}(0, s)\right)^{-\varepsilon-1} d\left(1+B_{\lambda}(0, s)\right) \\
& =\frac{\log \left(1+B_{\lambda}(0, t)\right)}{\varepsilon\left(1+B_{\lambda}(0, \delta t)\right)^{\varepsilon}}-\frac{\log \left(1+B_{\lambda}(0, t)\right)}{\varepsilon\left(1+B_{\lambda}(0, t)\right)^{\varepsilon}} \lesssim 1
\end{aligned}
$$

Consequently, we obtain the desired estimate

$$
\mathbf{H} \lesssim\left(1+B_{\lambda}(0, t)\right)^{-\frac{n}{2}\left(\frac{1}{m}-\frac{1}{2}\right)-\frac{\sigma}{2}} .
$$

Therefore, from (3.4.15) and (3.4.18) we arrive at the estimate

$$
\left\||D|^{\sigma} u^{\mathrm{nl}}(t, \cdot)\right\|_{L^{2}} \lesssim\|u\|_{X(t)}^{p}\left(1+B_{\lambda}(0, t)\right)^{-\frac{n}{2}\left(\frac{1}{m}-\frac{1}{2}\right)-\frac{\sigma}{2}} .
$$

In the same way, following the proof of Theorem 3.3.1 one can prove

$$
\left\|u^{\mathrm{nl}}(t, \cdot)\right\|_{L^{2}} \lesssim\|u\|_{X(t)}^{p}\left(1+B_{\lambda}(0, t)\right)^{-\frac{n}{2}\left(\frac{1}{m}-\frac{1}{2}\right)}
$$

by the conditions $p>\hat{p}_{1, m}(n), p>\hat{p}_{2, m}(n)$ and (3.4.3). Moreover, again analogously to the proof of Theorem 3.3.1 one can also prove

$$
\begin{array}{r}
\left\|u_{t}^{\mathrm{nl}}(t, \cdot)\right\|_{L^{2}} \lesssim\|u\|_{X(t)}^{p} \max \left\{\frac{\lambda^{2}(t)}{\rho(\tau)}\left(1+B_{\lambda}(0, t)\right)^{-\frac{n}{2}\left(\frac{1}{m}-\frac{1}{2}\right)-1} ;\right. \\
\left.\lambda(t) F(\Lambda(t))\left(F^{2}(\Lambda(t))\right)^{-\frac{n}{2}\left(\frac{1}{m}-\frac{1}{2}\right)-1}\right\},
\end{array}
$$

provided that the conditions $p>\hat{p}_{1, m}(n), p>\hat{p}_{3, m}(n)$ and (3.4.3) are satisfied.

Now let us continue to estimate the norm $\left\||D|^{\sigma-1} u_{t}^{\mathrm{nl}}(t, \cdot)\right\|_{L^{2}}$. We get

$$
\left\||D|^{\sigma-1} u_{t}^{\mathrm{nl}}(t, \cdot)\right\|_{L^{2}} \lesssim \int_{0}^{t}\left\||D|^{\sigma-1}\left(K_{1}(t, s, x) *_{(x)}|u(s, x)|^{p}\right)\right\|_{L^{m} \cap L^{2}} d s .
$$


Proceeding in the same way as we did to estimate the norm $\left\||D|^{\sigma} u^{\mathrm{nl}}(t, \cdot)\right\|_{L^{2}}$, we obtain the following estimate:

$$
\begin{aligned}
\left\||D|^{\sigma-1} u_{t}^{\mathrm{nl}}(t, \cdot)\right\|_{L^{2}} \lesssim & \int_{0}^{\delta t} \Phi_{2, m}(s, t) \frac{\Lambda(s)}{\lambda(s)}\left\||u(s, \cdot)|^{p}\right\|_{L^{m} \cap L^{2} \cap \dot{H}^{\sigma-1}} d s \\
& +\int_{\delta t}^{t} \Phi_{2}(s, t) \frac{\Lambda(s)}{\lambda(s)}\left\||u(s, \cdot)|^{p}\right\|_{L^{2} \cap \dot{H}^{\sigma-1}} d s,
\end{aligned}
$$

where $\Phi_{2, m}=\Phi_{2, m}(s, t)$ and $\Phi_{2}=\Phi_{2}(s, t)$ are defined in (3.1.15) and (3.1.16), respectively. Summarizing, using the estimates of the norm $\left\||u(s, \cdot)|^{p}\right\|_{L^{m} \cap L^{2} \cap \dot{H}^{\sigma-1}}$ from (3.4.7), (3.4.8) and (3.4.10) with no other requirements to the admissible exponents $p$ and $\|u\|_{X(s)} \leq\|u\|_{X(t)}$ for $0 \leq s \leq t$ we arrive at

$$
\begin{aligned}
\left\||D|^{\sigma-1} u_{t}^{\mathrm{nl}}(t, \cdot)\right\|_{L^{2}} \lesssim & \|u\|_{X(t)}^{p} \underbrace{\int_{0}^{\delta t} \Phi_{2, m}(s, t) \frac{\Lambda(s)}{\lambda(s)}\left(1+B_{\lambda}(0, s)\right)^{-\frac{n}{2 m} p+\frac{n}{2 m}} d s}_{\mathbf{K}} \\
& +\|u\|_{X(t)}^{p} \underbrace{\int_{\delta t}^{t} \Phi_{2}(s, t) \frac{\Lambda(s)}{\lambda(s)}\left(1+B_{\lambda}(0, s)\right)^{-\frac{n}{2 m} p+\frac{n}{4}} d s}_{\mathbf{L}} .
\end{aligned}
$$

Let us consider the integral $\mathbf{K}$. In this case we have

$$
\mathbf{K} \lesssim \Phi_{2, m}(0, t) \underbrace{\int_{0}^{\delta t} \frac{\Lambda(s)}{\lambda(s)}\left(1+B_{\lambda}(0, s)\right)^{-\frac{n}{2 m} p+\frac{n}{2 m}} d s}_{K_{1}(t)},
$$

where we used (3.1.18). From $G_{1}=G_{1}(t)$ in (3.4.14) we may conclude that after using $p>\hat{p}_{1, m}(n)$ the integral $K_{1}=K_{1}(t)$ is uniformly bounded, too. Thus, we obtain

$$
\mathbf{K} \lesssim \Phi_{2, m}(0, t) .
$$

We consider now the integral $\mathbf{L}$, that is,

$$
\mathbf{L}=\int_{\delta t}^{t} \Phi_{2}(s, t) \frac{\Lambda(s)}{\lambda(s)}\left(1+B_{\lambda}(0, s)\right)^{-\alpha_{2}(p)} d s,
$$

where $\alpha_{2}(p):=\frac{n}{2 m} p-\frac{n}{4}$. We see that we have a competition between the influence on the estimates of both components of $\Phi_{2}=\Phi_{2}(s, t)$ in (3.1.16) for all $s \in[\delta t, t]$. Then, analogously to the proof of Theorem 3.3.1, condition (C1) comes into play which helps to avoid some difficulties in the treatment. That is, we are only interested in the following two cases, which implies that we have either the first component or the second component is dominant in $\Phi_{2}=\Phi_{2}(s, t)$ for all $s \in[\delta t, t]:$

Case a: $\Phi_{2}(s, t)=\frac{\lambda^{2}(t)}{\rho(t)}\left(1+B_{\lambda}(s, t)\right)^{-\frac{\sigma-1}{2}-1}$. In this case we have

$$
\begin{aligned}
\mathbf{L}= & \frac{\lambda^{2}(t)}{\rho(t)} \int_{\delta t}^{t} \frac{\Lambda(s)}{\lambda(s)}\left(1+B_{\lambda}(s, t)\right)^{-\frac{\sigma-1}{2}-1}\left(1+B_{\lambda}(0, s)\right)^{-\alpha_{2}(p)} d s \\
= & -\frac{\lambda^{2}(t)}{\rho(t)} \int_{\delta t}^{t} \frac{\mu(s)}{\lambda^{2}(s)}\left(1+B_{\lambda}(0, s)\right)^{-\alpha_{2}(p)}\left(1+B_{\lambda}(s, t)\right)^{-\frac{\sigma-1}{2}-1} d\left(1+B_{\lambda}(s, t)\right) \\
= & \left.\frac{2}{\sigma-1} \frac{\lambda^{2}(t)}{\rho(t)} \frac{\mu(s)}{\lambda^{2}(s)}\left(1+B_{\lambda}(0, s)\right)^{-\alpha_{2}(p)}\left(1+B_{\lambda}(s, t)\right)^{-\frac{\sigma-1}{2}}\right|_{\delta t} ^{t} \\
& -\frac{2}{\sigma-1} \frac{\lambda^{2}(t)}{\rho(t)} \int_{\delta t}^{t}\left(1+B_{\lambda}(s, t)\right)^{-\frac{\sigma-1}{2}} d\left(\frac{\mu(s)}{\lambda^{2}(s)}\left(1+B_{\lambda}(0, s)\right)^{-\alpha_{2}(p)}\right)
\end{aligned}
$$


Using Lemma 3.1.5, it holds

$$
\begin{aligned}
\frac{\rho(t)}{\lambda^{2}(t)} \mathbf{L} \lesssim & \frac{\mu(t)}{\lambda^{2}(t)}\left(1+B_{\lambda}(0, t)\right)^{-\alpha_{2}(p)}-\frac{\mu(\delta t)}{\lambda^{2}(\delta t)}\left(1+B_{\lambda}(0, \delta t)\right)^{-\alpha_{2}(p)}\left(1+B_{\lambda}(\delta t, t)\right)^{-\frac{\sigma-1}{2}} \\
& +\int_{\delta t}^{t} \frac{\mu(s)}{\lambda^{2}(s)}\left(1+B_{\lambda}(0, s)\right)^{-\alpha_{2}(p)}\left(1+B_{\lambda}(s, t)\right)^{-\frac{\sigma-1}{2}} \frac{d\left(1+B_{\lambda}(0, s)\right)}{1+B_{\lambda}(0, s)} .
\end{aligned}
$$

Then, we have

$$
\begin{aligned}
\frac{\rho(t)}{\lambda^{2}(t)}(1 & \left.+B_{\lambda}(0, t)\right)^{\frac{n}{2}\left(\frac{1}{m}-\frac{1}{2}\right)+\frac{\sigma-1}{2}+1} \mathbf{L} \\
\lesssim & \underbrace{\frac{\mu(t)}{\lambda^{2}(t)}\left(1+B_{\lambda}(0, t)\right)^{\frac{n}{2}\left(\frac{1}{m}-\frac{1}{2}\right)+\frac{\sigma-1}{2}+1-\alpha_{2}(p)}}_{L_{1}(t)} \\
& +\underbrace{\int_{\delta t}^{t} \frac{\mu(s)}{\lambda^{2}(s)}\left(1+B_{\lambda}(0, s)\right)^{-\alpha_{2}(p)}\left(1+B_{\lambda}(0, t)\right)^{\frac{n}{2}\left(\frac{1}{m}-\frac{1}{2}\right)+\frac{\sigma-1}{2}+1} \frac{d\left(1+B_{\lambda}(0, s)\right)}{1+B_{\lambda}(0, s)}}_{L_{2}(t)} .
\end{aligned}
$$

We begin with $L_{1}=L_{1}(t)$. Using $\alpha_{2}(p)=\alpha_{1}(p)+\frac{n}{2}\left(\frac{1}{m}-\frac{1}{2}\right)$ we have

$$
L_{1}(t):=\frac{\mu(t)}{\lambda^{2}(t)}\left(1+B_{\lambda}(0, t)\right)^{\frac{\sigma+1}{2}-\alpha_{1}(p)} .
$$

If we take the derivative of this function, then it follows

$$
\begin{aligned}
L_{1}^{\prime}(t) & =\left(\frac{\mu(t)}{\lambda^{2}(t)}\right)^{\prime}\left(1+B_{\lambda}(0, t)\right)^{\frac{\sigma+1}{2}-\alpha_{1}(p)}+\left(\frac{\sigma+1}{2}-\alpha_{1}(p)\right) \frac{\mu(t)}{\lambda^{2}(t)} \frac{\lambda^{2}(t)}{\rho(t)}\left(1+B_{\lambda}(0, t)\right)^{\frac{\sigma-1}{2}-\alpha_{1}(p)} \\
& \leq\left[-\beta_{1} \frac{\mu(t)}{\lambda(t) \Lambda(t)}\left(1+B_{\lambda}(0, t)\right)+\left(\frac{\sigma+1}{2}-\alpha_{1}(p)\right) \frac{\Lambda(t)}{\lambda(t)}\right]\left(1+B_{\lambda}(0, t)\right)^{\frac{\sigma-1}{2}-\alpha_{1}(p)} \\
& \leq\left[-\frac{\beta_{1}}{2+R}+\frac{\sigma+1}{2}-\alpha_{1}(p)\right] \frac{\Lambda(t)}{\lambda(t)}\left(1+B_{\lambda}(0, t)\right)^{\frac{\sigma-1}{2}-\alpha_{1}(p)} .
\end{aligned}
$$

We get $L_{1}^{\prime}(t)<0$, namely, $L_{1}=L_{1}(t)$ is decreasing if

$$
-\frac{\beta_{1}}{2+R}+\frac{\sigma+1}{2}-\alpha_{1}(p)<0,
$$

which can be concluded from $p>\hat{p}_{1, m}(n)$.

Let us continue now with $L_{2}=L_{2}(t)$. We choose an arbitrarily small positive constant $\varepsilon$ such that it holds

$$
\frac{\mu(\delta t)}{\lambda^{2}(\delta t)}\left(1+B_{\lambda}(0, \delta t)\right)^{-\alpha_{2}(p)}\left(1+B_{\lambda}(0, t)\right)^{\frac{n}{2}\left(\frac{1}{m}-\frac{1}{2}\right)+\frac{\sigma-1}{2}+1} \lesssim\left(1+B_{\lambda}(0, \delta t)\right)^{-\varepsilon} .
$$

Using (3.1.19) we find

$$
\begin{aligned}
& \frac{\mu(\delta t)}{\lambda^{2}(\delta t)}\left(1+B_{\lambda}(0, \delta t)\right)^{-\alpha_{2}(p)+\varepsilon}\left(1+B_{\lambda}(0, t)\right)^{\frac{n}{2}\left(\frac{1}{m}-\frac{1}{2}\right)+\frac{\sigma-1}{2}+1} \\
& \quad \leq \frac{\mu(\delta t)}{\lambda^{2}(\delta t)}\left(1+B_{\lambda}(0, t)\right)^{\frac{n}{2}\left(\frac{1}{m}-\frac{1}{2}\right)+\frac{\sigma-1}{2}+1-\alpha_{2}(p)+\varepsilon}\left(\frac{\Lambda(\delta t)}{\Lambda(t)}\right)^{(2+R)\left(-\alpha_{2}(p)+\varepsilon\right)}:=\mathcal{L}_{2}(t) .
\end{aligned}
$$

From $p>\hat{p}_{3, m}(n)$ we may conclude that $\mathcal{L}_{2}^{\prime}(t)<0$ following the same considerations we have done for $H_{1}=H_{1}(t)$ and $H_{2}=H_{2}(t)$. Then, this gives the uniformly boundedness of $L_{2}=L_{2}(t)$. Therefore, we obtain

$$
\mathbf{L} \lesssim \frac{\lambda^{2}(t)}{\rho(t)}\left(1+B_{\lambda}(0, t)\right)^{-\frac{n}{2}\left(\frac{1}{m}-\frac{1}{2}\right)-\frac{\sigma-1}{2}-1}
$$


Case b: $\Phi_{2}(s, t)=\lambda(t) F(\Lambda(t))\left(F^{2}(\Lambda(t))\right)^{-\frac{\sigma-1}{2}-1}$. In this case we have

$$
\begin{aligned}
\mathbf{L} & =\lambda(t) F(\Lambda(t))\left(F^{2}(\Lambda(t))\right)^{-\frac{\sigma-1}{2}-1} \int_{\delta t}^{t} \frac{\Lambda(s)}{\lambda(s)}\left(1+B_{\lambda}(0, s)\right)^{-\alpha_{2}(p)} d s \\
& =\lambda(t) F(\Lambda(t))\left(F^{2}(\Lambda(t))\right)^{-\frac{\sigma-1}{2}-1} \int_{\delta t}^{t} \frac{\mu(s)}{\lambda^{2}(s)}\left(1+B_{\lambda}(0, s)\right)^{-\alpha_{2}(p)} d\left(1+B_{\lambda}(0, s)\right) .
\end{aligned}
$$

Following the the proof of Theorem 3.3.1 in both cases, namely Case b.1: $\alpha_{2}(p)>1$ and Case b.2: $\alpha_{2}(p)<1$, we find the desired estimates with the conditions $p>\hat{p}_{1, m}(n)$ and $p>1+\left(1-\frac{\delta}{\vartheta(\delta)} \frac{\beta_{1}}{2}\right) \frac{2 m}{n}$. Moreover, by the condition (3.3.2) it holds $\frac{\beta_{1}}{\frac{n}{2}\left(\frac{1}{m}-\frac{1}{2}\right)}>2 \frac{\vartheta(\delta)}{\delta}$ which gives the desired estimate in the Case b.3 $: \alpha_{2}(p)=1$. Hence, we get

$$
\mathbf{L} \lesssim \lambda(t) F(\Lambda(t))\left(F^{2}(\Lambda(t))\right)^{-\frac{n}{2}\left(\frac{1}{m}-\frac{1}{2}\right)-\frac{\sigma-1}{2}-1} .
$$

Consequently, the estimates (3.4.20) and (3.4.21) yield

$$
\begin{aligned}
\left\||D|^{\sigma-1} u_{t}^{\mathrm{nl}}(t, \cdot)\right\|_{L^{2}} \lesssim\|u\|_{X(t)}^{p} \max \left\{\frac{\lambda^{2}(t)}{\rho(t)}\left(1+B_{\lambda}(0, t)\right)^{-\frac{n}{2}\left(\frac{1}{m}-\frac{1}{2}\right)-\frac{\sigma-1}{2}-1} ;\right. \\
\left.\lambda(t) F(\Lambda(t))\left(F^{2}(\Lambda(t))\right)^{-\frac{n}{2}\left(\frac{1}{m}-\frac{1}{2}\right)-\frac{\sigma-1}{2}-1}\right\} .
\end{aligned}
$$

All together yields

$$
\left\|u^{\mathrm{nl}}\right\|_{X(t)} \lesssim\|u\|_{X(t)}^{p} .
$$

From (3.4.6) and (3.4.22), we get (3.4.4).

To prove (3.4.5) let us firstly recall

$$
\|N u-N v\|_{X(t)}=\left\|\int_{0}^{t} K_{1}(t, s, x) *_{(x)}\left(|u(s, x)|^{p}-|v(s, x)|^{p}\right) d s\right\|_{X(t)} .
$$

We only show how to estimate the norm $\left\||D|^{\sigma}(N u-N v)(t, \cdot)\right\|_{L^{2}}$. In the same way we estimate the other terms appearing in the norm $\|N u-N v\|_{X(t)}$. It holds

$$
\begin{aligned}
\||D|^{\sigma} \int_{0}^{t} & K_{1}(t, s, x) *_{(x)}\left(|u(s, x)|^{p}-|v(s, x)|^{p}\right) d s \|_{L^{2}} \\
\lesssim & \int_{0}^{\delta t} \frac{\Lambda(s)}{\lambda(s)}\left(1+B_{\lambda}(s, t)\right)^{-\frac{n}{2}\left(\frac{1}{m}-\frac{1}{2}\right)-\frac{\sigma}{2}}\left\||u(s, x)|^{p}-|v(s, x)|^{p}\right\|_{L^{m} \cap L^{2} \cap \dot{H}^{\sigma-1}} \\
& \quad+\int_{\delta t}^{t} \frac{\Lambda(s)}{\lambda(s)}\left(1+B_{\lambda}(s, t)\right)^{-\frac{\sigma}{2}}\left\||u(s, x)|^{p}-|v(s, x)|^{p}\right\|_{L^{2} \cap \dot{H}^{\sigma-1}} d s
\end{aligned}
$$

By Hölder's inequality we conclude for $k=m, 2$ the estimates

$$
\left\||u(s, \cdot)|^{p}-|v(s, \cdot)|^{p}\right\|_{L^{k}} \lesssim\|u(s, \cdot)-v(s, \cdot)\|_{L^{k p}}\left(\|u(s, \cdot)\|_{L^{k p}}^{p-1}+\|v(s, \cdot)\|_{L^{k p}}^{p-1}\right) .
$$

We apply the classical Gagliardo-Nirenberg inequality to the following terms:

$$
\|u(s, \cdot)-v(s, \cdot)\|_{L^{k p}}, \quad\|u(s, \cdot)\|_{L^{k p}} \quad \text { and } \quad\|v(s, \cdot)\|_{L^{k p}} .
$$

Summarizing, we arrive at the following inequalities for $\|u(s, \cdot)-v(s, \cdot)\|_{L^{k p}}$ :

$$
\begin{aligned}
\left\||u(s, \cdot)|^{p}-|v(s, \cdot)|^{p}\right\|_{L^{2}} & \lesssim\left(1+B_{\lambda}(0, s)\right)^{-\frac{n}{2 m} p+\frac{n}{4}}\|u-v\|_{X(s)}\left(\|u\|_{X(s)}^{p-1}+\|v\|_{X(s)}^{p-1}\right) \\
\left\||u(s, \cdot)|^{p}-|v(s, \cdot)|^{p}\right\|_{L^{m}} & \lesssim\left(1+B_{\lambda}(0, s)\right)^{-\frac{n}{2 m} p+\frac{n}{2 m}}\|u-v\|_{X(s)}\left(\|u\|_{X(s)}^{p-1}+\|v\|_{X(s)}^{p-1}\right) .
\end{aligned}
$$


Let us turn now to the estimate of the norm $\left\||u(s, \cdot)|^{p}-|v(s, \cdot)|^{p}\right\|_{\dot{H}^{\sigma-1}}$. By using the integral representation

$$
\begin{aligned}
|u(s, x)|^{p}-|v(s, x)|^{p} & =\int_{0}^{1} \frac{d}{d w}|w u(s, x)+(1-w) v(s, x)|^{p} d w \\
& =p \int_{0}^{1}(u(s, x)-v(s, x)) G(w u(s, x)+(1-w) v(s, x)) d w,
\end{aligned}
$$

where $G(u)=u|u|^{p-2}$, we obtain

$$
\left\||u(s, \cdot)|^{p}-|v(s, \cdot)|^{p}\right\|_{\dot{H}^{\sigma-1}} \lesssim \int_{0}^{1}\left\||D|^{\sigma-1}((u(s, \cdot)-v(s, \cdot)) G(w u(s, \cdot)+(1-w) v(s, \cdot)))\right\|_{L^{2}} d w .
$$

Using the fractional Leibniz rule from Proposition A.7.3 we can proceed as follows:

$$
\begin{aligned}
\left\||u(s, \cdot)|^{p}-|v(s, \cdot)|^{p}\right\|_{H^{\sigma-1}} & \\
\lesssim & \left\||D|^{\sigma-1}(u(s, \cdot)-v(s, \cdot))\right\|_{L^{r_{1}}} \int_{0}^{1}\|G(w u(s, \cdot)+(1-w) v(s, \cdot))\|_{L^{r_{2}}} d w \\
& \quad+\|u(s, \cdot)-v(s, \cdot)\|_{L^{r_{3}}} \int_{0}^{1}\left\||D|^{\sigma-1} G(w u(s, \cdot)+(1-w) v(s, \cdot))\right\|_{L^{r_{4}}} d w \\
\lesssim & \left\||D|^{\sigma-1}(u(s, \cdot)-v(s, \cdot))\right\|_{L^{r_{1}}}\left(\|u(s, \cdot)\|_{L^{r_{2}(p-1)}}^{p-1}+\|v(s, \cdot)\|_{L^{r_{2}(p-1)}}^{p-1}\right) \\
& \quad+\|u(s, \cdot)-v(s, \cdot)\|_{L^{r_{3}}} \int_{0}^{1}\left\||D|^{\sigma-1} G(w u(s, \cdot)+(1-w) v(s, \cdot))\right\|_{L^{r_{4}}} d w,
\end{aligned}
$$

where $\frac{1}{2}=\frac{1}{r_{1}}+\frac{1}{r_{2}}=\frac{1}{r_{3}}+\frac{1}{r_{4}}$. Employing the fractional Gargliardo-Nirenberg inequality from Corollary A.7.3 we get

$$
\begin{aligned}
\left\||D|^{\sigma-1}(u(s, \cdot)-v(s, \cdot))\right\|_{L^{r_{1}}} & \lesssim\|u(s, \cdot)-v(s, \cdot)\|_{\dot{H}^{\sigma}}^{\theta_{\sigma-\sigma}\left(r_{1}\right)}\|u(s, \cdot)-v(s, \cdot)\|_{L^{2}}^{1-\theta_{\sigma-1, \sigma}\left(r_{1}\right)} \\
& \lesssim\left(1+B_{\lambda}(0, s)\right)^{-\frac{n}{2 m}+\frac{n}{2 r_{1}}-\frac{\sigma-1}{2}}\|u-v\|_{X(s)} \\
\|u(s, \cdot)\|_{L^{r_{2}(p-1)}} & \lesssim\|u(s, \cdot)\|_{H^{\sigma}}^{\theta_{\sigma}\left(r_{2}(p-1)\right)}\|u(s, \cdot)\|_{L^{2}}^{1-\theta_{\sigma}\left(r_{2}(p-1)\right)} \\
& \lesssim\left(1+B_{\lambda}(0, s)\right)^{-\frac{n}{2 m}+\frac{n}{2 r_{2}(p-1)}}\|u\|_{X(s)}
\end{aligned}
$$

where $\theta_{\sigma-1, \sigma}\left(r_{1}\right)=\frac{n}{\sigma}\left(\frac{1}{2}-\frac{1}{r_{1}}+\frac{\sigma-1}{n}\right) \in\left[\frac{\sigma-1}{\sigma}, 1\right]$ and $\theta_{\sigma}\left(r_{2}(p-1)\right)=\frac{n}{\sigma}\left(\frac{1}{2}-\frac{1}{r_{2}(p-1)}\right) \in[0,1]$. For the choices of $r_{1}$ and $r_{2}$ we have the following conditions:

$$
\frac{1}{r_{1}}+\frac{1}{r_{2}}=\frac{1}{2} \quad \text { and } \quad\left\{\begin{array}{lll}
\frac{1}{r_{1}} \leq \frac{1}{2}, & \frac{1}{r_{2}} \leq \frac{p-1}{2} & \text { if } n \leq 2, \\
\frac{n-2}{2 n} \leq \frac{1}{r_{1}} \leq \frac{1}{2}, & \frac{1}{r_{2}} \leq \frac{p-1}{2} & \text { if } 2<n \leq 2 \sigma, \\
\frac{n-2}{2 n} \leq \frac{1}{r_{1}} \leq \frac{1}{2}, & \frac{n-2 \sigma}{2 n}(p-1) \leq \frac{1}{r_{2}} \leq \frac{p-1}{2} & \text { if } n>2 \sigma .
\end{array}\right.
$$

We can see immediately that we can repeat exactly the same arguments as we did for $q_{1}$ and $q_{2}$ in (3.4.12), choosing $\frac{1}{r_{1}}$ instead of $\frac{1}{q_{2}}$ and $\frac{1}{r_{2}}$ instead of $\frac{p-1}{q_{1}}$. Therefore, we get again the condition (3.4.13).

After getting (3.4.25) and (3.4.26) we may conclude the estimate

$$
\begin{aligned}
\||D|^{\sigma-1}(u(s, \cdot)- & v(s, \cdot)) \|_{L^{r_{1}}}\left(\|u(s, \cdot)\|_{L^{r_{2}(p-1)}}^{p-1}+\|v(s, \cdot)\|_{L^{r_{2}(p-1)}}^{p-1}\right) \\
& \lesssim\left(1+B_{\lambda}(0, s)\right)^{-\frac{n}{2 m} p+\frac{n}{4}-\frac{\sigma-1}{2}}\|u-v\|_{X(s)}\left(\|u\|_{X(s)}^{p-1}+\|v\|_{X(s)}^{p-1}\right) .
\end{aligned}
$$

Now we turn to estimate the remaining terms, that is, the second summand of the last inequality in (3.4.24). For the first term of this summand applying the classical GagliardoNirenberg inequality we get the estimate

$$
\begin{aligned}
\|u(s, \cdot)-v(s, \cdot)\|_{L^{r_{3}}} & \lesssim\|u(s, \cdot)-v(s, \cdot)\|_{H_{\sigma}^{\sigma}\left(r_{3}\right)}^{\theta^{\sigma}}\|u(s, \cdot)-v(s, \cdot)\|_{L^{2}}^{1-\theta_{\sigma}\left(r_{3}\right)} \\
& \lesssim\left(1+B_{\lambda}(0, s)\right)^{-\frac{n}{2 m}+\frac{n}{2 r_{3}}}\|u-v\|_{X(s)},
\end{aligned}
$$


where $\theta_{\sigma}\left(r_{3}\right)=\frac{n}{\sigma}\left(\frac{1}{2}-\frac{1}{r_{3}}\right) \in[0,1]$. On the other hand, let us devote to estimate the second term. Using the fractional chain rule from Proposition A.7.7 for $p-1>\lceil\sigma-1\rceil$ we obtain the estimate

$$
\begin{aligned}
\||D|^{\sigma-1} G & (w u(s, \cdot)+(1-w) v(s, \cdot)) \|_{L^{r_{4}}} \\
& \lesssim\|w u(s, \cdot)+(1-w) v(s, \cdot)\|_{L^{r_{5}}}^{p-2}\left\||D|^{\sigma-1}(w u(s, \cdot)+(1-w) v(s, \cdot))\right\|_{L^{r_{6}}},
\end{aligned}
$$

where $\frac{1}{r_{4}}=\frac{p-2}{r_{5}}+\frac{1}{r_{6}}$. For the first term on the right-hand side of the last inequality, after using the classical Gagliardo-Nirenberg inequality we find

$$
\begin{aligned}
\| w u(s, \cdot) & +(1-w) v(s, \cdot) \|_{L^{r_{5}}}^{p-2} \\
& \lesssim\|w u(s, \cdot)+(1-w) v(s, \cdot)\|_{\dot{H}^{\sigma}}^{(p-2) \theta_{\sigma}\left(r_{5}\right)}\|w u(s, \cdot)+(1-w) v(s, \cdot)\|_{L^{2}}^{(p-2)\left(1-\theta_{\sigma}\left(r_{5}\right)\right)} \\
& \lesssim\left(1+B_{\lambda}(0, s)\right)^{-\frac{n}{2 m}(p-2)+\frac{n}{2 r_{5}(p-2)}\|w u+(1-w) v\|_{X(s)}^{p-2},}
\end{aligned}
$$

where $\theta_{\sigma}\left(r_{5}\right)=\frac{n}{\sigma}\left(\frac{1}{2}-\frac{1}{r_{5}}\right) \in[0,1]$. Moreover, for the second term applying the fractional Gagliardo-Nirenberg inequality, we may conclude the estimate

$$
\begin{aligned}
\||D|^{\sigma-1}(w u(s, \cdot) & +(1-w) v(s, \cdot)) \|_{L^{r_{6}}} \\
& \lesssim\|w u(s, \cdot)+(1-w) v(s, \cdot)\|_{\dot{H}^{\sigma}}^{\theta_{\sigma-1, \sigma}\left(r_{6}\right)}\|w u(s, \cdot)+(1-w) v(s, \cdot)\|_{L^{2}}^{1-\theta_{\sigma-1, \sigma}\left(r_{6}\right)} \\
& \lesssim\left(1+B_{\lambda}(0, s)\right)^{-\frac{n}{2 m}+\frac{n}{2 r_{6}}-\frac{\sigma-1}{2}}\|w u+(1-w) v\|_{X(s)},
\end{aligned}
$$

where $\theta_{\sigma-1, \sigma}\left(r_{6}\right)=\frac{n}{\sigma}\left(\frac{1}{2}-\frac{1}{r_{6}}+\frac{\sigma-1}{n}\right) \in\left[\frac{\sigma-1}{\sigma}, 1\right]$.

Consequently, from the last two estimates we arrive at the estimate

$$
\begin{aligned}
\||D|^{\sigma-1} G(w u & (s, \cdot)+(1-w) v(s, \cdot)) \|_{L^{r_{4}}} \\
& \lesssim\left(1+B_{\lambda}(0, s)\right)^{-\frac{n}{2 m}(p-2)+\frac{n}{2 r_{5}}(p-2)-\frac{n}{2 m}+\frac{n}{2 r_{6}}-\frac{\sigma-1}{2}}\|w u+(1-w) v\|_{X(s)}^{p-1} \\
& =\left(1+B_{\lambda}(0, s)\right)^{-\frac{n}{2 m}(p-1)+\frac{n}{2 r_{4}}-\frac{\sigma-1}{2}}\|w u+(1-w) v\|_{X(s)}^{p-1} .
\end{aligned}
$$

Now we should verify that we can choose in a suitable way all parameters $r_{3}, r_{4}, r_{5}$ and $r_{6}$ such that $\theta_{\sigma}\left(r_{3}\right), \theta_{\sigma}\left(r_{5}\right)$ and $\theta_{\sigma-1, \sigma}\left(r_{6}\right)$ are in a suitable ranges and the conditions

$$
\frac{1}{r_{3}}+\frac{1}{r_{4}}=\frac{1}{2} \text { and } \quad \frac{p-2}{r_{5}}+\frac{1}{r_{6}}=\frac{1}{r_{4}}
$$

are fulfilled.

Let us begin with the parameters $r_{3}$ and $r_{4}$. From the conditions of $\theta_{\sigma}\left(r_{3}\right) \in[0,1]$ and using $\frac{1}{r_{4}}=\frac{1}{2}-\frac{1}{r_{3}}$ we get the following conditions for $r_{3}$ and $r_{4}$ :

$$
\left\{\begin{array} { l l } 
{ \frac { 1 } { r _ { 3 } } \in ( 0 , \frac { 1 } { 2 } ] } & { \text { if } n \leq 2 \sigma , } \\
{ \frac { 1 } { r _ { 3 } } \in [ \frac { 1 } { 2 } - \frac { \sigma } { n } , \frac { 1 } { 2 } ] } & { \text { if } n > 2 \sigma , }
\end{array} \quad \left\{\begin{array}{ll}
\frac{1}{r_{4}} \in\left[0, \frac{1}{2}\right) & \text { if } n \leq 2 \sigma, \\
\frac{1}{r_{4}} \in\left[0, \frac{\sigma}{n}\right] & \text { if } n>2 \sigma .
\end{array}\right.\right.
$$

Therefore, we can choose in a suitable way the parameters $r_{3}$ and $r_{4}$, which satisfy the previous conditions.

Now let us continue with the parameters $r_{5}$ and $r_{6}$ taking account of the conditions $\theta_{\sigma}\left(r_{5}\right) \in$ $[0,1]$ and $\theta_{\sigma-1, \sigma}\left(r_{6}\right) \in[0,1]$. Then, we have

$$
\left\{\begin{array} { l l } 
{ \frac { 1 } { r _ { 5 } } \in ( 0 , \frac { 1 } { 2 } ] } & { \text { if } n \leq 2 \sigma , } \\
{ \frac { 1 } { r _ { 5 } } \in [ \frac { 1 } { 2 } - \frac { \sigma } { n } , \frac { 1 } { 2 } ] } & { \text { if } n > 2 \sigma , }
\end{array} \quad \left\{\begin{array}{ll}
\frac{1}{r_{6}} \in\left(0, \frac{1}{2}\right] & \text { if } n \leq 2, \\
\frac{1}{r_{6}} \in\left[\frac{1}{2}-\frac{1}{n}, \frac{1}{2}\right] & \text { if } n>2 .
\end{array}\right.\right.
$$

Moreover, using the relation $\frac{1}{r_{6}}=\frac{1}{r_{4}}-\frac{p-2}{r_{5}}$ and the obtained conditions for $\frac{1}{r_{4}}$, we can express the condition on $r_{6}$ in the following way after using the condition for $r_{5}$ :

$$
\begin{cases}\frac{1}{r_{6}} \in\left[\frac{1}{r_{4}}-\frac{p-2}{2}, \frac{1}{r_{4}}\right] & \text { if } n \leq 2 \sigma, \\ \frac{1}{r_{6}} \in\left[\frac{1}{r_{4}}-\frac{p-2}{2}, \frac{1}{r_{4}}-\left(\frac{1}{2}-\frac{\sigma}{n}\right)(p-2)\right] & \text { if } n>2 \sigma .\end{cases}
$$


Hence, in order to ensure that we get a non-empty range for the parameters $r_{5}$ and $r_{6}$ we will consider the following cases:

Case 1: $n>2 \sigma$ : For choosing in a suitable way $r_{5}$ and $r_{6}$ we require

$$
\left[\frac{1}{2}-\frac{1}{n}, \frac{1}{2}\right] \cap\left[\frac{1}{r_{4}}-\frac{p-2}{2}, \frac{1}{r_{4}}-\left(\frac{1}{2}-\frac{\sigma}{n}\right)(p-2)\right] \neq \emptyset,
$$

or equivalently

$$
\frac{1}{r_{4}} \in\left[\left(\frac{1}{2}-\frac{\sigma}{n}\right)(p-2)+\frac{1}{2}-\frac{1}{n}, \frac{p-1}{2}\right]
$$

Thus, we get a second condition for $r_{4}$. Therefore, ensuring a non-empty range for $r_{4}$, we get on the one hand, the possibility to find a suitable $r_{3}$ and, on the other hand, we guarantee the possibility to choose in a suitable way $r_{6}$, and in turn, $r_{5}$. So, a non-empty range for $r_{4}$ is given in the following way:

$$
\left[0, \frac{\sigma}{n}\right] \cap\left[\left(\frac{1}{2}-\frac{\sigma}{n}\right)(p-2)+\frac{1}{2}-\frac{1}{n}, \frac{p-1}{2}\right] \neq \emptyset,
$$

which is equivalent to $1 \leq p \leq 1+\frac{2}{n-2 \sigma}$.

Case 2: $2<n \leq 2 \sigma$ : In order to choose a non-empty range for $r_{6}$, we have to guarantee

$$
\left[\frac{1}{2}-\frac{1}{n}, \frac{1}{2}\right] \cap\left[\frac{1}{r_{4}}-\frac{p-2}{2}, \frac{1}{r_{4}}\right] \neq \emptyset
$$

or equivalently, since $p \geq 2$ we can write

$$
\frac{1}{r_{4}} \in\left(\frac{1}{2}-\frac{1}{n}, \frac{p-1}{2}\right] .
$$

Therefore, in the same way as in the previous case a non-empty range for $r_{4}$ is given by

$$
\left(\frac{1}{2}-\frac{1}{n}, \frac{p-1}{2}\right] \cap\left[0, \frac{1}{2}\right) \neq \emptyset,
$$

which is equivalent to $p \geq 1$.

Case 3: $n \leq 2$ : In this case we should guarantee that the range for $r_{6}$ is not empty, that is,

$$
\left(0, \frac{1}{2}\right] \cap\left[\frac{1}{r_{4}}-\frac{p-2}{2}, \frac{1}{r_{4}}\right] \neq \emptyset,
$$

or, in an equivalent way

$$
\frac{1}{r_{4}} \in\left(0, \frac{p-1}{2}\right],
$$

which implies $p>1$.

Consequently, as in the paper [28] we show that the condition (3.4.13) is not only a sufficient condition but also a necessary condition for a suitable choice of these parameters.

Now, using the estimates from (3.4.27) and (3.4.28) we arrive at the estimate

$$
\begin{aligned}
\| u(s, \cdot) & -v(s, \cdot)\left\|_{L^{q_{3}}}\right\||D|^{\sigma-1} G(w u(s, \cdot)+(1-w) v(s, \cdot)) \|_{L^{r_{4}}} \\
& \lesssim\left(1+B_{\lambda}(0, s)\right)^{-\frac{n}{2 m} p+\frac{n}{4}-\frac{\sigma-1}{2}}\|u-v\|_{X(s)}\|w u+(1-w) v\|_{X(s)}^{p-1} .
\end{aligned}
$$

Thus, we obtain

$$
\begin{aligned}
\left\||u(s, \cdot)|^{p}-|v(s, \cdot)|^{p}\right\|_{\dot{H}^{\sigma-1}} & \lesssim \int_{0}^{1}\left(1+B_{\lambda}(0, s)\right)^{-\frac{n}{2 m} p+\frac{n}{4}-\frac{\sigma-1}{2}}\|u-v\|_{X(s)}\|w u+(1-w) v\|_{X(s)}^{p-1} d w \\
& \lesssim \int_{0}^{1}\left(1+B_{\lambda}(0, s)\right)^{-\frac{n}{2 m} p+\frac{n}{4}-\frac{\sigma-1}{2}}\|u-v\|_{X(s)}\left(\|u\|_{X(s)}^{p-1}+\|v\|_{X(s)}^{p-1}\right) d w \\
& \lesssim\left(1+B_{\lambda}(0, s)\right)^{-\frac{n}{2 m} p+\frac{n}{4}-\frac{\sigma-1}{2}}\|u-v\|_{X(s)}\left(\|u\|_{X(s)}^{p-1}+\|v\|_{X(s)}^{p-1}\right) .
\end{aligned}
$$


Taking account of (3.4.23) and $\|u\|_{X(s)} \leq\|u\|_{X(t)}$ with $0 \leq s \leq t$, we find

$$
\begin{gathered}
\left\||D|^{\sigma} \int_{0}^{t} K_{1}(t, s, x) *(x)\left(|u(s, x)|^{p}-|v(s, x)|^{p}\right) d s\right\|_{L^{2}} \lesssim\left(\|u\|_{X(t)}^{p-1}+\|v\|_{X(t)}^{p-1}\right)\|u-v\|_{X(t)} \\
\times\left(\int_{0}^{\delta t} \frac{\Lambda(s)}{\lambda(s)}\left(1+B_{\lambda}(s, t)\right)^{-\frac{n}{2}\left(\frac{1}{m}-\frac{1}{2}\right)-\frac{\sigma}{2}}\left(1+B_{\lambda}(0, s)\right)^{-\frac{n}{2 m} p+\frac{n}{2 m}} d s\right. \\
\left.\quad+\int_{\delta t}^{t} \frac{\Lambda(s)}{\lambda(s)}\left(1+B_{\lambda}(s, t)\right)^{-\frac{\sigma}{2}}\left(1+B_{\lambda}(0, s)\right)^{-\frac{n}{2 m} p+\frac{n}{4}} d s\right) .
\end{gathered}
$$

Following the same steps to estimate the term $\left\||D|^{\sigma} u^{\mathrm{nl}}(t, \cdot)\right\|_{L^{2}}$, we can immediately conclude

$$
\left\||D|^{\sigma}(N u-N v)(t, \cdot)\right\|_{L^{2}} \lesssim\left(1+B_{\lambda}(0, t)\right)^{-\frac{n}{2}\left(\frac{1}{m}-\frac{1}{2}\right)-\frac{\sigma}{2}}\|u-v\|_{X(t)}\left(\|u\|_{X(t)}^{p-1}+\|v\|_{X(t)}^{p-1}\right) .
$$

In the same way, with no other requirements to the range of admissible exponents $p$ one can derive

$$
\begin{aligned}
\|(N u-N v)(t, \cdot)\|_{L^{2}} & \lesssim\left(1+B_{\lambda}(0, t)\right)^{-\frac{n}{2}\left(\frac{1}{m}-\frac{1}{2}\right)}\|u-v\|_{X(t)}\left(\|u\|_{X(t)}^{p-1}+\|v\|_{X(t)}^{p-1}\right), \\
\left\|\partial_{t}(N u-N v)(t, \cdot)\right\|_{L^{2}} & \lesssim \Phi_{1, m}(0, t)\|u-v\|_{X(t)}\left(\|u\|_{X(t)}^{p-1}+\|v\|_{X(t)}^{p-1}\right), \\
\left\||D|^{\sigma-1} \partial_{t}(N u-N v)(t, \cdot)\right\|_{L^{2}} & \lesssim \Phi_{2, m}(0, t)\|u-v\|_{X(t)}\left(\|u\|_{X(t)}^{p-1}+\|v\|_{X(t)}^{p-1}\right),
\end{aligned}
$$

where $\Phi_{1, m}=\Phi_{1, m}(0, t)$ and $\Phi_{2, m}=\Phi_{1, m}(0, t)$ are defined in (3.1.13) and (3.1.15) for $\tau=t$, respectively. The proof is completed if we replace all these estimates into the definition of the norm of the solution space $X(t)$ to get (3.4.5).

\subsubsection{Case of super-exponential propagation speed}

Now we will consider the case of super-exponential propagation speed with suitable higher regularity of the data and additional $L^{m}$ regularity, $m \in[1,2)$, similarly as in the previous section. We have the following statement.

Theorem 3.4.2. We assume that the Hypotheses (A1) to (A5), (B1) to (B6), (C1), (C3) and (C4) hold. Let $\nu(\delta)=\infty$ and $\vartheta(\delta)=\infty$ and the following condition for the functions $\nu=\nu(\delta, t)$ and $\vartheta=\vartheta(\delta, t)$ (see (3.1.2) and (3.1.3)) hold:

$$
\max \{\nu(\delta, t) ; \vartheta(\delta, t)\}=o(\log \Lambda(t))
$$

We consider the data $\left(u_{0}, u_{1}\right) \in \mathcal{D}_{m}^{\sigma}$ with $\sigma \in\left(1,1+\frac{n}{2}\right]$ and $m \in[1,2)$, and that the exponent $p$ satisfies

$$
p>\max \left\{\lceil\sigma\rceil ; \frac{2}{m}\right\} \quad \text { and } \quad p \leq 1+\frac{2}{n-2 \sigma} \quad \text { if } \quad n>2 \sigma
$$

Then, there exists a constant $\varepsilon_{0}>0$ such that for all $\left(u_{0}, u_{1}\right) \in \mathcal{D}_{m}^{\sigma}$ with $\left\|\left(u_{0}, u_{1}\right)\right\|_{\mathcal{D}_{m}^{\sigma}} \leq \varepsilon_{0}$ there is a uniquely determined energy solution

$$
u \in \mathcal{C}\left([0, \infty), H^{\sigma}\right) \cap \mathcal{C}^{1}\left([0, \infty), H^{\sigma-1}\right)
$$

to the Cauchy problem (3.1.1).

Furthermore, there exists a constant $C>0$ such that the solution satisfies the following 
estimates:

$$
\begin{aligned}
&\|u(t, \cdot)\|_{L^{2}} \leq C\left(1+B_{\lambda}(0, t)\right)^{-\frac{n}{2}\left(\frac{1}{m}-\frac{1}{2}\right)}\left\|\left(u_{0}, u_{1}\right)\right\|_{\mathcal{D}_{m}^{\sigma}}, \\
&\left\||D|^{\sigma} u(t, \cdot)\right\|_{L^{2}} \leq C\left(1+B_{\lambda}(0, t)\right)^{-\frac{n}{2}\left(\frac{1}{m}-\frac{1}{2}\right)-\frac{\sigma}{2}}\left\|\left(u_{0}, u_{1}\right)\right\|_{\mathcal{D}_{m}^{\sigma}}, \\
&\left\|u_{t}(t, \cdot)\right\|_{L^{2}} \leq C \max \left\{\frac{\lambda^{2}(t)}{\rho(t)}\left(1+B_{\lambda}(0, t)\right)^{-\frac{n}{2}\left(\frac{1}{m}-\frac{1}{2}\right)-1} ;\right. \\
&\left.\lambda(t) F(\Lambda(t))\left(F^{2}(\Lambda(t))\right)^{-\frac{n}{2}\left(\frac{1}{m}-\frac{1}{2}\right)-1}\right\}\left\|\left(u_{0}, u_{1}\right)\right\|_{\mathcal{D}_{m}^{\sigma}}, \\
&\left\||D|^{\sigma-1} u_{t}(t, \cdot)\right\|_{L^{2}} \leq C \max \left\{\frac{\lambda^{2}(t)}{\rho(t)}\left(1+B_{\lambda}(0, t)\right)^{-\frac{n}{2}\left(\frac{1}{m}-\frac{1}{2}\right)-\frac{\sigma-1}{2}-1} ;\right. \\
&\left.\lambda(t) F(\Lambda(t))\left(F^{2}(\Lambda(t))\right)^{-\frac{n}{2}\left(\frac{1}{m}-\frac{1}{2}\right)-\frac{\sigma-1}{2}-1}\right\}\left\|\left(u_{0}, u_{1}\right)\right\|_{\mathcal{D}_{m}^{\sigma}} .
\end{aligned}
$$

Proof. We proceed analogously to the proof of Theorem 3.4.1. We introduce in the same way the space of solutions $X(t)$ by

$$
X(t)=\mathcal{C}\left([0, t], H^{\sigma}\right) \cap \mathcal{C}^{1}\left([0, t], H^{\sigma-1}\right)
$$

and its norm $\|u\|_{X(t)}$ as follows:

$$
\begin{aligned}
\|u(\tau, \cdot)\|_{X(t)}=\sup _{0 \leq \tau \leq t}[ & \left(1+B_{\lambda}(0, \tau)\right)^{\frac{n}{2}\left(\frac{1}{m}-\frac{1}{2}\right)}\|u(\tau, \cdot)\|_{L^{2}} \\
& +\left(1+B_{\lambda}(0, \tau)\right)^{\frac{n}{2}\left(\frac{1}{m}-\frac{1}{2}\right)+\frac{\sigma}{2}}\left\||D|^{\sigma} u(\tau, \cdot)\right\|_{L^{2}} \\
& \left.+\Phi_{1, m}^{-1}(0, \tau)\left\|u_{t}(\tau, \cdot)\right\|_{L^{2}}+\Phi_{2, m}^{-1}(0, \tau)\left\||D|^{\sigma-1} u_{t}(\tau, \cdot)\right\|_{L^{2}}\right] .
\end{aligned}
$$

Here $\Phi_{1, m}=\Phi_{1, m}(0, \tau)$ and $\Phi_{2, m}=\Phi_{2, m}(0, \tau)$ are defined in (3.1.13) and (3.1.15), respectively for $s=0$ and $t=\tau$.

Our aim is again to prove the following two inequalities:

$$
\begin{aligned}
\|N u\|_{X(t)} & \lesssim\left\|\left(u_{0}, u_{1}\right)\right\|_{\mathcal{D}_{m}^{\sigma}}+\|u\|_{X(t)}^{p}, \\
\|N u-N v\|_{X(t)} & \lesssim\|u-v\|_{X(t)}\left(\|u\|_{X(t)}^{p-1}+\|v\|_{X(t)}^{p-1}\right) .
\end{aligned}
$$

Let us begin to the proof of inequality (3.4.31). By Theorem 3.1.2 it is clear that

$$
\left\|u^{\operatorname{lin}}\right\|_{X(t)}=\left\|K_{0}(t, 0, x) *_{(x)} u_{0}(x)+K_{1}(t, 0, x) *_{(x)} u_{1}(x)\right\|_{X(t)} \lesssim\left\|\left(u_{0}, u_{1}\right)\right\|_{D_{m}^{\sigma}}
$$

To complete the proof of (3.4.31) it remains to estimate the integral term

$$
u^{\mathrm{nl}}(t, x)=\int_{0}^{t} K_{1}(t, s, x) *(x)|u(s, x)|^{p} d s
$$

as in the proof of Theorem 3.3.2. Therefore, we consider the following norms:

$$
\left\|u^{\mathrm{nl}}(t, \cdot)\right\|_{L^{2}}, \quad\left\||D|^{\sigma} u^{\mathrm{nl}}(t, \cdot)\right\|_{L^{2}}, \quad\left\|u_{t}^{\mathrm{nl}}(t, \cdot)\right\|_{L^{2}} \quad \text { and } \quad\left\||D|^{\sigma-1} u_{t}^{\mathrm{nl}}(t, \cdot)\right\|_{L^{2}} \cdot
$$

We start to estimate the norm $\left\||D|^{\sigma} u^{\mathrm{nl}}(t, \cdot)\right\|_{L^{2}}$. As we derived in the proof of Theorem 3.4.1 employing fractional chain rule (cf. Proposition A.7.7) and the fractional GagliardoNirenberg inequality (cf. Corollary A.7.3), respectively, we may conclude the estimate

$$
\begin{aligned}
\left\||D|^{\sigma} u^{\mathrm{nl}}(t, \cdot)\right\|_{L^{2}} \lesssim & \|u\|_{X(t)}^{p} \underbrace{\int_{0}^{\delta t} \frac{\Lambda(s)}{\lambda(s)}\left(1+B_{\lambda}(s, t)\right)^{-\frac{n}{2}\left(\frac{1}{m}-\frac{1}{2}\right)-\frac{\sigma}{2}}\left(1+B_{\lambda}(0, s)\right)^{-\frac{n}{2 m} p+\frac{n}{2 m}} d s}_{\mathbf{G}} \\
& +\|u\|_{X(t)}^{p} \underbrace{\int_{\delta t}^{t} \frac{\Lambda(s)}{\lambda(s)}\left(1+B_{\lambda}(s, t)\right)^{-\frac{\sigma}{2}}\left(1+B_{\lambda}(0, s)\right)^{-\frac{n}{2 m} p+\frac{n}{4}} d s}_{\mathbf{H}}
\end{aligned}
$$


with the same requirements to the admissible exponent $p$

$$
1<p \leq 1+\frac{2}{n-2 \sigma} \text { and } p>\lceil\sigma-1\rceil .
$$

Similarly as we did in the proof of Theorems 3.2.2 and 3.3.2, now we want to estimate the integrals $\mathbf{G}$ and $\mathbf{H}$ by using the estimates for $B_{\lambda}(s, t)$ from Lemma 3.1.4 by the aid of condition (C3).

Firstly, let us consider the integral G. It holds

$$
\mathbf{G} \lesssim\left(1+B_{\lambda}(0, t)\right)^{-\frac{n}{2}\left(\frac{1}{m}-\frac{1}{2}\right)-\frac{\sigma}{2}} \underbrace{\int_{0}^{\delta t} \frac{\Lambda(s)}{\lambda(s)}\left(1+B_{\lambda}(0, s)\right)^{-\frac{n}{2 m} p+\frac{n}{2 m}} d s}_{\bar{G}_{1}(t)}
$$

where we used (3.1.21). We have shown in the proof of Theorem 3.2.2 that the integral $\bar{A}_{1}=\bar{A}_{1}(t)$ in (3.2.26), which coincides with $\bar{G}_{1}=\bar{G}_{1}(t)$, is bounded for large time $t$ in the cases $\alpha_{1}(p) \neq 1$ and $\alpha_{1}(p)=1$, respectively. Therefore, we may conclude the estimate

$$
\mathbf{G} \lesssim\left(1+B_{\lambda}(0, t)\right)^{-\frac{n}{2}\left(\frac{1}{m}-\frac{1}{2}\right)-\frac{\sigma}{2}} .
$$

Next, we consider the integral $\mathbf{H}$. Defining $\alpha_{2}(p)=\frac{n}{2 m} p-\frac{n}{4}$ it yields

$$
\begin{aligned}
\mathbf{H}= & -\int_{\delta t}^{t} \frac{\mu(s)}{\lambda^{2}(s)}\left(1+B_{\lambda}(0, s)\right)^{-\alpha_{2}(p)}\left(1+B_{\lambda}(s, t)\right)^{-\frac{\sigma}{2}} d\left(1+B_{\lambda}(s, t)\right) \\
= & \left.\frac{-1}{1-\frac{\sigma}{2}} \frac{\mu(s)}{\lambda^{2}(s)}\left(1+B_{\lambda}(0, s)\right)^{-\alpha_{2}(p)}\left(1+B_{\lambda}(s, t)\right)^{-\frac{\sigma}{2}+1}\right|_{\delta t} ^{t} \\
& +\frac{1}{1-\frac{\sigma}{2}} \int_{\delta t}^{t}\left(1+B_{\lambda}(s, t)\right)^{-\frac{\sigma}{2}+1} d\left(\frac{\mu(s)}{\lambda^{2}(s)}\left(1+B_{\lambda}(0, s)\right)^{-\alpha_{2}(p)}\right) .
\end{aligned}
$$

We will distinguish between the following cases:

Case 1: $-\frac{\sigma}{2}+1>0$. It yields

$$
\begin{aligned}
\mathbf{H} \lesssim & -\frac{\mu(t)}{\lambda^{2}(t)}\left(1+B_{\lambda}(0, t)\right)^{-\alpha_{2}(p)}+\frac{\mu(\delta t)}{\lambda^{2}(\delta t)}\left(1+B_{\lambda}(0, \delta t)\right)^{-\alpha_{2}(p)}\left(1+B_{\lambda}(\delta t, t)\right)^{-\frac{\sigma}{2}+1} \\
& +\int_{\delta t}^{t} \frac{\mu(s)}{\lambda^{2}(s)}\left(1+B_{\lambda}(0, s)\right)^{-\alpha_{2}(p)}\left(1+B_{\lambda}(s, t)\right)^{-\frac{\sigma}{2}+1} \frac{d\left(1+B_{\lambda}(0, s)\right)}{1+B_{\lambda}(0, s)}
\end{aligned}
$$

where we used Lemma 3.1.5. Then, it follows

$$
\begin{aligned}
\left(1+B_{\lambda}(0,\right. & t))^{\frac{n}{2}\left(\frac{1}{m}-\frac{1}{2}\right)+\frac{\sigma}{2}} \mathbf{H} \\
& \lesssim \underbrace{\frac{\mu(\delta t)}{\lambda^{2}(\delta t)}\left(1+B_{\lambda}(0, \delta t)\right)^{-\alpha_{2}(p)}\left(1+B_{\lambda}(0, t)\right)^{\frac{n}{2}\left(\frac{1}{m}-\frac{1}{2}\right)+1}}_{\bar{H}_{1}(t)} \\
& +\underbrace{\int_{\delta t}^{t} \frac{\mu(s)}{\lambda^{2}(s)}\left(1+B_{\lambda}(0, s)\right)^{-\alpha_{2}(p)}\left(1+B_{\lambda}(0, t)\right)^{\frac{n}{2}\left(\frac{1}{m}-\frac{1}{2}\right)+1} \frac{d\left(1+B_{\lambda}(0, s)\right)}{1+B_{\lambda}(0, s)}}_{\widetilde{H}_{2}(t)} .
\end{aligned}
$$

In order to derive the desired estimate for $\mathbf{H}$ we suppose that there exists a positive constant $\varepsilon$ such that it holds

$$
\frac{\mu(\delta t)}{\lambda^{2}(\delta t)}\left(1+B_{\lambda}(0, \delta t)\right)^{-\alpha_{2}(p)}\left(1+B_{\lambda}(0, t)\right)^{\frac{n}{2}\left(\frac{1}{m}-\frac{1}{2}\right)+1} \lesssim\left(1+B_{\lambda}(0, \delta t)\right)^{-\varepsilon} .
$$


Then, from the previous estimate it is sufficient to consider the monotonicity of the function

$$
\overline{\mathcal{H}}_{1}(t):=\frac{\mu(\delta t)}{\lambda^{2}(\delta t)}\left(1+B_{\lambda}(0, \delta t)\right)^{-\alpha_{2}(p)+\varepsilon}\left(1+B_{\lambda}(0, t)\right)^{\frac{n}{2}\left(\frac{1}{m}-\frac{1}{2}\right)+1} .
$$

From (3.2.30) we can see immediately that $\overline{\mathcal{H}}_{1}^{\prime}(t)<0$ by the condition (3.4.29). This gives that $\bar{H}_{1}=\bar{H}_{1}(t)$ and $\bar{H}_{2}=\bar{H}_{2}(t)$ are both uniformly bounded. For this reason we obtain

$$
\mathbf{H} \lesssim\left(1+B_{\lambda}(0, t)\right)^{-\frac{n}{2}\left(\frac{1}{m}-\frac{1}{2}\right)-\frac{\sigma}{2}} .
$$

Case 2: $-\frac{\sigma}{2}+1<0$. It holds

$$
\begin{aligned}
\mathbf{H} \lesssim & \frac{\mu(t)}{\lambda^{2}(t)}\left(1+B_{\lambda}(0, t)\right)^{-\alpha_{2}(p)}-\frac{\mu(\delta t)}{\lambda^{2}(\delta t)}\left(1+B_{\lambda}(0, \delta t)\right)^{-\alpha_{2}(p)}\left(1+B_{\lambda}(\delta t, t)\right)^{-\frac{\sigma}{2}+1} \\
& +\int_{\delta t}^{t} \frac{\mu(s)}{\lambda^{2}(s)}\left(1+B_{\lambda}(0, s)\right)^{-\alpha_{2}(p)}\left(1+B_{\lambda}(s, t)\right)^{-\frac{\sigma}{2}+1} \frac{d\left(1+B_{\lambda}(0, s)\right)}{1+B_{\lambda}(0, s)}
\end{aligned}
$$

where again we used Lemma 3.1.5. Then, we get

$$
\begin{aligned}
\left(1+B_{\lambda}(0, t)\right)^{\frac{n}{2}\left(\frac{1}{m}-\frac{1}{2}\right)+\frac{\sigma}{2}} \mathbf{H} & \lesssim \\
\lesssim & \underbrace{\frac{\mu(t)}{\lambda^{2}(t)}\left(1+B_{\lambda}(0, t)\right)^{-\alpha_{2}(p)+\frac{n}{2}\left(\frac{1}{m}-\frac{1}{2}\right)+\frac{\sigma}{2}}}_{\bar{H}_{3}(t)} \\
& +\underbrace{\int_{\delta t}^{t} \frac{\mu(s)}{\lambda^{2}(s)}\left(1+B_{\lambda}(0, s)\right)^{-\alpha_{2}(p)}\left(1+B_{\lambda}(0, t)\right)^{\frac{n}{2}\left(\frac{1}{m}-\frac{1}{2}\right)+\frac{\sigma}{2}} \frac{d\left(1+B_{\lambda}(0, s)\right)}{1+B_{\lambda}(0, s)}}_{\bar{H}_{4}(t)} .
\end{aligned}
$$

For $\bar{H}_{3}=\bar{H}_{3}(t)$, using $\alpha_{2}(p)=\alpha_{1}(p)+\frac{n}{2}\left(\frac{1}{m}-\frac{1}{2}\right)$ we find

$$
\bar{H}_{3}(t)=\frac{\mu(t)}{\lambda^{2}(t)}\left(1+B_{\lambda}(0, t)\right)^{-\alpha_{1}(p)+\frac{\sigma}{2}} .
$$

Then, it follows

$$
\begin{aligned}
\bar{H}_{3}^{\prime}(t) & =\left(\frac{\mu(t)}{\lambda^{2}(t)}\right)^{\prime}\left(1+B_{\lambda}(0, t)\right)^{-\alpha_{1}(p)+\frac{\sigma}{2}}+\left(-\alpha_{1}(p)+\frac{\sigma}{2}\right) \frac{\mu(t)}{\lambda^{2}(t)} \frac{\lambda^{2}(t)}{\rho(t)}\left(1+B_{\lambda}(0, t)\right)^{-\alpha_{1}(p)+\frac{\sigma}{2}-1} \\
& \leq\left[-\beta_{1} \frac{\mu(t)}{\lambda(t) \Lambda(t)}\left(1+B_{\lambda}(0, t)\right)+\left(-\alpha_{1}(p)+\frac{\sigma}{2}\right) \frac{\Lambda(t)}{\lambda(t)}\right]\left(1+B_{\lambda}(0, t)\right)^{-\alpha_{1}(p)+\frac{\sigma}{2}-1} \\
& \leq\left[-\frac{\beta_{1}}{\alpha+2} \log \Lambda(t)-\alpha_{1}(p)+\frac{\sigma}{2}\right] \frac{\Lambda(t)}{\lambda(t)}\left(1+B_{\lambda}(0, t)\right)^{-\alpha_{1}(p)+\frac{\sigma}{2}-1},
\end{aligned}
$$

where we used condition (C4) and (3.1.20), respectively. Then, for large time $t$ we obtain $\bar{H}_{3}^{\prime}(t)<0$. As for $\bar{H}_{4}=\bar{H}_{4}(t)$, we may conclude the desired estimates similarly to $\bar{H}_{1}=\bar{H}_{1}(t)$ and $\bar{H}_{2}=\bar{H}_{2}(t)$. Hence, we arrive at the desired estimate

$$
\mathbf{H} \lesssim\left(1+B_{\lambda}(0, t)\right)^{-\frac{n}{2}\left(\frac{1}{m}-\frac{1}{2}\right)-\frac{\sigma}{2}} .
$$

Case 3: $-\frac{\sigma}{2}+1=0$. In this case we have

$$
\begin{aligned}
\mathbf{H}= & \int_{\delta t}^{t} \frac{\Lambda(s)}{\lambda(s)}\left(1+B_{\lambda}(s, t)\right)^{-1}\left(1+B_{\lambda}(0, s)\right)^{-\alpha_{2}(p)} d s \\
= & -\int_{\delta t}^{t} \frac{\mu(s)}{\lambda^{2}(s)}\left(1+B_{\lambda}(0, s)\right)^{-\alpha_{2}(p)} \frac{d\left(1+B_{\lambda}(s, t)\right)}{1+B_{\lambda}(s, t)} \\
= & -\left.\frac{\mu(s)}{\lambda^{2}(s)}\left(1+B_{\lambda}(0, s)\right)^{-\alpha_{2}(p)} \log \left(1+B_{\lambda}(s, t)\right)\right|_{\delta t} ^{t} \\
& +\int_{\delta t}^{t} \log \left(1+B_{\lambda}(s, t)\right) d\left(\frac{\mu(s)}{\lambda^{2}(s)}\left(1+B_{\lambda}(0, s)\right)^{-\alpha_{2}(p)}\right) .
\end{aligned}
$$


Applying Lemma 3.1 .5 and since $\frac{\sigma}{2}=1$ we have

$$
\begin{aligned}
& \left(1+B_{\lambda}(0, t)\right)^{\frac{n}{2}\left(\frac{1}{m}-\frac{1}{2}\right)+\frac{\sigma}{2}} \mathbf{H} \\
& \lesssim \underbrace{\mu(\delta t)}_{\bar{H}_{5}(t)}\left(1+B_{\lambda}(0, \delta t)\right)^{-\alpha_{2}(p)}\left(1+B_{\lambda}(0, t)\right)^{\frac{n}{2}\left(\frac{1}{m}-\frac{1}{2}\right)+1} \log \left(1+B_{\lambda}(0, t)\right) \\
& +\underbrace{\int_{\delta t}^{t} \frac{\mu(s)}{\lambda^{2}(s)}\left(1+B_{\lambda}(0, s)\right)^{-\alpha_{2}(p)}\left(1+B_{\lambda}(0, t)\right)^{\frac{n}{2}\left(\frac{1}{m}-\frac{1}{2}\right)+1} \log \left(1+B_{\lambda}(0, t)\right) \frac{d\left(1+B_{\lambda}(0, s)\right)}{1+B_{\lambda}(0, s)}}_{\bar{H}_{6}(t)} .
\end{aligned}
$$

For the boundedness of $\bar{H}_{5}=\bar{H}_{5}(t)$ we have

$$
\bar{H}_{5}(t)=\frac{\log \left(1+B_{\lambda}(0, t)\right)}{\left(1+B_{\lambda}(0, \delta t)\right)^{\varepsilon}} \overline{\mathcal{H}}_{1}(t) \lesssim \frac{\log \left(1+B_{\lambda}(0, t)\right)}{\left(1+B_{\lambda}(0, \delta t)\right)^{\varepsilon}} \lesssim 1
$$

for a positive constant $\varepsilon>1$ and for large time $t$. Here we have used the boundedness of $\overline{\mathcal{H}}_{1}=\overline{\mathcal{H}}_{1}(t)$ which is given in (3.4.35) and l'Hospital's rule (cf. condition (B4)) for

$$
\begin{aligned}
\lim _{t \rightarrow \infty} \sup \frac{\log \left(1+B_{\lambda}(0, t)\right)}{\left(1+B_{\lambda}(0, \delta t)\right)^{\varepsilon}} & =\lim _{t \rightarrow \infty} \sup \frac{\frac{\lambda(t) \Lambda(t)}{\mu(t)}}{\varepsilon \delta\left(1+B_{\lambda}(0, t)\right) \frac{\lambda(\delta t) \Lambda(\delta t)}{\mu(\delta t)}\left(1+B_{\lambda}(0, \delta t)\right)^{\varepsilon-1}} \\
& \lesssim \lim _{t \rightarrow \infty} \sup \frac{\frac{\lambda(t)}{\Lambda(t)} \phi(t)}{\phi(t) \log \Lambda(t) \frac{\lambda(\delta t)}{\Lambda(\delta t)} \phi(\delta t)\left(1+B_{\lambda}(0, \delta t)\right)^{\varepsilon-1}} \\
& \lesssim \lim _{t \rightarrow \infty} \sup \frac{\lambda(t)}{\Lambda(t)} \frac{\Lambda(\delta t)}{\lambda(\delta t)} \frac{1}{\phi(\delta t) \log \Lambda(t)} \frac{1}{\left(1+B_{\lambda}(0, \delta t)\right)^{\varepsilon-1}} \lesssim 1 .
\end{aligned}
$$

On the other hand, for $\bar{H}_{6}=\bar{H}_{6}(t)$ after using $\overline{\mathcal{H}}_{1}=\overline{\mathcal{H}}_{1}(t)$ and (3.4.36), respectively, we have

$$
\begin{aligned}
\bar{H}_{6}(t) & \lesssim \log \left(1+B_{\lambda}(0, t)\right) \int_{\delta t}^{t}\left(1+B_{\lambda}(0, s)\right)^{-\varepsilon-1} d\left(1+B_{\lambda}(0, s)\right) \\
& =\frac{\log \left(1+B_{\lambda}(0, t)\right)}{\varepsilon\left(1+B_{\lambda}(0, \delta t)\right)^{\varepsilon}}-\frac{\log \left(1+B_{\lambda}(0, t)\right)}{\varepsilon\left(1+B_{\lambda}(0, t)\right)^{\varepsilon}} \lesssim 1 .
\end{aligned}
$$

Therefore, we obtain the desired estimate

$$
\mathbf{H} \lesssim\left(1+B_{\lambda}(0, t)\right)^{-\frac{n}{2}\left(\frac{1}{m}-\frac{1}{2}\right)-\frac{\sigma}{2}} .
$$

Taking into consideration the derived estimates for $\mathbf{G}$ and $\mathbf{H}$ we arrive at

$$
\left\||D|^{\sigma} u^{\mathrm{nl}}(t, \cdot)\right\|_{L^{2}} \lesssim\|u\|_{X(t)}^{p}\left(1+B_{\lambda}(0, t)\right)^{-\frac{n}{2}\left(\frac{1}{m}-\frac{1}{2}\right)-\frac{\sigma}{2}} .
$$

In the same way, following the proof of Theorem 3.3.2 one can prove

$$
\begin{aligned}
\left\|u^{\mathrm{nl}}(t, \cdot)\right\|_{L^{2}} & \lesssim\|u\|_{X(t)}^{p}\left(1+B_{\lambda}(0, t)\right)^{-\frac{n}{2}\left(\frac{1}{m}-\frac{1}{2}\right)}, \\
\left\|u_{t}^{\mathrm{nl}}(t, \cdot)\right\|_{L^{2}} & \lesssim\|u\|_{X(t)}^{p} \max \left\{\frac{\lambda^{2}(t)}{\rho(t)}\left(1+B_{\lambda}(0, t)\right)^{-\frac{n}{2}\left(\frac{1}{m}-\frac{1}{2}\right)-1} ; \lambda(t) F(\Lambda(t))\left(F^{2}(\Lambda(t))\right)^{-\frac{n}{2}\left(\frac{1}{m}-\frac{1}{2}\right)-1}\right\} .
\end{aligned}
$$

Now let us continue with the estimate of the norm $\left\||D|^{\sigma-1} u_{t}^{\mathrm{nl}}(t, \cdot)\right\|_{L^{2}}$. Proceeding in the same way as we did to estimate the norm $\left\||D|^{\sigma} u^{\mathrm{nl}}(t, \cdot)\right\|_{L^{2}}$ with no other requirements to the 
admissible exponents $p$ we have the following estimate:

$$
\begin{aligned}
\left\||D|^{\sigma-1} u_{t}^{\mathrm{nl}}(t, \cdot)\right\|_{L^{2}} \lesssim & \|u\|_{X(t)}^{p} \underbrace{\int_{0}^{\delta t} \Phi_{2, m}(s, t) \frac{\Lambda(s)}{\lambda(s)}\left(1+B_{\lambda}(0, s)\right)^{-\frac{n}{2 m} p+\frac{n}{2 m}} d s}_{\mathbf{K}} \\
& +\|u\|_{X(t)}^{p} \underbrace{\int_{\delta t}^{t} \Phi_{2}(s, t) \frac{\Lambda(s)}{\lambda(s)}\left(1+B_{\lambda}(0, s)\right)^{-\frac{n}{2 m} p+\frac{n}{4}} d s}_{\mathbf{L}} .
\end{aligned}
$$

Here $\Phi_{2, m}=\Phi_{2, m}(s, t)$ and $\Phi_{2}=\Phi_{2}(s, t)$ are defined in (3.1.15) and (3.1.16), respectively. Firstly, we consider the integral $\mathbf{K}$. We have

$$
\mathbf{K} \lesssim \Phi_{2, m}(0, t) \underbrace{\int_{0}^{\delta t} \frac{\Lambda(s)}{\lambda(s)}\left(1+B_{\lambda}(0, s)\right)^{-\frac{n}{2 m} p+\frac{n}{2 m}} d s}_{\bar{K}_{1}(t)}
$$

where we used (3.1.21) to $B_{\lambda}(s, t)$. We have shown in the proof of Theorem 3.2.2 that the function $\bar{A}_{1}=\bar{A}_{1}(t)$ in (3.2.26), which coincides with $\bar{K}_{1}=\bar{K}_{1}(t)$, is bounded for large $t$. Thus, we have

$$
\mathbf{K} \lesssim \Phi_{2, m}(0, t)
$$

Now we consider the integral L. Defining $\alpha_{2}(p):=\frac{n}{2 m} p-\frac{n}{4}$, we restrict our considerations to the following two cases only, for all $s \in[\delta t, t]$ by condition $(\mathbf{C 1})$, as we did in the proof of Theorem 3.3.2.

Case a: $\Phi_{2}(s, t)=\frac{\lambda^{2}(t)}{\rho(t)}\left(1+B_{\lambda}(s, t)\right)^{-\frac{\sigma-1}{2}-1}$. In this case we have

$$
\begin{aligned}
\mathbf{L}= & \frac{\lambda^{2}(t)}{\rho(t)} \int_{\delta t}^{t} \frac{\Lambda(s)}{\lambda(s)}\left(1+B_{\lambda}(s, t)\right)^{-\frac{\sigma-1}{2}-1}\left(1+B_{\lambda}(0, s)\right)^{-\alpha_{2}(p)} d s \\
= & -\frac{\lambda^{2}(t)}{\rho(t)} \int_{\delta t}^{t} \frac{\mu(s)}{\lambda^{2}(s)}\left(1+B_{\lambda}(0, s)\right)^{-\alpha_{2}(p)}\left(1+B_{\lambda}(s, t)\right)^{-\frac{\sigma-1}{2}-1} d\left(1+B_{\lambda}(s, t)\right) \\
= & \left.\frac{2}{\sigma-1} \frac{\lambda^{2}(t)}{\rho(t)} \frac{\mu(s)}{\lambda^{2}(s)}\left(1+B_{\lambda}(0, s)\right)^{-\alpha_{2}(p)}\left(1+B_{\lambda}(s, t)\right)^{-\frac{\sigma-1}{2}}\right|_{\delta t} ^{t} \\
& -\frac{2}{\sigma-1} \frac{\lambda^{2}(t)}{\rho(t)} \int_{\delta t}^{t}\left(1+B_{\lambda}(s, t)\right)^{-\frac{\sigma-1}{2}} d\left(\frac{\mu(s)}{\lambda^{2}(s)}\left(1+B_{\lambda}(0, s)\right)^{-\alpha_{2}(p)}\right)
\end{aligned}
$$

After using Lemma 3.1.5 it follows

$$
\begin{aligned}
\frac{\rho(t)}{\lambda^{2}(t)} \mathbf{L} \lesssim & \frac{\mu(t)}{\lambda^{2}(t)}\left(1+B_{\lambda}(0, t)\right)^{-\alpha_{2}(p)}-\frac{\mu(\delta t)}{\lambda^{2}(\delta t)}\left(1+B_{\lambda}(0, \delta t)\right)^{-\alpha_{2}(p)}\left(1+B_{\lambda}(\delta t, t)\right)^{-\frac{\sigma-1}{2}} \\
& +\int_{\delta t}^{t} \frac{\mu(s)}{\lambda^{2}(s)}\left(1+B_{\lambda}(0, s)\right)^{-\alpha_{2}(p)}\left(1+B_{\lambda}(s, t)\right)^{-\frac{\sigma-1}{2}} \frac{d\left(1+B_{\lambda}(0, s)\right)}{1+B_{\lambda}(0, s)} .
\end{aligned}
$$

So, we have

$$
\begin{aligned}
\frac{\rho(t)}{\lambda^{2}(t)}(1 & \left.+B_{\lambda}(0, t)\right)^{\frac{n}{2}\left(\frac{1}{m}-\frac{1}{2}\right)+\frac{\sigma-1}{2}+1} \mathbf{L} \\
& \underbrace{\frac{\mu(t)}{\lambda^{2}(t)}\left(1+B_{\lambda}(0, t)\right)^{\frac{n}{2}\left(\frac{1}{m}-\frac{1}{2}\right)+\frac{\sigma-1}{2}+1-\alpha_{2}(p)}}_{\bar{L}_{1}(t)} \\
& +\underbrace{\int_{\delta t}^{t} \frac{\mu(s)}{\lambda^{2}(s)}\left(1+B_{\lambda}(0, s)\right)^{-\alpha_{2}(p)}\left(1+B_{\lambda}(0, t)\right)^{\frac{n}{2}\left(\frac{1}{m}-\frac{1}{2}\right)+\frac{\sigma-1}{2}+1} \frac{d\left(1+B_{\lambda}(0, s)\right)}{1+B_{\lambda}(0, s)}}_{\bar{L}_{2}(t)} .
\end{aligned}
$$


We begin with $\bar{L}_{1}=\bar{L}_{1}(t)$. Using $\alpha_{2}(p)=\alpha_{1}(p)+\frac{n}{2}\left(\frac{1}{m}-\frac{1}{2}\right)$ we get

$$
\bar{L}_{1}(t)=\frac{\mu(t)}{\lambda^{2}(t)}\left(1+B_{\lambda}(0, t)\right)^{\frac{\sigma+1}{2}-\alpha_{1}(p)} .
$$

So, we may conclude

$$
\begin{aligned}
\bar{L}_{1}^{\prime}(t) & =\left(\frac{\mu(t)}{\lambda^{2}(t)}\right)^{\prime}\left(1+B_{\lambda}(0, t)\right)^{\frac{\sigma+1}{2}-\alpha_{1}(p)}+\left(\frac{\sigma+1}{2}-\alpha_{1}(p)\right) \frac{\mu(t)}{\lambda^{2}(t)} \frac{\lambda^{2}(t)}{\rho(t)}\left(1+B_{\lambda}(0, t)\right)^{\frac{\sigma-1}{2}-\alpha_{1}(p)} \\
& \leq\left[-\beta_{1} \frac{\mu(t)}{\lambda(t) \Lambda(t)}\left(1+B_{\lambda}(0, t)\right)+\left(\frac{\sigma+1}{2}-\alpha_{1}(p)\right) \frac{\Lambda(t)}{\lambda(t)}\right]\left(1+B_{\lambda}(0, t)\right)^{\frac{\sigma-1}{2}-\alpha_{1}(p)} \\
& \leq\left[-\frac{\beta_{1}}{\alpha+2}+\left(\frac{\sigma+1}{2}-\alpha_{1}(p)\right) \frac{1}{\log \Lambda(t)}\right] \frac{\Lambda(t)}{\lambda(t)} \log \Lambda(t)\left(1+B_{\lambda}(0, t)\right)^{\frac{\sigma-1}{2}-\alpha_{1}(p)},
\end{aligned}
$$

where we used again condition (C4) and (3.1.20), respectively. Then, for a large time $t$ we get $\bar{L}_{1}^{\prime}(t)<0$. Therefore, this shows that $\bar{L}_{1}=\bar{L}_{1}(t)$ is uniformly bounded. For $\bar{L}_{2}=\bar{L}_{2}(t)$, we assume that there exists a constant $\varepsilon>0$ such that

$$
\frac{\mu(\delta t)}{\lambda^{2}(\delta t)}\left(1+B_{\lambda}(0, \delta t)\right)^{-\alpha_{2}(p)}\left(1+B_{\lambda}(0, t)\right)^{\frac{n}{2}\left(\frac{1}{m}-\frac{1}{2}\right)+\frac{\sigma-1}{2}+1} \lesssim\left(1+B_{\lambda}(0, \delta t)\right)^{-\varepsilon} .
$$

This implies to introduce

$$
\overline{\mathcal{L}}_{2}(t):=\frac{\mu(\delta t)}{\lambda^{2}(\delta t)}\left(1+B_{\lambda}(0, \delta t)\right)^{-\alpha_{2}(p)+\varepsilon}\left(1+B_{\lambda}(0, t)\right)^{\frac{n}{2}\left(\frac{1}{m}-\frac{1}{2}\right)+\frac{\sigma-1}{2}+1} .
$$

Then, analogously as in the proof of Theorem 3.2.2 for deriving the decreasing behavior of $\overline{\mathcal{B}}_{1}=\overline{\mathcal{B}}_{1}(t)$ in (3.2.30), employing the condition (3.4.29) we may also conclude that $\overline{\mathcal{L}}_{2}^{\prime}(t)<0$. This gives the desired estimates for $\bar{L}_{2}=\bar{L}_{2}(t)$. Therefore, we obtain

$$
\mathbf{L} \lesssim \frac{\lambda^{2}(t)}{\rho(t)}\left(1+B_{\lambda}(0, t)\right)^{-\frac{n}{2}\left(\frac{1}{m}-\frac{1}{2}\right)-\frac{\sigma-1}{2}-1}
$$

Consequently, in the Case a from (3.4.37) and (3.4.38) we obtain

$$
\left\||D|^{\sigma-1} u_{t}^{\mathrm{nl}}(t, \cdot)\right\|_{L^{2}} \lesssim\|u\|_{X(t)}^{p} \frac{\lambda^{2}(t)}{\rho(t)}\left(1+B_{\lambda}(0, t)\right)^{-\frac{n}{2}\left(\frac{1}{m}-\frac{1}{2}\right)-\frac{\sigma-1}{2}-1} .
$$

Case b: $\Phi(s, t)=\lambda(t) F(\Lambda(t))\left(F^{2}(\Lambda(t))\right)^{-\frac{\sigma-1}{2}-1}$. In this case we have

$$
\begin{aligned}
\mathbf{L} & =\lambda(t) F(\Lambda(t))\left(F^{2}(\Lambda(t))\right)^{-\frac{\sigma-1}{2}-1} \int_{\delta t}^{t} \frac{\Lambda(s)}{\lambda(s)}\left(1+B_{\lambda}(0, s)\right)^{-\alpha_{2}(p)} d s \\
& =\lambda(t) F(\Lambda(t))\left(F^{2}(\Lambda(t))\right)^{-\frac{\sigma-1}{2}-1} \int_{\delta t}^{t} \frac{\mu(s)}{\lambda^{2}(s)}\left(1+B_{\lambda}(0, s)\right)^{-\alpha_{2}(p)} d\left(1+B_{\lambda}(0, s)\right) .
\end{aligned}
$$

To get the desired estimate for $\mathbf{L}$ we follow the proof of Theorem 3.3.2 in the Case b.1: $\alpha_{2}(p) \neq 1$ and in the Case b.2: $\alpha_{2}(p)=1$ by using (3.4.29). Thus, we may conclude

$$
\mathbf{L} \lesssim \lambda(t) F(\Lambda(t))\left(F^{2}(\Lambda(t))\right)^{-\frac{n}{2}\left(\frac{1}{m}-\frac{1}{2}\right)-\frac{\sigma-1}{2}-1} .
$$

Consequently, in the Case $b$ from (3.4.37) and (3.4.40) we have

$$
\left\||D|^{\sigma-1} u_{t}^{\mathrm{nl}}(t, \cdot)\right\|_{L^{2}} \lesssim\|u\|_{X(t)}^{p} \lambda(t) F(\Lambda(t))\left(F^{2}(\Lambda(t))\right)^{-\frac{n}{2}\left(\frac{1}{m}-\frac{1}{2}\right)-\frac{\sigma-1}{2}-1} .
$$

Together with the estimates (3.4.39) and (3.4.41) it yields the desired estimate

$$
\begin{array}{r}
\left\||D|^{\sigma-1} u_{t}^{\mathrm{nl}}(t, \cdot)\right\|_{L^{2}} \lesssim\|u\|_{X(t)}^{p} \max \left\{\frac{\lambda^{2}(t)}{\rho(t)}\left(1+B_{\lambda}(0, t)\right)^{-\frac{n}{2}\left(\frac{1}{m}-\frac{1}{2}\right)-\frac{\sigma-1}{2}-1} ;\right. \\
\left.\lambda(t) F(\Lambda(t))\left(F^{2}(\Lambda(t))\right)^{-\frac{n}{2}\left(\frac{1}{m}-\frac{1}{2}\right)-\frac{\sigma-1}{2}-1}\right\} .
\end{array}
$$


Taking into consideration the norm of the solution space $X(t)$, all together the derived estimates for the norms $\left\|u^{\mathrm{nl}}(t, \cdot)\right\|_{L^{2}},\left\||D|^{\sigma} u^{\mathrm{nl}}(t, \cdot)\right\|_{L^{2}},\left\|u_{t}^{\mathrm{nl}}(t, \cdot)\right\|_{L^{2}}$ and $\left\||D|^{\sigma-1} u_{t}^{\mathrm{nl}}(t, \cdot)\right\|_{L^{2}}$ yield

$$
\left\|u^{\mathrm{nl}}(t, \cdot)\right\|_{X(t)} \lesssim\|u\|_{X(t)}^{p} .
$$

From (3.4.33) and (3.4.42), we get (3.4.31).

Let us consider now the estimate (3.4.32). We proceed as we did in the proof of Theorem 3.4.1. The goal is to estimate the norm $\left\||D|^{\sigma}(N u-N v)(t, \cdot)\right\|_{L^{2}}$. It holds

$$
\begin{aligned}
\||D|^{\sigma} \int_{0}^{t} & K_{1}(t, s, x) *_{(x)}\left(|u(s, x)|^{p}-|v(s, x)|^{p}\right) d s \|_{L^{2}} \\
\lesssim & \int_{0}^{\delta t} \frac{\Lambda(s)}{\lambda(s)}\left(1+B_{\lambda}(s, t)\right)^{-\frac{n}{2}\left(\frac{1}{m}-\frac{1}{2}\right)-\frac{\sigma}{2}}\left\||u(s, x)|^{p}-|v(s, x)|^{p}\right\|_{L^{m} \cap L^{2} \cap \dot{H}^{\sigma-1}} \\
& +\int_{\delta t}^{t} \frac{\Lambda(s)}{\lambda(s)}\left(1+B_{\lambda}(s, t)\right)^{-\frac{\sigma}{2}}\left\||u(s, x)|^{p}-|v(s, x)|^{p}\right\|_{L^{2} \cap \dot{H}^{\sigma-1}} d s
\end{aligned}
$$

Employing the tools which we explained in Appendix A.7 and applied in the same way as in the proof of Theorem 3.4.1, by the conditions

$$
1<p \leq 1+\frac{2}{n-2 \sigma} \text { with } n>2 \sigma \text { and } p>\lceil\sigma\rceil
$$

we may conclude the estimate

$$
\begin{gathered}
\left\||D|^{\sigma} \int_{0}^{t} K_{1}(t, s, x) *_{(x)}\left(|u(s, x)|^{p}-|v(s, x)|^{p}\right) d s\right\|_{L^{2}} \lesssim\left(\|u\|_{X(t)}^{p-1}+\|v\|_{X(t)}^{p-1}\right)\|u-v\|_{X(t)} \\
\times\left(\int_{0}^{\delta t} \frac{\Lambda(s)}{\lambda(s)}\left(1+B_{\lambda}(s, t)\right)^{-\frac{n}{2}\left(\frac{1}{m}-\frac{1}{2}\right)-\frac{\sigma}{2}}\left(1+B_{\lambda}(0, s)\right)^{-\frac{n}{2 m} p+\frac{n}{2 m}} d s\right. \\
\left.\quad+\int_{\delta t}^{t} \frac{\Lambda(s)}{\lambda(s)}\left(1+B_{\lambda}(s, t)\right)^{-\frac{\sigma}{2}}\left(1+B_{\lambda}(0, s)\right)^{-\frac{n}{2 m} p+\frac{n}{4}} d s\right) .
\end{gathered}
$$

Following the same steps to estimate the norm $\left\||D|^{\sigma} u^{\mathrm{nl}}(t, \cdot)\right\|_{L^{2}}$ we obtain

$$
\left\||D|^{\sigma}(N u-N v)(t, \cdot)\right\|_{L^{2}} \lesssim\left(1+B_{\lambda}(0, t)\right)^{-\frac{n}{2}\left(\frac{1}{m}-\frac{1}{2}\right)-\frac{\sigma}{2}}\|u-v\|_{X(t)}\left(\|u\|_{X(t)}^{p-1}+\|v\|_{X(t)}^{p-1}\right) .
$$

In the same way, with no other requirements to the admissible exponents $p$ we can derive the estimates $\|(N u-N v)(t, \cdot)\|_{L^{2}},\left\|\partial_{t}(N u-N v)(t, \cdot)\right\|_{L^{2}}$ and $\left\||D|^{\sigma-1} \partial_{t}(N u-N v)(t, \cdot)\right\|_{L^{2}}$. Finally, we replace all these estimates into the definition of the norm of the solution space $X(t)$ to get (3.4.32), and this concludes the proof.

Remark 3.4.1. From the condition (3.4.2) of Theorem 3.4.1 and from condition (3.4.30) of Theorem 3.4.2, if $p \in\left(\lceil\sigma\rceil, 1+\frac{2}{n-2 \sigma}\right]$ when $n>2 \sigma$, then this condition implies that we have $(n-2 \sigma)(\lceil\sigma\rceil-1)<2$. Therefore, the admissible range for $p$ is not empty for $\sigma$ close to 1 in low space dimensions, whereas higher space dimensions are allowed to suppose if $\sigma$ close to $\frac{n}{2}$.

Remark 3.4.2. In the proof of the Theorem 3.4.1 and Theorem 3.4.2, if we choose $\sigma=2$, then we have $\left\||u(s, \cdot)|^{p}-|v(s, \cdot)|^{p}\right\|_{\dot{H}^{1}}$. Therefore, we can easily handle this norm without using the fractional chain rule from Proposition A.7.7. Because, in this case the differential operator $\nabla$ is a local operator which appears in the proof of the Lipschitz condition. Therefore, we avoid the lower bound $\lceil\sigma\rceil$ of the admissible range for $p$.

Remark 3.4.3. Our goal was to weaken the upper bound of admissible exponents $p$ by supposing a suitable higher regularity of the data. Indeed, for $\sigma>1$ we have

$$
\frac{n}{n-2}<1+\frac{n}{n-2 \sigma} \text { if } n>2 \sigma
$$

which clarifies the expectation. 
Remark 3.4.4 (Choice of the parameters $q_{1}, q_{2}$ and $r_{1}, \cdots, r_{6}$ ). One choice for the parameters $q_{1}, q_{2}$ and $r_{1}, \cdots, r_{6}$ in the proof of Theorem 3.4.1 may be given as follows:

$$
q_{1}=\frac{2 n}{n-2 \sigma}, q_{2}=\frac{2 n}{n-2}, r_{1}=\frac{2 n}{n-2}, r_{2}=\frac{2 n}{n-2 \sigma} \frac{1}{p-1},
$$

and

$$
r_{3}=\frac{2 n}{n-2 \sigma}, r_{4}=\frac{n}{\sigma}, r_{5}=\frac{2 n}{n-2 \sigma}, r_{6}=\frac{2 n}{n-2} .
$$

\subsection{Large regular data}

We consider once again the Cauchy problem (3.1.1), i.e.,

$$
\begin{cases}u_{t t}-\lambda^{2}(t) \omega^{2}(t) \Delta u+\rho(t) \omega(t) u_{t}=|u|^{p}, & (t, x) \in[0, \infty) \times \mathbb{R}^{n}, \\ u(0, x)=u_{0}(x), \quad u_{t}(0, x)=u_{1}(x), & x \in \mathbb{R}^{n} .\end{cases}
$$

In this section we discuss the global (in time) existence of small data solutions with large regular data. In this case we suppose for the data the embedding

$$
H^{\sigma-1} \hookrightarrow L^{\infty}, \quad \sigma>1+\frac{n}{2}
$$

Therefore, in order to estimate the norms $\left\||u(s, \cdot)|^{p}\right\|_{\dot{H}^{\sigma-1}}$ and $\left\||u(s, \cdot)|^{p}-|v(s, \cdot)|^{p}\right\|_{\dot{H}^{\sigma-1}}$ we are able to use fractional powers rules instead of fractional Leibniz rule and fractional chain rule as we did in Theorems 3.4.1 and 3.4.2. Finally, if $\sigma>1+\frac{n}{2}$, then by using fractional powers rules the condition $p>\lceil\sigma\rceil$ may be improved to $p>\sigma$.

\subsubsection{Case of sub-exponential propagation speed}

We define the parameters $\hat{p}_{1, m}(n), \hat{p}_{2, m}(n)$ and $\hat{p}_{3, m}(n)$ as we defined in Section 3.4.1, and $\hat{p}_{m}(n):=\max \left\{\hat{p}_{1, m}(n) ; \hat{p}_{2, m}(n) ; \hat{p}_{3, m}(n)\right\}$.

We have the following statement for the case of sub-exponential propagation speed.

Theorem 3.5.1. We assume that the Hypotheses (A1) to (A5), (B1) to (B6), (C1), (C2) and $(\boldsymbol{C} 4)$ are satisfied. Let us consider $\nu(\delta) \lesssim 1$ and $\vartheta(\delta) \lesssim 1$, where the functions $\nu=\nu(\delta)$ and $\vartheta=\vartheta(\delta)$ are defined in (3.1.2) and (3.1.3), respectively. Let the data $\left(u_{0}, u_{1}\right) \in \mathcal{D}_{m}^{\sigma}$ with $\sigma>1+\frac{n}{2}$ and $m \in[1,2)$. Moreover, we assume that the exponent $p$ satisfies

$$
p>\hat{p}_{m}(n) \quad \text { and } \quad p>\max \left\{\sigma ; \frac{2}{m}\right\} .
$$

Finally, we suppose that the following condition holds:

$$
\frac{\beta_{1}}{\frac{n}{2}\left(\frac{1}{m}-\frac{1}{2}\right)}>\max \left\{(2+R) \frac{\nu(\delta)}{\delta} ; 2 \frac{\vartheta(\delta)}{\delta}\right\},
$$

where $\beta_{1}$ is from condition (C4) and $R$ is from (3.1.17).

Then, there exists a constant $\varepsilon_{0}>0$ such that for all $\left(u_{0}, u_{1}\right) \in \mathcal{D}_{m}^{\sigma}$ with $\left\|\left(u_{0}, u_{1}\right)\right\|_{\mathcal{D}_{m}^{\sigma}} \leq \varepsilon_{0}$ there is a uniquely determined energy solution

$$
u \in \mathcal{C}\left([0, \infty), H^{\sigma}\right) \cap \mathcal{C}^{1}\left([0, \infty), H^{\sigma-1}\right)
$$

to the Cauchy problem (3.1.1). 
Furthermore, there exists a constant $C>0$ such that the solution satisfies the estimates

$$
\begin{aligned}
&\|u(t, \cdot)\|_{L^{2}} \leq C\left(1+B_{\lambda}(0, t)\right)^{-\frac{n}{2}\left(\frac{1}{m}-\frac{1}{2}\right)}\left\|\left(u_{0}, u_{1}\right)\right\|_{\mathcal{D}_{m}^{\sigma}}, \\
&\left\||D|^{\sigma} u(t, \cdot)\right\|_{L^{2}} \leq C\left(1+B_{\lambda}(0, t)\right)^{-\frac{n}{2}\left(\frac{1}{m}-\frac{1}{2}\right)-\frac{\sigma}{2}}\left\|\left(u_{0}, u_{1}\right)\right\|_{\mathcal{D}_{m}^{\sigma}}, \\
&\left\|u_{t}(t, \cdot)\right\|_{L^{2}} \leq C \max \left\{\frac{\lambda^{2}(t)}{\rho(t)}\left(1+B_{\lambda}(0, t)\right)^{-\frac{n}{2}\left(\frac{1}{m}-\frac{1}{2}\right)-1} ;\right. \\
&\left.\lambda(t) F(\Lambda(t))\left(F^{2}(\Lambda(t))\right)^{-\frac{n}{2}\left(\frac{1}{m}-\frac{1}{2}\right)-1}\right\}\left\|\left(u_{0}, u_{1}\right)\right\|_{\mathcal{D}_{m}^{\sigma}}, \\
&\left\||D|^{\sigma-1} u_{t}(t, \cdot)\right\|_{L^{2}} \leq C \max \left\{\frac{\lambda^{2}(t)}{\rho(t)}\left(1+B_{\lambda}(0, t)\right)^{-\frac{n}{2}\left(\frac{1}{m}-\frac{1}{2}\right)-\frac{\sigma-1}{2}-1} ;\right. \\
&\left.\lambda(t) F(\Lambda(t))\left(F^{2}(\Lambda(t))\right)^{-\frac{n}{2}\left(\frac{1}{m}-\frac{1}{2}\right)-\frac{\sigma-1}{2}-1}\right\}\left\|\left(u_{0}, u_{1}\right)\right\|_{\mathcal{D}_{m}^{\sigma}} .
\end{aligned}
$$

Proof. We will follow the same approach used in the proof of Theorem 3.4.1 to prove this theorem. The definition of the evolution space $X(t)$ and its norm $\|u\|_{X(t)}$ are just the same as in the proof of Theorem 3.4.1. Therefore, it is sufficient to verify the following two inequalities:

$$
\begin{aligned}
\|N u\|_{X(t)} & \lesssim\left\|\left(u_{0}, u_{1}\right)\right\|_{\mathcal{D}_{m}^{\sigma}}+\|u\|_{X(t)}^{p}, \\
\|N u-N v\|_{X(t)} & \lesssim\|u-v\|_{X(t)}\left(\|u\|_{X(t)}^{p-1}+\|v\|_{X(t)}^{p-1}\right) .
\end{aligned}
$$

To treat the "nonlinear part" we estimate on the one hand the norms

$$
\left\||u(s, \cdot)|^{p}\right\|_{L^{q}} \text { and }\left\||u(s, \cdot)|^{p}-|v(s, \cdot)|^{p}\right\|_{L^{q}}
$$

with $q=m$ and $q=2$, as we did exactly in the proof of Theorem 3.4.1. So, from GagliardoNirenberg inequality we have the following estimates for $p \geq \frac{2}{m}$ :

$$
\begin{aligned}
\|u(s, \cdot)\|_{L^{m p}}^{p} & \lesssim\left(1+B_{\lambda}(0, s)\right)^{-\frac{n}{2 m} p+\frac{n}{2 m}}\|u\|_{X(s)}^{p}, \\
\|u(s, \cdot)\|_{L^{2 p}}^{p} & \lesssim\left(1+B_{\lambda}(0, s)\right)^{-\frac{n}{2 m} p+\frac{n}{4}}\|u\|_{X(s)}^{p} .
\end{aligned}
$$

On the other hand, we turn to estimate the norms $\left\||u(s, \cdot)|^{p}\right\|_{\dot{H}^{\sigma-1}}$ and $\||u(s, \cdot)|^{p}-$ $|v(s, \cdot)|^{p} \|_{\dot{H}^{\sigma-1}}$ by using results on fractional powers for $\sigma-1>\frac{n}{2}$.

Firstly, let us begin to estimate the norm $\left\||u(s, \cdot)|^{p}\right\|_{\dot{H}^{\sigma-1}}$. We apply Corollary A.7.10 with $\sigma-1 \in\left(\frac{n}{2}, p\right)$ and Proposition A.7.13 with a suitable $\sigma^{*}<\frac{n}{2}$, from Section A.7 in the appendix, respectively. Therefore, we obtain

$$
\begin{aligned}
\left\||u(s, \cdot)|^{p}\right\|_{\dot{H}^{\sigma-1}} & \lesssim\|u(s, \cdot)\|_{\dot{H}^{\sigma-1}}\|u(s, \cdot)\|_{L^{\infty}}^{p-1} \\
& \lesssim\|u(s, \cdot)\|_{\dot{H}^{\sigma-1}}\left(\|u(s, \cdot)\|_{\dot{H}^{\sigma^{*}}}+\|u(s, \cdot)\|_{\dot{H}^{\sigma-1}}\right)^{p-1} \\
& \lesssim\|u(s, \cdot)\|_{\dot{H}^{\sigma-1}}\|u(s, \cdot)\|_{\dot{H}^{\sigma^{*}}}^{p-1}+\|u(s, \cdot)\|_{\dot{H}^{\sigma-1}}^{p} .
\end{aligned}
$$

Applying the fractional Gagliardo-Nirenberg inequality and using the definition of the norm of the solution space $X(t)$ we derive

$$
\begin{aligned}
\|u(s, \cdot)\|_{\dot{H}^{\sigma-1}} & \lesssim\|u(s, \cdot)\|_{\dot{H}^{\sigma}}^{\theta_{\sigma-1, \sigma}}\|u(s, \cdot)\|_{L^{2}}^{1-\theta_{\sigma-1, \sigma}} \\
& \lesssim\left(1+B_{\lambda}(0, s)\right)^{-\frac{n}{2}\left(\frac{1}{m}-\frac{1}{2}\right)-\frac{\sigma-1}{2}}\|u\|_{X(s)}, \\
\|u(s, \cdot)\|_{\dot{H}^{\sigma^{*}}} & \lesssim\|u(s, \cdot)\|_{\dot{H}^{\sigma}}^{\theta_{\sigma^{*}, \sigma}}\|u(s, \cdot)\|_{L^{2}}^{1-\theta_{\sigma^{*}, \sigma}} \\
& \lesssim\left(1+B_{\lambda}(0, s)\right)^{-\frac{n}{2}\left(\frac{1}{m}-\frac{1}{2}\right)-\frac{\sigma^{*}}{2}}\|u\|_{X(s)},
\end{aligned}
$$

where $\theta_{\sigma-1, \sigma}=1-\frac{1}{\sigma}$ and $\theta_{\sigma^{*}, \sigma}=\frac{\sigma^{*}}{\sigma}$. Hence, by choosing the parameter $\sigma^{*}=\frac{n}{2}-\varepsilon<\frac{n}{2}$ with a sufficiently small positive $\varepsilon$ we have

$$
\left\||u(s, \cdot)|^{p}\right\|_{\dot{H}^{\sigma-1}} \lesssim\left(1+B_{\lambda}(0, s)\right)^{-\frac{n}{2}\left(\frac{1}{m}-\frac{1}{2}\right) p-\frac{\sigma-1}{2}-\frac{\sigma^{*}}{2}(p-1)}\|u\|_{X(s)}^{p} .
$$


For this reason, in order to get the desired estimates from the estimate

$$
\begin{aligned}
\left\||D|^{\sigma} u^{\mathrm{nl}}(t, \cdot)\right\|_{L^{2}} \lesssim & \int_{0}^{\delta t} \frac{\Lambda(s)}{\lambda(s)}\left(1+B_{\lambda}(s, t)\right)^{-\frac{n}{2}\left(\frac{1}{m}-\frac{1}{2}\right)-\frac{\sigma}{2}}\left\||u(s, \cdot)|^{p}\right\|_{L^{m} \cap L^{2} \cap \dot{H}^{\sigma-1}} d s \\
& +\int_{\delta t}^{t} \frac{\Lambda(s)}{\lambda(s)}\left(1+B_{\lambda}(s, t)\right)^{-\frac{\sigma}{2}}\left\||u(s, \cdot)|^{p}\right\|_{L^{2} \cap \dot{H}^{\sigma-1}} d s,
\end{aligned}
$$

after taking into consideration (3.5.5), (3.5.6), (3.5.7), $\sigma^{*}<\frac{n}{2}$ and

$$
-\frac{n}{2 m} p+\frac{n}{2 m}>-\frac{n}{2 m} p+\frac{n}{4}>-\frac{n}{2}\left(\frac{1}{m}-\frac{1}{2}\right) p-\frac{\sigma-1}{2}-\frac{\sigma^{*}}{2}(p-1),
$$

where we choose $\sigma^{*}=\frac{n}{2}-\varepsilon<\frac{n}{2}$ and $\|u\|_{X(s)} \leq\|u\|_{X(t)}$ with $0 \leq s \leq t$, we arrive at the estimate

$$
\begin{aligned}
\left\||D|^{\sigma} u^{\mathrm{nl}}(t, \cdot)\right\|_{L^{2}} \lesssim & \|u\|_{X(t)}^{p} \underbrace{\int_{0}^{\delta t} \frac{\Lambda(s)}{\lambda(s)}\left(1+B_{\lambda}(s, t)\right)^{-\frac{n}{2}\left(\frac{1}{m}-\frac{1}{2}\right)-\frac{\sigma}{2}}\left(1+B_{\lambda}(0, s)\right)^{-\frac{n}{2 m} p+\frac{n}{2 m}} d s}_{\mathbf{G}} \\
& +\|u\|_{X(t)}^{p} \underbrace{\int_{\delta t}^{t} \frac{\Lambda(s)}{\lambda(s)}\left(1+B_{\lambda}(s, t)\right)^{-\frac{\sigma}{2}}\left(1+B_{\lambda}(0, s)\right)^{-\frac{n}{2 m} p+\frac{n}{4}} d s}_{\mathbf{H}} .
\end{aligned}
$$

Proceeding analogously to the proof of Theorem 3.4.1 we have

$$
\mathbf{G} \lesssim\left(1+B_{\lambda}(0, t)\right)^{-\frac{n}{2}\left(\frac{1}{m}-\frac{1}{2}\right)-\frac{\sigma}{2}} \underbrace{\int_{0}^{\delta t} \frac{\Lambda(s)}{\lambda(s)}\left(1+B_{\lambda}(0, s)\right)^{-\frac{n}{2 m} p+\frac{n}{2 m}} d s}_{G_{1}(t)}
$$

We may conclude by using

$$
p>1+\left(\frac{\sigma+1}{2}-\frac{\beta_{1}}{2+R}\right) \frac{2 m}{n}>1+\left(1-\frac{\beta_{1}}{2+R}\right) \frac{2 m}{n}
$$

that $G_{1}=G_{1}(t)$ is uniformly bounded. Therefore, with $p>\hat{p}_{1, m}(n)$ we obtain the desired estimate

$$
\mathbf{G} \lesssim\left(1+B_{\lambda}(0, t)\right)^{-\frac{n}{2}\left(\frac{1}{m}-\frac{1}{2}\right)-\frac{\sigma}{2}} .
$$

Now, let us consider the integral H. Defining $\alpha_{2}(p):=\frac{n}{2 m} p-\frac{n}{4}$ we have $($ for $\sigma \neq 2$ )

$$
\begin{aligned}
\mathbf{H}= & -\int_{\delta t}^{t} \frac{\mu(s)}{\lambda^{2}(s)}\left(1+B_{\lambda}(0, s)\right)^{-\alpha_{2}(p)}\left(1+B_{\lambda}(s, t)\right)^{-\frac{\sigma}{2}} d\left(1+B_{\lambda}(s, t)\right) \\
= & \left.\frac{-1}{1-\frac{\sigma}{2}} \frac{\mu(s)}{\lambda^{2}(s)}\left(1+B_{\lambda}(0, s)\right)^{-\alpha_{2}(p)}\left(1+B_{\lambda}(s, t)\right)^{-\frac{\sigma}{2}+1}\right|_{\delta t} ^{t} \\
& +\frac{1}{1-\frac{\sigma}{2}} \int_{\delta t}^{t}\left(1+B_{\lambda}(s, t)\right)^{-\frac{\sigma}{2}+1} d\left(\frac{\mu(s)}{\lambda^{2}(s)}\left(1+B_{\lambda}(0, s)\right)^{-\alpha_{2}(p)}\right) .
\end{aligned}
$$

To get the desired estimate in this case we distinguish between the following cases and we summarize the conditions for the exponent $p$ from the proof of Theorem 3.4.1. Let us point out that $\sigma>1+\frac{n}{2}$ leads to some conditions for the space dimension $n$.

- Case 1: $-\frac{\sigma}{2}+1>0$. This case comes into play only if $n=1$ and by $p>\hat{p}_{3, m}(n)$ we obtain the desired estimate for $\mathbf{H}$.

- Case 2: $-\frac{\sigma}{2}+1<0$. This case appears if $n \geq 2$ and by the conditions $p>\hat{p}_{1, m}(n)$ and $p>\hat{p}_{3, m}(n)$ we get the desired estimate for $\mathbf{H}$. 
- Case 3: $-\frac{\sigma}{2}+1=0$. Similarly, this case holds only if $n=1$ and by $p>\hat{p}_{3, m}(n)$ we find the desired estimate for $\mathbf{H}$.

Consequently, we arrive at the estimate

$$
\mathbf{H} \lesssim\left(1+B_{\lambda}(0, t)\right)^{-\frac{n}{2}\left(\frac{1}{m}-\frac{1}{2}\right)-\frac{\sigma}{2}} .
$$

Therefore, from (3.5.8) and (3.5.9) we have the estimate

$$
\left\||D|^{\sigma} u^{\mathrm{nl}}(t, \cdot)\right\|_{L^{2}} \lesssim\|u\|_{X(t)}^{p}\left(1+B_{\lambda}(0, t)\right)^{-\frac{n}{2}\left(\frac{1}{m}-\frac{1}{2}\right)-\frac{\sigma}{2}} .
$$

To complete the proof of (3.5.3) one can prove in the same way

$$
\left\|u^{\mathrm{nl}}(t, \cdot)\right\|_{L^{2}} \lesssim\|u\|_{X(t)}^{p}\left(1+B_{\lambda}(0, t)\right)^{-\frac{n}{2}\left(\frac{1}{m}-\frac{1}{2}\right)}
$$

by the conditions $p>\hat{p}_{1, m}(n), p>\hat{p}_{2, m}(n)$ and (3.5.2), respectively.

Now let us continue to estimate the norm $\left\||D|^{\sigma-1} u_{t}^{\mathrm{nl}}(t, \cdot)\right\|_{L^{2}}$. We have

$$
\left\||D|^{\sigma-1} u_{t}^{\mathrm{nl}}(t, \cdot)\right\|_{L^{2}} \lesssim \int_{0}^{t}\left\||D|^{\sigma-1}\left(K_{1}(t, s, x) *_{(x)}|u(s, x)|^{p}\right)\right\|_{L^{m} \cap L^{2}} d s .
$$

Proceeding in the same way as we did to estimate the norm $\left\||D|^{\sigma} u^{\mathrm{nl}}(t, \cdot)\right\|_{L^{2}}$ we have the following estimate:

$$
\begin{aligned}
\left\||D|^{\sigma-1} u_{t}^{\mathrm{nl}}(t, \cdot)\right\|_{L^{2}} \lesssim & \int_{0}^{\delta t} \Phi_{2, m}(s, t) \frac{\Lambda(s)}{\lambda(s)}\left\||u(s, \cdot)|^{p}\right\|_{L^{m} \cap L^{2} \cap \dot{H}^{\sigma-1}} d s \\
& +\int_{\delta t}^{t} \Phi_{2}(s, t) \frac{\Lambda(s)}{\lambda(s)}\left\||u(s, \cdot)|^{p}\right\|_{L^{2} \cap \dot{H}^{\sigma-1}} d s,
\end{aligned}
$$

where $\Phi_{2, m}=\Phi_{2, m}(s, t)$ and $\Phi_{2}=\Phi_{2}(s, t)$ are defined in (3.1.15) and (3.1.16), respectively. Summarizing, using the estimates of the norm $\left\||u(s, \cdot)|^{p}\right\|_{L^{m} \cap L^{2} \cap \dot{H}^{\sigma-1}}$ from (3.5.5), (3.5.6) and (3.5.7) with no other requirements to the admissible exponents $p$ we arrive at

$$
\begin{aligned}
\left\||D|^{\sigma-1} u_{t}^{\mathrm{nl}}(t, \cdot)\right\|_{L^{2}} \lesssim & \|u\|_{X(t)}^{p} \underbrace{\int_{0}^{\delta t} \Phi_{2, m}(s, t) \frac{\Lambda(s)}{\lambda(s)}\left(1+B_{\lambda}(0, s)\right)^{-\frac{n}{2 m} p+\frac{n}{2 m}} d s}_{\mathbf{K}} \\
& +\|u\|_{X(t)}^{p} \underbrace{\int_{\delta t}^{t} \Phi_{2}(s, t) \frac{\Lambda(s)}{\lambda(s)}\left(1+B_{\lambda}(0, s)\right)^{-\frac{n}{2 m} p+\frac{n}{4}} d s}_{\mathbf{L}} .
\end{aligned}
$$

In order to obtain the desired estimates for $\mathbf{K}$ and $\mathbf{L}$ we follow exactly the same considerations from the proof of Theorem 3.4.1. Then, by the conditions $p>\hat{p}_{1, m}(n), p>\hat{p}_{3, m}(n)$ and (3.5.2) we obtain the estimate

$$
\begin{aligned}
&\left\||D|^{\sigma-1} u_{t}^{\mathrm{nl}}(t, \cdot)\right\|_{L^{2}} \lesssim\|u\|_{X(t)}^{p} \max \{ \frac{\lambda^{2}(t)}{\rho(t)}\left(1+B_{\lambda}(0, t)\right)^{-\frac{n}{2}\left(\frac{1}{m}-\frac{1}{2}\right)-\frac{\sigma-1}{2}-1} \\
&\left.\lambda(t) F(\Lambda(t))\left(F^{2}(\Lambda(t))\right)^{-\frac{n}{2}\left(\frac{1}{m}-\frac{1}{2}\right)-\frac{\sigma-1}{2}-1}\right\} .
\end{aligned}
$$

Moreover, analogously one can prove

$$
\begin{array}{r}
\left\|u_{t}^{\mathrm{nl}}(t, \cdot)\right\|_{L^{2}} \lesssim\|u\|_{X(t)}^{p} \max \left\{\frac{\lambda^{2}(\tau)}{\rho(\tau)}\left(1+B_{\lambda}(0, \tau)\right)^{-\frac{n}{2}\left(\frac{1}{m}-\frac{1}{2}\right)-1} ;\right. \\
\left.\lambda(\tau) F(\Lambda(\tau))\left(F^{2}(\Lambda(\tau))\right)^{-\frac{n}{2}\left(\frac{1}{m}-\frac{1}{2}\right)-1}\right\},
\end{array}
$$


provided that the conditions $p>\hat{p}_{1, m}(n), p>\hat{p}_{3, m}(n)$ and (3.5.2) are fulfilled.

Consequently, using the norm of the solution space $X(t)$, from (3.5.10), (3.5.11), (3.5.12) and (3.5.13) it follows

$$
\left\|u^{\mathrm{n} 1}\right\|_{X(t)} \lesssim\|u\|_{X(t)}^{p} \cdot
$$

Now we want to prove (3.5.4). By the definition of the solution space $X(t)$ and its norm we have

$$
\begin{aligned}
\left\||u(s, \cdot)|^{p}-|v(s, \cdot)|^{p}\right\|_{L^{2}} & \lesssim\left(1+B_{\lambda}(0, s)\right)^{-\frac{n}{2 m} p+\frac{n}{4}}\|u-v\|_{X(s)}\left(\|u\|_{X(s)}^{p-1}+\|v\|_{X(s)}^{p-1}\right), \\
\left\||u(s, \cdot)|^{p}-|v(s, \cdot)|^{p}\right\|_{L^{m}} & \lesssim\left(1+B_{\lambda}(0, s)\right)^{-\frac{n}{2 m} p+\frac{n}{2 m}}\|u-v\|_{X(s)}\left(\|u\|_{X(t)}^{p-1}+\|v\|_{X(s)}^{p-1}\right) .
\end{aligned}
$$

On the other hand, to estimate the norm

we use the integral representation

$$
\left\||u(s, \cdot)|^{p}-|v(s, \cdot)|^{p}\right\|_{\dot{H}^{\sigma-1}}
$$

$$
\begin{aligned}
|u(s, x)|^{p}-|v(s, x)|^{p} & =\int_{0}^{1} \frac{d}{d w}|w u(s, x)+(1-w) v(s, x)|^{p} d w \\
& =p \int_{0}^{1}(u(s, x)-v(s, x)) G(w u(s, x)+(1-w) v(s, x)) d w,
\end{aligned}
$$

where $G(u)=u|u|^{p-2}$. Then, we have

$$
\left\||u(s, \cdot)|^{p}-|v(s, \cdot)|^{p}\right\|_{\dot{H}^{\sigma-1}} \lesssim \int_{0}^{1}\left\||D|^{\sigma-1}((u(s, \cdot)-v(s, \cdot)) G(w u(s, \cdot)+(1-w) v(s, \cdot)))\right\|_{L^{2}} d w .
$$

Now we apply Corollary A.7.12 in order to estimate the last statement. Therefore, we get

$$
\begin{aligned}
\||u(s, \cdot)|^{p}- & |v(s, \cdot)|^{p} \|_{\dot{H}^{\sigma-1}} \\
\lesssim & \int_{0}^{1}\|u(s, \cdot)-v(s, \cdot)\|_{\dot{H}^{\sigma-1}}\|G(w u(s, \cdot)+(1-w) v(s, \cdot))\|_{L^{\infty}} d w \\
& +\int_{0}^{1}\|u(s, \cdot)-v(s, \cdot)\|_{L^{\infty}}\|G(w u(s, \cdot)+(1-w) v(s, \cdot))\|_{\dot{H}^{\sigma-1}} d w .
\end{aligned}
$$

Employing the fractional powers rules from Corollary A.7.10 and Proposition A.7.13, respectively, we obtain

$$
\begin{aligned}
\|G(w u(s, \cdot)+(1-w) v(s, \cdot))\|_{\dot{H}^{\sigma-1}} \\
\lesssim\|w u(s, \cdot)+(1-w) v(s, \cdot)\|_{\dot{H}^{\sigma-1}}\|w u(s, \cdot)+(1-w) v(s, \cdot)\|_{L^{\infty}}^{p-2} \\
\lesssim\|w u(s, \cdot)+(1-w) v(s, \cdot)\|_{\dot{H}^{\sigma-1}} \\
\quad \times\left(\|w u(s, \cdot)+(1-w) v(s, \cdot)\|_{\dot{H}^{\sigma^{*}}}+\|w u(s, \cdot)+(1-w) v(s, \cdot)\|_{\dot{H}^{\sigma-1}}\right)^{p-2} \\
\quad \lesssim\|w u(s, \cdot)+(1-w) v(s, \cdot)\|_{\dot{H}^{\sigma-1}}\|w u(s, \cdot)+(1-w) v(s, \cdot)\|_{\dot{H}^{\sigma^{*}}}^{p-2} \\
\quad+\|w u(s, \cdot)+(1-w) v(s, \cdot)\|_{\dot{H}^{\sigma-1}}^{p-1} .
\end{aligned}
$$

To conclude the estimate for the previous statement we need the following estimates:

$$
\begin{aligned}
\|w u(s, \cdot)+(1-w) v(s, \cdot)\|_{\dot{H}^{\sigma-1}} & \lesssim\left(1+B_{\lambda}(0, s)\right)^{-\frac{n}{2 m}+\frac{n}{4}-\frac{\sigma-1}{2}}\|w u+(1-w) v\|_{X(s)}, \\
\|w u(s, \cdot)+(1-w) v(s, \cdot)\|_{\dot{H}^{\sigma^{*}}}^{p-2} & \lesssim\left(1+B_{\lambda}(0, s)\right)^{-\frac{n}{2 m}(p-2)+\frac{n}{4}(p-2)-\frac{\sigma^{*}}{2}(p-2)}\|w u+(1-w) v\|_{X(s)}^{p-2} .
\end{aligned}
$$

Therefore, we arrive at the estimates

$$
\begin{aligned}
\| w u(s, \cdot) & +(1-w) v(s, \cdot)\left\|_{\dot{H}^{\sigma-1}}\right\| w u(s, \cdot)+(1-w) v(s, \cdot) \|_{\dot{H}^{\sigma^{*}}}^{p-2} \\
& \lesssim\left(1+B_{\lambda}(0, s)\right)^{-\frac{n}{2 m}(p-1)+\frac{n}{4}(p-1)-\frac{\sigma-1}{2}-\frac{\sigma^{*}}{2}(p-2)}\|w u+(1-w) v\|_{X(s)}^{p-1}, \\
\| w u(s, \cdot) & +(1-w) v(s, \cdot) \|_{\dot{H}^{\sigma-1}}^{p-1} \\
& \lesssim\left(1+B_{\lambda}(0, s)\right)^{-\frac{n}{2 m}(p-1)+\frac{n}{4}(p-1)-\frac{\sigma-1}{2}(p-1)}\|w u+(1-w) v\|_{X(s)}^{p-1}
\end{aligned}
$$


where by $\sigma^{*}<\frac{n}{2}$ it holds

$$
-\frac{n}{2 m}(p-1)+\frac{n}{4}(p-1)-\frac{\sigma-1}{2}-\frac{\sigma^{*}}{2}(p-2)>-\frac{n}{2 m}(p-1)+\frac{n}{4}(p-1)-\frac{\sigma-1}{2}(p-1) .
$$

This implies

$$
\begin{aligned}
\| G(w u(s, \cdot) & +(1-w) v(s, \cdot)) \|_{\dot{H}^{\sigma-1}} \\
& \lesssim\left(1+B_{\lambda}(0, s)\right)^{-\frac{n}{2 m}(p-1)+\frac{n}{4}(p-1)-\frac{\sigma-1}{2}-\frac{\sigma^{*}}{2}(p-2)}\|w u+(1-w) v\|_{X(s)}^{p-1} .
\end{aligned}
$$

On the other hand, applying Proposition A.7.13 with $\sigma^{*}<\frac{n}{2}$ we have

$$
\begin{aligned}
\| G(w u(s, \cdot)+ & (1-w) v(s, \cdot)) \|_{L^{\infty}} \\
\lesssim & \|w u(s, \cdot)+(1-w) v(s, \cdot)\|_{\dot{H}^{\sigma^{*}}}^{p-1}+\|w u(s, \cdot)+(1-w) v(s, \cdot)\|_{\dot{H}^{\sigma-1}}^{p-1} \\
\lesssim & \left(1+B_{\lambda}(0, s)\right)^{-\frac{n}{2 m}(p-1)+\frac{n}{4}(p-1)-\frac{\sigma^{*}}{2}(p-1)}\|w u+(1-w) v\|_{X(s)}^{p-1} \\
& +\left(1+B_{\lambda}(0, s)\right)^{-\frac{n}{2 m}(p-1)+\frac{n}{4}(p-1)-\frac{\sigma-1}{2}(p-1)}\|w u+(1-w) v\|_{X(s)}^{p-1} \\
\lesssim & \left(1+B_{\lambda}(0, s)\right)^{-\frac{n}{2 m}(p-1)+\frac{n}{4}(p-1)-\frac{\sigma^{*}}{2}(p-1)}\|w u+(1-w) v\|_{X(s)}^{p-1} .
\end{aligned}
$$

Consequently, we arrive at the estimates

$$
\begin{aligned}
\| u(s, \cdot) & -v(s, \cdot)\left\|_{\dot{H}^{\sigma-1}}\right\| G(w u(s, \cdot)+(1-w) v(s, \cdot)) \|_{L^{\infty}} \\
& \lesssim\left(1+B_{\lambda}(0, s)\right)^{-\frac{n}{2 m} p+\frac{n}{4} p-\frac{\sigma-1}{2}-\frac{\sigma^{*}}{2}(p-1)}\|u-v\|_{X(s)}\|w u+(1-w) v\|_{X(s)}^{p-1}, \\
\| u(s, \cdot) & -v(s, \cdot)\left\|_{L^{\infty}}\right\| G(w u(s, \cdot)+(1-w) v(s, \cdot)) \|_{\dot{H}^{\sigma-1}} \\
& \lesssim\left(1+B_{\lambda}(0, s)\right)^{-\frac{n}{2 m} p+\frac{n}{4} p-\frac{\sigma-1}{2}-\frac{\sigma^{*}}{2}(p-1)}\|u-v\|_{X(s)}\|w u+(1-w) v\|_{X(s)}^{p-1} .
\end{aligned}
$$

Thus, we obtain

$$
\begin{aligned}
\left\||u(s, \cdot)|^{p}-|v(s, \cdot)|^{p}\right\|_{\dot{H}^{\sigma-1}} & \\
& \lesssim\left(1+B_{\lambda}(0, s)\right)^{-\frac{n}{2 m} p+\frac{n}{4} p-\frac{\sigma-1}{2}-\frac{\sigma^{*}}{2}(p-1)} \int_{0}^{1}\|u-v\|_{X(s)}\|w u+(1-w) v\|_{X(s)}^{p-1} d w \\
& \lesssim\left(1+B_{\lambda}(0, s)\right)^{-\frac{n}{2 m} p+\frac{n}{4} p-\frac{\sigma-1}{2}-\frac{\sigma^{*}}{2}(p-1)} \int_{0}^{1}\|u-v\|_{X(s)}\left(\|u\|_{X(s)}^{p-1}+\|v\|_{X(s)}^{p-1}\right) d w \\
& \lesssim\left(1+B_{\lambda}(0, s)\right)^{-\frac{n}{2 m} p+\frac{n}{4} p-\frac{\sigma-1}{2}-\frac{\sigma^{*}}{2}(p-1)}\|u-v\|_{X(s)}\left(\|u\|_{X(s)}^{p-1}+\|v\|_{X(s)}^{p-1}\right) .
\end{aligned}
$$

Taking into consideration (3.5.7), $\sigma^{*}=\frac{n}{2}-\varepsilon<\frac{n}{2}$ with $\varepsilon>0$ and $\|u\|_{X(s)} \leq\|u\|_{X(t)}$ with $0 \leq s \leq t$ we find

$$
\begin{gathered}
\left\||D|^{\sigma} \int_{0}^{t} K_{1}(t, s, x) *_{(x)}\left(|u(s, x)|^{p}-|v(s, x)|^{p}\right) d s\right\|_{L^{2}} \lesssim\left(\|u\|_{X(t)}^{p-1}+\|v\|_{X(t)}^{p-1}\right)\|u-v\|_{X(t)} \\
\times\left(\int_{0}^{\delta t} \frac{\Lambda(s)}{\lambda(s)}\left(1+B_{\lambda}(s, t)\right)^{-\frac{n}{2}\left(\frac{1}{m}-\frac{1}{2}\right)-\frac{\sigma}{2}}\left(1+B_{\lambda}(0, s)\right)^{-\frac{n}{2 m} p+\frac{n}{2 m}} d s\right. \\
\left.\quad+\int_{\delta t}^{t} \frac{\Lambda(s)}{\lambda(s)}\left(1+B_{\lambda}(s, t)\right)^{-\frac{\sigma}{2}}\left(1+B_{\lambda}(0, s)\right)^{-\frac{n}{2 m} p+\frac{n}{4}} d s\right) .
\end{gathered}
$$

In order to conclude the proof of the estimate (3.5.4) we can follow the same steps of the proof of (3.5.3). Hence, we arrive at the estimate

$$
\left\||D|^{\sigma}(N u-N v)(t, \cdot)\right\|_{L^{2}} \lesssim\left(1+B_{\lambda}(0, t)\right)^{-\frac{n}{2}\left(\frac{1}{m}-\frac{1}{2}\right)-\frac{\sigma}{2}}\|u-v\|_{X(t)}\left(\|u\|_{X(t)}^{p-1}+\|v\|_{X(t)}^{p-1}\right) .
$$


In the same way, with no other requirements to the admissible exponents $p$ one can derive

$$
\begin{aligned}
\|(N u-N v)(t, \cdot)\|_{L^{2}} & \lesssim\left(1+B_{\lambda}(0, s)\right)^{-\frac{n}{2}\left(\frac{1}{m}-\frac{1}{2}\right)}\|u-v\|_{X(t)}\left(\|u\|_{X(t)}^{p-1}+\|v\|_{X(t)}^{p-1}\right), \\
\left\|\partial_{t}(N u-N v)(t, \cdot)\right\|_{L^{2}} & \lesssim \Phi_{1, m}(0, t)\|u-v\|_{X(t)}\left(\|u\|_{X(t)}^{p-1}+\|v\|_{X(t)}^{p-1}\right), \\
\left\||D|^{\sigma-1} \partial_{t}(N u-N v)(t, \cdot)\right\|_{L^{2}} & \lesssim \Phi_{2, m}(0, t)\|u-v\|_{X(t)}\left(\|u\|_{X(t)}^{p-1}+\|v\|_{X(t)}^{p-1}\right),
\end{aligned}
$$

where $\Phi_{1, m}=\Phi_{1, m}(0, t)$ and $\Phi_{2, m}=\Phi_{2, m}(0, t)$ are defined in (3.1.13) and (3.1.15) for $\tau=t$, respectively. Thus, from the definition of the norm of the solution space $X(t)$ we may conclude the proof of (3.5.4), which completes the proof of the theorem.

\subsubsection{Case of super-exponential propagation speed}

Finally, we conclude this section with the treatment of the case of super-exponential propagation speed for large regular data belonging to $L^{\infty}$. For this reason, we choose the regularity parameter $\sigma$ from the interval $\sigma \in\left(1+\frac{n}{2}, \infty\right)$. We have the following statement.

Theorem 3.5.2. We assume that the Hypotheses (A1) to (A5), (B1) to (B6), (C1), (C3) and (C4) hold. Let $\nu(\delta)=\infty$ and $\vartheta(\delta)=\infty$, where the functions $\nu=\nu(\delta)$ and $\vartheta=\vartheta(\delta)$ are defined in (3.1.2) and (3.1.3), respectively. Moreover, the following condition for the functions $\nu$ and $\vartheta$ holds:

$$
\max \{\nu(\delta, t) ; \vartheta(\delta, t)\}=o(\log \Lambda(t)) .
$$

We choose the data $\left(u_{0}, u_{1}\right) \in \mathcal{D}_{m}^{\sigma}$ with $\sigma>1+\frac{n}{2}$ and $m \in[1,2)$. Finally, the exponent $p$ satisfies $p>\max \left\{\sigma ; \frac{2}{m}\right\}$.

Then, there exists a constant $\varepsilon_{0}>0$ such that for all $\left(u_{0}, u_{1}\right) \in \mathcal{D}_{m}^{\sigma}$ with $\left\|\left(u_{0}, u_{1}\right)\right\|_{\mathcal{D}_{m}^{\sigma}} \leq \varepsilon_{0}$ there is a uniquely determined energy solution

$$
u \in \mathcal{C}\left([0, \infty), H^{\sigma}\right) \cap \mathcal{C}^{1}\left([0, \infty), H^{\sigma-1}\right)
$$

to the Cauchy problem (3.1.1).

Furthermore, there exists a constant $C>0$ such that the solution satisfies the following estimates:

$$
\begin{aligned}
&\|u(t, \cdot)\|_{L^{2}} \leq C\left(1+B_{\lambda}(0, t)\right)^{-\frac{n}{2}\left(\frac{1}{m}-\frac{1}{2}\right)}\left\|\left(u_{0}, u_{1}\right)\right\|_{\mathcal{D}_{m}^{\sigma}}, \\
&\left\||D|^{\sigma} u(t, \cdot)\right\|_{L^{2}} \leq C\left(1+B_{\lambda}(0, t)\right)^{-\frac{n}{2}\left(\frac{1}{m}-\frac{1}{2}\right)-\frac{\sigma}{2}}\left\|\left(u_{0}, u_{1}\right)\right\|_{\mathcal{D}_{m}^{\sigma}}, \\
&\left\|u_{t}(t, \cdot)\right\|_{L^{2}} \leq C \max \left\{\frac{\lambda^{2}(t)}{\rho(t)}\left(1+B_{\lambda}(0, t)\right)^{-\frac{n}{2}\left(\frac{1}{m}-\frac{1}{2}\right)-1} ;\right. \\
&\left.\lambda(t) F(\Lambda(t))\left(F^{2}(\Lambda(t))\right)^{-\frac{n}{2}\left(\frac{1}{m}-\frac{1}{2}\right)-1}\right\}\left\|\left(u_{0}, u_{1}\right)\right\|_{\mathcal{D}_{m}^{\sigma}}, \\
&\left\||D|^{\sigma-1} u_{t}(t, \cdot)\right\|_{L^{2}} \leq C \max \left\{\frac{\lambda^{2}(t)}{\rho(t)}\left(1+B_{\lambda}(0, t)\right)^{-\frac{n}{2}\left(\frac{1}{m}-\frac{1}{2}\right)-\frac{\sigma-1}{2}-1} ;\right. \\
&\left.\lambda(t) F(\Lambda(t))\left(F^{2}(\Lambda(t))\right)^{-\frac{n}{2}\left(\frac{1}{m}-\frac{1}{2}\right)-\frac{\sigma-1}{2}-1}\right\}\left\|\left(u_{0}, u_{1}\right)\right\|_{\mathcal{D}_{m}^{\sigma}} .
\end{aligned}
$$

Proof. To prove this theorem we will proceed in an analogous way as in the proofs of Theorems 3.4.2 and 3.5.1. We want to prove the following two inequalities:

$$
\begin{aligned}
\|N u\|_{X(t)} & \lesssim\left\|\left(u_{0}, u_{1}\right)\right\|_{\mathcal{D}_{m}^{\sigma}}+\|u\|_{X(t)}^{p}, \\
\|N u-N v\|_{X(t)} & \lesssim\|u-v\|_{X(t)}\left(\|u\|_{X(t)}^{p-1}+\|v\|_{X(t)}^{p-1}\right) .
\end{aligned}
$$

Let us derive estimates of the following norms:

$$
\left\|u^{\mathrm{nl}}(t, \cdot)\right\|_{L^{2}}, \quad\left\||D|^{\sigma} u^{\mathrm{nl}}(t, \cdot)\right\|_{L^{2}}, \quad\left\|u_{t}^{\mathrm{nl}}(t, \cdot)\right\|_{L^{2}} \quad \text { and } \quad\left\||D|^{\sigma-1} u_{t}^{\mathrm{nl}}(t, \cdot)\right\|_{L^{2}} \cdot
$$


We begin to estimate the norm $\left\||D|^{\sigma} u^{\mathrm{nl}}(t, \cdot)\right\|_{L^{2}}$. In the same way as we derived in the proof of Theorem 3.5.1, employing the fractional powers rules from Corollary A.7.10 with $\sigma-1 \in\left(\frac{n}{2}, p\right)$ and Proposition A.7.13 with $\sigma^{*}<\frac{n}{2}$ we obtain

$$
\begin{aligned}
\left\||D|^{\sigma} u^{\mathrm{nl}}(t, \cdot)\right\|_{L^{2}} \lesssim & \|u\|_{X(t)}^{p} \underbrace{\int_{0}^{\delta t} \frac{\Lambda(s)}{\lambda(s)}\left(1+B_{\lambda}(s, t)\right)^{-\frac{n}{2}\left(\frac{1}{m}-\frac{1}{2}\right)-\frac{\sigma}{2}}\left(1+B_{\lambda}(0, s)\right)^{-\frac{n}{2 m} p+\frac{n}{2 m}} d s}_{\mathbf{G}} \\
& +\|u\|_{X(t)}^{p} \underbrace{\int_{\delta t}^{t} \frac{\Lambda(s)}{\lambda(s)}\left(1+B_{\lambda}(s, t)\right)^{-\frac{\sigma}{2}}\left(1+B_{\lambda}(0, s)\right)^{-\frac{n}{2 m} p+\frac{n}{4}} d s}_{\mathbf{H}} .
\end{aligned}
$$

Now we want to estimate the integrals $\mathbf{G}$ and $\mathbf{H}$, as we did in the proof of Theorem 3.4.2.

Setting $\alpha_{1}(p):=\frac{n}{2 m} p-\frac{n}{2 m}$ the integral $\mathbf{G}$ can be estimated as follows:

$$
\mathbf{G} \lesssim\left(1+B_{\lambda}(0, t)\right)^{-\frac{n}{2}\left(\frac{1}{m}-\frac{1}{2}\right)-\frac{\sigma}{2}} \underbrace{\int_{0}^{\delta t} \frac{\Lambda(s)}{\lambda(s)}\left(1+B_{\lambda}(0, s)\right)^{-\alpha_{1}(p)} d s}_{\bar{G}_{1}(t)}
$$

where we used (3.1.20). We have shown in the proof of Theorem 3.2.2 that the integral $\bar{A}_{1}=\bar{A}_{1}(t)$, which is defined in (3.2.26) and coincides with the integral $\bar{G}_{1}=\bar{G}_{1}(t)$, is bounded for large time $t$ in the cases $\alpha_{1}(p) \neq 1$ and $\alpha_{1}(p)=1$, respectively. Therefore, we may conclude the estimate

$$
\mathbf{G} \lesssim\left(1+B_{\lambda}(0, t)\right)^{-\frac{n}{2}\left(\frac{1}{m}-\frac{1}{2}\right)-\frac{\sigma}{2}} .
$$

Now we consider the integral $\mathbf{H}$. Introducing $\alpha_{2}(p):=\frac{n}{2 m} p-\frac{n}{4}$ in the case $\sigma \neq 2$ it follows

$$
\begin{aligned}
\mathbf{H}= & -\int_{\delta t}^{t} \frac{\mu(s)}{\lambda^{2}(s)}\left(1+B_{\lambda}(0, s)\right)^{-\alpha_{2}(p)}\left(1+B_{\lambda}(s, t)\right)^{-\frac{\sigma}{2}} d\left(1+B_{\lambda}(s, t)\right) \\
= & \left.\frac{-1}{1-\frac{\sigma}{2}} \frac{\mu(s)}{\lambda^{2}(s)}\left(1+B_{\lambda}(0, s)\right)^{-\alpha_{2}(p)}\left(1+B_{\lambda}(s, t)\right)^{-\frac{\sigma}{2}+1}\right|_{\delta t} ^{t} \\
& +\frac{1}{1-\frac{\sigma}{2}} \int_{\delta t}^{t}\left(1+B_{\lambda}(s, t)\right)^{-\frac{\sigma}{2}+1} d\left(\frac{\mu(s)}{\lambda^{2}(s)}\left(1+B_{\lambda}(0, s)\right)^{-\alpha_{2}(p)}\right) .
\end{aligned}
$$

To get the desired estimate for $\mathbf{H}$ we shall distinguish between the following cases from the proof of Theorem 3.5.1. These cases give us some conditions for the space dimension $n$, since $\sigma>1+\frac{n}{2}$. Then, by using the condition (3.5.14) we may summarize it in the following way:

- Case 1: $-\frac{\sigma}{2}+1>0$. This case comes into play only if $n=1$ and we obtain the desired estimate for $\mathbf{H}$.

- Case 2: $-\frac{\sigma}{2}+1<0$. This case appears if $n \geq 2$ and we get the desired estimate for $\mathbf{H}$.

- Case 3: $-\frac{\sigma}{2}+1=0$. Similarly, this case holds only if $n=1$ and we find the desired estimate for $\mathbf{H}$.

Therefore, we arrive at the estimate

$$
\mathbf{H} \lesssim\left(1+B_{\lambda}(0, t)\right)^{-\frac{n}{2}\left(\frac{1}{m}-\frac{1}{2}\right)-\frac{\sigma}{2}} .
$$

Taking into consideration the derived estimates for $\mathbf{G}$ and $\mathbf{H}$ we get

$$
\left\||D|^{\sigma} u^{\mathrm{nl}}(t, \cdot)\right\|_{L^{2}} \lesssim\left(1+B_{\lambda}(0, t)\right)^{-\frac{n}{2}\left(\frac{1}{m}-\frac{1}{2}\right)-\frac{\sigma}{2}}\|u\|_{X(t)}^{p} .
$$


Similarly, one can derive

$$
\left\|u^{\mathrm{nl}}(t, \cdot)\right\|_{L^{2}} \lesssim\left(1+B_{\lambda}(0, t)\right)^{-\frac{n}{2}\left(\frac{1}{m}-\frac{1}{2}\right)}\|u\|_{X(t)}^{p} .
$$

Now let us continue to estimate the norm $\left\||D|^{\sigma-1} u_{t}^{\mathrm{nl}}(t, \cdot)\right\|_{L^{2}}$. Proceeding in the same way as we did to estimate the norm $\left\||D|^{\sigma} u^{\mathrm{nl}}(t, \cdot)\right\|_{L^{2}}$ we have the following estimate:

$$
\begin{aligned}
\left\||D|^{\sigma-1} u_{t}^{\mathrm{nl}}(t, \cdot)\right\|_{L^{2}} \lesssim & \|u\|_{X(t)}^{p} \underbrace{\int_{0}^{\delta t} \Phi_{2, m}(s, t) \frac{\Lambda(s)}{\lambda(s)}\left(1+B_{\lambda}(0, s)\right)^{-\frac{n}{2 m} p+\frac{n}{2 m}} d s}_{\mathbf{K}} \\
& +\|u\|_{X(t)}^{p} \underbrace{\int_{\delta t}^{t} \Phi_{2}(s, t) \frac{\Lambda(s)}{\lambda(s)}\left(1+B_{\lambda}(0, s)\right)^{-\frac{n}{2 m} p+\frac{n}{4}} d s}_{\mathbf{L}} .
\end{aligned}
$$

Here $\Phi_{2, m}=\Phi_{2, m}(s, t)$ and $\Phi_{2}=\Phi_{2}(s, t)$ are defined in (3.1.15) and (3.1.16), respectively. In order to obtain the desired estimates for $\mathbf{K}$ and $\mathbf{L}$ we follow exactly the same considerations from the proof of Theorem 3.5.1. Then, we obtain the estimate

$$
\begin{aligned}
\left\||D|^{\sigma-1} u_{t}^{\mathrm{nl}}(t, \cdot)\right\|_{L^{2}} \lesssim\|u\|_{X(t)}^{p} \max \{ & \frac{\lambda^{2}(t)}{\rho(t)}\left(1+B_{\lambda}(0, t)\right)^{-\frac{n}{2}\left(\frac{1}{m}-\frac{1}{2}\right)-\frac{\sigma-1}{2}-1} ; \\
& \left.\lambda(t) F(\Lambda(t))\left(F^{2}(\Lambda(t))\right)^{-\frac{n}{2}\left(\frac{1}{m}-\frac{1}{2}\right)-\frac{\sigma-1}{2}-1}\right\} .
\end{aligned}
$$

Moreover, analogously we can also prove

$$
\begin{aligned}
\left\|u_{t}^{\mathrm{nl}}(t, \cdot)\right\|_{L^{2}} \lesssim\|u\|_{X(t)}^{p} \max \left\{\frac{\lambda^{2}(\tau)}{\rho(\tau)}\left(1+B_{\lambda}(0, \tau)\right)^{-\frac{n}{2}\left(\frac{1}{m}-\frac{1}{2}\right)-1} ;\right. \\
\left.\lambda(\tau) F(\Lambda(\tau))\left(F^{2}(\Lambda(\tau))\right)^{-\frac{n}{2}\left(\frac{1}{m}-\frac{1}{2}\right)-1}\right\} .
\end{aligned}
$$

Consequently, using the norm of the solution space $X(t)$, from (3.5.17), (3.5.18), (3.5.19) and (3.5.20) we arrive at

$$
\left\|u^{\mathrm{nl}}(t, \cdot)\right\|_{X(t)} \lesssim\|u\|_{X(t)}^{p} \cdot
$$

Let us consider now the estimate (3.5.16) analogously as we did in the proof of Theorem 3.5.1. The goal is to estimate the norm $\left\||D|^{\sigma}(N u-N v)(t, \cdot)\right\|_{L^{2}}$. Employing the tools which we explained in Appendix A.7 and applied in the same way as in the proof of Theorem 3.5.1 we may conclude the estimate

$$
\begin{gathered}
\left\||D|^{\sigma} \int_{0}^{t} K_{1}(t, s, x) *_{(x)}\left(|u(s, x)|^{p}-|v(s, x)|^{p}\right) d s\right\|_{L^{2}} \lesssim\left(\|u\|_{X(t)}^{p-1}+\|v\|_{X(t)}^{p-1}\right)\|u-v\|_{X(t)} \\
\times\left(\int_{0}^{\delta t} \frac{\Lambda(s)}{\lambda(s)}\left(1+B_{\lambda}(s, t)\right)^{-\frac{n}{2}\left(\frac{1}{m}-\frac{1}{2}\right)-\frac{\sigma}{2}}\left(1+B_{\lambda}(0, s)\right)^{-\frac{n}{2 m} p+\frac{n}{2 m}} d s\right. \\
\left.\quad+\int_{\delta t}^{t} \frac{\Lambda(s)}{\lambda(s)}\left(1+B_{\lambda}(s, t)\right)^{-\frac{\sigma}{2}}\left(1+B_{\lambda}(0, s)\right)^{-\frac{n}{2 m} p+\frac{n}{4}} d s\right) .
\end{gathered}
$$

Following the same steps to estimate the norm $\left\||D|^{\sigma} u^{\mathrm{nl}}(t, \cdot)\right\|_{L^{2}}$ we obtain

$$
\left\||D|^{\sigma}(N u-N v)(t, \cdot)\right\|_{L^{2}} \lesssim\left(1+B_{\lambda}(0, t)\right)^{-\frac{n}{2}\left(\frac{1}{m}-\frac{1}{2}\right)-\frac{\sigma}{2}}\|u-v\|_{X(t)}\left(\|u\|_{X(t)}^{p-1}+\|v\|_{X(t)}^{p-1}\right) .
$$

In the same way, with no other requirements we can derive the estimates for $\|(N u-$ $N v)(t, \cdot)\left\|_{L^{2}},\right\| \partial_{t}(N u-N v)(t, \cdot) \|_{L^{2}}$ and $\left\||D|^{\sigma-1} \partial_{t}(N u-N v)(t, \cdot)\right\|_{L^{2}}$. The proof is completed if we replace all these estimates into the definition of the norm of solution space $X(t)$ to get (3.5.16). 



\section{Global in time existence results for damped wave models with different power nonlinearities}

\subsection{Introduction}

In this chapter we consider the following Cauchy problem:

$$
\begin{cases}u_{t t}-\lambda^{2}(t) \omega^{2}(t) \Delta u+\rho(t) \omega(t) u_{t}=f\left(u_{t},|D|^{a} u\right), & (t, x) \in[0, \infty) \times \mathbb{R}^{n}, \\ u(0, x)=u_{0}(x), \quad u_{t}(0, x)=u_{1}(x), & x \in \mathbb{R}^{n},\end{cases}
$$

with some different power nonlinearities on the right-hand side. Our goal is to discuss again the global (in time) existence of small data Sobolev solutions with source power nonlinearities $\left|u_{t}\right|^{p}$ and $\left.\left.|| D\right|^{a} u\right|^{p}, a \in(0,1]$, respectively. The main approach to deal with these kind of source terms is similar to the one we used to treat the Cauchy problem (3.1.1).

Let us recall again the following auxiliary functions from Chapter 3 under condition (C1):

$$
\begin{aligned}
& \Phi_{1, m}(s, t)=\max \left\{\frac{\lambda^{2}(t)}{\rho(t)}\left(1+B_{\lambda}(s, t)\right)^{-\frac{n}{2}\left(\frac{1}{m}-\frac{1}{2}\right)-1} ;\right. \\
&\left.\lambda(t) F(\Lambda(t))\left(F^{2}(\Lambda(t))\right)^{-\frac{n}{2}\left(\frac{1}{m}-\frac{1}{2}\right)-1}\right\}, \\
& \Phi_{1}(s, t)=\max \left\{\frac{\lambda^{2}(t)}{\rho(t)}\left(1+B_{\lambda}(s, t)\right)^{-1} ; \lambda(t) F(\Lambda(t))\left(F^{2}(\Lambda(t))\right)^{-1}\right\}, \\
& \Phi_{2, m}(s, t)=\max \left\{\frac{\lambda^{2}(t)}{\rho(t)}\left(1+B_{\lambda}(s, t)\right)^{-\frac{n}{2}\left(\frac{1}{m}-\frac{1}{2}\right)-\frac{\sigma-1}{2}-1} ;\right. \\
&\left.\lambda(t) F(\Lambda(t))\left(F^{2}(\Lambda(t))\right)^{-\frac{n}{2}\left(\frac{1}{m}-\frac{1}{2}\right)-\frac{\sigma-1}{2}-1}\right\}, \\
& \Phi_{2}(s, t)=\max \left\{\frac{\lambda^{2}(t)}{\rho(t)}\left(1+B_{\lambda}(s, t)\right)^{-\frac{\sigma-1}{2}-1} ; \lambda(t) F(\Lambda(t))\left(F^{2}(\Lambda(t))\right)^{-\frac{\sigma-1}{2}-1}\right\} .
\end{aligned}
$$

\subsubsection{Philosophy of our approach}

In this section, we will apply the estimates for the solutions to the family of linear parameterdependent Cauchy problems from Theorem 3.1.1 to prove the global (in time) existence of small data Sobolev solutions to the semi-linear model (4.1.1). Denoting by $K_{0}=K_{0}(t, 0, x)$ and $K_{1}=K_{1}(t, 0, x)$ the fundamental solutions to the corresponding linear equation with vanishing right-hand side, we write the solutions in the form

$$
u^{\operatorname{lin}}(t, x):=K_{0}(t, 0, x) *_{(x)} u_{0}(x)+K_{1}(t, 0, x) *_{(x)} u_{1}(x) .
$$

On the other hand, applying Duhamel's principle leads to the following formal implicit representation of the solution to (4.1.1):

$$
\begin{aligned}
N u(t, x)= & K_{0}(t, 0, x) *_{(x)} u_{0}(x)+K_{1}(t, 0, x) *_{(x)} u_{1}(x) \\
& +\int_{0}^{t} K_{1}(t, s, x) *_{(x)} f\left(u_{t},|D|^{a} u\right)(s, x) d s=: u^{\operatorname{lin}}(t, x)+u^{\mathrm{nl}}(t, x),
\end{aligned}
$$


where $f\left(u_{t},|D|^{a} u\right)=\left.\left.|| D\right|^{a} u\right|^{p}, a \in(0,1]$ or $\left|u_{t}\right|^{p}$ and $K_{1}=K_{1}(t, s, x)$ is the fundamental solution to the corresponding parameter-dependent linear Cauchy problem (3.1.4). Now we introduce the operator $N$ for any $u \in X(t)$ by

$$
N: u \in X(t) \rightarrow N u=N u(t, x):=u^{\operatorname{lin}}(t, x)+u^{\mathrm{nl}}(t, x),
$$

and try to find a fixed point $u=N u$ of the mapping $N: X(t) \rightarrow X(t)$. We will show that the mapping $N$ satisfies the following two estimates:

$$
\begin{aligned}
\|N u\|_{X(t)} & \lesssim\left\|\left(u_{0}, u_{1}\right)\right\|_{\mathcal{D}_{m}^{\sigma}}+\|u\|_{X(t)}^{p}, \\
\|N u-N v\|_{X(t)} & \lesssim\|u-v\|_{X(t)}\left(\|u\|_{X(t)}^{p-1}+\|v\|_{X(t)}^{p-1}\right) .
\end{aligned}
$$

These estimates indicate the existence of a unique solution of $u=N u$. Therefore, to complete the proof it remains to establish (4.1.6) and (4.1.7).

Remark 4.1.1. From the estimates in Theorem 3.1.1 and the definition of the norm in $X(t)$ we may easily conclude

$$
\left\|u^{\operatorname{lin}}\right\|_{X(t)} \lesssim\left\|\left(u_{0}, u_{1}\right)\right\|_{D_{m}^{\sigma}} .
$$

For this reason, to complete the proof of (4.1.6) we need to show

$$
\left\|u^{\mathrm{nl}}\right\|_{X(t)} \lesssim\|u\|_{X(t)}^{p} .
$$

After that we shall prove (4.1.7). Then, from the estimates of Theorem 3.1.1 it is necessary to estimate the term $|u(s, \cdot)|^{p}-|v(s, \cdot)|^{p}$ in $L^{m} \cap L^{2} \cap \dot{H}^{\sigma-1}$. In general, straight-forward calculations yield the desired estimate of the $L^{m}$ norm and $L^{2}$ norm. However, to estimate the $\dot{H}^{\sigma-1}$ norm we use tools from the Appendix A.7.

\subsection{Semilinear damped wave models of the derivative type power nonlinearity $\left|u_{t}\right|^{p}$}

We study the Cauchy problem for the following semilinear damped wave model:

$$
\begin{cases}u_{t t}-\lambda^{2}(t) \omega^{2}(t) \Delta u+\rho(t) \omega(t) u_{t}=\left|u_{t}\right|^{p}, & (t, x) \in[0, \infty) \times \mathbb{R}^{n}, \\ u(0, x)=u_{0}(x), \quad u_{t}(0, x)=u_{1}(x), & x \in \mathbb{R}^{n},\end{cases}
$$

where $\left(u_{0}, u_{1}\right) \in \mathcal{D}_{m}^{\sigma}$ with $\sigma>1+\frac{n}{2}$ and $m \in[1,2)$. In this case we have the embedding $H^{\sigma-1} \hookrightarrow L^{\infty}$. So, we obtain large regular solutions to (4.2.1) by using the fractional powers rules. Let us point out that, in order to use fractional powers the condition $p>\sigma$ is necessary to suppose.

\section{The case of sub-exponential propagation speed}

In this case we assume the following condition.

(D1) The function $\frac{\mu(t)}{\lambda^{2}(t)} \frac{\lambda^{2 p}(t)}{\rho^{p}(t)}$ satisfies the following conditions:

- $\frac{\mu(t)}{\lambda^{2}(t)} \frac{\lambda^{2 p}(t)}{\rho^{p}(t)}$ is increasing,

- there exist positive constants $d_{1}$ and $\tilde{d}_{1}$ such that the following inequalities hold:

$$
\tilde{d}_{1} \frac{\mu(t)}{\lambda(t) \Lambda(t)} \frac{\lambda^{2 p}(t)}{\rho^{p}(t)} \leq\left(\frac{\mu(t)}{\lambda^{2}(t)} \frac{\lambda^{2 p}(t)}{\rho^{p}(t)}\right)^{\prime} \leq d_{1} \frac{\mu(t)}{\lambda(t) \Lambda(t)} \frac{\lambda^{2 p}(t)}{\rho^{p}(t)} .
$$


Remark 4.2.1. By using conditions (A1), (C2) and (D1) we obtain the following estimate for $p>2$ and $n \geq 2$ :

$$
\begin{aligned}
\left(\frac{\mu(t)}{\lambda^{2}(t)} \frac{\lambda^{2 p}(t)}{\rho^{p}(t)}\right)^{\prime} & =\left(\frac{\lambda^{p-2}(t) \Lambda^{p}(t)}{\mu^{p-1}(t)}\right)^{\prime} \\
& \geq \frac{(p-2) \lambda_{0} \lambda^{p-1}(t) \Lambda^{p-1}(t)+p \lambda^{p-1}(t) \Lambda^{p-1}(t)-(p-1) \mu_{1} \lambda^{p-1}(t) \Lambda^{p-1}(t)}{\mu^{p-1}(t)} \\
& =\left((p-2) \lambda_{0}+p-(p-1) \mu_{1}\right)\left(\frac{\lambda(t) \Lambda(t)}{\mu(t)}\right)^{p-1} \\
& =\left((p-2) \lambda_{0}+p-(p-1) \mu_{1}\right) \frac{\mu(t)}{\lambda(t) \Lambda(t)} \frac{\lambda^{2 p}(t)}{\rho^{p}(t)} .
\end{aligned}
$$

Here we assume $(p-2) \lambda_{0}+p-(p-1) \mu_{1} \geq 0$ with $\lambda_{0}-\mu_{1}+1>0$, which implies

$$
p>2>\frac{2 \lambda_{0}-\mu_{1}}{\lambda_{0}-\mu_{1}+1} .
$$

Example 4.2.1. Let us consider $\lambda(t)=(\alpha+1)(1+t)^{\alpha}, \alpha>0$. Then, we get

$$
\Lambda(t)=(1+t)^{\alpha+1} \text { and } \Theta(t)=(1+t)^{\gamma+1}, \quad-1<\gamma<\alpha .
$$

Moreover, we choose

$$
\mu(t)=(1+t)^{\beta}, \quad \alpha-\gamma<\beta<2 \alpha+2,
$$

which implies

$$
\rho(t)=(\alpha+1)(1+t)^{\beta-1},
$$

where due to condition (C2) we define $\beta:=\mu_{1}(\alpha+1)$ with $\mu_{1} \in[0,2)$. Then, we have

$$
\begin{aligned}
\left(\frac{\mu(t)}{\lambda^{2}(t)} \frac{\lambda^{2 p}(t)}{\rho^{p}(t)}\right)^{\prime} & =\left(\frac{\lambda^{p-2}(t) \Lambda^{p}(t)}{\mu^{p-1}(t)}\right)^{\prime}=\left((\alpha+1)^{p-2}(1+t)^{p(2 \alpha-\beta+1)+\beta-2 \alpha}\right)^{\prime} \\
& =(\alpha+1)^{p-2}((p-1)(2 \alpha-\beta+1)+1)(1+t)^{(p-1)(2 \alpha-\beta+1)} .
\end{aligned}
$$

Here with $2 \alpha-\beta+1>0$ we find

$$
(p-1)(2 \alpha-\beta+1)+1>0, \quad \text { that is, } \quad p>2>\frac{2 \alpha-\beta}{2 \alpha-\beta+1} .
$$

Example 4.2.2. Let us choose $\lambda(t)=e^{t}$. Then, we obtain

$$
\Lambda(t)=e^{t} \quad \text { and } \quad \Theta(t)=e^{r t}, \quad 0<r<1 .
$$

Moreover, we take

$$
\mu(t)=\rho(t)=e^{\mu_{1} t}, \quad 1-r<\mu_{1}<2 .
$$

Then, we find

$$
\left(\frac{\mu(t)}{\lambda^{2}(t)} \frac{\lambda^{2 p}(t)}{\rho^{p}(t)}\right)^{\prime}=\left(\frac{\lambda^{p-2}(t) \Lambda^{p}(t)}{\mu^{p-1}(t)}\right)^{\prime}=\left(e^{(p-1)\left(2-\mu_{1}\right) t}\right)^{\prime}=(p-1)\left(2-\mu_{1}\right) e^{(p-1)\left(2-\mu_{1}\right) t},
$$

where $(p-1)\left(2-\mu_{1}\right)>0$, that is, $\frac{\mu(t)}{\lambda^{2}(t)} \frac{\lambda^{2 p}(t)}{\rho^{p}(t)}$ is increasing.

\section{The case of super-exponential propagation speed}

In this case we assume the following condition.

(D2) The function $\frac{\mu(t)}{\lambda^{2}(t)} \frac{\lambda^{2 p}(t)}{\rho^{p}(t)}$ satisfies the following conditions:

- $\frac{\mu(t)}{\lambda^{2}(t)} \frac{\lambda^{2 p}(t)}{\rho^{p}(t)}$ is increasing, 
- there exist positive constants $d_{2}$ and $\tilde{d}_{2}$ such that the following inequalities hold:

$$
\tilde{d}_{2} \frac{\mu(t)}{\lambda(t) \Lambda(t)} \frac{\lambda^{2 p}(t)}{\rho^{p}(t)} \frac{1}{\log \Lambda(t)} \leq\left(\frac{\mu(t)}{\lambda^{2}(t)} \frac{\lambda^{2 p}(t)}{\rho^{p}(t)}\right)^{\prime} \leq d_{2} \frac{\mu(t)}{\lambda(t) \Lambda(t)} \frac{\lambda^{2 p}(t)}{\rho^{p}(t)} \frac{1}{\log \Lambda(t)} .
$$

Example 4.2.3. We choose $\lambda(t)=e^{t} e^{e^{t}}$. Then, we have

$$
\Lambda(t)=e^{e^{t}}, \quad \Theta(t)=e^{r e^{t}}, \quad 0<r<1 .
$$

Moreover, since $\phi(t)=\frac{\Lambda^{2}(t)}{\mu(t)}$ is an increasing function from condition (C3) choosing

$$
\mu(t)=\frac{e^{2 e^{t}}}{e^{\alpha t}}, \quad \alpha>0 \quad \text { we get } \quad \rho(t)=\mu(t) e^{t}=e^{(1-\alpha) t} e^{2 e^{t}} .
$$

Therefore, we find

$$
\left(\frac{\mu(t)}{\lambda^{2}(t)} \frac{\lambda^{2 p}(t)}{\rho^{p}(t)}\right)^{\prime}=\left(\frac{\lambda^{p-2}(t) \Lambda^{p}(t)}{\mu^{p-1}(t)}\right)^{\prime}=\left(e^{(p-2+\alpha p-\alpha) t}\right)^{\prime}=(p-2+\alpha p-\alpha) e^{(p-2+\alpha p-\alpha) t}
$$

which implies the condition $p-2+\alpha(p-1)>0$ to conclude that $\frac{\mu(t)}{\lambda^{2}(t)} \frac{\lambda^{2 p}(t)}{\rho^{p}(t)}$ is increasing.

We introduce now statements for both cases for proving global (in time) existence theorems.

Lemma 4.2.1. We assume that conditions (D1) and (D2) hold. Then, from Lemma 3.1.3 and Lemma 3.1.4, respectively, we have the following estimates for all $s \in[0, t]$ :

$$
\begin{aligned}
& \frac{d\left(\frac{\mu(s)}{\lambda^{2}(s)} \frac{\lambda^{2 p}(t)}{\rho^{p}(t)}\left(1+B_{\lambda}(0, s)\right)^{-\alpha_{4}(p)}\right)}{d s} \lesssim \frac{\mu(s)}{\lambda^{2}(s)} \frac{\lambda^{2 p}(s)}{\rho^{p}(s)}\left(1+B_{\lambda}(0, s)\right)^{-\alpha_{4}(p)-1} \frac{d\left(1+B_{\lambda}(0, s)\right)}{d s}, \\
& \frac{d\left(\frac{\mu(s)}{\lambda^{2}(s)} \frac{\lambda^{2 p}(t)}{\rho^{p}(t)}\left(1+B_{\lambda}(0, s)\right)^{-\alpha_{4}(p)}\right)}{d s} \gtrsim-\frac{\mu(s)}{\lambda^{2}(s)} \frac{\lambda^{2 p}(s)}{\rho^{p}(s)}\left(1+B_{\lambda}(0, s)\right)^{-\alpha_{4}(p)-1} \frac{d\left(1+B_{\lambda}(0, s)\right)}{d s},
\end{aligned}
$$

where $\alpha_{4}(p)=\frac{n}{2 m} p-\frac{n}{4}+p>0$.

Now we introduce the following conditions which come into play if the second component of the functions $\Phi_{1, m}=\Phi_{1, m}(s, t), \Phi_{1}=\Phi_{1}(s, t)$ and $\Phi_{2, m}=\Phi_{2, m}(s, t), \Phi_{2}=\Phi_{2}(s, t)$ is dominant.

(D3) We assume that the following estimate holds with $k_{1} \geq 0$ :

$$
\Xi^{\prime}(t) \leq k_{1} \Xi(t) \frac{\lambda(t)}{\Lambda(t)} .
$$

Then, by using conditions (A1) and (D3) there exists a positive constant $r$ such that

$$
\begin{aligned}
\left(\lambda^{p}(t) \Lambda(t) \Xi^{2}(t)\right)^{\prime} & \leq p \lambda_{1} \lambda^{p-1}(t) \frac{\lambda^{2}(t)}{\Lambda(t)} \Lambda(t) \Xi^{2}(t)+\lambda^{p+1}(t) \Xi^{2}(t)+2 k_{1} \lambda^{p}(t) \Lambda(t) \Xi^{2}(t) \frac{\lambda(t)}{\Lambda(t)} \\
& \leq\left(p \lambda_{1}+1+2 k_{1}\right) \lambda^{p+1}(t) \Xi^{2}(t) \leq r \lambda^{p+1}(t) \Xi^{2}(t) .
\end{aligned}
$$

(D4) We suppose that the following estimate is satisfied:

$$
\frac{F(\Lambda(t))}{F^{\prime}(\Lambda(t))} \leq \Lambda(t)
$$




\subsubsection{Case of sub-exponential propagation speed}

We define the following parameters:

$$
\begin{aligned}
& p_{1}(m, n, \sigma):=\max \left\{\frac{n}{n+2 m}+\left(\frac{\sigma+1}{2}+\frac{d_{1}}{2-\mu_{1}}\right) \frac{2 m}{n+2 m} ; \frac{m r+n-m}{n+m}\right\}, \\
& p_{2}(m, n, \sigma):=\max \left\{\frac{n m}{2(n+2 m)}+\frac{\frac{n m}{n+2 m}\left(\frac{1}{m}-\frac{1}{2}\right)}{1-\frac{2+R}{2-\mu_{1}}+\frac{2+R}{2-\mu_{1}} \frac{\delta}{\nu(\delta)}}+\left(\frac{\frac{\sigma+1}{2}+\frac{d_{1}}{2-\mu_{1}} \frac{\delta}{\nu(\delta)}}{1-\frac{2+R}{2-\mu_{1}}+\frac{2+R}{2-\mu_{1}} \frac{\delta}{\nu(\delta)}}\right) \frac{2 m}{n+2 m} ;\right. \\
&\left.\frac{m(n-2)}{2(n+m)}+\frac{1}{\vartheta(\delta)} \frac{r m}{n+m}+\frac{\nu(\delta)}{\delta}\left(\frac{n}{2}\left(\frac{1}{m}-\frac{1}{2}\right)+\frac{\sigma}{2}\right)(2+R) \frac{m}{n+m}\right\},
\end{aligned}
$$

where we set

$$
p(m, n, \sigma):=\max \left\{p_{1}(m, n, \sigma) ; p_{2}(m, n, \sigma)\right\} .
$$

We have the following result in the case of sub-exponential propagation speed.

Theorem 4.2.2. We assume that the Hypotheses $(\boldsymbol{A} 1)$ to $(\boldsymbol{A} 5),(\boldsymbol{B} 1)$ to $(\boldsymbol{B} 6)$, (C1), (C2), (D1), (D3) and (D4) are satisfied. Let us suppose $\nu(\delta) \lesssim 1$ and $\vartheta(\delta) \lesssim 1$, where the functions $\nu=\nu(\delta)$ and $\vartheta=\vartheta(\delta)$ are defined in (3.1.2) and (3.1.3), respectively. Let the data $\left(u_{0}, u_{1}\right) \in \mathcal{D}_{m}^{\sigma}$ with $\sigma>1+\frac{n}{2}, n \geq 2$ and $m \in[1,2)$. Moreover, we assume that the exponent $p$ satisfies $p>p(m, n, \sigma)$ and $p>\sigma$.

Then, there exists a constant $\varepsilon_{0}>0$ such that for all $\left(u_{0}, u_{1}\right) \in \mathcal{D}_{m}^{\sigma}$ with $\left\|\left(u_{0}, u_{1}\right)\right\|_{\mathcal{D}_{m}^{\sigma}} \leq \varepsilon_{0}$ there is a uniquely determined energy solution

$$
u \in \mathcal{C}\left([0, \infty), H^{\sigma}\right) \cap \mathcal{C}^{1}\left([0, \infty), H^{\sigma-1}\right)
$$

to the Cauchy problem (4.2.1). Furthermore, the solution satisfies the estimates

$$
\begin{aligned}
& \|u(t, \cdot)\|_{L^{2}} \lesssim\left(1+B_{\lambda}(0, t)\right)^{-\frac{n}{2}\left(\frac{1}{m}-\frac{1}{2}\right)}\left\|\left(u_{0}, u_{1}\right)\right\|_{\mathcal{D}_{m}^{\sigma}} \\
& \left\||D|^{\sigma} u(t, \cdot)\right\|_{L^{2}} \lesssim\left(1+B_{\lambda}(0, t)\right)^{-\frac{n}{2}\left(\frac{1}{m}-\frac{1}{2}\right)-\frac{\sigma}{2}}\left\|\left(u_{0}, u_{1}\right)\right\|_{\mathcal{D}_{m}^{\sigma}} \\
& \left\|u_{t}(t, \cdot)\right\|_{L^{2}} \\
& \lesssim \max \left\{\frac{\lambda^{2}(t)}{\rho(t)}\left(1+B_{\lambda}(0, t)\right)^{-\frac{n}{2}\left(\frac{1}{m}-\frac{1}{2}\right)-1} ; \lambda(t) F(\Lambda(t))\left(F^{2}(\Lambda(t))\right)^{-\frac{n}{2}\left(\frac{1}{m}-\frac{1}{2}\right)-1}\right\}\left\|\left(u_{0}, u_{1}\right)\right\|_{\mathcal{D}_{m}^{\sigma}} \\
& \left\||D|^{\sigma-1} u_{t}(t, \cdot)\right\|_{L^{2}} \\
& \lesssim \max \left\{\frac{\lambda^{2}(t)}{\rho(t)}\left(1+B_{\lambda}(0, t)\right)^{-\frac{n}{2}\left(\frac{1}{m}-\frac{1}{2}\right)-\frac{\sigma-1}{2}-1} ; \lambda(t) F(\Lambda(t))\left(F^{2}(\Lambda(t))\right)^{-\frac{n}{2}\left(\frac{1}{m}-\frac{1}{2}\right)-\frac{\sigma-1}{2}-1}\right\}\left\|\left(u_{0}, u_{1}\right)\right\|_{\mathcal{D}_{m}^{\sigma}}
\end{aligned}
$$

Proof. We introduce for all $t>0$ the functions spaces

$$
X(t)=\mathcal{C}\left([0, t], H^{\sigma}\right) \cap \mathcal{C}^{1}\left([0, t], H^{\sigma-1}\right)
$$

with the norm

$$
\begin{aligned}
\|u(\tau, \cdot)\|_{X(t)}=\sup _{0 \leq \tau \leq t}[ & \left(1+B_{\lambda}(0, \tau)\right)^{\frac{n}{2}\left(\frac{1}{m}-\frac{1}{2}\right)}\|u(\tau, \cdot)\|_{L^{2}} \\
& +\left(1+B_{\lambda}(0, \tau)\right)^{\frac{n}{2}\left(\frac{1}{m}-\frac{1}{2}\right)+\frac{\sigma}{2}}\left\||D|^{\sigma} u(\tau, \cdot)\right\|_{L^{2}} \\
& \left.+\Phi_{1, m}^{-1}(0, \tau)\left\|u_{t}(\tau, \cdot)\right\|_{L^{2}}+\Phi_{2, m}^{-1}(0, \tau)\left\||D|^{\sigma-1} u_{t}(\tau, \cdot)\right\|_{L^{2}}\right] .
\end{aligned}
$$

Here $\Phi_{1, m}=\Phi_{1, m}(0, \tau)$ and $\Phi_{2, m}=\Phi_{1, m}(0, \tau)$ are defined in (4.1.2) and (4.1.4), respectively for $s=0$ and $t=\tau$. In order to prove (4.1.6) let us begin with the "nonlinear part" of the solution $u$, namely, with

$$
u^{\mathrm{nl}}(t, x)=\int_{0}^{t} K_{1}(t, s, x) *_{(x)}\left|u_{t}(s, x)\right|^{p} d s .
$$


For this reason we have to estimate the norms

$$
\left\|u^{\mathrm{nl}}(t, \cdot)\right\|_{L^{2}}, \quad\left\||D|^{\sigma} u^{\mathrm{nl}}(t, \cdot)\right\|_{L^{2}}, \quad\left\|u_{t}^{\mathrm{nl}}(t, \cdot)\right\|_{L^{2}} \quad \text { and } \quad\left\||D|^{\sigma-1} u_{t}^{\mathrm{nl}}(t, \cdot)\right\|_{L^{2}} \cdot
$$

Case 1: Let us assume that the first components are dominant, that is,

$$
\begin{aligned}
& \Phi_{1, m}(s, t)=\frac{\lambda^{2}(t)}{\rho(t)}\left(1+B_{\lambda}(s, t)\right)^{-\frac{n}{2}\left(\frac{1}{m}-\frac{1}{2}\right)-1} \quad \text { and } \Phi_{1}(s, t)=\frac{\lambda^{2}(t)}{\rho(t)}\left(1+B_{\lambda}(s, t)\right)^{-1} \\
& \Phi_{2, m}(s, t)=\frac{\lambda^{2}(t)}{\rho(t)}\left(1+B_{\lambda}(s, t)\right)^{-\frac{n}{2}\left(\frac{1}{m}-\frac{1}{2}\right)-\frac{\sigma-1}{2}-1} \text { and } \Phi_{2}(s, t)=\frac{\lambda^{2}(t)}{\rho(t)}\left(1+B_{\lambda}(s, t)\right)^{-\frac{\sigma-1}{2}-1},
\end{aligned}
$$

for all $t \geq s \geq 0$. In order to estimate the norm $\left\||D|^{\sigma} u^{\mathrm{nl}}(t, \cdot)\right\|_{L^{2}}$ we use the $\left(L^{m} \cap L^{2}\right)-L^{2}$ estimates if $s \in[0, \delta t]$ and $L^{2}-L^{2}$ estimates if $s \in[\delta t, t]$ from Theorem 3.1.1. Therefore, we have the following estimate:

$$
\begin{aligned}
\left\||D|^{\sigma} u^{\mathrm{nl}}(t, \cdot)\right\|_{L^{2}} \lesssim & \int_{0}^{\delta t} \frac{\Lambda(s)}{\lambda(s)}\left(1+B_{\lambda}(s, t)\right)^{-\frac{n}{2}\left(\frac{1}{m}-\frac{1}{2}\right)-\frac{\sigma}{2}}\left\|\left|u_{t}(s, \cdot)\right|^{p}\right\|_{L^{m} \cap L^{2} \cap \dot{H}^{\sigma-1}} d s \\
& +\int_{\delta t}^{t} \frac{\Lambda(s)}{\lambda(s)}\left(1+B_{\lambda}(s, t)\right)^{-\frac{\sigma}{2}}\left\|\left|u_{t}(s, \cdot)\right|^{p}\right\|_{L^{2} \cap \dot{H}^{\sigma-1}} d s .
\end{aligned}
$$

It remains to estimate the following norms:

$$
\left\|\left|u_{t}(s, \cdot)\right|^{p}\right\|_{L^{m}}, \quad\left\|\left|u_{t}(s, \cdot)\right|^{p}\right\|_{L^{2}} \quad \text { and } \quad\left\|\left|u_{t}(s, \cdot)\right|^{p}\right\|_{\dot{H}^{\sigma-1}} \cdot
$$

For the first two norms, by using the fractional Gagliardo-Nirenberg inequality one can get for $p \geq \frac{2}{m}$ the estimates

$$
\begin{aligned}
\left\|u_{t}(s, \cdot)\right\|_{L^{m p}}^{p} & \lesssim\left\||D|^{\sigma} u_{t}(s, \cdot)\right\|_{L^{2}}^{p \theta_{\sigma}(m p)}\left\|u_{t}(s, \cdot)\right\|_{L^{2}}^{p\left(1-\theta_{\sigma}(m p)\right)} \\
& \lesssim \frac{\lambda^{2 p}(s)}{\rho^{p}(s)}\left(1+B_{\lambda}(0, s)\right)^{-\frac{n}{2 m} p+\frac{n}{2 m}-p}\|u\|_{X(s)}^{p}, \\
\left\|u_{t}(s, \cdot)\right\|_{L^{2 p}}^{p} & \lesssim\left\||D|^{\sigma} u_{t}(s, \cdot)\right\|_{L^{2}}^{p \theta_{\sigma}(2 p)}\left\|u_{t}(s, \cdot)\right\|_{L^{2}}^{p\left(1-\theta_{\sigma}(2 p)\right)} \\
& \lesssim \frac{\lambda^{2 p}(s)}{\rho^{p}(s)}\left(1+B_{\lambda}(0, s)\right)^{-\frac{n}{2 m} p+\frac{n}{4}-p}\|u\|_{X(s)}^{p} .
\end{aligned}
$$

Now, we turn to estimate the norm $\left\|\left|u_{t}(s, \cdot)\right|^{p}\right\|_{\dot{H}^{\sigma-1}}$ by using results on fractional powers for $\sigma-1>\frac{n}{2}$. We apply Corollary A.7.10 with $\sigma-1 \in\left(\frac{n}{2}, p\right)$ and Proposition A.7.13 with a suitable $\sigma^{*}<\frac{n}{2}$, from Section A.7 in the Appendix, respectively. Therefore, we obtain

$$
\begin{aligned}
\left\|\left|u_{t}(s, \cdot)\right|^{p}\right\|_{\dot{H}^{\sigma-1}} & \lesssim\left\|u_{t}(s, \cdot)\right\|_{\dot{H}^{\sigma-1}}\left\|u_{t}(s, \cdot)\right\|_{L^{\infty}}^{p-1} \\
& \lesssim\left\|u_{t}(s, \cdot)\right\|_{\dot{H}^{\sigma-1}}\left(\left\|u_{t}(s, \cdot)\right\|_{\dot{H}^{\sigma^{*}}}+\left\|u_{t}(s, \cdot)\right\|_{\dot{H}^{\sigma-1}}\right)^{p-1} \\
& \lesssim\left\|u_{t}(s, \cdot)\right\|_{\dot{H}^{\sigma-1}}\left\|u_{t}(s, \cdot)\right\|_{\dot{H}^{\sigma^{*}}}^{p-1}+\left\|u_{t}(s, \cdot)\right\|_{\dot{H}^{\sigma-1}}^{p} .
\end{aligned}
$$

Applying the fractional Gagliardo-Nirenberg inequality and using the definition of the norm of the solution space $X(t)$, we derive

$$
\begin{aligned}
\left\|u_{t}(s, \cdot)\right\|_{\dot{H}^{\sigma-1}} & \lesssim\left\|u_{t}(s, \cdot)\right\|_{\dot{H}^{\sigma}}^{\theta_{\sigma-1, \sigma}}\left\|u_{t}(s, \cdot)\right\|_{L^{2}}^{1-\theta_{\sigma-1, \sigma}} \\
& \lesssim \frac{\lambda^{2}(s)}{\rho(s)}\left(1+B_{\lambda}(0, s)\right)^{-\frac{n}{2}\left(\frac{1}{m}-\frac{1}{2}\right)-\frac{\sigma-1}{2}-1}\|u\|_{X(s)}, \\
\left\|u_{t}(s, \cdot)\right\|_{\dot{H}^{\sigma^{*}}} & \lesssim\left\|u_{t}(s, \cdot)\right\|_{\dot{H}^{\sigma}, \sigma}^{\theta_{\sigma^{*}}}\left\|u_{t}(s, \cdot)\right\|_{L^{2}}^{1-\theta_{\sigma^{*}, \sigma}} \\
& \lesssim \frac{\lambda^{2}(s)}{\rho(s)}\left(1+B_{\lambda}(0, s)\right)^{-\frac{n}{2}\left(\frac{1}{m}-\frac{1}{2}\right)-\frac{\sigma^{*}}{2}-1}\|u\|_{X(s)},
\end{aligned}
$$


where $\theta_{\sigma-1, \sigma}=1-\frac{1}{\sigma}$ and $\theta_{\sigma^{*}, \sigma}=\frac{\sigma^{*}}{\sigma}$. Hence, by choosing the parameter $\sigma^{*}=\frac{n}{2}-\varepsilon<\frac{n}{2}$ with a sufficiently small positive $\varepsilon$ we have

$$
\left\|\left|u_{t}(s, \cdot)\right|^{p}\right\|_{\dot{H}^{\sigma-1}} \lesssim \frac{\lambda^{2 p}(s)}{\rho^{p}(s)}\left(1+B_{\lambda}(0, s)\right)^{-\frac{n}{2}\left(\frac{1}{m}-\frac{1}{2}\right) p-\frac{\sigma-1}{2}-\frac{\sigma^{*}}{2}(p-1)-p}\|u\|_{X(s)}^{p} .
$$

For this reason, in order to get the desired estimates after taking into consideration (4.2.2), (4.2.3), (4.2.4), $\sigma^{*}<\frac{n}{2}$ and

$$
-\frac{n}{2 m} p+\frac{n}{2 m}-p>-\frac{n}{2 m} p+\frac{n}{4}-p>-\frac{n}{2}\left(\frac{1}{m}-\frac{1}{2}\right) p-\frac{\sigma-1}{2}-\frac{\sigma^{*}}{2}(p-1)-p,
$$

where we choose $\sigma^{*}=\frac{n}{2}-\varepsilon<\frac{n}{2}$ and $\|u\|_{X(s)} \leq\|u\|_{X(t)}$ with $0 \leq s \leq t$, we may conclude

$$
\begin{aligned}
& \left\||D|^{\sigma} u^{\mathrm{nl}}(t, \cdot)\right\|_{L^{2}} \\
& \lesssim\|u\|_{X(t)}^{p} \underbrace{\int_{0}^{\delta t} \frac{\Lambda(s)}{\lambda(s)} \frac{\lambda^{2 p}(s)}{\rho^{p}(s)}\left(1+B_{\lambda}(s, t)\right)^{-\frac{n}{2}\left(\frac{1}{m}-\frac{1}{2}\right)-\frac{\sigma}{2}}\left(1+B_{\lambda}(0, s)\right)^{-\frac{n}{2 m} p+\frac{n}{2 m}-p} d s}_{\mathbf{M}} \\
& +\|u\|_{X(t)}^{p} \underbrace{\int_{\delta t}^{t} \frac{\Lambda(s)}{\lambda(s)} \frac{\lambda^{2 p}(s)}{\rho^{p}(s)}\left(1+B_{\lambda}(s, t)\right)^{-\frac{\sigma}{2}}\left(1+B_{\lambda}(0, s)\right)^{-\frac{n}{2 m} p+\frac{n}{4}-p} d s}_{\mathbf{N}} .
\end{aligned}
$$

Let us consider the integral M. Using (3.1.18), we have the estimate

$$
\mathbf{M} \lesssim\left(1+B_{\lambda}(0, t)\right)^{-\frac{n}{2}\left(\frac{1}{m}-\frac{1}{2}\right)-\frac{\sigma}{2}} \underbrace{\int_{0}^{\delta t} \frac{\Lambda(s)}{\lambda(s)} \frac{\lambda^{2 p}(s)}{\rho^{p}(s)}\left(1+B_{\lambda}(0, s)\right)^{-\alpha_{3}(p)} d s}_{M_{1}(t)},
$$

where $\alpha_{3}(p):=\frac{n}{2 m} p-\frac{n}{2 m}+p$. Then, it follows

$$
\begin{aligned}
M_{1}(t)= & \int_{0}^{\delta t} \frac{\mu(s)}{\lambda^{2}(s)} \frac{\lambda^{2 p}(s)}{\rho^{p}(s)}\left(1+B_{\lambda}(0, s)\right)^{-\alpha_{3}(p)} d\left(1+B_{\lambda}(0, s)\right) \\
= & \left.\frac{1}{1-\alpha_{3}(p)} \frac{\mu(s)}{\lambda^{2}(s)} \frac{\lambda^{2 p}(s)}{\rho^{p}(s)}\left(1+B_{\lambda}(0, s)\right)^{-\alpha_{3}(p)+1}\right|_{0} ^{\delta t} \\
& -\frac{1}{1-\alpha_{3}(p)} \int_{0}^{\delta t}\left(1+B_{\lambda}(0, s)\right)^{-\alpha_{3}(p)+1} d\left(\frac{\mu(s)}{\lambda^{2}(s)} \frac{\lambda^{2 p}(s)}{\rho^{p}(s)}\right) .
\end{aligned}
$$

We consider the integral $M_{1}=M_{1}(t)$ only in the case $\alpha_{3}(p)>1$. In this case it holds

$$
\begin{aligned}
M_{1}(t) \leq & \left.\frac{1}{1-\alpha_{3}(p)} \frac{\mu(s)}{\lambda^{2}(s)} \frac{\lambda^{2 p}(s)}{\rho^{p}(s)}\left(1+B_{\lambda}(0, s)\right)^{-\alpha_{3}(p)+1}\right|_{0} ^{\delta t} \\
& +\frac{d_{1}}{\alpha_{3}(p)-1} \int_{0}^{\delta t}\left(1+B_{\lambda}(0, s)\right)^{-\alpha_{3}(p)}\left(1+B_{\lambda}(0, s)\right) \frac{\mu(s)}{\lambda(s) \Lambda(s)} \frac{\lambda^{2 p}(s)}{\rho^{p}(s)} d s \\
\leq & \left.\frac{1}{1-\alpha_{3}(p)} \frac{\mu(s)}{\lambda^{2}(s)} \frac{\lambda^{2 p}(s)}{\rho^{p}(s)}\left(1+B_{\lambda}(0, s)\right)^{-\alpha_{3}(p)+1}\right|_{0} ^{\delta t} \\
& +\frac{d_{1}}{\left(\alpha_{3}(p)-1\right)\left(2-\mu_{1}\right)} \underbrace{\int_{0}^{\delta t} \frac{\Lambda(s)}{\lambda(s)} \frac{\lambda^{2 p}(s)}{\rho^{p}(s)}\left(1+B_{\lambda}(0, s)\right)^{-\alpha_{3}(p)} d s}_{M_{1}(t)},
\end{aligned}
$$

where we used condition (D1) and (3.1.17), respectively. Thus, we obtain

$$
\left(1-\frac{d_{1}}{\left(\alpha_{3}(p)-1\right)\left(2-\mu_{1}\right)}\right) M_{1}(t) \lesssim \frac{\mu(0)}{\lambda^{2}(0)} \frac{\lambda^{2 p}(0)}{\rho^{p}(0)}-\frac{\mu(\delta t)}{\lambda^{2}(\delta t)} \frac{\lambda^{2 p}(\delta t)}{\rho^{p}(\delta t)}\left(1+B_{\lambda}(0, \delta t)\right)^{-\alpha_{3}(p)+1} .
$$


We can guarantee that $M_{1}=M_{1}(t)$ is bounded by

$$
1-\frac{d_{1}}{\left(\alpha_{3}(p)-1\right)\left(2-\mu_{1}\right)}>0, \quad \text { that is, } \quad p>1+\left(\frac{d_{1}}{2-\mu_{1}}\right) \frac{2 m}{n+2 m} .
$$

Therefore, we obtain the desired estimate

$$
\mathbf{M} \lesssim\left(1+B_{\lambda}(0, t)\right)^{-\frac{n}{2}\left(\frac{1}{m}-\frac{1}{2}\right)-\frac{\sigma}{2}} .
$$

Now, let us consider the integral N. Defining $\alpha_{4}(p):=\frac{n}{2 m} p-\frac{n}{4}+p$ we have

$$
\begin{aligned}
\mathbf{N}= & -\int_{\delta t}^{t} \frac{\mu(s)}{\lambda^{2}(s)} \frac{\lambda^{2 p}(s)}{\rho^{p}(s)}\left(1+B_{\lambda}(0, s)\right)^{-\alpha_{4}(p)}\left(1+B_{\lambda}(s, t)\right)^{-\frac{\sigma}{2}} d\left(1+B_{\lambda}(s, t)\right) \\
= & -\left.\frac{1}{1-\frac{\sigma}{2}} \frac{\mu(s)}{\lambda^{2}(s)} \frac{\lambda^{2 p}(s)}{\rho^{p}(s)}\left(1+B_{\lambda}(0, s)\right)^{-\alpha_{4}(p)}\left(1+B_{\lambda}(s, t)\right)^{-\frac{\sigma}{2}+1}\right|_{\delta t} ^{t} \\
& +\frac{1}{1-\frac{\sigma}{2}} \int_{\delta t}^{t}\left(1+B_{\lambda}(s, t)\right)^{-\frac{\sigma}{2}+1} d\left(\frac{\mu(s)}{\lambda^{2}(s)} \frac{\lambda^{2 p}(s)}{\rho^{p}(s)}\left(1+B_{\lambda}(0, s)\right)^{-\alpha_{4}(p)}\right) .
\end{aligned}
$$

Here we consider only $1-\frac{\sigma}{2}<0$, because $p>\sigma>2$ with $n \geq 2$. Then, applying Lemma 4.2.1 we have

$$
\begin{aligned}
&\left(1+B_{\lambda}(0, t)\right)^{\frac{n}{2}\left(\frac{1}{m}-\frac{1}{2}\right)+\frac{\sigma}{2}} \mathbf{N} \\
& \lesssim \underbrace{\frac{\mu(t)}{\lambda^{2}(t)} \frac{\lambda^{2 p}(t)}{\rho^{p}(t)}\left(1+B_{\lambda}(0, t)\right)^{-\alpha_{4}(p)+\frac{n}{2}\left(\frac{1}{m}-\frac{1}{2}\right)+\frac{\sigma}{2}}}_{N_{1}(t)} \\
&+\underbrace{\int_{\delta t}^{t} \frac{\mu(s)}{\lambda^{2}(s)} \frac{\lambda^{2 p}(s)}{\rho^{p}(s)}\left(1+B_{\lambda}(0, s)\right)^{-\alpha_{4}(p)}\left(1+B_{\lambda}(0, t)\right)^{\frac{n}{2}\left(\frac{1}{m}-\frac{1}{2}\right)+\frac{\sigma}{2}} \frac{d\left(1+B_{\lambda}(0, s)\right)}{1+B_{\lambda}(0, s)}}_{N_{2}(t)} .
\end{aligned}
$$

Using $\alpha_{4}(p)=\alpha_{3}(p)+\frac{n}{2}\left(\frac{1}{m}-\frac{1}{2}\right)$ we find

$$
N_{1}(t)=\frac{\mu(t)}{\lambda^{2}(t)} \frac{\lambda^{2 p}(t)}{\rho^{p}(t)}\left(1+B_{\lambda}(0, t)\right)^{-\alpha_{3}(p)+\frac{\sigma}{2}} .
$$

In order to show that $N_{1}=N_{1}(t)$ is uniformly bounded we study the monotonicity of this function as follows:

$$
\begin{aligned}
N_{1}^{\prime}(t) & \leq\left[d_{1} \frac{\mu(t)}{\lambda(t) \Lambda(t)}\left(1+B_{\lambda}(0, t)\right)+\left(-\alpha_{3}(p)+\frac{\sigma}{2}\right) \frac{\Lambda(t)}{\lambda(t)}\right] \frac{\lambda^{2 p}(t)}{\rho^{p}(t)}\left(1+B_{\lambda}(0, t)\right)^{-\alpha_{3}(p)+\frac{\sigma}{2}-1} \\
& \leq\left[\frac{d_{1}}{2-\mu_{1}} \frac{\mu(t)}{\lambda(t) \Lambda(t)} \frac{\Lambda^{2}(t)}{\mu(t)}+\left(-\alpha_{3}(p)+\frac{\sigma}{2}\right) \frac{\Lambda(t)}{\lambda(t)}\right] \frac{\lambda^{2 p}(t)}{\rho^{p}(t)}\left(1+B_{\lambda}(0, t)\right)^{-\alpha_{3}(p)+\frac{\sigma}{2}-1} \\
& \leq\left[\frac{d_{1}}{2-\mu_{1}}-\alpha_{3}(p)+\frac{\sigma}{2}\right] \frac{\lambda^{2 p}(t)}{\rho^{p}(t)} \frac{\Lambda(t)}{\lambda(t)}\left(1+B_{\lambda}(0, t)\right)^{-\alpha_{3}(p)+\frac{\sigma}{2}-1},
\end{aligned}
$$

where we used condition (D1) and (3.1.17), respectively. Then, we get $N_{1}^{\prime}(t)<0$ if

$$
\frac{d_{1}}{2-\mu_{1}}-\alpha_{3}(p)+\frac{\sigma}{2}<0, \quad \text { that is, } \quad p>\frac{n}{n+2 m}+\left(\frac{\sigma}{2}+\frac{d_{1}}{2-\mu_{1}}\right) \frac{2 m}{n+2 m} .
$$

Namely, by $p>p_{1}(m, n, \sigma)$ we obtain that $N_{1}=N_{1}(t)$ is decreasing. In order to show that $N_{2}=N_{2}(t)$ is uniformly bounded we suppose that there exists a positive constant $\varepsilon$ such that we have

$$
\frac{\mu(\delta t)}{\lambda^{2}(\delta t)} \frac{\lambda^{2 p}(\delta t)}{\rho^{p}(\delta t)}\left(1+B_{\lambda}(0, \delta t)\right)^{-\alpha_{4}(p)}\left(1+B_{\lambda}(0, t)\right)^{\frac{n}{2}\left(\frac{1}{m}-\frac{1}{2}\right)+\frac{\sigma}{2}} \lesssim\left(1+B_{\lambda}(0, \delta t)\right)^{-\varepsilon} .
$$


Employing (3.1.19), from the previous estimate we have

$$
\begin{aligned}
& \frac{\mu(\delta t)}{\lambda^{2}(\delta t)} \frac{\lambda^{2 p}(\delta t)}{\rho^{p}(\delta t)}\left(1+B_{\lambda}(0, \delta t)\right)^{-\alpha_{4}(p)+\varepsilon}\left(1+B_{\lambda}(0, t)\right)^{\frac{n}{2}\left(\frac{1}{m}-\frac{1}{2}\right)+\frac{\sigma}{2}} \\
& \leq \frac{\mu(\delta t)}{\lambda^{2}(\delta t)} \frac{\lambda^{2 p}(\delta t)}{\rho^{p}(\delta t)}\left(1+B_{\lambda}(0, t)\right)^{\frac{n}{2}\left(\frac{1}{m}-\frac{1}{2}\right)+\frac{\sigma}{2}-\alpha_{4}(p)+\varepsilon}\left(\frac{\Lambda(\delta t)}{\Lambda(t)}\right)^{(2+R)\left(-\alpha_{4}(p)+\varepsilon\right)}:=\mathcal{N}_{2}(t) .
\end{aligned}
$$

Analogously as we did in the proof of Theorem 3.2.1 for deriving the decreasing behavior of the function $\mathcal{B}_{1}=\mathcal{B}_{1}(t)$, which is given in (3.2.15), we may conclude that $\mathcal{N}_{2}^{\prime}(t)<0$ by the condition

$$
\delta \frac{d_{1}}{2-\mu_{1}}+\left(\frac{n}{2}\left(\frac{1}{m}-\frac{1}{2}\right)+\frac{\sigma}{2}-\alpha_{4}(p)\right) \nu(\delta)+\alpha_{4}(p) \frac{2+R}{2-\mu_{1}}(\nu(\delta)-\delta)<0
$$

which may be concluded from the condition $p>p_{2}(m, n, \sigma)$. All together gives the expected estimate for $N_{2}=N_{2}(t)$. Consequently, we obtain

$$
\mathbf{N} \lesssim\left(1+B_{\lambda}(0, t)\right)^{-\frac{n}{2}\left(\frac{1}{m}-\frac{1}{2}\right)-\frac{\sigma}{2}} .
$$

Therefore, from (4.2.6) and (4.2.8) we arrive at the estimate

$$
\left\||D|^{\sigma} u^{\mathrm{nl}}(t, \cdot)\right\|_{L^{2}} \lesssim\|u\|_{X(t)}^{p}\left(1+B_{\lambda}(0, t)\right)^{-\frac{n}{2}\left(\frac{1}{m}-\frac{1}{2}\right)-\frac{\sigma}{2}} .
$$

To complete the proof of (4.1.6), by using the conditions $p>p_{1}(m, n, \sigma)$ and $p>p_{2}(m, n, \sigma)$ one can prove in the same way

$$
\left\|u^{\mathrm{nl}}(t, \cdot)\right\|_{L^{2}} \lesssim\|u\|_{X(t)}^{p}\left(1+B_{\lambda}(0, t)\right)^{-\frac{n}{2}\left(\frac{1}{m}-\frac{1}{2}\right)} .
$$

Now let us turn to estimate the norm $\left\||D|^{\sigma-1} u_{t}^{\mathrm{nl}}(t, \cdot)\right\|_{L^{2}}$. Proceeding in the same way as we did to estimate the norm $\left\||D|^{\sigma} u^{\mathrm{nl}}(t, \cdot)\right\|_{L^{2}}$, we obtain the following estimate:

$$
\begin{aligned}
\left\||D|^{\sigma-1} u_{t}^{\mathrm{nl}}(t, \cdot)\right\|_{L^{2}} \lesssim & \int_{0}^{\delta t} \frac{\lambda^{2}(t)}{\rho(t)} \frac{\Lambda(s)}{\lambda(s)}\left(1+B_{\lambda}(s, t)\right)^{-\frac{n}{2}\left(\frac{1}{m}-\frac{1}{2}\right)-\frac{\sigma-1}{2}-1}\left\|\left|u_{t}(s, \cdot)\right|^{p}\right\|_{L^{m} \cap L^{2} \cap \dot{H}^{\sigma-1}} d s \\
& +\int_{\delta t}^{t} \frac{\lambda^{2}(t)}{\rho(t)} \frac{\Lambda(s)}{\lambda(s)}\left(1+B_{\lambda}(s, t)\right)^{-\frac{\sigma-1}{2}-1}\left\|\left|u_{t}(s, \cdot)\right|^{p}\right\|_{L^{2} \cap \dot{H}^{\sigma-1}} d s .
\end{aligned}
$$

Then, using the estimates of the norm $\left\|\left|u_{t}(s, \cdot)\right|^{p}\right\|_{L^{m} \cap L^{2} \cap \dot{H}^{\sigma-1}}$ from (4.2.2), (4.2.3) and (4.2.4) with no other requirements to the admissible exponents $p$ we arrive at

$$
\begin{aligned}
& \left\||D|^{\sigma-1} u_{t}^{\mathrm{nl}}(t, \cdot)\right\|_{L^{2}} \\
& \lesssim\|u\|_{X(t)}^{p} \underbrace{\int_{0}^{\delta t} \frac{\lambda^{2}(t)}{\rho(t)} \frac{\Lambda(s)}{\lambda(s)} \frac{\lambda^{2 p}(s)}{\rho^{p}(s)}\left(1+B_{\lambda}(s, t)\right)^{-\frac{n}{2}\left(\frac{1}{m}-\frac{1}{2}\right)-\frac{\sigma-1}{2}-1}\left(1+B_{\lambda}(0, s)\right)^{-\frac{n}{2 m} p+\frac{n}{2 m}-p} d s}_{\mathbf{P}} \\
& +\|u\|_{X(t)}^{p} \underbrace{\int_{\delta t}^{t} \frac{\lambda^{2}(t)}{\rho(t)} \frac{\Lambda(s)}{\lambda(s)} \frac{\lambda^{2 p}(s)}{\rho^{p}(s)}\left(1+B_{\lambda}(s, t)\right)^{-\frac{\sigma-1}{2}-1}\left(1+B_{\lambda}(0, s)\right)^{-\frac{n}{2 m} p+\frac{n}{4}-p} d s}_{\mathbf{R}} .
\end{aligned}
$$

In order to obtain the desired estimates for $\mathbf{P}$ and $\mathbf{R}$ we follow the same considerations from the proof of Theorem 3.4.1. In this way we obtain

$$
\mathbf{P} \lesssim \frac{\lambda^{2}(t)}{\rho(t)}\left(1+B_{\lambda}(0, t)\right)^{-\frac{n}{2}\left(\frac{1}{m}-\frac{1}{2}\right)-\frac{\sigma-1}{2}-1} \underbrace{\int_{0}^{\delta t} \frac{\Lambda(s)}{\lambda(s)} \frac{\lambda^{2 p}(s)}{\rho^{p}(s)}\left(1+B_{\lambda}(0, s)\right)^{-\frac{n}{2 m} p+\frac{n}{2 m}-p} d s}_{P_{1}(t)}
$$


where we used (3.1.18). From the treatment of $M_{1}=M_{1}(t)$ in (4.2.5) we may conclude that the integral $P_{1}=P_{1}(t)$ is uniformly bounded, too. Thus, we obtain

$$
\mathbf{P} \lesssim \frac{\lambda^{2}(t)}{\rho(t)}\left(1+B_{\lambda}(0, t)\right)^{-\frac{n}{2}\left(\frac{1}{m}-\frac{1}{2}\right)-\frac{\sigma-1}{2}-1} .
$$

Now we treat the integral $\mathbf{R}$ as follows:

$$
\begin{aligned}
\mathbf{R}= & -\frac{\lambda^{2}(t)}{\rho(t)} \int_{\delta t}^{t} \frac{\mu(s)}{\lambda^{2}(s)} \frac{\lambda^{2 p}(s)}{\rho^{p}(s)}\left(1+B_{\lambda}(0, s)\right)^{-\alpha_{4}(p)}\left(1+B_{\lambda}(s, t)\right)^{-\frac{\sigma-1}{2}-1} d\left(1+B_{\lambda}(s, t)\right) \\
= & \left.\frac{2}{\sigma-1} \frac{\lambda^{2}(t)}{\rho(t)} \frac{\mu(s)}{\lambda^{2}(s)} \frac{\lambda^{2 p}(s)}{\rho^{p}(s)}\left(1+B_{\lambda}(0, s)\right)^{-\alpha_{4}(p)}\left(1+B_{\lambda}(s, t)\right)^{-\frac{\sigma-1}{2}}\right|_{\delta t} ^{t} \\
& -\frac{2}{\sigma-1} \frac{\lambda^{2}(t)}{\rho(t)} \int_{\delta t}^{t}\left(1+B_{\lambda}(s, t)\right)^{-\frac{\sigma-1}{2}} d\left(\frac{\mu(s)}{\lambda^{2}(s)} \frac{\lambda^{2 p}(s)}{\rho^{p}(s)}\left(1+B_{\lambda}(0, s)\right)^{-\alpha_{4}(p)}\right),
\end{aligned}
$$

where $\alpha_{4}(p):=\frac{n}{2 m} p-\frac{n}{4}+p$. Using Lemma 4.2.1, it holds

$$
\begin{aligned}
\frac{\rho(t)}{\lambda^{2}(t)}(1 & \left.+B_{\lambda}(0, t)\right)^{\frac{n}{2}\left(\frac{1}{m}-\frac{1}{2}\right)+\frac{\sigma-1}{2}+1} \mathbf{R} \\
& \lesssim \underbrace{\frac{\mu(t)}{\lambda^{2}(t)} \frac{\lambda^{2 p}(t)}{\rho^{p}(t)}\left(1+B_{\lambda}(0, t)\right)^{\frac{n}{2}\left(\frac{1}{m}-\frac{1}{2}\right)+\frac{\sigma-1}{2}+1-\alpha_{4}(p)}}_{R_{1}(t)} \\
& +\underbrace{\int_{\delta t}^{t} \frac{\mu(s)}{\lambda^{2}(s)} \frac{\lambda^{2 p}(s)}{\rho^{p}(s)}\left(1+B_{\lambda}(0, s)\right)^{-\alpha_{4}(p)}\left(1+B_{\lambda}(0, t)\right)^{\frac{n}{2}\left(\frac{1}{m}-\frac{1}{2}\right)+\frac{\sigma-1}{2}+1} \frac{d\left(1+B_{\lambda}(0, s)\right)}{1+B_{\lambda}(0, s)}}_{R_{2}(t)} .
\end{aligned}
$$

We begin to estimate $R_{1}=R_{1}(t)$. Using $\alpha_{4}(p)=\alpha_{3}(p)+\frac{n}{2}\left(\frac{1}{m}-\frac{1}{2}\right)$ we have

$$
R_{1}(t):=\frac{\mu(t)}{\lambda^{2}(t)} \frac{\lambda^{2 p}(t)}{\rho^{p}(t)}\left(1+B_{\lambda}(0, t)\right)^{\frac{\sigma+1}{2}-\alpha_{3}(p)} .
$$

If we take the derivative of this function, then it follows

$$
\begin{aligned}
R_{1}^{\prime}(t) & \leq\left[d_{1} \frac{\mu(t)}{\lambda(t) \Lambda(t)}\left(1+B_{\lambda}(0, t)\right)+\left(\frac{\sigma+1}{2}-\alpha_{3}(p)\right) \frac{\Lambda(t)}{\lambda(t)}\right] \frac{\lambda^{2 p}(t)}{\rho^{p}(t)}\left(1+B_{\lambda}(0, t)\right)^{\frac{\sigma-1}{2}-\alpha_{3}(p)} \\
& \leq\left[\frac{d_{1}}{2-\mu_{1}} \frac{\mu(t)}{\lambda(t) \Lambda(t)} \frac{\Lambda^{2}(t)}{\mu(t)}+\left(\frac{\sigma+1}{2}-\alpha_{3}(p)\right) \frac{\Lambda(t)}{\lambda(t)}\right] \frac{\lambda^{2 p}(t)}{\rho^{p}(t)}\left(1+B_{\lambda}(0, t)\right)^{\frac{\sigma-1}{2}-\alpha_{3}(p)} \\
& \leq\left[\frac{d_{1}}{2-\mu_{1}}+\frac{\sigma+1}{2}-\alpha_{3}(p)\right] \frac{\Lambda(t)}{\lambda(t)} \frac{\lambda^{2 p}(t)}{\rho^{p}(t)}\left(1+B_{\lambda}(0, t)\right)^{\frac{\sigma-1}{2}-\alpha_{3}(p)} .
\end{aligned}
$$

We get $R_{1}^{\prime}(t)<0$, that is, $R_{1}=R_{1}(t)$ is decreasing, if $\frac{d_{1}}{2-\mu_{1}}+\frac{\sigma+1}{2}-\alpha_{3}(p)<0, \quad$ this is equivalent to $\quad p>\frac{n}{n+2 m}+\left(\frac{\sigma+1}{2}+\frac{d_{1}}{2-\mu_{1}}\right) \frac{2 m}{n+2 m}$, which can be concluded from $p>p_{1}(m, n, \sigma)$. Let us turn to estimate $R_{2}=R_{2}(t)$. We choose an arbitrarily small positive constant $\varepsilon$ such that it holds

$$
\frac{\mu(\delta t)}{\lambda^{2}(\delta t)} \frac{\lambda^{2 p}(\delta t)}{\rho^{p}(\delta t)}\left(1+B_{\lambda}(0, \delta t)\right)^{-\alpha_{4}(p)}\left(1+B_{\lambda}(0, t)\right)^{\frac{n}{2}\left(\frac{1}{m}-\frac{1}{2}\right)+\frac{\sigma-1}{2}+1} \lesssim\left(1+B_{\lambda}(0, \delta t)\right)^{-\varepsilon} .
$$

Using (3.1.19) we find

$$
\begin{aligned}
& \frac{\mu(\delta t)}{\lambda^{2}(\delta t)} \frac{\lambda^{2 p}(\delta t)}{\rho^{p}(\delta t)}\left(1+B_{\lambda}(0, \delta t)\right)^{-\alpha_{4}(p)+\varepsilon}\left(1+B_{\lambda}(0, t)\right)^{\frac{n}{2}\left(\frac{1}{m}-\frac{1}{2}\right)+\frac{\sigma-1}{2}+1} \\
& \quad \leq \frac{\mu(\delta t)}{\lambda^{2}(\delta t)} \frac{\lambda^{2 p}(\delta t)}{\rho^{p}(\delta t)}\left(1+B_{\lambda}(0, t)\right)^{\frac{n}{2}\left(\frac{1}{m}-\frac{1}{2}\right)+\frac{\sigma-1}{2}+1-\alpha_{4}(p)+\varepsilon}\left(\frac{\Lambda(\delta t)}{\Lambda(t)}\right)^{(2+R)\left(-\alpha_{4}(p)+\varepsilon\right)}:=\mathcal{R}_{2}(t) .
\end{aligned}
$$


Analogously as we did in the proof of Theorem 3.2.1 for deriving the decreasing behavior of the function $\mathcal{B}_{1}=\mathcal{B}_{1}(t)$, which is given in (3.2.15), we may conclude that $\mathcal{R}_{2}^{\prime}(t)<0$ by

$$
\delta \frac{d_{1}}{2-\mu_{1}}+\left(\frac{n}{2}\left(\frac{1}{m}-\frac{1}{2}\right)+\frac{\sigma+1}{2}-\alpha_{4}(p)\right) \nu(\delta)+\alpha_{4}(p) \frac{2+R}{2-\mu_{1}}(\nu(\delta)-\delta)<0,
$$

which is concluded by $p>p_{2}(m, n, \sigma)$ and this gives the uniformly boundedness of $R_{2}=R_{2}(t)$. Therefore, we obtain

$$
\mathbf{R} \lesssim \frac{\lambda^{2}(t)}{\rho(t)}\left(1+B_{\lambda}(0, t)\right)^{-\frac{n}{2}\left(\frac{1}{m}-\frac{1}{2}\right)-\frac{\sigma-1}{2}-1} .
$$

Consequently, the estimates (4.2.11) and (4.2.12) imply

$$
\left\||D|^{\sigma-1} u_{t}^{\mathrm{nl}}(t, \cdot)\right\|_{L^{2}} \lesssim\|u\|_{X(t)}^{p} \frac{\lambda^{2}(t)}{\rho(t)}\left(1+B_{\lambda}(0, t)\right)^{-\frac{n}{2}\left(\frac{1}{m}-\frac{1}{2}\right)-\frac{\sigma-1}{2}-1} .
$$

Similarly, one can also prove

$$
\left\|u_{t}^{\mathrm{nl}}(t, \cdot)\right\|_{L^{2}} \lesssim\|u\|_{X(t)}^{p} \frac{\lambda^{2}(t)}{\rho(t)}\left(1+B_{\lambda}(0, t)\right)^{-\frac{n}{2}\left(\frac{1}{m}-\frac{1}{2}\right)-1} .
$$

Now we want to prove (4.1.7) in the Case 1. By the definition of the solution space $X(t)$ and its norm we have

$$
\begin{aligned}
\left\|\left|u_{t}(s, \cdot)\right|^{p}-\left|v_{t}(s, \cdot)\right|^{p}\right\|_{L^{2}} & \lesssim \frac{\lambda^{2 p}(s)}{\rho^{p}(s)}\left(1+B_{\lambda}(0, s)\right)^{-\frac{n}{2 m} p+\frac{n}{4}-p}\|u-v\|_{X(s)}\left(\|u\|_{X(s)}^{p-1}+\|v\|_{X(s)}^{p-1}\right), \\
\left\|\left|u_{t}(s, \cdot)\right|^{p}-\left|v_{t}(s, \cdot)\right|^{p}\right\|_{L^{m}} & \lesssim \frac{\lambda^{2 p}(s)}{\rho^{p}(s)}\left(1+B_{\lambda}(0, s)\right)^{-\frac{n}{2 m} p+\frac{n}{2 m}-p}\|u-v\|_{X(s)}\left(\|u\|_{X(s)}^{p-1}+\|v\|_{X(s)}^{p-1}\right) .
\end{aligned}
$$

On the other hand, to estimate the norm $\left\|\left|u_{t}(s, \cdot)\right|^{p}-\left|v_{t}(s, \cdot)\right|^{p}\right\|_{\dot{H}^{\sigma-1}}$ we use the integral representation

$$
\begin{aligned}
\left|u_{t}(s, x)\right|^{p}-\left|v_{t}(s, x)\right|^{p} & =\int_{0}^{1} \frac{d}{d w}\left|w u_{t}(s, x)+(1-w) v_{t}(s, x)\right|^{p} d w \\
& =p \int_{0}^{1}\left(u_{t}(s, x)-v_{t}(s, x)\right) G\left(w u_{t}(s, x)+(1-w) v_{t}(s, x)\right) d w,
\end{aligned}
$$

where $G(u)=u_{t}\left|u_{t}\right|^{p-2}$. Then, we have

$$
\begin{aligned}
\|\left|u_{t}(s, \cdot)\right|^{p} & -\left|v_{t}(s, \cdot)\right|^{p} \|_{\dot{H}^{\sigma-1}} \\
& \lesssim \int_{0}^{1}\left\||D|^{\sigma-1}\left(\left(u_{t}(s, \cdot)-v_{t}(s, \cdot)\right) G\left(w u_{t}(s, \cdot)+(1-w) v_{t}(s, \cdot)\right)\right)\right\|_{L^{2}} d w .
\end{aligned}
$$

Now we apply Corollary A.7.12 in order to estimate the last integral. Then, we get

$$
\begin{aligned}
\|\left|u_{t}(s, \cdot)\right|^{p}- & \left|v_{t}(s, \cdot)\right|^{p} \|_{\dot{H}^{\sigma-1}} \\
\lesssim & \int_{0}^{1}\left\|u_{t}(s, \cdot)-v_{t}(s, \cdot)\right\|_{\dot{H}^{\sigma-1}}\left\|G\left(w u_{t}(s, \cdot)+(1-w) v_{t}(s, \cdot)\right)\right\|_{L^{\infty}} d w \\
& +\int_{0}^{1}\left\|u_{t}(s, \cdot)-v_{t}(s, \cdot)\right\|_{L^{\infty}}\left\|G\left(w u_{t}(s, \cdot)+(1-w) v_{t}(s, \cdot)\right)\right\|_{\dot{H}^{\sigma-1}} d w .
\end{aligned}
$$

Employing the fractional powers rules from Corollary A.7.10 and Proposition A.7.13, respectively, we obtain

$$
\begin{aligned}
& \left\|G\left(w u_{t}(s, \cdot)+(1-w) v_{t}(s, \cdot)\right)\right\|_{\dot{H}^{\sigma-1}} \\
& \lesssim\left\|w u_{t}(s, \cdot)+(1-w) v_{t}(s, \cdot)\right\|_{\dot{H}^{\sigma-1}}\left\|w u_{t}(s, \cdot)+(1-w) v_{t}(s, \cdot)\right\|_{L^{\infty}}^{p-2} \\
& \lesssim\left\|w u_{t}(s, \cdot)+(1-w) v_{t}(s, \cdot)\right\|_{\dot{H}^{\sigma-1}} \\
& \quad \times\left(\left\|w u_{t}(s, \cdot)+(1-w) v_{t}(s, \cdot)\right\|_{\dot{H}^{\sigma^{*}}}+\left\|w u_{t}(s, \cdot)+(1-w) v_{t}(s, \cdot)\right\|_{\dot{H}^{\sigma-1}}\right)^{p-2} \\
& \quad \lesssim\left\|w u_{t}(s, \cdot)+(1-w) v_{t}(s, \cdot)\right\|_{\dot{H}^{\sigma-1}}\left\|w u_{t}(s, \cdot)+(1-w) v_{t}(s, \cdot)\right\|_{\dot{H}^{\sigma^{*}}}^{p-2} \\
& \quad+\left\|w u_{t}(s, \cdot)+(1-w) v_{t}(s, \cdot)\right\|_{\dot{H}^{\sigma-1}}^{p-1} .
\end{aligned}
$$


To derive a suitable estimate for the last norm we apply the following estimates:

$$
\begin{aligned}
\left\|w u_{t}(s, \cdot)+(1-w) v_{t}(s, \cdot)\right\|_{\dot{H}^{\sigma-1}} & \\
& \lesssim \frac{\lambda^{2}(s)}{\rho(s)}\left(1+B_{\lambda}(0, s)\right)^{-\frac{n}{2 m}+\frac{n}{4}-\frac{\sigma-1}{2}-1}\|w u+(1-w) v\|_{X(s)}, \\
\left\|w u_{t}(s, \cdot)+(1-w) v_{t}(s, \cdot)\right\|_{\dot{H}^{\sigma^{*}}}^{p-2} & \\
& \lesssim \frac{\lambda^{2(p-2)}(s)}{\rho^{p-2}(s)}\left(1+B_{\lambda}(0, s)\right)^{-\frac{n}{2 m}(p-2)+\frac{n}{4}(p-2)-\frac{\sigma^{*}}{2}(p-2)-(p-2)}\|w u+(1-w) v\|_{X(s)}^{p-2} .
\end{aligned}
$$

Therefore, we arrive at the estimates

$$
\begin{aligned}
& \left\|w u_{t}(s, \cdot)+(1-w) v_{t}(s, \cdot)\right\|_{\dot{H}^{\sigma-1}}\left\|w u_{t}(s, \cdot)+(1-w) v_{t}(s, \cdot)\right\|_{\dot{H}^{\sigma^{*}}}^{p-2} \\
& \quad \lesssim \frac{\lambda^{2(p-1)}(s)}{\rho^{p-1}(s)}\left(1+B_{\lambda}(0, s)\right)^{-\frac{n}{2 m}(p-1)+\frac{n}{4}(p-1)-\frac{\sigma-1}{2}-\frac{\sigma^{*}}{2}(p-2)-(p-1)}\|w u+(1-w) v\|_{X(s)}^{p-1}, \\
& \left\|w u_{t}(s, \cdot)+(1-w) v_{t}(s, \cdot)\right\|_{\dot{H}^{\sigma-1}}^{p-1} \\
& \quad \lesssim \frac{\lambda^{2(p-1)}(s)}{\rho^{p-1}(s)}\left(1+B_{\lambda}(0, s)\right)^{-\frac{n}{2 m}(p-1)+\frac{n}{4}(p-1)-\frac{\sigma-1}{2}(p-1)-(p-1)}\|w u+(1-w) v\|_{X(s)}^{p-1},
\end{aligned}
$$

where due to $\sigma^{*}<\frac{n}{2}$ it holds

$-\frac{n}{2 m}(p-1)+\frac{n}{4}(p-1)-\frac{\sigma-1}{2}-\frac{\sigma^{*}}{2}(p-2)-(p-1)>-\frac{n}{2 m}(p-1)+\frac{n}{4}(p-1)-\frac{\sigma-1}{2}(p-1)-(p-1)$.

This implies

$$
\begin{aligned}
& \left\|G\left(w u_{t}(s, \cdot)+(1-w) v_{t}(s, \cdot)\right)\right\|_{\dot{H}^{\sigma-1}} \\
& \quad \lesssim \frac{\lambda^{2(p-1)}(s)}{\rho^{p-1}(s)}\left(1+B_{\lambda}(0, s)\right)^{-\frac{n}{2 m}(p-1)+\frac{n}{4}(p-1)-\frac{\sigma-1}{2}-\frac{\sigma^{*}}{2}(p-2)-(p-1)}\|w u+(1-w) v\|_{X(s)}^{p-1} .
\end{aligned}
$$

On the other hand, applying Proposition A.7.13 with $\sigma^{*}<\frac{n}{2}$ we have

$$
\begin{aligned}
& \left\|G\left(w u_{t}(s, \cdot)+(1-w) v_{t}(s, \cdot)\right)\right\|_{L^{\infty}} \\
& \quad \lesssim\left\|w u_{t}(s, \cdot)+(1-w) v_{t}(s, \cdot)\right\|_{\dot{H}^{\sigma^{*}}}^{p-1}+\left\|w u_{t}(s, \cdot)+(1-w) v_{t}(s, \cdot)\right\|_{\dot{H}^{\sigma-1}}^{p-1} \\
& \quad \lesssim \frac{\lambda^{2(p-1)}(s)}{\rho^{p-1}(s)}\left(1+B_{\lambda}(0, s)\right)^{-\frac{n}{2 m}(p-1)+\frac{n}{4}(p-1)-\frac{\sigma^{*}}{2}(p-1)-(p-1)}\|w u+(1-w) v\|_{X(s)}^{p-1}
\end{aligned}
$$

Consequently, we arrive at the estimates

$$
\begin{aligned}
& \left\|u_{t}(s, \cdot)-v_{t}(s, \cdot)\right\|_{\dot{H}^{\sigma-1}}\left\|G\left(w u_{t}(s, \cdot)+(1-w) v_{t}(s, \cdot)\right)\right\|_{L^{\infty}} \\
& \quad \lesssim \frac{\lambda^{2 p}(s)}{\rho^{p}(s)}\left(1+B_{\lambda}(0, s)\right)^{-\frac{n}{2 m} p+\frac{n}{4} p-\frac{\sigma-1}{2}-\frac{\sigma^{*}}{2}(p-1)-(p-1)}\|u-v\|_{X(s)}\|w u+(1-w) v\|_{X(s)}^{p-1}, \\
& \left\|u_{t}(s, \cdot)-v_{t}(s, \cdot)\right\|_{L^{\infty}}\left\|G\left(w u_{t}(s, \cdot)+(1-w) v_{t}(s, \cdot)\right)\right\|_{\dot{H}^{\sigma-1}} \\
& \quad \lesssim \frac{\lambda^{2 p}(s)}{\rho^{p}(s)}\left(1+B_{\lambda}(0, s)\right)^{-\frac{n}{2 m} p+\frac{n}{4} p-\frac{\sigma-1}{2}-\frac{\sigma^{*}}{2}(p-1)-(p-1)}\|u-v\|_{X(s)}\|w u+(1-w) v\|_{X(s)}^{p-1} .
\end{aligned}
$$

Thus, we obtain

$$
\begin{aligned}
& \left\|\left|u_{t}(s, \cdot)\right|^{p}-\left|v_{t}(s, \cdot)\right|^{p}\right\|_{\dot{H}^{\sigma-1}} \\
& \quad \lesssim \frac{\lambda^{2 p}(s)}{\rho^{p}(s)}\left(1+B_{\lambda}(0, s)\right)^{-\frac{n}{2 m} p+\frac{n}{4} p-\frac{\sigma-1}{2}-\frac{\sigma^{*}}{2}(p-1)-(p-1)} \int_{0}^{1}\|u-v\|_{X(s)}\|w u+(1-w) v\|_{X(s)}^{p-1} d w \\
& \quad \lesssim \frac{\lambda^{2 p}(s)}{\rho^{p}(s)}\left(1+B_{\lambda}(0, s)\right)^{-\frac{n}{2 m} p+\frac{n}{4} p-\frac{\sigma-1}{2}-\frac{\sigma^{*}}{2}(p-1)-(p-1)} \int_{0}^{1}\|u-v\|_{X(s)}\left(\|u\|_{X(s)}^{p-1}+\|v\|_{X(s)}^{p-1}\right) d w \\
& \lesssim \frac{\lambda^{2 p}(s)}{\rho^{p}(s)}\left(1+B_{\lambda}(0, s)\right)^{-\frac{n}{2 m} p+\frac{n}{4} p-\frac{\sigma-1}{2}-\frac{\sigma^{*}}{2}(p-1)-(p-1)}\|u-v\|_{X(s)}\left(\|u\|_{X(s)}^{p-1}+\|v\|_{X(s)}^{p-1}\right) .
\end{aligned}
$$


Taking into consideration (4.2.4), $\sigma^{*}=\frac{n}{2}-\varepsilon<\frac{n}{2}$ with $\varepsilon>0$ and $\|u\|_{X(s)} \leq\|u\|_{X(t)}$ with $0 \leq s \leq t$ we find

$$
\begin{gathered}
\left\||D|^{\sigma} \int_{0}^{t} K_{1}(t, s, x) *_{(x)}\left(\left|u_{t}(s, x)\right|^{p}-\left|v_{t}(s, x)\right|^{p}\right) d s\right\|_{L^{2}} \lesssim\left(\|u\|_{X(t)}^{p-1}+\|v\|_{X(t)}^{p-1}\right)\|u-v\|_{X(t)} \\
\times\left(\int_{0}^{\delta t} \frac{\Lambda(s)}{\lambda(s)} \frac{\lambda^{2 p}(s)}{\rho^{p}(s)}\left(1+B_{\lambda}(s, t)\right)^{-\frac{n}{2}\left(\frac{1}{m}-\frac{1}{2}\right)-\frac{\sigma}{2}}\left(1+B_{\lambda}(0, s)\right)^{-\frac{n}{2 m} p+\frac{n}{2 m}-p} d s\right. \\
\left.\quad+\int_{\delta t}^{t} \frac{\Lambda(s)}{\lambda(s)} \frac{\lambda^{2 p}(s)}{\rho^{p}(s)}\left(1+B_{\lambda}(s, t)\right)^{-\frac{\sigma}{2}}\left(1+B_{\lambda}(0, s)\right)^{-\frac{n}{2 m} p+\frac{n}{4}-p} d s\right) .
\end{gathered}
$$

In order to conclude the proof of the estimate (4.1.7) we can follow the same steps of the proof of (4.1.6). Hence, we arrive at the estimate

$$
\left\||D|^{\sigma}(N u-N v)(t, \cdot)\right\|_{L^{2}} \lesssim\left(1+B_{\lambda}(0, t)\right)^{-\frac{n}{2}\left(\frac{1}{m}-\frac{1}{2}\right)-\frac{\sigma}{2}}\|u-v\|_{X(t)}\left(\|u\|_{X(t)}^{p-1}+\|v\|_{X(t)}^{p-1}\right) .
$$

In the same way, with no other requirements to the admissible exponents $p$ one can derive

$$
\begin{aligned}
&\|(N u-N v)(t, \cdot)\|_{L^{2}} \lesssim\left(1+B_{\lambda}(0, s)\right)^{-\frac{n}{2}\left(\frac{1}{m}-\frac{1}{2}\right)}\|u-v\|_{X(t)}\left(\|u\|_{X(t)}^{p-1}+\|v\|_{X(t)}^{p-1}\right), \\
&\left\|\partial_{t}(N u-N v)(t, \cdot)\right\|_{L^{2}} \lesssim\left(1+B_{\lambda}(0, s)\right)^{-\frac{n}{2}\left(\frac{1}{m}-\frac{1}{2}\right)-1}\|u-v\|_{X(t)}\left(\|u\|_{X(t)}^{p-1}+\|v\|_{X(t)}^{p-1}\right), \\
&\left\||D|^{\sigma-1} \partial_{t}(N u-N v)(t, \cdot)\right\|_{L^{2}} \lesssim\left(1+B_{\lambda}(0, s)\right)^{-\frac{n}{2}\left(\frac{1}{m}-\frac{1}{2}\right)-\frac{\sigma-1}{2}-1}\|u-v\|_{X(t)}\left(\|u\|_{X(t)}^{p-1}+\|v\|_{X(t)}^{p-1}\right) .
\end{aligned}
$$

Thus, from the definition of the norm of the solution space $X(t)$ we may conclude the proof of (4.1.7) in the Case 1.

Case 2: Let us assume that the second component of the functions $\Phi_{1, m}=\Phi_{1, m}(s, t), \Phi_{1}=$ $\Phi_{1}(s, t)$ and $\Phi_{2, m}=\Phi_{2, m}(s, t), \Phi_{2}=\Phi_{2}(s, t)$ is dominant, that is,

$$
\begin{array}{ll}
\Phi_{1, m}(s, t)=\lambda(t) F(\Lambda(t))\left(F^{2}(\Lambda(t))\right)^{-\frac{n}{2}\left(\frac{1}{m}-\frac{1}{2}\right)-1}, & \Phi_{1}(s, t)=\lambda(t) F(\Lambda(t))\left(F^{2}(\Lambda(t))\right)^{-1}, \\
\Phi_{2, m}(s, t)=\lambda(t) F(\Lambda(t))\left(F^{2}(\Lambda(t))\right)^{-\frac{n}{2}\left(\frac{1}{m}-\frac{1}{2}\right)-\frac{\sigma-1}{2}-1}, & \Phi_{2}(s, t)=\lambda(t) F(\Lambda(t))\left(F^{2}(\Lambda(t))\right)^{-\frac{\sigma-1}{2}-1}
\end{array}
$$

for all $t \geq s \geq 0$.

In order to control the norm $\left\||D|^{\sigma} u^{\mathrm{nl}}(t, \cdot)\right\|_{L^{2}}$ we use the $\left(L^{m} \cap L^{2}\right)-L^{2}$ estimates if $s \in[0, \delta t]$ and the $L^{2}-L^{2}$ estimates if $s \in[\delta t, t]$ from Theorem 3.1.1. Therefore, we have the following estimate:

$$
\begin{aligned}
\left\||D|^{\sigma} u^{\mathrm{nl}}(t, \cdot)\right\|_{L^{2}} \lesssim & \int_{0}^{\delta t} \frac{\Lambda(s)}{\lambda(s)}\left(1+B_{\lambda}(s, t)\right)^{-\frac{n}{2}\left(\frac{1}{m}-\frac{1}{2}\right)-\frac{\sigma}{2}}\left\|\left|u_{t}(s, \cdot)\right|^{p}\right\|_{L^{m} \cap L^{2} \cap \dot{H}^{\sigma-1}} d s \\
& +\int_{\delta t}^{t} \frac{\Lambda(s)}{\lambda(s)}\left(1+B_{\lambda}(s, t)\right)^{-\frac{\sigma}{2}}\left\|\left|u_{t}(s, \cdot)\right|^{p}\right\|_{L^{2} \cap \dot{H}^{\sigma-1}} d s .
\end{aligned}
$$

Now we have to estimate the following norms:

$$
\left\|\left|u_{t}(s, \cdot)\right|^{p}\right\|_{L^{m}}, \quad\left\|\left|u_{t}(s, \cdot)\right|^{p}\right\|_{L^{2}} \quad \text { and } \quad\left\|\left|u_{t}(s, \cdot)\right|^{p}\right\|_{\dot{H}^{\sigma-1}} \cdot
$$

For the first two norms, by using the fractional Gagliardo-Nirenberg inequality one can get for $p \geq \frac{2}{m}$ the estimates

$$
\begin{aligned}
\left\|u_{t}(s, \cdot)\right\|_{L^{m p}}^{p} & \lesssim\left\||D|^{\sigma} u_{t}(s, \cdot)\right\|_{L^{2}}^{p \theta_{\sigma}(m p)}\left\|u_{t}(s, \cdot)\right\|_{L^{2}}^{p\left(1-\theta_{\sigma}(m p)\right)} \\
& \lesssim \lambda^{p}(s) F^{p}(\Lambda(s))\left(F^{2}(\Lambda(s))\right)^{-\frac{n}{2 m} p+\frac{n}{2 m}-p}\|u\|_{X(s)}^{p}, \\
\left\|u_{t}(s, \cdot)\right\|_{L^{2 p}}^{p} & \lesssim\left\||D|^{\sigma} u_{t}(s, \cdot)\right\|_{L^{2}}^{p \theta_{\sigma}(2 p)}\left\|u_{t}(s, \cdot)\right\|_{L^{2}}^{p\left(1-\theta_{\sigma}(2 p)\right)} \\
& \lesssim \lambda^{p}(s) F^{p}(\Lambda(s))\left(F^{2}(\Lambda(s))\right)^{-\frac{n}{2 m} p+\frac{n}{4}-p}\|u\|_{X(s)}^{p} .
\end{aligned}
$$


Now, we turn to estimate the norm $\left\|\left|u_{t}(s, \cdot)\right|^{p}\right\|_{\dot{H}^{\sigma-1}}$ by using results on fractional powers for $\sigma-1>\frac{n}{2}$. Firstly, let us begin to estimate the norm $\left\|\left|u_{t}(s, \cdot)\right|^{p}\right\|_{\dot{H}^{\sigma-1}}$. We apply Corollary A.7.10 with $\sigma-1 \in\left(\frac{n}{2}, p\right)$ and Proposition A.7.13 with a suitable $\sigma^{*}<\frac{n}{2}$, from Section A.7 in the Appendix, respectively. Therefore, we obtain

$$
\begin{aligned}
\left\|\left|u_{t}(s, \cdot)\right|^{p}\right\|_{\dot{H}^{\sigma-1}} & \lesssim\left\|u_{t}(s, \cdot)\right\|_{\dot{H}^{\sigma-1}}\left\|u_{t}(s, \cdot)\right\|_{L^{\infty}}^{p-1} \\
& \lesssim\left\|u_{t}(s, \cdot)\right\|_{\dot{H}^{\sigma-1}}\left\|u_{t}(s, \cdot)\right\|_{\dot{H}^{\sigma^{*}}}^{p-1}+\left\|u_{t}(s, \cdot)\right\|_{\dot{H}^{\sigma-1}}^{p} .
\end{aligned}
$$

Applying the fractional Gagliardo-Nirenberg inequality and using the definition of the norm of the solution space $X(t)$ we derive

$$
\begin{aligned}
\left\|u_{t}(s, \cdot)\right\|_{\dot{H}^{\sigma-1}} & \lesssim\left\|u_{t}(s, \cdot)\right\|_{\dot{H}^{\sigma}}^{\theta_{\sigma-1, \sigma}}\|u(s, \cdot)\|_{L^{2}}^{1-\theta_{\sigma-1, \sigma}} \\
& \lesssim \lambda^{p}(s) F^{p}(\Lambda(s))\left(F^{2}(\Lambda(s))\right)^{-\frac{n}{2}\left(\frac{1}{m}-\frac{1}{2}\right)-\frac{\sigma-1}{2}-1}\|u\|_{X(s)}, \\
\left\|u_{t}(s, \cdot)\right\|_{\dot{H}^{\sigma^{*}}} & \lesssim\left\|u_{t}(s, \cdot)\right\|_{\dot{H}^{\sigma}}^{\theta_{\sigma^{*}, \sigma}}\|u(s, \cdot)\|_{L^{2}}^{1-\theta_{\sigma^{*}, \sigma}} \\
& \lesssim \lambda^{p}(s) F^{p}(\Lambda(s))\left(F^{2}(\Lambda(s))\right)^{-\frac{n}{2}\left(\frac{1}{m}-\frac{1}{2}\right)-\frac{\sigma^{*}}{2}-1}\|u\|_{X(s)},
\end{aligned}
$$

where $\theta_{\sigma-1, \sigma}=1-\frac{1}{\sigma}$ and $\theta_{\sigma^{*}, \sigma}=\frac{\sigma^{*}}{\sigma}$. Hence, by choosing the parameter $\sigma^{*}=\frac{n}{2}-\varepsilon<\frac{n}{2}$ with a sufficiently small positive $\varepsilon$ we have

$$
\left\|\left|u_{t}(s, \cdot)\right|^{p}\right\|_{\dot{H}^{\sigma-1}} \lesssim \lambda^{p}(s) F^{p}(\Lambda(s))\left(F^{2}(\Lambda(s))\right)^{-\frac{n}{2}\left(\frac{1}{m}-\frac{1}{2}\right) p-\frac{\sigma-1}{2}-\frac{\sigma^{*}}{2}(p-1)-p}\|u\|_{X(s)}^{p} .
$$

For this reason, in order to get the desired estimates after taking into consideration (4.2.13), $(4.2 .14),(4.2 .15), \sigma^{*}<\frac{n}{2}$ and

$$
-\frac{n}{2 m} p+\frac{n}{2 m}-p>-\frac{n}{2 m} p+\frac{n}{4}-p>-\frac{n}{2}\left(\frac{1}{m}-\frac{1}{2}\right) p-\frac{\sigma-1}{2}-\frac{\sigma^{*}}{2}(p-1)-p,
$$

where we take account of $\|u\|_{X(s)} \leq\|u\|_{X(t)}$ for $0 \leq s \leq t$, we arrive at the estimate

$$
\begin{aligned}
& \left\||D|^{\sigma} u^{\mathrm{nl}}(t, \cdot)\right\|_{L^{2}} \\
& \lesssim\|u\|_{X(t)}^{p} \underbrace{\int_{0}^{\delta t} \frac{\Lambda(s)}{\lambda(s)} \lambda^{p}(s) F^{p}(\Lambda(s))\left(1+B_{\lambda}(s, t)\right)^{-\frac{n}{2}\left(\frac{1}{m}-\frac{1}{2}\right)-\frac{\sigma}{2}}\left(F^{2}(\Lambda(s))\right)^{-\frac{n}{2 m} p+\frac{n}{2 m}-p} d s}_{\mathbf{S}} \\
& \quad+\|u\|_{X(t)}^{p} \underbrace{\int_{\delta t}^{t} \frac{\Lambda(s)}{\lambda(s)} \lambda^{p}(s) F^{p}(\Lambda(s))\left(1+B_{\lambda}(s, t)\right)^{-\frac{\sigma}{2}}\left(F^{2}(\Lambda(s))\right)^{-\frac{n}{2 m} p+\frac{n}{4}-p} d s}_{\mathbf{T}} .
\end{aligned}
$$

Let us consider the integral $\mathbf{S}$. Using the property (3.1.18), we have the estimate

$$
\mathbf{S} \lesssim\left(1+B_{\lambda}(0, t)\right)^{-\frac{n}{2}\left(\frac{1}{m}-\frac{1}{2}\right)-\frac{\sigma}{2}} \underbrace{\int_{0}^{\delta t} \Lambda(s) \lambda^{p-1}(s) F(\Lambda(s))^{-\frac{n}{m} p+\frac{n}{m}-p} d s}_{S_{1}(t)} .
$$

Then, introducing $\alpha_{5}(p):=\frac{n}{m} p-\frac{n}{m}+p$ it follows

$$
\begin{aligned}
S_{1}(t)= & \int_{0}^{\delta t} \lambda^{p}(s) \Lambda(s) \Xi^{2}(s) F(\Lambda(s))^{-\alpha_{5}(p)-2} d(F(\Lambda(s))) \\
= & \left.\frac{-1}{\alpha_{5}(p)+1} \lambda^{p}(s) \Lambda(s) \Xi^{2}(s) F(\Lambda(s))^{-\alpha_{5}(p)-1}\right|_{0} ^{\delta t} \\
& +\frac{1}{\alpha_{5}(p)+1} \int_{0}^{\delta t} F(\Lambda(s))^{-\alpha_{5}(p)-1} d\left(\lambda^{p}(s) \Lambda(s) \Xi^{2}(s)\right),
\end{aligned}
$$


where from condition (A5) we used $F^{\prime}(\Lambda(s)) \lambda(s)=\frac{F^{2}(\Lambda(s))}{\lambda(s) \Xi^{2}(s)}$. Since $\alpha_{5}(p)+1>0$, then, it holds

$$
\begin{aligned}
S_{1}(t) \leq & -\left.\frac{1}{\alpha_{5}(p)+1} \lambda^{p}(s) \Lambda(s) \Xi^{2}(s) F(\Lambda(s))^{-\alpha_{5}(p)-1}\right|_{0} ^{\delta t} \\
& +\frac{r}{\alpha_{5}(p)+1} \int_{0}^{\delta t} F(\Lambda(s))^{-\alpha_{5}(p)-1} \lambda^{p+1}(s) \Xi^{2}(s) d s \\
= & -\left.\frac{1}{\alpha_{5}(p)+1} \lambda^{p}(s) \Lambda(s) \Xi^{2}(s) F(\Lambda(s))^{-\alpha_{5}(p)-1}\right|_{0} ^{\delta t} \\
& +\frac{r}{\alpha_{5}(p)+1} \int_{0}^{\delta t} F(\Lambda(s))^{-\alpha_{5}(p)} \frac{F(\Lambda(s))}{F^{\prime}(\Lambda(s))} \lambda^{p-1}(s) d s \\
\leq & -\left.\frac{1}{\alpha_{5}(p)+1} \lambda^{p}(s) \Lambda(s) \Xi^{2}(s) F(\Lambda(s))^{-\alpha_{5}(p)-1}\right|_{0} ^{\delta t} \\
& +\frac{r}{\alpha_{5}(p)+1} \underbrace{\int_{0}^{\delta t} \Lambda(s) \lambda^{p-1}(s) F(\Lambda(s))^{-\alpha_{5}(p)} d s}_{S_{1}(t)}
\end{aligned}
$$

where firstly we used condition (D3). Then, from conditions (A5) and (D4) we used $\Xi^{2}(t)=$ $\frac{F^{2}(\Lambda(t))}{\lambda^{2}(t) F^{\prime}(\Lambda(t))}$ and $\frac{F(\Lambda(t))}{F^{\prime}(\Lambda(t))} \leq \Lambda(t)$, respectively. Thus, from the last estimate of $S_{1}=S_{1}(t)$ we obtain

$$
\left(1-\frac{r}{\alpha_{5}(p)+1}\right) S_{1}(t) \lesssim \lambda^{p}(0) \Lambda(0) \Xi^{2}(0) F(\Lambda(0))^{-\alpha_{5}(p)-1}-\lambda^{p}(\delta t) \Lambda(\delta t) \Xi^{2}(\delta t) F(\Lambda(\delta t))^{-\alpha_{5}(p)-1} .
$$

This implies that with

$$
1-\frac{r}{\alpha_{5}(p)+1}>0, \quad \text { that is, } \quad p>\frac{m r+n-m}{n+m}
$$

gives the desired estimate

$$
\mathbf{S} \lesssim\left(1+B_{\lambda}(0, t)\right)^{-\frac{n}{2}\left(\frac{1}{m}-\frac{1}{2}\right)-\frac{\sigma}{2}} .
$$

Now let us consider the integral $\mathbf{T}$. We have

$$
\mathbf{T} \leq \underbrace{\int_{\delta t}^{t} \frac{\Lambda(s)}{\lambda(s)} \lambda^{p}(s) F^{p}(\Lambda(s))\left(F^{2}(\Lambda(s))\right)^{-\frac{n}{2 m} p+\frac{n}{4}-p} d s}_{T_{1}(t)} .
$$

Then, proceeding in the same way as we did to estimate the integral $S_{1}=S_{1}(t)$ and introducing $\alpha_{6}(p)=\frac{n}{m} p-\frac{n}{2}+p$ we get

$$
\begin{aligned}
T_{1}(t)= & \int_{\delta t}^{t} \lambda^{p}(s) \Lambda(s) \Xi^{2}(s) F(\Lambda(s))^{-\alpha_{6}(p)-2} d(F(\Lambda(s))) \\
= & \left.\frac{-1}{\alpha_{6}(p)+1} \lambda^{p}(s) \Lambda(s) \Xi^{2}(s) F(\Lambda(s))^{-\alpha_{6}(p)-1}\right|_{\delta t} ^{t} \\
& +\frac{1}{\alpha_{6}(p)+1} \int_{\delta t}^{t} F(\Lambda(s))^{-\alpha_{6}(p)-1} d\left(\lambda^{p}(s) \Lambda(s) \Xi^{2}(s)\right) \\
\leq & \left.\frac{-1}{\alpha_{6}(p)+1} \lambda^{p}(s) \Lambda(s) \Xi^{2}(s) F(\Lambda(s))^{-\alpha_{6}(p)-1}\right|_{\delta t} ^{t} \\
& +\frac{r}{\alpha_{6}(p)+1} \underbrace{\int_{\delta t}^{t} \Lambda(s) \lambda^{p-1}(s) F(\Lambda(s))^{-\alpha_{6}(p)} d s}_{T_{1}(t)}
\end{aligned}
$$


Then, we find

$$
\left(1-\frac{r}{\alpha_{6}(p)+1}\right) T_{1}(t) \lesssim \lambda^{p}(\delta t) \Lambda(\delta t) \Xi^{2}(\delta t) F(\Lambda(\delta t))^{-\alpha_{6}(p)-1}-\lambda^{p}(t) \Lambda(t) \Xi^{2}(t) F(\Lambda(t))^{-\alpha_{6}(p)-1} .
$$

We know that by using $p>p_{1}(m, n, \sigma)$ we have $1-\frac{r}{\alpha_{6}(p)+1}>0$. In order to get the desired estimate we have

$$
\begin{aligned}
\left(1+B_{\lambda}\right. & (0, t))^{\frac{n}{2}\left(\frac{1}{m}-\frac{1}{2}\right)+\frac{\sigma}{2}} T_{1}(t) \\
& \lesssim \lambda^{p}(\delta t) \Lambda(\delta t) \Xi^{2}(\delta t) F(\Lambda(\delta t))^{-\alpha_{6}(p)-1}\left(1+B_{\lambda}(0, t)\right)^{\frac{n}{2}\left(\frac{1}{m}-\frac{1}{2}\right)+\frac{\sigma}{2}}:=\mathcal{T}_{1}(t) .
\end{aligned}
$$

We will show that the function $\mathcal{T}_{1}=\mathcal{T}_{1}(t)$ is uniformly bounded. Indeed, we get

$$
\begin{aligned}
\mathcal{T}_{1}^{\prime}(t) \leq & {\left[r \delta \lambda^{p+1}(\delta t) \Xi^{2}(\delta t)-\delta\left(\alpha_{6}(p)+1\right) \lambda^{p+1}(\delta t) \Xi^{2}(\delta t) \Lambda(\delta t) \frac{F^{\prime}(\Lambda(\delta t))}{F(\Lambda(\delta t))}\right.} \\
& \left.+\left(\frac{n}{2}\left(\frac{1}{m}-\frac{1}{2}\right)+\frac{\sigma}{2}\right) \lambda^{p+1}(\delta t) \Xi^{2}(\delta t) \frac{\Lambda(\delta t)}{\lambda(\delta t)} \frac{\lambda(t) \Lambda(t)}{\mu(t)} \frac{1}{1+B_{\lambda}(0, t)}\right] \\
& \times F(\Lambda(\delta t))^{-\alpha_{6}(p)-1}\left(1+B_{\lambda}(0, t)\right)^{\frac{n}{2}\left(\frac{1}{m}-\frac{1}{2}\right)+\frac{\sigma}{2}} \\
\leq & {\left[r \delta-\delta\left(\alpha_{6}(p)+1\right) \frac{\Lambda(\delta t)}{\lambda(\delta t)} \frac{F(\Lambda(\delta t))}{\lambda(\delta t) \Xi^{2}(\delta t)}+\left(\frac{n}{2}\left(\frac{1}{m}-\frac{1}{2}\right)+\frac{\sigma}{2}\right)(2+R) \frac{\Lambda(\delta t)}{\lambda(\delta t)} \frac{\lambda(t)}{\Lambda(t)}\right] } \\
& \times \lambda^{p+1}(\delta t) \Xi^{2}(\delta t) F(\Lambda(\delta t))^{-\alpha_{6}(p)-1}\left(1+B_{\lambda}(0, t)\right)^{\frac{n}{2}\left(\frac{1}{m}-\frac{1}{2}\right)+\frac{\sigma}{2}} \\
\leq & {\left[r-\left(\alpha_{6}(p)+1\right) \vartheta(\delta, t)+\left(\frac{n}{2}\left(\frac{1}{m}-\frac{1}{2}\right)+\frac{\sigma}{2}\right)(2+R) \frac{\nu(\delta, t)}{\delta}\right] } \\
& \times \delta \lambda^{p+1}(\delta t) \Xi^{2}(\delta t) F(\Lambda(\delta t))^{-\alpha_{6}(p)-1}\left(1+B_{\lambda}(0, t)\right)^{\frac{n}{2}\left(\frac{1}{m}-\frac{1}{2}\right)+\frac{\sigma}{2}} .
\end{aligned}
$$

Then, for large time $t$ after using $p>p_{2}(m, n, \sigma)$ we have

$$
r-\left(\alpha_{6}(p)+1\right) \vartheta(\delta)+\left(\frac{n}{2}\left(\frac{1}{m}-\frac{1}{2}\right)+\frac{\sigma}{2}\right)(2+R) \frac{\nu(\delta)}{\delta}<0 .
$$

Therefore, we arrive at the estimate

$$
T_{1}(t) \lesssim\left(1+B_{\lambda}(0, t)\right)^{-\frac{n}{2}\left(\frac{1}{m}-\frac{1}{2}\right)-\frac{\sigma}{2}}
$$

This estimate together with (4.2.17) concludes

$$
\left\||D|^{\sigma} u^{\mathrm{nl}}(t, \cdot)\right\|_{L^{2}} \lesssim\left(1+B_{\lambda}(0, t)\right)^{-\frac{n}{2}\left(\frac{1}{m}-\frac{1}{2}\right)-\frac{\sigma}{2}} .
$$

Now let us continue to estimate the norm $\left\||D|^{\sigma-1} u_{t}^{\mathrm{nl}}(t, \cdot)\right\|_{L^{2}}$ in the Case 2. Proceeding in the same way as we did to estimate the norm $\left\||D|^{\sigma} u^{\mathrm{nl}}(t, \cdot)\right\|_{L^{2}}$, we obtain the following estimate:

$\left\||D|^{\sigma-1} u_{t}^{\mathrm{nl}}(t, \cdot)\right\|_{L^{2}} \lesssim \int_{0}^{t} \frac{\Lambda(s)}{\lambda(s)} \lambda(t) F(\Lambda(t))\left(F^{2}(\Lambda(t))\right)^{-\frac{n}{2}\left(\frac{1}{m}-\frac{1}{2}\right)-\frac{\sigma-1}{2}-1}\left\|\left|u_{t}(s, \cdot)\right|^{p}\right\|_{L^{m} \cap L^{2} \cap \dot{H}^{\sigma-1}} d s$.

Summarizing, using the estimates of the norm $\left\|\left|u_{t}(s, \cdot)\right|^{p}\right\|_{L^{m} \cap L^{2} \cap \dot{H}^{\sigma-1}}$ from (4.2.13), (4.2.14) and (4.2.15) with no other requirements to the admissible exponents $p$ we arrive at

$$
\begin{aligned}
& \left\||D|^{\sigma-1} u_{t}^{\mathrm{nl}}(t, \cdot)\right\|_{L^{2}} \\
& \lesssim\|u\|_{X(t)}^{p} \lambda(t) F(\Lambda(t))\left(F^{2}(\Lambda(t))\right)^{-\frac{n}{2}\left(\frac{1}{m}-\frac{1}{2}\right)-\frac{\sigma-1}{2}-1} \int_{0}^{t} \frac{\Lambda(s)}{\lambda(s)} \lambda^{p}(s) F^{p}(\Lambda(s))\left(F^{2}(\Lambda(s))\right)^{-\frac{n}{2 m} p+\frac{n}{2 m}-p} d s \\
& =\|u\|_{X(t)}^{p} \lambda(t) F(\Lambda(t))\left(F^{2}(\Lambda(t))\right)^{-\frac{n}{2}\left(\frac{1}{m}-\frac{1}{2}\right)-\frac{\sigma-1}{2}-1} \underbrace{\int_{0}^{t} \lambda^{p-1}(s) \Lambda(s) F(\Lambda(s))^{-\frac{n}{m} p+\frac{n}{m}-p} d s}_{\mathbf{U}} .
\end{aligned}
$$


Similarly to the treatment of $S_{1}=S_{1}(t)$ and $T_{1}=T_{1}(t)$, we obtain

$$
\begin{aligned}
\mathbf{U}= & \int_{0}^{t} \lambda^{p}(s) \Lambda(s) \Xi^{2}(s) F(\Lambda(s))^{-\alpha_{5}(p)-2} d(F(\Lambda(s))) \\
= & \left.\frac{-1}{\alpha_{5}(p)+1} \lambda^{p}(s) \Lambda(s) \Xi^{2}(s) F(\Lambda(s))^{-\alpha_{5}(p)-1}\right|_{0} ^{t} \\
& +\frac{1}{\alpha_{5}(p)+1} \int_{0}^{t} F(\Lambda(s))^{-\alpha_{5}(p)-1} d\left(\lambda^{p}(s) \Lambda(s) \Xi^{2}(s)\right),
\end{aligned}
$$

where $\alpha_{5}(p)=\frac{n}{m} p-\frac{n}{m}+p$ and $\alpha_{5}(p)+1>0$. In this case it holds

$$
\begin{aligned}
\mathbf{U} \leq & -\left.\frac{1}{\alpha_{5}(p)+1} \lambda^{p}(s) \Lambda(s) \Xi^{2}(s) F(\Lambda(s))^{-\alpha_{5}(p)-1}\right|_{0} ^{t} \\
& +\frac{r}{\alpha_{5}(p)+1} \underbrace{\int_{0}^{t} \Lambda(s) \lambda^{p-1}(s) F(\Lambda(s))^{-\alpha_{5}(p)} d s}_{\mathbf{U}}
\end{aligned}
$$

where from condition (A5) we used $\Xi^{2}(t)=\frac{F^{2}(\Lambda(t))}{\lambda^{2}(t) F^{\prime}(\Lambda(t))}$ and from condition (D4) we used $\frac{F(\Lambda(s))}{F^{\prime}(\Lambda(s))} \leq \Lambda(s)$, respectively. Thus, from the last estimate of $\mathbf{U}$ we obtain

$$
\left(1-\frac{r}{\alpha_{5}(p)+1}\right) \mathbf{U} \lesssim \lambda^{p}(0) \Lambda(0) \Xi^{2}(0) F(\Lambda(0))^{-\alpha_{5}(p)-1}-\lambda^{p}(t) \Lambda(t) \Xi^{2}(t) F(\Lambda(t))^{-\alpha_{5}(p)-1} .
$$

Since we have $1-\frac{r}{\alpha_{5}(p)+1}>0$, we may conclude the estimate

$$
\left\||D|^{\sigma-1} u_{t}^{\mathrm{nl}}(t, \cdot)\right\|_{L^{2}} \lesssim\|u\|_{X(t)}^{p} \lambda(t) F(\Lambda(t))\left(F^{2}(\Lambda(t))\right)^{-\frac{n}{2}\left(\frac{1}{m}-\frac{1}{2}\right)-\frac{\sigma-1}{2}-1} .
$$

Now we want to prove (4.1.7) in the Case 2. By the definition of the solution space $X(t)$ and its norm we have

$$
\begin{aligned}
\|\left|u_{t}(s, \cdot)\right|^{p}-\mid & \left.v_{t}(s, \cdot)\right|^{p} \|_{L^{2}} \\
& \lesssim \lambda^{p}(s) F^{p}(\Lambda(s))\left(F^{2}(\Lambda(s))\right)^{-\frac{n}{2 m} p+\frac{n}{4}-p}\|u-v\|_{X(s)}\left(\|u\|_{X(t)}^{p-1}+\|v\|_{X(s)}^{p-1}\right), \\
\|\left|u_{t}(s, \cdot)\right|^{p}-\mid & \left.v_{t}(s, \cdot)\right|^{p} \|_{L^{m}} \\
& \lesssim \lambda^{p}(s) F^{p}(\Lambda(s))\left(F^{2}(\Lambda(s))\right)^{-\frac{n}{2 m} p+\frac{n}{2 m}-p}\|u-v\|_{X(s)}\left(\|u\|_{X(s)}^{p-1}+\|v\|_{X(s)}^{p-1}\right) .
\end{aligned}
$$

On the other hand, to estimate the norm $\left\|\left|u_{t}(s, \cdot)\right|^{p}-\left|v_{t}(s, \cdot)\right|^{p}\right\|_{\dot{H}^{\sigma-1}}$ we follow the considerations from the Case 1. Thus, we obtain

$$
\begin{aligned}
& \left\|\left|u_{t}(s, \cdot)\right|^{p}-\left|v_{t}(s, \cdot)\right|^{p}\right\|_{\dot{H}^{\sigma-1}} \\
& \lesssim \lambda^{p}(s) F^{p}(\Lambda(s))\left(F^{2}(\Lambda(s))\right)^{-\frac{n}{2 m} p+\frac{n}{4} p-\frac{\sigma-1}{2}-\frac{\sigma^{*}}{2}(p-1)-(p-1)} \int_{0}^{1}\|u-v\|_{X(s)}\|w u+(1-w) v\|_{X(s)}^{p-1} d w \\
& \lesssim \lambda^{p}(s) F^{p}(\Lambda(s))\left(F^{2}(\Lambda(s))\right)^{-\frac{n}{2 m} p+\frac{n}{4} p-\frac{\sigma-1}{2}-\frac{\sigma^{*}}{2}(p-1)-(p-1)}\|u-v\|_{X(s)}\left(\|u\|_{X(s)}^{p-1}+\|v\|_{X(s)}^{p-1}\right),
\end{aligned}
$$

where $\sigma^{*}<\frac{n}{2}$. Taking into consideration (4.2.15), $\sigma^{*}=\frac{n}{2}-\varepsilon<\frac{n}{2}$ with $\varepsilon>0$ and $\|u\|_{X(s)} \leq$ $\|u\|_{X(t)}$ with $0 \leq s \leq t$ we find

$$
\begin{gathered}
\left\||D|^{\sigma} \int_{0}^{t} K_{1}(t, s, x) *_{(x)}\left(\left|u_{t}(s, x)\right|^{p}-\left|v_{t}(s, x)\right|^{p}\right) d s\right\|_{L^{2}} \lesssim\left(\|u\|_{X(t)}^{p-1}+\|v\|_{X(t)}^{p-1}\right)\|u-v\|_{X(t)} \\
\times\left(\int_{0}^{\delta t} \frac{\Lambda(s)}{\lambda(s)} \lambda^{p}(s) F^{p}(\Lambda(s))\left(1+B_{\lambda}(s, t)\right)^{-\frac{n}{2}\left(\frac{1}{m}-\frac{1}{2}\right)-\frac{\sigma}{2}}\left(F^{2}(\Lambda(s))\right)^{-\frac{n}{2 m} p+\frac{n}{2 m}-p} d s\right. \\
\left.\quad+\int_{\delta t}^{t} \frac{\Lambda(s)}{\lambda(s)} \frac{\Lambda(s)}{\lambda(s)}\left(1+B_{\lambda}(s, t)\right)^{-\frac{\sigma}{2}}\left(F^{2}(\Lambda(s))\right)^{-\frac{n}{2 m} p+\frac{n}{4}-p} d s\right) .
\end{gathered}
$$


In order to conclude the proof of the estimate (4.1.7) we can follow the same steps of the proof of (4.1.6). Hence, we arrive at the estimate

$$
\left\||D|^{\sigma}(N u-N v)(t, \cdot)\right\|_{L^{2}} \lesssim\left(1+B_{\lambda}(0, t)\right)^{-\frac{n}{2}\left(\frac{1}{m}-\frac{1}{2}\right)-\frac{\sigma}{2}}\|u-v\|_{X(t)}\left(\|u\|_{X(t)}^{p-1}+\|v\|_{X(t)}^{p-1}\right) .
$$

In the same way, with no other requirements to the admissible exponents $p$ one can derive

$$
\begin{aligned}
& \|(N u-N v)(t, \cdot)\|_{L^{2}} \\
& \quad \lesssim\left(1+B_{\lambda}(0, s)\right)^{-\frac{n}{2}\left(\frac{1}{m}-\frac{1}{2}\right)}\|u-v\|_{X(t)}\left(\|u\|_{X(t)}^{p-1}+\|v\|_{X(t)}^{p-1}\right) \\
& \left\|\partial_{t}(N u-N v)(t, \cdot)\right\|_{L^{2}} \\
& \quad \lesssim \lambda(t) F(\Lambda(t))\left(F^{2}(\Lambda(t))\right)^{-\frac{n}{2}\left(\frac{1}{m}-\frac{1}{2}\right)-1}\|u-v\|_{X(t)}\left(\|u\|_{X(t)}^{p-1}+\|v\|_{X(t)}^{p-1}\right), \\
& \left\||D|^{\sigma-1} \partial_{t}(N u-N v)(t, \cdot)\right\|_{L^{2}} \\
& \quad \lesssim \lambda(t) F(\Lambda(t))\left(F^{2}(\Lambda(t))\right)^{-\frac{n}{2}\left(\frac{1}{m}-\frac{1}{2}\right)-\frac{\sigma-1}{2}-1}\|u-v\|_{X(t)}\left(\|u\|_{X(t)}^{p-1}+\|v\|_{X(t)}^{p-1}\right) .
\end{aligned}
$$

Thus, from the definition of the norm of the solution space $X(t)$ we may conclude the proof of (4.1.7), which completes the proof of the theorem.

\subsubsection{Case of super-exponential propagation speed}

We define the following parameters:

$$
\begin{aligned}
& \tilde{p}_{1}(m, n, \sigma):=\max \left\{\frac{n}{n+2 m}+\left(\frac{\sigma+1}{2}+d_{2}\right) \frac{2 m}{n+2 m} ; \frac{m r-m+n}{m+n}\right\}, \\
& \tilde{p}_{2}(m, n, \sigma):=\frac{n m}{2 n+4 m}+d_{2} \delta(\alpha+2) \frac{2 m}{n+2 m},
\end{aligned}
$$

where we set

$$
\tilde{p}(m, n, \sigma):=\max \left\{\tilde{p}_{1}(m, n, \sigma) ; \tilde{p}_{2}(m, n, \sigma)\right\} .
$$

We consider now the case of super-exponential propagation speed for large regular data belonging to $L^{\infty}$. For this reason, we choose the regularity parameter $\sigma$ from the interval $\sigma \in\left(1+\frac{n}{2}, \infty\right)$ with $n \geq 2$. We have the following statement.

Theorem 4.2.3. We assume that the Hypotheses (A1) to (A5), (B1) to (B6), (C1), (C3), (D2) and (D3) hold. Let $\nu(\delta)=\infty$ and $\vartheta(\delta)=\infty$, where the functions $\nu=\nu(\delta)$ and $\vartheta=\vartheta(\delta)$ are defined in (3.1.2) and (3.1.3), respectively. Moreover, the following condition for the function $\nu$ is supposed to be satisfied:

$$
\nu(\delta, t)=o(\log \Lambda(t)) .
$$

We choose the data $\left(u_{0}, u_{1}\right) \in \mathcal{D}_{m}^{\sigma}$ with $\sigma>1+\frac{n}{2}$ and $m \in[1,2)$. Finally, the exponent $p$ satisfies $p>\sigma$ and $p>\tilde{p}(m, n, \sigma)$. Then, there exists a constant $\varepsilon_{0}>0$ such that for all $\left(u_{0}, u_{1}\right) \in \mathcal{D}_{m}^{\sigma}$ with $\left\|\left(u_{0}, u_{1}\right)\right\|_{\mathcal{D}_{m}^{\sigma}} \leq \varepsilon_{0}$ there is a uniquely determined energy solution

$$
u \in \mathcal{C}\left([0, \infty), H^{\sigma}\right) \cap \mathcal{C}^{1}\left([0, \infty), H^{\sigma-1}\right)
$$

to the Cauchy problem (3.1.1). Furthermore, the solution satisfies the following estimates:

$$
\begin{aligned}
& \|u(t, \cdot)\|_{L^{2}} \lesssim\left(1+B_{\lambda}(0, t)\right)^{-\frac{n}{2}\left(\frac{1}{m}-\frac{1}{2}\right)}\left\|\left(u_{0}, u_{1}\right)\right\|_{\mathcal{D}_{m}^{\sigma}}, \\
& \left\||D|^{\sigma} u(t, \cdot)\right\|_{L^{2}} \lesssim\left(1+B_{\lambda}(0, t)\right)^{-\frac{n}{2}\left(\frac{1}{m}-\frac{1}{2}\right)-\frac{\sigma}{2}}\left\|\left(u_{0}, u_{1}\right)\right\|_{\mathcal{D}_{m}^{\sigma}}, \\
& \left\|u_{t}(t, \cdot)\right\|_{L^{2}} \\
& \quad \lesssim \max \left\{\frac{\lambda^{2}(t)}{\rho(t)}\left(1+B_{\lambda}(0, t)\right)^{-\frac{n}{2}\left(\frac{1}{m}-\frac{1}{2}\right)-1} ; \lambda(t) F(\Lambda(t))\left(F^{2}(\Lambda(t))\right)^{-\frac{n}{2}\left(\frac{1}{m}-\frac{1}{2}\right)-1}\right\}\left\|\left(u_{0}, u_{1}\right)\right\|_{\mathcal{D}_{m}^{\sigma}}, \\
& \left\||D|^{\sigma-1} u_{t}(t, \cdot)\right\|_{L^{2}} \\
& \quad \lesssim \max \left\{\frac{\lambda^{2}(t)}{\rho(t)}\left(1+B_{\lambda}(0, t)\right)^{-\frac{n}{2}\left(\frac{1}{m}-\frac{1}{2}\right)-\frac{\sigma-1}{2}-1} ; \lambda(t) F(\Lambda(t))\left(F^{2}(\Lambda(t))\right)^{-\frac{n}{2}\left(\frac{1}{m}-\frac{1}{2}\right)-\frac{\sigma-1}{2}-1}\right\}\left\|\left(u_{0}, u_{1}\right)\right\|_{\mathcal{D}_{m}^{\sigma}} .
\end{aligned}
$$


Proof. To prove this theorem we will proceed in an analogous way as in the proof of Theorem 4.2.2. We want to prove the inequalities (4.1.6) and (4.1.7). Let us only derive estimates of the following norms:

$$
\left\|u^{\mathrm{nl}}(t, \cdot)\right\|_{L^{2}}, \quad\left\||D|^{\sigma} u^{\mathrm{nl}}(t, \cdot)\right\|_{L^{2}}, \quad\left\|u_{t}^{\mathrm{nl}}(t, \cdot)\right\|_{L^{2}} \quad \text { and } \quad\left\||D|^{\sigma-1} u_{t}^{\mathrm{nl}}(t, \cdot)\right\|_{L^{2}} \cdot
$$

Case 1: Let us assume that the first components are dominant, that is,

$$
\begin{aligned}
& \Phi_{1, m}(s, t)=\frac{\lambda^{2}(t)}{\rho(t)}\left(1+B_{\lambda}(s, t)\right)^{-\frac{n}{2}\left(\frac{1}{m}-\frac{1}{2}\right)-1} \quad \text { and } \Phi_{1}(s, t)=\frac{\lambda^{2}(t)}{\rho(t)}\left(1+B_{\lambda}(s, t)\right)^{-1}, \\
& \Phi_{2, m}(s, t)=\frac{\lambda^{2}(t)}{\rho(t)}\left(1+B_{\lambda}(s, t)\right)^{-\frac{n}{2}\left(\frac{1}{m}-\frac{1}{2}\right)-\frac{\sigma-1}{2}-1} \text { and } \Phi_{2}(s, t)=\frac{\lambda^{2}(t)}{\rho(t)}\left(1+B_{\lambda}(s, t)\right)^{-\frac{\sigma-1}{2}-1},
\end{aligned}
$$

for all $t \geq s \geq 0$.

We begin to estimate the norm $\left\||D|^{\sigma} u^{\mathrm{nl}}(t, \cdot)\right\|_{L^{2}}$. In the same way as we did in the proof of Theorem 4.2.2, employing the fractional powers rules from Corollary A.7.10 with $\sigma-1 \in\left(\frac{n}{2}, p\right)$ and Proposition A.7.13 with $\sigma^{*}<\frac{n}{2}$ we obtain

$$
\begin{aligned}
& \left\||D|^{\sigma} u^{\mathrm{nl}}(t, \cdot)\right\|_{L^{2}} \\
& \lesssim\|u\|_{X(t)}^{p} \underbrace{\int_{0}^{\delta t} \frac{\Lambda(s)}{\lambda(s)} \frac{\lambda^{2 p}(s)}{\rho^{p}(s)}\left(1+B_{\lambda}(s, t)\right)^{-\frac{n}{2}\left(\frac{1}{m}-\frac{1}{2}\right)-\frac{\sigma}{2}}\left(1+B_{\lambda}(0, s)\right)^{-\frac{n}{2 m} p+\frac{n}{2 m}-p} d s}_{\mathbf{M}} \\
& +\|u\|_{X(t)}^{p} \underbrace{\int_{\delta t}^{t} \frac{\Lambda(s)}{\lambda(s)} \frac{\lambda^{2 p}(s)}{\rho^{p}(s)}\left(1+B_{\lambda}(s, t)\right)^{-\frac{\sigma}{2}}\left(1+B_{\lambda}(0, s)\right)^{-\frac{n}{2 m} p+\frac{n}{4}-p} d s}_{\mathbf{N}} .
\end{aligned}
$$

Let us consider the integral M. Using condition (3.1.21) we have the estimate

$$
\mathbf{M} \lesssim\left(1+B_{\lambda}(0, t)\right)^{-\frac{n}{2}\left(\frac{1}{m}-\frac{1}{2}\right)-\frac{\sigma}{2}} \underbrace{\int_{0}^{\delta t} \frac{\Lambda(s)}{\lambda(s)} \frac{\lambda^{2 p}(s)}{\rho^{p}(s)}\left(1+B_{\lambda}(0, s)\right)^{-\alpha_{3}(p)} d s}_{\bar{M}_{1}(t)}
$$

where $\alpha_{3}(p):=\frac{n}{2 m} p-\frac{n}{2 m}+p$. Then, by (D2) it follows

$$
\begin{aligned}
\bar{M}_{1}(t)= & \int_{0}^{\delta t} \frac{\mu(s)}{\lambda^{2}(s)} \frac{\lambda^{2 p}(s)}{\rho^{p}(s)}\left(1+B_{\lambda}(0, s)\right)^{-\alpha_{3}(p)} d\left(1+B_{\lambda}(0, s)\right) \\
= & \left.\frac{1}{1-\alpha_{3}(p)} \frac{\mu(s)}{\lambda^{2}(s)} \frac{\lambda^{2 p}(s)}{\rho^{p}(s)}\left(1+B_{\lambda}(0, s)\right)^{-\alpha_{3}(p)+1}\right|_{0} ^{\delta t} \\
& -\frac{1}{1-\alpha_{3}(p)} \int_{0}^{\delta t}\left(1+B_{\lambda}(0, s)\right)^{-\alpha_{3}(p)+1} d\left(\frac{\mu(s)}{\lambda^{2}(s)} \frac{\lambda^{2 p}(s)}{\rho^{p}(s)}\right) \\
\leq & \left.\frac{1}{1-\alpha_{3}(p)} \frac{\mu(s)}{\lambda^{2}(s)} \frac{\lambda^{2 p}(s)}{\rho^{p}(s)}\left(1+B_{\lambda}(0, s)\right)^{-\alpha_{3}(p)+1}\right|_{0} ^{\delta t} \\
& +\frac{d_{2}}{\alpha_{3}(p)-1} \int_{0}^{\delta t}\left(1+B_{\lambda}(0, s)\right)^{-\alpha_{3}(p)}\left(1+B_{\lambda}(0, s)\right) \frac{\mu(s)}{\lambda(s) \Lambda(s)} \frac{\lambda^{2 p}(s)}{\rho^{p}(s)} \frac{1}{\log \Lambda(s)} d s \\
\leq & \left.\frac{1}{1-\alpha_{3}(p)} \frac{\mu(s)}{\lambda^{2}(s)} \frac{\lambda^{2 p}(s)}{\rho^{p}(s)}\left(1+B_{\lambda}(0, s)\right)^{-\alpha_{3}(p)+1}\right|_{0} ^{\delta t} \\
& +\frac{d_{2}}{\alpha_{3}(p)-1} \underbrace{\int_{0}^{\delta t} \frac{\Lambda(s)}{\lambda(s)} \frac{\lambda^{2 p}(s)}{\rho^{p}(s)}\left(1+B_{\lambda}(0, s)\right)^{-\alpha_{3}(p)} d s}_{\bar{M}_{1}(t)},
\end{aligned}
$$

where $\alpha_{3}(p)>1$. Then, it follows

$$
\left(1-\frac{d_{2}}{\alpha_{3}(p)-1}\right) \bar{M}_{1}(t) \lesssim \frac{\mu(0)}{\lambda^{2}(0)} \frac{\lambda^{2 p}(0)}{\rho^{p}(0)}-\frac{\mu(\delta t)}{\lambda^{2}(\delta t)} \frac{\lambda^{2 p}(\delta t)}{\rho^{p}(\delta t)}\left(1+B_{\lambda}(0, \delta t)\right)^{-\alpha_{3}(p)+1} .
$$


We can guarantee that $\bar{M}_{1}=\bar{M}_{1}(t)$ is bounded by

$$
1-\frac{d_{2}}{\alpha_{3}(p)-1}>0, \quad \text { or equivalent, } \quad p>1+d_{2} \frac{2 m}{n+2 m},
$$

which can be concluded from the condition $p>\tilde{p}_{1}(m, n, \sigma)$. In this case we arrive at the following desired estimate:

$$
\mathbf{M} \lesssim\left(1+B_{\lambda}(0, t)\right)^{-\frac{n}{2}\left(\frac{1}{m}-\frac{1}{2}\right)-\frac{\sigma}{2}} .
$$

Now let us consider the integral N. Defining $\alpha_{4}(p):=\frac{n}{2 m} p-\frac{n}{4}+p$ it holds

$$
\begin{aligned}
\mathbf{N}= & \int_{\delta t}^{t} \frac{\Lambda(s)}{\lambda(s)} \frac{\lambda^{2 p}(s)}{\rho^{p}(s)}\left(1+B_{\lambda}(s, t)\right)^{-\frac{\sigma}{2}}\left(1+B_{\lambda}(0, s)\right)^{-\alpha_{4}(p)} d s \\
= & -\int_{\delta t}^{t} \frac{\mu(s)}{\lambda^{2}(s)} \frac{\lambda^{2 p}(s)}{\rho^{p}(s)}\left(1+B_{\lambda}(0, s)\right)^{-\alpha_{4}(p)}\left(1+B_{\lambda}(s, t)\right)^{-\frac{\sigma}{2}} d\left(1+B_{\lambda}(s, t)\right) \\
= & -\left.\frac{1}{1-\frac{\sigma}{2}} \frac{\mu(s)}{\lambda^{2}(s)} \frac{\lambda^{2 p}(s)}{\rho^{p}(s)}\left(1+B_{\lambda}(0, s)\right)^{-\alpha_{4}(p)}\left(1+B_{\lambda}(s, t)\right)^{-\frac{\sigma}{2}+1}\right|_{\delta t} ^{t} \\
& +\frac{1}{1-\frac{\sigma}{2}} \int_{\delta t}^{t}\left(1+B_{\lambda}(s, t)\right)^{-\frac{\sigma}{2}+1} d\left(\frac{\mu(s)}{\lambda^{2}(s)} \frac{\lambda^{2 p}(s)}{\rho^{p}(s)}\left(1+B_{\lambda}(0, s)\right)^{-\alpha_{4}(p)}\right) .
\end{aligned}
$$

Here we consider only the case $1-\frac{\sigma}{2}<0$, because $p>\sigma>2$ with $n \geq 2$. Then, employing Lemma 4.2.1 we get

$$
\begin{aligned}
\left(1+B_{\lambda}(0, t)\right)^{\frac{n}{2}\left(\frac{1}{m}-\frac{1}{2}\right)+\frac{\sigma}{2}} \mathbf{N} & \lesssim \underbrace{\frac{\mu(t)}{\lambda^{2}(t)} \frac{\lambda^{2 p}(t)}{\rho^{p}(t)}\left(1+B_{\lambda}(0, t)\right)^{-\alpha_{4}(p)+\frac{n}{2}\left(\frac{1}{m}-\frac{1}{2}\right)+\frac{\sigma}{2}}}_{\bar{N}_{1}(t)} \\
& +\underbrace{\int_{\delta t}^{t} \frac{\mu(s)}{\lambda^{2}(s)} \frac{\lambda^{2 p}(s)}{\rho^{p}(s)}\left(1+B_{\lambda}(0, s)\right)^{-\alpha_{4}(p)}\left(1+B_{\lambda}(0, t)\right)^{\frac{n}{2}\left(\frac{1}{m}-\frac{1}{2}\right)+\frac{\sigma}{2}} \frac{d\left(1+B_{\lambda}(0, s)\right)}{1+B_{\lambda}(0, s)}}_{\bar{N}_{2}(t)} .
\end{aligned}
$$

For $\bar{N}_{1}=\bar{N}_{1}(t)$, using $\alpha_{4}(p)=\alpha_{3}(p)+\frac{n}{2}\left(\frac{1}{m}-\frac{1}{2}\right)$ we find

$$
\bar{N}_{1}(t)=\frac{\mu(t)}{\lambda^{2}(t)} \frac{\lambda^{2 p}(t)}{\rho^{p}(t)}\left(1+B_{\lambda}(0, t)\right)^{-\alpha_{3}(p)+\frac{\sigma}{2}} .
$$

Then, it follows

$$
\begin{aligned}
& \bar{N}_{1}^{\prime}(t) \\
& \leq\left[d_{2} \frac{\mu(t)}{\lambda(t) \Lambda(t)} \frac{1}{\log \Lambda(t)}\left(1+B_{\lambda}(0, t)\right)+\left(-\alpha_{3}(p)+\frac{\sigma}{2}\right) \frac{\Lambda(t)}{\lambda(t)}\right] \frac{\lambda^{2 p}(t)}{\rho^{p}(t)}\left(1+B_{\lambda}(0, t)\right)^{-\alpha_{3}(p)+\frac{\sigma}{2}-1} \\
& \leq\left[d_{2}-\alpha_{3}(p)+\frac{\sigma}{2}\right] \frac{\lambda^{2 p}(t)}{\rho^{p}(t)} \frac{\Lambda(t)}{\lambda(t)}\left(1+B_{\lambda}(0, t)\right)^{-\alpha_{3}(p)+\frac{\sigma}{2}-1},
\end{aligned}
$$

where we used condition (D2) and (3.1.20), respectively. Then, for large time $t$ we obtain $\bar{N}_{1}^{\prime}(t)<0$ by $p>\tilde{p}_{1}(m, n, \sigma)$. In order to derive the desired estimate for $\bar{N}_{2}=\bar{N}_{2}(t)$ we suppose that there exists a positive constant $\varepsilon$ such that it holds

$$
\frac{\mu(\delta t)}{\lambda^{2}(\delta t)} \frac{\lambda^{2 p}(\delta t)}{\rho^{p}(\delta t)}\left(1+B_{\lambda}(0, \delta t)\right)^{-\alpha_{4}(p)}\left(1+B_{\lambda}(0, t)\right)^{\frac{n}{2}\left(\frac{1}{m}-\frac{1}{2}\right)+\frac{\sigma}{2}} \lesssim\left(1+B_{\lambda}(0, \delta t)\right)^{-\varepsilon} .
$$

Then, from the previous estimate it is sufficient to consider the monotonicity of the function

$$
\overline{\mathcal{N}}_{2}(t):=\frac{\mu(\delta t)}{\lambda^{2}(\delta t)} \frac{\lambda^{2 p}(\delta t)}{\rho^{p}(\delta t)}\left(1+B_{\lambda}(0, \delta t)\right)^{-\alpha_{4}(p)+\varepsilon}\left(1+B_{\lambda}(0, t)\right)^{\frac{n}{2}\left(\frac{1}{m}-\frac{1}{2}\right)+\frac{\sigma}{2}} .
$$


Indeed, by taking the derivative of $\overline{\mathcal{N}}_{2}=\overline{\mathcal{N}}_{2}(t)$ we get

$$
\begin{aligned}
& \overline{\mathcal{N}}_{2}^{\prime}(t) \\
& \leq\left[\left(d_{2} \delta+\frac{-\alpha_{4}(p)+\varepsilon}{\alpha+2}\right) \frac{1}{\log \Lambda(\delta t)}+\left(\frac{n}{2}\left(\frac{1}{m}-\frac{1}{2}\right)+\frac{\sigma}{2}\right) \frac{\lambda(t)}{\Lambda(t)} \frac{\Lambda(\delta t)}{\lambda(\delta t)} \frac{1}{\log \Lambda(t)}\right] \frac{\lambda^{2 p}(\delta t)}{\rho^{p}(\delta t)} \frac{\mu(\delta t)}{\lambda^{2}(\delta t)} \\
& \quad \times \phi(t) \phi(\delta t) \log \Lambda(t) \log \Lambda(\delta t)\left(1+B_{\lambda}(0, \delta t)\right)^{-\alpha_{4}(p)+\varepsilon-1}\left(1+B_{\lambda}(0, t)\right)^{\frac{n}{2}\left(\frac{1}{m}-\frac{1}{2}\right)+\frac{\sigma}{2}-1} \frac{\lambda(\delta t)}{\Lambda(\delta t)}
\end{aligned}
$$

where we have used condition (D2) and (3.1.20). We can see that $\overline{\mathcal{N}}_{2}^{\prime}(t)<0$ by the condition (4.2.18) and

$$
d_{2} \delta-\frac{\alpha_{4}(p)}{\alpha+2}<0, \quad \text { that is, } \quad p>\frac{n m}{2 n+4 m}+d_{2} \delta(\alpha+2) \frac{2 m}{n+2 m} .
$$

This gives that $\bar{N}_{2}=\bar{N}_{2}(t)$ is uniformly bounded. For this reason we obtain

$$
\mathbf{N} \lesssim\left(1+B_{\lambda}(0, t)\right)^{-\frac{n}{2}\left(\frac{1}{m}-\frac{1}{2}\right)-\frac{\sigma}{2}}
$$

Taking into consideration the derived estimates for $\mathbf{M}$ and $\mathbf{N}$ we arrive at

$$
\left\||D|^{\sigma} u^{\mathrm{nl}}(t, \cdot)\right\|_{L^{2}} \lesssim\|u\|_{X(t)}^{p}\left(1+B_{\lambda}(0, t)\right)^{-\frac{n}{2}\left(\frac{1}{m}-\frac{1}{2}\right)-\frac{\sigma}{2}} .
$$

In the same way, one can prove

$$
\begin{aligned}
\left\|u^{\mathrm{nl}}(t, \cdot)\right\|_{L^{2}} & \lesssim\|u\|_{X(t)}^{p}\left(1+B_{\lambda}(0, t)\right)^{-\frac{n}{2}\left(\frac{1}{m}-\frac{1}{2}\right)} \\
\left\|u_{t}^{\mathrm{nl}}(t, \cdot)\right\|_{L^{2}} & \lesssim\|u\|_{X(t)}^{p} \frac{\lambda^{2}(t)}{\rho(t)}\left(1+B_{\lambda}(0, t)\right)^{-\frac{n}{2}\left(\frac{1}{m}-\frac{1}{2}\right)-1} .
\end{aligned}
$$

Now let us continue with the estimate of the norm $\left\||D|^{\sigma-1} u_{t}^{\mathrm{nl}}(t, \cdot)\right\|_{L^{2}}$. Proceeding in the same way as we did to estimate the norm $\left\||D|^{\sigma} u^{\mathrm{nl}}(t, \cdot)\right\|_{L^{2}}$ with no other requirements to the admissible exponents $p$ we have the following estimate:

$$
\begin{aligned}
& \left\||D|^{\sigma-1} u_{t}^{\mathrm{nl}}(t, \cdot)\right\|_{L^{2}} \\
& \lesssim\|u\|_{X(t)}^{p} \underbrace{\int_{0}^{\delta t} \frac{\lambda^{2}(t)}{\rho(t)} \frac{\Lambda(s)}{\lambda(s)} \frac{\lambda^{2 p}(s)}{\rho^{p}(s)}\left(1+B_{\lambda}(s, t)\right)^{-\frac{n}{2}\left(\frac{1}{m}-\frac{1}{2}\right)-\frac{\sigma-1}{2}-1}\left(1+B_{\lambda}(0, s)\right)^{-\frac{n}{2 m} p+\frac{n}{2 m}-p} d s}_{\mathbf{P}} \\
& \quad+\|u\|_{X(t)}^{p} \underbrace{\int_{\delta t}^{t} \frac{\lambda^{2}(t)}{\rho(t)} \frac{\Lambda(s)}{\lambda(s)} \frac{\lambda^{2 p}(s)}{\rho^{p}(s)}\left(1+B_{\lambda}(s, t)\right)^{-\frac{\sigma-1}{2}-1}\left(1+B_{\lambda}(0, s)\right)^{-\frac{n}{2 m} p+\frac{n}{4}-p} d s}_{\mathbf{R}} .
\end{aligned}
$$

Firstly, we consider the integral $\mathbf{P}$. We have

$$
\mathbf{P} \lesssim \frac{\lambda^{2}(t)}{\rho(t)}\left(1+B_{\lambda}(0, t)\right)^{-\frac{n}{2}\left(\frac{1}{m}-\frac{1}{2}\right)-\frac{\sigma-1}{2}-1} \underbrace{\int_{0}^{\delta t} \frac{\Lambda(s)}{\lambda(s)} \frac{\lambda^{2 p}(s)}{\rho^{p}(s)}\left(1+B_{\lambda}(0, s)\right)^{-\frac{n}{2 m} p+\frac{n}{2 m}-p} d s}_{\bar{P}_{1}(t)}
$$

where we used (3.1.21) to estimate $B_{\lambda}(s, t)$. Similarly as we did to estimate $\bar{M}_{1}(t)$ in $(4.2 .19)$ one can show that $\bar{P}_{1}=\bar{P}_{1}(t)$, is bounded by the condition $p>\tilde{p}_{1}(m, n, \sigma)$. Thus, we have

$$
\mathbf{P} \lesssim \frac{\lambda^{2 p}(t)}{\rho^{p}(t)}\left(1+B_{\lambda}(0, t)\right)^{-\frac{n}{2}\left(\frac{1}{m}-\frac{1}{2}\right)-\frac{\sigma-1}{2}-1} .
$$


Now we consider the integral R. Defining $\alpha_{4}(p):=\frac{n}{2 m} p-\frac{n}{4}+p$, we find

$$
\begin{aligned}
\mathbf{R}= & -\frac{\lambda^{2}(t)}{\rho(t)} \int_{\delta t}^{t} \frac{\mu(s)}{\lambda^{2}(s)} \frac{\lambda^{2 p}(s)}{\rho^{p}(s)}\left(1+B_{\lambda}(0, s)\right)^{-\alpha_{4}(p)}\left(1+B_{\lambda}(s, t)\right)^{-\frac{\sigma-1}{2}-1} d\left(1+B_{\lambda}(s, t)\right) \\
= & \left.\frac{2}{\sigma-1} \frac{\lambda^{2}(t)}{\rho(t)} \frac{\mu(s)}{\lambda^{2}(s)} \frac{\lambda^{2 p}(s)}{\rho^{p}(s)}\left(1+B_{\lambda}(0, s)\right)^{-\alpha_{4}(p)}\left(1+B_{\lambda}(s, t)\right)^{-\frac{\sigma-1}{2}}\right|_{\delta t} ^{t} \\
& -\frac{2}{\sigma-1} \frac{\lambda^{2}(t)}{\rho(t)} \int_{\delta t}^{t}\left(1+B_{\lambda}(s, t)\right)^{-\frac{\sigma-1}{2}} d\left(\frac{\mu(s)}{\lambda^{2}(s)} \frac{\lambda^{2 p}(s)}{\rho^{p}(s)}\left(1+B_{\lambda}(0, s)\right)^{-\alpha_{4}(p)}\right) .
\end{aligned}
$$

After using Lemma 4.2.1 it follows

$$
\begin{aligned}
\frac{\rho(t)}{\lambda^{2}(t)}(1 & \left.+B_{\lambda}(0, t)\right)^{\frac{n}{2}\left(\frac{1}{m}-\frac{1}{2}\right)+\frac{\sigma-1}{2}+1} \mathbf{R} \\
\lesssim & \underbrace{\frac{\mu(t)}{\lambda^{2}(t)} \frac{\lambda^{2 p}(t)}{\rho^{p}(t)}\left(1+B_{\lambda}(0, t)\right)^{\frac{n}{2}\left(\frac{1}{m}-\frac{1}{2}\right)+\frac{\sigma-1}{2}+1-\alpha_{4}(p)}}_{\bar{R}_{1}(t)} \\
& +\underbrace{\int_{\delta t}^{t} \frac{\mu(s)}{\lambda^{2}(s)} \frac{\lambda^{2 p}(s)}{\rho^{p}(s)}\left(1+B_{\lambda}(0, s)\right)^{-\alpha_{4}(p)}\left(1+B_{\lambda}(0, t)\right)^{\frac{n}{2}\left(\frac{1}{m}-\frac{1}{2}\right)+\frac{\sigma-1}{2}+1} \frac{d\left(1+B_{\lambda}(0, s)\right)}{1+B_{\lambda}(0, s)}}_{\bar{R}_{2}(t)} .
\end{aligned}
$$

We begin to estimate $\bar{R}_{1}=\bar{R}_{1}(t)$. Using $\alpha_{4}(p)=\alpha_{3}(p)+\frac{n}{2}\left(\frac{1}{m}-\frac{1}{2}\right)$ we get

$$
\bar{R}_{1}(t)=\frac{\mu(t)}{\lambda^{2}(t)} \frac{\lambda^{2 p}(t)}{\rho^{p}(t)}\left(1+B_{\lambda}(0, t)\right)^{\frac{\sigma+1}{2}-\alpha_{3}(p)} .
$$

So, we may conclude

$$
\begin{aligned}
& \bar{R}_{1}^{\prime}(t) \\
& \leq\left[d_{2} \frac{\mu(t)}{\lambda(t) \Lambda(t)} \frac{1}{\log \Lambda(t)}\left(1+B_{\lambda}(0, t)\right)+\left(\frac{\sigma+1}{2}-\alpha_{3}(p)\right) \frac{\Lambda(t)}{\lambda(t)}\right] \frac{\lambda^{2 p}(t)}{\rho^{p}(t)}\left(1+B_{\lambda}(0, t)\right)^{\frac{\sigma-1}{2}-\alpha_{3}(p)} \\
& \leq\left[d_{2}+\frac{\sigma+1}{2}-\alpha_{3}(p)\right] \frac{\Lambda(t)}{\lambda(t)}\left(1+B_{\lambda}(0, t)\right)^{\frac{\sigma-1}{2}-\alpha_{3}(p)},
\end{aligned}
$$

where we used again condition (D2) and (3.1.20), respectively. Then, for a large time $t$ we get $\bar{R}_{1}^{\prime}(t)<0$ by

$$
d_{2}+\frac{\sigma+1}{2}-\alpha_{3}(p)<0, \quad \text { that is, } \quad p>\frac{n}{n+2 m}+\left(\frac{\sigma+1}{2}+d_{2}\right) \frac{2 m}{n+2 m},
$$

which follows from the condition $p>\tilde{p}_{1}(m, n, \sigma)$. Therefore, $\bar{R}_{1}=\bar{R}_{1}(t)$ is uniformly bounded. For $\bar{R}_{2}=\bar{R}_{2}(t)$, we assume that there exists a constant $\varepsilon>0$ such that

$$
\frac{\mu(\delta t)}{\lambda^{2}(\delta t)} \frac{\lambda^{2 p}(\delta t)}{\rho^{p}(\delta t)}\left(1+B_{\lambda}(0, \delta t)\right)^{-\alpha_{4}(p)}\left(1+B_{\lambda}(0, t)\right)^{\frac{n}{2}\left(\frac{1}{m}-\frac{1}{2}\right)+\frac{\sigma-1}{2}+1} \lesssim\left(1+B_{\lambda}(0, \delta t)\right)^{-\varepsilon} .
$$

This motivates to introduce

$$
\overline{\mathcal{R}}_{2}(t):=\frac{\mu(\delta t)}{\lambda^{2}(\delta t)} \frac{\lambda^{2 p}(\delta t)}{\rho^{p}(\delta t)}\left(1+B_{\lambda}(0, \delta t)\right)^{-\alpha_{4}(p)+\varepsilon}\left(1+B_{\lambda}(0, t)\right)^{\frac{n}{2}\left(\frac{1}{m}-\frac{1}{2}\right)+\frac{\sigma-1}{2}+1} .
$$

Then, analogously as we did to estimate $\overline{\mathcal{N}}_{2}=\overline{\mathcal{N}}_{2}(t)$ in (4.2.21) we may also conclude that $\overline{\mathcal{R}}_{2}^{\prime}(t)<0$ by the condition $p>\tilde{p}_{2}(m, n, \sigma)$. This gives the desired estimates for $\bar{R}_{2}=\bar{R}_{2}(t)$. Therefore, we obtain

$$
\mathbf{R} \lesssim \frac{\lambda^{2}(t)}{\rho(t)}\left(1+B_{\lambda}(0, t)\right)^{-\frac{n}{2}\left(\frac{1}{m}-\frac{1}{2}\right)-\frac{\sigma-1}{2}-1} .
$$


In order to conclude the proof of the estimate (4.1.7), proceeding in the same way as in the proof of Theorem 4.2.2 and following the same steps of the proof of (4.1.6) we obtain the estimate

$$
\left\||D|^{\sigma}(N u-N v)(t, \cdot)\right\|_{L^{2}} \lesssim\left(1+B_{\lambda}(0, t)\right)^{-\frac{n}{2}\left(\frac{1}{m}-\frac{1}{2}\right)-\frac{\sigma}{2}}\|u-v\|_{X(t)}\left(\|u\|_{X(t)}^{p-1}+\|v\|_{X(t)}^{p-1}\right) .
$$

In the same way, with no other requirements to the admissible exponents $p$ one can derive the estimates for $\|(N u-N v)(t, \cdot)\|_{L^{2}},\left\|\partial_{t}(N u-N v)(t, \cdot)\right\|_{L^{2}}$ and $\left\||D|^{\sigma-1} \partial_{t}(N u-N v)(t, \cdot)\right\|_{L^{2}}$. Thus, from the definition of the norm of the solution space $X(t)$ we may conclude the proof of (4.1.7) in the Case 1.

Case 2: Let us assume that the second component of the functions $\Phi_{1, m}=\Phi_{1, m}(s, t), \Phi_{1}=$ $\Phi_{1}(s, t)$ and $\Phi_{2, m}=\Phi_{2, m}(s, t), \Phi_{2}=\Phi_{2}(s, t)$ is dominant, that is,

$$
\begin{array}{ll}
\Phi_{1, m}(s, t)=\lambda(t) F(\Lambda(t))\left(F^{2}(\Lambda(t))\right)^{-\frac{n}{2}\left(\frac{1}{m}-\frac{1}{2}\right)-1}, & \Phi_{1}(s, t)=\lambda(t) F(\Lambda(t))\left(F^{2}(\Lambda(t))\right)^{-1}, \\
\Phi_{2, m}(s, t)=\lambda(t) F(\Lambda(t))\left(F^{2}(\Lambda(t))\right)^{-\frac{n}{2}\left(\frac{1}{m}-\frac{1}{2}\right)-\frac{\sigma-1}{2}-1}, & \Phi_{2}(s, t)=\lambda(t) F(\Lambda(t))\left(F^{2}(\Lambda(t))\right)^{-\frac{\sigma-1}{2}-1},
\end{array}
$$

for all $t \geq s \geq 0$. From Theorem 4.2.2 we have

$$
\begin{aligned}
& \left\||D|^{\sigma} u^{\mathrm{nl}}(t, \cdot)\right\|_{L^{2}} \\
& \lesssim\|u\|_{X(t)}^{p} \underbrace{\int_{0}^{\delta t} \frac{\Lambda(s)}{\lambda(s)} \lambda^{p}(s) F^{p}(\Lambda(s))\left(1+B_{\lambda}(s, t)\right)^{-\frac{n}{2}\left(\frac{1}{m}-\frac{1}{2}\right)-\frac{\sigma}{2}}\left(F^{2}(\Lambda(s))\right)^{-\frac{n}{2 m} p+\frac{n}{2 m}-p} d s}_{\mathbf{S}} \\
& \quad+\|u\|_{X(t)}^{p} \underbrace{\int_{\delta t}^{t} \frac{\Lambda(s)}{\lambda(s)} \lambda^{p}(s) F^{p}(\Lambda(s))\left(1+B_{\lambda}(s, t)\right)^{-\frac{\sigma}{2}}\left(F^{2}(\Lambda(s))\right)^{-\frac{n}{2 m} p+\frac{n}{4}-p} d s}_{\mathbf{T}} .
\end{aligned}
$$

Let us consider the integral S. Using (3.1.18), we have the estimate

$$
\mathbf{S} \lesssim\left(1+B_{\lambda}(0, t)\right)^{-\frac{n}{2}\left(\frac{1}{m}-\frac{1}{2}\right)-\frac{\sigma}{2}} \underbrace{\int_{0}^{\delta t} \Lambda(s) \lambda^{p-1}(s) F(\Lambda(s))^{-\frac{n}{m} p+\frac{n}{m}-p} d s}_{S_{1}(t)} .
$$

Following the proof of Theorem 4.2.2 from (4.2.16), we may conclude the desired estimate

$$
\mathbf{S} \lesssim\left(1+B_{\lambda}(0, t)\right)^{-\frac{n}{2}\left(\frac{1}{m}-\frac{1}{2}\right)-\frac{\sigma}{2}}
$$

by the condition

$$
p>\frac{m r+n-m}{n+m} .
$$

Now let us consider the integral $\mathbf{T}$. We have

$$
\mathbf{T} \leq \underbrace{\int_{\delta t}^{t} \frac{\Lambda(s)}{\lambda(s)} \lambda^{p}(s) F^{p}(\Lambda(s))\left(F^{2}(\Lambda(s))\right)^{-\frac{n}{2 m} p+\frac{n}{4}-p} d s}_{\bar{T}_{1}(t)} .
$$

Then, proceeding in the same way to estimate the integral $S_{1}=S_{1}(t)$ and introducing $\alpha_{6}(p)=\frac{n}{m} p-\frac{n}{2}+p$ we find

$$
\begin{aligned}
\left(1+B_{\lambda}(0, t)\right)^{\frac{n}{2}\left(\frac{1}{m}-\frac{1}{2}\right)+\frac{\sigma}{2}} \bar{T}_{1}(t) & \\
& \lesssim \lambda^{p}(\delta t) \Lambda(\delta t) \Xi^{2}(\delta t) F(\Lambda(\delta t))^{-\alpha_{6}(p)-1}\left(1+B_{\lambda}(0, t)\right)^{\frac{n}{2}\left(\frac{1}{m}-\frac{1}{2}\right)+\frac{\sigma}{2}}:=\overline{\mathcal{T}}_{1}(t) .
\end{aligned}
$$


We want to show that the function $\overline{\mathcal{T}}_{1}=\overline{\mathcal{T}}_{1}(t)$ is uniformly bounded. Therefore, we estimate the derivative of this function as follows:

$$
\begin{aligned}
\overline{\mathcal{T}}_{1}^{\prime}(t) \leq[ & r \delta \lambda^{p+1}(\delta t) \Xi^{2}(\delta t)-\delta\left(\alpha_{6}(p)+1\right) \lambda^{p+1}(\delta t) \Xi^{2}(\delta t) \Lambda(\delta t) \frac{F^{\prime}(\Lambda(\delta t))}{F(\Lambda(\delta t))} \\
& \left.+\left(\frac{n}{2}\left(\frac{1}{m}-\frac{1}{2}\right)+\frac{\sigma}{2}\right) \lambda^{p+1}(\delta t) \Xi^{2}(\delta t) \frac{\Lambda(\delta t)}{\lambda(\delta t)} \frac{\lambda(t) \Lambda(t)}{\mu(t)} \frac{1}{1+B_{\lambda}(0, t)}\right] \\
& \times F(\Lambda(\delta t))^{-\alpha_{6}(p)-1}\left(1+B_{\lambda}(0, t)\right)^{\frac{n}{2}\left(\frac{1}{m}-\frac{1}{2}\right)+\frac{\sigma}{2}} \\
\leq[ & r \delta-\delta\left(\alpha_{6}(p)+1\right) \frac{\Lambda(\delta t)}{\lambda(\delta t)} \frac{F(\Lambda(\delta t))}{\lambda(\delta t) \Xi^{2}(\delta t)} \\
& \left.+\left(\frac{n}{2}\left(\frac{1}{m}-\frac{1}{2}\right)+\frac{\sigma}{2}\right)(\alpha+2) \frac{\Lambda(\delta t)}{\lambda(\delta t)} \frac{\lambda(t) \Lambda(t)}{\mu(t)} \frac{\mu(t)}{\Lambda^{2}(t)} \frac{1}{\log \Lambda(t)}\right] \\
& \times \lambda^{p+1}(\delta t) \Xi^{2}(\delta t) F(\Lambda(\delta t))^{-\alpha_{6}(p)-1}\left(1+B_{\lambda}(0, t)\right)^{\frac{n}{2}\left(\frac{1}{m}-\frac{1}{2}\right)+\frac{\sigma}{2}} \\
\leq[ & \left.r \delta-\delta\left(\alpha_{6}(p)+1\right) \vartheta(\delta, t)+\left(\frac{n}{2}\left(\frac{1}{m}-\frac{1}{2}\right)+\frac{\sigma}{2}\right)(\alpha+2) \frac{\nu(\delta, t)}{\log \Lambda(t)}\right] \\
& \times \lambda^{p+1}(\delta t) \Xi^{2}(\delta t) F(\Lambda(\delta t))^{-\alpha_{6}(p)-1}\left(1+B_{\lambda}(0, t)\right)^{\frac{n}{2}\left(\frac{1}{m}-\frac{1}{2}\right)+\frac{\sigma}{2}} .
\end{aligned}
$$

Then, for large time $t$ we have $\overline{\mathcal{T}}_{1}^{\prime}(t)<0$. Therefore, we arrive at the estimate

$$
\bar{T}_{1}(t) \lesssim\left(1+B_{\lambda}(0, t)\right)^{-\frac{n}{2}\left(\frac{1}{m}-\frac{1}{2}\right)-\frac{\sigma}{2}} .
$$

This estimate together with (4.2.25) concludes

$$
\left\||D|^{\sigma} u^{\mathrm{nl}}(t, \cdot)\right\|_{L^{2}} \lesssim\left(1+B_{\lambda}(0, t)\right)^{-\frac{n}{2}\left(\frac{1}{m}-\frac{1}{2}\right)-\frac{\sigma}{2}} .
$$

To estimate the norm $\left\||D|^{\sigma-1} u_{t}^{\mathrm{nl}}(t, \cdot)\right\|_{L^{2}}$ in the Case 2 we follow the proof of Theorem 4.2.2. Then, by the condition

$$
p>\frac{m r+n-m}{n+m}
$$

we may conclude the estimate

$$
\left\||D|^{\sigma-1} u_{t}^{\mathrm{nl}}(t, \cdot)\right\|_{L^{2}} \lesssim\|u\|_{X(t)}^{p} \lambda(t) F(\Lambda(t))\left(F^{2}(\Lambda(t))\right)^{-\frac{n}{2}\left(\frac{1}{m}-\frac{1}{2}\right)-\frac{\sigma-1}{2}-1} .
$$

Moreover, to prove (4.1.7) in the Case 2 again we follow the proof of Theorem 4.1.6 which completes the proof.

\subsection{Semilinear damped wave models of the generalized type power nonlinearity $\left.\left.|| D\right|^{a} u\right|^{p}$ and $a \in(0,1]$}

Now we consider the following semilinear Cauchy problem:

$$
\begin{cases}u_{t t}-\lambda^{2}(t) \omega^{2}(t) \Delta u+\rho(t) \omega(t) u_{t}=\left.\left.|| D\right|^{a} u\right|^{p}, & (t, x) \in[0, \infty) \times \mathbb{R}^{n}, \\ u(0, x)=u_{0}(x), \quad u_{t}(0, x)=u_{1}(x), & x \in \mathbb{R}^{n},\end{cases}
$$

where $a \in(0,1]$ and the data $\left(u_{0}, u_{1}\right) \in \mathcal{D}_{m}^{1}$ and $m \in[1,2)$.

Here the pseudo-differential operator $|D|^{a}, a>0$ is defined as follows:

$$
|D|^{a} u=F^{-1}\left(|\xi|^{a} F(u)\right) \text { for all } u \in H^{-\infty} .
$$

So, it is a nonlocal operator for $a \in(0,1]$. 


\subsubsection{Case of sub-exponential propagation speed in energy space}

We define the following parameters:

$$
\begin{aligned}
& p_{1}(a, m, n):=\frac{n}{n+a m}+\left(1-\frac{\beta_{1}}{2+R}\right) \frac{2 m}{n+a m} \\
& p_{2}(a, m, n):=\frac{n}{n+a m}+\left(1-\frac{\delta}{\nu(\delta)} \frac{\beta_{1}}{2+R}\right) \frac{2 m}{n+a m} \\
& p_{3}(a, m, n) \\
& :=\max \left\{\frac{n m}{2(n+a m)}+\frac{n\left(1-\frac{m}{2}\right)}{(n+a m)\left(1-\frac{2+R}{2-\mu_{1}}+\frac{2+R}{2-\mu_{1}} \frac{\delta}{\nu(\delta)}\right)}+\left(\frac{1-\frac{\beta_{1}}{2+R} \frac{\delta}{\nu(\delta)}}{1-\frac{2+R}{2-\mu_{1}}+\frac{2+R}{2-\mu_{1}} \frac{\delta}{\nu(\delta)}}\right) \frac{2 m}{n+a m} ;\right. \\
& \left.\frac{n}{n+a m}+\left(1-\frac{\beta_{1}}{2} \frac{\delta}{\vartheta(\delta)}\right) \frac{2 m}{n+a m}\right\}
\end{aligned}
$$

where we set

$$
p(a, m, n):=\max \left\{p_{1}(a, m, n) ; p_{2}(a, m, n) ; p_{3}(a, m, n)\right\} .
$$

Theorem 4.3.1. We assume that the Hypotheses (A1) to (A5), (B1) to (B6), (C1), (C2) and $\left(C_{4}\right)$ are satisfied. Let us consider $\nu(\delta) \lesssim 1$ and $\vartheta(\delta) \lesssim 1$, where the functions $\nu=\nu(\delta)$ and $\vartheta=\vartheta(\delta)$ are defined in (3.1.2) and (3.1.3), respectively. Let the data $\left(u_{0}, u_{1}\right) \in \mathcal{D}_{m}^{1}$ and $m \in[1,2)$. Moreover, we assume that the exponent $p$ satisfies

$$
p>p(a, m, n) \quad \text { and } \quad p>\frac{2}{m} .
$$

Finally, we consider the following condition:

$$
\frac{\beta_{1}}{\frac{n}{2}\left(\frac{1}{m}-\frac{1}{2}\right)}>\max \left\{(2+R) \frac{\nu(\delta)}{\delta} ; 2 \frac{\vartheta(\delta)}{\delta}\right\},
$$

where the parameters $\beta_{1}$ and $R$ are from condition $(\boldsymbol{C} 4)$ and (3.1.17), respectively.

Then, there exists a constant $\varepsilon_{0}>0$ such that for all $\left(u_{0}, u_{1}\right) \in \mathcal{D}_{m}^{\sigma}$ with $\left\|\left(u_{0}, u_{1}\right)\right\|_{\mathcal{D}_{m}^{\sigma}} \leq \varepsilon_{0}$, there is a uniquely determined energy solution

$$
u \in \mathcal{C}\left([0, \infty), H^{1}\right) \cap \mathcal{C}^{1}\left([0, \infty), L^{2}\right)
$$

to the Cauchy problem (4.3.1). Furthermore, there the solution satisfies the estimates

$$
\begin{aligned}
\|u(t, \cdot)\|_{L^{2}} & \lesssim\left(1+B_{\lambda}(0, t)\right)^{-\frac{n}{2}\left(\frac{1}{m}-\frac{1}{2}\right)}\left\|\left(u_{0}, u_{1}\right)\right\|_{\mathcal{D}_{m}^{1}} \\
\||D| u(t, \cdot)\|_{L^{2}} & \lesssim\left(1+B_{\lambda}(0, t)\right)^{-\frac{n}{2}\left(\frac{1}{m}-\frac{1}{2}\right)-\frac{1}{2}}\left\|\left(u_{0}, u_{1}\right)\right\|_{\mathcal{D}_{m}^{1}} \\
\left\|u_{t}(t, \cdot)\right\|_{L^{2}} & \lesssim \max \left\{\frac{\lambda^{2}(t)}{\rho(t)}\left(1+B_{\lambda}(0, t)\right)^{-\frac{n}{2}\left(\frac{1}{m}-\frac{1}{2}\right)-1} ; \lambda(t) F(\Lambda(t))\left(F^{2}(\Lambda(t))\right)^{-\frac{n}{2}\left(\frac{1}{m}-\frac{1}{2}\right)-1}\right\}\left\|\left(u_{0}, u_{1}\right)\right\|_{\mathcal{D}_{m}^{1}} .
\end{aligned}
$$

Proof. We introduce for all $t>0$ the functions spaces

$$
X(t)=\mathcal{C}\left([0, t], H^{1}\right) \cap \mathcal{C}^{1}\left([0, t], L^{2}\right)
$$

with the norm

$$
\begin{aligned}
\|u(\tau, \cdot)\|_{X(t)}=\sup _{0 \leq \tau \leq t}[ & \left(1+B_{\lambda}(0, \tau)\right)^{\frac{n}{2}\left(\frac{1}{m}-\frac{1}{2}\right)}\|u(\tau, \cdot)\|_{L^{2}} \\
& \left.+\left(1+B_{\lambda}(0, \tau)\right)^{\frac{n}{2}\left(\frac{1}{m}-\frac{1}{2}\right)+\frac{1}{2}}\||D| u(\tau, \cdot)\|_{L^{2}}+\Phi_{1, m}^{-1}(0, \tau)\left\|u_{t}(\tau, \cdot)\right\|_{L^{2}}\right] .
\end{aligned}
$$

Here $\Phi_{1, m}=\Phi_{1, m}(0, \tau)$ is defined in (4.1.2) with $s=0$ and $t=\tau$. 
To complete the proof of (4.1.6) we have to estimate $\left\|u^{\mathrm{nl}}\right\|_{X(t)}$. For this reason we use

$$
\left\||D|^{j} \partial_{t}^{\ell} u^{\mathrm{nl}}(t, \cdot)\right\|_{L^{2}} \lesssim \int_{0}^{t}\left\||D|^{j} \partial_{t}^{\ell}\left(\left.\left.K_{1}(t, s, x) *(x)|| D\right|^{a} u(s, x)\right|^{p}\right)\right\|_{L^{2}} d s
$$

for $j+\ell=0,1$. If $s \in[0, \delta t]$, then we use Theorem 3.1.1 with $m \in[1,2)$ and if $s \in[\delta t, t]$ we use it for $m=2$ only. So, it follows

$$
\begin{aligned}
\left\||D|^{j} \partial_{t}^{\ell} u^{\mathrm{nl}}(t, \cdot)\right\|_{L^{2}} \lesssim & \int_{0}^{\delta t} \Phi_{1, m}^{\ell}(s, t) \frac{\Lambda(s)}{\lambda(s)}\left(1+B_{\lambda}(s, t)\right)^{(\ell-1) \frac{n}{2}\left(\frac{1}{m}-\frac{1}{2}\right)-\frac{j}{2}}\left\|\left.\left.|| D\right|^{a} u(s, \cdot)\right|^{p}\right\|_{L^{m} \cap L^{2}} d s \\
& +\int_{\delta t}^{t} \Phi_{1}^{\ell}(s, t) \frac{\Lambda(s)}{\lambda(s)}\left(1+B_{\lambda}(s, t)\right)^{-\frac{j}{2}}\left\|\left.\left.|| D\right|^{a} u(s, \cdot)\right|^{p}\right\|_{L^{2}} d s
\end{aligned}
$$

for $j+\ell=0,1$. Here in the case $\ell=0$ we define $\Phi_{1, m}^{0}(s, t) \equiv 1$ and $\Phi_{1}^{0}(s, t) \equiv 1$ and, in the case $\ell=1$ the functions $\Phi_{1, m}^{1}(s, t)=\Phi_{1, m}(s, t)$ and $\Phi_{1}^{1}(s, t)=\Phi_{1}(s, t)$ are defined in (4.1.2) and (4.1.3), respectively.

Taking account of

$$
\left\|\left.\left.|| D\right|^{a} u(s, \cdot)\right|^{p}\right\|_{L^{m} \cap L^{2}} \lesssim\left\||D|^{a} u(s, \cdot)\right\|_{L^{m p}}^{p}+\left\||D|^{a} u(s, \cdot)\right\|_{L^{2 p}}^{p}
$$

and using the fractional Gagliardo-Nirenberg inequality we get for $p \geq \frac{2}{m}$ the estimates

$$
\begin{aligned}
\left\||D|^{a} u(s, \cdot)\right\|_{L^{m p}}^{p} & \lesssim\left\||D|^{\sigma} u(s, \cdot)\right\|_{L^{2}}^{p \theta_{\sigma, a}(m p)}\|u(s, \cdot)\|_{L^{2}}^{p\left(1-\theta_{\sigma, a}(m p)\right)} \\
& \lesssim\left(1+B_{\lambda}(0, s)\right)^{-\frac{n}{2 m} p+\frac{n}{2 m}-\frac{a}{2} p}\|u\|_{X(s)}^{p}, \\
\left\||D|^{a} u(s, \cdot)\right\|_{L^{2 p}}^{p} & \lesssim\left\||D|^{\sigma} u(s, \cdot)\right\|_{L^{2}}^{p \theta_{\sigma, a}(2 p)}\|u(s, \cdot)\|_{L^{2}}^{p\left(1-\theta_{\sigma, a}(2 p)\right)} \\
& \lesssim\left(1+B_{\lambda}(0, s)\right)^{-\frac{n}{2 m} p+\frac{n}{4}-\frac{a}{2} p}\|u\|_{X(s)}^{p},
\end{aligned}
$$

where $\theta_{\sigma, a}(q)=\frac{n}{\sigma}\left(\frac{1}{2}-\frac{1}{q}+\frac{a}{n}\right)$ and $\frac{a}{\sigma} \leq \theta_{\sigma, a}(q) \leq 1$.

Now we are able to estimate $\left\|\left.\left.|| D\right|^{a} u(s, \cdot)\right|^{p}\right\|_{L^{m} \cap L^{2}}$ by using (4.3.3) and (4.3.4). Therefore, using $\|u\|_{X(s)} \leq\|u\|_{X(t)}$ for $0 \leq s \leq t$ we find

$$
\begin{aligned}
& \left\||D|^{j} \partial_{t}^{\ell} u^{\mathrm{nl}}(t, \cdot)\right\|_{L^{2}} \\
& \lesssim\|u\|_{X(t)}^{p} \underbrace{\int_{0}^{\delta t} \Phi_{1, m}^{\ell}(s, t) \frac{\Lambda(s)}{\lambda(s)}\left(1+B_{\lambda}(s, t)\right)^{(\ell-1) \frac{n}{2}\left(\frac{1}{m}-\frac{1}{2}\right)-\frac{j}{2}}\left(1+B_{\lambda}(0, s)\right)^{-\frac{n}{2 m} p+\frac{n}{2 m}-\frac{a}{2} p} d s}_{\mathbf{V}} \\
& \quad+\|u\|_{X(t)}^{p} \underbrace{\int_{\delta t}^{t} \Phi_{1}^{\ell}(s, t) \frac{\Lambda(s)}{\lambda(s)}\left(1+B_{\lambda}(s, t)\right)^{-\frac{j}{2}}\left(1+B_{\lambda}(0, s)\right)^{-\frac{n}{2 m} p+\frac{n}{4}-\frac{a}{2} p}}_{\mathbf{Y}} d s .
\end{aligned}
$$

Now we want to estimate the integral terms $\mathbf{V}$ and $\mathbf{Y}$ in order to get the desired estimates. We have

$$
\mathbf{V} \lesssim \Phi_{1, m}^{\ell}(0, t)\left(1+B_{\lambda}(0, t)\right)^{(\ell-1) \frac{n}{2}\left(\frac{1}{m}-\frac{1}{2}\right)-\frac{j}{2}} \underbrace{\int_{0}^{\delta t} \frac{\Lambda(s)}{\lambda(s)}\left(1+B_{\lambda}(0, s)\right)^{-\frac{n}{2 m} p+\frac{n}{2 m}-\frac{a}{2} p} d s}_{V_{1}(t)},
$$

where we used (3.1.18). Denoting $\alpha_{1, a}(p):=\frac{n}{2 m} p-\frac{n}{2 m}+\frac{a}{2} p$ we get for $\alpha_{1, a}(p) \neq 1$ the relation

$$
\begin{aligned}
V_{1}(t)= & \int_{0}^{\delta t} \frac{\mu(s)}{\lambda^{2}(s)}\left(1+B_{\lambda}(0, s)\right)^{-\alpha_{1, a}(p)} d\left(1+B_{\lambda}(0, s)\right) \\
= & \left.\frac{1}{1-\alpha_{1, a}(p)} \frac{\mu(s)}{\lambda^{2}(s)}\left(1+B_{\lambda}(0, s)\right)^{-\alpha_{1, a}(p)+1}\right|_{0} ^{\delta t} \\
& -\frac{1}{1-\alpha_{1, a}(p)} \int_{0}^{\delta t}\left(1+B_{\lambda}(0, s)\right)^{-\alpha_{1, a}(p)+1} d\left(\frac{\mu(s)}{\lambda^{2}(s)}\right) .
\end{aligned}
$$


Now we shall distinguish between three cases.

Case 1: $\alpha_{1, a}(p)>1$, i.e., $p>\frac{2 m+n}{a m+n}$. In this case it holds

$$
\begin{aligned}
V_{1}(t) \leq & \left.\frac{1}{1-\alpha_{1, a}(p)} \frac{\mu(s)}{\lambda^{2}(s)}\left(1+B_{\lambda}(0, s)\right)^{-\alpha_{1, a}(p)+1}\right|_{0} ^{\delta t} \\
& -\frac{\beta_{1}}{\alpha_{1, a}(p)-1} \int_{0}^{\delta t}\left(1+B_{\lambda}(0, s)\right)^{-\alpha_{1, a}(p)}\left(1+B_{\lambda}(0, s)\right) \frac{\mu(s)}{\lambda(s) \Lambda(s)} d s \\
\leq & \left.\frac{1}{1-\alpha_{1, a}(p)} \frac{\mu(s)}{\lambda^{2}(s)}\left(1+B_{\lambda}(0, s)\right)^{-\alpha_{1, a}(p)+1}\right|_{0} ^{\delta t} \\
& -\frac{\beta_{1}}{\left(\alpha_{1, a}(p)-1\right)(2+R)} \underbrace{\int_{0}^{\delta t} \frac{\Lambda(s)}{\lambda(s)}\left(1+B_{\lambda}(0, s)\right)^{-\alpha_{1, a}(p)} d s}_{V_{1}(t)},
\end{aligned}
$$

where we used condition (C4) and (3.1.17), respectively. Thus, we obtain

$$
V_{1}(t) \lesssim \frac{\mu(0)}{\lambda^{2}(0)}-\frac{\mu(\delta t)}{\lambda^{2}(\delta t)}\left(1+B_{\lambda}(0, \delta t)\right)^{-\alpha_{1, a}(p)+1} \lesssim 1
$$

Case 2: $\alpha_{1, a}(p)<1$. In this case we have the estimate

$$
\begin{aligned}
V_{1}(t) \geq & \left.\frac{1}{1-\alpha_{1, a}(p)} \frac{\mu(s)}{\lambda^{2}(s)}\left(1+B_{\lambda}(0, s)\right)^{-\alpha_{1, a}(p)+1}\right|_{0} ^{\delta t} \\
& +\frac{\beta_{1}}{1-\alpha_{1, a}(p)} \int_{0}^{\delta t}\left(1+B_{\lambda}(0, s)\right)^{-\alpha_{1, a}(p)}\left(1+B_{\lambda}(0, s)\right) \frac{\mu(s)}{\lambda(s) \Lambda(s)} d s \\
\geq & \left.\frac{1}{1-\alpha_{1, a}(p)} \frac{\mu(s)}{\lambda^{2}(s)}\left(1+B_{\lambda}(0, s)\right)^{-\alpha_{1, a}(p)+1}\right|_{0} ^{\delta t} \\
& +\frac{\beta_{1}}{\left(1-\alpha_{1, a}(p)\right)(2+R)} \underbrace{\int_{0}^{\delta t} \frac{\Lambda(s)}{\lambda(s)}\left(1+B_{\lambda}(0, s)\right)^{-\alpha_{1, a}(p)} d s}_{V_{1}(t)} .
\end{aligned}
$$

Then, it follows

$$
\left(\frac{\beta_{1}}{\left(1-\alpha_{1, a}(p)\right)(2+R)}-1\right) V_{1} \lesssim \frac{\mu(0)}{\lambda^{2}(0)}-\frac{\mu(\delta t)}{\lambda^{2}(\delta t)}\left(1+B_{\lambda}(0, \delta t)\right)^{-\alpha_{1, a}(p)+1} .
$$

We can guarantee that $V_{1}=V_{1}(t)$ is bounded by the condition $p>p_{1}(a, m, n)$. Because,

$$
\frac{\beta_{1}}{\left(1-\alpha_{1, a}(p)\right)(2+R)}-1>0, \quad \text { follows from } \quad p>\frac{n}{n+a m}+\left(1-\frac{\beta_{1}}{2+R}\right) \frac{2 m}{n+a m},
$$

which we have assumed in the statement of the theorem. Otherwise, we need to show that

$$
\mathcal{V}_{1}(\delta t):=\frac{\mu(\delta t)}{\lambda^{2}(\delta t)}\left(1+B_{\lambda}(0, \delta t)\right)^{-\alpha_{1, a}(p)+1}
$$

is strictly decreasing in $t$. Indeed, taking the derivative of the function $\mathcal{V}_{1}=\mathcal{V}_{1}(t)$ we get

$$
\mathcal{V}_{1}^{\prime}(t) \leq\left[-\frac{\beta_{1}}{2+R}-\alpha_{1, a}(p)+1\right] \frac{\Lambda(t)}{\lambda(t)}\left(1+B_{\lambda}(0, t)\right)^{-\alpha_{1, a}(p)}
$$


The condition $p>p_{1}(a, m, n)$ implies that $\mathcal{V}_{1}^{\prime}(t)<0$. This shows us that the right-hand side of (4.3.7) is bounded.

Case 3: $\alpha_{1, a}(p)=1$. In this final case we have

$$
\begin{aligned}
V_{1}(t) & =\int_{0}^{\delta t} \frac{\Lambda(s)}{\lambda(s)}\left(1+B_{\lambda}(0, s)\right)^{-1} d s \lesssim \int_{0}^{\delta t} \frac{\Lambda(s)}{\lambda(s)} \frac{\mu(s)}{\Lambda^{2}(s)} d s=\int_{0}^{\delta t} \frac{\mu(s)}{\lambda^{2}(s)} \frac{\lambda(s)}{\Lambda(s)} d s \\
& =\left.\frac{\mu(s)}{\lambda^{2}(s)} \log \Lambda(s)\right|_{0} ^{\delta t}-\int_{0}^{\delta t} \log \Lambda(s) d\left(\frac{\mu(s)}{\lambda^{2}(s)}\right) \\
& \left.\lesssim \frac{\mu(s)}{\lambda^{2}(s)} \log \Lambda(s)\right|_{0} ^{\delta t}+\int_{0}^{\delta t} \frac{\mu(s)}{\lambda^{2}(s)} \log \Lambda(s) \frac{\lambda(s)}{\Lambda(s)} d s
\end{aligned}
$$

where we have used (3.1.17) and condition (C4), respectively. Now we will show that for a sufficiently small positive constant $\varepsilon$ we have for large $t$ the estimate

$$
\frac{\mu(t)}{\lambda^{2}(t)} \log \Lambda(t) \lesssim \Lambda^{-\varepsilon}(t)
$$

Therefore, we form the derivative of the function

$$
\mathcal{V}_{2}(t):=\frac{\mu(t)}{\lambda^{2}(t)} \Lambda^{\varepsilon}(t) \log \Lambda(t)
$$

Using condition (C4) it holds

$$
\mathcal{V}_{2}^{\prime}(t) \leq\left[-\beta_{1}+\varepsilon+\frac{1}{\log \Lambda(t)}\right] \frac{\mu(t)}{\lambda(t) \Lambda(t)} \log \Lambda(t) \Lambda^{\varepsilon}(t) .
$$

Hence, for a sufficiently large time $t$ and a sufficiently small constant $\varepsilon>0$ we get $\mathcal{V}_{2}^{\prime}(t)<0$. This shows the decreasing behavior of $\mathcal{V}_{2}=\mathcal{V}_{2}(t)$ for large $t$. Hence, we obtain that $V_{1}=V_{1}(t)$ is uniformly bounded. Summarizing, from the above three cases for $j+\ell=0,1$ we get our desired estimate

$$
\mathbf{V} \lesssim \Phi_{1, m}^{\ell}(0, t)\left(1+B_{\lambda}(0, t)\right)^{(\ell-1) \frac{n}{2}\left(\frac{1}{m}-\frac{1}{2}\right)-\frac{j}{2}} .
$$

Now let us consider the integral $\mathbf{Y}$. We have

$$
\begin{aligned}
\mathbf{Y} & =\int_{\delta t}^{t} \Phi_{1}^{\ell}(s, t) \frac{\Lambda(s)}{\lambda(s)}\left(1+B_{\lambda}(s, t)\right)^{-\frac{j}{2}}\left(1+B_{\lambda}(0, s)\right)^{-\alpha_{2, a}(p)} d s \\
& =-\int_{\delta t}^{t} \Phi_{1}^{\ell}(s, t) \frac{\mu(s)}{\lambda^{2}(s)}\left(1+B_{\lambda}(0, s)\right)^{-\alpha_{2, a}(p)}\left(1+B_{\lambda}(s, t)\right)^{-\frac{j}{2}} d\left(1+B_{\lambda}(s, t)\right),
\end{aligned}
$$

where $\alpha_{2, a}(p):=\frac{n}{2 m} p-\frac{n}{4}+\frac{a}{2} p$. To obtain the desired estimates we consider separately the cases $j+\ell=0,1$.

For $j=\ell=0$ and $\alpha_{2, a}(p) \neq 1$ we get

$$
\begin{aligned}
\mathbf{Y}= & \int_{\delta t}^{t} \frac{\mu(s)}{\lambda^{2}(s)}\left(1+B_{\lambda}(0, s)\right)^{-\alpha_{2, a}(p)} d\left(1+B_{\lambda}(0, s)\right) \\
= & \left.\frac{1}{1-\alpha_{2, a}(p)} \frac{\mu(s)}{\lambda^{2}(s)}\left(1+B_{\lambda}(0, s)\right)^{-\alpha_{2, a}(p)+1}\right|_{\delta t} ^{t} \\
& -\frac{1}{1-\alpha_{2, a}(p)} \int_{\delta t}^{t}\left(1+B_{\lambda}(0, s)\right)^{-\alpha_{2, a}(p)+1} d\left(\frac{\mu(s)}{\lambda^{2}(s)}\right) .
\end{aligned}
$$


Case 1: $\alpha_{2, a}(p)>1$. In this case it holds

$$
\begin{aligned}
\mathbf{Y} \leq & \left.\frac{1}{1-\alpha_{2}(p)} \frac{\mu(s)}{\lambda^{2}(s)}\left(1+B_{\lambda}(0, s)\right)^{-\alpha_{2, a}(p)+1}\right|_{\delta t} ^{t} \\
& +\frac{\beta_{1}}{1-\alpha_{2, a}(p)} \int_{\delta t}^{t}\left(1+B_{\lambda}(0, s)\right)^{-\alpha_{2, a}(p)}\left(1+B_{\lambda}(0, s)\right) \frac{\mu(s)}{\lambda(s) \Lambda(s)} d s \\
\leq & \left.\frac{1}{1-\alpha_{2, a}(p)} \frac{\mu(s)}{\lambda^{2}(s)}\left(1+B_{\lambda}(0, s)\right)^{-\alpha_{2, a}(p)+1}\right|_{\delta t} ^{t} \\
& +\frac{\beta_{1}}{\left(1-\alpha_{2, a}(p)\right)(2+R)} \underbrace{\int_{\delta t}^{t} \frac{\Lambda(s)}{\lambda(s)}\left(1+B_{\lambda}(0, s)\right)^{-\alpha_{2, a}(p)} d s}_{\mathbf{Y}},
\end{aligned}
$$

where we used condition (C4) and (3.1.17), respectively. Then, we get

$$
\mathbf{Y} \lesssim \frac{\mu(\delta t)}{\lambda^{2}(\delta t)}\left(1+B_{\lambda}(0, \delta t)\right)^{-\alpha_{2, a}(p)+1}-\frac{\mu(t)}{\lambda^{2}(t)}\left(1+B_{\lambda}(0, t)\right)^{-\alpha_{2, a}(p)+1} .
$$

Case 2: $\alpha_{2, a}(p)<1$. In this case we have the estimates

$$
\begin{aligned}
\mathbf{Y} \geq & \left.\frac{1}{1-\alpha_{2, a}(p)} \frac{\mu(s)}{\lambda^{2}(s)}\left(1+B_{\lambda}(0, s)\right)^{-\alpha_{2, a}(p)+1}\right|_{\delta t} ^{t} \\
& +\frac{\beta_{1}}{1-\alpha_{2, a}(p)} \int_{\delta t}^{t}\left(1+B_{\lambda}(0, s)\right)^{-\alpha_{2, a}(p)}\left(1+B_{\lambda}(0, s)\right) \frac{\mu(s)}{\lambda(s) \Lambda(s)} d s \\
\geq & \left.\frac{1}{1-\alpha_{2, a}(p)} \frac{\mu(s)}{\lambda^{2}(s)}\left(1+B_{\lambda}(0, s)\right)^{-\alpha_{2, a}(p)+1}\right|_{\delta t} ^{t} \\
& +\frac{\beta_{1}}{\left(1-\alpha_{2, a}(p)\right)(2+R)} \underbrace{\int_{\delta t}^{t} \frac{\Lambda(s)}{\lambda(s)}\left(1+B_{\lambda}(0, s)\right)^{-\alpha_{2, a}(p)} d s}_{\mathbf{Y}},
\end{aligned}
$$

where we have used (3.1.17). This implies

$$
\left(\frac{\beta_{1}}{\left(1-\alpha_{2, a}(p)\right)(2+R)}-1\right) \mathbf{Y} \lesssim \frac{\mu(\delta t)}{\lambda^{2}(\delta t)}\left(1+B_{\lambda}(0, \delta t)\right)^{-\alpha_{2, a}(p)+1}-\frac{\mu(t)}{\lambda^{2}(t)}\left(1+B_{\lambda}(0, t)\right)^{-\alpha_{2, a}(p)+1} .
$$

Here we can guarantee that

$$
\frac{\beta_{1}}{\left(1-\alpha_{2, a}(p)\right)(2+R)}-1>0, \quad \text { or equivalently } \quad p>\frac{n m}{2(a m+n)}+\left(1-\frac{\beta_{1}}{2+R}\right) \frac{2 m}{a m+n} .
$$

It is clear that the above condition for $p$ is satisfied by the condition $p>p_{1}(a, m, n)$. Then, for all $p>p_{1}(a, m, n)$ it holds

$$
\mathbf{Y} \lesssim \frac{\mu(\delta t)}{\lambda^{2}(\delta t)}\left(1+B_{\lambda}(0, \delta t)\right)^{-\alpha_{2, a}(p)+1}-\frac{\mu(t)}{\lambda^{2}(t)}\left(1+B_{\lambda}(0, t)\right)^{-\alpha_{2, a}(p)+1} .
$$

Now to get the desired estimate for $\mathbf{Y}$ in the case $j=\ell=0$ we will show the following estimate:

$$
\begin{aligned}
& \left(1+B_{\lambda}(0, t)\right)^{\frac{n}{2}\left(\frac{1}{m}-\frac{1}{2}\right)} \mathbf{Y} \\
& \lesssim \frac{\mu(\delta t)}{\lambda^{2}(\delta t)}\left(1+B_{\lambda}(0, t)\right)^{\frac{n}{2}\left(\frac{1}{m}-\frac{1}{2}\right)}\left(1+B_{\lambda}(0, \delta t)\right)^{-\alpha_{2, a}(p)+1}-\frac{\mu(t)}{\lambda^{2}(t)}\left(1+B_{\lambda}(0, t)\right)^{-\alpha_{1, a}(p)+1} \lesssim 1,
\end{aligned}
$$

where we used the fact that $\alpha_{2, a}(p)=\alpha_{1, a}(p)+\frac{n}{2}\left(\frac{1}{m}-\frac{1}{2}\right)$. The second summand on the right-hand side of previous estimate, that is,

$$
\mathcal{Y}_{1}(t):=\frac{\mu(t)}{\lambda^{2}(t)}\left(1+B_{\lambda}(0, t)\right)^{-\alpha_{1, a}(p)+1}
$$


is bounded due to the condition $p>p_{1}(a, m, n)$. Indeed, we have

$$
\mathcal{Y}_{1}^{\prime}(t) \leq\left[-\frac{\beta_{1}}{2+R}-\alpha_{1, a}(p)+1\right] \frac{\Lambda(t)}{\lambda(t)}\left(1+B_{\lambda}(0, t)\right)^{-\alpha_{1, a}(p)} .
$$

Then, we can get

$$
\frac{-\beta_{1}}{2+R}-\alpha_{1, a}(p)+1<0, \quad \text { if } \quad p>\frac{n}{n+a m}+\left(1-\frac{\beta_{1}}{2+R}\right) \frac{2 m}{n+a m} .
$$

Hence, we have $\mathcal{Y}_{1}^{\prime}(t)<0$ by $p>p_{1}(a, m, n)$, namely, $\mathcal{Y}_{1}=\mathcal{Y}_{1}(t)$ is decreasing. Therefore, it remains to ensure the boundedness of the term

$$
\frac{\mu(\delta t)}{\lambda^{2}(\delta t)}\left(1+B_{\lambda}(0, t)\right)^{\frac{n}{2}\left(\frac{1}{m}-\frac{1}{2}\right)}\left(1+B_{\lambda}(0, \delta t)\right)^{-\alpha_{2, a}(p)+1}
$$

in both cases $1-\alpha_{2, a}(p)>0$ and $1-\alpha_{2, a}(p)<0$. In the case $1-\alpha_{2, a}(p)>0$ we have

$$
\begin{aligned}
& \frac{\mu(\delta t)}{\lambda^{2}(\delta t)}\left(1+B_{\lambda}(0, t)\right)^{\frac{n}{2}\left(\frac{1}{m}-\frac{1}{2}\right)}\left(1+B_{\lambda}(0, \delta t)\right)^{-\alpha_{2, a}(p)+1} \\
& \quad \lesssim \frac{\mu(\delta t)}{\lambda^{2}(\delta t)}\left(1+B_{\lambda}(0, t)\right)^{\frac{n}{2}\left(\frac{1}{m}-\frac{1}{2}\right)-\alpha_{2, a}(p)+1}=\frac{\mu(\delta t)}{\lambda^{2}(\delta t)}\left(1+B_{\lambda}(0, t)\right)^{-\alpha_{1, a}(p)+1}:=\mathcal{Y}_{2}(t) .
\end{aligned}
$$

To guarantee that the function $\mathcal{Y}_{2}=\mathcal{Y}_{2}(t)$ is decreasing we estimate its derivative as follows:

$$
\mathcal{Y}_{2}^{\prime} \leq\left[-\frac{\delta \beta_{1}}{2+R}+\left(-\alpha_{1, a}(p)+1\right) \nu(\delta, t)\right] \frac{\mu(\delta t)}{\lambda^{2}(\delta t)} \frac{\lambda(\delta t)}{\Lambda(\delta t)} \frac{\Lambda^{2}(t)}{\mu(t)}\left(1+B_{\lambda}(0, t)\right)^{-\alpha_{1, a}(p)} .
$$

Then, for a large time $t$ we can guarantee that $\mathcal{Y}_{2}^{\prime}(t)<0$ by

$$
\frac{-\delta \beta_{1}}{2+R}+\left(-\alpha_{1, a}(p)+1\right) \nu(\delta)<0,
$$

and this can be concluded from $p>p_{2}(a, m, n)$. In the other case $1-\alpha_{2, a}(p)<0$, our desired estimate can be obtained directly from the case $1-\alpha_{2, a}(p)>0$. Therefore, for all $p>p_{1}(a, m, n)$ and $p>p_{2}(a, m, n)$ we arrive at the expected estimate

$$
\mathbf{Y} \lesssim\left(1+B_{\lambda}(0, t)\right)^{-\frac{n}{2}\left(\frac{1}{m}-\frac{1}{2}\right)} .
$$

Case 3: $\alpha_{2, a}(p)=1$. In this case it holds for large $t$

$$
\begin{aligned}
\mathbf{Y} & =\int_{\delta t}^{t} \frac{\Lambda(s)}{\lambda(s)}\left(1+B_{\lambda}(0, s)\right)^{-1} d s \lesssim \int_{\delta t}^{t} \frac{\Lambda(s)}{\lambda(s)} \frac{\mu(s)}{\Lambda^{2}(s)} d s \\
& =\int_{\delta t}^{t} \frac{\mu(s)}{\lambda^{2}(s)} \frac{\lambda(s)}{\Lambda(s)} d s=\left.\frac{\mu(s)}{\lambda^{2}(s)} \log \Lambda(s)\right|_{\delta t} ^{t}-\int_{\delta t}^{t} \log \Lambda(s) d\left(\frac{\mu(s)}{\lambda^{2}(s)}\right) \\
& \lesssim \frac{\mu(t)}{\lambda^{2}(t)} \log \Lambda(t)-\frac{\mu(\delta t)}{\lambda^{2}(\delta t)} \log \Lambda(\delta t)+\int_{\delta t}^{t} \frac{\mu(s)}{\lambda^{2}(s)} \log \Lambda(s) \frac{\lambda(s)}{\Lambda(s)} d s
\end{aligned}
$$

where we have used (3.1.17) and condition (C4), respectively. Then, it follows

$$
\begin{aligned}
\left(1+B_{\lambda}(0, t)\right)^{\frac{n}{2}\left(\frac{1}{m}-\frac{1}{2}\right)} \mathbf{Y} \lesssim & \frac{\mu(t)}{\lambda^{2}(t)}\left(1+B_{\lambda}(0, t)\right)^{\frac{n}{2}\left(\frac{1}{m}-\frac{1}{2}\right)} \log \Lambda(t) \\
& +\int_{\delta t}^{t} \frac{\mu(s)}{\lambda^{2}(s)}\left(1+B_{\lambda}(0, t)\right)^{\frac{n}{2}\left(\frac{1}{m}-\frac{1}{2}\right)} \log \Lambda(s) \frac{\lambda(s)}{\Lambda(s)} d s .
\end{aligned}
$$

In order to prove that $\left(1+B_{\lambda}(0, t)\right)^{\frac{n}{2}\left(\frac{1}{m}-\frac{1}{2}\right)} \mathbf{Y}$ is bounded, we will show that for a sufficiently small positive constant $\varepsilon$ we have the estimate

$$
\frac{\mu(\delta t)}{\lambda^{2}(\delta t)}\left(1+B_{\lambda}(0, t)\right)^{\frac{n}{2}\left(\frac{1}{m}-\frac{1}{2}\right)} \log \Lambda(\delta t) \lesssim \Lambda^{-\varepsilon}(\delta t)
$$


So, it suffices to estimate the derivative of the function

$$
\mathcal{Y}_{3}(t):=\frac{\mu(\delta t)}{\lambda^{2}(\delta t)} \Lambda^{\varepsilon}(\delta t)\left(1+B_{\lambda}(0, t)\right)^{\frac{n}{2}\left(\frac{1}{m}-\frac{1}{2}\right)} \log \Lambda(\delta t) .
$$

Indeed, it holds

$$
\begin{aligned}
\mathcal{Y}_{3}^{\prime}(t) \leq & {\left[-\beta_{1}+\varepsilon+(2+R) \frac{n}{2}\left(\frac{1}{m}-\frac{1}{2}\right) \frac{\nu(\delta, t)}{\delta}+\frac{1}{\log \Lambda(\delta t)}\right] } \\
& \times \delta \frac{\mu(\delta t)}{\lambda^{2}(\delta t)} \frac{\lambda(\delta t)}{\Lambda(\delta t)} \Lambda^{\varepsilon}(\delta t)\left(1+B_{\lambda}(0, t)\right)^{\frac{n}{2}\left(\frac{1}{m}-\frac{1}{2}\right)} \log \Lambda(\delta t) .
\end{aligned}
$$

Then, for sufficiently large time $t$ and a sufficiently small constant $\varepsilon>0$, if

$$
(2+R) \frac{n}{2}\left(\frac{1}{m}-\frac{1}{2}\right) \frac{\nu(\delta)}{\delta}-\beta_{1}<0,
$$

which is related to condition $(4.3 .2)$, we get $\mathcal{Y}_{3}^{\prime}(t)<0$. This shows the decreasing behavior of $\mathcal{Y}_{3}=\mathcal{Y}_{3}(t)$. Hence, we obtain

$$
\int_{\delta t}^{t} \frac{\mu(s)}{\lambda^{2}(s)}\left(1+B_{\lambda}(0, t)\right)^{\frac{n}{2}\left(\frac{1}{m}-\frac{1}{2}\right)} \log \Lambda(s) \frac{\lambda(s)}{\Lambda(s)} d s \lesssim \int_{\delta t}^{t} \Lambda^{-\varepsilon-1}(s) \lambda(s) d s \lesssim 1 .
$$

In the same way, by condition (4.3.2) we can also derive for large $t$ the estimates

$$
\frac{\mu(t)}{\lambda^{2}(t)}\left(1+B_{\lambda}(0, t)\right)^{\frac{n}{2}\left(\frac{1}{m}-\frac{1}{2}\right)} \log \Lambda(t) \lesssim \frac{\mu(\delta t)}{\lambda^{2}(\delta t)}\left(1+B_{\lambda}(0, t)\right)^{\frac{n}{2}\left(\frac{1}{m}-\frac{1}{2}\right)} \log \Lambda(t) \lesssim \Lambda^{-\varepsilon}(\delta t) .
$$

Consequently, in the case $j=\ell=0$ we obtain the desired estimate

$$
\mathbf{Y} \lesssim\left(1+B_{\lambda}(0, t)\right)^{-\frac{n}{2}\left(\frac{1}{m}-\frac{1}{2}\right)} .
$$

Now let us consider the case $j=1$ and $\ell=0$ for the integral $\mathbf{Y}$. In this case we have

$$
\begin{aligned}
\mathbf{Y}= & -\int_{\delta t}^{t} \frac{\mu(s)}{\lambda^{2}(s)}\left(1+B_{\lambda}(0, s)\right)^{-\alpha_{2, a}(p)}\left(1+B_{\lambda}(s, t)\right)^{-\frac{1}{2}} d\left(1+B_{\lambda}(s, t)\right) \\
= & -\left.2 \frac{\mu(s)}{\lambda^{2}(s)}\left(1+B_{\lambda}(0, s)\right)^{-\alpha_{2, a}(p)}\left(1+B_{\lambda}(s, t)\right)^{\frac{1}{2}}\right|_{\delta t} ^{t} \\
& +2 \int_{\delta t}^{t}\left(1+B_{\lambda}(s, t)\right)^{\frac{1}{2}} d\left(\frac{\mu(s)}{\lambda^{2}(s)}\left(1+B_{\lambda}(0, s)\right)^{-\alpha_{2, a}(p)}\right) \\
\lesssim & -\left.\frac{\mu(s)}{\lambda^{2}(s)}\left(1+B_{\lambda}(0, s)\right)^{-\alpha_{2, a}(p)}\left(1+B_{\lambda}(s, t)\right)^{\frac{1}{2}}\right|_{\delta t} ^{t} \\
& +\int_{\delta t}^{t} \frac{\mu(s)}{\lambda^{2}(s)}\left(1+B_{\lambda}(0, s)\right)^{-\alpha_{2, a}(p)}\left(1+B_{\lambda}(s, t)\right)^{\frac{1}{2}} \frac{d\left(1+B_{\lambda}(0, s)\right)}{1+B_{\lambda}(0, s)},
\end{aligned}
$$

where we used Lemma 3.1.5. Then, we get

$$
\begin{aligned}
&\left(1+B_{\lambda}(0, t)\right)^{\frac{n}{2}\left(\frac{1}{m}-\frac{1}{2}\right)+\frac{1}{2}} \mathbf{Y} \\
& \lesssim \underbrace{\frac{\mu(\delta t)}{\lambda^{2}(\delta t)}\left(1+B_{\lambda}(0, \delta t)\right)^{-\alpha_{2, a}(p)}\left(1+B_{\lambda}(0, t)\right)^{\frac{n}{2}\left(\frac{1}{m}-\frac{1}{2}\right)+1}}_{Y_{1}(t)} \\
&+\underbrace{\int_{\delta t}^{t} \frac{\mu(s)}{\lambda^{2}(s)}\left(1+B_{\lambda}(0, s)\right)^{-\alpha_{2, a}(p)}\left(1+B_{\lambda}(0, t)\right)^{\frac{n}{2}\left(\frac{1}{m}-\frac{1}{2}\right)+1} \frac{d\left(1+B_{\lambda}(0, s)\right)}{1+B_{\lambda}(0, s)}}_{Y_{2}(t)}
\end{aligned}
$$


In order to prove that the functions $Y_{1}=Y_{1}(t)$ and $Y_{2}=Y_{2}(t)$ are uniformly bounded it is sufficient to show that for small positive constant $\varepsilon$ we have

$$
\frac{\mu(\delta t)}{\lambda^{2}(\delta t)}\left(1+B_{\lambda}(0, \delta t)\right)^{-\alpha_{2, a}(p)}\left(1+B_{\lambda}(0, t)\right)^{\frac{n}{2}\left(\frac{1}{m}-\frac{1}{2}\right)+1} \lesssim\left(1+B_{\lambda}(0, \delta t)\right)^{-\varepsilon} .
$$

Then, employing (3.1.19) we get

$$
\begin{aligned}
& \frac{\mu(\delta t)}{\lambda^{2}(\delta t)}\left(1+B_{\lambda}(0, \delta t)\right)^{-\alpha_{2, a}(p)+\varepsilon}\left(1+B_{\lambda}(0, t)\right)^{\frac{n}{2}\left(\frac{1}{m}-\frac{1}{2}\right)+1} \\
& \quad \leq \frac{\mu(\delta t)}{\lambda^{2}(\delta t)}\left(1+B_{\lambda}(0, t)\right)^{\frac{n}{2}\left(\frac{1}{m}-\frac{1}{2}\right)+1-\alpha_{2, a}(p)+\varepsilon}\left(\frac{\Lambda(\delta t)}{\Lambda(t)}\right)^{(2+R)\left(-\alpha_{2, a}(p)+\varepsilon\right)}:=\mathcal{Y}_{4}(t) .
\end{aligned}
$$

We may conclude immediately that the function $\mathcal{Y}_{4}=\mathcal{Y}_{4}(t)$ is bounded by the aid of the condition $p>p_{3}(a, m, n)$, following the considerations for the function $\mathcal{B}_{1}=\mathcal{B}_{1}(t)$ in (3.2.15). Consequently, in the case $j=1$ and $\ell=0$ we obtain the estimate

$$
\mathbf{Y} \lesssim\left(1+B_{\lambda}(0, t)\right)^{-\frac{n}{2}\left(\frac{1}{m}-\frac{1}{2}\right)-\frac{1}{2}} .
$$

Finally, it remains to consider the case $j=0$ and $\ell=1$ for the integral $\mathbf{Y}$. We have

$$
\mathbf{Y}=\int_{\delta t}^{t} \Phi_{1}(s, t) \frac{\Lambda(s)}{\lambda(s)}\left(1+B_{\lambda}(0, s)\right)^{-\frac{n}{2 m} p+\frac{n}{4}-\frac{a}{2} p} d s
$$

where $\Phi_{1}=\Phi_{1}(s, t)$ is defined in (4.1.3). Due to the competition between the estimates for $\Phi_{1}(s, t)$, from condition (C1) we are interested in the following two cases only for all $s \in[\delta t, t]:$

Case a: $\Phi_{1}(s, t)=\frac{\lambda^{2}(t)}{\rho(t)}\left(1+B_{\lambda}(s, t)\right)^{-1}$. Then, it follows

$$
\begin{aligned}
\mathbf{Y}= & -\frac{\lambda^{2}(t)}{\rho(t)} \int_{\delta t}^{t} \frac{\mu(s)}{\lambda^{2}(s)}\left(1+B_{\lambda}(0, s)\right)^{-\alpha_{2, a}(p)} \frac{d\left(1+B_{\lambda}(s, t)\right)}{1+B_{\lambda}(s, t)} \\
= & -\left.\frac{\lambda^{2}(t)}{\rho(t)} \frac{\mu(s)}{\lambda^{2}(s)}\left(1+B_{\lambda}(0, s)\right)^{-\alpha_{2, a}(p)} \log \left(1+B_{\lambda}(s, t)\right)\right|_{\delta t} ^{t} \\
& +\frac{\lambda^{2}(t)}{\rho(t)} \int_{\delta t}^{t} \log \left(1+B_{\lambda}(s, t)\right) d\left(\frac{\mu(s)}{\lambda^{2}(s)}\left(1+B_{\lambda}(0, s)\right)^{-\alpha_{2, a}(p)}\right) \\
\lesssim & -\left.\frac{\lambda^{2}(t)}{\rho(t)} \frac{\mu(s)}{\lambda^{2}(s)}\left(1+B_{\lambda}(0, s)\right)^{-\alpha_{2, a}(p)} \log \left(1+B_{\lambda}(s, t)\right)\right|_{\delta t} ^{t} \\
& +\frac{\lambda^{2}(t)}{\rho(t)} \int_{\delta t}^{t} \frac{\mu(s)}{\lambda^{2}(s)}\left(1+B_{\lambda}(0, s)\right)^{-\alpha_{2, a}(p)} \log \left(1+B_{\lambda}(s, t)\right) \frac{d\left(1+B_{\lambda}(0, s)\right)}{1+B_{\lambda}(0, s)} .
\end{aligned}
$$

Thus, we have

$$
\begin{aligned}
& \left(1+B_{\lambda}(0, t)\right)^{\frac{n}{2}\left(\frac{1}{m}-\frac{1}{2}\right)+1} \frac{\rho(t)}{\lambda^{2}(t)} \mathbf{Y} \\
& \lesssim \underbrace{\frac{\mu(\delta t)}{\lambda^{2}(\delta t)}\left(1+B_{\lambda}(0, \delta t)\right)^{-\alpha_{2, a}(p)}\left(1+B_{\lambda}(0, t)\right)^{\frac{n}{2}\left(\frac{1}{m}-\frac{1}{2}\right)+1} \log \left(1+B_{\lambda}(0, t)\right)}_{Y_{3}(t)} \\
& +\underbrace{\int_{\delta t}^{t} \frac{\mu(s)}{\lambda^{2}(s)}\left(1+B_{\lambda}(0, s)\right)^{-\alpha_{2, a}(p)}\left(1+B_{\lambda}(0, t)\right)^{\frac{n}{2}\left(\frac{1}{m}-\frac{1}{2}\right)+1} \log \left(1+B_{\lambda}(0, t)\right) \frac{d\left(1+B_{\lambda}(0, s)\right)}{1+B_{\lambda}(0, s)}}_{Y_{4}(t)} .
\end{aligned}
$$


In order to prove that the functions $Y_{3}=Y_{3}(t)$ and $Y_{4}=Y_{4}(t)$ are bounded we will show that for a small positive constant $\varepsilon$ we have

$$
\frac{\mu(\delta t)}{\lambda^{2}(\delta t)}\left(1+B_{\lambda}(0, \delta t)\right)^{-\alpha_{2, a}(p)}\left(1+B_{\lambda}(0, t)\right)^{\frac{n}{2}\left(\frac{1}{m}-\frac{1}{2}\right)+1} \log \left(1+B_{\lambda}(0, t)\right) \lesssim\left(1+B_{\lambda}(0, \delta t)\right)^{-\varepsilon} .
$$

This implies to deal with

$$
\frac{\mu(\delta t)}{\lambda^{2}(\delta t)}\left(1+B_{\lambda}(0, \delta t)\right)^{-\alpha_{2, a}(p)+\varepsilon}\left(1+B_{\lambda}(0, t)\right)^{\frac{n}{2}\left(\frac{1}{m}-\frac{1}{2}\right)+1} \log \left(1+B_{\lambda}(0, t)\right) .
$$

Hence, for showing the boundedness of $Y_{3}=Y_{3}(t)$ we have

$$
Y_{3}(t) \lesssim \frac{\log \left(1+B_{\lambda}(0, t)\right)}{\left(1+B_{\lambda}(0, \delta t)\right)^{\varepsilon}} \mathcal{Y}_{4}(t) \lesssim \frac{\log \left(1+B_{\lambda}(0, t)\right)}{\left(1+B_{\lambda}(0, \delta t)\right)^{\varepsilon}} \lesssim 1
$$

for large $t$. Here we used the boundedness of $\mathcal{Y}_{4}=\mathcal{Y}_{4}(t)$ which is given in (4.3.12) and l'Hospital's rule (cf. condition (B4)) for

$$
\lim _{t \rightarrow \infty} \sup \frac{\log \left(1+B_{\lambda}(0, t)\right)}{\left(1+B_{\lambda}(0, \delta t)\right)^{\varepsilon}} \lesssim \lim _{t \rightarrow \infty} \sup \frac{\nu(\delta, t)}{\left(1+B_{\lambda}(0, \delta t)\right)^{\varepsilon}} \lesssim 1,
$$

respectively. On the other hand, for $Y_{4}=Y_{4}(t)$ we have

$$
Y_{4} \lesssim \log \left(1+B_{\lambda}(0, t)\right) \int_{\delta t}^{t}\left(1+B_{\lambda}(0, s)\right)^{-\varepsilon-1} d\left(1+B_{\lambda}(0, s)\right) \lesssim \frac{\log \left(1+B_{\lambda}(0, t)\right)}{\varepsilon\left(1+B_{\lambda}(0, \delta t)\right)^{\varepsilon}} \lesssim 1,
$$

where we used (4.3.14). Therefore, in the Case $a$ we obtain the desired estimate

$$
\mathbf{Y} \lesssim \frac{\lambda^{2}(t)}{\rho(t)}\left(1+B_{\lambda}(0, t)\right)^{-\frac{n}{2}\left(\frac{1}{m}-\frac{1}{2}\right)-1}
$$

Summarizing, in the Case $a$ from (4.3.10), (4.3.11), (4.3.13) and (4.3.15) for $j+\ell=0,1$ we arrive at

$$
\mathbf{Y} \lesssim \frac{\lambda^{2 \ell}(t)}{\rho^{\ell}(t)}\left(1+B_{\lambda}(0, t)\right)^{-\frac{n}{2}\left(\frac{1}{m}-\frac{1}{2}\right)-\frac{j}{2}-\ell}
$$

Case b: $\Phi_{1}(s, t)=\frac{\lambda(t)}{F(\Lambda(t))}$. In this case we have

$$
\mathbf{Y}=\frac{\lambda(t)}{F(\Lambda(t))} \int_{\delta t}^{t} \frac{\Lambda(s)}{\lambda(s)}\left(1+B_{\lambda}(0, s)\right)^{-\alpha_{2, a}(p)} d s .
$$

Case b.1: $\alpha_{2, a}(p)>1$. In a similar way as we did in the Case 1 we have

$$
\frac{F(\Lambda(t))}{\lambda(t)} \mathbf{Y} \lesssim \frac{\mu(\delta t)}{\lambda^{2}(\delta t)}\left(1+B_{\lambda}(0, \delta t)\right)^{-\alpha_{2, a}(p)+1}-\frac{\mu(t)}{\lambda^{2}(t)}\left(1+B_{\lambda}(0, t)\right)^{-\alpha_{2, a}(p)+1} .
$$

Case b.2: $\alpha_{2, a}(p)<1$. Similarly as in the Case 2 for $p>p_{1}(a, m, n)$ we conclude

$$
\frac{F(\Lambda(t))}{\lambda(t)} \mathbf{Y} \lesssim \frac{\mu(\delta t)}{\lambda^{2}(\delta t)}\left(1+B_{\lambda}(0, \delta t)\right)^{-\alpha_{2, a}(p)+1}-\frac{\mu(t)}{\lambda^{2}(t)}\left(1+B_{\lambda}(0, t)\right)^{-\alpha_{2, a}(p)+1} .
$$

Then, it follows

$$
\frac{F(\Lambda(t))}{\lambda(t)}\left(F^{2}(\Lambda(t))\right)^{\frac{n}{2}\left(\frac{1}{m}-\frac{1}{2}\right)} \mathbf{Y} \lesssim \frac{\mu(\delta t)}{\lambda^{2}(\delta t)}\left(F^{2}(\Lambda(t))\right)^{\frac{n}{2}\left(\frac{1}{m}-\frac{1}{2}\right)}\left(1+B_{\lambda}(0, \delta t)\right)^{-\alpha_{2, a}(p)+1},
$$


where we used the condition (B6), that is, $B_{\lambda}(0, t) \lesssim F^{2}(\Lambda(t))$. We consider the right-hand side of the last inequality in both cases $\alpha_{2, a}(p)<1$ and $\alpha_{2, a}(p)>1$. In the case $\alpha_{2, a}(p)<1$ we have

$$
\begin{aligned}
& \frac{\mu(\delta t)}{\lambda^{2}(\delta t)}\left(F^{2}(\Lambda(t))\right)^{\frac{n}{2}\left(\frac{1}{m}-\frac{1}{2}\right)}\left(1+B_{\lambda}(0, \delta t)\right)^{-\alpha_{2, a}(p)+1} \\
& \quad \lesssim \frac{\mu(\delta t)}{\lambda^{2}(\delta t)}\left(F^{2}(\Lambda(t))\right)^{\frac{n}{2}\left(\frac{1}{m}-\frac{1}{2}\right)-\alpha_{2, a}(p)+1}=\frac{\mu(\delta t)}{\lambda^{2}(\delta t)}\left(F^{2}(\Lambda(t))\right)^{-\alpha_{1, a}(p)+1}:=\mathcal{Y}_{5}(t),
\end{aligned}
$$

where again we used condition (B6) and $\alpha_{2, a}(p)=\alpha_{1, a}(p)+\frac{n}{2}\left(\frac{1}{m}-\frac{1}{2}\right)$. Now if we estimate the derivative of the function $\mathcal{Y}_{5}=\mathcal{Y}_{5}(t)$ we get

$$
\mathcal{Y}_{5}^{\prime}(t) \leq\left[-\delta \beta_{1}+2\left(-\alpha_{1, a}(p)+1\right) \vartheta(\delta, t)\right] \frac{\mu(\delta t)}{\lambda^{2}(\delta t)} \frac{\lambda(\delta t)}{\Lambda(\delta t)} F(\Lambda(t))^{-2 \alpha_{1, a}(p)+2},
$$

where from condition (A5) we used $F^{\prime}(\Lambda(t))=\frac{F^{2}(\Lambda(t))}{\lambda^{2}(t) \Xi^{2}(t)}$. Then, for a large time $t$ by using $p>p_{3}(a, m, n)$ we obtain

$$
-\delta \beta_{1}+2\left(-\alpha_{1, a}(p)+1\right) \vartheta(\delta)<0,
$$

which implies due to condition $(4.3 .2)$ the estimate $\mathcal{Y}_{5}^{\prime}(t)<0$. Similarly, in the case $\alpha_{2, a}(p)>$ 1 the desired estimate can be derived as in the case $\alpha_{2, a}(p)<1$. For this reason, we obtain the expected estimate

$$
\mathbf{Y} \lesssim \lambda(t) F(\Lambda(t))\left(F^{2}(\Lambda(t))\right)^{-\frac{n}{2}\left(\frac{1}{m}-\frac{1}{2}\right)-1} .
$$

Case b.3: $\alpha_{2}(p)=1$. In the same way as in the Case 3 we obtain

$$
\frac{F(\Lambda(t))}{\lambda(t)} \mathbf{Y} \lesssim \frac{\mu(t)}{\lambda^{2}(t)} \log \Lambda(t)-\frac{\mu(\delta t)}{\lambda^{2}(\delta t)} \log \Lambda(\delta t)+\int_{\delta t}^{t} \frac{\mu(s)}{\lambda^{2}(s)} \log \Lambda(s) \frac{\lambda(s)}{\Lambda(s)} d s .
$$

Then, we get

$$
\begin{aligned}
\frac{F(\Lambda(t))}{\lambda(t)}\left(F^{2}(\Lambda(t))\right)^{\frac{n}{2}\left(\frac{1}{m}-\frac{1}{2}\right)} \mathbf{Y} \lesssim & \frac{\mu(t)}{\lambda^{2}(t)}\left(F^{2}(\Lambda(t))\right)^{\frac{n}{2}\left(\frac{1}{m}-\frac{1}{2}\right)} \log \Lambda(t) \\
& +\int_{\delta t}^{t} \frac{\mu(s)}{\lambda^{2}(s)}\left(F^{2}(\Lambda(t))\right)^{\frac{n}{2}\left(\frac{1}{m}-\frac{1}{2}\right)} \log \Lambda(s) \frac{\lambda(s)}{\Lambda(s)} d s .
\end{aligned}
$$

In order to show the desired estimate we assume that there exists a sufficiently small positive constant $\varepsilon$ such that we have the estimate

$$
\frac{\mu(\delta t)}{\lambda^{2}(\delta t)}\left(F^{2}(\Lambda(t))\right)^{\frac{n}{2}\left(\frac{1}{m}-\frac{1}{2}\right)} \log \Lambda(\delta t) \lesssim \Lambda^{-\varepsilon}(\delta t) .
$$

Thus, it is sufficient to consider the derivative of the function

$$
\mathcal{Y}_{6}(t):=\frac{\mu(\delta t)}{\lambda^{2}(\delta t)} \Lambda^{\varepsilon}(\delta t)\left(F^{2}(\Lambda(t))\right)^{\frac{n}{2}\left(\frac{1}{m}-\frac{1}{2}\right)} \log \Lambda(\delta t) .
$$

It follows

$\mathcal{Y}_{6}^{\prime}(t) \leq\left[-\beta_{1}+\varepsilon+n\left(\frac{1}{m}-\frac{1}{2}\right) \frac{\vartheta(\delta, t)}{\delta}+\frac{1}{\log \Lambda(\delta t)}\right] \delta \frac{\mu(\delta t)}{\lambda^{2}(\delta t)} \frac{\lambda(\delta t)}{\Lambda(\delta t)} \Lambda^{\varepsilon}(\delta t) F(\Lambda(t))^{n\left(\frac{1}{m}-\frac{1}{2}\right)} \log \Lambda(\delta t)$.

Here from condition (A5) we used $F^{\prime}(\Lambda(t))=\frac{F^{2}(\Lambda(t))}{\lambda^{2}(t) \Xi^{2}(t)}$. Hence, for a small constant $\varepsilon>0$ and for large time $t$ after taking account of

$$
-\beta_{1}+\varepsilon+n\left(\frac{1}{m}-\frac{1}{2}\right) \frac{\vartheta(\delta)}{\delta}<0
$$


which is related to condition (4.3.2), we have $\mathcal{Y}_{6}^{\prime}(t)<0$. Analogously, using the decreasing behavior of the function $\frac{\mu(t)}{\lambda^{2}(t)}$ one can also prove by (4.3.2) the estimate

$$
\frac{\mu(t)}{\lambda^{2}(t)}\left(F^{2}(\Lambda(t))\right)^{\frac{n}{2}\left(\frac{1}{m}-\frac{1}{2}\right)} \log \Lambda(t) \lesssim \frac{\mu(\delta t)}{\lambda^{2}(\delta t)}\left(F^{2}(\Lambda(t))\right)^{\frac{n}{2}\left(\frac{1}{m}-\frac{1}{2}\right)} \log \Lambda(t) \lesssim \Lambda^{-\varepsilon}(\delta t) .
$$

Thus, we get

$$
\mathbf{Y} \lesssim \lambda(t) F(\Lambda(t))\left(F^{2}(\Lambda(t))\right)^{-\frac{n}{2}\left(\frac{1}{m}-\frac{1}{2}\right)-1}
$$

Summarizing, from (4.3.16), (4.3.17) and (4.3.18) for $j=0$ and $\ell=1$ we get

$$
\mathbf{Y} \lesssim \max \left\{\frac{\lambda^{2}(t)}{\rho(t)}\left(1+B_{\lambda}(0, t)\right)^{-\frac{n}{2}\left(\frac{1}{m}-\frac{1}{2}\right)-1} ; \lambda(t) F(\Lambda(t))\left(F^{2}(\Lambda(t))\right)^{-\frac{n}{2}\left(\frac{1}{m}-\frac{1}{2}\right)-1}\right\} .
$$

Consequently, from (4.3.9), (4.3.16) and (4.3.19) we arrive at the statement

$$
\left\||D|^{j} \partial_{t}^{\ell} u^{\mathrm{nl}}(t, \cdot)\right\|_{L^{2}} \lesssim\|u\|_{X(t)}^{p} \Phi_{1, m}^{\ell}(0, t)\left(1+B_{\lambda}(0, t)\right)^{(\ell-1) \frac{n}{2}\left(\frac{1}{m}-\frac{1}{2}\right)-\frac{j}{2}},
$$

where $\Phi_{1, m}^{1}(0, t)=\Phi_{1, m}(0, t)$ is defined in (4.1.2) for $s=0$ and $\Phi_{1, m}^{0}(0, t)=1$. Replacing the estimates for $j+\ell=0,1$ in the norm of the solution space $X(t)$ we obtain

$$
\left\|u^{\mathrm{nl}}\right\|_{X(t)} \lesssim\|u\|_{X(t)}^{p} .
$$

Now let us prove (4.1.7). Thanks to the estimates for the solutions to the family of linear parameter dependent Cauchy problems with vanishing right-hand side we can estimate

$$
\begin{aligned}
& \left\||D|^{j} \partial_{t}^{\ell} K_{1}(t, s, x) *_{(x)}\left(\left.\left.|| D\right|^{a} u(s, x)\right|^{p}-\left.\left.|| D\right|^{a} v(s, x)\right|^{p}\right)\right\|_{L^{2}} \\
& \lesssim \int_{0}^{\delta t} \Phi_{1, m}^{\ell}(s, t) \frac{\Lambda(s)}{\lambda(s)}\left(1+B_{\lambda}(s, t)\right)^{(\ell-1) \frac{n}{2}\left(\frac{1}{m}-\frac{1}{2}\right)-\frac{j}{2}}\left\|\left.\left.|| D\right|^{a} u(s, x)\right|^{p}-\left.|| D\right|^{a} v(s, x)||^{p}\right\|_{L^{m} \cap L^{2}} d s \\
& \quad+\int_{\delta t}^{t} \Phi_{1}^{\ell}(s, t) \frac{\Lambda(s)}{\lambda(s)}\left(1+B_{\lambda}(s, t)\right)^{-\frac{j}{2}}\left\|\left.\left.|| D\right|^{a} u(s, x)\right|^{p}-\left.|D|^{a} v(s, x)\right|^{p}\right\|_{L^{2}} d s .
\end{aligned}
$$

By Hölder's inequality we obtain

$$
\begin{aligned}
\|\left.\left.|| D\right|^{a} u(s, \cdot)\right|^{p} & -\left.\left.|| D\right|^{a} v(s, \cdot)\right|^{p} \|_{L^{m}} \\
& \lesssim\left\||D|^{a} u(s, \cdot)-|D|^{a} v(s, \cdot)\right\|_{L^{m p}}\left(\left\||D|^{a} u(s, \cdot)\right\|_{L^{m p}}^{p-1}+\left\||D|^{a} v(s, \cdot)\right\|_{L^{m p}}^{p-1}\right) \\
\|\left.|| D\right|^{a}|u(s, \cdot)|^{p} & -\left.\left.|| D\right|^{a} v(s, \cdot)\right|^{p} \|_{L^{2}} \\
& \lesssim\left\||D|^{a} u(s, \cdot)-|D|^{a} v(s, \cdot)\right\|_{L^{2 p}}\left(\left\||D|^{a} u(s, \cdot)\right\|_{L^{2 p}}^{p-1}+\left\||D|^{a} v(s, \cdot)\right\|_{L^{2 p}}^{p-1}\right) .
\end{aligned}
$$

In a similar way to the proof of (4.1.6) we use again Gagliardo-Nirenberg inequality to the following terms:

$$
\left\||D|^{a} u(s, \cdot)-|D|^{a} v(s, \cdot)\right\|_{L^{q}}, \quad\left\||D|^{a} u(s, \cdot)\right\|_{L^{q}}, \quad\left\||D|^{a} v(s, \cdot)\right\|_{L^{q}}
$$

with $q=m p$ and $q=2 p$. Summarizing, we arrive at the following inequality:

$$
\begin{aligned}
\left\||D|^{j} \partial_{t}^{\ell}(N u-N v)\right\|_{L^{2}} \lesssim\left\||D|^{a} u-|D|^{a} v\right\|_{X(t)}\left(\left\||D|^{a} u\right\|_{X(t)}^{p-1}+\left\||D|^{a} v\right\|_{X(t)}^{p-1}\right) \\
\times\left(\int_{0}^{\delta t} \Phi_{1, m}^{\ell}(s, t) \frac{\Lambda(s)}{\lambda(s)}\left(1+B_{\lambda}(s, t)\right)^{(\ell-1) \frac{n}{2}\left(\frac{1}{m}-\frac{1}{2}\right)-\frac{j}{2}}\left(1+B_{\lambda}(0, s)\right)^{-\frac{n}{2 m} p+\frac{n}{2 m}-\frac{a}{2} p} d s\right. \\
\left.\quad+\int_{\delta t}^{t} \Phi_{1}^{\ell}(s, t) \frac{\Lambda(s)}{\lambda(s)}\left(1+B_{\lambda}(s, t)\right)^{-\frac{j}{2}}\left(1+B_{\lambda}(0, s)\right)^{-\frac{n}{2 m} p+\frac{n}{4}-\frac{a}{2} p} d s\right) .
\end{aligned}
$$

Thus, we can repeat the same arguments as we used in the proof of (4.1.6) in order to estimate these two integrals employing the conditions $p>p(a, m, n)$ and (4.3.2). Finally, we may conclude the proof of the statement (4.1.7) from the definition of the norm of $X(t)$. In this way the proof of the theorem is completed. 


\subsubsection{Case of super-exponential propagation speed in energy space}

Theorem 4.3.2. We assume that the Hypotheses (A1) to (A5), (B1) to (B6), (C1), (C3) and (C4) hold. Let $\nu(\delta)=\infty$ and $\vartheta(\delta)=\infty$, and we suppose that the following condition for the functions $\nu=\nu(\delta, t)$ and $\vartheta=\vartheta(\delta, t)$ (see (3.1.2) and (3.1.3)) holds:

$$
\max \{\nu(\delta, t) ; \vartheta(\delta, t)\}=o(\log \Lambda(t)) .
$$

We choose data $\left(u_{0}, u_{1}\right) \in \mathcal{D}_{m}^{1}$ with $m \in[1,2), n \leq \frac{4}{2-m}$ and suppose that the exponent $p$ satisfies

$$
\begin{cases}\frac{2}{m} \leq p<\infty & \text { if } n=1,2, \\ \frac{2}{m} \leq p \leq p_{G N}(n)=\frac{n}{n-2} & \text { if } n \geq 3 .\end{cases}
$$

Then, there exists a constant $\varepsilon_{0}>0$ such that for all $\left(u_{0}, u_{1}\right) \in \mathcal{D}_{m}^{1}$ with $\left\|\left(u_{0}, u_{1}\right)\right\|_{\mathcal{D}_{m}^{1}} \leq \varepsilon_{0}$, there is a uniquely determined globally (in time) energy solution to the Cauchy problem (4.3.1) in

$$
\mathcal{C}\left([0, \infty), H^{1}\right) \cap \mathcal{C}^{1}\left([0, \infty), L^{2}\right) .
$$

Furthermore, the solution satisfies the following estimates:

$$
\begin{aligned}
\|u(t, \cdot)\|_{L^{2}} & \lesssim\left(1+B_{\lambda}(0, t)\right)^{-\frac{n}{2}\left(\frac{1}{m}-\frac{1}{2}\right)}\left\|\left(u_{0}, u_{1}\right)\right\|_{\mathcal{D}_{m}^{1}}, \\
\||D| u(t, \cdot)\|_{L^{2}} & \lesssim\left(1+B_{\lambda}(0, t)\right)^{-\frac{n}{2}\left(\frac{1}{m}-\frac{1}{2}\right)-\frac{1}{2}}\left\|\left(u_{0}, u_{1}\right)\right\|_{\mathcal{D}_{m}^{1}}, \\
\left\|u_{t}(t, \cdot)\right\|_{L^{2}} & \lesssim \max \left\{\frac{\lambda^{2}(t)}{\rho(t)}\left(1+B_{\lambda}(0, t)\right)^{-\frac{n}{2}\left(\frac{1}{m}-\frac{1}{2}\right)-1} ; \lambda(t) F(\Lambda(t))\left(F^{2}(\Lambda(t))\right)^{-\frac{n}{2}\left(\frac{1}{m}-\frac{1}{2}\right)-1}\right\}\left\|\left(u_{0}, u_{1}\right)\right\|_{\mathcal{D}_{m}^{1}} .
\end{aligned}
$$

Proof. We proceed in the same way as we did in the proof of Theorem 4.3.1 to verify that the integrals $\mathbf{V}$ and $\mathbf{Y}$, which are given in (4.3.5), fulfill the desired estimates. For this reason our goal is only to prove the inequalities (4.1.6) and (4.1.7).

To complete the proof of (4.1.6) we have to estimate $\left\|u^{\mathrm{nl}}(t, \cdot)\right\|_{X(t)}$. For this reason, we begin with the estimate of the integral $\mathbf{V}$ for $j+\ell=0,1$, that is, with

$$
\mathbf{V} \lesssim \Phi_{1, m}^{\ell}(0, t)\left(1+B_{\lambda}(0, t)\right)^{(\ell-1) \frac{n}{2}\left(\frac{1}{m}-\frac{1}{2}\right)-\frac{j}{2}} \underbrace{\int_{0}^{\delta t} \frac{\Lambda(s)}{\lambda(s)}\left(1+B_{\lambda}(0, s)\right)^{-\frac{n}{2 m} p+\frac{n}{2 m}-\frac{a}{2} p} d s}_{\bar{V}_{1}(t)},
$$

where we used (3.1.21). Here $\Phi_{1, m}^{0}(0, t) \equiv 1$ and $\Phi_{1, m}^{1}(0, t)=\Phi_{1, m}(0, t)$ is defined in (4.1.2) for $s=0$. Let us define $\alpha_{1, a}(p):=\frac{n}{2 m} p-\frac{n}{2 m}+\frac{a}{2}$ and discuss the following cases:

Case 1: $\alpha_{1, a}(p) \neq 1$. In this case, it holds

$$
\begin{aligned}
\bar{V}_{1}(t)= & \int_{0}^{\delta t} \frac{\mu(s)}{\lambda^{2}(s)}\left(1+B_{\lambda}(0, s)\right)^{-\alpha_{1, a}(p)} d\left(1+B_{\lambda}(0, s)\right) \\
= & \left.\frac{1}{1-\alpha_{1, a}(p)} \frac{\mu(s)}{\lambda^{2}(s)}\left(1+B_{\lambda}(0, s)\right)^{-\alpha_{1}(p)+1}\right|_{0} ^{\delta t} \\
& -\frac{1}{1-\alpha_{1, a}(p)} \int_{0}^{\delta t}\left(1+B_{\lambda}(0, s)\right)^{-\alpha_{1, a}(p)+1} d\left(\frac{\mu(s)}{\lambda^{2}(s)}\right) \\
\lesssim & \frac{1}{1-\alpha_{1, a}(p)}\left(\frac{\mu(\delta t)}{\lambda^{2}(\delta t)}\left(1+B_{\lambda}(0, \delta t)\right)^{-\alpha_{1, a}(p)+1}-\frac{\mu(0)}{\lambda^{2}(0)}\right) \\
& +\frac{1}{1-\alpha_{1, a}(p)} \int_{0}^{\delta t} \frac{\mu(s)}{\lambda^{2}(s)}\left(1+B_{\lambda}(0, s)\right)^{-\alpha_{1, a}(p)+1} \frac{\lambda(s)}{\Lambda(s)} d s
\end{aligned}
$$

where we used the condition (C4). Now we shall show that the integral $\bar{V}_{1}=\bar{V}_{1}(t)$ is bounded. For this reason, by choosing an arbitrary small positive constant $\varepsilon$ we want to verify the inequality

$$
\frac{\mu(t)}{\lambda^{2}(t)}\left(1+B_{\lambda}(0, t)\right)^{-\alpha_{1, a}(p)+1} \lesssim \Lambda(t)^{-\varepsilon}
$$


Then, we show that the following function is bounded:

$$
\overline{\mathcal{V}}_{1}(t):=\frac{\mu(t)}{\lambda^{2}(t)} \Lambda(t)^{\varepsilon}\left(1+B_{\lambda}(0, t)\right)^{-\alpha_{1, a}(p)+1} .
$$

Let us consider the monotonicity of $\overline{\mathcal{V}}_{1}=\overline{\mathcal{V}}_{1}(t)$ by estmating its derivative. Indeed, by using condition (C4) and (3.1.20) we have

$$
\overline{\mathcal{V}}_{1}^{\prime}(t) \leq\left[\left(\frac{-\beta_{1}+\varepsilon}{\alpha+2}\right) \log \Lambda(t)+\left(1-\alpha_{1, a}(p)\right)\right]\left(1+B_{\lambda}(0, t)\right)^{-\alpha_{1, a}(p)} \frac{\Lambda(t)}{\lambda(t)} \Lambda(t)^{\varepsilon} .
$$

This inequality implies $\overline{\mathcal{V}}_{1}^{\prime}(t)<0$ for a small positive constant $\varepsilon$ and for large $t$. Hence, we get

$$
\int_{0}^{\delta t} \frac{\mu(s)}{\lambda^{2}(s)}\left(1+B_{\lambda}(0, s)\right)^{-\alpha_{1, a}(p)+1} \frac{\lambda(s)}{\Lambda(s)} d s \lesssim \int_{0}^{\delta t} \Lambda(s)^{-\varepsilon-1} \lambda(s) d s \lesssim 1 .
$$

Therefore, we may conclude that $\bar{V}_{1}=\bar{V}_{1}(t)$ is uniformly bounded.

Case 2: $\alpha_{1, a}(p)=1$. In this case we have

$$
\begin{aligned}
\bar{V}_{1}(t) & =\int_{0}^{\delta t} \frac{\Lambda(s)}{\lambda(s)}\left(1+B_{\lambda}(0, s)\right)^{-1} d s \lesssim \int_{0}^{\delta t} \frac{\Lambda(s)}{\lambda(s)} \frac{\mu(s)}{\Lambda^{2}(s)} \frac{1}{\log \Lambda(s)} d s \\
& \leq \int_{0}^{\delta t} \frac{\mu(s)}{\lambda^{2}(s)} \frac{\lambda(s)}{\Lambda(s)} d s=\left.\frac{\mu(s)}{\lambda^{2}(s)} \log \Lambda(s)\right|_{0} ^{\delta t}-\int_{0}^{\delta t} \log \Lambda(s) d\left(\frac{\mu(s)}{\lambda^{2}(s)}\right) \\
& \leq\left.\frac{\mu(s)}{\lambda^{2}(s)} \log \Lambda(s)\right|_{0} ^{\delta t}+\beta_{0} \int_{0}^{\delta t} \frac{\mu(s)}{\lambda^{2}(s)} \log \Lambda(s) \frac{\lambda(s)}{\Lambda(s)} d s,
\end{aligned}
$$

where we used (3.1.20) and condition (C4), respectively. Then, we show that for a sufficiently small constant $\varepsilon>0$ we have the estimate

$$
\frac{\mu(t)}{\lambda^{2}(t)} \log \Lambda(t) \lesssim \Lambda^{-\varepsilon}(t)
$$

Therefore, let us compute the derivative of the function

$$
\overline{\mathcal{V}}_{2}(t):=\frac{\mu(t)}{\lambda^{2}(t)} \Lambda^{\varepsilon}(t) \log \Lambda(t)
$$

It follows

$$
\overline{\mathcal{V}}_{2}^{\prime}(t) \leq\left[\left(-\beta_{1}+\varepsilon\right) \log \Lambda(t)+1\right] \frac{\mu(t)}{\lambda(t) \Lambda(t)} \Lambda^{\varepsilon}(t)
$$

Then, for sufficiently large time $t$ and sufficiently small $\varepsilon>0$ we get $\overline{\mathcal{V}}_{2}^{\prime}(t)<0$. This shows the decreasing behavior of $\overline{\mathcal{V}}_{2}=\overline{\mathcal{V}}_{2}(t)$. Hence, we obtain that $\bar{V}_{1}=\bar{V}_{1}(t)$ is uniformly bounded. Therefore, we may conclude the following desired estimate for $j+\ell=0,1$ :

$$
\mathbf{V} \lesssim \Phi_{1, m}^{\ell}(0, t)\left(1+B_{\lambda}(0, t)\right)^{(\ell-1) \frac{n}{2}\left(\frac{1}{m}-\frac{1}{2}\right)-\frac{j}{2}} .
$$

Now let us consider the integral $\mathbf{Y}$, that is,

$$
\begin{aligned}
\mathbf{Y} & =\int_{\delta t}^{t} \Phi_{1}^{\ell}(s, t) \frac{\Lambda(s)}{\lambda(s)}\left(1+B_{\lambda}(s, t)\right)^{-\frac{j}{2}}\left(1+B_{\lambda}(0, s)\right)^{-\frac{n}{2 m} p+\frac{n}{4}-\frac{a}{2} p} d s \\
& =-\int_{\delta t}^{t} \Phi_{1}^{\ell}(s, t) \frac{\mu(s)}{\lambda^{2}(s)}\left(1+B_{\lambda}(0, s)\right)^{-\alpha_{2, a}(p)}\left(1+B_{\lambda}(s, t)\right)^{-\frac{j}{2}} d\left(1+B_{\lambda}(s, t)\right),
\end{aligned}
$$

where $\alpha_{2, a}(p):=\frac{n}{2 m} p-\frac{n}{4}+\frac{a}{2} p$. We define for $\ell=0$ the function $\Phi_{1}^{0}(s, t) \equiv 1$. For $\ell=1$ the function $\Phi_{1}^{1}(s, t)=\Phi_{1}(s, t)$ is defined in (4.1.3). 
For $j=\ell=0$ we will discuss the cases $\alpha_{2, a}(p) \neq 1$ and $\alpha_{2, a}(p)=1$, respectively.

Case 1: $\alpha_{2, a}(p) \neq 1$. In this case, by using condition (C4) we have

$$
\begin{aligned}
& \mathbf{Y}=\int_{\delta t}^{t} \frac{\Lambda(s)}{\lambda(s)}\left(1+B_{\lambda}(0, s)\right)^{-\alpha_{2, a}(p)} d s=\int_{\delta t}^{t} \frac{\mu(s)}{\lambda^{2}(s)}\left(1+B_{\lambda}(0, s)\right)^{-\alpha_{2, a}(p)} d\left(1+B_{\lambda}(0, s)\right) \\
& =\frac{1}{1-\alpha_{2, a}(p)}\left[\left.\frac{\mu(s)}{\lambda^{2}(s)}\left(1+B_{\lambda}(0, s)\right)^{-\alpha_{2, a}(p)+1}\right|_{\delta t} ^{t}-\int_{\delta t}^{t}\left(1+B_{\lambda}(0, s)\right)^{-\alpha_{2, a}(p)+1} d\left(\frac{\mu(s)}{\lambda^{2}(s)}\right)\right] \\
& \lesssim \frac{1}{1-\alpha_{2, a}(p)}\left[\left.\frac{\mu(s)}{\lambda^{2}(s)}\left(1+B_{\lambda}(0, s)\right)^{-\alpha_{2, a}(p)+1}\right|_{\delta t} ^{t}+\int_{\delta t}^{t} \frac{\mu(s)}{\lambda^{2}(s)}\left(1+B_{\lambda}(0, s)\right)^{-\alpha_{2, a}(p)+1} \frac{\lambda(s)}{\Lambda(s)} d s\right] .
\end{aligned}
$$

Then, it follows

$$
\begin{aligned}
\left(1+B_{\lambda}(0, t)\right)^{\frac{n}{2}\left(\frac{1}{m}-\frac{1}{2}\right)} \mathbf{Y} \lesssim & \underbrace{\frac{\mu(t)}{\lambda^{2}(t)}\left(1+B_{\lambda}(0, t)\right)^{-\alpha_{2, a}(p)+1}\left(1+B_{\lambda}(0, t)\right)^{\frac{n}{2}\left(\frac{1}{m}-\frac{1}{2}\right)}}_{\bar{Y}_{1}(t)} \\
& +\underbrace{\frac{\mu(\delta t)}{\lambda^{2}(\delta t)}\left(1+B_{\lambda}(0, \delta t)\right)^{-\alpha_{2, a}(p)+1}\left(1+B_{\lambda}(0, t)\right)^{\frac{n}{2}\left(\frac{1}{m}-\frac{1}{2}\right)}}_{\bar{Y}_{2}(t)} \\
& +\underbrace{\int_{\delta t}^{t} \frac{\mu(s)}{\lambda^{2}(s)}\left(1+B_{\lambda}(0, s)\right)^{-\alpha_{2, a}(p)+1}\left(1+B_{\lambda}(0, t)\right)^{\frac{n}{2}\left(\frac{1}{m}-\frac{1}{2}\right)} \frac{\lambda(s)}{\Lambda(s)} d s}_{\bar{Y}_{3}(t)} .
\end{aligned}
$$

Since $\alpha_{2, a}(p)=\alpha_{1, a}(p)+\frac{n}{2}\left(\frac{1}{m}-\frac{1}{2}\right)$, we may conclude for large $t$ the relation

$$
\bar{Y}_{1}(t)=\frac{\mu(t)}{\lambda^{2}(t)}\left(1+B_{\lambda}(0, t)\right)^{-\alpha_{1, a}(p)+1}=\overline{\mathcal{Y}}_{1}(t) \Lambda(t)^{-\varepsilon} \lesssim \Lambda(t)^{-\varepsilon} \lesssim 1
$$

where $\overline{\mathcal{Y}}_{1}=\overline{\mathcal{Y}}_{1}(t)$ is defined by

$$
\overline{\mathcal{Y}}_{1}(t):=\frac{\mu(t)}{\lambda^{2}(t)} \Lambda^{\varepsilon}(t)\left(1+B_{\lambda}(0, t)\right)^{-\alpha_{1, a}(p)+1}
$$

Let us consider the monotonicity of $\overline{\mathcal{Y}}_{1}=\overline{\mathcal{Y}}_{1}(t)$ by taking its derivative. Indeed, by using condition (C4) and (3.1.20) we have

$$
\overline{\mathcal{Y}}_{1}^{\prime}(t) \leq\left[\left(\frac{-\beta_{1}+\varepsilon}{\alpha+2}\right) \log \Lambda(t)+\left(1-\alpha_{1, a}(p)\right)\right]\left(1+B_{\lambda}(0, t)\right)^{-\alpha_{1, a}(p)} \frac{\Lambda(t)}{\lambda(t)} \Lambda(t)^{\varepsilon} .
$$

This inequality implies $\overline{\mathcal{Y}}_{1}^{\prime}(t)<0$ for a small positive constant $\varepsilon$ and for large $t$. Hence, we get

$$
\int_{0}^{\delta t} \frac{\mu(s)}{\lambda^{2}(s)}\left(1+B_{\lambda}(0, s)\right)^{-\alpha_{1, a}(p)+1} \frac{\lambda(s)}{\Lambda(s)} d s \lesssim \int_{0}^{\delta t} \Lambda(s)^{-\varepsilon-1} \lambda(s) d s \lesssim 1 .
$$

Therefore, we may conclude that $\bar{Y}_{1}=\bar{Y}_{1}(t)$ is uniformly bounded.

Now in order to prove that $\bar{Y}_{2}=\bar{Y}_{2}(t)$ and $\bar{Y}_{3}=\bar{Y}_{3}(t)$ are both uniformly bounded it is sufficient to show that for a small positive constant $\varepsilon$ we have

$$
\frac{\mu(\delta t)}{\lambda^{2}(\delta t)}\left(1+B_{\lambda}(0, \delta t)\right)^{-\alpha_{2, a}(p)+1}\left(1+B_{\lambda}(0, t)\right)^{\frac{n}{2}\left(\frac{1}{m}-\frac{1}{2}\right)} \lesssim \Lambda(\delta t)^{-\varepsilon} .
$$

Therefore, we will consider the monotonicity of the function

$$
\overline{\mathcal{Y}}_{2}(t):=\frac{\mu(\delta t)}{\lambda^{2}(\delta t)} \Lambda(\delta t)^{\varepsilon}\left(1+B_{\lambda}(0, \delta t)\right)^{-\alpha_{2, a}(p)+1}\left(1+B_{\lambda}(0, t)\right)^{\frac{n}{2}\left(\frac{1}{m}-\frac{1}{2}\right)}
$$


After performing the derivative of the function $\overline{\mathcal{Y}}_{2}=\overline{\mathcal{Y}}_{2}(t)$ we obtain the estimate

$$
\begin{gathered}
\overline{\mathcal{Y}}_{2}^{\prime}(t) \leq\left[-\delta \beta_{1}+\varepsilon \delta+\left(-\alpha_{2, a}(p)+1\right) \frac{1}{\log \Lambda(\delta t)}+\frac{n}{2}\left(\frac{1}{m}-\frac{1}{2}\right) \frac{\lambda(t)}{\Lambda(t)} \frac{\Lambda(\delta t)}{\lambda(\delta t)} \frac{1}{\log \Lambda(t)}\right] \frac{\lambda(\delta t)}{\Lambda(\delta t)} \\
\times \phi(t) \log \Lambda(t) \phi(\delta t) \log \Lambda(\delta t) \frac{\mu(\delta t)}{\lambda^{2}(\delta t)} \Lambda(\delta t)^{\varepsilon}\left(1+B_{\lambda}(0, \delta t)\right)^{-\alpha_{2, a}(p)}\left(1+B_{\lambda}(0, t)\right)^{\frac{n}{2}\left(\frac{1}{m}-\frac{1}{2}\right)-1}
\end{gathered}
$$

Here we used the condition (C4) and (3.1.20) with condition (C3), respectively. Now employing the condition (4.3.20), we immediately get $\overline{\mathcal{Y}}_{2}^{\prime}(t)<0$ for large $t$. This implies that $\bar{Y}_{2}=\bar{Y}_{2}(t)$ is bounded and using (4.3.25) we find

$$
\bar{Y}_{3}(t) \lesssim \int_{\delta t}^{t} \Lambda(s)^{-\varepsilon-1} \lambda(s) d s \lesssim 1
$$

Hence, we may conclude in the case $j=\ell=0$ the estimate

$$
\mathbf{Y} \lesssim\left(1+B_{\lambda}(0, t)\right)^{-\frac{n}{2}\left(\frac{1}{m}-\frac{1}{2}\right)}
$$

Case 2: $\alpha_{2, a}(p)=1$. In this case it follows

$$
\begin{aligned}
\mathbf{Y} & =\int_{\delta t}^{t} \frac{\Lambda(s)}{\lambda(s)}\left(1+B_{\lambda}(0, s)\right)^{-1} d s=\int_{\delta t}^{t} \frac{\mu(s)}{\lambda^{2}(s)} d\left(\log \left(1+B_{\lambda}(0, s)\right)\right) \\
& =\left.\frac{\mu(s)}{\lambda^{2}(s)} \log \left(1+B_{\lambda}(0, s)\right)\right|_{\delta t} ^{t}-\int_{\delta t}^{t} \log \left(1+B_{\lambda}(0, s)\right) d\left(\frac{\mu(s)}{\lambda^{2}(s)}\right) \\
& \lesssim \frac{\mu(t)}{\lambda^{2}(t)} \log \left(1+B_{\lambda}(0, t)\right)-\frac{\mu(\delta t)}{\lambda^{2}(\delta t)} \log \left(1+B_{\lambda}(0, \delta t)\right)+\int_{\delta t}^{t} \frac{\mu(s)}{\lambda^{2}(s)} \log \left(1+B_{\lambda}(0, s)\right) \frac{\lambda(s)}{\Lambda(s)} d s .
\end{aligned}
$$

Then, it holds

$$
\begin{aligned}
\left(1+B_{\lambda}(0, t)\right)^{\frac{n}{2}\left(\frac{1}{m}-\frac{1}{2}\right)} \mathbf{Y} \lesssim & \frac{\mu(t)}{\lambda^{2}(t)} \log \left(1+B_{\lambda}(0, t)\right)\left(1+B_{\lambda}(0, t)\right)^{\frac{n}{2}\left(\frac{1}{m}-\frac{1}{2}\right)} \\
& +\int_{\delta t}^{t} \frac{\mu(s)}{\lambda^{2}(s)} \log \left(1+B_{\lambda}(0, s)\right)\left(1+B_{\lambda}(0, t)\right)^{\frac{n}{2}\left(\frac{1}{m}-\frac{1}{2}\right)} \frac{\lambda(s)}{\Lambda(s)} d s
\end{aligned}
$$

Similarly, in order to prove $\left(1+B_{\lambda}(0, t)\right)^{\frac{n}{2}\left(\frac{1}{m}-\frac{1}{2}\right)} \mathbf{Y}$ is uniformly bounded we will show that for a sufficiently small positive constant $\varepsilon$ we have the estimate

$$
\frac{\mu(\delta t)}{\lambda^{2}(\delta t)} \log \left(1+B_{\lambda}(0, \delta t)\right)\left(1+B_{\lambda}(0, t)\right)^{\frac{n}{2}\left(\frac{1}{m}-\frac{1}{2}\right)} \lesssim \Lambda(\delta t)^{-\varepsilon}
$$

Then, for an arbitrary positive constant $r$ we have

$$
\begin{aligned}
\frac{\mu(\delta t)}{\lambda^{2}(\delta t)} & \Lambda(\delta t)^{\varepsilon} \log \left(1+B_{\lambda}(0, \delta t)\right)\left(1+B_{\lambda}(0, t)\right)^{\frac{n}{2}\left(\frac{1}{m}-\frac{1}{2}\right)} \\
& \lesssim \frac{\mu(\delta t)}{\lambda^{2}(\delta t)} \Lambda(\delta t)^{\varepsilon}\left(1+B_{\lambda}(0, \delta t)\right)^{r}\left(1+B_{\lambda}(0, t)\right)^{\frac{n}{2}\left(\frac{1}{m}-\frac{1}{2}\right)}
\end{aligned}
$$

where we have employed l'Hospital rule (cf. condition (B4)) as follows:

$$
\lim _{t \rightarrow \infty} \sup \frac{\log \left(1+B_{\lambda}(0, \delta t)\right)}{\left(1+B_{\lambda}(0, \delta t)\right)^{r}}=\lim _{t \rightarrow \infty} \sup \frac{1}{r \delta\left(1+B_{\lambda}(0, \delta t)\right)^{r}} \lesssim 1 .
$$

Therefore, taking account of the monotonic behavior of $\overline{\mathcal{Y}}_{2}=\overline{\mathcal{Y}}_{2}(t)$ we get the desired estimate

$$
\mathbf{Y} \lesssim\left(1+B_{\lambda}(0, t)\right)^{-\frac{n}{2}\left(\frac{1}{m}-\frac{1}{2}\right)}
$$


Now let us consider (4.3.24) in the case $j=1$ and $\ell=0$. Then, we get

$$
\begin{aligned}
\mathbf{Y}= & -\int_{\delta t}^{t} \frac{\mu(s)}{\lambda^{2}(s)}\left(1+B_{\lambda}(0, s)\right)^{-\alpha_{2, a}(p)}\left(1+B_{\lambda}(s, t)\right)^{-\frac{1}{2}} d\left(1+B_{\lambda}(s, t)\right) \\
= & -\left.2 \frac{\mu(s)}{\lambda^{2}(s)}\left(1+B_{\lambda}(0, s)\right)^{-\alpha_{2, a}(p)}\left(1+B_{\lambda}(s, t)\right)^{\frac{1}{2}}\right|_{\delta t} ^{t} \\
& +2 \int_{\delta t}^{t}\left(1+B_{\lambda}(s, t)\right)^{\frac{1}{2}} d\left(\frac{\mu(s)}{\lambda^{2}(s)}\left(1+B_{\lambda}(0, s)\right)^{-\alpha_{2, a}(p)}\right) \\
\lesssim & -\left.\frac{\mu(s)}{\lambda^{2}(s)}\left(1+B_{\lambda}(0, s)\right)^{-\alpha_{2, a}(p)}\left(1+B_{\lambda}(s, t)\right)^{\frac{1}{2}}\right|_{\delta t} ^{t} \\
& +\int_{\delta t}^{t} \frac{\mu(s)}{\lambda^{2}(s)}\left(1+B_{\lambda}(0, s)\right)^{-\alpha_{2, a}(p)}\left(1+B_{\lambda}(s, t)\right)^{\frac{1}{2}} \frac{d\left(1+B_{\lambda}(0, s)\right)}{1+B_{\lambda}(0, s)}
\end{aligned}
$$

where we have used Lemma 3.1.5. Multiplying both sides of the last estimate by the function $\left(1+B_{\lambda}(0, t)\right)^{\frac{n}{2}\left(\frac{1}{m}-\frac{1}{2}\right)+\frac{1}{2}}$, we find

$$
\begin{aligned}
\left(1+B_{\lambda}(0, t)\right)^{\frac{n}{2}\left(\frac{1}{m}-\frac{1}{2}\right)+\frac{1}{2}} \mathbf{Y} & \\
\lesssim & \frac{\mu(\delta t)}{\lambda^{2}(\delta t)}\left(1+B_{\lambda}(0, \delta t)\right)^{-\alpha_{2, a}(p)}\left(1+B_{\lambda}(0, t)\right)^{\frac{n}{2}\left(\frac{1}{m}-\frac{1}{2}\right)+1} \\
& +\int_{\delta t}^{t} \frac{\mu(s)}{\lambda^{2}(s)}\left(1+B_{\lambda}(0, s)\right)^{-\alpha_{2, a}(p)}\left(1+B_{\lambda}(0, t)\right)^{\frac{n}{2}\left(\frac{1}{m}-\frac{1}{2}\right)+1} \frac{d\left(1+B_{\lambda}(0, s)\right)}{1+B_{\lambda}(0, s)} .
\end{aligned}
$$

To derive the desired estimates for the two summands on the right-hand side of the previous inequality, employing (4.3.20), we proceed in the same way as we did in the proof of Theorem 3.2.2 to estimate the terms $\bar{B}_{1}=\bar{B}_{1}(t)$ and $\bar{B}_{2}=\bar{B}_{2}(t)$. Summarizing, we arrive at the estimate

$$
\mathbf{Y} \lesssim\left(1+B_{\lambda}(0, t)\right)^{-\frac{n}{2}\left(\frac{1}{m}-\frac{1}{2}\right)-\frac{1}{2}}
$$

Finally, we will consider (4.3.24) in the case $j=0$ and $\ell=1$. So, we have

$$
\mathbf{Y}=\int_{\delta t}^{t} \Phi_{1}(s, t) \frac{\Lambda(s)}{\lambda(s)}\left(1+B_{\lambda}(0, s)\right)^{-\alpha_{2, a}(p)} d s,
$$

where $\Phi_{1}=\Phi_{1}(s, t)$ is defined in (4.1.3). Employing condition (C1) we are only interested in the following two cases, which explains that we have that either the first component or the second component is dominant in $\Phi_{1}=\Phi_{1}(s, t)$ for all $s \in[\delta t, t]$.

Case a: $\Phi_{1}(s, t)=\frac{\lambda^{2}(t)}{\rho(t)}\left(1+B_{\lambda}(s, t)\right)^{-1}$. In this case it holds

$$
\begin{aligned}
\mathbf{Y}= & -\frac{\lambda^{2}(t)}{\rho(t)} \int_{\delta t}^{t} \frac{\mu(s)}{\lambda^{2}(s)}\left(1+B_{\lambda}(0, s)\right)^{-\alpha_{2, a}(p)} \frac{d\left(1+B_{\lambda}(s, t)\right)}{1+B_{\lambda}(s, t)} \\
= & -\left.\frac{\lambda^{2}(t)}{\rho(t)} \frac{\mu(s)}{\lambda^{2}(s)}\left(1+B_{\lambda}(0, s)\right)^{-\alpha_{2, a}(p)} \log \left(1+B_{\lambda}(s, t)\right)\right|_{\delta t} ^{t} \\
& +\frac{\lambda^{2}(t)}{\rho(t)} \int_{\delta t}^{t} \log \left(1+B_{\lambda}(s, t)\right) d\left(\frac{\mu(s)}{\lambda^{2}(s)}\left(1+B_{\lambda}(0, s)\right)^{-\alpha_{2, a}(p)}\right) \\
\lesssim & -\left.\frac{\lambda^{2}(t)}{\rho(t)} \frac{\mu(s)}{\lambda^{2}(s)}\left(1+B_{\lambda}(0, s)\right)^{-\alpha_{2, a}(p)} \log \left(1+B_{\lambda}(s, t)\right)\right|_{\delta t} ^{t} \\
& +\frac{\lambda^{2}(t)}{\rho(t)} \int_{\delta t}^{t} \frac{\mu(s)}{\lambda^{2}(s)}\left(1+B_{\lambda}(0, s)\right)^{-\alpha_{2, a}(p)} \log \left(1+B_{\lambda}(s, t)\right) \frac{d\left(1+B_{\lambda}(0, s)\right)}{1+B_{\lambda}(0, s)} .
\end{aligned}
$$


Then, we have

$$
\begin{aligned}
\frac{\rho(t)}{\lambda^{2}(t)}(1 & \left.+B_{\lambda}(0, t)\right)^{\frac{n}{2}\left(\frac{1}{m}-\frac{1}{2}\right)+1} \mathbf{Y} \\
& \lesssim \underbrace{\frac{\mu(\delta t)}{\lambda^{2}(\delta t)}\left(1+B_{\lambda}(0, \delta t)\right)^{-\alpha_{2}(p)} \log \left(1+B_{\lambda}(0, t)\right)\left(1+B_{\lambda}(0, t)\right)^{\frac{n}{2}\left(\frac{1}{m}-\frac{1}{2}\right)+1}}_{\widetilde{Y}_{4}(t)} \\
& +\underbrace{\int_{\delta t}^{t} \frac{\mu(s)}{\lambda^{2}(s)} \frac{\log \left(1+B_{\lambda}(0, t)\right)\left(1+B_{\lambda}(0, t)\right)^{\frac{n}{2}\left(\frac{1}{m}-\frac{1}{2}\right)+1}}{\left(1+B_{\lambda}(0, s)\right)^{\alpha_{2}(p)}} \frac{d\left(1+B_{\lambda}(0, s)\right)}{1+B_{\lambda}(0, s)}}_{\bar{Y}_{5}(t)} .
\end{aligned}
$$

After applying the l'Hospital rule for $\gamma>1$ (cf. condition (B4)) we find

$$
\begin{aligned}
\lim _{t \rightarrow \infty} \sup \frac{\log \left(1+B_{\lambda}(0, t)\right)}{\left(1+B_{\lambda}(0, \delta t)\right)^{\gamma}} & =\lim _{t \rightarrow \infty} \sup \frac{\frac{\lambda(t) \Lambda(t)}{\mu(t)}}{\gamma \delta\left(1+B_{\lambda}(0, t)\right) \frac{\lambda(\delta t) \Lambda(\delta t)}{\mu(\delta t)}\left(1+B_{\lambda}(0, \delta t)\right)^{\gamma-1}} \\
& \lesssim \lim _{t \rightarrow \infty} \sup \frac{\lambda(t)}{\Lambda(t)} \frac{\Lambda(\delta t)}{\lambda(\delta t)} \frac{1}{\phi(\delta t) \log \Lambda(t)} \frac{1}{\left(1+B_{\lambda}(0, \delta t)\right)^{\gamma-1}} \lesssim 1,
\end{aligned}
$$

where we used (3.1.20) with condition (C3). Therefore, to prove that the functions $\bar{Y}_{4}=\bar{Y}_{4}(t)$ and $\bar{Y}_{5}=\bar{Y}_{5}(t)$ are bounded we use the estimate

$$
\frac{\mu(\delta t)}{\lambda^{2}(\delta t)}\left(1+B_{\lambda}(0, \delta t)\right)^{-\alpha_{2, a}(p)}\left(1+B_{\lambda}(0, t)\right)^{\frac{n}{2}\left(\frac{1}{m}-\frac{1}{2}\right)+1} \lesssim\left(1+B_{\lambda}(0, \delta t)\right)^{-\gamma} .
$$

Then, we have

$$
\overline{\mathcal{Y}}_{4}(t):=\frac{\mu(\delta t)}{\lambda^{2}(\delta t)}\left(1+B_{\lambda}(0, \delta t)\right)^{-\alpha_{2, a}(p)+\gamma}\left(1+B_{\lambda}(0, t)\right)^{\frac{n}{2}\left(\frac{1}{m}-\frac{1}{2}\right)+1} \lesssim 1 .
$$

To prove the boundedness of the function $\overline{\mathcal{Y}}_{4}=\overline{\mathcal{Y}}_{4}(t)$ we follow the proof of $\overline{\mathcal{B}}_{1}=\overline{\mathcal{B}}_{1}(t)$ which is defined in (3.2.30). Hence, by using (4.3.29) we get

$$
\begin{aligned}
\bar{Y}_{4}(t) & =\frac{\log \left(1+B_{\lambda}(0, t)\right)}{\left(1+B_{\lambda}(0, \delta t)\right)^{\gamma}} \frac{\mu(\delta t)}{\lambda^{2}(\delta t)}\left(1+B_{\lambda}(0, \delta t)\right)^{-\alpha_{2, a}(p)+\gamma}\left(1+B_{\lambda}(0, t)\right)^{\frac{n}{2}\left(\frac{1}{m}-\frac{1}{2}\right)+1} \\
& =\frac{\log \left(1+B_{\lambda}(0, t)\right)}{\left(1+B_{\lambda}(0, \delta t)\right)^{\gamma}} \overline{\mathcal{Y}}_{4}(t) \lesssim \frac{\log \left(1+B_{\lambda}(0, t)\right)}{\left(1+B_{\lambda}(0, \delta t)\right)^{\gamma}} \lesssim 1
\end{aligned}
$$

for large $t$. Moreover, for $\bar{Y}_{5}=\bar{Y}_{5}(t)$ using (4.3.30) and (4.3.31), respectively, we obtain

$$
\bar{Y}_{5}(t) \lesssim \log \left(1+B_{\lambda}(0, t)\right) \int_{\delta t}^{t}\left(1+B_{\lambda}(0, s)\right)^{-\gamma-1} d\left(1+B_{\lambda}(0, s)\right) \lesssim \frac{\log \left(1+B_{\lambda}(0, t)\right)}{\left(1+B_{\lambda}(0, \delta t)\right)^{\gamma}} \lesssim 1,
$$

where we used again the l'Hospital rule from (4.3.29). Consequently, we obtain that $\bar{Y}_{4}=\bar{Y}_{4}(t)$ and $\bar{Y}_{5}=\bar{Y}_{5}(t)$ are uniformly bounded. This implies

$$
\mathbf{Y} \lesssim \frac{\lambda^{2}(t)}{\rho(t)}\left(1+B_{\lambda}(0, t)\right)^{-\frac{n}{2}\left(\frac{1}{m}-\frac{1}{2}\right)-1} .
$$

Taking account of (4.3.26), (4.3.27), (4.3.28) and (4.3.32), in the Case $a$ and $j+\ell=0,1$ we arrive at the estimate

$$
\mathbf{Y} \lesssim \frac{\lambda^{2 \ell}(t)}{\rho^{\ell}(t)}\left(1+B_{\lambda}(0, t)\right)^{-\frac{n}{2}\left(\frac{1}{m}-\frac{1}{2}\right)-\frac{j}{2}-\ell}
$$


Case b: $\Phi_{1}(s, t)=\frac{\lambda(t)}{F(\Lambda(t))}$. In this case we have

$$
\mathbf{Y}=\frac{\lambda(t)}{F(\Lambda(t))} \int_{\delta t}^{t} \frac{\mu(s)}{\lambda^{2}(s)}\left(1+B_{\lambda}(0, s)\right)^{-\alpha_{2, a}(p)} d\left(1+B_{\lambda}(0, s)\right)
$$

Now we distinguish between two cases.

Case b.1: $\alpha_{2, a}(p) \neq 1$. It holds

$$
\begin{aligned}
\frac{F(\Lambda(t))}{\lambda(t)}\left(F^{2}(\Lambda(t))\right)^{\frac{n}{2}\left(\frac{1}{m}-\frac{1}{2}\right)} \mathbf{Y} & \\
\lesssim & \underbrace{\frac{\mu(t)}{\lambda^{2}(t)}\left(1+B_{\lambda}(0, t)\right)^{-\alpha_{2}(p)+1}\left(F^{2}(\Lambda(t))\right)^{\frac{n}{2}\left(\frac{1}{m}-\frac{1}{2}\right)}}_{\bar{Z}_{1}(t)} \\
& +\underbrace{\frac{\mu(\delta t)}{\lambda^{2}(\delta t)}\left(1+B_{\lambda}(0, \delta t)\right)^{-\alpha_{2}(p)+1}\left(F^{2}(\Lambda(t))\right)^{\frac{n}{2}\left(\frac{1}{m}-\frac{1}{2}\right)}}_{\bar{Z}_{2}(t)} \\
& +\underbrace{\int_{\delta t}^{t} \frac{\mu(s)}{\lambda^{2}(s)}\left(1+B_{\lambda}(0, s)\right)^{-\alpha_{2}(p)+1}\left(F^{2}(\Lambda(t))\right)^{\frac{n}{2}\left(\frac{1}{m}-\frac{1}{2}\right)} \frac{\lambda(s)}{\Lambda(s)} d s}_{\bar{Z}_{3}(t)} .
\end{aligned}
$$

Let us begin to estimate $\bar{Z}_{1}=\bar{Z}_{1}(t)$ for both cases $\alpha_{2, a}(p)>1$ and $\alpha_{2, a}(p)<1$. If $\alpha_{2, a}(p)>1$, then we have

$$
\bar{Z}_{1}(t) \leq \frac{\mu(t)}{\lambda^{2}(t)}\left(F^{2}(\Lambda(t))\right)^{\frac{n}{2}\left(\frac{1}{m}-\frac{1}{2}\right)} \leq \frac{\mu(\delta t)}{\lambda^{2}(\delta t)}\left(F^{2}(\Lambda(t))\right)^{\frac{n}{2}\left(\frac{1}{m}-\frac{1}{2}\right)}:=\overline{\mathcal{Z}}_{1}(t),
$$

where we used the decreasing behavior of the function $\frac{\mu(t)}{\lambda^{2}(t)}$ from condition (C4). Now we estimate the derivative of the function $\overline{\mathcal{Z}}_{1}=\overline{\mathcal{Z}}_{1}(t)$. It follows

$$
\overline{\mathcal{Z}}_{1}^{\prime}(t) \leq\left[-\delta \beta_{1} \frac{1}{\log \Lambda(t)}+n\left(\frac{1}{m}-\frac{1}{2}\right) \vartheta(\delta, t) \frac{1}{\log \Lambda(t)}\right] \frac{\mu(\delta t)}{\lambda^{2}(\delta t)} \frac{\lambda(\delta t)}{\Lambda(\delta t)} \log \Lambda(t) F(\Lambda(t))^{n\left(\frac{1}{m}-\frac{1}{2}\right)},
$$

where from condition (A5) we used $F^{\prime}(\Lambda(t))=\frac{F^{2}(\Lambda(t))}{\lambda^{2}(t) \Xi^{2}(t)}$. Therefore, by using (4.3.20) we find $\overline{\mathcal{Z}}_{1}^{\prime}(t)<0$ for large $t$. Analogously, if $\alpha_{2, a}(p)<1$, by using condition (B6) we get

$$
\begin{aligned}
\bar{Z}_{1}(t) & \leq \frac{\mu(t)}{\lambda^{2}(t)}\left(F^{2}(\Lambda(t))\right)^{-\alpha_{2, a}(p)+1}\left(F^{2}(\Lambda(t))\right)^{\frac{n}{2}\left(\frac{1}{m}-\frac{1}{2}\right)} \\
& \leq \frac{\mu(t)}{\lambda^{2}(t)}\left(F^{2}(\Lambda(t))\right)^{-\alpha_{1, a}(p)+1} \leq \frac{\mu(\delta t)}{\lambda^{2}(\delta t)}\left(F^{2}(\Lambda(t))\right)^{-\alpha_{1, a}(p)+1}:=\overline{\mathcal{Z}}_{2}(t) .
\end{aligned}
$$

Hence, in the same way as above we obtain

$$
\overline{\mathcal{Z}}_{2}^{\prime}(t) \leq\left[-\frac{\delta \beta_{1}}{\log \Lambda(t)}+2\left(-\alpha_{1, a}(p)+1\right) \frac{\vartheta(\delta, t)}{\log \Lambda(t)}\right] \frac{\mu(\delta t)}{\lambda^{2}(\delta t)} \frac{\lambda(\delta t)}{\Lambda(\delta t)} \log \Lambda(t)(F(\Lambda(t)))^{-2 \alpha_{1, a}(p)+2} .
$$

Thus, if $\alpha_{1, a}(p)<1$, employing the condition (4.3.20) we may conclude that $\bar{Z}_{1}=\bar{Z}_{1}(t)$ is uniformly bounded for large $t$. Now, in order to show that the functions $\bar{Z}_{2}=\bar{Z}_{2}(t)$ and $\bar{Z}_{3}=\bar{Z}_{3}(t)$ are uniformly bounded we verify

$$
\frac{\mu(\delta t)}{\lambda^{2}(\delta t)}\left(1+B_{\lambda}(0, \delta t)\right)^{-\alpha_{1, a}(p)+1}\left(F^{2}(\Lambda(t))\right)^{\frac{n}{2}\left(\frac{1}{m}-\frac{1}{2}\right)} \lesssim \Lambda(\delta t)^{-\varepsilon}
$$


with an arbitrary small positive constant $\varepsilon$. Then, we may conclude

$$
\frac{\mu(\delta t)}{\lambda^{2}(\delta t)} \Lambda(\delta t)^{\varepsilon}\left(1+B_{\lambda}(0, \delta t)\right)^{-\alpha_{2, a}(p)+1}\left(F^{2}(\Lambda(t))\right)^{\frac{n}{2}\left(\frac{1}{m}-\frac{1}{2}\right)} \lesssim 1
$$

in both cases $\alpha_{2, a}(p)<1$ and $\alpha_{2, a}(p)>1$ as well. In the case $\alpha_{2, a}(p)<1$ we have

$$
\begin{aligned}
& \frac{\mu(\delta t)}{\lambda^{2}(\delta t)} \Lambda(\delta t)^{\varepsilon}\left(1+B_{\lambda}(0, \delta t)\right)^{-\alpha_{2, a}(p)+1}\left(F^{2}(\Lambda(t))\right)^{\frac{n}{2}\left(\frac{1}{m}-\frac{1}{2}\right)} \\
& \quad \lesssim \frac{\mu(\delta t)}{\lambda^{2}(\delta t)} \Lambda(\delta t)^{\varepsilon}\left(F^{2}(\Lambda(t))\right)^{\frac{n}{2}\left(\frac{1}{m}-\frac{1}{2}\right)-\alpha_{2, a}(p)+1}=\frac{\mu(\delta t)}{\lambda^{2}(\delta t)} \Lambda(\delta t)^{\varepsilon}\left(F^{2}(\Lambda(t))\right)^{-\alpha_{1, a}(p)+1}:=\overline{\mathcal{Z}}_{3}(t),
\end{aligned}
$$

where we used condition (B6). We can estimate the derivative of the function $\overline{\mathcal{Z}}_{3}=\overline{\mathcal{Z}}_{3}(t)$ as follows:

$$
\begin{aligned}
\overline{\mathcal{Z}}_{3}^{\prime}(t) \leq[ & \left.-\delta\left(\beta_{1}-\varepsilon\right) \frac{1}{\log \Lambda(t)}+2\left(-\alpha_{1, a}(p)+1\right) \vartheta(\delta, t) \frac{1}{\log \Lambda(t)}\right] \\
& \times \frac{\mu(\delta t)}{\lambda^{2}(\delta t)} \frac{\lambda(\delta t)}{\Lambda(\delta t)} \log \Lambda(t) \Lambda(\delta t)^{\varepsilon}(F(\Lambda(t)))^{-2 \alpha_{1, a}(p)+2} .
\end{aligned}
$$

Therefore, employing (4.3.20) we obtain that $\overline{\mathcal{Z}}_{3}^{\prime}(t)<0$ for large $t$. On the other hand, if $\alpha_{2, a}(p)>1$, then one can derive for large $t$ the estimate

$$
\frac{\mu(\delta t)}{\lambda^{2}(\delta t)} \Lambda(\delta t)^{\varepsilon}\left(1+B_{\lambda}(0, \delta t)\right)^{-\alpha_{2, a}(p)+1}\left(F^{2}(\Lambda(t))\right)^{\frac{n}{2}\left(\frac{1}{m}-\frac{1}{2}\right)} \lesssim \frac{\mu(\delta t)}{\lambda^{2}(\delta t)} \Lambda(\delta t)^{\epsilon}\left(F^{2}(\Lambda(t))\right)^{\frac{n}{2}\left(\frac{1}{m}-\frac{1}{2}\right)} \lesssim 1 .
$$

Summarizing, in the case $\alpha_{2, a}(p) \neq 1$ we obtain the desired estimate

$$
\mathbf{Y} \lesssim \lambda(t) F(\Lambda(t))\left(F^{2}(\Lambda(t))\right)^{-\frac{n}{2}\left(\frac{1}{m}-\frac{1}{2}\right)-1}
$$

Case b.2: $\alpha_{2, a}(p)=1$. In this case it holds

$$
\begin{aligned}
& \frac{F(\Lambda(t))}{\lambda(t)}\left(F^{2}(\Lambda(t))\right)^{\frac{n}{2}\left(\frac{1}{m}-\frac{1}{2}\right)} \mathbf{Y} \\
& \lesssim \underbrace{\frac{\mu(t)}{\lambda^{2}(t)} \log \left(1+B_{\lambda}(0, t)\right)\left(F^{2}(\Lambda(t))\right)^{\frac{n}{2}\left(\frac{1}{m}-\frac{1}{2}\right)}}_{\bar{Z}_{4}(t)} \\
&+\underbrace{\frac{\mu(\delta t)}{\lambda^{2}(\delta t)} \log \left(1+B_{\lambda}(0, \delta t)\right)\left(F^{2}(\Lambda(t))\right)^{\frac{n}{2}\left(\frac{1}{m}-\frac{1}{2}\right)}}_{\bar{Z}_{5}(t)} \\
&+\underbrace{\int_{\delta t}^{t} \frac{\mu(s)}{\lambda^{2}(s)} \log \left(1+B_{\lambda}(0, s)\right)\left(F^{2}(\Lambda(t))\right)^{\frac{n}{2}\left(\frac{1}{m}-\frac{1}{2}\right)} \frac{\lambda(s)}{\Lambda(s)} d s}_{\bar{Z}_{6}(t)} .
\end{aligned}
$$

Applying the l'Hospital rule for $r>1$ (cf. condition (B4)) we find

$$
\begin{aligned}
\lim _{t \rightarrow \infty} \sup \frac{\log \left(1+B_{\lambda}(0, t)\right)}{\left(1+B_{\lambda}(0, \delta t)\right)^{r}} & =\lim _{t \rightarrow \infty} \sup \frac{\frac{\lambda(t) \Lambda(t)}{\mu(t)}}{r \delta\left(1+B_{\lambda}(0, t)\right) \frac{\lambda(\delta t) \Lambda(\delta t)}{\mu(\delta t)}\left(1+B_{\lambda}(0, \delta t)\right)^{r-1}} \\
& \lesssim \lim _{t \rightarrow \infty} \sup \frac{\lambda(t)}{\Lambda(t)} \frac{\Lambda(\delta t)}{\lambda(\delta t)} \frac{1}{\phi(\delta t) \log \Lambda(t)} \frac{1}{\left(1+B_{\lambda}(0, \delta t)\right)^{r-1}} \lesssim 1
\end{aligned}
$$


where we used (3.1.20) with condition (C3). Therefore, in order to estimate $\bar{Z}_{4}=\bar{Z}_{4}(t)$, by using the estimates (4.3.35) we find

$$
\begin{aligned}
\bar{Z}_{4}(t) & \lesssim \frac{\mu(\delta t)}{\lambda^{2}(\delta t)} \frac{\log \left(1+B_{\lambda}(0, t)\right)}{\left(1+B_{\lambda}(0, \delta t)\right)^{r}}\left(1+B_{\lambda}(0, \delta t)\right)^{r}\left(F^{2}(\Lambda(t))\right)^{\frac{n}{2}\left(\frac{1}{m}-\frac{1}{2}\right)} \\
& \lesssim \frac{\mu(\delta t)}{\lambda^{2}(\delta t)}\left(1+B_{\lambda}(0, \delta t)\right)^{r}\left(F^{2}(\Lambda(t))\right)^{\frac{n}{2}\left(\frac{1}{m}-\frac{1}{2}\right)} .
\end{aligned}
$$

Now, using condition (B6) we continue in the same way as we did to estimate $\overline{\mathcal{Z}}_{2}=\overline{\mathcal{Z}}_{2}(t)$ and we may conclude

$$
\bar{Z}_{4}(t) \lesssim \frac{\mu(\delta t)}{\lambda^{2}(\delta t)}\left(1+B_{\lambda}(0, \delta t)\right)^{r}\left(F^{2}(\Lambda(t))\right)^{\frac{n}{2}\left(\frac{1}{m}-\frac{1}{2}\right)} \lesssim \frac{\mu(\delta t)}{\lambda^{2}(\delta t)}\left(F^{2}(\Lambda(t))\right)^{\frac{n}{2}\left(\frac{1}{m}-\frac{1}{2}\right)+r} \lesssim 1 .
$$

Now in order to estimate the functions $\bar{Z}_{5}=\bar{Z}_{5}(t)$ and $\bar{Z}_{6}=\bar{Z}_{6}(t)$ we verify the estimate

$$
\frac{\mu(\delta t)}{\lambda^{2}(\delta t)} \log \left(1+B_{\lambda}(0, \delta t)\right)\left(F^{2}(\Lambda(t))\right)^{\frac{n}{2}\left(\frac{1}{m}-\frac{1}{2}\right)} \lesssim \Lambda(\delta t)^{-\varepsilon},
$$

with an arbitrary small positive constant $\varepsilon$. Then, using again (4.3.35) and proceeding in the same way as we did to estimate $\overline{\mathcal{Z}}_{3}=\overline{\mathcal{Z}}_{3}(t)$ for large $t$, we have

$$
\begin{aligned}
\frac{\mu(\delta t)}{\lambda^{2}(\delta t)} & \Lambda(\delta t)^{\varepsilon} \log \left(1+B_{\lambda}(0, \delta t)\right)\left(F^{2}(\Lambda(t))\right)^{\frac{n}{2}\left(\frac{1}{m}-\frac{1}{2}\right)} \\
& =\frac{\mu(\delta t)}{\lambda^{2}(\delta t)} \Lambda(\delta t)^{\varepsilon} \frac{\log \left(1+B_{\lambda}(0, t)\right)}{\left(1+B_{\lambda}(0, \delta t)\right)^{r}}\left(1+B_{\lambda}(0, \delta t)\right)^{r}\left(F^{2}(\Lambda(t))\right)^{\frac{n}{2}\left(\frac{1}{m}-\frac{1}{2}\right)} \\
& \leq \frac{\mu(\delta t)}{\lambda^{2}(\delta t)} \Lambda(\delta t)^{\varepsilon}\left(F^{2}(\Lambda(t))\right)^{\frac{n}{2}\left(\frac{1}{m}-\frac{1}{2}\right)+r} \lesssim 1 .
\end{aligned}
$$

Consequently, in the case $\alpha_{2}(p)=1$ we obtain the desired estimate

$$
\mathbf{Y} \lesssim \lambda(t) F(\Lambda(t))\left(F^{2}(\Lambda(t))\right)^{-\frac{n}{2}\left(\frac{1}{m}-\frac{1}{2}\right)-1} .
$$

Now taking account of (4.3.26), (4.3.27), (4.3.28), (4.3.34) and (4.3.36) in the Case $b$ and $j+\ell=0,1$ we obtain the estimate

$$
\mathbf{Y} \lesssim \lambda^{\ell}(t) F^{\ell}(\Lambda(t))\left(1+B_{\lambda}(0, t)\right)^{-\frac{n}{2}\left(\frac{1}{m}-\frac{1}{2}\right)-\frac{j}{2}-\ell} .
$$

Combining (4.3.23) and (4.3.37) we may conclude

$$
\left\||D|^{j} \partial_{t}^{\ell} u^{\mathrm{nl}}(t, \cdot)\right\|_{L^{2}} \lesssim\|u\|_{X(t)}^{p} \Phi_{1, m}^{\ell}(0, t)\left(1+B_{\lambda}(0, t)\right)^{(\ell-1) \frac{n}{2}\left(\frac{1}{m}-\frac{1}{2}\right)-\frac{j}{2}},
$$

where $\Phi_{1, m}^{1}(0, t)=\Phi_{1, m}(0, t)$ is defined in (4.1.2) for $s=0$. Replacing the estimates for $j+\ell=0,1$ in the norm of the solution space $X(t)$ we obtain the expected estimate.

In order to prove (4.1.7) we proceed in the same way as in the proof of Theorem 4.3.1 and repeat the same arguments as in the proof of (4.1.6). Finally, we may conclude the proof of the statement (4.1.7) from the definition of the norm of $X(t)$. This completes the proof. 


\section{5. $L^{p}-L^{q}$ estimates for wave equations with strong time-dependent oscillations}

\subsection{Introduction}

Let us consider the following Cauchy problem for the wave equation with time-dependent speed of propagation:

$$
\begin{cases}u_{t t}-a^{2}(t) \Delta u=0, & (t, x) \in[0, \infty) \times \mathbb{R}^{n}, \\ u(0, x)=u_{0}(x), \quad u_{t}(0, x)=u_{1}(x), & x \in \mathbb{R}^{n}\end{cases}
$$

where $a=a(t)$ is a positive function. In the case $a=a(t)$ is a positive constant, then $L^{p}-L^{q}$ decay estimates, or Strichartz estimates are well-known in [41, 42] (cf. [45, 5, 29]). In a precise formulation such $L^{p}-L^{q}$ decay estimates on the conjugate line read as follows:

$$
\left\|u_{t}(t, \cdot), \nabla_{x} u(t, \cdot)\right\|_{L^{q}} \leq C(1+t)^{-\frac{n-1}{2}\left(\frac{1}{p}-\frac{1}{q}\right)}\left(\left\|u_{0}\right\|_{W_{p}^{N_{p}+1}}+\left\|u_{1}\right\|_{W_{p}^{N_{p}}}\right)
$$

with $n \geq 2, p \in(1,2], \frac{1}{p}+\frac{1}{q}=1$ and $N_{p} \geq n\left(\frac{1}{p}-\frac{1}{q}\right)$. Here $W_{p}^{N_{p}}$ denotes the standard Sobolev space over $L^{p}$ with regularity $N_{p} \in \mathbb{N}$.

The treatment of the Cauchy problem (5.1.1) with time-dependent propagation speed is quite delicate. In a series of papers, the authors $[34,35,36,37]$ began to study the question for $L^{p}-L^{q}$ decay estimates on the conjugate line $(p+q=p q)$ for wave equations with time-dependent coefficients. The authors generalized the question for such decay estimates to the wave equation with $a(t)=\lambda(t) \omega(t)$, where $\lambda=\lambda(t)$ is a strictly increasing function and $\omega=\omega(t)$ is a bounded oscillating function. They proposed a classification of oscillations based on the interplay between $\lambda$ and $\omega$ in the following way.

Definition 5.1.1. We assume that there exists a real $\gamma \in[0,1]$ such that the following estimate is satisfied:

$$
\left|\omega^{(k)}(t)\right| \leq C_{k}\left(\frac{\lambda(t)}{\Lambda(t)}(\log \Lambda(t))^{\gamma}\right)^{k}, \quad \text { for large } t, k \in \mathbb{N},
$$

where $\Lambda=\Lambda(t)$ is defined by $\Lambda(t):=\int_{0}^{t} \lambda(\tau) d \tau$. The parameter $\gamma$ controls the oscillations of $\omega$ as follows:

- if $\gamma=0$, then $\omega$ has very slow oscillations,

- if $0<\gamma<1$, then $\omega$ has slow oscillations,

- if $\gamma=1$, then $\omega$ has fast oscillations,

- and if the condition (5.1.3) is not satisfied for $\gamma=1$, then $\omega$ has very fast oscillations.

If (5.1.3) holds for $\gamma \in[0,1]$, then $L^{p}-L^{q}$ estimates to the solution $u=u(t, x)$ in (5.1.1) with $a(t)=\lambda(t) \omega(t)$ are given by

$$
\left\|\left(u_{t}, \lambda(t) \nabla_{x} u\right)(t, \cdot)\right\|_{L^{q}} \leq C \sqrt{\lambda(t)}(\Lambda(t))^{\gamma_{0}-\frac{n-1}{2}\left(\frac{1}{p}-\frac{1}{q}\right)}\left(\left\|u_{0}\right\|_{W_{p}^{N_{p}+1}}+\left\|u_{1}\right\|_{W_{p}^{N_{p}}}\right),
$$

where the real value $\gamma_{0} \geq 0$ depends on $\gamma$. Here $n \geq 2, p \in(1,2], \frac{1}{p}+\frac{1}{q}=1$ and $N_{p} \geq n\left(\frac{1}{p}-\frac{1}{q}\right)$. Moreover, if the condition (5.1.3) does not hold for $\gamma=1$, then no such estimate can be 
found: a counterexample is constructed in [34]. There is a connection between the oscillating behavior of $\omega$ and the loss of decay, that is, the size of $\gamma_{0}$ describes how the decay rate differs from the classical one in (5.1.2). This means, if the oscillations of $\omega$ are very slow, slow or fast by assuming some suitable assumptions on the smooth coefficient $\lambda=\lambda(t)$ one can expect $L^{p}-L^{q}$ estimates as shown above. If the oscillations are very fast, then one can not expect such type of estimates.

Then, in the paper [33] the authors proved $L^{p}-L^{q}$ decay estimates for Sobolev solutions to (5.1.1) in the case that the coefficient $a=a(t)$ is bounded, sufficiently smooth and an oscillating function. Here let us point out that the authors studied the limit case of (5.1.3), where $\lambda(t) \equiv 1$ which is not covered by the above cited results. The main idea of their approach is to gain the WKB representation of solutions to the ordinary differential equation corresponding to (5.1.1) through the Fourier transform, and apply the stationary phase method to the Fourier multipliers.

Our main goal in this chapter is to derive $L^{p}-L^{q}$ estimates for Sobolev solutions to the Cauchy problem (5.1.1) in the case that the coefficient has very fast oscillations. To do this we want to modify the conditions to allow stronger oscillations. More precisely, we want to study the remaining case of the above cited papers. Our approach follows similar arguments used in the papers [34, 35, 36, 37] and [33], and based on explicit representations of solutions. There are two major differences to the situation of the cited papers: the hyperbolic zone is smaller (which makes it necessary to invoke a new argument for small frequencies) and the weaker assumptions for derivatives make it necessary to perform more diagonalization steps. In consequence, representations by Fourier integrals contain an inhomogeneous phase function.

We know that the time-dependent coefficients makes the problem more difficult, especially oscillating coefficients. Therefore, if one proves some decay estimates, then we should consider a balance between the coefficients. It turns out that it is useful to write

$$
a(t)=\lambda(t) \omega(t)
$$

where

- $\lambda=\lambda(t)$ describes the increasing behavior (improving influence on $L^{p}-L^{q}$ decay estimates),

- $\omega=\omega(t)$ describes the oscillating behavior (deteriorating influence on $L^{p}-L^{q}$ decay estimates).

Some tools are used from the paper [19]. In this paper the authors only studied $L^{2}-L^{2}$ estimates of energy solutions to (5.1.1) with $a(t)=\lambda(t) \omega(t)$, where $\lambda=\lambda(t)$ is a strictly increasing function and $\omega=\omega(t)$ is a bounded function. By using the $\mathcal{C}^{M}$ property of $\lambda=\lambda(t)$ and $\omega=\omega(t)$ and the idea of a stabilization condition they proved the two sided energy estimate

$$
C_{0} \leq \frac{1}{\lambda(t)} \mathbb{E}_{\lambda}(u)(t) \leq C_{1},
$$

where the nonnegative constants $C_{0}$ and $C_{1}$ depend on the data and $\mathbb{E}_{\lambda}(u)(t)$ is defined as

$$
\mathbb{E}_{\lambda}(u)(t):=\frac{1}{2}\left(\lambda^{2}(t)\left\|\nabla_{x} u(t, \cdot)\right\|_{L^{2}}^{2}+\left\|u_{t}(t, \cdot)\right\|_{L^{2}}^{2}\right) .
$$

\subsection{Representation of solutions}

We consider the following Cauchy problem:

$$
\begin{cases}u_{t t}-\lambda^{2}(t) \omega^{2}(t) \Delta u=0, & (t, x) \in[0, \infty) \times \mathbb{R}^{n} \\ u(0, x)=u_{0}(x), \quad u_{t}(0, x)=u_{1}(x), & x \in \mathbb{R}^{n} .\end{cases}
$$

Here $\lambda=\lambda(t)$ is an increasing shape function and $\omega=\omega(t)$ is a bounded oscillating function. Then, one has to explain the interplay between both parts. This can be given by the following conditions. 


\subsubsection{Assumptions}

We suppose that the smooth time-dependent coefficients $\lambda=\lambda(t)$ and $\omega=\omega(t)$ satisfy the following conditions:

(A1) $\lambda(t)>0$ and $\lambda^{\prime}(t)>0$ for all times $t$ and the derivatives of $\lambda$ satisfy the conditions

$$
\lambda_{0} \frac{\lambda(t)}{\Lambda(t)} \leq \frac{\lambda^{\prime}(t)}{\lambda(t)} \leq \lambda_{1} \frac{\lambda(t)}{\Lambda(t)}, \quad\left|d_{t}^{k} \lambda(t)\right| \leq \lambda_{k} \lambda(t)\left(\frac{\lambda(t)}{\Lambda(t)}\right)^{k}, \text { for all } k=1,2, \cdots,
$$

where $\lambda_{0}$ and all $\lambda_{k}$ are positive constants and $\Lambda(t)=1+\int_{0}^{t} \lambda(\tau) d \tau$ is a primitive of $\lambda(t)$

(A2) $\vartheta(t)>0$ and $\vartheta^{\prime}(t)>0$ for all times $t$ and the derivatives of $\vartheta$ satisfy the conditions

$$
\vartheta_{0} \frac{\vartheta(t)}{\Theta(t)} \leq \frac{\vartheta^{\prime}(t)}{\vartheta(t)} \leq \vartheta_{1} \frac{\vartheta(t)}{\Theta(t)}, \quad\left|d_{t}^{k} \vartheta(t)\right| \leq \vartheta_{k} \vartheta(t)\left(\frac{\vartheta(t)}{\Theta(t)}\right)^{k}, \text { for all } k=1,2, \cdots,
$$

where $\vartheta_{0}$ and all $\vartheta_{k}$ are positive constants and $\Theta(t)=1+\int_{0}^{t} \vartheta(\tau) d \tau$ is a primitive of $\vartheta(t)$

(A3) for $M \geq 2$ the following estimate holds:

$$
\int_{t}^{\infty} \lambda(\tau)^{1-M} \Xi(\tau)^{-M} d \tau \lesssim \Theta(t)^{1-M}
$$

(A4) $0<c_{0} \leq \omega(t) \leq c_{1}$ and the derivatives of $\omega$ satisfy the conditions

$$
\left|d_{t}^{k} \omega(t)\right| \lesssim \Xi(t)^{-k}, \text { for all } k=1,2, \cdots,
$$

where $\Xi=\Xi(t)$ is a positive and continuous function satisfying the compatibility condition

$$
\frac{\Theta(t)}{\lambda(t)} \lesssim \Xi(t) \lesssim \frac{\Lambda(t)}{\lambda(t)}
$$

here $\Theta=\Theta(t)$ is a strictly increasing continuous function with $\Theta(0)=1$;

(A5) the function $\frac{\lambda(t)}{\Lambda(t)} \Xi(t)$ is decreasing.

In order to develop $L^{p}-L^{q}$ decay estimates for the solution $u$ and its partial derivatives $u_{t}$, and $\nabla_{x} u$ to $(5.2 .1)$ we investigate solution representations by using Fourier multipliers. For this reason we apply the partial Fourier transformation with respect to spatial variables to (5.2.1). Then, we get that $\hat{v}(t, \xi)=\mathcal{F}_{x \rightarrow \xi}(u(t, x))(t, \xi)$ solves the Cauchy problem

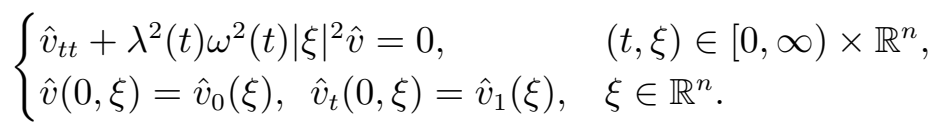

In this chapter we apply a diagonalization procedure to the Cauchy problem (5.2.1) with sufficiently smooth time-dependent coefficients $\lambda=\lambda(t)$ and $\omega=\omega(t)$ aiming to find a representation for the solution by Fourier multipliers. The core of this strategy was given in [49] and further developed in [36, 37, 33, 16, 19] for the treatment of the large-time behaviour of uniformly strictly hyperbolic systems. 


\section{Division of the extended phase space}

To find a WKB-representation of the solution to (5.2.2) we divide the extended phase space $[0, \infty) \times \mathbb{R}_{\xi}^{n}$ into three zones: the hyperbolic zone, the oscillation subzone and the pseudodifferential zone. This division enables us to use the hyperbolicity of our starting problem (5.2.1) and tools from hyperbolic theory in the hyperbolic zone.

For a given $N>0$, these zones are defined as follows:

- hyperbolic zone:

$$
Z_{\mathrm{hyp}}(N)=\left\{(t, \xi) \in[0, \infty) \times \mathbb{R}_{\xi}^{n}: \Theta(t)|\xi| \geq N\right\}
$$

- oscillation subzone:

$$
Z_{\mathrm{osc}}(N)=\left\{(t, \xi) \in[0, \infty) \times \mathbb{R}_{\xi}^{n}: \Theta(t)|\xi| \leq N \leq \Lambda(t)|\xi|\right\},
$$

- pseudo-differential zone:

$$
Z_{\mathrm{pd}}(N)=\left\{(t, \xi) \in[0, \infty) \times \mathbb{R}_{\xi}^{n}: \Lambda(t)|\xi| \leq N\right\} .
$$

We introduce separating lines between these zones as follows:

- by $t_{|\xi|}=t(|\xi|)$, we denote the separating line between the oscillation subzone and the pseudo-differential zone, which solves $\Lambda\left(t_{|\xi|}\right)|\xi|=N$;

- by $\tilde{t}_{|\xi|}=\tilde{t}(|\xi|)$, we denote the separating line between the hyperbolic zone and the oscillation subzone, which solves $\Theta\left(\tilde{t}_{|\xi|}\right)|\xi|=N$.

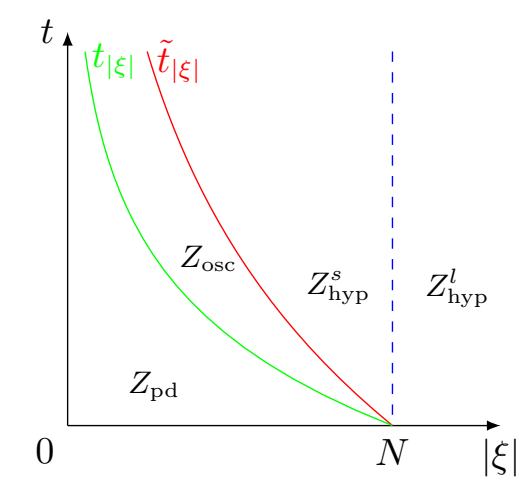

Fig. 5.1.: Division of extended phase space into zones

Remark 5.2.1. We have that the hyperbolic zone is $Z_{\text {hyp }}(N)=Z_{\text {hyp }}^{s}(N) \cup Z_{\text {hyp }}^{l}(N)$. In the zones $Z_{\text {hyp }}^{s}(N), Z_{\text {osc }}(N)$ and $Z_{\mathrm{pd}}(N)$ we consider only small frequencies and in the zone $Z_{\text {hyp }}^{l}(N)$ we consider only large frequencies. The zones $Z_{\text {hyp }}^{s}(N)$ and $Z_{\text {hyp }}^{l}(N)$ will be defined and considered in the next section.

\subsubsection{Considerations in the pseudo-differential zone}

We define $V(t, \xi)=\left(\lambda(t)|\xi| \hat{v}, D_{t} \hat{v}\right)^{T}$. Then, from (5.2.2) it follows

$$
D_{t} V=\underbrace{\left(\begin{array}{cc}
\frac{D_{t} \lambda(t)}{\lambda(t)} & \lambda(t)|\xi| \\
\lambda(t) \omega^{2}(t)|\xi| & 0
\end{array}\right)}_{A(t, \xi)} V .
$$


We denote by $E_{\mathrm{pd}}=E_{\mathrm{pd}}(t, s, \xi)$ the fundamental solutions of $(5.2 .2)$ for any $0 \leq s \leq t$, that is, the matrix-valued functions solving the Cauchy problem

$$
D_{t} E_{\mathrm{pd}}(t, s, \xi)=A(t, \xi) E_{\mathrm{pd}}(t, s, \xi), \quad E_{\mathrm{pd}}(s, s, \xi)=I .
$$

Hence, we can write $V(t, \xi)=E_{\mathrm{pd}}(t, 0, \xi) V_{0}(\xi)$. The fundamental solution $E_{\mathrm{pd}}=E_{\mathrm{pd}}(t, s, \xi)$ can be written as an infinite sum via Peano-Baker series

$$
E_{\mathrm{pd}}(t, s, \xi)=I+\sum_{j=1}^{\infty} \int_{s}^{t} A\left(t_{1}, \xi\right) \int_{s}^{t_{1}} A\left(t_{2}, \xi\right) \cdots \int_{s}^{t_{j-1}} A\left(t_{j}, \xi\right) d t_{j} \cdots d t_{1} .
$$

For $(t, \xi) \in Z_{\mathrm{pd}}(N)$ we have

$$
\int_{s}^{t}|A(\tau, \xi)| d \tau \leq C|\xi| \int_{s}^{t} \lambda(\tau) d \tau+\int_{s}^{t} \frac{\lambda^{\prime}(\tau)}{\lambda(\tau)} d \tau \leq C \Lambda(t)|\xi|+\ln \frac{\lambda(t)}{\lambda(s)} \leq C N+\ln \frac{\lambda(t)}{\lambda(s)},
$$

where we used the Euclidian norm of the matrix $A=A(t, \xi)$. Hence, we get

$$
\exp \left(\int_{s}^{t}|A(\tau, \xi)| d \tau\right) \lesssim \frac{\lambda(t)}{\lambda(s)}
$$

Following the considerations from the paper [19] we have the following two statements.

Proposition 5.2.1. Assume that $\lambda=\lambda(t)$ satisfies $(\boldsymbol{A} 1)$. Then, there exists a real $\delta \in(0,1)$ such that

$$
\lambda(t)^{\delta} \int_{0}^{t} \lambda(\tau)^{1-\delta} d \tau \lesssim \Lambda(t)
$$

Proposition 5.2.2. We assume that conditions $(\boldsymbol{A} 1)$ and $(\boldsymbol{A} 4)$ are satisfied. Then, the fundamental solution $E_{p d}=E_{p d}(t, s, \xi)$ satisfies the following estimates for $0 \leq s \leq t \leq t_{|\xi|}$ :

$$
\left(\left|E_{p d}(t, s, \xi)\right|\right) \lesssim\left(\begin{array}{cc}
\lambda(t) & |\xi| \lambda(t)^{1-\delta} \Lambda(t) \\
|\xi| K(t) & \lambda(t)^{1-\delta}
\end{array}\right)
$$

where $K(t)=\int_{0}^{t} \lambda^{2}(\tau) d \tau \leq \lambda(t) \Lambda(t)$

\subsubsection{Considerations in the oscillation subzone}

In $Z_{\text {osc }}(N)$ we will carry out only two steps of diagonalization. Two steps of diagonalization allow us in the study of Fourier multipliers to apply the Hardy-Littlewood theorem not only in $Z_{\mathrm{pd}}(N)$, but also in $Z_{\mathrm{osc}}(N)$.

Definition 5.2.1. The time-dependent amplitude function $f=f(t, \xi) \in \mathcal{C}^{\infty}\left(Z_{\text {osc }}(N)\right)$ belongs to the symbol class $S_{N}\left\{m_{1}, m_{2}, m_{3}\right\}$ with infinite smoothness, if it satisfies the symbollike estimates

$$
\left|D_{\xi}^{\alpha} D_{t}^{k} f(t, \xi)\right| \leq C_{\alpha, k}|\xi|^{m_{1}-|\alpha|} \lambda(t)^{m_{2}} \Xi(t)^{-m_{3}-k}
$$

for all $(t, \xi) \in Z_{\text {osc }}(N)$ and all multi-indices $\alpha$ and all $k$. Here the constants $C_{\alpha, k}$ are independent of $N$.

We introduce $V(t, \xi)=\left(\lambda(t)|\xi| \hat{v}, D_{t} \hat{v}\right)^{T}$. Then, it holds

$$
D_{t} V=\left(\begin{array}{cc}
0 & \lambda(t)|\xi| \\
\lambda(t) \omega^{2}(t)|\xi| & 0
\end{array}\right) V+\left(\begin{array}{cc}
\frac{D_{t} \lambda(t)}{\lambda(t)} & 0 \\
0 & 0
\end{array}\right) V .
$$

Let us carry out the first step of the diagonalization procedure. The eigenvalues of the first matrix are $\pm \lambda(t) \omega(t)|\xi|$. Thus, the matrix of eigenvectors $P=P(t)$ and its inverse $P^{-1}=P^{-1}(t)$ are

$$
P(t)=\frac{1}{\omega(t)}\left(\begin{array}{cc}
1 & -1 \\
\omega(t) & \omega(t)
\end{array}\right), \quad P^{-1}(t)=\frac{1}{2}\left(\begin{array}{cc}
\omega(t) & 1 \\
-\omega(t) & 1
\end{array}\right) .
$$


Defining $V^{(1)}:=P^{-1}(t) V$, we get the transformed system

$$
D_{t} V^{(1)}=\left[\mathcal{D}_{0}(t, \xi)+\mathcal{R}_{0}(t, \xi)\right] V^{(1)}
$$

where

$$
\mathcal{D}_{0}(t, \xi):=\left(\begin{array}{cc}
\lambda(t) \omega(t)|\xi| & 0 \\
0 & -\lambda(t) \omega(t)|\xi|
\end{array}\right) \in S_{N}\{1,1,0\}
$$

and

$$
\mathcal{R}_{0}(t, \xi):=\frac{D_{t}(\lambda(t) \omega(t))}{2 \lambda(t) \omega(t)}\left(\begin{array}{cc}
1 & -1 \\
-1 & 1
\end{array}\right) \in S_{N}\{0,0,1\}
$$

We can write

$$
\left(D_{t}-\mathcal{D}_{1}(t, \xi)-\mathcal{R}_{1}(t, \xi)\right) V^{(1)}(t, \xi)=0,
$$

where

$$
\mathcal{D}_{1}(t, \xi):=\left(\begin{array}{cc}
\lambda(t) \omega(t)|\xi|+\frac{D_{t}(\lambda(t) \omega(t))}{2 \lambda(t) \omega(t)} & 0 \\
0 & -\lambda(t) \omega(t)|\xi|+\frac{D_{t}(\lambda(t) \omega(t))}{2 \lambda(t) \omega(t)}
\end{array}\right)
$$

and

$$
\mathcal{R}_{1}(t, \xi):=\left(\begin{array}{cc}
0 & -\frac{D_{t}(\lambda(t) \omega(t))}{2 \lambda(t) \omega(t)} \\
-\frac{D_{t}(\lambda(t) \omega(t))}{2 \lambda(t) \omega(t)} & 0
\end{array}\right) .
$$

Note that $\mathcal{R}_{1}(t, \xi) \in S_{N}\{0,0,1\}$. The next lemma shows that it is reasonable to carry out one more step of the diagolization procedure in $Z_{\mathrm{osc}}(N)$.

Lemma 5.2.3. Assume $(\boldsymbol{A} 1)$ to (A3). Then, there exist matrix-valued functions $N_{1}=$ $N_{1}(t, \xi) \in S_{N}\{0,0,0\}$ and $\mathcal{R}_{2}=\mathcal{R}_{2}(t, \xi) \in S_{N}\{-1,-1,2\}$ such that the following operatorvalued identity holds:

$$
\left(D_{t}-\mathcal{D}_{1}(t, \xi)-\mathcal{R}_{1}(t, \xi)\right) N_{1}(t, \xi)=N_{1}(t, \xi)\left(D_{t}-\mathcal{D}_{1}(t, \xi)-\mathcal{R}_{2}(t, \xi)\right),
$$

where $N_{1}=N_{1}(t, \xi)$ is invertible and belongs together with its inverse $N_{1}^{-1}=N_{1}^{-1}(t, \xi)$ to $S_{N}\{0,0,0\}$.

Proof. Let us carry out the second step of diagonalization procedure. Therefore, we introduce

$$
N^{(1)}(t, \xi)=:\left(\begin{array}{cc}
0 & -\frac{\mathcal{R}_{1}^{(12)}}{\tau_{1}^{-}-\tau_{1}^{+}} \\
\frac{\mathcal{R}_{1}^{(21)}}{\tau_{1}^{-}-\tau_{1}^{+}} & 0
\end{array}\right)=\left(\begin{array}{cc}
0 & -\frac{D_{t}(\lambda(t) \omega(t))}{4 \lambda^{2}(t) \omega^{2}(t)|\xi|} \\
\frac{D_{t}(\lambda(t) \omega(t))}{4 \lambda^{2}(t) \omega^{2}(t)|\xi|} & 0
\end{array}\right) \in S_{N}\{-1,-1,1\},
$$

$N_{1}(t, \xi)=I+N^{(1)}(t, \xi) \in S_{N}\{0,0,0\}$. For sufficiently large time $t$ the matrix $N_{1}=N_{1}(t, \xi)$ is invertible with uniformly bounded inverse $N^{-1}=N^{-1}(t, \xi)$. Now we can follow the usual procedure of the diagonalization. Let

$$
\begin{aligned}
B^{(1)}(t, \xi) & =D_{t} N^{(1)}(t, \xi)-\mathcal{R}_{1}(t, \xi) N^{(1)}(t, \xi) \in S_{N}\{-1,-1,2\}, \\
\mathcal{R}_{2}(t, \xi) & =-N^{-1}(t, \xi) B^{(1)}(t, \xi) \in S_{N}\{-1,-1,2\} .
\end{aligned}
$$

Then, we may conclude

$$
\left(D_{t}-\mathcal{D}_{1}(t, \xi)-\mathcal{R}_{1}(t, \xi)\right) N_{1}(t, \xi)=N_{1}(t, \xi)\left(D_{t}-\mathcal{D}_{1}(t, \xi)-\mathcal{R}_{2}(t, \xi)\right) .
$$

This we wanted to have.

Remark 5.2.2. If $\mathcal{R}_{2}=\mathcal{R}_{2}(t, \xi) \in S_{N}\{-1,-1,2\}$, then we have for all $t_{|\xi|} \leq t \leq \tilde{t}_{|\xi|}$ the estimates

$$
\int_{t_{|\xi|}}^{t} \mathcal{R}_{2}(\tau, \xi) d \tau \lesssim \int_{t_{|\xi|}}^{t}|\xi|^{-1} \lambda(\tau)^{-1} \Xi(\tau)^{-2} d \tau \lesssim \Lambda\left(t_{|\xi|}\right) \int_{t_{|\xi|}}^{t} \lambda(\tau)^{-1} \Xi(\tau)^{-2} d \tau \lesssim \frac{\Lambda\left(t_{|\xi|}\right)}{\Theta\left(t_{|\xi|}\right)},
$$

where we used $\Lambda\left(t_{|\xi|}\right)|\xi|=N$ and condition (A3). 
Example 5.2.1. Let $\lambda(t)=(\alpha+1)(1+t)^{\alpha}$ with $\alpha>0$. Then, we get

$$
\Lambda(t)=(1+t)^{\alpha+1} \quad \text { and } \quad \Theta(t)=(1+t)^{\gamma+1}, \quad 0<\gamma<\alpha .
$$

Moreover, for $M=2$ we have

$$
\Xi(t)=(1+t)^{\kappa}, \quad 1>\kappa \geq 1+\frac{\gamma-\alpha}{2} .
$$

Then, from $\mathcal{R}_{2}(t, \xi) \in S_{N}\{-1,-1,2\}$ and $\Lambda\left(t_{|\xi|}\right)|\xi|=N$ we find

$$
\begin{aligned}
\int_{t_{|\xi|}}^{t} \mathcal{R}_{2}(\tau, \xi) d \tau & \lesssim \int_{t_{|\xi|}}^{t}|\xi|^{-1} \lambda(\tau)^{-1} \Xi(\tau)^{-2} d \tau=\frac{1}{N} \Lambda\left(t_{|\xi|}\right) \int_{t_{|\xi|}}^{t} \lambda(\tau)^{-1} \Xi(\tau)^{-2} d \tau \\
& =\frac{\alpha+1}{N}\left(1+t_{|\xi|}\right)^{\alpha+1} \int_{t_{|\xi|}}^{t}(1+\tau)^{-\alpha-2 \kappa} d \tau \\
& =\frac{\alpha+1}{N(-\alpha-2 \kappa+1)}\left(\left(1+t_{|\xi|}\right)^{\alpha+1}(1+t)^{-\alpha-2 \kappa+1}-\left(1+t_{|\xi|}\right)^{-2 \kappa+2}\right) \\
& \lesssim\left(1+t_{|\xi|}\right)^{-2 \kappa+2} .
\end{aligned}
$$

Here we used the following relations:

$$
\begin{aligned}
\kappa<1 \quad \text { iff } & -2 \kappa+2>0, \\
\kappa \geq 1+\frac{\gamma-\alpha}{2} \quad \text { iff } & -1 \geq-\alpha-2 \kappa+1+\gamma .
\end{aligned}
$$

Example 5.2.2. We consider $\lambda(t)=e^{t}$. Then, we obtain

$$
\Lambda(t)=e^{t} \quad \text { and } \quad \Theta(t)=e^{r t}, \quad 0<r<1 .
$$

Moreover, by $M=2$ we have

$$
\Xi(t)=e^{\kappa t}, \quad 0>\kappa \geq \frac{r-1}{2} .
$$

Now, from $\mathcal{R}_{2}(t, \xi) \in S_{N}\{-1,-1,2\}$ and $\Lambda\left(t_{|\xi|}\right)|\xi|=N$ we find

$$
\begin{aligned}
\int_{t_{|\xi|}}^{t} \mathcal{R}_{2}(\tau, \xi) d \tau & \lesssim \int_{t_{|\xi|}}^{t}|\xi|^{-1} \lambda(\tau)^{-1} \Xi(\tau)^{-2} d \tau \\
& =\frac{1}{N} \Lambda\left(t_{|\xi|}\right) \int_{t_{|\xi|}}^{t} \lambda(\tau)^{-1} \Xi(\tau)^{-2} d \tau=\frac{1}{N} e^{t_{|\xi|}} \int_{t_{|\xi|}}^{t} e^{(-1-2 \kappa) \tau} d \tau \\
& =\frac{1}{N(-1-2 \kappa)}\left(e^{t_{|\xi|}} e^{(-1-2 \kappa) t}-e^{-2 \kappa t_{|\xi|}}\right) \lesssim e^{-2 \kappa t_{|\xi|}},
\end{aligned}
$$

where we used

$$
0>\kappa \geq \frac{r-1}{2} \quad \text { iff } \quad 0>-r>-1-2 \kappa .
$$

Now we want to construct the fundamental solution $E_{\mathrm{osc}}=E_{\mathrm{osc}}(t, s, \xi), t_{|\xi|} \leq s \leq t \leq \tilde{t}_{|\xi|}$, for the operator

$$
D_{t}-\mathcal{D}_{1}(t, \xi)-\mathcal{R}_{1}(t, \xi) .
$$

For this reason after two steps of diagonalization it is sufficient to construct the fundamental solution satisfying the system

$$
D_{t} E_{2}(t, s, \xi)=\left(\mathcal{D}_{1}(t, \xi)+\mathcal{R}_{2}(t, \xi)\right) E_{2}(t, s, \xi), \quad E_{2}(s, s, \xi)=I .
$$

We begin to solve the diagonal system

$$
D_{t} \mathcal{E}_{2}(t, s, \xi)=\mathcal{D}_{1}(t, \xi) \mathcal{E}_{2}(t, s, \xi), \quad \mathcal{E}_{2}(s, s, \xi)=I, \quad 0 \leq s \leq t .
$$


Its fundamental solution is represented by

$$
\mathcal{E}_{2}(t, s, \xi)=\exp \left(i \int_{s}^{t} \mathcal{D}_{1}(\theta, \xi) d \theta\right)=\operatorname{diag}\left(e^{i \int_{s}^{t} \tau_{2}^{+}(\theta, \xi) d \theta}, e^{i \int_{s}^{t} \tau_{2}^{-}(\theta, \xi) d \theta}\right) .
$$

We make the ansatz $E_{2}(t, s, \xi)=\mathcal{E}_{2}(t, s, \xi) \mathcal{Q}_{2}(t, s, \xi)$ with a uniformly bounded and invertible matrix $\mathcal{Q}_{2}=\mathcal{Q}_{2}(t, s, \xi)$. It follows that the matrix $\mathcal{Q}_{2}=\mathcal{Q}_{2}(t, s, \xi)$ satisfies the system

$$
D_{t} \mathcal{Q}_{2}(t, s, \xi)=R_{2}(t, s, \xi) \mathcal{Q}_{2}(t, s, \xi), \quad \mathcal{Q}_{2}(s, s, \xi)=I
$$

with the coefficient matrix

$$
R_{2}(t, s, \xi)=\mathcal{E}_{2}(s, t, \xi) \mathcal{R}_{2}(t, \xi) \mathcal{E}_{2}(t, s, \xi) .
$$

Taking account of $\mathcal{R}_{2}(t, \xi) \in S_{N}\{-1,-1,2\}$ we obtain

$$
\left|R_{2}(t, s, \xi)\right|=\left|\mathcal{R}_{2}(t, \xi)\right| \lesssim|\xi|^{-1} \lambda(t)^{-1} \Xi(t)^{-2} .
$$

The solution $\mathcal{Q}_{2}=\mathcal{Q}_{2}(t, s, \xi)$ can be represented as Peano-Baker series

$$
\mathcal{Q}_{2}(t, s, \xi)=I+\sum_{k=1}^{\infty} i^{k} \int_{s}^{t} R_{2}\left(t_{1}, s, \xi\right) \int_{s}^{t_{1}} R_{2}\left(t_{2}, s, \xi\right) \cdots \int_{s}^{t_{k-1}} R_{2}\left(t_{k}, s, \xi\right) d t_{k} \cdots d t_{1} .
$$

Due to the representation of $R_{2}(t, s, \xi)$ in (5.2.5) we have

$$
\begin{aligned}
R_{2}\left(t, t_{|\xi|}, \xi\right) & =\mathcal{E}_{2}\left(t_{|\xi|}, t, \xi\right) \mathcal{R}_{2}(t, \xi) \mathcal{E}_{2}\left(t, t_{|\xi|}, \xi\right) \\
& =\left(\begin{array}{cc}
r_{2}^{(11)} & r_{2}^{(12)} e^{-2 i|\xi| \int_{t_{|\xi|}}^{t} \lambda(\tau) \omega(\tau) d \tau} \\
r_{2}^{(21)} e^{2 i|\xi| \int_{t|\xi|}^{t} \lambda(\tau) \omega(\tau) d \tau} & r_{2}^{(22)}
\end{array}\right) .
\end{aligned}
$$

Applying Lemma 5.2.3 we deduce

$$
R_{2}=R_{2}\left(t, t_{|\xi|}, \xi\right) \in S_{N}\{-1,-1,2\}
$$

The standard construction of $\mathcal{Q}_{2}=\mathcal{Q}_{2}(t, s, \xi)$ in terms of a Peano-Baker series implies uniform bounds for this matrix as follows:

$$
\begin{aligned}
\left|\mathcal{Q}_{2}\left(t, t_{|\xi|}, \xi\right)\right| & \lesssim \exp \left(\int_{t_{|\xi|}}^{t}|\xi|^{-1} \lambda(\theta)^{-1} \Xi(\theta)^{-2} d \theta\right) \\
& \lesssim \exp \left(\Lambda\left(t_{|\xi|}\right) \int_{t_{|\xi|}}^{t} \lambda(\theta)^{-1} \Xi(\theta)^{-2} d \theta\right) \lesssim \exp \left(\frac{\Lambda\left(t_{|\xi|}\right)}{\Theta\left(t_{|\xi|}\right)}\right),
\end{aligned}
$$

where we used $\Lambda\left(t_{|\xi|}\right)|\xi|=N$.

Remark 5.2.3. The micro-energy $V(t, \xi)=\left(\lambda(t)|\xi| \hat{v}, D_{t} \hat{v}\right)^{T}$ can be represented as

$$
V(t, \xi)=\frac{\sqrt{\lambda(t)}}{\sqrt{\lambda(s)}} P(t) N_{2}(t, \xi) \mathcal{E}_{2}(t, s, \xi) \mathcal{Q}_{2}(t, s, \xi) N_{2}(s, \xi)^{-1} P(s)^{-1} V(s, \xi),
$$

with uniformly bounded coefficient matrices $N_{2}, N_{2}^{-1} \in S_{N}\{0,0,0\}$.

Proposition 5.2.4. We assume the conditions (A1) to (A3). Then, the fundamental solution $E_{2}=E_{2}(t, s, \xi)$ to

$$
\left(D_{t}-\mathcal{D}_{1}(t, \xi)-\mathcal{R}_{2}(t, \xi)\right) E_{2}(t, s, \xi)=0, \quad E_{2}(s, s, \xi)=I
$$

can be represented as

$$
E_{2}(t, s, \xi)=\frac{\sqrt{\lambda(t)}}{\sqrt{\lambda(s)}} \mathcal{E}_{2}(t, s, \xi) \mathcal{Q}_{2}(t, s, \xi) \quad \text { for all } \quad t_{|\xi|} \leq s \leq t \leq \tilde{t}_{|\xi|}
$$

with an amplitude $\mathcal{Q}_{2}=\mathcal{Q}_{2}(t, s, \xi)$ satisfying the following estimates:

$$
\left|\mathcal{Q}_{2}(t, s, \xi)\right| \lesssim \exp \left(\frac{\Lambda\left(t_{|\xi|}\right)}{\Theta(s)}\right) \quad \text { for all } \quad t_{|\xi|} \leq s \leq t \leq \tilde{t}_{|\xi|}
$$




\subsubsection{Considerations in the hyperbolic zone}

In $Z_{\text {hyp }}(N)$ for the further considerations we need suitable classes of symbols.

Definition 5.2.2. The time-dependent amplitude function $f=f(t, \xi) \in \mathcal{C}^{\infty}\left(Z_{\text {hyp }}(N)\right)$ belongs to the symbol class $S_{N}\left\{m_{1}, m_{2}, m_{3}\right\}$ with infinite smoothness, if it satisfies the symbollike estimates

$$
\left|D_{\xi}^{\alpha} D_{t}^{k} f(t, \xi)\right| \leq C_{\alpha, k}|\xi|^{m_{1}-|\alpha|} \lambda(t)^{m_{2}} \Xi(t)^{-m_{3}-k}
$$

for all $(t, \xi) \in Z_{\mathrm{hyp}}(N)$ and all multi-indices $\alpha$ and all $k$. Here the constants $C_{\alpha, k}$ are independent of $N$.

Proposition 5.2.5. The classes $S_{N}\left\{m_{1}, m_{2}, m_{3}\right\}$ have the following properties:

1. $S_{N}\left\{m_{1}, m_{2}, m_{3}\right\}$ is a vector space;

2. $S_{N}\left\{m_{1}, m_{2}, m_{3}\right\} \hookrightarrow S_{N}\left\{m_{1}+k, m_{2}+k, m_{3}-k\right\}$ for all $k \geq 0$;

3. $S_{N}\left\{m_{1}, m_{2}, m_{3}\right\} \cdot S_{N}\left\{m_{1}^{\prime}, m_{2}^{\prime}, m_{3}^{\prime}\right\} \hookrightarrow S_{N}\left\{m_{1}+m_{1}^{\prime}, m_{2}+m_{2}^{\prime}, m_{3}+m_{3}^{\prime}\right\}$;

4. $D_{t}^{k} D_{\xi}^{\alpha} S_{N}\left\{m_{1}, m_{2}, m_{3}\right\} \hookrightarrow S_{N}\left\{m_{1}-|\alpha|, m_{2}, m_{3}+k\right\}$;

5. $S_{N}\{-M,-M, M+1\} \hookrightarrow L_{\xi}^{\infty} L_{t}^{1}\left(Z_{h y p}(N)\right)$.

Let us introduce the micro-energy $V(t, \xi)=\left(\lambda(t)|\xi| \hat{v}, D_{t} \hat{v}\right)^{T}$. Then, we get

$$
D_{t} V=\left(\begin{array}{cc}
0 & \lambda(t)|\xi| \\
\lambda(t) \omega^{2}(t)|\xi| & 0
\end{array}\right) V+\left(\begin{array}{cc}
\frac{D_{t} \lambda(t)}{\lambda(t)} & 0 \\
0 & 0
\end{array}\right) V .
$$

Thus, the matrix of eigenvectors $P=P(t)$ and its inverse $P^{-1}=P(t)^{-1}$ are

$$
P(t)=\frac{1}{\omega(t)}\left(\begin{array}{cc}
1 & -1 \\
\omega(t) & \omega(t)
\end{array}\right), \quad P(t)^{-1}=\frac{1}{2}\left(\begin{array}{cc}
\omega(t) & 1 \\
-\omega(t) & 1
\end{array}\right) .
$$

Setting $V^{(1)}:=P(t)^{-1} V$, we get the transformed system

$$
D_{t} V^{(1)}=\left[\mathcal{D}_{0}(t, \xi)+\mathcal{R}_{0}(t, \xi)\right] V^{(1)},
$$

where

$$
\mathcal{D}_{0}(t, \xi):=\left(\begin{array}{cc}
\lambda(t) \omega(t)|\xi| & 0 \\
0 & -\lambda(t) \omega(t)|\xi|
\end{array}\right) \in S_{N}\{1,1,0\}
$$

and

$$
\mathcal{R}_{0}(t, \xi):=\frac{D_{t}(\lambda(t) \omega(t))}{2 \lambda(t) \omega(t)}\left(\begin{array}{cc}
1 & -1 \\
-1 & 1
\end{array}\right) \in S_{N}\{0,0,1\}
$$

We can write

$$
\left(D_{t}-\mathcal{D}_{1}(t, \xi)-\mathcal{R}_{1}(t, \xi)\right) V^{(1)}(t, \xi)=0
$$

where

$$
\mathcal{D}_{1}(t, \xi):=\left(\begin{array}{cc}
\lambda(t) \omega(t)|\xi|+\frac{D_{t}(\lambda(t) \omega(t))}{2 \lambda(t) \omega(t)} & 0 \\
0 & -\lambda(t) \omega(t)|\xi|+\frac{D_{t}(\lambda(t) \omega(t))}{2 \lambda(t) \omega(t)}
\end{array}\right)
$$

and

$$
\mathcal{R}_{1}(t, \xi):=\left(\begin{array}{cc}
0 & -\frac{D_{t}(\lambda(t) \omega(t))}{2 \lambda(t) \omega(t)} \\
-\frac{D_{t}(\lambda(t) \omega(t))}{2 \lambda(t) \omega(t)} & 0
\end{array}\right) .
$$

Note that $\mathcal{R}_{1}(t, \xi) \in S_{N}\{0,0,1\}$. Now we want to carry out further steps of the diagonalization procedure. The goal is to transform the previous system such that the new matrix has diagonal structure and the new remainder belongs to a hyperbolic symbol class. To prove $L^{p}-L^{q}$ estimates we need diagonalization $\bmod \mathcal{R}_{M} \in S_{N}\{1-M, 1-M, M\}$. 
Lemma 5.2.6. Assume the conditions (A1) to (A3). Then, there exists a zone constant $N>0$ such that we can find matrices with the following properties:

- the matrices $N_{k}=N_{k}(t, \xi) \in S_{N}\{0,0,0\}$ are invertible and $N_{k}^{-1}=N_{k}^{-1}(t, \xi) \in S_{N}\{0,0,0\}$ for all $(t, \xi) \in Z_{\text {hyp }}(N)$;

- the matrices $\mathcal{D}_{k}=\mathcal{D}_{k}(t, \xi) \in S_{N}\{1,1,0\}$ are diagonal and

$$
\mathcal{D}_{k}=\mathcal{D}_{k}(t, \xi)=\operatorname{diag}\left(\tau_{k}^{+}(t, \xi), \tau_{k}^{-}(t, \xi)\right)
$$

with $\left|\tau_{k}^{+}(t, \xi)-\tau_{k}^{-}(t, \xi)\right| \geq C_{k} \lambda(t)|\xi| ;$

- the matrices $\mathcal{R}_{k}=\mathcal{R}_{k}(t, \xi) \in S_{N}\{1-k, 1-k, k\}$ are antidiagonal;

such that the following operator-valued identity holds:

$$
\left(D_{t}-\mathcal{D}_{k}(t, \xi)-\mathcal{R}_{k}(t, \xi)\right) N_{k}(t, \xi)=N_{k}(t, \xi)\left(D_{t}-\mathcal{D}_{k+1}(t, \xi)-\mathcal{R}_{k+1}(t, \xi)\right)
$$

for all $(t, \xi) \in Z_{\text {hyp }}(N)$.

Proof. Here we refer to [19]. We denote the difference of the diagonal entries by

$$
\delta_{k}(t, \xi)=\tau_{k}^{+}(t, \xi)-\tau_{k}^{-}(t, \xi) .
$$

Assume that we have given a system by $D_{t} V^{(k)}=\left(\mathcal{D}_{k}(t, \xi)+\mathcal{R}_{k}(t, \xi)\right) V^{(k)}$ with

$$
\mathcal{D}_{k}(t, \xi)=\operatorname{diag}\left(\tau_{k}^{+}(t, \xi), \tau_{k}^{-}(t, \xi)\right) \in S_{N}\{1,1,0\}
$$

satisfying

$$
\left|\delta_{k}(t, \xi)\right|=\left|\tau_{k}^{+}(t, \xi)-\tau_{k}^{-}(t, \xi)\right| \geq C_{k} \lambda(t)|\xi|
$$

and an antidiagonal remainder $\mathcal{R}_{k}=\mathcal{R}_{k}(t, \xi) \in S_{N}\{1-k, 1-k, k\}$. Then, we set

$$
N^{(k)}(t, \xi)=\left(\begin{array}{cc}
0 & -\frac{\mathcal{R}_{k}^{(12)}}{\delta_{k}} \\
\frac{\mathcal{R}_{k}^{(21)}}{\delta_{k}} & 0
\end{array}\right) \in S_{N}\{-k,-k, k\},
$$

such that $N_{k}(t, \xi)=I+N^{(k)}(t, \xi)$ is invertible for a sufficiently large zone constant $N$. Indeed,

$$
\left|N^{(k)}(t, \xi)\right| \lesssim|\xi|^{-k} \lambda(t)^{-k} \Xi(t)^{-k} \lesssim \frac{1}{|\xi|^{k} \Theta(t)^{k}} \leq \frac{1}{N^{k}} \rightarrow 0 \text { as } N \rightarrow \infty .
$$

Furthermore, by construction $\left[\mathcal{D}_{k}(t, \xi), N_{k}(t, \xi)\right]=-\mathcal{R}_{k}(t, \xi)$. So, we may conclude

$$
\begin{aligned}
B^{(k+1)}(t, \xi) & =\left(D_{t}-\mathcal{D}_{k}(t, \xi)-\mathcal{R}_{k}(t, \xi)\right) N_{k}(t, \xi)-N_{k}(t, \xi)\left(D_{t}-\mathcal{D}_{k}(t, \xi)\right) \\
& =D_{t} N_{k}(t, \xi)-\left[\mathcal{D}_{k}(t, \xi), N_{k}(t, \xi)\right]-\mathcal{R}_{k}(t, \xi) N_{k}(t, \xi) \\
& =D_{t} N_{k}(t, \xi)-\mathcal{R}_{k}(t, \xi)\left(N_{k}(t, \xi)-I\right) \in S_{N}^{M-k-1}\{-k,-k, k+1\}
\end{aligned}
$$

After defining

$$
\begin{aligned}
& \mathcal{D}_{k+1}(t, \xi)=\mathcal{D}_{k}(t, \xi)-\operatorname{diag}\left(N_{k}^{-1}(t, \xi) B^{(k+1)}(t, \xi)\right), \\
& \mathcal{R}_{k+1}(t, \xi)=\operatorname{diag}\left(N_{k}^{-1}(t, \xi) B^{(k+1)}(t, \xi)\right)-N_{k}^{-1}(t, \xi) B^{(k+1)}(t, \xi),
\end{aligned}
$$

we obtain the operator equation

$$
\left(D_{t}-\mathcal{D}_{k}(t, \xi)-\mathcal{R}_{k}(t, \xi)\right) N_{k}(t, \xi)=N_{k}(t, \xi)\left(D_{t}-\mathcal{D}_{k+1}(t, \xi)-\mathcal{R}_{k+1}(t, \xi)\right)
$$

with $\mathcal{D}_{k+1} \in S_{N}\{1,1,0\}$ and $\mathcal{R}_{k+1} \in S_{N}\{-k,-k, k+1\}$. The estimate for $B^{(k+1)}(t, \xi)$ implies that

$$
\left|\tau_{k+1}^{+}(t, \xi)-\tau_{k+1}^{-}(t, \xi)\right| \geq\left|\tau_{k}^{+}(t, \xi)-\tau_{k}^{-}(t, \xi)\right|-\lambda(t)|\xi| \frac{C}{N} .
$$

If we choose $N$ sufficiently large enough, then the statement is proved. 
Finally, we obtain for $k=M$ a remainder $\mathcal{R}_{M}=\mathcal{R}_{M}(t, \xi) \in S_{N}\{1-M, 1-M, M\}$, which is uniformly integrable over the hyperbolic zone.

Lemma 5.2.7. The difference of the diagonal entries of $\mathcal{D}_{k}=\mathcal{D}_{k}(t, \xi)$ is real for all $k=$ $1, \cdots, M$.

Proof. Here we refer to [19]. We proceed by induction over $k$ and follow the diagonalization scheme. We will show that the above statement and the conjectures

$\left(H_{k}\right) \mathcal{R}_{k}=\mathcal{R}_{k}(t, \xi)$ has the form $\mathcal{R}_{k}=i\left(\begin{array}{cc}0 & \overline{\beta_{k}} \\ \beta_{k} & 0\end{array}\right)$ with complex-valued $\beta_{k}=\beta_{k}(t, \xi)$ for all $k=1, \cdots, M$,

are both true.

For $k=1$, by the definition of $\mathcal{D}_{1}$ and $\mathcal{R}_{1}$ we see that the assertion $\left(H_{1}\right)$ is satisfied with

$$
\beta_{1}=\beta_{1}(t, \xi)=\frac{(\lambda(t) \omega(t))^{\prime}}{2 \lambda(t) \omega(t)} \text { and } \tau_{1}^{ \pm}=\tau_{1}^{ \pm}(t, \xi)= \pm \lambda(t) \omega(t)|\xi|-i \frac{(\lambda(t) \omega(t))^{\prime}}{2 \lambda(t) \omega(t)} .
$$

Now we assume that $\left(H_{k}\right)$ is true and we will show that $\left(H_{k}\right)$ implies $\left(H_{k+1}\right)$. The construction implies

$$
N^{(k)}(t, \xi)=\frac{i}{\delta_{k}}\left(\begin{array}{cc}
0 & -\overline{\beta_{k}} \\
\beta_{k} & 0
\end{array}\right) \quad \text { with } \operatorname{det} N^{(k)}=\frac{\left|\beta_{k}\right|^{2}}{\delta_{k}^{2}} \leq c<1
$$

with a suitable choice of the zone constant $N$. Following $[16,18,19]$ and setting

$$
d_{k}=d_{k}(t, \xi):=\frac{\left|\beta_{k}(t, \xi)\right|^{2}}{\delta_{k}^{2}(t, \xi)}
$$

we obtain

$$
N_{k}^{-1}\left(\mathcal{D}_{k}+\mathcal{R}_{k}\right) N_{k}=\frac{1}{1-d_{k}}\left(\operatorname{diag}\left(\tau_{k}^{+}-d_{k} \tau_{k}^{+}-\delta_{k} d_{k}, \tau_{k}^{-}-d_{k} \tau_{k}^{-}+\delta_{k} d_{k}\right)+d_{k} \mathcal{R}_{k}\right)
$$

and

$$
N_{k}^{-1}\left(D_{t} N_{k}\right)=\frac{1}{1-d_{k}}\left[\left(\begin{array}{cc}
i \frac{\overline{\beta_{k}}}{\delta_{k}} \partial_{t} \frac{\beta_{k}}{\delta_{k}} & 0 \\
0 & i \frac{\beta_{k}}{\delta_{k}} \partial_{t} \frac{\overline{\beta_{k}}}{\delta_{k}}
\end{array}\right)+\left(\begin{array}{cc}
0 & -\partial_{t} \overline{\frac{\beta_{k}}{\delta_{k}}} \\
\partial_{t} \frac{\beta_{k}}{\delta_{k}} & 0
\end{array}\right)\right]
$$

such that

$$
\operatorname{Re}\left(\frac{\beta_{k}}{\delta_{k}} \partial_{t} \frac{\overline{\beta_{k}}}{\delta_{k}}\right)=\frac{1}{2}\left(\frac{\beta_{k}}{\delta_{k}} \partial_{t} \frac{\overline{\beta_{k}}}{\delta_{k}}+\frac{\overline{\beta_{k}}}{\delta_{k}} \partial_{t} \frac{\beta_{k}}{\delta_{k}}\right)=\frac{1}{2} \partial_{t} d_{k}=\operatorname{Re}\left(\frac{\overline{\beta_{k}}}{\delta_{k}} \partial_{t} \frac{\beta_{k}}{\delta_{k}}\right)
$$

implies

$$
\tau_{k+1}^{ \pm}=\tau_{k}^{ \pm} \mp \frac{1}{1-d_{k}}\left(d_{k} \delta_{k}+\operatorname{Im}\left(\frac{\beta_{k}}{\delta_{k}} \partial_{t} \frac{\overline{\beta_{k}}}{\delta_{k}}\right)\right)-i \frac{\partial_{t} d_{k}}{2\left(d_{k}-1\right)} .
$$

Hence $\delta_{k+1}$ is real again and $\mathcal{R}_{k+1}$ satisfies $\left(H_{k+1}\right)$. Therefore, both statements are true for all $k=1, \cdots, M$.

\subsubsection{Some auxiliary estimates}

In order to apply the stationary phase method we need some symbol-like estimates for the amplitudes of suitable Fourier multipliers. Let us begin with the following important remark to simplify the calculations. 
Remark 5.2.4. Let $g=g(|\xi|)$ be a sufficiently smooth function satisfying the estimates

$$
\left|D_{|\xi|}^{|\alpha|} g(|\xi|)\right| \lesssim|\xi|^{-|\alpha|}
$$

for all $\xi \in \mathbb{R}^{n}$. Then, we have for all multi-indices $\alpha$ the estimates

$$
\left|D_{\xi}^{\alpha} g(|\xi|)\right| \lesssim|\xi|^{-|\alpha|} .
$$

Indeed, applying Faá di Bruno's formula we get that

$$
\begin{aligned}
\left|D_{\xi}^{\alpha} g(|\xi|)\right| & =\left|\sum_{j=1}^{|\alpha|} \sum_{\beta_{1}+\cdots+\beta_{j}=\alpha} C_{\beta_{1}, \cdots, \beta_{j}} g^{(j)}(|\xi|) \prod_{i=1}^{j} D_{\xi}^{\beta_{i}}\right| \xi|| \\
& \leq \sum_{j=1}^{|\alpha|} \sum_{\beta_{1}+\cdots+\beta_{j}=\alpha}\left|C_{\beta_{1}, \cdots, \beta_{j}}\right|\left|g^{(j)}(|\xi|)\right|\left|\prod_{i=1}^{j} D_{\xi}^{\beta_{i}}\right| \xi|| \\
& \leq \sum_{j=1}^{|\alpha|} \sum_{\beta_{1}+\cdots+\beta_{j}=\alpha}\left|C_{\beta_{1}, \cdots, \beta_{j}}\right||\xi|^{-j}|\xi|^{-\left(\left|\beta_{1}\right|+\cdots+\left|\beta_{j}\right|\right)+j} \\
& \leq C_{\alpha}|\xi|^{-|\alpha|} .
\end{aligned}
$$

Proposition 5.2.8. Let us assume that $t_{|\xi|}$ is the separating line between the oscillation subzone $Z_{o s c}(N)$ and the pseudo-differential zone $Z_{p d}(N)$. Then, we have the following estimates:

$$
\left|D_{\xi}^{\alpha} t_{|\xi|}\right| \lesssim \frac{1}{\lambda\left(t_{|\xi|}\right)}|\xi|^{-1-|\alpha|}
$$

for all multi-indices $\alpha$ with $|\alpha|>0$.

Proof. Let us consider the first derivative with respect to $\xi$ of $t_{|\xi|}$ as follows:

$$
\Lambda\left(t_{|\xi|}\right)|\xi|=N \quad \text { implies } \quad d_{|\xi|} t_{|\xi|} \lambda\left(t_{|\xi|}\right)|\xi|+\Lambda\left(t_{|\xi|}\right)=0
$$

Then, we obtain

$$
d_{|\xi|} t_{|\xi|}=-\frac{\Lambda\left(t_{|\xi|}\right)}{\lambda\left(t_{|\xi|}\right)|\xi|}=-\frac{N}{\lambda\left(t_{|\xi|}\right)}|\xi|^{-2}
$$

Now, we assume that

$$
\left|D_{|\xi|}^{k} t_{|\xi|}\right| \lesssim \frac{1}{\lambda\left(t_{|\xi|}\right)}|\xi|^{-1-k}
$$

holds for all $1 \leq k \leq M$. Multiplying (5.2.11) by $\lambda\left(t_{|\xi|}\right)$ and taking $M$ derivatives with respect to $|\xi|$ gives

$$
\begin{aligned}
& d_{|\xi|}^{M}\left(d_{|\xi|} t_{|\xi|} \lambda\left(t_{|\xi|}\right)\right)=-d_{|\xi|}^{M}\left(\frac{N}{|\xi|^{2}}\right) \\
& \sum_{k=0}^{M} C_{M}^{k} d_{|\xi|}^{k+1} t_{|\xi|} d_{|\xi|}^{M-k} \lambda\left(t_{|\xi|}\right)=(-1)^{M+1}(M+1) ! \frac{N}{|\xi|^{M+2}}
\end{aligned}
$$

respectively. So, we have

$$
\left|d_{|\xi|}^{M+1} t_{|\xi|} \lambda\left(t_{|\xi|}\right)\right| \lesssim \frac{1}{|\xi|^{M+2}}+\sum_{k=0}^{M-1}\left|d_{|\xi|}^{k+1} t_{|\xi|} d_{|\xi|}^{M-k} \lambda\left(t_{|\xi|}\right)\right| .
$$

Applying Faà di Bruno's formula we get

$$
d_{|\xi|}^{n} \lambda\left(t_{|\xi|}\right)=\sum \frac{n !}{m_{1} ! 1 !^{m_{1}} m_{2} ! 2 !^{m_{2}} \cdots m_{n} ! n !^{m_{n}}} \lambda^{\left(m_{1}+m_{2}+\cdots+m_{n}\right)}\left(t_{|\xi|}\right) \prod_{j=1}^{n}\left(d_{|\xi|}^{j} t_{|\xi|}\right)^{m_{j}},
$$


where the sum is taken over all n-tuples of nonnegative integers $\left(m_{1}, m_{2}, \cdots, m_{n}\right)$ satisfying the constraint

$$
1 \cdot m_{1}+2 \cdot m_{2}+\cdots+n \cdot m_{n}=n .
$$

The assumption (A1) together with (5.2.12) yields the estimate

$$
\begin{aligned}
\left|d_{|\xi|}^{n} \lambda\left(t_{|\xi|}\right)\right| & \lesssim \sum \lambda\left(t_{|\xi|}\right)\left(\frac{\lambda\left(t_{|\xi|}\right)}{\Lambda\left(t_{|\xi|}\right)}\right)^{m_{1}+m_{2}+\cdots+m_{n}} \prod_{j=1}^{n}\left(\frac{1}{\lambda\left(t_{|\xi|}\right)|\xi|^{j+1}}\right)^{m_{j}} \\
& \lesssim \sum \lambda\left(t_{|\xi|}\right)\left(\frac{\lambda\left(t_{|\xi|}\right)}{\Lambda\left(t_{|\xi|}\right)}\right)^{m_{1}+m_{2}+\cdots+m_{n}} \frac{1}{\left(\lambda\left(t_{|\xi|}\right)|\xi|\right)^{m_{1}+m_{2}+\cdots+m_{n}}|\xi|^{m_{1}+2 m_{2}+\cdots+n m_{n}}} \\
& \lesssim \frac{\lambda\left(t_{|\xi|}\right)}{|\xi|^{n}}
\end{aligned}
$$

Combining (5.2.13) and (5.2.14) we obtain

$$
\left|d_{|\xi|}^{M+1} t_{|\xi|} \lambda\left(t_{|\xi|}\right)\right| \lesssim \frac{1}{|\xi|^{M+2}}+\sum_{k=0}^{M-1} \frac{1}{\lambda\left(t_{|\xi|}\right)|\xi|^{k+2}} \frac{\lambda\left(t_{|\xi|}\right)}{|\xi|^{M-k}} \lesssim \frac{1}{|\xi|^{M+2}}
$$

Then,

This completes the proof.

Proposition 5.2.9. Let us assume that $\tilde{t}_{|\xi|}$ is the separating line between the oscillation subzone $Z_{o s c}(N)$ and the hyperbolic zone $Z_{\text {hyp }}(N)$. Then, we have the following estimates:

$$
\left|D_{\xi}^{\alpha} \tilde{t}_{|\xi|}\right| \lesssim \frac{1}{\vartheta\left(\tilde{t}_{|\xi|}\right)}|\xi|^{-1-|\alpha|}
$$

for all multi-indices $\alpha$ with $|\alpha|>0$.

Proof. We will apply the principle of induction as we did in Proposition 5.2.8. Let us consider the first derivative with respect to $\xi$ of $\tilde{t}_{|\xi|}$ that can be obtained directly from the following calculations:

$$
\Theta\left(\tilde{t}_{|\xi|}\right)|\xi|=N \quad \text { implies } \quad d_{|\xi|} \tilde{t}_{|\xi|} \vartheta\left(\tilde{t}_{|\xi|}\right)|\xi|+\Theta\left(\tilde{t}_{|\xi|}\right)=0 .
$$

Hence, we obtain

$$
d_{|\xi|} \tilde{t}_{|\xi|}=-\frac{\Theta\left(\tilde{t}_{|\xi|}\right)}{\vartheta\left(\tilde{t}_{|\xi|}\right)|\xi|}=-\frac{N}{\vartheta\left(\tilde{t}_{|\xi|}\right)}|\xi|^{-2} .
$$

Now, let us assume that

$$
\left|D_{|\xi|}^{k} \tilde{t}_{|\xi|}\right| \lesssim \frac{1}{\vartheta\left(\tilde{t}_{|\xi|}\right)}|\xi|^{-1-k}
$$

holds for all $1 \leq k \leq M$. Then, following the same steps to the proof of Proposition 5.2.8 and using condition (A2) the proof is completed.

Proposition 5.2.10. We assume that $g=g(|\xi|)$ with $\xi \in \mathbb{R}^{n} \backslash\{0\}$ is an infinitely differentiable function. Then, it holds

$$
d_{|\xi|}^{m} e^{g(|\xi|)}=C\left(k_{1}, k_{2}, \cdots, k_{j}, j\right) e^{g(|\xi|)} \sum_{j=1}^{m} \sum_{k_{1}+\cdots+k_{j}=m} \prod_{i=1}^{j} d_{|\xi|}^{k_{i}} g(|\xi|)
$$

with $k_{i} \geq 1$. 
Proposition 5.2.11. Let us introduce $\Lambda_{\omega}(t)=\int_{0}^{t} \lambda(\tau) \omega(\tau) d \tau$. Then, the following estimates hold:

$$
\left|D_{\xi}^{\alpha}\left(\Lambda_{\omega}\left(t_{|\xi|}\right)|\xi|\right)\right| \leq C_{\alpha}|\xi|^{-|\alpha|}\left(\frac{\lambda\left(t_{|\xi|}\right)}{\Lambda\left(t_{|\xi|}\right)} \Xi\left(t_{|\xi|}\right)\right)^{1-|\alpha|},
$$

where $|\alpha| \geq 1$.

Proof. We start with the following identity:

$$
\left|D_{\xi}^{\alpha}\left(\Lambda_{\omega}\left(t_{|\xi|}\right)|\xi|\right)\right|=\left|\sum_{\beta_{1}+\beta_{2}=\alpha} C_{\beta_{1}, \beta_{2}} D_{\xi}^{\beta_{1}} \Lambda_{\omega}\left(t_{|\xi|}\right) D_{\xi}^{\beta_{2}}\right| \xi|| .
$$

Thus, we need to estimate $\left|D_{\xi}^{\beta_{1}} \Lambda_{\omega}\left(t_{|\xi|}\right)\right|$. It holds

$$
\begin{aligned}
\left|D_{\xi} \Lambda_{\omega}\left(t_{|\xi|}\right)\right| & =\left|D_{\xi} \int_{0}^{t_{|\xi|}} \lambda(\tau) \omega(\tau) d \tau\right| \\
& =\left|\lambda\left(t_{|\xi|}\right) \omega\left(t_{|\xi|}\right) D_{\xi} t_{|\xi|}\right| \leq \lambda\left(t_{|\xi|}\right) \omega\left(t_{|\xi|}\right)\left|D_{\xi} t_{|\xi|}\right| \lesssim|\xi|^{-2}
\end{aligned}
$$

Moreover, we have

$$
\begin{aligned}
\left|D_{\xi}^{2} \Lambda_{\omega}\left(t_{|\xi|}\right)\right| & =\left|D_{\xi}\left(\lambda\left(t_{|\xi|}\right) \omega\left(t_{|\xi|}\right) D_{\xi} t_{|\xi|}\right)\right| \\
& \leq\left|\lambda^{\prime}\left(t_{|\xi|}\right)\right| \omega\left(t_{|\xi|}\right)\left|D_{\xi} t_{|\xi|}\right|^{2}+\lambda\left(t_{|\xi|}\right)\left|\omega^{\prime}\left(t_{|\xi|}\right)\right|\left|D_{\xi} t_{|\xi|}\right|^{2}+\lambda\left(t_{|\xi|}\right) \omega\left(t_{|\xi|}\right)\left|D_{\xi}^{2} t_{|\xi|}\right| \\
& \lesssim|\xi|^{-3}+\Xi\left(t_{|\xi|}\right)^{-1} \frac{\Lambda\left(t_{|\xi|}\right)}{\lambda\left(t_{|\xi|}\right)}|\xi|^{-3} \lesssim \Xi\left(t_{|\xi|}\right)^{-1} \frac{\Lambda\left(t_{|\xi|}\right)}{\lambda\left(t_{|\xi|}\right)}|\xi|^{-3},
\end{aligned}
$$

where we used conditions (A1), (A4) and (A5). Therefore, by the induction principle we may conclude the estimate

$$
\left|D_{\xi}^{\beta_{1}} \Lambda_{\omega}\left(t_{|\xi|}\right)\right| \lesssim|\xi|^{-\beta_{1}-1}\left(\frac{\lambda\left(t_{|\xi|}\right)}{\Lambda\left(t_{|\xi|}\right)} \Xi\left(t_{|\xi|}\right)\right)^{1-\beta_{1}}
$$

On the other hand, we have

$$
\left|D_{\xi}^{\beta_{2}}\right| \xi|| \leq C_{\beta_{2}}|\xi|^{-\beta_{2}+1}
$$

The estimates (5.2.18) and (5.2.19) help us to conclude the desired inequality.

Definition 5.2.3. The function $h=h(t, \xi)$ belongs to symbol class $S_{N}^{l_{1}, l_{2}}\left\{m_{1}, m_{2}, m_{3}\right\}$ with restricted smoothness $l_{1}, l_{2}$ if it satisfies the following estimates:

$$
\left|D_{\xi}^{\alpha} D_{t}^{k} h(t, \xi)\right| \leq C_{\alpha, k}|\xi|^{m_{1}-|\alpha|} \lambda(t)^{m_{2}} \Xi(t)^{-m_{3}-k}
$$

in $Z_{\text {hyp }}(N)$ for all $|\alpha| \leq l_{2}$ and $k \leq l_{1}$.

Obviously, it holds

$$
S_{N}^{l_{1}, l_{2}}\left\{m_{1}, m_{2}, m_{3}\right\} \hookrightarrow S_{N}^{l_{1}^{\prime}, l_{2}^{\prime}}\left\{m_{1}, m_{2}, m_{3}\right\} \quad \text { for all } \quad l_{1}^{\prime} \leq l_{1}, \quad l_{2}^{\prime} \leq l_{2} .
$$

Using the definition of $Z_{\text {hyp }}(N)$ we have

$$
S_{N}^{l_{1}, l_{2}}\left\{m_{1}-k, m_{2}-k, m_{3}+k\right\} \hookrightarrow S_{N}^{l_{1}, l_{2}}\left\{m_{1}, m_{2}, m_{3}\right\} \text { for all } k \geq 0 .
$$

This property will be essentially used in the diagonalization scheme.

Proposition 5.2.12. The family of symbol classes $S_{N}^{l_{1}, l_{2}}\left\{m_{1}, m_{2}, m_{3}\right\}$ generates a hierarchy of symbol classes having the following properties:

- $S_{N}^{l_{1}, l_{2}}\left\{m_{1}, m_{2}, m_{3}\right\}$ is a vector space; 
- $S_{N}^{l_{1}, l_{2}}\left\{m_{1}, m_{2}, m_{3}\right\} S_{N}^{l_{1}, l_{2}}\left\{m_{1}^{\prime}, m_{2}^{\prime}, m_{3}^{\prime}\right\} \hookrightarrow S_{N}^{l_{1}, l_{2}}\left\{m_{1}+m_{1}^{\prime}, m_{2}+m_{2}^{\prime}, m_{3}+m_{3}^{\prime}\right\}$;

- $D_{t}^{k} D_{\xi}^{\alpha} S_{N}^{l_{1}, l_{2}}\left\{m_{1}, m_{2}, m_{3}\right\} \hookrightarrow S_{N}^{l_{1}-k, l_{2}-|\alpha|}\left\{m_{1}-|\alpha|, m_{2}, m_{3}+k\right\}$;

- $S_{N}^{0,0}\{1-M, 1-M, M\} \hookrightarrow L_{\xi}^{\infty} L_{t}^{1}\left(Z_{h y p}(N)\right)$.

The next proposition shows us that the multiplication by $e^{ \pm i|\xi| \Lambda_{\omega}\left(t_{|\xi|}\right)}$ is not a well-defined operation on the symbol classes $S_{N}\left\{m_{1}, m_{2}, m_{3}\right\}$, this means, that we remain in the hierarchy of symbol classes after deriving an estimate.

Proposition 5.2.13. The following relation holds for all $h=h(t, \xi) \in S_{N}\left\{m_{1}, m_{2}, m_{3}\right\}$ :

$$
\left|D_{t}^{k} D_{\xi}^{\alpha}\left(e^{ \pm i \Lambda_{\omega}\left(t_{|\xi|}\right)|\xi|} h(t, \xi)\right)\right| \leq C_{\alpha, k}|\xi|^{m_{1}-|\alpha|} \lambda(t)^{m_{2}} \Xi(t)^{-m_{3}-k}\left(\frac{\lambda\left(t_{|\xi|}\right)}{\Lambda\left(t_{|\xi|}\right)} \Xi\left(t_{|\xi|}\right)\right)^{-|\alpha|} .
$$

Proof. We choose $h=h(t, \xi) \in S_{N}\left\{m_{1}, m_{2}, m_{3}\right\}$. Then, it holds

$$
\left|D_{t}^{k} D_{\xi}^{\alpha}\left(e^{ \pm i \Lambda_{\omega}\left(t_{|\xi|}\right)|\xi|} h(t, \xi)\right)\right|=\left|\sum_{\alpha_{1}+\alpha_{2}=\alpha} C_{\alpha_{1}, \alpha_{2}} D_{\xi}^{\alpha_{1}} e^{ \pm i|\xi| \Lambda_{\omega}\left(t_{|\xi|}\right)} D_{t}^{k} D_{\xi}^{\alpha_{2}} h(t, \xi)\right| .
$$

By the aid of Proposition 5.2.10, it is enough to consider the following estimates:

$$
\begin{aligned}
& \left|\sum_{\alpha_{1}+\alpha_{2}=\alpha} C_{\alpha_{1}, \alpha_{2}} D_{|\xi|}^{\left|\alpha_{1}\right|} e^{ \pm i|\xi| \Lambda_{\omega}\left(t_{|\xi|}\right)} D_{t}^{k} D_{\xi}^{\alpha_{2}} h(t, \xi)\right| \\
& \leq \sum_{\alpha_{1}+\alpha_{2}=\alpha} C_{\alpha_{1}, \alpha_{2}}\left(C^{\prime}\left(\alpha_{1}\right)\left|e^{ \pm i|\xi| \Lambda_{\omega}\left(t_{|\xi|}\right)} \sum_{j=1}^{\left|\alpha_{1}\right|} \sum_{\ell_{1}+\cdots+\ell_{j}=\left|\alpha_{1}\right|} \prod_{i=1}^{j} d_{|\xi|}^{\ell_{i}}\left(|\xi| \Lambda_{\omega}\left(t_{|\xi|}\right)\right)\right|\right)\left|D_{t}^{k} D_{\xi}^{\alpha_{2}} h(t, \xi)\right| \\
& \leq \sum_{\alpha_{1}+\alpha_{2}=\alpha} C_{\alpha_{1}, \alpha_{2}}\left(C^{\prime}\left(\alpha_{1}\right) \sum_{j=1}^{\left|\alpha_{1}\right|} \sum_{\ell_{1}+\cdots+\ell_{j}=\left|\alpha_{1}\right|} \prod_{i=1}^{j}|\xi|^{-\ell_{i}}\left(\frac{\lambda\left(t_{|\xi|}\right)}{\Lambda\left(t_{|\xi|}\right)} \Xi\left(t_{|\xi|}\right)\right)^{1-\ell_{i}}\right)\left|D_{t}^{k} D_{\xi}^{\alpha_{2}} h(t, \xi)\right| \\
& \leq \sum_{\alpha_{1}+\alpha_{2}=\alpha} C_{\alpha_{1}, \alpha_{2}}\left(C^{\prime}\left(\alpha_{1}\right) \sum_{j=1}^{\left|\alpha_{1}\right|}\left(\frac{\lambda\left(t_{|\xi|}\right)}{\Lambda\left(t_{|\xi|}\right)} \Xi\left(t_{|\xi|}\right)^{j}|\xi|^{-\left|\alpha_{1}\right|}\left(\frac{\lambda\left(t_{|\xi|}\right)}{\Lambda\left(t_{|\xi|}\right)} \Xi\left(t_{|\xi|}\right)\right)^{-\left|\alpha_{1}\right|}\right)\left|D_{t}^{k} D_{\xi}^{\alpha_{2}} h(t, \xi)\right|\right. \\
& \leq \sum_{\alpha_{1}+\alpha_{2}=\alpha} \tilde{C}_{\alpha_{1}, \alpha_{2}}|\xi|^{-\left|\alpha_{1}\right|}\left(\frac{\lambda\left(t_{|\xi|}\right)}{\Lambda\left(t_{|\xi|}\right)} \Xi\left(t_{|\xi|}\right)\right)^{-\left|\alpha_{1}\right|}|\xi|^{m_{1}-\left|\alpha_{2}\right|} \lambda(t)^{m_{2}} \Xi(t)^{-m_{3}-k} \\
& \leq C_{\alpha}|\xi|^{m_{1}-|\alpha|} \lambda(t)^{m_{2}} \Xi(t)^{-m_{3}-k}\left(\frac{\lambda\left(t_{|\xi|}\right)}{\Lambda\left(t_{|\xi|}\right)} \Xi\left(t_{|\xi|}\right)\right)^{-|\alpha|},
\end{aligned}
$$

where in the third line we have used Proposition 5.2.11.

Remark 5.2.5. Proposition 5.2.13 tells us that it is allowed to extend the phase function

$$
\pm|\xi| \int_{t_{|\xi|}}^{t} \lambda(\tau) \omega(\tau) d \tau \quad \text { in } \quad \exp \left( \pm i|\xi| \int_{t_{|\xi|}}^{t} \lambda(\tau) \omega(\tau) d \tau\right)
$$

which we use later to get $L^{p}-L^{q}$ estimates to the phase function

$$
\pm|\xi| \int_{0}^{t} \lambda(\tau) \omega(\tau) d \tau \quad \text { in } \quad \exp \left( \pm i|\xi| \int_{0}^{t} \lambda(\tau) \omega(\tau) d \tau\right) .
$$

Here we use that the remainder term

$$
\exp \left( \pm i|\xi| \int_{0}^{t_{|\xi|}} \lambda(\tau) \omega(\tau) d \tau\right)
$$

satisfies

$$
\left|D_{|\xi|}^{\alpha} e^{ \pm i|\xi| \Lambda_{\omega}\left(t_{|\xi|}\right)}\right| \lesssim|\xi|^{-|\alpha|}\left(\frac{\lambda\left(t_{|\xi|}\right)}{\Lambda\left(t_{|\xi|}\right)} \Xi\left(t_{|\xi|}\right)\right)^{-|\alpha|} .
$$

Thus, we can put the term $e^{ \pm i|\xi| \Lambda_{\omega}\left(t_{|\xi|}\right)}$ into the amplitude. 


\section{Construction of the fundamental solution in $Z_{\text {hyp }}(N)$}

Now we want to construct the fundamental solution $E_{\text {hyp }}=E_{\text {hyp }}(t, s, \xi), \tilde{t}_{|\xi|} \leq s \leq t$ for small frequencies $\xi$ and $0 \leq s \leq t$ for large frequencies $\xi$, respectively, for the operator

$$
D_{t}-\mathcal{D}_{1}(t, \xi)-\mathcal{R}_{1}(t, \xi) .
$$

For this reason after $M$ steps of diagonalization it is sufficient to construct the fundamental solution satisfying the system

$$
D_{t} E_{M}(t, s, \xi)=\left(\mathcal{D}_{M}(t, \xi)+\mathcal{R}_{M}(t, \xi)\right) E_{M}(t, s, \xi), \quad E_{M}(s, s, \xi)=I .
$$

At first we solve the diagonal system

$$
D_{t} \mathcal{E}_{M}(t, s, \xi)=\mathcal{D}_{M}(t, \xi) \mathcal{E}_{M}(t, s, \xi), \quad \mathcal{E}_{M}(s, s, \xi)=I, \quad 0 \leq s \leq t .
$$

Its fundamental solution is given by

$$
\mathcal{E}_{M}(t, s, \xi)=\exp \left(i \int_{s}^{t} \mathcal{D}_{M}(\theta, \xi) d \theta\right)=\operatorname{diag}\left(e^{i \int_{s}^{t} \tau_{M}^{+}(\theta, \xi) d \theta}, e^{i \int_{s}^{t} \tau_{M}^{-}(\theta, \xi) d \theta}\right)
$$

We make the ansatz $E_{M}(t, s, \xi)=\mathcal{E}_{M}(t, s, \xi) \mathcal{Q}_{M}(t, s, \xi)$ with a uniformly bounded and invertible matrix $\mathcal{Q}_{M}=\mathcal{Q}_{M}(t, s, \xi)$. It follows that the matrix $\mathcal{Q}_{M}=\mathcal{Q}_{M}(t, s, \xi)$ satisfies the system

$$
D_{t} \mathcal{Q}_{M}(t, s, \xi)=R_{M}(t, s, \xi) \mathcal{Q}_{M}(t, s, \xi), \quad \mathcal{Q}_{M}(s, s, \xi)=I
$$

with the coefficient matrix

$$
R_{M}(t, s, \xi)=\mathcal{E}_{M}(s, t, \xi) \mathcal{R}_{M}(t, \xi) \mathcal{E}_{M}(t, s, \xi) .
$$

Taking account of $\mathcal{R}_{M}=\mathcal{R}_{M}(t, \xi) \in S_{N}\{1-M, 1-M, M\}$ we obtain

$$
\left|R_{M}(t, s, \xi)\right|=\left|\mathcal{R}_{M}(t, \xi)\right| \lesssim|\xi|^{1-M} \lambda(t)^{1-M} \Xi(t)^{-M} .
$$

The solution $\mathcal{Q}_{M}=\mathcal{Q}_{M}(t, s, \xi)$ can be represented as Peano-Baker series

$$
\mathcal{Q}_{M}(t, s, \xi)=I+\sum_{k=1}^{\infty} i^{k} \int_{s}^{t} R_{M}\left(t_{1}, s, \xi\right) \int_{s}^{t_{1}} R_{M}\left(t_{2}, s, \xi\right) \cdots \int_{s}^{t_{k-1}} R_{M}\left(t_{k}, s, \xi\right) d t_{k} \cdots d t_{1} .
$$

Due to the representation of $R_{M}=R_{M}(t, s, \xi)$ in (5.2.20) we have

$$
\begin{aligned}
R_{M}\left(t, \tilde{t}_{|\xi|}, \xi\right) & =\mathcal{E}_{M}\left(\tilde{t}_{|\xi|}, t, \xi\right) \mathcal{R}_{M}(t, \xi) \mathcal{E}_{M}\left(t, \tilde{t}_{|\xi|}, \xi\right) \\
& =\left(\begin{array}{cc}
r_{M}^{(11)} & r_{M}^{(12)} e^{-2 i|\xi| \int_{t_{|\xi|}}^{t} \lambda(\tau) \omega(\tau) d \tau} \\
r_{M}^{(21)} e^{2 i|\xi| \int_{\hat{t}}^{t} \lambda \mid} \lambda(\tau) \omega(\tau) d \tau & r_{M}^{(22)}
\end{array}\right) .
\end{aligned}
$$

Lemma 5.2.14. We assume conditions (A1) to (A4). Then, the fundamental solution $E_{M}=E_{M}(t, s, \xi)$ to

$$
\left(D_{t}-\mathcal{D}_{M}(t, \xi)-\mathcal{R}_{M}(t, \xi)\right) E_{M}(t, s, \xi)=0, \quad E_{M}(s, s, \xi)=I
$$

can be represented as

$$
E_{M}(t, s, \xi)=\frac{\sqrt{\lambda(t)}}{\sqrt{\lambda(s)}} \mathcal{E}_{M}(t, s, \xi) \mathcal{Q}_{M}(t, s, \xi)
$$

for all $t, s \geq \tilde{t}_{|\xi|}$ with an amplitude $\mathcal{Q}_{M}=\mathcal{Q}_{M}(t, s, \xi)$ satisfying the following estimates:

$$
\left|D_{\xi}^{\alpha-\beta} \mathcal{Q}_{M}\left(t, \tilde{t}_{|\xi|}, \xi\right) D_{\xi}^{\beta} \tilde{t}_{|\xi|}\right| \leq C_{\alpha, M, N}\left(\frac{\lambda(t)}{\vartheta(t)}\right)^{|\beta|}|\xi|^{-|\alpha|}
$$

for all $t \geq \tilde{t}_{|\xi|}$ and all multi-indices $\beta$ with $\beta \leq \alpha$ and $|\alpha| \leq M-1$. 
Proof. Let us consider the first statement. The standard construction of $\mathcal{Q}_{M}=\mathcal{Q}_{M}(t, s, \xi)$ in terms of a Peano-Baker series implies the uniform bounds for this matrix as follows:

$$
\begin{aligned}
\left|\mathcal{Q}_{M}\left(t, \tilde{t}_{|\xi|}, \xi\right)\right| & \leq \exp \left(\int_{\tilde{t}_{|\xi|}^{t}}^{t} C|\xi|^{1-M} \lambda(\theta)^{1-M} \Xi(\theta)^{-M} d \theta\right) \\
& \leq \exp \left(C|\xi|^{1-M} \int_{\tilde{t}_{|\xi|}^{\infty}}^{\infty} \lambda(\theta)^{1-M} \Xi(\theta)^{-M} d \theta\right) \\
& \leq \exp \left(\frac{C^{\prime}}{|\xi|^{M-1} \Theta\left(\tilde{t}_{|\xi|}\right)^{M-1}}\right)=\exp \left(\frac{C^{\prime}}{N^{M-1}}\right) \lesssim 1,
\end{aligned}
$$

where we used condition (A3) and the definition of $Z_{\text {hyp }}(N)$, respectively.

Now let us take $\alpha$ derivatives with respect to $\xi$ in the representation formula for $\mathcal{Q}_{M}\left(t, \tilde{t}_{|\xi|}, \xi\right)$ in (5.2.21). Then,

$D_{\xi}^{\alpha} \mathcal{Q}_{M}\left(t, \tilde{t}_{|\xi|}, \xi\right)=\sum_{k=1}^{\infty} i^{k} D_{\xi}^{\alpha}\left(\int_{\tilde{t}_{|\xi|}}^{t} R_{M}\left(t_{1}, \tilde{t}_{|\xi|}, \xi\right) \int_{\tilde{t}_{|\xi|}}^{t_{1}} R_{M}\left(t_{2}, \tilde{t}_{|\xi|}, \xi\right) \cdots \int_{\tilde{t}_{|\xi|}}^{t_{k-1}} R_{M}\left(t_{k}, \tilde{t}_{|\xi|}, \xi\right) d t_{k} \cdots d t_{1}\right)$.

Let us consider terms of the form

$$
\int_{\tilde{t}_{|\xi|}}^{t} D_{\xi}^{\alpha_{1}} R_{M}\left(t_{1}, \tilde{t}_{|\xi|}, \xi\right) \int_{\tilde{t}_{|\xi|}}^{t_{1}} D_{\xi}^{\alpha_{2}} R_{M}\left(t_{2}, \tilde{t}_{|\xi|}, \xi\right) \cdots \int_{\tilde{t}_{|\xi|}}^{t_{k-1}} D_{\xi}^{\alpha_{k}} R_{M}\left(t_{k}, \tilde{t}_{|\xi|}, \xi\right) d t_{k} \cdots d t_{1}
$$

with $\sum_{j=1}^{k} \alpha_{k}=|\alpha|$. Therefore, we have

$$
\begin{aligned}
C(\alpha, M, N) \int_{\tilde{t}_{|\xi|}}^{t}\left(|\xi|^{1-M-\left|\alpha_{1}\right|} \lambda\left(t_{1}\right)^{1-M} \Xi\left(t_{1}\right)^{-M}\right) \int_{\tilde{t}_{|\xi|}}^{t_{1}}\left(|\xi|^{1-M-\left|\alpha_{2}\right|} \lambda\left(t_{2}\right)^{1-M} \Xi\left(t_{2}\right)^{-M}\right) \\
\quad \times \cdots \int_{\tilde{t}_{|\xi|}^{t_{k-1}}}^{t^{1-M}}\left(|\xi|^{1-M-\left|\alpha_{k}\right|} \lambda\left(t_{k}\right)^{1-M} \Xi\left(t_{k}\right)^{-M}\right) d t_{k} \cdots d t_{1} \\
\leq C^{\prime}(\alpha, M, N)|\xi|^{-|\alpha|} .
\end{aligned}
$$

We have to care for derivatives of the lower integral bound $\tilde{t}_{|\xi|}$. Then, there arise terms as

$$
D_{\xi}^{\alpha-\beta} R_{M}\left(\tilde{t}_{|\xi|}, \tilde{t}_{|\xi|}, \xi\right) D_{\xi}^{\beta} \tilde{t}_{|\xi|} .
$$

For $|\beta|=1$ we can estimate as follows:

$$
\begin{aligned}
\mid D_{\xi}^{\alpha-\beta}( & \left.R_{M}\left(\tilde{t}_{|\xi|}, \tilde{t}_{|\xi|}, \xi\right) d_{|\xi|} \tilde{t}_{|\xi|}\right) \mid \\
& =\left|\sum_{\left|\alpha_{1}\right|+\left|\alpha_{2}\right|=|\alpha|-1} C_{\alpha_{1}, \alpha_{2}} D_{\xi}^{\alpha_{1}} R_{M}\left(\tilde{t}_{|\xi|}, \tilde{t}_{|\xi|}, \xi\right) D_{\xi}^{\alpha_{2}+\beta} \tilde{t}_{|\xi|}\right| \\
& \leq C(\alpha) \sum_{\left|\alpha_{1}\right|+\left|\alpha_{2}\right|=|\alpha|-1}|\xi|^{1-M-\left|\alpha_{1}\right|} \lambda\left(\tilde{t}_{|\xi|}\right)^{1-M} \Xi\left(\tilde{t}_{|\xi|}\right)^{-M} \vartheta\left(\tilde{t}_{|\xi|}\right)^{-1}|\xi|^{-\left|\alpha_{2}\right|-2} \\
& =C(\alpha) \sum_{\left|\alpha_{1}\right|+\left|\alpha_{2}\right|=|\alpha|-1}|\xi|^{-\left|\alpha_{1}\right|-\left|\alpha_{2}\right|-1-M} \lambda\left(\tilde{t}_{|\xi|}\right)^{-M} \Xi\left(\tilde{t}_{|\xi|}\right)^{-M} \frac{\lambda\left(\tilde{t}_{|\xi|}\right)}{\vartheta\left(\tilde{t}_{|\xi|}\right)} \\
& \leq C^{\prime}(\alpha)|\xi|^{-|\alpha|} \frac{1}{|\xi|^{M} \Theta\left(\tilde{t}_{|\xi|}\right)^{M}} \frac{\lambda\left(\tilde{t}_{|\xi|}\right)}{\vartheta\left(\tilde{t}_{|\xi|}\right)} \leq C(\alpha, N)|\xi|^{-|\alpha|} \frac{\lambda\left(\tilde{t}_{|\xi|}\right)}{\vartheta\left(\tilde{t}_{|\xi|}\right)}
\end{aligned}
$$

where we used Proposition 5.2.9 to estimate the terms $\left|D_{\xi}^{\alpha_{2}+\beta} \tilde{t}_{|\xi|}\right|$. We use that the terms $\left|D_{\xi}^{\alpha_{1}} R_{M}\left(\tilde{t}_{|\xi|}, \tilde{t}_{|\xi|}, \xi\right)\right|$ can be estimated in the following way:

$$
\left|D_{\xi}^{\alpha} R_{M}\left(\tilde{t}_{|\xi|}, \tilde{t}_{|\xi|}, \xi\right)\right| \lesssim|\xi|^{1-M-|\alpha|} \lambda\left(\tilde{t}_{|\xi|}\right)^{1-M} \Xi\left(\tilde{t}_{|\xi|}\right)^{-M} .
$$


Indeed, due to the representation of $R_{M}(t, s, \xi)$ we have

$$
R_{M}\left(\tilde{t}_{|\xi|}, \tilde{t}_{|\xi|}, \xi\right)=\mathcal{R}_{M}\left(\tilde{t}_{|\xi|}, \xi\right) \text {. }
$$

Applying the generalized version of Faá di Bruno's formula we obtain for the case $|\alpha|=n$ the relation

$$
D_{\xi}^{\alpha} \mathcal{R}_{M}\left(\tilde{t}_{|\xi|}, \xi\right)=\sum_{0} \sum_{1} \cdots \sum_{n} \frac{n !}{\prod_{i=1}^{n}(i !)^{k_{i}} \prod_{i=1}^{n} \prod_{j=1}^{2} q_{i j} !} \frac{\partial^{\kappa} \mathcal{R}_{M}}{\partial_{\tilde{t}_{|\xi|}}^{p_{1}} \partial_{\xi}^{\alpha_{2}}}\left(\tilde{t}_{|\xi|}, \xi\right) \prod_{i=1}^{n}\left(D_{\xi}^{i} \tilde{t}_{|\xi|}\right)^{q_{i 1}}\left(D_{\xi}^{i} \xi\right)^{q_{i 2}},
$$

where the respective sums are taken over all non-negative integer solutions of the Diophantine equations as follows:

$$
\begin{aligned}
& \sum_{0} \rightarrow k_{1}+2 k_{2}+\cdots+n k_{n}=n, \\
& \sum_{1} \rightarrow q_{11}+q_{12}=k_{1}, \\
& \vdots \\
& \sum_{n} \rightarrow q_{n 1}+q_{n 2}=k_{n},
\end{aligned}
$$

and

$$
\begin{gathered}
p_{1}=\sum_{i=1}^{n} q_{i 1}, \quad\left|\alpha_{2}\right|=\sum_{i=1}^{n} q_{i 2}, \\
|\kappa|=k_{1}+k_{2}+\cdots+k_{n}=p_{1}+\left|\alpha_{2}\right| .
\end{gathered}
$$

By virtue of $\partial_{\xi_{k}} \xi_{l}=\delta_{k l}$ we may conclude that $q_{i 2}=0$, for all $i \geq 2$ and $\left|\alpha_{2}\right|=q_{12}$. This yields the estimate

$$
\begin{aligned}
& \left|D_{\xi}^{\alpha} \mathcal{R}_{M}\left(\tilde{t}_{|\xi|}, \xi\right)\right| \\
& \lesssim \sum_{0} \sum_{1} \cdots \sum_{n}|\xi|^{1-M-\left|\alpha_{2}\right|} \lambda\left(\tilde{t}_{|\xi|}\right)^{1-M} \Xi\left(\tilde{t}_{|\xi|}\right)^{-M-p_{1}} \prod_{i=1}^{n} \vartheta\left(\tilde{t}_{|\xi|}\right)^{-q_{i 1}}|\xi|^{-(i+1) q_{i 1}}|\xi|^{(-i+1) q_{i 2}} \\
& =\sum_{0} \sum_{1} \cdots \sum_{n}|\xi|^{1-M-q_{12}} \lambda\left(\tilde{t}_{|\xi|}\right)^{1-M} \Xi\left(\tilde{t}_{|\xi|}\right)^{-M-p_{1}} \prod_{i=1}^{n} \vartheta\left(\tilde{t}_{|\xi|}\right)^{-p_{1}}|\xi|^{-p_{1}+q_{i 2}-i\left(q_{i 1}+q_{i 2}\right)} \\
& =\sum_{0} \sum_{1} \cdots \sum_{n}|\xi|^{1-M-q_{12}} \lambda\left(\tilde{t}_{|\xi|}\right)^{1-M} \Xi\left(\tilde{t}_{|\xi|}\right)^{-M-p_{1}} \vartheta\left(\tilde{t}_{|\xi|}\right)^{-p_{1}}|\xi|^{-p_{1}-n+q_{12}} \\
& =\sum_{0} \sum_{1} \cdots \sum_{n}|\xi|^{1-M-q_{12}-p_{1}+q_{12}-n} \lambda\left(\tilde{t}_{|\xi|}\right)^{1-M} \Xi\left(\tilde{t}_{|\xi|}\right)^{-M-p_{1}} \frac{1}{\vartheta\left(\tilde{t}_{|\xi|}\right)^{p_{1}}} \\
& =\sum_{0} \sum_{1} \cdots \sum_{n}|\xi|^{1-M-n} \lambda\left(\tilde{t}_{|\xi|}\right)^{1-M} \Xi\left(\tilde{t}_{|\xi|}\right)^{-M} \frac{1}{|\xi|^{p_{1}} \Xi\left(\tilde{t}_{|\xi|}\right)^{p_{1}}} \frac{1}{\vartheta\left(\tilde{t}_{|\xi|}\right)^{p_{1}}} \\
& \lesssim|\xi|^{1-M-n} \lambda\left(\tilde{t}_{|\xi|}\right)^{1-M} \Xi\left(\tilde{t}_{|\xi|}\right)^{-M} \frac{1}{|\xi|^{p_{1}} \Theta\left(\tilde{t}_{|\xi|}\right)^{p_{1}}} \frac{\lambda\left(\tilde{t}_{|\xi|}\right)^{p_{1}}}{\vartheta\left(\tilde{t}_{|\xi|}\right)^{p_{1}}} \\
& \lesssim|\xi|^{1-M-n} \lambda\left(\tilde{t}_{|\xi|}\right)^{1-M} \Xi\left(\tilde{t}_{|\xi|}\right)^{-M}\left(\frac{\lambda\left(\tilde{t}_{|\xi|}\right)}{\vartheta\left(\tilde{t}_{|\xi|}\right)}\right)^{p_{1}}
\end{aligned}
$$

where we used Proposition 5.2.9 and from condition (A4) the estimate $\Theta(t) \lesssim \lambda(t) \Xi(t)$. This completes the proof of this lemma.

Following a similar procedure as in the proof of the previous lemma, we may conclude the following proposition for the estimates of the derivatives with respect to $\xi$ in the representation formula (5.2.4) appearing in the treatment in the oscillation subzone. 
Proposition 5.2.15. We assume the conditions $(\boldsymbol{A} 1)$ to $(\boldsymbol{A} 3)$. Then, the amplitude $\mathcal{Q}_{2}=$ $\mathcal{Q}_{2}(t, s, \xi)$ from (5.2.4) satisfies the following estimates:

$$
\left|D_{\xi}^{\alpha-\beta} \mathcal{Q}_{2}\left(\tilde{t}_{|\xi|}, t_{|\xi|}, \xi\right) D_{\xi}^{\beta} t_{|\xi|}\right| \leq C_{\alpha, N}|\xi|^{-|\alpha|} \exp \left(\frac{\Lambda\left(t_{|\xi|}\right)}{\Theta\left(t_{|\xi|}\right)}\right)^{|\beta|+1}
$$

for all multi-indices $\beta$ with $\beta \leq \alpha$.

\section{Transforming back to the original problem}

In the hyperbolic zone we divide our considerations for large and small frequencies. We want to construct the fundamental solution $E_{\mathrm{hyp}}=E_{\mathrm{hyp}}(t, s, \xi), \tilde{t}_{|\xi|} \leq s \leq t$ for small frequencies $\xi$ and $0 \leq s \leq t$ for large frequencies $\xi$, respectively, for the operator

$$
D_{t}-\mathcal{D}_{1}(t, \xi)-\mathcal{R}_{1}(t, \xi) .
$$

After constructing the fundamental solution $E_{M}=E_{M}(t, s, \xi)$ we want to transform back to the original problem and get in the hyperbolic zone the representation that we are looking for. We know that,

$$
\left(D_{t}-\mathcal{D}_{1}(t, \xi)-\mathcal{R}_{1}(t, \xi)\right) N_{M}(t, \xi)=N_{M}(t, \xi)\left(D_{t}-\mathcal{D}_{1}(t, \xi)-\mathcal{R}_{M}(t, \xi)\right) .
$$

If $E_{M}=E_{M}(t, s, \xi)$ is the fundamental solution to

$$
N_{M}(t, \xi)\left(D_{t}-\mathcal{D}_{1}(t, \xi)-\mathcal{R}_{M}(t, \xi)\right),
$$

then

$$
\left(D_{t}-\mathcal{D}_{1}(t, \xi)-\mathcal{R}_{1}(t, \xi)\right) N_{M}(t, \xi) E_{M}(t, s, \xi)=0 .
$$

In this way we have that $\mathcal{E}_{M}(t, s, \xi) N_{M}(t, \xi)$ and $N_{M}(t, \xi) E_{M}(t, s, \xi)$ satisfy the same initial value problem.

Large frequencies. The representation of the fundamental solution in $Z_{\text {hyp }}^{l}(N)$ is

$$
\begin{aligned}
E_{\text {hyp }}(t, s, \xi) & =P(t) N_{M}(t, \xi) E_{M}(t, s, \xi) N_{M}(s, \xi)^{-1} P(s)^{-1} \\
& =\frac{\sqrt{\lambda(t)}}{\sqrt{\lambda(s)}} P(t) N_{M}(t, \xi) \mathcal{E}_{M}(t, s, \xi) \mathcal{Q}_{M}(t, s, \xi) N_{M}(s, \xi)^{-1} P(s)^{-1}
\end{aligned}
$$

for $\tilde{t}_{|\xi|} \leq s \leq t$ and with uniformly bounded coefficient matrices $N_{M}, N_{M}^{-1} \in S_{N}\{0,0,0\}$.

Remark 5.2.6. For large frequencies it holds

$$
V(t, \xi)=E_{\text {hyp }}(t, s, \xi) V(s, \xi) \text { for } \tilde{t}_{|\xi|} \leq s \leq t .
$$

Then, we have the following representation of the micro-energy $V(t, \xi)=\left(\lambda(t)|\xi| \hat{u}, D_{t} \hat{u}\right)^{T}$ in $Z_{\text {hyp }}^{l}(N)$ :

$$
V(t, \xi)=\frac{\sqrt{\lambda(t)}}{\sqrt{\lambda(s)}} P(t) N_{M}(t, \xi) \mathcal{E}(t, s, \xi) \mathcal{Q}_{M}(t, s, \xi) N_{M}(s, \xi)^{-1} P(t)^{-1} V(s, \xi) .
$$

Small frequencies. We will consider a different representation for small frequencies, because in this case we shall use a "gluing procedure". In $Z_{\text {hyp }}^{s}(N)$ we should remember that for $\tilde{t}_{|\xi|} \leq t_{|\xi|} \leq t$ we have

$$
E(t, 0, \xi)=E_{\mathrm{hyp}}\left(t, \tilde{t}_{|\xi|}, \xi\right) E_{\mathrm{osc}}\left(\tilde{t}_{|\xi|}, t_{|\xi|}, \xi\right) E_{\mathrm{pd}}\left(t_{|\xi|}, 0, \xi\right) .
$$

Then, taking into account $E_{\mathrm{hyp}}=E_{\mathrm{hyp}}\left(t, \tilde{t}_{|\xi|}, \xi\right)$ for $\tilde{t}_{|\xi|} \leq t_{|\xi|} \leq t$ we have

$$
\begin{aligned}
E_{\mathrm{hyp}}\left(t, \tilde{t}_{|\xi|}, \xi\right) & =P(t) N_{M}(t, \xi) E_{M}\left(t, \tilde{t}_{|\xi|}, \xi\right) N_{M}\left(\tilde{t}_{|\xi|}, \xi\right)^{-1} P\left(\tilde{t}_{|\xi|}\right)^{-1} \\
& =\frac{\sqrt{\lambda(t)}}{\sqrt{\lambda\left(\tilde{t}_{|\xi|}\right)}} P(t) N_{M}(t, \xi) \mathcal{E}_{M}\left(t, \tilde{t}_{|\xi|}, \xi\right) \mathcal{Q}_{M}\left(t, \tilde{t}_{|\xi|}, \xi\right) N_{M}\left(\tilde{t}_{|\xi|}, \xi\right)^{-1} P\left(\tilde{t}_{|\xi|}\right)^{-1} .
\end{aligned}
$$




\section{3. $L^{p}-L^{q}$ estimates on the conjugate line}

In this section we will use the representations for the solution to the Cauchy problem (5.2.1) in order to derive $L^{p}-L^{q}$ estimates on the conjugate line. To get these estimates we need to derive a representation of the solution to (5.2.1) by the aid of Fourier multipliers. Then one has to discuss mapping properties of these Fourier multipliers. This will be done after localizing the amplitudes by using a suitable dyadic decomposition related to the division into zones of the extended phase space. The part of the dyadic decomposition which belongs to the pseudo-differential zone and oscillation subzone generates Fourier multipliers which can be studied by the Hardy-Littlewood inequality. The part which belongs to the hyperbolic zone needs a Littman-type lemma. These are results for oscillating integrals with localized amplitude away from the origin.

The stationary phase method together with usual properties of the Fourier transformation leads to a $L^{1}-L^{\infty}$ estimate for Fourier multipliers with localized amplitude. After deriving a $L^{2}-L^{2}$ estimate some interpolation gives suitable $L^{p}-L^{q}$ estimates on the conjugate line. Gluing all these estimates for Fourier multipliers with localized amplitudes together leads to the desired $L^{p}-L^{q}$ estimates for the Fourier multiplier, the energy operator itself on the conjugate line for $p \in(1,2]$. The supposed regularity of the data we need to avoid constants depending on the parameter of the dyadic decomposition in the $L^{p}-L^{q}$ estimates for the Fourier multipliers with localized amplitudes.

Theorem 5.3.1 (Main theorem). We assume that the conditions (A1) to (A5) hold. Then, we have the following $L^{p}-L^{q}$ estimates for the kinetic and the "elastic" energy:

$$
\begin{aligned}
\| u_{t}(t, \cdot), & \lambda(t) \nabla_{x} u(t, \cdot) \|_{L^{q}} \\
\lesssim & \sqrt{\lambda(t)} \max \left\{\Theta(t)^{-n\left(\frac{1}{p}-\frac{1}{q}\right)} \exp \left(\frac{\Lambda(t)}{\Theta(t)}\right) ; \Lambda(t)^{-\frac{n-1}{2}\left(\frac{1}{p}-\frac{1}{q}\right)}\left(\frac{\lambda(t)}{\Lambda(t)} \Xi(t)\right)^{-M\left(\frac{1}{p}-\frac{1}{q}\right)}\right\} \\
& \times\left(\left\|u_{0}\right\|_{W_{p}^{N_{p}+1}}+\left\|u_{1}\right\|_{W_{p}^{N_{p}}}\right)
\end{aligned}
$$

with regularity $N_{p}=n\left(\frac{1}{p}-\frac{1}{q}\right), p \in(1,2]$ and $\frac{1}{p}+\frac{1}{q}=1$.

Proof. Let us consider the following zones in the extended phase space:

$$
\begin{aligned}
& Z_{\text {hyp }}^{s}(N)=\left\{(t, \xi) \in[0, \infty) \times \mathbb{R}_{\xi}^{n}: \Theta(t)|\xi| \geq N\right\} \cap\{|\xi| \leq N\}, \\
& Z_{\text {hyp }}^{l}(N)=\left\{(t, \xi) \in[0, \infty) \times \mathbb{R}_{\xi}^{n}:|\xi| \geq N\right\}, \\
& Z_{\text {osc }}(N)=\left\{(t, \xi) \in[0, \infty) \times \mathbb{R}_{\xi}^{n}: \Theta(t)|\xi| \leq N \leq \Lambda(t)|\xi|\right\}, \\
& Z_{\text {pd }}(N)=\left\{(t, \xi) \in[0, \infty) \times \mathbb{R}_{\xi}^{n}: \Lambda(t)|\xi| \leq N\right\} .
\end{aligned}
$$

More precisely, we introduce a cut-off function $\varphi=\varphi(t) \in \mathcal{C}_{0}^{\infty}([0, \infty))$ such that $\varphi(t) \equiv 1$ for $t \leq \frac{1}{2}, \varphi(t) \equiv 0$ for $t \geq 2$ and $\varphi^{\prime}(t) \leq 0$. Then, we can define the characteristic functions $\varphi_{\mathrm{pd}}$, $\varphi_{\text {osc }}, \varphi_{\text {hyp }}^{s}$ and $\varphi_{\text {hyp }}^{l}$ of the zones $Z_{\mathrm{pd}}(N), Z_{\text {osc }}(N), Z_{\text {hyp }}^{s}(N)$ and $Z_{\text {hyp }}^{l}(N)$, respectively, by

$$
\begin{aligned}
\varphi_{\text {pd }}(t, \xi) & =\varphi\left(\Theta(t)|\xi| N^{-1}\right) \varphi\left(\Lambda(t)|\xi| N^{-1}\right), \\
\varphi_{\text {osc }}(t, \xi) & =\varphi\left(\Theta(t)|\xi| N^{-1}\right)\left(1-\varphi\left(\Lambda(t)|\xi| N^{-1}\right)\right), \\
\varphi_{\text {hyp }}^{s}(t, \xi) & =\left(1-\varphi\left(\Theta(t)|\xi| N^{-1}\right)\right) \varphi\left(|\xi| N^{-1}\right), \\
\varphi_{\text {hyp }}^{l}(t, \xi) & =\left(1-\varphi\left(\Theta(t)|\xi| N^{-1}\right)\right)\left(1-\varphi\left(|\xi| N^{-1}\right)\right),
\end{aligned}
$$

such that $\varphi_{\text {pd }}+\varphi_{\text {osc }}+\varphi_{\text {hyp }}^{s}+\varphi_{\text {hyp }}^{l} \equiv 1$ on the whole extended phase space. 


\subsection{1. $L^{p}-L^{q}$ estimates for Fourier multipliers with amplitudes localized in $Z_{\text {pd }}(N)$}

In $Z_{\text {pd }}(N)$, we have

$$
V(t, \xi)=\varphi_{\mathrm{pd}}(t, \xi) E_{\mathrm{pd}}(t, 0, \xi) V(0, \xi) .
$$

For this reason, we consider the model Fourier multiplier

$$
\left\|\mathcal{F}^{-1}\left(\varphi_{\mathrm{pd}}(t, \xi) E_{\mathrm{pd}}^{(k l)}(t, 0, \xi) \mathcal{F}(v)\right)\right\|_{L^{q}}
$$

where $v \in \mathcal{S}, E_{\mathrm{pd}}^{(k l)}(t, 0, \xi), k, l=1,2$ are the entries of the fundamental solution $E_{\mathrm{pd}}(t, 0, \xi)$. The main tool is the Hardy-Littlewood inequality from Theorem A.6.1. It holds

$$
\begin{aligned}
\left\|\mathcal{F}^{-1}\left(\varphi_{\mathrm{pd}}(t, \xi) E_{\mathrm{pd}}^{(k l)}(t, 0, \xi) \mathcal{F}(v)\right)\right\|_{L^{q}} & \leq\left\|\varphi_{\mathrm{pd}}(t, \xi) E_{\mathrm{pd}}^{(k l)}(t, 0, \xi) \mathcal{F}(v)\right\|_{L^{p}} \\
& \leq\left\|\varphi_{\mathrm{pd}}(t, \xi)\right\|_{L^{\frac{p q}{q-p}}}\left\|E_{\mathrm{pd}}^{(k l)}(t, 0, \xi)\right\|_{L^{\infty}}\|\mathcal{F}(v)\|_{L^{q}} \\
& \lesssim \Lambda(t)^{-n\left(\frac{1}{p}-\frac{1}{q}\right)}\left\|E_{\mathrm{pd}}^{(k l)}(t, 0, \xi)\right\|_{L^{\infty}}\|v(\cdot)\|_{L^{p}} .
\end{aligned}
$$

Summarizing, from Proposition 5.2.2 with $\delta=\frac{1}{2}$ and $\max \{|\xi| \lambda(t) ; \sqrt{\lambda(t)}\}=\sqrt{\lambda(t)}$ we have

$$
\begin{gathered}
\left\|\mathcal{F}^{-1}\left(\varphi_{\mathrm{pd}}(t, \xi) \hat{v}_{t}(t, \cdot)\right)\right\|_{L^{q}}+\left\|\mathcal{F}^{-1}\left(\varphi_{\mathrm{pd}}(t, \xi) \lambda(t)|\xi| \hat{v}(t, \cdot)\right)\right\|_{L^{q}} \\
\lesssim \sqrt{\lambda(t)} \Lambda(t)^{-n\left(\frac{1}{p}-\frac{1}{q}\right)}\left(\left\|u_{0}(\cdot)\right\|_{L^{p}}+\left\|u_{1}(\cdot)\right\|_{L^{p}}\right) .
\end{gathered}
$$

\subsection{2. $L^{p}-L^{q}$ estimates for Fourier multipliers with amplitudes localized in $Z_{\text {osc }}(N)$}

In this zone we use the "gluing procedure" for $0 \leq t_{|\xi|} \leq t \leq \tilde{t}_{|\xi|}$ as follows:

$$
E(t, 0, \xi)=E_{\mathrm{osc}}\left(t, t_{|\xi|}, \xi\right) E_{\mathrm{pd}}\left(t_{|\xi|}, 0, \xi\right) .
$$

Then, we consider the Fourier multiplier

$$
\left\|\mathcal{F}^{-1}\left(\varphi_{\mathrm{osc}}(t, \xi) e^{ \pm i|\xi| \int_{t_{|\xi|}}^{t} \lambda(\tau) \omega(\tau) d \tau} \frac{\sqrt{\lambda(t)}}{\sqrt{\lambda\left(t_{|\xi|}\right)}} d(t, \xi) E_{\mathrm{pd}}^{(k l)}\left(t_{|\xi|}, 0, \xi\right) \mathcal{F}(v)\right)\right\|_{L^{q}}
$$

where used the estimate of $E_{\text {osc }}\left(t, t_{|\xi|}, \xi\right)$ from Proposition 5.2 .4 and $d=d(t, \xi)$ is related to the entries of $\mathcal{E}_{2}, \mathcal{Q}_{2}, N_{2}$ and $P$ from (5.2.7). Here $v \in L^{p}$ and $2 \leq q<\infty$. Moreover, $E_{\mathrm{pd}}^{(k l)}\left(t_{|\xi|}, 0, \xi\right), k, l=1,2$ are the entries of the fundamental solution $E_{\mathrm{pd}}\left(t_{|\xi|}, 0, \xi\right)$. Finally, the function $d=d(t, \xi)$ satisfies the following estimate from (5.2.6):

$$
|d(t, \xi)| \leq \exp \left(\frac{\Lambda\left(t_{|\xi|}\right)}{\Theta\left(t_{|\xi|}\right)}\right) .
$$

The main tool is again the Hardy-Littlewood inequality from Theorem A.6.1. So, we have

$$
\begin{aligned}
& \left\|\mathcal{F}^{-1}\left(\varphi_{\mathrm{osc}}(t, \xi) e^{ \pm i|\xi| \int_{t_{|\xi|}}^{t} \lambda(\tau) \omega(\tau) d \tau} \frac{\sqrt{\lambda(t)}}{\sqrt{\lambda\left(t_{|\xi|}\right)}} d(t, \xi) E_{\mathrm{pd}}^{(k l)}\left(t_{|\xi|}, 0, \xi\right) \mathcal{F}(v)\right)\right\|_{L^{q}} \\
& \quad \leq\left\|\mathcal{F}^{-1}\left(\varphi_{\mathrm{osc}}(t, \xi) e^{ \pm i|\xi| \int_{0}^{t} \lambda(\tau) \omega(\tau) d \tau} \sqrt{\lambda(t)} d_{1}(t, \xi) \mathcal{F}(v)\right)\right\|_{L^{q}} \\
& \quad \leq C_{N} \sqrt{\lambda(t)} \Theta(t)^{-n\left(\frac{1}{p}-\frac{1}{q}\right)} \exp \left(\frac{\Lambda\left(t_{|\xi|}\right)}{\Theta\left(t_{|\xi|}\right)}\right)\|v(\cdot)\|_{L^{p}} \\
& \quad \leq C_{N}^{\prime} \sqrt{\lambda(t)} \Theta(t)^{-n\left(\frac{1}{p}-\frac{1}{q}\right)} \exp \left(\frac{\Lambda(t)}{\Theta(t)}\right)\|v(\cdot)\|_{L^{p}},
\end{aligned}
$$


provided that $1<p \leq 2$ and $\frac{1}{p}+\frac{1}{q}=1$. Here $d_{1}=d_{1}(t, \xi)$ is given as follows:

$$
d_{1}(t, \xi)=\exp \left(\mp i|\xi| \int_{0}^{t_{|\xi|}} \lambda(\tau) \omega(\tau) d \tau\right) d(t, \xi)
$$

and we used the following estimate from (5.2.6):

$$
\left|d_{1}(t, \xi)\right| \leq \exp \left(\frac{\Lambda\left(t_{|\xi|}\right)}{\Theta\left(t_{|\xi|}\right)}\right) .
$$

Summarizing, we have shown

$$
\begin{aligned}
\| \mathcal{F}^{-1} & \left(\varphi_{\mathrm{osc}}(t, \xi) e^{ \pm i|\xi| \int_{0}^{t} \lambda(\tau) \omega(\tau) d \tau} \sqrt{\lambda(t)} d(t, \xi) \mathcal{F}\left(\lambda(0) \omega(0)|D| u(0, \cdot)+u_{t}(0, \cdot)\right)\right) \|_{L^{q}} \\
& \lesssim \sqrt{\lambda(t)} \Theta(t)^{-n\left(\frac{1}{p}-\frac{1}{q}\right)} \exp \left(\frac{\Lambda(t)}{\Theta(t)}\right)\left(\left\|u_{0}(\cdot)\right\|_{L^{p}}+\left\|u_{1}(\cdot)\right\|_{L^{p}}\right) .
\end{aligned}
$$

Therefore, we arrive at

$$
\begin{aligned}
& \left\|\mathcal{F}^{-1}\left(\varphi_{\mathrm{osc}}(t, \xi) \hat{v}_{t}(t, \cdot)\right)\right\|_{L^{q}}+\left\|\mathcal{F}^{-1}\left(\varphi_{\mathrm{osc}}(t, \xi) \lambda(t)|\xi| \hat{v}(t, \cdot)\right)\right\|_{L^{q}} \\
& \quad \lesssim \sqrt{\lambda(t)} \Theta(t)^{-n\left(\frac{1}{p}-\frac{1}{q}\right)} \exp \left(\frac{\Lambda(t)}{\Theta(t)}\right)\left(\left\|u_{0}(\cdot)\right\|_{L^{p}}+\left\|u_{1}(\cdot)\right\|_{L^{p}}\right) .
\end{aligned}
$$

\subsection{3. $L^{p}-L^{q}$ estimates for Fourier multipliers with amplitudes localized in $Z_{\text {hyp }}(N)$}

The micro-energy $V(t, \xi)=\left(\lambda(t)|\xi| \hat{v}, D_{t} \hat{v}\right)^{T}$ in $Z_{\text {hyp }}(N)$ is given by

$$
V(t, \xi)=\frac{\sqrt{\lambda(t)}}{\sqrt{\lambda(s)}} P(t) N_{M}(t, \xi) \mathcal{E}_{M}(t, s, \xi) \mathcal{Q}_{M}(t, s, \xi) N_{M}(s, \xi)^{-1} P(s)^{-1} V(s, \xi),
$$

with uniformly bounded coefficient matrices $N_{M}, N_{M}^{-1} \in S_{N}\{0,0,0\}$. The diagonal matrix $\mathcal{E}_{M}(t, s, \xi)$ has entries $e^{ \pm i|\xi| \int_{s}^{t} \lambda(\tau) \omega(\tau) d \tau}$. For this reason we will consider suitable localizations of the following Fourier multipliers:

$$
\mathcal{F}^{-1}\left(e^{ \pm i|\xi| \int_{0}^{t} \lambda(\tau) \omega(\tau) d \tau} \frac{\sqrt{\lambda(t)}}{\sqrt{\lambda(s)}} b(t, \xi)|\xi|^{-r} \mathcal{F}(v)\right)
$$

where $r$ is a real number and in the moment we suppose $v \in \mathcal{S}$. Moreover, here $b=b(t, \xi)$ is related to the entries of $\mathcal{E}_{M}, \mathcal{Q}_{M}, N_{M}$ and $P$. We will consider the last Fourier multiplier for large frequencies with $s=0$ and for small frequencies with $s=\tilde{t}_{|\xi|}$, respectively. For the small frequencies, in order to continue with the phase function $e^{ \pm i|\xi| \int_{0}^{t} \lambda(\tau) \omega(\tau) d \tau}$ we will shift the remainder terms in $b=b(t, \xi)$ (see Remark 5.2.5). The key tool in these two parts is a Littman type lemma from Lemma A.5.1.

\section{Estimates for large frequencies}

In this part of the extended phase space we will consider large frequencies $|\xi| \geq N$ only. We analyze

$$
\left\|\mathcal{F}^{-1}\left(\varphi_{\text {hyp }}^{l}(t, \xi) e^{ \pm i|\xi| \int_{0}^{t} \lambda(\tau) \omega(\tau) d \tau} \sqrt{\lambda(t)} b_{1}(t, \xi)|\xi|^{-r} \mathcal{F}(v)\right)\right\|_{L^{q}}
$$

with $2 \leq q<\infty$ and $v \in S$. Here the amplitude $b_{1}=b_{1}(t, \xi)$ is related to $b=b(t, \xi)$ from (5.3.3) with $s=0$. Then, from (5.2.22) it holds

$$
\left|D_{\xi}^{\alpha} b_{1}(t, \xi)\right| \leq C_{\alpha, M, N}|\xi|^{-|\alpha|}
$$


for $\beta=0$ and $|\alpha| \leq M-1$, because $N_{M}(t, \xi) \in S_{N}\{0,0,0\}$ and $\left|D_{\xi}^{\alpha} \mathcal{Q}_{M}(t, 0, \xi)\right| \leq C_{\alpha, M, N}|\xi|^{-|\alpha|}$.

Now we choose a non-negative function $\phi=\phi(s) \in \mathcal{C}_{0}^{\infty}([0, \infty))$ with $\operatorname{supp} \phi \subseteq\left[\frac{1}{2}, 1\right]$ such that $\sum_{j=-\infty}^{\infty} \phi\left(2^{-j} s\right)=1, s \neq 0$. Let us define a dyadic decomposition $\left\{\phi_{j}\right\}_{j \in \mathbb{Z}}$ by

$$
\phi_{j}(t, \xi)=\phi\left(2^{-j}|\xi| N^{-1}\right), \quad j \in \mathbb{Z} .
$$

Since $\varphi_{\text {hyp }}^{l}(t, \xi) \phi_{j}(t, \xi)=0$ for $j<0$, we have

$$
\varphi_{\text {hyp }}^{l}(t, \xi) \leq \sum_{j=0}^{\infty} \phi_{j}(t, \xi) .
$$

This allows us to investigate every sum stated in the right-hand side for $j \in \mathbb{N}$ separately. The goal here is to apply the Riesz-Thorin interpolation theorem from Theorem A.4.1. For every $j \in \mathbb{N}$ let us examine the oscillatory integral

$$
\mathcal{F}^{-1}\left(\varphi_{\text {hyp }}^{l}(t, \xi) \phi_{j}(t, \xi) e^{ \pm i|\xi| \int_{0}^{t} \lambda(\tau) \omega(\tau) d \tau} \sqrt{\lambda(t)}|\xi|^{-r} b_{1}(t, \xi) \mathcal{F}(v)\right)
$$

One basic assumption to apply this theorem is that the amplitude $b_{1}=b_{1}(t, \xi)$ is subjected to symbol-like estimates. The strategy of the proof in this part of the extended phase space is to obtain an $L^{p}-L^{q}$ estimate by interpolating $L^{1}-L^{\infty}$ and $L^{2}-L^{2}$ estimates with Riesz-Thorin interpolation theorem. We introduce

$$
\begin{aligned}
& I_{j}:=\left\|\mathcal{F}^{-1}\left(\varphi_{\text {hyp }}^{l}(t, \xi) \phi_{j}(t, \xi) e^{ \pm i|\xi| \int_{0}^{t} \lambda(\tau) \omega(\tau) d \tau} \sqrt{\lambda(t)}|\xi|^{-r} b_{1}(t, \xi)\right)\right\|_{L^{\infty}}, \\
& \tilde{I}_{j}:=\left\|\varphi_{\text {hyp }}^{l}(t, \xi) \phi_{j}(t, \xi) e^{ \pm i|\xi| \int_{0}^{t} \lambda(\tau) \omega(\tau) d \tau} \sqrt{\lambda(t)}|\xi|^{-r} b_{1}(t, \xi)\right\|_{L^{\infty}} .
\end{aligned}
$$

We want to derive the following estimates:

- a $L^{1}-L^{\infty}$ estimate for $I_{j}$,

- a $L^{2}-L^{2}$ estimate for $\tilde{I}_{j}$.

$L^{1}-L^{\infty}$ estimates. For all $j<0$ we have $I_{j}=\tilde{I}_{j}=0$. For $j \geq 0$ we perform the change of variables $\xi=2^{j} N \eta$ and conclude as follows:

$$
\begin{aligned}
I_{j} & \leq C 2^{j(n-r)}\left\|\mathcal{F}^{-1}\left(\phi\left(t, 2^{j} N \eta\right) e^{ \pm i 2^{j} N|\eta| \int_{0}^{t} \lambda(\tau) \omega(\tau) d \tau} \sqrt{\lambda(t)}|\eta|^{-r} b_{1}\left(t, 2^{j} N \eta\right)\right)\right\|_{L^{\infty}} \\
& \leq C 2^{j(n-r)}\left\|\mathcal{F}^{-1}\left(\phi\left(t, 2^{j} N \eta\right) e^{ \pm i 2^{j} N \tilde{C} \tilde{\Lambda}(t)|\eta|} \sqrt{\lambda(t)}|\eta|^{-r} b_{1}\left(t, 2^{j} N \eta\right)\right)\right\|_{L^{\infty}} \\
& \leq C 2^{j(n-r)}\left(1+2^{j} N \tilde{C} \tilde{\Lambda}(t)\right)^{-\frac{n-1}{2}} \sqrt{\lambda(t)} \sum_{|\alpha| \leq M}\left\|D_{\eta}^{\alpha}\left(\phi\left(t, 2^{j} N \eta\right)|\eta|^{-r} b_{1}\left(t, 2^{j} N \eta\right)\right)\right\|_{L^{\infty}} \\
& \leq C 2^{j(n-r)}\left(1+2^{j} N \tilde{C} \tilde{\Lambda}(t)\right)^{-\frac{n-1}{2}} \sqrt{\lambda(t)} \sum_{\left|\alpha_{1}+\alpha_{2}\right| \leq M} \sup _{1 / 2 \leq|\eta| \leq 2}|\eta|^{-r-\left|\alpha_{1}\right|}\left(2^{j} N\right)^{\left|\alpha_{2}\right|}\left(2^{j} N|\eta|\right)^{-\left|\alpha_{2}\right|} \\
& \leq C 2^{j(n-r)}(1+\Lambda(t))^{-\frac{n-1}{2}} \sqrt{\lambda(t)},
\end{aligned}
$$

where $\varphi_{\text {hyp }}(t, \xi) \equiv 1$ for $j \geq 2$ and $\tilde{\Lambda}(t):=\int_{0}^{t} \lambda(\tau) d \tau$. Moreover, in the second estimate we have used Lemma A.5.1 with a suitably positive constant $M$. The constant $M$ determines the necessary steps of diagonalization. Here we choose $M>\frac{n+5}{2}$. Additionally, we take advantage of $1+\Lambda(t) \lesssim 1+2^{j} N \tilde{C} \Lambda(t)$ for all $j \geq 0$ and $N$ sufficiently large. Thus, we get

$$
\begin{aligned}
I_{j} & =\left\|\mathcal{F}^{-1}\left(\varphi_{\text {hyp }}^{l}(t, \xi) \phi_{j}(t, \xi) e^{ \pm i|\xi| \int_{0}^{t} \lambda(\tau) \omega(\tau) d \tau} \sqrt{\lambda(t)}|\xi|^{-r} b_{1}(t, \xi) \mathcal{F}(v)\right)\right\|_{L^{\infty}} \\
& \lesssim 2^{j(n-r)} \sqrt{\lambda(t)}(1+\Lambda(t))^{-\frac{n-1}{2}}\|v(\cdot)\|_{L^{1}} .
\end{aligned}
$$


$L^{2}-L^{2}$ estimate. In order to have an $L^{2}-L^{2}$ estimate we shall estimate $\tilde{I}_{j}$. If we use the change of variables $\xi=2^{j} N \eta$, then we have

$$
\tilde{I}_{j} \leq C \sup _{1 / 2 \leq|\eta| \leq 2} \phi\left(t, 2^{j} N \eta\right) \sqrt{\lambda(t)}\left(2^{j} N|\eta|\right)^{-r}\left|b_{1}\left(t, 2^{j} N \eta\right)\right| \lesssim 2^{-j r} \sqrt{\lambda(t)}
$$

for $j \geq 0$. Consequently, we arrive at the following estimate:

$$
\left\|\mathcal{F}^{-1}\left(\varphi_{\text {hyp }}^{l}(t, \xi) \phi_{j}(t, \xi) e^{ \pm i|\xi| \int_{0}^{t} \lambda(\tau) \omega(\tau) d \tau} \sqrt{\lambda(t)}|\xi|^{-r} b_{1}(t, \xi) \mathcal{F}(v)\right)\right\|_{L^{2}} \lesssim 2^{-j r} \sqrt{\lambda(t)}\|v(\cdot)\|_{L^{2}} .
$$

$L^{p}-L^{q}$ estimates. Applying the Riesz-Thorin's interpolation theorem we have

$$
\begin{aligned}
& \left\|\mathcal{F}^{-1}\left(\varphi_{\text {hyp }}^{l}(t, \xi) \phi_{j}(t, \xi) e^{ \pm i|\xi| \int_{0}^{t} \lambda(\tau) \omega(\tau) d \tau} \sqrt{\lambda(t)}|\xi|^{-r} b_{1}(t, \xi)\right)\right\|_{L^{q}} \\
& \quad \lesssim 2^{j\left(n\left(\frac{1}{p}-\frac{1}{q}\right)-r\right)} \sqrt{\lambda(t)}(1+\Lambda(t))^{-\frac{n-1}{2}\left(\frac{1}{p}-\frac{1}{q}\right)} .
\end{aligned}
$$

Finally, we fix $r=N_{p}=n\left(\frac{1}{p}-\frac{1}{q}\right)$ and apply Theorem A.4.3 for $1<p \leq 2$. Then, we have

$$
\begin{aligned}
& \| \mathcal{F}^{-1}\left(\varphi _ { \text { hyp } } ^ { l } ( t , \xi ) e ^ { \pm i | \xi | \int _ { 0 } ^ { t } \lambda ( \tau ) \omega ( \tau ) d \tau } \sqrt { \lambda ( t ) } | \xi | ^ { - r } b _ { 1 } ( t , \xi ) \mathcal { F } \left(\lambda(0) \omega(0)|D|^{r+1} u(0, \cdot)\right.\right. \\
& \left.\left.+|D|^{r} u_{t}(0, \cdot)\right)\right) \|_{L^{q}} \\
& \lesssim \sqrt{\lambda(t)}(1+\Lambda(t))^{-\frac{n-1}{2}\left(\frac{1}{p}-\frac{1}{q}\right)}\left(\left\|u_{0}(\cdot)\right\|_{W_{p}^{N_{p}+1}}+\left\|u_{1}(\cdot)\right\|_{W_{p}^{N_{p}}} .\right.
\end{aligned}
$$

Thus, we may conclude

$$
\begin{aligned}
& \left\|\mathcal{F}^{-1}\left(\varphi_{\text {hyp }}^{l}(t, \xi) \hat{v}_{t}(t, \cdot)\right)\right\|_{L^{q}}+\left\|\mathcal{F}^{-1}\left(\varphi_{\text {hyp }}^{l}(t, \xi) \lambda(t)|\xi| \hat{v}(t, \cdot)\right)\right\|_{L^{q}} \\
& \quad \lesssim \sqrt{\lambda(t)}(1+\Lambda(t))^{-\frac{n-1}{2}\left(\frac{1}{p}-\frac{1}{q}\right)}\left(\left\|u_{0}(\cdot)\right\|_{W_{p}^{N_{p}+1}}+\left\|u_{1}(\cdot)\right\|_{W_{p}^{N_{p}}}\right)
\end{aligned}
$$

Using the density of $\mathcal{S}$ in $W_{p}^{N_{p}}$ we may assume $u_{0} \in W_{p}^{N_{p}+1}$ and $u_{1} \in W_{p}^{N_{p}}$ as well.

\section{Estimates for small frequencies}

For small frequencies we use the "gluing procedure" for $\tilde{t}_{|\xi|} \leq t$ as follows:

$$
E(t, 0, \xi)=E_{\mathrm{hyp}}\left(t, \tilde{t}_{|\xi|}, \xi\right) E_{\mathrm{osc}}\left(\tilde{t}_{|\xi|}, t_{|\xi|}, \xi\right) E_{\mathrm{pd}}\left(t_{|\xi|}, 0, \xi\right) .
$$

Then, we consider the model Fourier multiplier

$$
\begin{aligned}
& \mathcal{F}^{-1}\left(\varphi_{\text {hyp }}^{s}(t, \xi) e^{ \pm i|\xi| \int_{t_{|\xi|}^{t}}^{t} \lambda(\tau) \omega(\tau) d \tau} \frac{\sqrt{\lambda(t)}}{\sqrt{\lambda\left(\tilde{t}_{|\xi|}\right)}} b_{1}(t, \xi) e^{\mp i|\xi| \int_{t_{|\xi|}}^{\tilde{t}_{|\xi|}} \lambda(\tau) \omega(\tau) d \tau} \frac{\sqrt{\lambda\left(\tilde{t}_{|\xi|}\right)}}{\sqrt{\lambda\left(t_{|\xi|}\right)}} d\left(\tilde{t}_{|\xi|}, \xi\right)\right. \\
& \left.\times E_{\mathrm{pd}}^{(k l)}\left(t_{|\xi|}, 0, \xi\right)|\xi|^{-r} \mathcal{F}(v)\right)
\end{aligned}
$$

where $d=d(t, \xi)$ is related to the entries of $\mathcal{E}_{2}, \mathcal{Q}_{2}, N_{2}$ and $P$ from (5.2.7). So, we estimate the following norm:

$$
\left\|\mathcal{F}^{-1}\left(\varphi_{\text {hyp }}^{s}(t, \xi) e^{ \pm i|\xi| \int_{t_{|\xi|}}^{t} \lambda(\tau) \omega(\tau) d \tau} \frac{\sqrt{\lambda(t)}}{\sqrt{\lambda\left(t_{|\xi|}\right)}} b_{1}(t, \xi) d\left(\tilde{t}_{|\xi|}, \xi\right) E_{\mathrm{pd}}^{(k l)}\left(t_{|\xi|}, 0, \xi\right)|\xi|^{-r} \mathcal{F}(v)\right)\right\|_{L^{q}}
$$

where in the moment $v \in \mathcal{S}, E_{\mathrm{pd}}^{(k l)}\left(t_{|\xi|}, 0, \xi\right), k, l=1,2$, are the entries of the fundamental solution $E_{\mathrm{pd}}\left(t_{|\xi|}, 0, \xi\right)$ and $b_{1}=b_{1}(t, \xi), d=d\left(\tilde{t}_{|\xi|}, \xi\right)$ satisfy the following estimates from (5.2.22) and (5.2.23), respectively:

$$
\begin{aligned}
& \left|D_{\xi}^{\alpha} b_{1}(t, \xi)\right| \leq C_{\alpha, M, N}|\xi|^{-|\alpha|} \text { for } \tilde{t}_{|\xi|} \leq t \\
& \left|D_{\xi}^{\alpha} d\left(\tilde{t}_{|\xi|}, \xi\right)\right| \leq C_{\alpha, N}|\xi|^{-|\alpha|} \exp \left(\frac{\Lambda\left(\tilde{t}_{|\xi|}\right)}{\Theta\left(\tilde{t}_{|\xi|}\right)}\right) .
\end{aligned}
$$


Moreover, we define

$$
g(t, \xi):=\exp \left(\mp i|\xi| \int_{0}^{t_{|\xi|}} \lambda(\tau) \omega(\tau) d \tau\right) \frac{1}{\sqrt{\lambda\left(t_{|\xi|}\right)}} b_{1}(t, \xi) d\left(\tilde{t}_{|\xi|}, \xi\right) E_{\mathrm{pd}}^{(k l)}\left(t_{|\xi|}, 0, \xi\right)
$$

which satisfies the following estimate from Proposition 5.2.13:

$$
\left|D_{\xi}^{\alpha} g(t, \xi)\right| \leq C_{\alpha, M, N}\left(\frac{\lambda\left(t_{|\xi|}\right)}{\Lambda\left(t_{|\xi|}\right)} \Xi\left(t_{|\xi|}\right)\right)^{-|\alpha|}|\xi|^{-|\alpha|} \text { for all }|\alpha| \leq M-1 .
$$

We can rewrite the Fourier multiplier of our interest in the following form:

$$
\mathcal{F}^{-1}\left(\varphi_{\text {hyp }}^{s}(t, \xi) e^{ \pm i|\xi| \int_{0}^{t} \lambda(\tau) \omega(\tau) d \tau} \sqrt{\lambda(t)}|\xi|^{-r} g(t, \xi) \mathcal{F}(v)\right) .
$$

We use again a dyadic decomposition by defining

$$
\phi_{j}(t,|\xi|)=\phi\left(2^{-j} \Theta(t)|\xi| N^{-1}\right), \quad j \in \mathbb{Z},
$$

with $\phi \in \mathcal{C}_{0}^{\infty}([0, \infty))$. Thus, for $j \geq 0$ we will estimate the following $L^{\infty}$ norms:

$$
\begin{aligned}
& I_{j}:=\left\|\mathcal{F}^{-1}\left(\varphi_{\text {hyp }}^{s}(t, \xi) \phi_{j}(t,|\xi|) e^{ \pm i|\xi| \int_{0}^{t} \lambda(\tau) \omega(\tau) d \tau} \sqrt{\lambda(t)}|\xi|^{-r} \varphi\left(\Theta(t)|\xi| N^{-1}\right) g(t, \xi)\right)\right\|_{L^{\infty}} \\
& \tilde{I}_{j}:=\left\|\varphi_{\text {hyp }}^{s}(t, \xi) \phi_{j}(t,|\xi|) e^{ \pm i|\xi| \int_{0}^{t} \lambda(\tau) \omega(\tau) d \tau} \sqrt{\lambda(t)}|\xi|^{-r} \varphi\left(\Theta(t)|\xi| N^{-1}\right) g(t, \xi)\right\|_{L^{\infty}}
\end{aligned}
$$

Our goal is to derive

- a $L^{1}-L^{\infty}$ estimate for $I_{j}$,

- a $L^{2}-L^{2}$ estimate for $\tilde{I}_{j}$.

Hence, an interpolating argument yields $L^{p}-L^{q}$ estimates for small frequencies.

$L^{1}-L^{\infty}$ estimates. We perform the change of variables $\Lambda(t) \xi=2^{j} N \eta$ and get the estimates

$$
\begin{aligned}
I_{j} \leq & C 2^{j(n-r)} \Lambda(t)^{(r-n)}\left\|\mathcal{F}^{-1}\left(\phi\left(\frac{\Theta(t)|\eta|}{\Lambda(t)}\right) e^{ \pm i \tilde{C}^{j} N|\eta|} \sqrt{\lambda(t)}|\eta|^{-r} \varphi\left(\frac{2^{j} \Theta(t)|\eta|}{\Lambda(t)}\right) g\left(t, \frac{2^{j} N \eta}{\Lambda(t)}\right)\right)\right\|_{L^{\infty}} \\
\leq & C 2^{j(n-r)}\left(1+\tilde{C} 2^{j} N\right)^{-\frac{n-1}{2}} \Lambda(t)^{(r-n)} \sqrt{\lambda(t)} \\
& \times \sum_{|\alpha| \leq M}\left\|D_{\eta}^{\alpha} \phi\left(\frac{\Theta(t)|\eta|}{\Lambda(t)}\right)|\eta|^{-r} \varphi\left(\frac{2^{j} \Theta(t)|\eta|}{\Lambda(t)}\right) g\left(t, \frac{2^{j} N \eta}{\Lambda(t)}\right)\right\|_{L^{\infty}} \\
\leq & C 2^{j\left(\frac{n+1}{2}-r\right)} \Lambda(t)^{(r-n)} \sqrt{\lambda(t)} \\
& \quad \quad \sum_{\left|\alpha_{1}+\alpha_{2}\right| \leq M} \sup _{1 / 2 \leq|\eta| \leq 2}|\eta|^{-r-\left|\alpha_{1}\right|}\left(\frac{2^{j} N}{\Lambda(t)}\right)^{\left|\alpha_{2}\right|}\left(\frac{2^{j} N}{\Lambda(t)}\right)^{-\left|\alpha_{2}\right|}|\eta|^{-\left|\alpha_{2}\right|}\left(\frac{\lambda(t)}{\Lambda(t)} \Xi(t)\right)^{-\left|\alpha_{2}\right|} \\
\leq & C 2^{j\left(\frac{n+1}{2}-r\right)} \Lambda(t)^{(r-n)} \sqrt{\lambda(t)}\left(\frac{\lambda(t)}{\Lambda(t)} \Xi(t)\right)^{-M},
\end{aligned}
$$

where again we used in the second estimate Lemma A.5.1 with $M>\frac{n+5}{2}$. Thus, we have

$$
\begin{aligned}
& \left\|\mathcal{F}^{-1}\left(\varphi_{\text {hyp }}^{s}(t, \xi) \phi_{j}(t,|\xi|) e^{ \pm i|\xi| \int_{0}^{t} \lambda(\tau) \omega(\tau) d \tau} \sqrt{\lambda(t)}|\xi|^{-r} \varphi\left(\Theta(t)|\xi| N^{-1}\right) g(t, \xi) \mathcal{F}(v)(\cdot)\right)\right\|_{L^{\infty}} \\
& \leq C 2^{j\left(\frac{n+1}{2}-r\right)} \Lambda(t)^{(r-n)} \sqrt{\lambda(t)}\left(\frac{\lambda(t)}{\Lambda(t)} \Xi(t)\right)^{-M}\|v(\cdot)\|_{L^{1}}
\end{aligned}
$$

$L^{2}-L^{2}$ estimate. Now we shall estimate $\tilde{I}_{j}$. We have

$$
\begin{aligned}
\tilde{I}_{j} & \leq C \sup _{1 / 2 \leq|\eta| \leq 2} \phi\left(\frac{\Theta(t)|\eta|}{\Lambda(t)}\right) \sqrt{\lambda(t)}\left(\frac{2^{j} N|\eta|}{\Lambda(t)}\right)^{-r}\left|\varphi\left(\frac{2^{j} \Theta(t)|\eta|}{\Lambda(t)}\right)\right|\left|g\left(t, \frac{2^{j} N \eta}{\Lambda(t)}\right)\right| \\
& \lesssim 2^{-j r} \sqrt{\lambda(t)} \Lambda(t)^{r}
\end{aligned}
$$


for $j \geq 0$. This implies immediately

$$
\begin{gathered}
\left\|\mathcal{F}^{-1}\left(\varphi_{\text {hyp }}^{s}(t, \xi) \phi_{j}(t,|\xi|) e^{ \pm i|\xi| \int_{0}^{t} \lambda(\tau) \omega(\tau) d \tau} \sqrt{\lambda(t)}|\xi|^{-r} \varphi\left(\Theta(t)|\xi| N^{-1}\right) g(t, \xi) \mathcal{F}(v)(\cdot)\right)\right\|_{L^{2}} \\
\lesssim \sqrt{\lambda(t)} 2^{-j r} \Lambda(t)^{r}\|v(\cdot)\|_{L^{2}} .
\end{gathered}
$$

$L^{p}-L^{q}$ estimates. After applying again an interpolation argument it follows

$$
\begin{aligned}
& \left\|\mathcal{F}^{-1}\left(\varphi_{\text {hyp }}^{s}(t, \xi) \phi_{j}(t,|\xi|) e^{ \pm i|\xi| \int_{0}^{t} \lambda(\tau) \omega(\tau) d \tau} \sqrt{\lambda(t)}|\xi|^{-r} \varphi\left(\Theta(t)|\xi| N^{-1}\right) g(t, \xi) \mathcal{F}(v)(\cdot)\right)\right\|_{L^{q}} \\
& \quad \lesssim 2^{j\left(\frac{n+1}{2}\left(\frac{1}{p}-\frac{1}{q}\right)-r\right)} \sqrt{\lambda(t)}(1+\Lambda(t))^{r-n\left(\frac{1}{p}-\frac{1}{q}\right)}\left(\frac{\lambda(t)}{\Lambda(t)} \Xi(t)\right)^{-M\left(\frac{1}{p}-\frac{1}{q}\right)}\|v(\cdot)\|_{L^{p}} .
\end{aligned}
$$

Therefore, after the choice $r=\frac{n+1}{2}\left(\frac{1}{p}-\frac{1}{q}\right)$ and using Brenner's lemma from Lemma A.4.3 for $p \in(1,2]$ we may conclude the following estimate:

$$
\begin{aligned}
\| \mathcal{F}^{-1} & \left(\varphi_{\text {hyp }}^{s}(t, \xi) e^{ \pm i|\xi| \int_{0}^{t} \lambda(\tau) \omega(\tau) d \tau} \sqrt{\lambda(t)}|\xi|^{-r} g(t, \xi) \mathcal{F}(v)\right) \|_{L^{q}} \\
& \lesssim \sqrt{\lambda(t)}(1+\Lambda(t))^{-\frac{n-1}{2}\left(\frac{1}{p}-\frac{1}{q}\right)}\left(\frac{\lambda(t)}{\Lambda(t)} \Xi(t)\right)^{-M\left(\frac{1}{p}-\frac{1}{q}\right)}\|v(\cdot)\|_{L^{p}}
\end{aligned}
$$

uniformly for all $j \geq 0$. From the last estimate it follows

$$
\begin{aligned}
& \left\|\mathcal{F}^{-1}\left(\varphi_{\text {hyp }}^{s}(t, \xi) e^{ \pm i|\xi| \int_{0}^{t} \lambda(\tau) \omega(\tau) d \tau} \sqrt{\lambda(t)}|\xi|^{-r} h(t, \xi) \mathcal{F}\left(\lambda(0) \omega(0)|D|^{r+1} u(0, \cdot)+|D|^{r} u_{t}(0, \cdot)\right)\right)\right\|_{L^{q}} \\
& \lesssim \sqrt{\lambda(t)}(1+\Lambda(t))^{-\frac{n-1}{2}\left(\frac{1}{p}-\frac{1}{q}\right)}\left(\frac{\lambda(t)}{\Lambda(t)} \Xi(t)\right)^{-M\left(\frac{1}{p}-\frac{1}{q}\right)}\left(\left\|u_{0}(\cdot)\right\|_{W_{p}^{r+1}}+\left\|u_{1}(\cdot)\right\|_{W_{p}^{r}}\right) .
\end{aligned}
$$

Summarizing, we may conclude

$$
\begin{aligned}
& \left\|\mathcal{F}^{-1}\left(\varphi_{\text {hyp }}^{s}(t, \xi) \hat{u}_{t}(t, \cdot)\right)\right\|_{L^{q}}+\left\|\mathcal{F}^{-1}\left(\varphi_{\text {hyp }}^{s}(t, \xi) \lambda(t)|\xi| \hat{u}(t, \cdot)\right)\right\|_{L^{q}} \\
& \quad \lesssim \sqrt{\lambda(t)}(1+\Lambda(t))^{-\frac{n-1}{2}\left(\frac{1}{p}-\frac{1}{q}\right)}\left(\frac{\lambda(t)}{\Lambda(t)} \Xi(t)\right)^{-M\left(\frac{1}{p}-\frac{1}{q}\right)}\left(\left\|u_{0}(\cdot)\right\|_{W_{p}^{r+1}}+\left\|u_{1}(\cdot)\right\|_{W_{p}^{r}}\right) .
\end{aligned}
$$

Finally, we combine and compare the estimates from the four different parts of the extended phase space, i.e., the estimates from (5.3.1), (5.3.2), (5.3.4) and (5.3.5), respectively. Then, we see that from the point of view of decay the estimate in the pseudo-differential zone is better than those in the hyperbolic zone and oscillation subzone. The desired regularity of the data comes from the estimate for large frequencies in the hyperbolic zone. However, the desired estimate from the point of view of decay estimates comes from the estimates in the oscillation subzone and for small frequencies in the hyperbolic zone. In this way the proof is completed.

Remark 5.3.1. If we suppose that the function $\omega=\omega(t)$ has only very slow oscillations, as a representative we can put $\omega(t) \equiv 1$, then from (A4) we have $\Theta(t) \equiv \Lambda(t)$ and $\Xi(t) \equiv \frac{\Lambda(t)}{\lambda(t)}$. Therefore, we arrive at the following estimate from Theorem 5.3.1:

$$
\left\|u_{t}(t, \cdot), \lambda(t) \nabla_{x} u(t, \cdot)\right\|_{L^{q}} \lesssim \sqrt{\lambda(t)} \Lambda(t)^{-\frac{n-1}{2}\left(\frac{1}{p}-\frac{1}{q}\right)}\left(\left\|u_{0}\right\|_{W_{p}^{N_{p}+1}}+\left\|u_{1}\right\|_{W_{p}^{N_{p}}}\right)
$$

with regularity $N_{p}=n\left(\frac{1}{p}-\frac{1}{q}\right), p \in(1,2]$ and $\frac{1}{p}+\frac{1}{q}=1$.

Remark 5.3.2. The estimate (5.3.6) corresponds to the estimate in [33]. More details about $L^{p}-L^{q}$ decay estimates can be found in the paper [32].

Remark 5.3.3. If we choose in Theorem 5.3.1 $p=q=2$ and employ the stabilization condition

$$
\int_{0}^{t} \lambda(\tau)|\omega(\tau)-1| d \tau \lesssim \Theta(t)
$$

then our result corresponds to the $L^{2}-L^{2}$ estimate which is given in the paper [19]. Because, by using the stabilization condition we find that $\exp \left(\frac{\Lambda(t)}{\Theta(t)}\right)$ is estimated by a positive constant. 


\section{Further research topics}

In this short final chapter we give some problems arising in connection with the content of this thesis.

\subsection{Optimality}

We consider the Cauchy problem

$$
\begin{cases}u_{t t}-a^{2}(t) \Delta u=0, & (t, x) \in[0, \infty) \times \mathbb{R}^{n}, \\ u(0, x)=u_{0}(x), \quad u_{t}(0, x)=u_{1}(x), & x \in \mathbb{R}^{n} .\end{cases}
$$

In the paper [16], the author proved (GECL) to (6.1.1) provided that the following conditions to the coefficient $a=a(t)$ are satisfied:

$$
\begin{gathered}
0<a_{1} \leq a(t) \leq a_{2}, \\
\left|a^{(k)}(t)\right| \leq C_{k}(1+t)^{-k \alpha}, \quad k=1,2, \cdots, M, \\
\int_{0}^{t}\left|a(\tau)-a_{\infty}\right| d \tau \leq C(1+t)^{\beta},
\end{gathered}
$$

for some real $a_{\infty}$ and $\beta \in(0,1)$ with $\alpha \geq \beta+\frac{1-\beta}{M}$. Here (6.1.4) is a so-called stabilization condition and by this condition one can get some benefit of higher order regularity of $a=a(t)$.

Necessities of $\mathcal{C}^{M}$ property (6.1.3) and stabilization condition (6.1.4) are still interesting open problem. That is, there are no results to prove the sharpness of the assumptions and there are no counterexamples that show that these conditions really appear.

Let us give also a remark about condition (6.1.3). The control of the first derivative allows us to diagonalize the Fourier transformed system once. This yields a diagonal part and a remainder part. But this remainder part is too "bad" to be of any help. The advantage of the $\mathcal{C}^{2}$ theory is that we can diagonalize twice so that we get a remainder which is better in some hierarchies of symbol classes. In this way we get an advantage of $\mathcal{C}^{M}$ theory together with stabilization condition (6.1.4).

\subsection{Wave models with decreasing time dependent propagation speed}

Let us consider the following Cauchy problem:

$$
\begin{cases}u_{t t}-\lambda^{2}(t) \omega^{2}(t) \Delta u=0, & (t, x) \in[0, \infty) \times \mathbb{R}^{n}, \\ u(0, x)=u_{0}(x), \quad u_{t}(0, x)=u_{1}(x), & x \in \mathbb{R}^{n},\end{cases}
$$

with a decreasing time-dependent shape function $\lambda=\lambda(t)$ in the speed of propagation and a bounded positive oscillating function $\omega=\omega(t)$.

In several papers (see $[34,35,36,37,16]$ ) the authors studied special properties of Sobolev solutions to the Cauchy problem (6.2.1) with an increasing shape function $\lambda=\lambda(t)$.

An interesting research problem is to study $L^{p}-L^{q}$ decay estimates or generalized energy conservation law (GECL) under some suitable conditions for the time-dependent functions $\lambda, \omega$ and a stabilization condition which is related to the decreasing behavior of $\lambda$. 
As an example for this kind of model we can choose the anti-de Sitter model

$$
u_{t t}-e^{2 t} \Delta u=0 .
$$

If we inverse the time variable, $t \rightarrow-t$, it becomes

$$
u_{t t}-e^{-2 t} \Delta u=0 .
$$

This equation is called de Sitter model, but we consider it for positive time $t$. Hence, the Cauchy problem (6.2.1) may lead to study some new problems: de Sitter model with very fast oscillations.

\subsection{Damped wave models with different oscillation functions}

We consider the following Cauchy problem to the damped wave equation with time-dependent propagation speed and time-dependent damping:

$$
\begin{cases}u_{t t}-\lambda^{2}(t) \omega_{1}^{2}(t) \Delta u+\rho(t) \omega_{2}(t) u_{t}=f(u), & (t, x) \in[0, \infty) \times \mathbb{R}^{n} \\ u(0, x)=u_{0}(x), \quad u_{t}(0, x)=u_{1}(x), & x \in \mathbb{R}^{n},\end{cases}
$$

where the time-dependent functions $\lambda=\lambda(t), \rho=\rho(t), \omega_{1}=\omega_{1}(t)$ and $\omega_{2}=\omega_{2}(t)$ are smooth and strictly positive functions. In particular, $\lambda$ is a monotonously increasing nontrivial shape function in the propagation speed, $\rho$ is a nontrivial shape function in the damping term. The crucial point here is that $\omega_{1} \neq \omega_{2}$. Then, one can arrive at a generalization of a research problem to this thesis. Firstly one needs to study higher order energy estimates for Sobolev solutions to the Cauchy problem (6.3.1) with vanishing right-hand side.

\subsection{Weakly coupled systems of semilinear damped wave models}

Let us consider the following Cauchy problems for weakly coupled systems of semilinear damped wave models with time-dependent propagation speed and time-dependent damping:

$$
\begin{cases}u_{t t}-a^{2}(t) \Delta u+b(t) u_{t}=|v|^{p}, & v_{t t}-a^{2}(t) \Delta v+b(t) v_{t}=|u|^{q}, \\ u(0, x)=u_{0}(x), \quad u_{t}(0, x)=u_{1}(x), & v(0, x)=v_{0}(x), v_{t}(0, x)=v_{1}(x),\end{cases}
$$

where $a=a(t)>0$ is an increasing propagation speed and $b(t) u_{t}$ and $b(t) v_{t}$ are effective damping terms in the sense of [2] and [48]. Moreover, the time-dependent functions $a=a(t)$ and $b=b(t)$ have very slow oscillations only.

The corresponding linear model with vanishing right-hand sides is

$$
\left\{\begin{array}{l}
u_{t t}-a^{2}(t) \Delta u+b(t) u_{t}=0, \\
u(0, x)=u_{0}(x), u_{t}(0, x)=u_{1}(x) .
\end{array}\right.
$$

The first motivation of this problem is to get sharp $\left(L^{m} \cap L^{2}\right)-L^{2}$ estimates with $m \in[1,2)$ to the Sobolev solutions of the Cauchy problem (6.4.2) which has been already derived in [4]. Using these estimates plays a fundamental role in the treatment of corresponding semi-linear models (6.4.1). For this reason, the main motivation is to prove the global (in time) existence of small data Sobolev solutions to (6.4.1) by applying the obtained estimates to the linear Cauchy problem (6.4.2) and some developed tools from Harmonic Analysis (see, Section A.7 in Appendix). 


\section{A. Basic tools}

\section{A.1. Basic inequalities}

Lemma A.1.1 (Gronwall's inequality). Let $f$ and $h$ be continuous and nonnegative functions defined on $I=[a, b]$ and let $g$ be a continuous, positive and nondecreasing function defined on I. Then, the inequality

$$
f(t) \leq g(t)+\int_{a}^{t} h(r) f(r) d r, \quad t \in J
$$

implies that

$$
f(t) \leq g(t) \exp \left(\int_{a}^{t} h(r) d r\right), \quad t \in J
$$

For the proof see [26].

Proposition A.1.2 (Classical Gagliardo-Nirenberg inequality). Let $j, k \in \mathbb{N}$ with $j<k$ and $u \in \mathcal{C}_{0}^{k}\left(\mathbb{R}^{n}\right)$. Let us consider $\frac{j}{k} \leq \theta \leq 1$ and $1 \leq p, q, r \leq \infty$ such that

$$
j-\frac{n}{q}=\left(k-\frac{n}{r}\right) \theta-\frac{n}{p}(1-\theta) .
$$

Then, it holds

$$
\left\|\nabla^{j} u\right\|_{L^{q}} \lesssim\left\|\nabla^{k} u\right\|_{L^{r}}^{\theta}\|u\|_{L^{p}}^{1-\theta}
$$

provided that $\left(k-\frac{n}{r}\right)-j \notin \mathbb{N}$, that is, $\frac{n}{r}>k-j$ or $\frac{n}{r} \notin \mathbb{N}$. If $\left(k-\frac{n}{r}\right)-j \in \mathbb{N}$, then, (A.1.1) holds provided that $\frac{j}{k} \leq \theta<1$.

In the special case $j=0, k=1$ and $r=p=2$, applying a density argument, we obtain the following result.

Corollary A.1.3. Let us consider a finite $q \geq 2$ such that $q \leq \frac{2 n}{n-2}$ if $n \geq 3$. Then, it holds

$$
\|u\|_{L^{q}} \lesssim\|\nabla u\|_{L^{2}}^{\theta(q)}\|u\|_{L^{2}}^{1-\theta(q)}
$$

for any $u \in H^{1}\left(\mathbb{R}^{n}\right)$, where $\theta(q)=n\left(\frac{1}{2}-\frac{1}{q}\right)$.

For the proof see [12].

\section{A.2. The Peano-Baker formula}

Let us consider the following first order systems of ordinary differential equations:

$$
D_{t} U=A(t) U, \quad U(0)=U_{0} \in \mathbb{C}^{n}
$$

with $t \in[0, \infty)$. In order to solve this Cauchy problem, we find the fundamental solution $E=E(t, s)$ to the following matrix valued system:

$$
\frac{d}{d t} E(t, s)=A(t) E(t, s), \quad E(s, s)=I .
$$

Hence, it holds $U(t)=E(t, 0) U_{0}$. 
Theorem A.2.1. Let $A=A(t) \in L_{l o c}^{1}\left(\mathbb{R}, \mathbb{C}^{n \times n}\right)$. Then, the fundamental solution $E=E(t, s)$ to (A.2.1) is given by the Peano-Baker formula (or the matrizant representation)

$$
E(t, s)=I+\sum_{k=1}^{\infty} \int_{s}^{t} A\left(t_{1}\right) \int_{s}^{t_{1}} A\left(t_{2}\right) \cdots \int_{s}^{t_{k-1}} A\left(t_{k}\right) d t_{k} \cdots d t_{2} d t_{1} .
$$

The proof follows by differentiating the series term by term. To prove the convergence of the series and its formal derivative one uses the domination by the exponential series following next statement.

Proposition A.2.2. Assume $A=A(t) \in L_{l o c}^{1}(\mathbb{R})$. Then

$$
\int_{s}^{t}\left|A\left(t_{1}\right)\right| \int_{s}^{t_{1}}\left|A\left(t_{2}\right)\right| \cdots \int_{s}^{t_{k-1}}\left|A\left(t_{k}\right)\right| d t_{k} \cdots d t_{1} \leq \frac{1}{k !}\left(\int_{s}^{t}|A(\tau)| d \tau\right)^{k}
$$

for all $k \in \mathbb{N}$.

The proof follows by induction over $k$.

In several applications we need not only estimates for the fundamental solution, but also statements about its asymptotic behavior and invertibility. It is convenient to use the Theorem of Liouville in the following form.

Theorem A.2.3. Let $A=A(t) \in L_{\text {loc }}^{1}\left(\mathbb{R}, \mathbb{C}^{n \times n}\right)$. Then the fundamental solution $E=E(t, s)$ satisfies

$$
\operatorname{det} E(t, s)=\operatorname{det} E(s, s) \exp \left(\int_{s}^{t} \operatorname{tr} A(\tau) d \tau\right) .
$$

\section{A.3. Faà di Bruno's formula}

In this section we will recall the formula for derivatives of higher order of a compound function.

Proposition A.3.1. If functions of a real variable $f, g$ are $n$-times differentiable, then

$$
(f \circ g)^{(n)}=\sum_{k=0}^{n} \sum_{\begin{array}{c}
k_{1}+k_{2}+\cdots+k_{n}=k \\
k_{1}+2 k_{2}+\cdots+n k_{n}=n
\end{array}} \frac{n !}{k_{1} ! k_{2} ! \cdots k_{n} !}(f \circ g)^{(k)} \prod_{\ell=1}^{n}\left(\frac{g^{(\ell)}}{\ell !}\right)^{k_{\ell}} .
$$

For a proof of the previous formula one can see [39].

In several variables we have the following result proved in [15].

Proposition A.3.2. If a real-valued function $g=g\left(x_{1}, \cdots, x_{n}\right)$ and a function of real variable $f$ are $n$-times differentiable, then the following identity holds regardless of whether the $n$ variables are all distinct, or all identical, or partitioned into several distinguishable classes of indistinguishable variables:

$$
\frac{\partial^{n}}{\partial x_{1} \cdots \partial x_{n}} f(g)=\sum_{\pi \in \mathcal{P}} f^{(|\pi|)}(g) \prod_{B \in \pi} \frac{\partial^{|B|} g}{\prod_{j \in B} \partial x_{j}},
$$

where

- $\pi$ runs through the set $\mathcal{P}$ of all partitions of the set $\{1, \cdots, n\}$,

- $B \in \pi$ means that $B$ runs through the list of all blocks of the partition $\pi$,

- $|\pi|$ denotes the number of blocks in the partition $\pi$,

- $|B|$ denotes the cardinality of the set $B$.

Let us give some generalizations of Faà di Bruno's formula for a composite function with a vector-valued argument, see [25]. 
Theorem A.3.3. If $f$ and $t$ are scalars, $\boldsymbol{x}(t)=\left[x_{1}(t), x_{2}(t), \cdots, x_{r}(t)\right]^{T}$ is a r-vector and $f(\boldsymbol{x}(t))$ is a composite function for which all the necessary derivatives are defined, then

$D^{n} f(\boldsymbol{x}(t))=\sum_{0} \sum_{1} \cdots \sum_{n} \frac{n !}{\prod_{i=1}^{n}(i !)^{k_{i}} \prod_{i=1}^{n} \prod_{j=1}^{r} q_{i j} !} \frac{\partial^{k} f}{\partial x_{1}^{p_{1}} \partial x_{2}^{p_{2}} \cdots \partial x_{r}^{p_{r}}} \prod_{i=1}^{n}\left(x_{1}^{i}\right)^{q_{i 1}}\left(x_{2}^{i}\right)^{q_{i 2}} \cdots\left(x_{r}^{i}\right)^{q_{i r}}$,

where the respective sums are taken over all non-negative integer solution of the Diophantine equation as follows:

$$
\begin{gathered}
\sum_{0} \rightarrow k_{1}+2 k_{2}+\cdots+n k_{n}=n, \\
\sum_{1} \rightarrow q_{11}+q_{12}+\cdots+q_{1 r}=k_{1}, \\
\vdots \\
\sum_{n} \rightarrow q_{n 1}+q_{n 2}+\cdots+q_{n r}=k_{n},
\end{gathered}
$$

and $D=\frac{d}{d t}$ is the differential operator, $p_{j}$ is the order of the partial derivative with respect to $x_{j}$, and $k$ is the order of the partial derivative, more precisely,

$$
\begin{aligned}
p_{j} & =q_{1 j}+q_{2 j}+\cdots+q_{n j}, \quad j=1,2, \cdots, r, \\
k & =p_{1}+p_{2}+\cdots+p_{r}=k_{1}+k_{2}+\cdots+k_{n} .
\end{aligned}
$$

\section{A.4. Some results from Interpolation Theory}

In order to handle with $L^{p}-L^{q}$ estimates we state the important Riesz-Thorin interpolation theorem.

Theorem A.4.1 (Riesz-Thorin interpolation theorem). Let $1 \leq p_{0}, p_{1}, q_{0}, q_{1} \leq \infty$. If $T$ is a linear continuous operator in the space $\mathcal{L}\left(L^{p_{0}} \mapsto L^{q_{0}}\right) \cap \mathcal{L}\left(L^{p_{1}} \mapsto L^{q_{1}}\right)$, then

$$
T \in \mathcal{L}\left(L^{p_{\theta}} \mapsto L^{q_{\theta}}\right)
$$

for any $\theta \in(0,1)$, where

$$
\frac{1}{p_{\theta}}=\frac{1-\theta}{p_{0}}+\frac{\theta}{p_{1}} \quad \text { and } \quad \frac{1}{q_{\theta}}=\frac{1-\theta}{q_{0}}+\frac{\theta}{q_{1}} .
$$

Moreover, the following norm estimate holds:

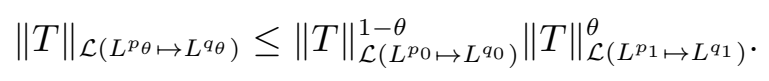

For the proof see [31, Appendix A].

In order to handle with $L^{1}-L^{\infty}$ and $L^{2}-L^{2}$ estimates, we have the following lemma.

Lemma A.4.2. Let $a \in L^{1}$. Then, we have

- If $\left\|\mathcal{F}^{-1} a\right\|_{L^{\infty}} \leq C_{0}$, then $\left\|\mathcal{F}^{-1}(a \mathcal{F} v)\right\|_{L^{\infty}} \leq C_{0}\|v\|_{L^{1}}$.

- If $\|a\|_{L^{\infty}} \leq C_{1}$, then $\left\|\mathcal{F}^{-1}(a \mathcal{F} v)\right\|_{L^{2}} \leq C_{1}\|v\|_{L^{2}}$.

- If $\left\|\mathcal{F}^{-1} a\right\|_{L^{\infty}} \leq C_{0}$ and $\|a\|_{L^{\infty}} \leq C_{1}$ are both satisfied, then

$$
\left\|\mathcal{F}^{-1}(a \mathcal{F} v)\right\|_{L^{p^{\prime}}} \leq C_{0}^{1-\theta} C_{1}^{\theta}\|v\|_{L^{p}}
$$

where $p \in[1,2], \frac{1}{p}+\frac{1}{p^{\prime}}=1$ and $\theta=\frac{2}{p^{\prime}}$. 
The proof of this lemma can be found in [5].

Let $\left\{\phi_{j}\right\}_{j \in \mathcal{Z}}$ denote a dyadic decomposition with $\phi_{j}(y)=\phi\left(2^{-j} y\right)$, where $\phi \in \mathcal{C}_{0}^{\infty}$ and $\operatorname{supp} \phi \subseteq\left[\frac{1}{2}, 2\right]$. By using Lemma A.4.2 and some embedding theorems concerning Besov spaces, one can prove the following result (see [5]).

Lemma A.4.3. Let $a \in L^{\infty}\left(\mathbb{R}^{n}\right)$ and assume that

$$
\left\|\mathcal{F}^{-1}\left(a \phi_{j} \mathcal{F} v\right)\right\|_{L^{q}} \leq C\|v\|_{L^{p}}
$$

uniformly for all $j \in \mathbb{Z}$ with $1<p \leq 2$ and $p q=p+q$. Then, there exists a constant $A$ independent of the function a such that

$$
\left\|\mathcal{F}^{-1}(a \mathcal{F} v)\right\|_{L^{q}} \leq A C\|v\|_{L^{p}} .
$$

\section{A.5. Littman type lemma}

If we want to obtain $L^{1}-L^{\infty}$ estimates for solutions of a given linear equation, then the following result might be useful.

Lemma A.5.1 (A Littman type lemma). We assume that $K=K(t)$ is a real-valued function and the amplitude function $d=d(t, \xi)$ is supposed to belong to $\mathcal{C}_{0}^{\infty}\left(\mathbb{R}_{\xi}^{n}\right)$. Then, the following $L^{\infty}-L^{\infty}$ estimate holds:

$$
\left\|\mathcal{F}^{-1}\left(e^{i K(t)|\xi|} d(t, \xi)\right)\right\|_{L^{\infty}} \leq C(1+K(t))^{-\frac{n-1}{2}} \sum_{|\alpha| \leq G}\left\|D_{\xi}^{\alpha} d(t, \xi)\right\|_{L^{\infty}},
$$

where $G>\frac{n+3}{2}$ and the constant $C$ is independent of $t$ and $\xi$.

The proof is based on the method of the stationary phase and can be found in [36] or [11, Section 16.3].

\section{A.6. Hardy-Littlewood inequality}

By $L_{p}^{q}$ we denote the space of tempered distributions $f \in \mathcal{S}^{\prime}$ satisfying the estimate

$$
\|f * u\|_{L^{q}} \leq C\|u\|_{L^{p}}
$$

for all $u \in \mathcal{S}$ with a constant $C$ which is independent of $u$. Thus, $L_{p}^{q}$ is isomorphic to a closed subspace of the Banach space of all bounded linear mappings of $L^{p}$ into $L^{q}$. The multiplier space $M_{p}^{q}$ is defined as the set of all Fourier transforms $\mathcal{F}(f)$ of distributions $f \in L_{p}^{q}$. The elements $\mathcal{F}(f) \in M_{p}^{q}$ are called multipliers of $(p, q)$. Thus, the Hardy-Littlewood inequaltiy is given as follows.

Theorem A.6.1 (Hardy-Littlewood inequality). Let $f$ be a measurable function. Moreover, we suppose the following relation with suitable positive constants $C, b \in(1, \infty)$ and all positive $\tau:$

$$
\text { meas }\left\{\xi \in \mathbb{R}^{n}:|f(\xi)| \geq \tau\right\} \leq C \tau^{-b}
$$

with $1<b<\infty$. Then, $f \in M_{p}^{q}$ if $1<p \leq 2 \leq q<\infty$ and $\frac{1}{p}-\frac{1}{q}=\frac{1}{b}$.

The proof can be found in [20, Theorem 1.11].

\section{A.7. New tools from Harmonic Analysis}

We present some results from the theory of Harmonic Analysis which are helpful and important tools for proving results on global (in time) existence of small data solutions for semilinear models with power nonlinearities. In particular, these tools allow to estimate power nonlinearities in homogeneous Sobolev spaces. 


\section{A.7.1. Bessel and Riesz potential spaces}

Let $s \in \mathbb{R}$ and $1<p<\infty$. Then,

$$
\begin{aligned}
& H_{p}^{s}\left(\mathbb{R}^{n}\right)=\left\{u \in \mathcal{S}^{\prime}\left(\mathbb{R}^{n}\right):\left\|\langle D\rangle^{s} u\right\|_{L^{p}}=\|u\|_{H_{p}^{s}}<\infty\right\}, \\
& \dot{H}_{p}^{s}\left(\mathbb{R}^{n}\right)=\left\{u \in \mathcal{Z}^{\prime}\left(\mathbb{R}^{n}\right):\left\||D|^{s} u\right\|_{L^{p}}=\|u\|_{\dot{H}_{p}^{s}}<\infty\right\}
\end{aligned}
$$

are called Bessel and Riesz potential spaces, respectively. If $p=2$, then we use the notations $H^{s}\left(\mathbb{R}^{n}\right)$ and $\dot{H}^{s}\left(\mathbb{R}^{n}\right)$, respectively. In the definition of the Riesz potential spaces we use the space of distributions $\mathcal{Z}^{\prime}\left(\mathbb{R}^{n}\right)$. This space of distributions can be identified with the factor space $\mathcal{S}^{\prime} / \mathcal{P}$, where $\mathcal{P}$ denotes the set of all polynomials.

For Bessel potential spaces we have the following embedding theorem.

Theorem A.7.1. Let $s>0$ and $1<p<\infty$. Then, the following embedding relations hold:

i) if $s p<n$, then $H_{p}^{s}\left(\mathbb{R}^{n}\right) \hookrightarrow L^{q}\left(\mathbb{R}^{n}\right)$ for any $p \leq q \leq p^{*}$, where $\frac{1}{p^{*}}=\frac{1}{p}-\frac{s}{n}$;

ii) if $s p=n$, then $H_{p}^{s}\left(\mathbb{R}^{n}\right) \hookrightarrow L_{l o c}^{q}\left(\mathbb{R}^{n}\right)$ for any $p \leq q<\infty$;

iii) if $s p>n$, then $H_{p}^{s}\left(\mathbb{R}^{n}\right) \hookrightarrow \mathcal{C}_{b}\left(\mathbb{R}^{n}\right)$.

\section{A.7.2. Fractional Gagliardo-Nirenberg inequality}

The first inequality is the fractional Gagliardo-Nirenberg inequality which is a generalization of the classical Gagliardo-Nirenberg inequality to the case of Sobolev spaces of fractional order.

Proposition A.7.2. Let $1<p, p_{0}, p_{1}<\infty$ and $s \in[0, \sigma)$. Then, it holds the following fractional Gagliardo-Nirenberg inequality for all $u \in L^{p_{0}}\left(\mathbb{R}^{n}\right) \cap \dot{H}_{p_{1}}^{\sigma}\left(\mathbb{R}^{n}\right)$ :

$$
\|u\|_{\dot{H}_{p}^{s}} \lesssim\|u\|_{L^{p_{0}}}^{1-\theta}\|u\|_{\dot{H}_{p_{1}}^{\sigma}}^{\theta}
$$

where $\theta=\theta_{s, \sigma}\left(p, p_{0}, p_{1}\right)=\frac{\frac{1}{p_{0}}-\frac{1}{p}+\frac{s}{n}}{\frac{1}{p_{0}}-\frac{1}{p_{1}}+\frac{\sigma}{n}}$ and $\frac{s}{\sigma} \leq \theta \leq 1$.

For the proof one can see [14].

Corollary A.7.3. Let $1<p, m<\infty, \sigma>0$ and $s \in[0, \sigma)$. Then, we have the following inequality for all $u \in H^{\sigma}\left(\mathbb{R}^{n}\right)$ :

$$
\left\||D|^{s} u\right\|_{L^{p}} \lesssim\|u\|_{L^{m}}^{1-\theta_{s, \sigma}(p, m)}\left\||D|^{\sigma} u\right\|_{L^{m}}^{\theta_{s, \sigma}(p, m)},
$$

where $\theta_{s, \sigma}(p, m)=\frac{n}{\sigma}\left(\frac{1}{m}-\frac{1}{p}+\frac{s}{n}\right)$ and $\frac{s}{\sigma} \leq \theta_{s, \sigma}(p, m) \leq 1$.

\section{A.7.3. Fractional Leibniz rule}

Proposition A.7.4. Let us assume $s>0,1 \leq r \leq \infty$ and $1<p_{1}, p_{2}, q_{1}, q_{2}<\infty$ satisfying the relation

$$
\frac{1}{r}=\frac{1}{p_{1}}+\frac{1}{p_{2}}=\frac{1}{q_{1}}+\frac{1}{q_{2}} .
$$

Then, the following fractional Leibniz rule holds:

$$
\left\||D|^{s}(u v)\right\|_{L^{r}} \lesssim\left\||D|^{s} u\right\|_{L^{p_{1}}}\|v\|_{L^{p_{2}}}+\|u\|_{L^{q_{1}}}\left\||D|^{s} v\right\|_{L^{q_{2}}}
$$

for any $u \in \dot{H}_{p_{1}}^{s}\left(\mathbb{R}^{n}\right) \cap L^{q_{1}}\left(\mathbb{R}^{n}\right)$ and $v \in \dot{H}_{q_{2}}^{s}\left(\mathbb{R}^{n}\right) \cap L^{p_{2}}\left(\mathbb{R}^{n}\right)$.

For the proof see [13]. 


\section{A.7.4. Fractional chain rule}

Proposition A.7.5. Let us choose $s \in(0,1), 1<r, r_{1}, r_{2}<\infty$ and a $\mathcal{C}^{1}$ function $F$ satisfying for any $\tau \in[0,1]$ and $u, v \in \mathbb{R}$ the inequality

$$
\left|F^{\prime}(\tau u+(1-\tau) v)\right| \leq \mu(\tau)(G(u)+G(v)),
$$

for some continuous and nonnegative function $G$ and some nonnegative function $\mu \in L^{1}([0,1])$.

Under these assumptions the following estimate is true:

$$
\|F(u)\|_{\dot{H}_{r}^{s}} \lesssim\|G(u)\|_{L^{r_{1}}}\|u\|_{\dot{H}_{r_{2}}^{s}}
$$

for any $u \in \dot{H}_{r_{2}}^{s}\left(\mathbb{R}^{n}\right)$ such that $G(u) \in L^{r_{1}}\left(\mathbb{R}^{n}\right)$, provided that

$$
\frac{1}{r}=\frac{1}{r_{1}}+\frac{1}{r_{2}}
$$
[27].

For the proof of this result one can see [6] or the proof in a slightly modified version in

In particular, we can apply Proposition A.7.5 for $F(u)=|u|^{p}$ or $F(u)= \pm u|u|^{p-1}$. After choosing $G(u)=\left|F^{\prime}(u)\right|$ and $\mu$ is a positive constant we obtain the following result.

Corollary A.7.6. Let $F(u)=|u|^{p}$ or $F(u)= \pm u|u|^{p-1}$ for $p>1, s \in(0,1)$ and $r, r_{1}, r_{2} \in$ $(1, \infty)$. Then,

$$
\|F(u)\|_{\dot{H}_{r}^{s}} \lesssim\|u\|_{L^{r_{1}}}^{p-1}\|u\|_{\dot{H}_{r_{2}}^{s}}
$$

for any $u \in L^{r_{1}} \cap H_{r_{2}}^{s}$, provided that

$$
\frac{1}{r}=\frac{p-1}{r_{1}}+\frac{1}{r_{2}}
$$

The last corollary can be found in [6] and [40] which is true only for $s \in(0,1)$. But we need to estimate the term $\|F(u)\|_{\dot{H}_{r}^{s}}$ for larger $s$. For this reason we recall the following result introduced and proved in [28].

Proposition A.7.7. Let us choose $s>0, p>\lceil s\rceil$ and $1<r, r_{1}, r_{2}<\infty$ satisfying

$$
\frac{1}{r}=\frac{p-1}{r_{1}}+\frac{1}{r_{2}} .
$$

Let us denote by $F(u)$ one of the functions $|u|^{p}, \pm|u|^{p-1} u$.

Then, it holds the following fractional chain rule:

$$
\left\||D|^{s} F(u)\right\|_{L^{r}} \lesssim\|u\|_{L^{r_{1}}}^{p-1}\left\||D|^{s} u\right\|_{L^{r_{2}}}
$$

for any $u \in L^{r_{1}}\left(\mathbb{R}^{n}\right) \cap \dot{H}_{r_{2}}^{s}\left(\mathbb{R}^{n}\right)$.

\section{A.7.5. Fractional homogeneous Sobolev embeddings}

Sometimes one can apply in proofs for global existence results for semilinear Cauchy problems, instead of the fractional Gagliardo-Nirenberg inequality the embedding of a homogeneous fractional Sobolev space with suitable order $\dot{H}^{\kappa}$ in $L^{q}$. Hence, we apply the following result.

Proposition A.7.8. Let $q \geq 2$ and $\kappa=n\left(\frac{1}{2}-\frac{1}{q}\right)$. Then, it holds the fractional Sobolev embedding

$$
\dot{H}^{\kappa}\left(\mathbb{R}^{n}\right) \hookrightarrow L^{q}\left(\mathbb{R}^{n}\right) .
$$

Therefore, there exists a constant $C=C(n, q)>0$ such that

$$
\|u\|_{L^{q}} \leq C\|u\|_{\dot{H}^{\kappa}}
$$

for any $u \in \dot{H}^{\kappa}\left(\mathbb{R}^{n}\right)$. 


\section{A.7.6. Fractional powers}

Sobolev embedding is very useful in estimates for $\left\||u|^{p}\right\|_{\dot{H}_{r}^{s}}$, where $s>\frac{n}{r}$. We apply a result from [38] for fractional powers.

Proposition A.7.9. Let $p>1,1<r<\infty$ and $u \in H_{r}^{s}\left(\mathbb{R}^{n}\right)$, where $s \in\left(\frac{n}{r}, p\right)$. Then, the following estimates hold:

$$
\begin{aligned}
\left\||u|^{p}\right\|_{H_{r}^{s}} & \lesssim\|u\|_{H_{r}^{s}}\|u\|_{L^{\infty}}^{p-1} \\
\left\|u|u|^{p-1}\right\|_{H_{r}^{s}} & \lesssim\|u\|_{H_{r}^{s}}\|u\|_{L^{\infty}}^{p-1}
\end{aligned}
$$

for any $u \in H_{r}^{s}\left(\mathbb{R}^{n}\right) \cap L^{\infty}\left(\mathbb{R}^{n}\right)$.

We can derive the following corollary from Proposition A.7.9.

Corollary A.7.10. Let $p>1,1<r<\infty$ and $u \in H_{r}^{s}$, where $s \in\left(\frac{n}{r}, p\right)$. Then, the following estimates hold:

$$
\begin{aligned}
\left\||u|^{p}\right\|_{\dot{H}_{r}^{s}} & \lesssim\|u\|_{\dot{H}_{r}^{s}}\|u\|_{L^{\infty}}^{p-1}, \\
\left\|u|u|^{p-1}\right\|_{\dot{H}_{r}^{s}} & \lesssim\|u\|_{\dot{H}_{r}^{s}}\|u\|_{L^{\infty}}^{p-1} .
\end{aligned}
$$

for any $u \in \dot{H}_{r}^{s}\left(\mathbb{R}^{n}\right) \cap L^{\infty}\left(\mathbb{R}^{n}\right)$.

For the proof see [11] or [38].

Proposition A.7.11. Let $r \in(1, \infty)$ and $s>0$. Then, it holds the following inequality:

$$
\|u v\|_{H_{r}^{s}} \lesssim\|u\|_{H_{r}^{s}}\|v\|_{L^{\infty}}+\|u\|_{L^{\infty}}\|v\|_{H_{r}^{s}}
$$

for any $u, v \in H_{r}^{s}\left(\mathbb{R}^{n}\right) \cap L^{\infty}\left(\mathbb{R}^{n}\right)$.

The proof can be found in [38].

Now, let us state the corresponding inequality in the homogeneous space $\dot{H}_{r}^{s}\left(\mathbb{R}^{n}\right)$.

Corollary A.7.12. Let $r \in(1, \infty)$ and $s>0$. Then, it holds the following inequality:

$$
\|u v\|_{\dot{H}_{r}^{s}} \lesssim\|u\|_{\dot{H}_{r}^{s}}\|v\|_{L^{\infty}}+\|u\|_{L^{\infty}}\|v\|_{\dot{H}_{r}^{s}}
$$

for any $u, v \in \dot{H}_{r}^{s}\left(\mathbb{R}^{n}\right) \cap L^{\infty}\left(\mathbb{R}^{n}\right)$.

Proposition A.7.13. Let $0<2 s^{*}<n<2 s$. Then, for any function $u \in \dot{H}^{s^{*}}\left(\mathbb{R}^{n}\right) \cap \dot{H}^{s}\left(\mathbb{R}^{n}\right)$ it holds the following inequality:

$$
\|u\|_{L^{\infty}} \lesssim\|u\|_{\dot{H}^{s^{*}}}+\|u\|_{\dot{H}^{s}} .
$$

The following proof taken from the paper [9].

Proof. Let us recall the following Sobolev's embeddings:

$$
\|u\|_{L^{\infty}} \lesssim\|u\|_{H_{q}^{\alpha}} \text { for } \quad \alpha q>n
$$

and

$$
\|u\|_{L^{\frac{2 n}{n-2 s^{*}}}} \lesssim\left\||D|^{s^{*}} u\right\|_{L^{2}} \quad \text { for } \quad 0<s^{*}<\frac{n}{2} .
$$

If we fix $q=\frac{2 n}{n-2 s^{*}}$ and $\alpha=s-s^{*}$, then we get

$$
\begin{aligned}
\|u\|_{L^{\infty}} & \lesssim\|u\|_{L^{\frac{2 n}{n-2 s^{*}}}}+\left\||D|^{\alpha} u\right\|_{L^{\frac{2 n}{n-s^{*}}}} \\
& \lesssim\left\||D|^{s^{*}} u\right\|_{L^{2}}+\left\||D|^{s+s^{*}} u\right\|_{L^{2}} .
\end{aligned}
$$

This completes the proof. 



\section{B. List of symbols and abbreviations}

\section{Symbols used throughout the thesis}

$|\cdot|$

$\langle\cdot\rangle$

$\lceil\cdot\rceil$

$[\cdot]_{+}$

$[\cdot, \cdot]$

$\operatorname{Re} z$

$\operatorname{Im} z$

$\nabla$

$\Delta$

$|D|^{\sigma}$

$D_{t}$

$\mathcal{F}_{x \rightarrow \xi}$

$\mathcal{F}_{\xi \rightarrow x}^{-1}$

$f \lesssim g$

$f \gtrsim g$

$f \approx g$

$f \simeq g$

$\operatorname{det} A$

$\operatorname{diag} A$

$\operatorname{supp} u$

$B_{1} \hookrightarrow B_{2}$

$\partial_{x}^{\alpha}$

$f *_{(x)} g$

$f \sim g$

$f(t)=O(g(t))$

$f(t)=o(g(t))$

$\delta_{i j}$ denotes the absolute value or the norm of a vector;

which stands for $\langle x\rangle=\sqrt{1+|x|^{2}}$;

denotes for a matrix the matrix of the absolute values of its entries;

the ceiling function, i.e., $\lceil x\rceil=\min \{m \in \mathbb{Z}: x \leq m\}$;

which stands for $[x]_{+}=\max \{x, 0\}, x \in \mathbb{R}$;

which stands the commutator for two matrices, i.e., $[A, B]=A B-B A$;

real part of $z \in \mathbb{C}$;

imaginary part of $z \in \mathbb{C}$;

spatial gradient, i.e, $\nabla=\nabla_{x}=\left(\partial_{x_{1}}, \cdots, \partial_{x_{n}}\right)$;

Laplace operator in $\mathbb{R}^{n}$, i.e., $\Delta=\Delta_{x}=\sum_{j=1}^{n} \partial_{x_{j}}^{2}$;

pseudo-differential operator with symbol $|\xi|^{\sigma}$;

denotes $D_{t}=\frac{1}{i} \partial_{t}$;

Fourier transformation $F_{x \rightarrow \xi}(u)(t, \xi)=(2 \pi)^{-\frac{n}{2}} \int_{\mathbb{R}^{n}} e^{-i x \cdot \xi} u(t, x) d x$;

inverse Fourier transformation $F_{\xi \rightarrow x}^{-1}(u)(t, \xi)=(2 \pi)^{-\frac{n}{2}} \int_{\mathbb{R}^{n}} e^{i x \cdot \xi} u(t, \xi) d \xi$;

if there exists a constant $C>0$ such that $f \leq C g$;

if there exists a constant $C>0$ such that $f \geq C g$;

if $f \lesssim g$ and $f \gtrsim g$;

if there exists a constant $C>0$ such that $f=C g$;

determinant of the matrix $A$;

diagonal matrix with entries $a_{j j} \in \mathbb{R}, j=1, \cdots, n$ on the diagonal;

support of the function $u$;

continuous embedding of $B_{1}$ in $B_{2}$;

denotes the partial derivatives $\partial_{x}^{\alpha}:=\partial_{x_{1}}^{\alpha_{1}} \cdots \partial_{x_{n}}^{\alpha_{n}}$ with a multi-index $\alpha=\left(\alpha_{1}, \cdots, \alpha_{n}\right) \in \mathbb{R}^{n}$, where $|\alpha|:=\alpha_{1}+\cdots \alpha_{n} ;$

convolution of the functions $f$ and $g$ with respect to spatial variables;

if $\lim _{t \rightarrow \infty} \frac{f(t)}{g(t)}=1$, i.e., $f$ and $g$ have the same asymptotic behavior;

if there exist constants $C>0$ and $t_{0}>0$ such that

$|f(t)| \leq C|g(t)|$ for all $t \geq t_{0}$;

if for every positive constant $\epsilon$ there exists a constant $t_{0}>0$ such that

$|f(t)| \leq \epsilon|g(t)|$ for all $t \geq t_{0}$;

denotes $\delta_{i j}=1$ for $i=j, \delta_{i j}=0$ for $i \neq j$ for $i, j \in \mathbb{N}$; 
$\Lambda(t)$

$\rho(t)$

$\delta(t)$

$\langle\xi\rangle_{\lambda(t), \omega(t)}$

$\gamma(t)$

$\sigma(t)$

$\eta(t)$

$B_{\lambda}(s, t)$

$\Pi_{\text {hyp }}$

$\Pi_{\text {ell }}$

$Z_{\text {hyp }}$

$Z_{\text {osc }}$

$Z_{\text {red }}$

$Z_{\text {ell }}$

$Z_{\text {diss }}$

$h_{1}(t, \xi)$

$h_{2}(t, \xi)$

$U(t, \xi)$

$V(t, \xi)$

$E(t, s, \xi)$

$E_{V}(t, s, \xi)$

$E_{\text {hyp }}(t, s, \xi)$

$E_{\mathrm{osc}}(t, s, \xi)$

$E_{\text {red }}(t, s, \xi)$

$E_{\text {ell }}(t, s, \xi)$

$E_{\text {diss }}(t, s, \xi)$

$t_{\text {diss }}$

$t_{\text {ell }}$

$t_{\text {red }}$

$t_{\mathrm{osc}}$
$\Lambda(t)=1+\int_{0}^{t} \lambda(\tau) d \tau ;$

$\rho(t)=\mu(t) \frac{\lambda(t)}{\Lambda(t)} ;$

$\delta(t)=\exp \left(\frac{1}{2} \int_{0}^{t} \rho(\tau) \omega(\tau) d \tau\right) ;$

$\langle\xi\rangle_{\lambda(t), \omega(t)}=\sqrt{\left.\left|\lambda^{2}(t) \omega^{2}(t)\right| \xi\right|^{2}-\frac{\rho^{2}(t) \omega^{2}(t)}{4} \mid} ;$

$\gamma(t)=\frac{\lambda(t)}{F(\Lambda(t))}$;

$\sigma(t)=\exp \left(\frac{1}{2} \int_{0}^{t} \rho(\tau) d \tau\right) ;$

$\eta(t)=\frac{\mu(t)}{2 \Lambda(t)}, \quad \eta_{\infty}=\lim _{t \rightarrow \infty} \eta(t) ;$

$B_{\lambda}(s, t)=\int_{s}^{t} \frac{\lambda^{2}(\tau)}{\rho(\tau)} d \tau$

hyperbolic region in the extended phase space;

elliptic region in the extended phase space;

hyperbolic zone in the extended phase space;

oscillation subzone in the extended phase space;

reduced zone in the extended phase space;

elliptic zone in the extended phase space;

dissipative zone in the extended phase space;

$h_{1}(t, \xi)=\chi(|\xi| F(\Lambda(t))) \frac{\lambda(t)}{F(\Lambda(t))}+(1-\chi(|\xi| F(\Lambda(t)))) \lambda(t)|\xi| ;$

$h_{2}(t, \xi)=\chi\left(\frac{\langle\xi\rangle_{\lambda(t), \omega(t)}}{\varepsilon \frac{\rho(t) \omega(t)}{2}}\right) \varepsilon \frac{\rho(t) \omega(t)}{2}+\left(1-\chi\left(\frac{\langle\xi\rangle_{\lambda(t), \omega(t)}}{\varepsilon \frac{\rho(t) \omega(t)}{2}}\right)\right)\langle\xi\rangle_{\lambda(t), \omega(t)} ;$

micro-energy $U(t, \xi)=\left(h_{1}(t, \xi) \hat{u}(t, \xi), D_{t} \hat{u}(t, \xi)\right)^{\mathrm{T}}$ satisfies $D_{t} U=A(t, \xi) U$;

micro-energy $V(t, \xi)=\left(h_{2}(t, \xi) v(t, \xi), D_{t} v(t, \xi)\right)^{\mathrm{T}}$ satisfies $D_{t} V=A_{V}(t, \xi) V$;

fundamental solution to $D_{t} U=A(t, \xi) U$, i.e., the matrix-valued

solution to $D_{t} E=A(t, \xi) E, E(s, s, \xi)=I$;

fundamental solution to $D_{t} V=A_{V}(t, \xi) V$, i.e., the matrix-valued

solution to $D_{t} E_{V}=A_{V}(t, \xi) E_{V}, E_{V}(s, s, \xi)=I$ in Section 2.4;

fundamental solutions in the hyperbolic zone;

fundamental solutions in the oscillation zone;

fundamental solutions in the reduced zone;

fundamental solutions in the elliptic zone;

fundamental solutions in the dissipative zone;

separating line between the dissipative zone and elliptic zone

which solves $F\left(\Lambda\left(t_{\text {diss }}\right)\right)|\xi|=d_{0}$;

separating line between the elliptic zone and reduced zone

which solves $\langle\xi\rangle_{\lambda\left(t_{\text {ell }}\right), \omega\left(t_{\text {ell }}\right)}=\varepsilon \frac{\rho\left(t_{\text {ell }}\right) \omega\left(t_{\text {ell }}\right)}{2}$;

separating line between the reduced zone and oscillation subzone zone

which solves $\langle\xi\rangle_{\lambda\left(t_{\mathrm{ell}}\right), \omega\left(t_{\mathrm{ell}}\right)}=N \frac{\rho\left(t_{\mathrm{ell}}\right) \omega\left(t_{\mathrm{ell}}\right)}{2}$ and $\Lambda\left(t_{\mathrm{red}}\right)|\xi|=N$;

separating line between the oscillation subzone and hyperbolic zone

which solves $\langle\xi\rangle_{\lambda\left(t_{\mathrm{osc}}\right), \omega\left(t_{\mathrm{osc}}\right)}=N \frac{\rho\left(t_{\mathrm{osc}}\right) \omega\left(t_{\mathrm{osc}}\right)}{2}$ and $\Theta\left(t_{\mathrm{osc}}\right)|\xi|=N$; 


\begin{tabular}{|c|c|}
\hline$E^{Y}(t, s, \xi)$ & $\begin{array}{l}\text { fundamental solution to } D_{t} Y=A^{Y}(t, \xi) Y \text {, i.e., the matrix- } \\
\text { valued solution to } D_{t} E^{Y}=A^{Y}(t, \xi) E^{Y} \cdot E^{Y}(s, s, \xi)=I \text { : }\end{array}$ \\
\hline $\mathcal{E}^{1}(t, s, \xi)$ & $\mathcal{E}^{1}(t, s, \xi)=E(t, s, \xi)$ \\
\hline $\mathcal{E}^{2}(t, s, \xi)$ & $\mathcal{E}^{2}(t, s, \xi)=E^{Y}(t, s, \xi)$ \\
\hline$\hat{K}_{0}(t, 0, \xi)$ & solutions to the corresponding linear Cauchy problems with \\
\hline$\hat{K}_{1}(t, s, \xi)$ & $\begin{array}{l}\text { initial data } \hat{u}(0, \xi)=1 \text { and } \hat{u}_{t}(0, \xi)=0 ; \\
\text { solutions to the corresponding linear Cauchy problems with }\end{array}$ \\
\hline$\hat{k}_{0}(t, 0, \xi)$ & $\begin{array}{l}\text { initial data } \hat{u}(s, \xi)=0 \text { and } \hat{u}_{t}(s, \xi)=1 \text {; } \\
\text { solutions to the corresponding linear Cauchy problems with } \\
\text { initial data } \hat{y}(0, \xi)=1 \text { and } \hat{y}_{t}(0, \xi)=0 ;\end{array}$ \\
\hline$\hat{k}_{1}(t, s, \xi)$ & solutions to the corresponding linear Cauchy problems with \\
\hline$t_{|\xi|}$ & $\begin{array}{l}\text { initial data } \hat{y}(s, \xi)=0 \text { and } \hat{y}_{t}(s, \xi)=1 \\
\text { separating curve between } \Pi_{\text {ell }}\left(d_{0}, \varepsilon\right) \text { and } \Pi_{\text {hyp }}(N, \varepsilon)\end{array}$ \\
\hline$\nu(\delta, t)$ & $\begin{array}{l}\text { which solves } \eta^{2}\left(t_{|\xi|}\right)-|\xi|^{2}=\varepsilon^{2} \eta^{2}\left(t_{|\xi|}\right) \text { in Section } 2.5 \\
\nu(\delta, t)=\frac{\lambda(t)}{\Lambda(t)} \frac{\Lambda(\delta t)}{\lambda(\delta t)}, \quad \nu_{\infty}=\lim \sup _{t \rightarrow \infty} \nu(\delta, t)\end{array}$ \\
\hline$\vartheta(\delta, t)$ & $\vartheta(\delta, t)=\frac{F(\Lambda(t))}{\lambda(t) \Xi^{2}(t)} \frac{\Lambda(\delta t)}{\lambda(\delta t)}, \quad \vartheta_{\infty}=\lim \sup _{t \rightarrow \infty} \vartheta(\delta, t)$ \\
\hline$\phi(t)$ & $\phi(t)=\frac{\Lambda^{2}(t)}{\mu(t)}$ \\
\hline$\alpha_{1}(p)$ & $\alpha_{1}(p)=\frac{n}{2 m} p-\frac{n}{2 m}$ \\
\hline$\alpha_{2}(p)$ & $\alpha_{2}(p)=\frac{n}{2 m} p-\frac{n}{4}$ \\
\hline$p_{\mathrm{Fuj}}(n)=1+\frac{2}{n}$ & Fujita exponent; \\
\hline$p_{\mathrm{Fuj}, m}(n)=1+\frac{2 m}{n}$ & modified Fujita exponent; \\
\hline$p_{\mathrm{GN}}(n)=\frac{n}{n-2}$ & Gagliardo-Nirenberg exponent; \\
\hline$p_{\mathrm{GN}, \sigma}(n)=\frac{n}{n-2 \sigma}$ & modified Gagliardo-Nirenberg exponent; \\
\hline$\theta_{s, \sigma}(q)=\frac{n}{\sigma}\left(\frac{1}{2}-\frac{1}{q}+\frac{s}{n}\right)$ & exponent which appears in the fractional Gagliardo-Nirenberg \\
\hline$\theta_{\sigma}(q)=\frac{n}{\sigma}\left(\frac{1}{2}-\frac{1}{q}\right)$ & $\begin{array}{l}\text { inequality, } 0 \leq s<\sigma \\
\text { special case } s=0\end{array}$ \\
\hline
\end{tabular}

\section{Function spaces}

$\mathcal{C}^{k}\left(\mathbb{R}^{n}\right)$

$\mathcal{C}^{\infty}\left(\mathbb{R}^{n}\right)$

$\mathcal{C}_{0}^{\infty}\left(\mathbb{R}^{n}\right)$

$L^{p}\left(\mathbb{R}^{n}\right)$

$\|\cdot\|_{L^{p}}$

$L^{\infty}\left(\mathbb{R}^{n}\right)$

$\|\cdot\|_{L^{\infty}}$

$W_{p}^{m}\left(\mathbb{R}^{n}\right)$

$H_{p}^{s}\left(\mathbb{R}^{n}\right)$

$H^{s}\left(\mathbb{R}^{n}\right)=H_{2}^{s}\left(\mathbb{R}^{n}\right)$

$\dot{H}_{p}^{s}\left(\mathbb{R}^{n}\right)$

$\dot{H}^{s}\left(\mathbb{R}^{n}\right)=\dot{H}_{2}^{s}\left(\mathbb{R}^{n}\right)$ space of $k$-times continuously differentiable functions; space of infinitely continuously differentiable functions; space of functions belonging to $\mathcal{C}^{\infty}\left(\mathbb{R}^{n}\right)$ with compact support; $L^{p}\left(\mathbb{R}^{n}\right)=\left\{u:\left.\mathbb{R}^{n} \rightarrow \mathbb{R}\left|\int_{\mathbb{R}^{n}}\right| u(x)\right|^{p} d x<\infty\right\} ;$ denote $L^{p}$-norms, where $\|u\|_{L^{p}}=\left(\int_{\mathbb{R}^{n}}|u(x)|^{p} d x\right)^{1 / p}$, where the functions $u$ are Lebesgue measurable and $1 \leq p<\infty$; $L^{\infty}\left(\mathbb{R}^{n}\right)=\left\{u: \mathbb{R}^{n} \rightarrow \mathbb{R}\left|\operatorname{ess}_{\sup } \in \mathbb{R}^{n}\right| u(x) \mid<\infty\right\} ;$ denotes the $L^{\infty}$-norm, where $\|u\|_{L^{\infty}}=\operatorname{ess}_{\sup _{x \in \mathbb{R}^{n}}}|u(x)|$; Sobolev spaces based on $L^{p}\left(\mathbb{R}^{n}\right), 1 \leq p \leq \infty, m \in \mathbb{N}$; Sobolev spaces of fractional order or Bessel potential space; Sobolev spaces of fractional order based on $L^{2}\left(\mathbb{R}^{n}\right)$; homogeneous Sobolev spaces of fractional order; homogeneous Sobolev spaces of fractional order based on $L^{2}\left(\mathbb{R}^{n}\right)$. 



\section{Bibliography}

[1] T. Bao Ngoc Bui. Semi-linear waves with time-dependent speed and dissipation. PhD thesis, TU Bergakademie Freiberg, 2013, 154 pp.

[2] T. Bao Ngoc Bui, M. Reissig. The interplay between time-dependent speed of propagation and dissipation in wave models. Fourier Analysis, 9-45, Trends Math., Birkhäuser/Springer, 2014.

[3] T. Bao Ngoc Bui, M. Reissig. Global existence of small data solutions for wave models with sub-exponential propagation speed. Nonlinear Anal. 129 (2015), 173-188.

[4] T. Bao Ngoc Bui, M. Reissig. Global existence of small data solutions for wave models with super-exponential propagation speed. Nonlinear Anal. 121 (2015), 82-100.

[5] P. Brenner. On $L^{p}-L^{q}$ estimates for the wave-equation. Math. Z. 145 (1975), no. 3, 251-254.

[6] F. Christ, M. Weinstein. Dispersion of small-amplitude solutions of the generalized Korteweg-de Vries equation. J. Funct. Anal. 100 (1991), no. 1, 87-109.

[7] F.Colombini. Energy estimates at infinity for hyperbolic equations with oscillating coefficients. J. Differential Equations 231 (2006), no. 2, 598-610.

[8] M. D'Abbicco, S. Lucente. A modified test function method for damped wave equations. Adv. Nonlinear Stud. 13 (2013), no. 4, 867-892.

[9] M. D'Abbicco, M. R. Ebert, S. Lucente. Self-similar asymptotic profile of the solution to a nonlinear evolution equation with critical dissipation. Math. Methods Appl. Sci. 40 (2017), no. 18, 6480-6494.

[10] M. D'Abbicco, S. Lucente, M.Reissig. Semi-linear wave equations with effective damping. Chin. Ann. Math. Ser. B 34 (2013), no. 3, 345-380.

[11] M. R. Ebert, M. Reissig. Methods for partial differential equations. Qualitative properties of solutions, phase space analysis, semilinear models. Birkhäuser/Springer, 2018.

[12] A. Friedman. Partial Differential Equations. Krieger, New York, 1976.

[13] L. Grafakos. Classical and modern Fourier analysis. Second edition. Springer, New York, 2009.

[14] H. Hajaiej, L. Molinet, T. Ozawa, B. Wang. Necessary and sufficient conditions for the fractional Gagliardo-Nirenberg inequalities and applications to Navier-Stokes and generalized boson equations. Harmonic analysis and nonlinear partial differential equations, 159175, RIMS Kôkyûroku Bessatsu, B26, Res. Inst. Math. Sci. (RIMS), Kyoto, 2011.

[15] M. Hardy. Combinatorics of partial derivatives. Electron. J. Combin. 13 (2006), no. 1, Research Paper 1, 13 pp.

[16] F. Hirosawa. On the asymptotic behavior of the energy for the wave equation with time depending coefficients. Math. Ann. 339 (2007), no. 4, 819-838.

[17] F. Hirosawa. Energy estimates for wave equations with time dependent propagation speeds in the Gevrey class. J. Differential Equations 248 (2010), no. 12, 2972-2993. 
[18] F. Hirosawa, J. Wirth. $C^{m}$-theory of damped wave equations with stabilisation. J. Math. Anal. Appl. 343 (2008), no. 2, 1022-1035.

[19] F. Hirosawa, J. Wirth. Generalised energy conservation law for wave equations with variable propagation speed. J. Math. Anal. Appl. 358 (2009), no. 1, 56-74.

[20] L. Hörmander. Estimates for translation invariant operators in $l^{p}$ spaces. Acta Math. 104 (1960), 93140.

[21] R. Ikehata, K. Tanizawa. Global existence of solutions for semilinear damped wave equations in $\mathbf{R}^{N}$ with noncompactly supported initial data. Nonlinear Anal. 61 (2005), no. $7,1189-1208$.

[22] J. Lin, K. Nishihara, J. Zhai. Critical exponent for the semilinear wave equation with time-dependent damping. Discrete Contin. Dyn. Syst. 32 (2012), no. 12, 4307-4320.

[23] A. Matsumura. On the asymptotic behavior of solutions of semi-linear wave equations. Publ. Res. Inst. Math. Sci. 12 (1976/77), no. 1, 169-189.

[24] A. Matsumura. Energy decay of solutions of dissipative wave equations. Proc. Japan Acad. Ser. A Math. Sci. 53 (1977), no. 7, 232-236.

[25] R. Mishkov. Generalization fo the Formula of Fa di Bruno for a composite function with a vector argument. Int. J. Math. Math. Sci. 24 (2000), no. 7, 481-491.

[26] B. G. Pachpatte. Inequalities for differential and integral equations. Mathematics in Science and Engineering, 197. Academic Press, Inc., San Diego, CA, 1998.

[27] A. Palmieri. Global in time existence and blow-up results for a semilinear wave equation with scale-invariant damping and mass. PhD Thesis, TU Bergakademie Freiberg, 2018, 279 pp.

[28] A. Palmieri, M. Reissig. Semi-linear wave models with power non-linearity and scaleinvariant time-dependent mass and dissipation, II. Math. Nachr. 291 (2018), no. 11-12, 1859-1892.

[29] H. Pecher. $L^{p}$-Abschätzungen und klassische Lösungen für nichtlineare Wellengleichungen. I. Math. Z. 150 (1976), no.2, 159-183.

[30] G. Ponce. Global existence of small solutions to a class of nonlinear evolution equations. Nonlinear Anal. 9 (1985), no. 5, 399-418.

[31] R. Racke. Lectures on nonlinear evolution equations. Initial value problems. Second edition. Birkhäuser/Springer, 2015.

[32] M. Reissig. $L_{p}-L_{q}$ decay estimates for wave equations with time-dependent coefficients. J. Nonlinear Math. Phys. 11 (2004), no. 4, 534-548.

[33] M. Reissig, J. Smith. $L^{p}-L^{q}$ estimate for wave equation with bounded time-dependent coefficient. Hokkaido Math. J. 34 (2005), no. 3, 541-586.

[34] M. Reissig, K. Yagdjian. One application of Floquet's theory to $L_{p}-L_{q}$ estimates for hyperbolic equations with very fast oscillations. Math. Methods Appl. Sci. 22 (1999), no. $11,937-951$.

[35] M. Reissig, K. Yagdjian. About the influence of oscillations on Strichartz-type decay estimates. Partial differential operators (Torino, 2000). Rem. Sem. Mat. Univ. Politec. Torino 58 (2000), no. 3, 375-388.

[36] M. Reissig, K. Yagdjian. $L_{p}-L_{q}$ decay estimates for the solutions of strictly hyperbolic equations of second order with increasing in time coefficients. Math. Nachr. 214 (2000), 71-104. 
[37] M. Reissig, K. Yagdjian. $L_{p}-L_{q}$ decay estimates for hyperbolic equations with oscillations in coefficients. Chinese Ann. Math. Ser. B 21 (2000), no. 2, 153-164.

[38] T. Runst, W. Sickel. Sobolev spaces of fractional order, Nemytskij operators, and nonlinear partial differential equations. De Gruyter Series in Nonlinear Analysis and Applications, 3. Walter de Gruyter \& Co., Berlin, 1996.

[39] K. Spindler. A short proof of the formula of Faà di Bruno. Elem. Math. 60 (2005), no. $1,33-35$.

[40] G. Staffilani. The initial value problem for some dispersive differential equations. PhD Thesis, University of Chicago, 1995, 88 pp.

[41] R. S. Strichartz. A priori estimates for the wave equation and some applications. $J$. Functional Analysis 5 (1970), 218-235.

[42] R. S. Strichartz. Convolutions with kernels having singularities of a sphere. Trans. Amer. Math. Soc. 148 (1970), 461-471.

[43] H. Takamura, K. Wakasa. Almost global solutions of semilinear wave equations with the critical exponent in high dimensions. Nonlinear Anal. 109 (2014), 187-229.

[44] G. Todorova, B. Yordanov. Critical exponent for a nonlinear wave equation with damping. J. Differential Equations 174 (2001), no. 2, 464-489.

[45] W. von Wahl. $L^{p}$-decay rates for homogeneous wave-equations. Math. Z. 120 (1971), 93-106.

[46] J. Wirth. Asymptotic properties of solutions to wave equations with time-dependent dissipation. PhD thesis, TU Bergakademie Freiberg, 2004, 146 pp.

[47] J. Wirth. Wave equations with time-dependent dissipation I. Non-Effective dissipation. J. Differential Equations 222 (2006), no. 2, 487-514.

[48] J. Wirth. Wave equations with time-dependent dissipation II. Effective dissipation. $J$. Differential Equations 232 (2007) no. 1, 74-103.

[49] K. Yagdjian. The Cauchy problem for hyperbolic operators. Multiple characteristics. Micro-local approach. Mathematical Topics, 12. Akademie Verlag, Berlin, 1997.

[50] Q. S. Zhang. A blow-up result for a nonlinear wave equation with damping: the critical case. C. R. Acad. Sci. Paris Sér. I Math. 333 (2001), no. 2, 109-114. 



\section{Acknowledgments}

I would like to express my most sincere gratitude to the supervisor of this thesis Prof. Michael Reissig for his continuous support throughout my $\mathrm{PhD}$ studies, for his patience, motivation, and immense knowledge. I am truly pleased to have been his student. Without his valuable suggestions and countless mathematical discussions during the last three years, this thesis would have never been possible. His guidance has been a precious help in all the time of research and writing of this thesis.

I am very grateful to Prof. Fumihiko Hirosawa (Yamaguchi University), for the readiness as referee in reading the thesis, for his comments, suggestions and several fruitful mathematical discussions.

I express my gratitude to Prof. Rainer Picard (Technical University Dresden) as well, for his readiness to act as a referee, for his comments and suggestions.

A special thank goes to Prof. Necat Polat, who gave me the opportunity to work on some exciting topics of PDEs and also for his support in many important decisions during my studies at Dicle University.

Furthermore, I would like to thank my former and present colleagues and friends Maximilian Reich, Abdelhamid Mohammed-Djaouti, Alessandro Palmieri, Daniel Lorenz, Wenhui Chen and Tuan Anh Dao for their assistance, suggestions, constructive comments and mathematical discussions.

My PhD studies at Technical University Bergakademie Freiberg were financially supported by a scholarship (Landesgraduiertenstipendium) of the Ministry of Sciences and Arts Saxony, Germany. I am very grateful to the staff of Freiberg University, in particular, to the Institute of Applied Analysis for the excellent working conditions.

Finally, I want to express my sincere thanks to my parents and family members for their permanent support and encouragement during my studies, everything that I have achieved I owe them. 